QA: QA

TDR-NBS-HS-000017 REV00

August 2006

\title{
Chlorine-36 Validation Study at Yucca Mountain, Nevada
}

\author{
Prepared for: \\ U.S. Department of Energy \\ Office of Civilian Radioactive Waste Management \\ Office of Repository Development \\ P.O. Box 364629 \\ North Las Vegas, Nevada 89036 \\ Prepared by: \\ U.S. Geological Survey \\ Yucca Mountain Project Branch \\ P.O. Box 25046, MS 963 \\ Denver Federal Center \\ Denver, Colorado 80225 \\ Under Interagency Agreement \\ DE-AI28-02RW12167
}


INTENTIONALLY LEFT BLANK 
3. Technical Report Title

Chlorine-36 Validation Study at Yucca Mountain, Nevada

4. DI (including Rev. No.)

TDR-NBS-HS-000017 REV00

\begin{tabular}{|l|l|l|}
\hline \multirow{2}{*}{ 5. Originator(s) } & \multicolumn{1}{|c|}{ Printed Name } \\
\hline $\begin{array}{l}\text { 6. Checker } \\
\text { 7. QER }\end{array}$ & James B. Paces & Robert C. Roback \\
\hline $\begin{array}{l}\text { 8. Lead or Supervisor } \\
\text { 9. Responsible Manager or } \\
\text { Project Engineer }\end{array}$ & Schön S. Levy & Gerard (Jerry) Heaney \\
\hline $\begin{array}{l}\text { 10. Remarks } \\
\text { Deuglas J. Weaver }\end{array}$ &
\end{tabular}

Change History

\begin{tabular}{|l|l|}
\hline 11. Revision No. & 12. Description of Change \\
\hline REV OO & INITIAL ISSUE \\
\hline & \\
\hline & \\
\hline
\end{tabular}


INTENTIONALLY LEFT BLANK 


\section{EXECUTIVE SUMMARY}

Chlorine-36 $\left({ }^{36} \mathrm{Cl}\right)$ data were collected by Los Alamos National Laboratory (LANL) during the late 1990s using leachates of rock samples collected from the walls of the Exploratory Studies Facility (ESF) in the unsaturated zone (UZ) at Yucca Mountain, Nevada, to test whether the Paintbrush Tuff nonwelded hydrogeologic unit (PTn) represents an effective barrier to vertical flow, whether water in the matrix of the Topopah Spring welded hydrogeologic unit (TSw) is essentially stagnant, and whether fast pathways transporting water to the proposed repository horizon occur at discrete locations associated with fault structures. Thirteen percent of the ${ }^{36} \mathrm{Cl}$ measurements ( 37 of 288 samples) showed elevated values for ratios of ${ }^{36} \mathrm{Cl}$ to total chloride $\left({ }^{36} \mathrm{Cl} / \mathrm{Cl}\right)$ at the level of the proposed repository, indicating that small amounts of water carrying bomb-pulse ${ }^{36} \mathrm{Cl}$ (i.e., ${ }^{36} \mathrm{Cl} / \mathrm{Cl}$ ratios greater than $1250 \times 10^{-15}$ resulting from ${ }^{36} \mathrm{Cl}$ produced by atmospheric testing of nuclear devices during the 1950s and early 1960s) had percolated through welded and nonwelded tuffs to depths of 200 to 300 meters $(\mathrm{m})$ beneath the land surface over the past 50 years. Because of the implications of short travel times to the performance of the proposed repository, the U.S. Department of Energy (DOE), Office of Civilian Radioactive Waste Management (OCRWM), Office of Repository Development (ORD) decided to verify the ${ }^{36} \mathrm{Cl} / \mathrm{Cl}$ data with an independent validation study.

DOE asked the U.S. Geological Survey (USGS) to design and implement a validation study that would include ${ }^{36} \mathrm{Cl}$ and tritium $\left({ }^{3} \mathrm{H}\right)$ analyses. Study participants included the USGS, Lawrence Livermore National Laboratory (LLNL), Atomic Energy of Canada Limited (AECL), and LANL. Core samples were taken from 50 new boreholes drilled across two zones in the ESF where a substantial number of samples with elevated ${ }^{36} \mathrm{Cl} / \mathrm{Cl}$ ratios had been identified previously. Also, core intervals from the Sample Management Facility (SMF) were acquired for water extraction and ${ }^{3} \mathrm{H}$ analyses.

The ${ }^{36} \mathrm{Cl}$ validation study was conducted in three phases. Results from Phase I of the work conducted at LLNL indicated that active leaching pulverized the rock samples and extracted too much rock chloride relative to meteoric chloride $\left({ }^{36} \mathrm{Cl} / \mathrm{Cl}\right.$ ratios range from $47 \times 10^{-15}$ to $248 \times 10^{-15}$; all values but one are less than $156 \times 10^{-15}$ ). Results from Phase I of the work conducted at LANL on validation core samples from the Sundance fault zone yielded ${ }^{36} \mathrm{Cl} / \mathrm{Cl}$ values consistent with analyses from previous LANL studies. Following a detailed series of leaching experiments in Phase II of the validation study, a 1-hour passive leaching protocol was established for processing samples in Phase III of the study. The passive leaching process extracted less rock chloride relative to meteoric chloride.

USGS-LLNL ${ }^{36} \mathrm{Cl} / \mathrm{Cl}$ values for leachates of 34 samples of core from validation study boreholes across an area that includes the Sundance fault zone range from $137 \times 10^{-15}$ to $615 \times 10^{-15}$, with a mean value of $326 \times 10^{-15}$. These are lower than bomb-pulse values previously reported for feature-based tunnel-wall samples in the same area. ${ }^{36} \mathrm{Cl} / \mathrm{Cl}$ ratios for passive leachates of validation study core samples prepared at the USGS and processed separately at LLNL and LANL agree within analytical error. The reproducibility of results also was tested at USGS-LLNL and LANL using available core from Niche \#1, a short drift that was driven from the ESF to access the Sundance fault by drilling. LLNL analyses of six Niche \#1 core samples prepared at the USGS are statistically indistinguishable from validation study borehole data. $\left({ }^{36} \mathrm{Cl} / \mathrm{Cl}\right.$ ratios range from $226 \times 10^{-15}$ to $\left.717 \times 10^{-15}\right)$. LANL ${ }^{36} \mathrm{Cl} / \mathrm{Cl}$ validation results for seven 
Niche \#1 core samples yielded bomb-pulse values that are comparable to previous LANL ${ }^{36} \mathrm{Cl}$ data $\left(1,016 \times 10^{-15}\right.$ to $\left.8,558 \times 10^{-15}\right)$. One LANL validation study analysis and several previous analyses of samples from the Enhanced Characterization of the Repository Block (ECRB) Cross Drift also show large ${ }^{36} \mathrm{Cl} / \mathrm{Cl}$ values.

Tritium concentrations in pore water extracted from validation study core samples across the Drill Hole Wash fault zone and the Sundance fault zone range from less than 0.1 to 2.6 tritium units (TU). Tritium concentrations in pore water extracted from samples from areas of known faulting in the ESF indicate the presence of modern water (i.e., water that entered the Yucca Mountain UZ after 1952, thus indicating fast pathways). Tritium concentrations in pore water extracted from core samples from the ECRB Cross Drift range from less than 0.1 to $10.3 \mathrm{TU}$. The USGS and LANL established different thresholds for interpreting ${ }^{3} \mathrm{H}$ values as indicators of modern water (2.0 TU and $1.4 \mathrm{TU}$, respectively). The lower LANL threshold allows for the presence of modern water in a larger number of locations in the ESF and ECRB Cross Drift.

The validation study work conducted by USGS-LLNL did not confirm previously reported bomb-pulse ${ }^{36} \mathrm{Cl} / \mathrm{Cl}$ ratios in the Sundance fault zone, but new analyses at LANL of Niche \#1 core samples and ECRB Cross Drift tunnel-wall samples were consistent with results from previous studies. Consequently, a number of issues were identified that need to be addressed. Recommendations include a detailed evaluation of potential field contamination and sample handling and processing, including a rigorous evaluation of crushing blanks; additional ${ }^{36} \mathrm{Cl} / \mathrm{Cl}$ analyses of validation study core samples; confirmation of young water in high ${ }^{3} \mathrm{H}$ samples by analyzing the same core samples for ${ }^{36} \mathrm{Cl}$; and an independent validation study using new samples. 


\section{CONTENTS}

EXECUTIVE SUMMARY $\mathrm{v}$

ACRONYMS, ABBREVIATIONS, AND SYMBOLS ..................................................... xvii

STRATIGRAPHIC AND HYDROGEOLOGIC NAMES....................................................... xix

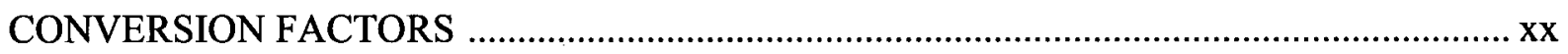

REPORTING OF UNCERTAINTIES AND PARAMETER VARIABILITIES ....................... $\mathrm{xx}$

NOTATION OF CHLORINE-36/CHLORIDE RATIOS IN TEXT, TABLES, AND

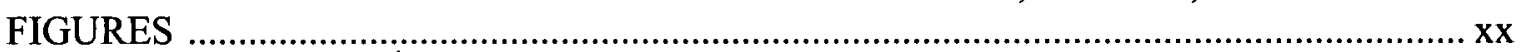

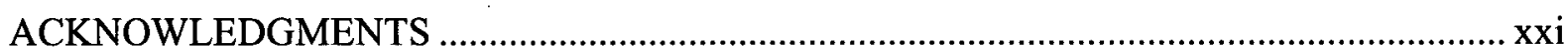

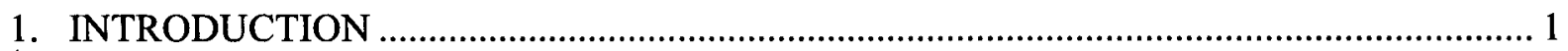

1.1 PURPOSE

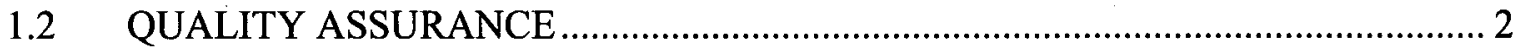

1.3 ORGANIZATION OF THE REPORT ………………………………………... 2

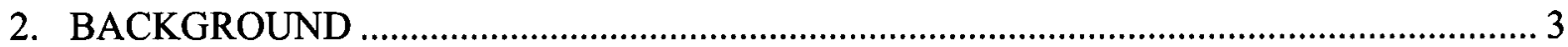

2.1 STUDIES OF CHLORINE-36 AND FRACTURE MINERALS IN THE EXPLORATORY STUDIES FACILITY........................................................... 4

2.1.1 Results from Previous Chlorine-36 Studies................................................... 4

2.1.2 Fracture Mineral Studies.........................................................................

2.2 PREVIOUS STUDIES OF OTHER BOMB-PULSE ISOTOPES ............................... 7

2.3 PEER REVIEW OF CHLORINE-36 STUDIES ………...................................... 8

3. DESIGN AND IMPLEMENTATION OF THE VALIDATION STUDY …………............. 11

3.1 DESIGN OF SAMPLING PROTOCOL …………………………………….... 11

3.2 DESCRIPTION AND ALLOCATION OF VALIDATION STUDY CORE ............. 13

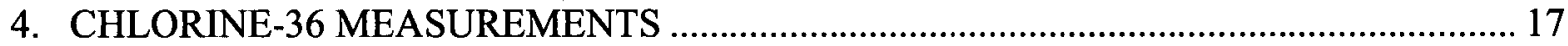

4.1 PHASE I: MEASUREMENTS MADE AT LLNL …………………………..... 17

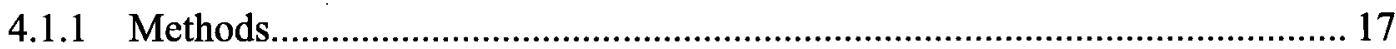

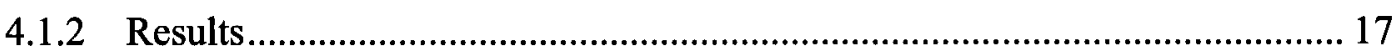

4.2 PHASE I: MEASUREMENTS MADE AT LANL ……………………………….... 18

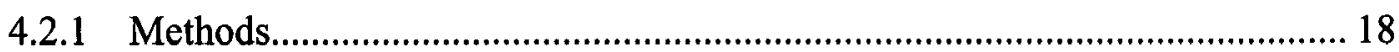

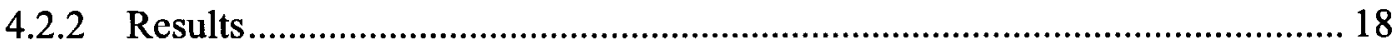

4.3 PHASE II: LEACHING EXPERIMENTS …………....................................... 19

4.3.1 Preparation of the Reference Sample......................................................... 19

4.3.2 Leaching Experiments Conducted at LANL............................................. 20

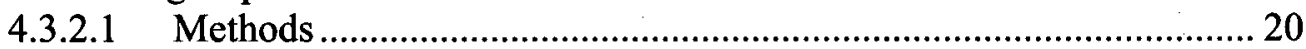

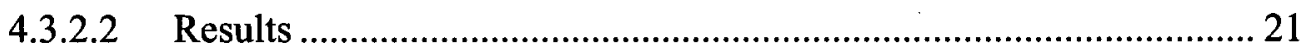

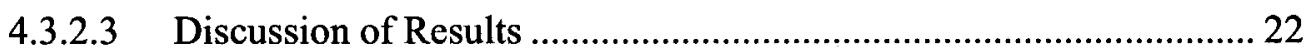

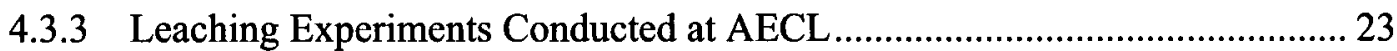

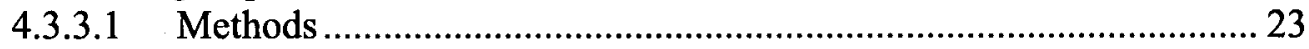

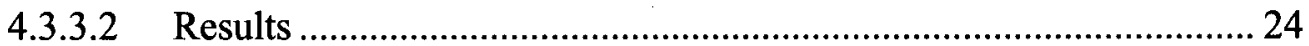

4.3.3.3 Discussion of Results .............................................................. 26 
4.3.4 Conclusions from the Phase II Leaching Experiments ............................... 27

4.4 PHASE III: MEASUREMENTS MADE AT USGS-LLNL …................................. 27

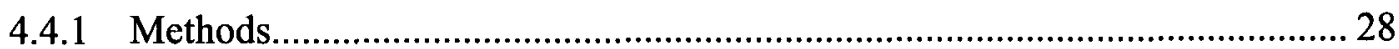

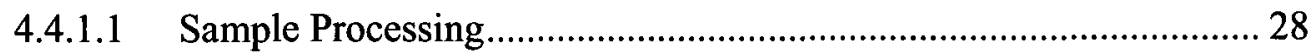

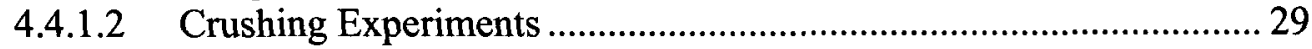

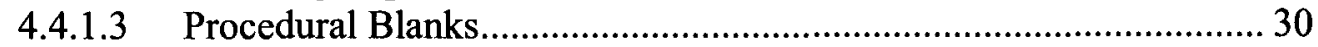

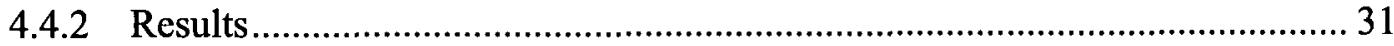

4.4.2.1 Anions in Leachates of Validation Study Core ............................... 31

4.4.2.2 Chlorine-36 in Leachates of Validation Study Core ........................ 32

4.4.2.3 Re-Analysis of Niche \#1 Core for Chlorine-36.............................. 33

4.5 PHASE III: MEASUREMENTS MADE AT LANL ............................................. 34

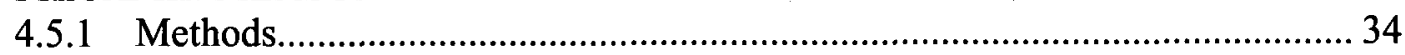

4.5.1.1 Sample Processing ..................................................................... 34

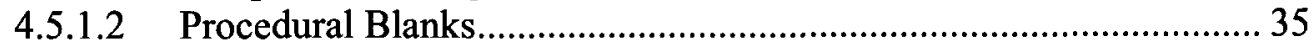

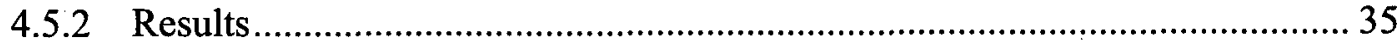

4.5.2.1 Chlorine-36 in Leachates of Validation Study Core ....................... 35

4.5.2.2 Chlorine-36 in ECRB Cross Drift Tunnel-Wall Samples ................. 36

4.5.2.3 Re-Analysis of Niche \#1 Core for Chlorine-36............................... 36

4.6 DISCUSSION OF THE CHLORINE-36 MEASUREMENTS ................................ 37

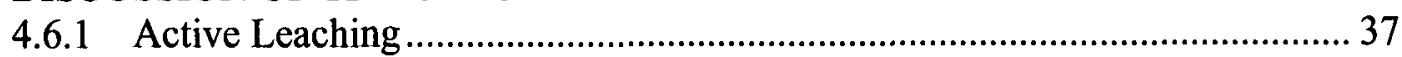

4.6.2 Chloride Sources and Leaching Experiments ........................................... 37

4.6.3 Procedural Blanks and Detection Limits for the Total Chloride and

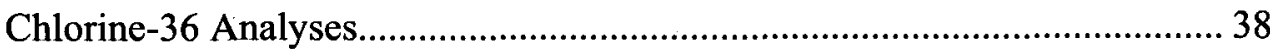

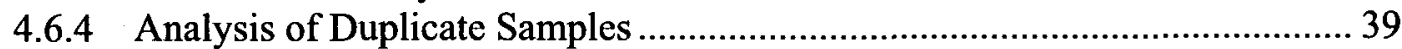

4.6.5 LANL Data from the ECRB Cross Drift ................................................... 40

4.6.6 Comparison of Validation Study Data with Previous Chlorine-36 Data........ 40

4.6.6.1 Sundance Fault Zone ...................................................................... 40

4.6.6.2 Southern Exploratory Studies Facility ......................................... 41

4.6.7 Comparison of USGS-LLNL Niche \#1 Data and LANL-LLNL

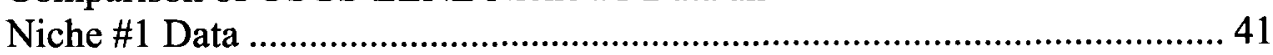

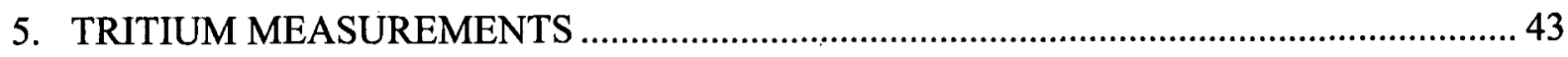

5.1 POTENTIAL SOURCES OF TRITIUM IN CORE SAMPLES FROM THE YUCCA MOUNTAIN UNSATURATED ZONE............................................ 43

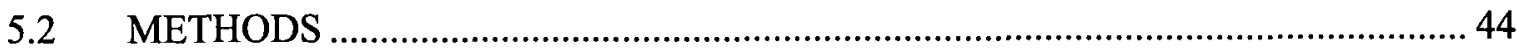

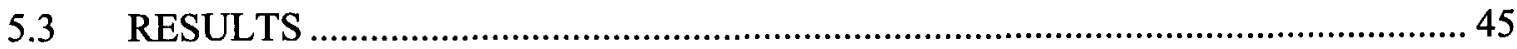

5.3.1 Tritium in Validation Study Core Samples............................................ 45

5.3.2 Tritium in Other Core Samples from the Exploratory Studies Facility .......... 45

5.3.3 Tritium in Core Samples from the ECRB Cross Drift................................... 46

5.4 THRESHOLD VALUES FOR DETECTING MODERN WATER ......................... 47

5.4.1 USGS Establishment of a Threshold for Identifying Modern Water ............. 47

5.4.2 LANL Establishment of a Threshold for Identifying Modern Water............. 48

5.5 INTERPRETATION OF THE TRITIUM MEASUREMENTS …......................... 51

5.5.1 USGS Interpretation of the Tritium Measurements..................................... 51

5.5.2 LANL Interpretation of the Tritium Measurements .................................. 51

6. SUMMARY OF RESULTS, CONCLUSIONS, REMAINING ISSUES, AND RECOMMENDATIONS 


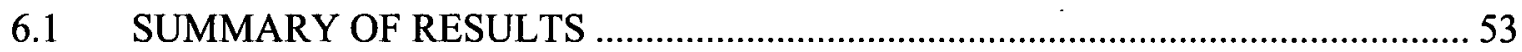

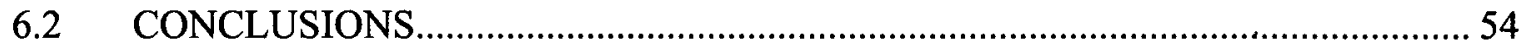

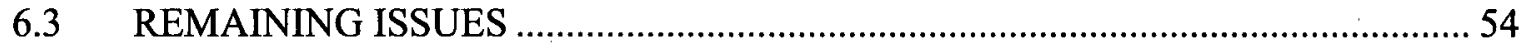

6.3.1 Absence of Elevated Chlorine-36/Chloride Ratios in USGS-LLNL

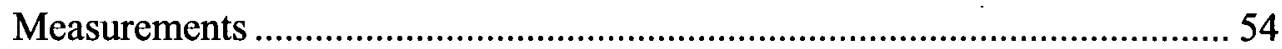

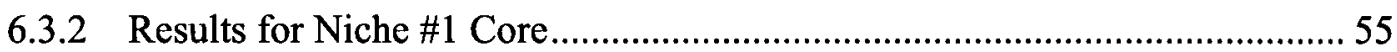

6.3.3 Spatial Distribution of Elevated Chlorine-36 Values and Tritium

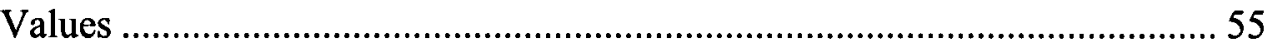

6.3.3.1 USGS Interpretation of the Spatial Distribution of Elevated

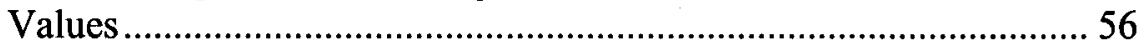

6.3.3.2 LANL Interpretation of the Spatial Distribution of Elevated

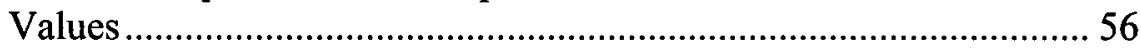

6.3.4 Potential Contamination from Field and Laboratory Environments................57

6.3.4.1 USGS Interpretation of the Potential for Contamination from Field and Laboratory Environments................................................ 57

6.3.4.2 LANL Interpretation of the Potential for Contamination from Field and Laboratory Environments.................................................5 58

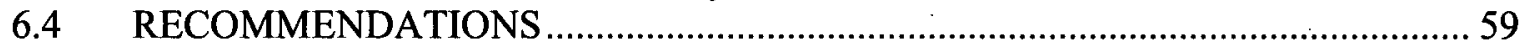

6.4.1 Evaluation of Field Contamination.................................................................. 60

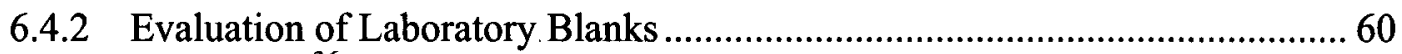

6.4.3 Additional ${ }^{36} \mathrm{Cl} / \mathrm{Cl}$ Analyses of Validation Study Core and ECRB

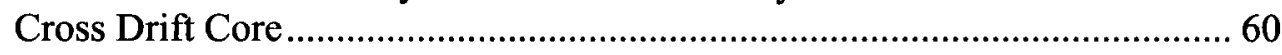

6.4.4 Independent Validation Study Using New Samples.............................................. 60

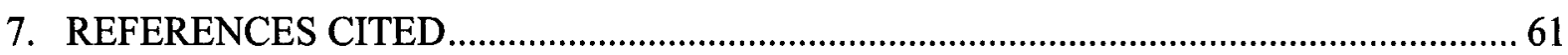

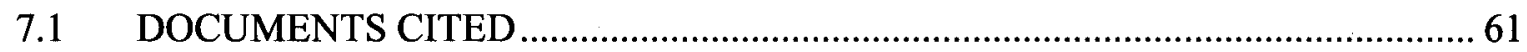

7.2 CODES, STANDARDS, REGULATIONS, AND PROCEDURES .............................6 68

7.3 SOURCE DATA, LISTED BY DATA TRACKING NUMBER ……………............6 68

APPENDIX A -CHLORIDE CONCENTRATIONS AND CHLORINE-36/CHLORIDE

RATIOS IN SALTS LEACHED FROM ESF ROCK SAMPLES AT LOS ALAMOS

NATIONAL LABORATORY AS OF SEPTEMBER 8, 1998

APPENDIX B -VIDEO-LOG OBSERVATIONS FROM VALIDATION STUDY

BOREHOLES 
INTENTIONALLY LEFT BLANK 


\section{FIGURES}

1-1. Generalized Map of Central Yucca Mountain (A) and Schematic Geologic Section along the ESF Showing the Sundance Fault Zone Validation Study Area (B)

2-1. Distribution of Chloride Concentrations (A) and ${ }^{36} \mathrm{Cl} / \mathrm{Cl}$ Ratios (B) in the ESF, as Reported by LANL in 1996, 1997, and 1998

2-2. Relations between Fault/Shear Intensity as Mapped in the ESF and ${ }^{36} \mathrm{Cl} / \mathrm{Cl}$ Ratios for Samples Described as Localities Associated with Faults or Shears

3-1. Distribution of ${ }^{36} \mathrm{Cl}$ along the. Drill Hole Wash Fault Zone in the ESF, between 1,500 and 2.500 meters (A) and between 1,880 and 1,980 meters (B), as Reported by LANL in 1996

3-2. Distribution of ${ }^{36} \mathrm{Cl}$ in and adjacent to the Sundance Fault in the ESF, as Reported by LANL in 1996 and 1998

3-3. Distribution of Fracture Densities in the ESF

3-4. Histograms Showing the Linear Spacing (A) and Log Spacing (B) between Fractures and Cooling Joints Longer than 1 Meter, Measured from Detailed Line Surveys between ESF Stations $16+00$ and $21+00$

3-5. Histograms Showing the Linear Spacing (A) and Log Spacing (B) between Fractures and Cooling Joints Longer than 1 Meter, Measured from Detailed Line Surveys between ESF Stations $34+00$ and $36+00$

3-6. Schematic Map Showing General Relations of Niche \#1 to the ESF Main Drift and Sundance Fault, and the Orientations of Boreholes Used for the Validation Study

3-7. Distribution of Niche \#1 Core Intervals Used for the Validation Study

4-1. Chloride Concentrations and ${ }^{36} \mathrm{Cl} / \mathrm{Cl}$ Ratios in Active Leachates of Validation Study Samples Processed and Analyzed at LLNL during Phase I

4-2. Distribution of Chloride Concentrations (A) and ${ }^{36} \mathrm{Cl} / \mathrm{Cl}$ Ratios (B) in Active Leachates of Validation Study. Samples Processed and Analyzed at LLNL during Phase I

4-3. Relations between Reciprocal Chloride Concentrations and ${ }^{36} \mathrm{Cl} / \mathrm{Cl}$ Ratios in Active Leachates of Validation Study Samples Processed and Analyzed at LLNL during Phase I (A), and for Passive Leachates of ESF Samples Reported Previously by LANL (B)

4-4. Relations between Chloride Concentrations (A) and Cumulative Chloride Concentrations (B) Plotted against Leach Duration for Sequential Leachates of Reference Sample EVAL001 Leached at LANL by Passive and Active Methods during Phase II

4-5. Relations between ${ }^{36} \mathrm{Cl} / \mathrm{Cl}$ Ratios (A) and Cumulative ${ }^{36} \mathrm{Cl} / \mathrm{Cl}$ Ratios (B) Plotted against Leach Duration for Sequential Leachates of Reference Sample EVAL001 Leached at LANL by Passive and Active Methods during Phase II 
4-6. Relations between Chloride Concentrations (A, showing all data) and Cumulative Chloride Concentrations ( $B$, showing a subset of the data at a larger scale) Plotted against Leach Duration for Sequential Passive Leachates of the 6.3- to 12.5-mm Fraction of Six Samples from the ECRB Cross Drift Analyzed at LANL during Phase II

4-7. Relations between ${ }^{36} \mathrm{Cl} / \mathrm{Cl}$ Ratios (A) and Cumulative ${ }^{36} \mathrm{Cl} / \mathrm{Cl}$ Ratios (B) Plotted against Leach Duration for: Sequential Passive Leachates of the 6.3- to 12.5-mm Fraction of Six Samples from the ECRB Cross Drift Analyzed at LANL during Phase II

4-8. Relations between Chloride Concentrations (A) and Cumulative Chloride Concentrations (B) Plotted against Leach Duration for Passive Leachates of Different Size Fractions of ECRB: Cross Drift Sample EXD-069 Analyzed at LANL during Phase II

4-9. Relations between ${ }^{36} \mathrm{Cl} / \mathrm{Cl}$ Ratios (A) and Cumulative ${ }^{36} \mathrm{Cl} / \mathrm{Cl}$ Ratios (B) Plotted against Leach Duration for Passive Leachates of Different Size Fractions of ECRB Cross Drift Sample EXD-069 Analyzed at LANL during Phase II

4-10. Relations between ${ }^{36} \mathrm{Cl} / \mathrm{Cl}$ Ratios and Reciprocal Chloride Concentrations in Sequential Leachates of Reference Sample EVAL001 and ECRB Cross Drift Samples Analyzed at LANL during Phase II

4-11. Effect of Particle Size on Leach Duration and Chloride Concentration for Two Size Fractions of Tuff from Unfractured (CT and FT series, \#2) and Relatively Unfractured (2CT series, \#14) Core Samples Analyzed at AECL during Phase II

4-12. Detail from Figure 4-11 Showing the Changes in Chloride Concentrations in the First Few Hours of Two Leaching Tests on the Coarse Tuff

4-13. Effect of Particle Size on Chloride Concentrations in Phase II Leachates of Intact Core from Borehole ESF-SD-CIV\#2 (GS series in Table 4-6) and Broken Core from Borehole ESF-SD-ClV\#14 (2A2 series in Table 4-6)

4-14. Effect of Particle Size and Leach Duration on Rubblized Core Fragments from Borehole ESF-SD-ClV\#9 (BT series in Table 4-6)

4-15. Comparison of Chloride Concentrations in Phase II Leachates of Core Samples from ESF-SD-CIV and Niche \#1 Boreholes in the Sundance Fault Zone

4-16. Relations between Chloride Concentrations and ${ }^{36} \mathrm{Cl} / \mathrm{Cl}$ Ratios in Phase III Leachates of Core Samples from Borehole ESF-SAD-GTB\#1

4-17. Relations between ${ }^{36} \mathrm{Cl} / \mathrm{Cl}$ Ratios and Chloride Concentrations (A) and Reciprocal Chloride Concentrations (B) in Phase III Leachates of Validation Study Samples Leached at the USGS and Analyzed at LLNL

4-18. Box Plots of Chloride Concentration Data Comparing Phase III Leachates of Core Samples from the Drill Hole Wash and Sundance Fault Zones (A), and from Different Samples within the Sundance Fault Zone (B)

4-19. Concentrations of Chloride Determined by Ion Chromatography in Phase III Leachates of Validation Study Core Samples and Niche \#1 Core Samples from the Sundance Fault Zone (A) and Drill Hole Wash Fault Zone (B) 
4-20. Comparison of Chloride Concentrations in Phase III Leachates of Validation Study Core Leached at the USGS, with $\mathrm{NO}_{3}$ Concentrations (A) and $\mathrm{SO}_{4}$ Concentrations (B)

4-21. Comparison of Chloride Concentrations in Phase III Leachates of Validation Study Samples Analyzed by Ion Chromatography at the USGS and by Isotope Dilution at LLNL

4-22. Histograms Showing Chloride Concentrations (A) and ${ }^{36} \mathrm{Cl} / \mathrm{Cl}$ Ratios (B) in Phase III Leachates of Validation Study Samples Leached at the USGS and Analyzed at LLNL

4-23. Relations between Sample Locations in the ESF and Chloride Concentrations (A) and ${ }^{36} \mathrm{Cl} / \mathrm{Cl}$ Ratios (B) in Phase III Leachates of Validation Study Samples Leached at the USGS and Analyzed at LLNL

4-24. Relations between Borehole Completion Dates and ${ }^{36} \mathrm{Cl} / \mathrm{Cl}$ Ratios in Phase III Leachates of Validation Study Samples Leached at the USGS and Analyzed at LLNL

4-25. Histogram Showing ${ }^{36} \mathrm{Cl} / \mathrm{Cl}$ Ratios in Phase III Leachates of ESF-SD-ClV and Niche \#1 Core Samples Prepared at the USGS and Analyzed at LLNL

4-26. Relations between Reciprocal Chloride Concentrations and ${ }^{36} \mathrm{Cl} / \mathrm{Cl}$ Ratios in Phase III Leachates of Niche \#1 Core Samples as Linear (A) and Semi-Log (B) Plots

4-27. Comparison of Reciprocal Chloride Concentrations and ${ }^{36} \mathrm{Cl} / \mathrm{Cl}$ Ratios in Phase III Leachates of Samples from ESF Tunnel Walls (Sundance Fault Zone between Stations 34+28 and 37+00) and Niche \#1 Core

4-28. Relations between Reciprocal Chloride Concentrations and ${ }^{36} \mathrm{Cl} / \mathrm{Cl}$ Ratios in Phase III Leachates of Validation Study Samples from the Sundance Fault Zone within the ESF

4-29. Conceptual Model of the Isotopic Evolution of ${ }^{36} \mathrm{Cl} / \mathrm{Cl}$ Ratios in Passively Leached Solutions with Time

4-30. Comparison of Chloride Concentrations (A) and ${ }^{36} \mathrm{Cl} / \mathrm{Cl}$ Ratios (B) in Aliquots of Validation Study Samples Passively Leached for 1 Hour at the USGS and Sent to LLNL and LANL for AgCl Target Preparation

4-31. Frequency Distribution (A) and Box Plot (B) of ${ }^{36} \mathrm{Cl} / \mathrm{Cl}$ Ratios in Leachates of Validation Study Core Leached at the USGS and Sent to LLNL and LANL for $\mathrm{AgCl}$ Precipitation and Analysis

4-32. Relations between ${ }^{36} \mathrm{Cl} / \mathrm{Cl}$ Ratios Determined at LANL and Distance in the ECRB Cross Drift

4-33. Distribution of Chloride Concentrations (A) and ${ }^{36} \mathrm{Cl} / \mathrm{Cl}$ Ratios (B) in Leachates of Samples from the Sundance Fault Zone within the ESF

4-34. Relations between Reciprocal Chloride Concentrations and ${ }^{36} \mathrm{Cl} / \mathrm{Cl}$ Ratios in Leachates of Samples from the Sundance Fault Zone

4-35. Distribution of Chloride Concentrations (A) and ${ }^{36} \mathrm{Cl} / \mathrm{Cl}$ Ratios (B) in Leachates of USGS-LLNL Samples from the Sundance Fault Zone and LANL Samples from the Southern ESF 
5-1. Distribution of Tritium Concentrations in Samples of Pore Water Extracted from Validation Study Core along the Drill Hole Wash Fault Zone (A) and Sundance Fault Zone (B)

5-2. Frequency Distribution of Tritium Concentrations in Pore Water from Validation Study Core Samples

5-3. Distribution of Tritium Concentrations Plotted at Full Scale (A) and at a Reduced Scale (B) in Samples of Pore Water Extracted from Drill Core throughout the ESF

5-4. Frequency Distribution of Tritium Concentrations in Pore Water from Boreholes along the ESF South Ramp

5-5. Geologic Section of the ESF South Ramp Showing Locations of Samples Analyzed for Tritium

5-6. Distribution of Tritium Concentrations in Samples of Pore Water Extracted from Drill Core along the ECRB Cross Drift

5-7. Frequency Distribution of Tritium Concentrations in Pore Water from ECRB Cross Drift Drill Core

5-8. Application of Chauvenet's Criterion to Establish a Cutoff Tritium Concentration for Identifying the Presence of Bomb-Pulse Tritium in Samples from the ESF and ECRB Cross Drift (USGS)

5-9. Application of Chauvenet's Criterion to Establish a Cutoff Tritium Concentration for Identifying the Presence of Bomb-Pulse Tritium in Validation Study Boreholes and ECRB Cross Drift Samples (LANL)

6-1. Relations between ${ }^{36} \mathrm{Cl} / \mathrm{Cl}$ Ratios in Validation Study Samples from the Sundance Fault Zone and ${ }^{36} \mathrm{Cl} / \mathrm{Cl}$ Ratios in Samples from the Same Area Reported by LANL in 1996, 1997, and 1998 


\section{TABLES}

3-1. Chronology of Locations and Personnel Directly Involved in the Preparation and Analysis of LANL ${ }^{36} \mathrm{Cl}$ Samples

3-2. Validation Study Boreholes

3-3. Core Samples from Niche \#1 Boreholes

4-1. Chloride Concentrations and ${ }^{36} \mathrm{Cl} / \mathrm{Cl}$ Ratios in Active Leachates Prepared and Analyzed at LLNL during Phase I

4-2. Chloride, Bromide, and Sulfate Concentrations, and ${ }^{36} \mathrm{Cl} / \mathrm{Cl}$ Ratios in Leachates of Validation Study Core Samples Analyzed at LANL during Phase I

4-3. Chloride Concentrations and ${ }^{36} \mathrm{Cl} / \mathrm{Cl}$ Ratios in Sequential Leachates of Reference Sample EVAL001 and Six Samples from the ECRB Cross Drift Analyzed at LANL during Phase II

4-4. Possible Sources for ${ }^{36} \mathrm{Cl} / \mathrm{Cl}$ Ratios in Tuff Samples from Yucca Mountain

4-5. Dry-Drilled Core Samples Used in Chloride Leaching Experiments Conducted at AECL during Phase II

4-6. Summary of Data for Core Samples Analyzed at AECL during Phase II

4-7. Processing History of Validation Study Core Samples Leached at the USGS during Phase III

4-8. Validation Study Core Intervals Chosen for Passive Leaching at the USGS during Phase III

4-9. Chloride Concentrations and ${ }^{36} \mathrm{Cl} / \mathrm{Cl}$ Ratios in Core Samples Leached and Analyzed at USGS-LLNL during Phase III

4-10. Concentrations and Chloride Isotopic Compositions of Procedural Blanks Obtained for Passive Leaching at the USGS and Chloride Precipitation and Analysis at LLNL during Phase III

4-11. Chloride Concentrations and ${ }^{36} \mathrm{Cl} / \mathrm{Cl}$ Ratios Measured during Phase III at USGS-LLNL in Silicon Crushing Blanks, System Process Blanks, and a Composite Sample of Niche \#1 Core Crushed and Sieved at LANL

4-12. Chloride Concentrations and ${ }^{36} \mathrm{Cl} / \mathrm{Cl}$ Ratios in Leachates of Validation Study Samples Analyzed at LANL during Phase III

4-13. Concentrations of Anions in Leachates of Validation Study Samples Analyzed by Ion Chromatography at the USGS during Phase III

4-14. Summary of Anion Concentrations in Leachates of Validation Study Samples Analyzed by Ion Chromatography at the USGS during Phase III

4-15. Summary of Chloride Concentrations and ${ }^{36} \mathrm{Cl} / \mathrm{Cl}$ Ratios in Core Samples Leached and Analyzed at USGS-LLNL during Phase III

4-16. Mass of Total Chloride, ${ }^{36} \mathrm{Cl} / \mathrm{Cl}$ Ratios, and Mass of ${ }^{36} \mathrm{Cl}$ Present in Validation Study Blanks Processed at LANL during Phase III

4-17. Chloride, Bromide, and Sulfate Concentrations, and ${ }^{36} \mathrm{Cl} / \mathrm{Cl}$ Ratios in Leachates of ECRB Cross Drift Samples Analyzed at LANL during Phase III

4-18. Chloride Concentrations and ${ }^{36} \mathrm{Cl} / \mathrm{Cl}$ Ratios in Duplicate Analyses Used to Calculate External Error in ${ }^{36} \mathrm{Cl} / \mathrm{Cl}$ Ratios during Phase III 
5-1. Tritium Concentrations in Water Standards with Known Values

5-2. Tritium Concentrations in Pore Water Extracted from Validation Study Core Samples

5-3. Tritium Concentrations in Pore Water Extracted from ESF Core Samples

5-4. Tritium Concentrations in Pore Water Extracted from ECRB Cross Drift Core Samples 


\section{ACRONYMS, ABBREVIATIONS, AND SYMBOLS}

$\begin{array}{ll}{ }^{40} \mathrm{Ca} & \text { calcium-40 } \\ { }^{137} \mathrm{Cs} & \text { cesium-137 } \\ { }^{35} \mathrm{Cl} & \text { chlorine-35 } \\ \text { chlorine-36 } \\ { }^{36} \mathrm{Cl} & \text { chlorine-37 } \\ { }^{37} \mathrm{Cl} & \text { deuterium } \\ { }^{2} \mathrm{H} & \text { tritium } \\ { }^{3} \mathrm{H} & \text { lithium-6 } \\ { }^{6} \mathrm{Li} & \text { oxygen-18 } \\ { }^{18} \mathrm{O} & \text { strontium-87/strontium-86 } \\ { }^{87} \mathrm{Sr} /{ }^{86} \mathrm{Sr} & \text { technetium-99 } \\ { }^{99} \mathrm{Tc} & \text { sigma } \\ & 1 \text { standard deviation } \\ \sigma & 2 \text { standard deviations }\end{array}$

A ampere

AECL Atomic Energy of Canada Limited

$\mathrm{AgCl}$ silver chloride

AMS accelerator mass spectrometry

$\mathrm{Br} \quad$ bromine

BSC Bechtel SAIC Company

CAMS Center for Accelerator Mass Spectrometry, Lawrence Livermore National Laboratory

$\mathrm{CHn}$

$\mathrm{Ci}$ Calico Hills nonwelded hydrogeologic unit

$\mathrm{Cl} \quad$ chlorine

cm centimeter

CRWMS Civilian Radioactive Waste Management System

DIRS Document Input Reference System

DOE U.S. Department of Energy

ECRB Enhanced Characterization of the Repository Block

ESF Exploratory Studies Facility

F fluorine

g gram

IBM International Business Machines Corporation 
ka

$\mathrm{kg}$

$\mathrm{km}$

L

LANL Los Alamos National Laboratory

LLNL Lawrence Livermore National Laboratory

LRL laboratory reporting level

LT-MDL long-term method detection limit

$\mu \mathrm{m} \quad$ micrometer

m meter

$\mathrm{M} \& \mathrm{O} \quad$ management and operating (contractor)

MDL method detection limit

mg milligram

mm millimeter

ML minimum level of quantitation

$\mathrm{NO}_{3} \quad$ nitrate

NWQL National Water Quality Laboratory, U.S. Geological Survey

NWTRB Nuclear Waste Technical Review Board

OCRWM Office of Civilian Radioactive Waste Management

ORD

pCi

PRIME Lab Purdue Rare Isotope Measurement Laboratory

psi

PTn

SE

SMF

$\mathrm{SO}_{4}$

TCw

TDMS

TIMS

TIP

TSw

TU

USEPA

USGS

UZ

YMPB
Office of Repository Development

picocurie

pounds per square inch

Paintbrush Tuff nonwelded hydrogeologic unit

standard error

Sample Management Facility

sulfate

Tiva Canyon welded hydrogeologic unit

Technical Data Management System

thermal ionization mass spectrometry

technical implementation procedure

Topopah Spring welded hydrogeologic unit

tritium unit

U.S. Environmental Protection Agency

U.S. Geological Survey

unsaturated zone

Yucca Mountain Project Branch, U.S. Geological Survey 


\section{STRATIGRAPHIC AND HYDROGEOLOGIC NAMES}

Yucca Mountain consists of north-trending fault-block ridges composed of gently dipping Miocene ash-flow tuffs (Scott and Bonk 1984). Differences in the hydrologic character of the welded and nonwelded tuffs led Montazer and Wilson (1984) and Ortiz et al. (1985) to develop a hydrogeologic classification of the volcanic rocks. Because these units are based on hydrologic properties, they do not correspond exactly with the stratigraphic units described by Sawyer et al. (1994). For example, as shown below, the Paintbrush Tuff nonwelded hydrogeologic unit (PTn) consists of the nonwelded basal part of the Tiva Canyon Tuff, the entire Yucca Mountain and Pah Canyon Tuffs and associated but unnamed bedded tuffs, and the nonwelded upper part of the Topopah Spring Tuff. Both nomenclatures are used in this report.

\begin{tabular}{|c|c|c|}
\hline & Stratigraphic Unit & Hydrogeologic Unit \\
\hline & Alluvium & Alluvium \\
\hline \multirow{6}{*}{ 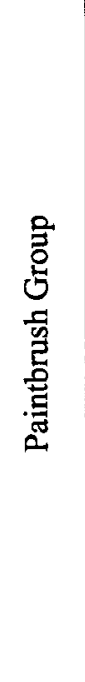 } & Tiva Canyon Tuff & $\begin{array}{l}\text { Tiva Canyon welded } \\
\text { (TCw) }\end{array}$ \\
\hline & Yucca Mountain Tuff & \multirow{4}{*}{$\begin{array}{c}\text { Paintbrush Tuff } \\
\text { nonwelded (PTn) }\end{array}$} \\
\hline & bedded tuff & \\
\hline & Pah Canyon Tuff & \\
\hline & bedded tuff & \\
\hline & Topopah Spring Tuff & $\begin{array}{l}\text { Topopah Spring } \\
\text { welded (TSw) }\end{array}$ \\
\hline \multicolumn{2}{|r|}{ Calico Hills Formation } & \multirow{2}{*}{$\begin{array}{l}\text { Calico Hills nonwelded } \\
\text { (CHn) }\end{array}$} \\
\hline \multirow{2}{*}{$\begin{array}{l}\text { 吾 } \\
\text { 离 } \\
\text { 总 }\end{array}$} & \multirow[t]{2}{*}{ Prow Pass Tuff } & \\
\hline & & $\begin{array}{c}\text { Crater Flat } \\
\text { undifferentiated }(\mathrm{CFu})\end{array}$ \\
\hline
\end{tabular}

Modified from Montazer and Wilson (1984) 


\section{CONVERSION FACTORS}

$\begin{array}{lll}\text { Multiply } & \text { by } & \text { To obtain } \\ \text { centimeter }(\mathrm{cm}) & 0.3937 & \text { inch (in.) } \\ \text { millimeter }(\mathrm{mm}) & 0.03937 & \text { inch (in.) } \\ \text { micrometer }(\mu \mathrm{m}) & 3.937 \times 10^{-5} & \text { inch (in.) } \\ \text { meter }(\mathrm{m}) & 3.281 & \text { foot }(\mathrm{ft}) \\ \text { kilometer } & 0.6214 & \text { mile (mi) } \\ \text { liter }(\mathrm{L}) & 33.82 & \text { ounce }(\mathrm{oz}) \\ \text { liter }(\mathrm{L}) & 1.0567 & \text { quart }(\mathrm{qt}) \\ \text { kilogram }(\mathrm{kg}) & 2.205 & \text { pound }(\mathrm{lb}) \\ \text { milligram }(\mathrm{mg}) & 2.205 \times 10^{-6} & \text { pound }(\mathrm{lb}) \\ \text { milligram }(\mathrm{mg}) & 3.527 \times 10^{-5} & \text { ounce }(\mathrm{oz})\end{array}$

\section{REPORTING OF UNCERTAINTIES AND PARAMETER VARIABILITIES}

Throughout this report uncertainties are cited for individual measurements and means of multiple measurements. For individual measurements, the uncertainty is expressed as 2 standard deviations $(2 \sigma)$, unless otherwise specified. One standard deviation $(1 \sigma)$ is used to express natural variability of measured parameters, such as concentrations and isotope ratios, within a group of samples.

For averages of multiple measurements, uncertainty is expressed as standard error (SE), which is $1 \sigma$ divided by the square root of the number of measurements. Weighted averages were calculated for multiple measurements with highly variable errors (for example, process blanks), using reciprocals of squared individual $1 \sigma$ uncertainties as weighting factors.

\section{NOTATION OF CHLORINE-36/CHLORIDE RATIOS IN TEXT, TABLES, AND FIGURES}

In the text of this report, ${ }^{36} \mathrm{Cl} / \mathrm{Cl}$ ratios are given as a value multiplied by $10^{-15}$. For example, a ratio of 0.000000000000666 is cited as " $666 \times 10^{-15}$." To simplify the tabulation of the data and the labels for the graphs, these ratios have been multiplied by $10^{15}$. Thus, the example ${ }^{36} \mathrm{Cl} / \mathrm{Cl}$ ratio will be given as " 666 " in a table where the column heading indicates ${ }^{36} \mathrm{Cl} / \mathrm{Cl} \times 10^{15}$." 


\section{ACKNOWLEDGMENTS}

L.A. Neymark (USGS), Z.E. Peterman (USGS), B.D. Marshall (USGS), G.J. Nimz (LLNL), M. Gascoyne (AECL and Gascoyne GeoProjects, Inc., Pinawa, Manitoba), and G.L. Patterson (USGS) contributed to this report. 
INTENTIONALLY LEFT BLANK 


\section{INTRODUCTION}

The amount, spatial distribution, and velocity of water percolating through the unsaturated zone (UZ) at Yucca Mountain, Nevada, are important issues for assessing the performance of the proposed deep geologic repository for spent nuclear fuel and high-level radioactive waste. To help characterize the nature and history of UZ flow, isotopic studies were initiated in 1995, using rock samples collected from the Miocene ash-flow tuffs in the Exploratory Studies Facility (ESF), an 8-km-long tunnel constructed along the north-south extent of the repository block, and the Enhanced Characterization of the Repository Block (ECRB) Cross Drift, a 2.5-km-long tunnel constructed across the repository block (Figure 1-1, Sources: Modified from DOE 2002 [Figure 1-14] and USBR 1996). Scientists from Los Alamos National Laboratory (LANL) analyzed for chlorine-36 $\left({ }^{36} \mathrm{Cl}\right)$ in salts leached from whole-rock samples collected from tunnel walls and subsurface boreholes, and scientists from the U.S. Geological Survey (USGS) analyzed for isotopes of oxygen, carbon, uranium, lead, thorium, and strontium in secondary minerals collected from subsurface fractures and lithophysal cavities. Elevated values for ratios of ${ }^{36} \mathrm{Cl}$ to total chloride $\left({ }^{36} \mathrm{Cl} / \mathrm{Cl}\right)$ at the level of the proposed repository indicated that small amounts of water carrying bomb-pulse ${ }^{36} \mathrm{Cl}$ (i.e., ${ }^{36} \mathrm{Cl} / \mathrm{Cl}$ ratios greater than $1250 \times 10^{-15}$ resulting from ${ }^{36} \mathrm{Cl}$ produced by atmospheric testing of nuclear devices during the 1950 s and early $1960 \mathrm{~s}$ ) had percolated through welded and nonwelded tuffs to depths of 200 to 300 meters $(\mathrm{m})$ beneath the land surface over the past 50 years. Because of the implications of short travel times to the performance of the proposed repository, the U.S. Department of Energy (DOE)/Office of Civilian Radioactive Waste Management (OCRWM), Office of Repository Development (ORD), decided to verify the ${ }^{36} \mathrm{Cl} / \mathrm{Cl}$ data with an independent validation study.

DOE asked the USGS to design and implement a validation study that would include ${ }^{36} \mathrm{Cl}$ and tritium $\left({ }^{3} \mathrm{H}\right)$ analyses. Core samples were taken from 50 new boreholes drilled across two zones in the ESF where a substantial number of samples with elevated ${ }^{36} \mathrm{Cl} / \mathrm{Cl}$ ratios had been identified previously. Also, core intervals from the Sample Management Facility (SMF) were acquired for water extraction and ${ }^{3} \mathrm{H}$ analyses.

\subsection{PURPOSE}

This report documents the background and history of the validation study and presents the results of the ${ }^{36} \mathrm{Cl}$ to total chloride $\left({ }^{36} \mathrm{Cl} / \mathrm{Cl}\right)$ and ${ }^{3} \mathrm{H}$ analyses. The study was funded by the DOE/OCRWM ORD to attempt to validate elevated ${ }^{36} \mathrm{Cl} / \mathrm{Cl}$ values reported by LANL, and to apply other isotopic methods to identify evidence of rapid flow in the UZ at Yucca Mountain. This report was prepared as part of activities being conducted under Technical Work Plan for: Performance Assessment Unsaturated Zone (BSC 2002) and Test Plan for: Chlorine-36 Validation (USGS 2002). Study participants included the USGS, Lawrence Livermore National Laboratory (LLNL), Atomic Energy of Canada Limited (AECL), and LANL. LANL was funded to analyze ${ }^{36} \mathrm{Cl}$ in some of the validation study samples. The Purdue Rare Isotope Measurement Laboratory (PRIME Lab) and Rosenstiel School of Marine and Atmospheric Science Laboratory at the University of Miami performed ${ }^{36} \mathrm{Cl}$ and ${ }^{3} \mathrm{H}$ analyses, respectively, and Phillips Enterprises, L.L.C. in Golden, Colorado prepared the reference sample that was used to standardize the leaching procedure. 


\subsection{QUALITY ASSURANCE}

The Yucca Mountain Project activities and data summarized in this report were subject to the revision of the Quality Assurance Requirements and Description that was in place at the time the work was completed (current Revision 16: DOE 2004). The quality assurance status (qualified ["Q"] or unqualified ["UQ"]) of the data presented in this report is determined by the activities under which they were generated. Although this is a " $Q$ " document, not all data presented are "Q" data. The qualification status of the data is indicated in Section 7.3 of this report and in the electronic Document Input Reference System (DIRS) database.

This report has been prepared in accordance with PA-PRO-0313, Technical Reports. It is a summary report, with no technical outputs that could be used as input to another Yucca Mountain Project technical report.

Commercial, off-the-shelf software (i.e., Microsoft Excel 2000 running under the Microsoft Windows XP operating system on an International Business Machines Corporation [IBM]-compatible personal computer) was used for data compilation, reduction, computation, and graphical representation of output in the figures and tables contained in this report.

\subsection{ORGANIZATION OF THE REPORT}

The background for initiating the ${ }^{36} \mathrm{Cl}$ validation study is given in Section 2 of this report, along with a summary of previous ${ }^{36} \mathrm{Cl}$ studies. Section 3 describes the design and implementation of the validation study. Chlorine-36 results from the validation study are presented in Section 4 . Section 5 describes ${ }^{3} \mathrm{H}$ measurements, which also may be used to identify rapid percolation. Section 6 summarizes the results of the validation study, presents the main conclusions, and describes the important analytical issues that remain unresolved. Section 6 also gives recommendations for a path forward that will help resolve these issues. Publications and data cited in the report are listed in Section 7. Supporting information is contained in the appendixes, including a compilation of previous ${ }^{36} \mathrm{Cl}$ results (Appendix A), video logs for the validation study boreholes (Appendix B), and a description of accelerator mass spectrometry (AMS) analytical methods (Appendix C). 


\section{BACKGROUND}

Chlorine-36 is the only naturally occurring radioactive isotope of chlorine. It is produced by cosmic ray-induced reactions in the atmosphere and in minerals at and near the earth's surface. Chlorine-36 also is produced in the subsurface by reactions with neutrons from the natural decay of uranium- and thorium-series elements. Large amounts of ${ }^{36} \mathrm{Cl}$, relative to natural abundances, were produced during atmospheric thermonuclear tests in the western Pacific Ocean during the 1950s and early 1960s (Phillips 2000, p. 318).

Chlorine-36 in rocks and water at Yucca Mountain derives from multiple sources. Meteoric ${ }^{36} \mathrm{Cl}$ produced by cosmic radiation in the upper atmosphere is rapidly transferred to the land surface by dry-fall or by incorporation into precipitation. At Yucca Mountain, meteoric ${ }^{36} \mathrm{Cl} / \mathrm{Cl}$ ratios have been about $500 \times 10^{-15}$ throughout the Holocene (CRWMS M\&O 2000, Table 25, Section 6.6.3.1), but ${ }^{36} \mathrm{Cl} / \mathrm{Cl}$ ratios have varied in the past due to several factors. Production rates of ${ }^{36} \mathrm{Cl}$ vary inversely with the intensity of the geomagnetic field (CRWMS M\&O 2000, Figure 31, Section 6.6.3.1). Theoretical reconstructions and measurements of fossil urine from pack-rat middens indicate that meteoric ${ }^{36} \mathrm{Cl} / \mathrm{Cl}$ ratios prior to about 10 thousand years ago (ka) were appreciably larger (Fabryka-Martin, Wolfsberg et al. 1996, Figure 2-2; Plummer et al. 1997 , Figure 2), with average late Pleistocene ${ }^{36} \mathrm{Cl} / \mathrm{Cl}$ ratios of about $900 \times 10^{-15}$ and peak values as high as about $1,100 \times 10^{-15}$ (Fabryka-Martin et al. 1997, p. 3-3).

High concentrations of atmospheric ${ }^{36} \mathrm{Cl}$ produced during atmospheric thermonuclear tests resulted in ${ }^{36} \mathrm{Cl} / \mathrm{Cl}$ ratios of meteoric water and soil water ranging from $10^{-12}$ to $10^{-10}$ (FabrykaMartin et al. 1997, p. 3-5). Atmospheric concentrations of ${ }^{36} \mathrm{Cl}$ have since returned to pre-bombpulse values (Phillips 2000, Figure 10.8). Infiltration has carried this bomb-pulse ${ }^{36} \mathrm{Cl}$ into the subsurface. In alluvium in arid regions where infiltration is low, most of the bomb-pulse ${ }^{36} \mathrm{Cl}$ has remained within a few meters of the land surface (Tyler et al. 1996, p. 1489; Norris et al. 1987, p. 377).

In situ production of ${ }^{36} \mathrm{Cl}$ from natural neutron fluxes in the tuffs at Yucca Mountain results in low ${ }^{36} \mathrm{Cl} / \mathrm{Cl}$ values. An equilibrium ${ }^{36} \mathrm{Cl} / \mathrm{Cl}$ value of about $40 \times 10^{-15}$ was calculated by FabrykaMartin et al. (1997, Section 3.4.1). Large chloride concentrations of 7.6 to 17.6 milligrams per kilogram (mg/kg) and small ${ }^{36} \mathrm{Cl} / \mathrm{Cl}$ values of $43 \times 10^{-15}$ to $57 \times 10^{-15}$ were measured in leachates of powdered rock samples after most of the meteoric chloride components had been removed (Fabryka-Martin, Wolfsberg, et al. 1996, Table 5-4).

Cosmogenic production of ${ }^{36} \mathrm{Cl}$ also takes place in rocks within the upper few meters of the land surface, dominantly through spallation of ${ }^{40} \mathrm{Ca}$ in calcium-rich soils (Stone et al. 1996, Section 4.1). Spallation-derived ${ }^{36} \mathrm{Cl}$ may contribute elevated ${ }^{36} \mathrm{Cl} / \mathrm{Cl}$ values to infiltration under wetter climate conditions when old soil carbonate may dissolve and re-crystallize, releasing the accumulated ${ }^{36} \mathrm{Cl}$ to soil water. Also, radioactive decay will result in lowering the ${ }^{36} \mathrm{Cl}$ values, regardless of original sources. The 301,000 -year half-life of ${ }^{36} \mathrm{Cl}$ (Phillips 2000, p. 299) is sufficiently long so that decay will not considerably affect processes less than about 50,000 years old, but must be taken into account when considering older geologic and hydrologic processes. 


\subsection{STUDIES OF CHLORINE-36 AND FRACTURE MINERALS IN THE EXPLORATORY STUDIES FACILITY}

The ESF was constructed between September 1994 and April 1997, through Miocene ash-flow tuffs, using a tunnel boring machine (DOE 2001, p. 1-16). $\mathrm{A}{ }^{36} \mathrm{Cl}$ study was initiated by LANL in 1995 to test whether the Paintbrush Tuff nonwelded hydrogeologic unit (PTn) is an effective barrier to vertical flow, whether water in the matrix of the Topopah Spring welded hydrogeologic unit (TSw) is essentially stagnant, and whether fast paths transporting water to the proposed repository horizon occur at discrete locations associated primarily with fault structures (Fabryka-Martin, Wolfsberg et al. 1996, p. 1). During this time, the USGS began isotopic and geochronologic studies of low-temperature minerals in fractures and lithophysal cavities to evaluate the history of fracture flow over the past 500,000 years (Paces et al. 2001, p. 3). Early sampling for both ${ }^{36} \mathrm{Cl}$ and fracture mineral studies followed advances of the tunnel boring machine through the ESF. One of the objectives of the early work was to evaluate the effectiveness of lateral diversion of percolating water in the PTn (Montazer and Wilson 1984, p. 14). Several nonwelded and mostly vitric pyroclastic units lie between the lower, densely welded part of the overlying Tiva Canyon welded hydrogeologic unit ( $\mathrm{TCw}$ ) and the top of the underlying, crystal-rich vitrophyre of the TSw (Moyer et al. 1996, p. 1). The moderate-to-high porosity and permeability of the PTn and the relatively sharp upper and lower contacts may influence downward percolation into the TSw (Montazer and Wilson 1984, p. 47; Kwicklis et al. 1994, p. 2341; Moyer et al. 1996, p. 2).

\subsubsection{Results from Previous Chlorine-36 Studies}

Analyses of ${ }^{36} \mathrm{Cl} / \mathrm{Cl}$ ratios in salts leached from ESF samples were presented in a series of milestone reports (Fabryka-Martin, Wolfsberg et al. 1996; Fabryka-Martin et al. 1997; CRWMS M\&O 1998). Data collected through September 1998 are tabulated in Appendix A. Because sampling followed tunnel advances, analytical results were obtained progressively in time and space (Figure 2-1). ${ }^{36} \mathrm{Cl} / \mathrm{Cl}$ ratios obtained for samples from the northern ESF, reported in 1996 (Fabryka-Martin, Wolfsberg et al. 1996, Table 5-3), differ from values for samples from the southern ESF, reported in 1997 (Fabryka-Martin et al. 1997, Appendix B). Most ${ }^{36} \mathrm{Cl} / \mathrm{Cl}$ ratios from the northern ESF are greater than $500 \times 10^{-15}$, the value generally accepted for Holocene meteoric input (Fabryka-Martin et al. 1993, Section IV.A; Fabryka-Martin, Wolfsberg et al. 1996, p. 3; Fabryka-Martin et al. 1997, Section 3.1.1). About one fifth of the data from the northern ESF (up to station $45+00^{1}$, obtained through the Summer of 1996) are either sporadic or clustered ${ }^{36} \mathrm{Cl} / \mathrm{Cl}$ values greater than $1,250 \times 10^{-15}$ (Fabryka-Martin et al. 1997, p. 4-15, Figure 4-6), the cutoff value established by statistical methods as an upper limit of the normal distribution of background samples. Samples with ${ }^{36} \mathrm{Cl} / \mathrm{Cl}$ ratios above this cutoff were interpreted to contain a component of bomb-pulse ${ }^{36} \mathrm{Cl}$. Samples from the southern ESF (beyond station $45+00$ ) have ${ }^{36} \mathrm{Cl} / \mathrm{Cl}$ ratios less than $1,250 \times 10^{-15}$ and some are less than the $500 \times 10^{-15}$ Holocene meteoric value.

Later efforts focused on samples from near the Sundance fault zone in Niche \#1 (equivalent to Niche 3566 in other publications) and the Ghost Dance fault zone in Alcoves \#6 and \#7. Five

${ }^{1}$ ESF station numbers are equivalent to distances, in hundreds of meters from a point outside the north portal of the ESF, defined as station $00+00$. Thus, ESF station $45+00$ is $4,500 \mathrm{~m}$ from the north portal. 
samples from the walls of Niche \#1, including a damp breccia, showed ${ }^{36} \mathrm{Cl} / \mathrm{Cl}$ ratios between $540 \times 10^{-15}$ and $635 \times 10^{-15}$ (CRWMS M\&O 1998, Table 3-2). Core samples from Niche \#1 produced ${ }^{36} \mathrm{Cl} / \mathrm{Cl}$ values from $997 \times 10^{15}$ to $2,038 \times 10^{15}$ (CRWMS M\&O 1998, Table 3-4). ${ }^{36} \mathrm{Cl} / \mathrm{Cl}$ ratios in eight of 20 samples from the walls of the northern Ghost Dance fault zone (Alcove \#6) were greater than $1,000 \times 10^{-15}$, although most samples directly from the Ghost Dance fault exposed in alcove walls were within analytical uncertainty of the Holocene meteoric input value of $500 \times 10^{-15}$ (CRWMS M\&O 1998, Table 3-2). ${ }^{36} \mathrm{Cl} / \mathrm{Cl}$ ratios for samples from the southern Ghost Dance fault zone (Alcove \#7) did not exceed $644 \times 10^{-15}$.

The elevated ${ }^{36} \mathrm{Cl} / \mathrm{Cl}$ ratios in samples from the northern ESF were of immediate interest because of the implications of fast pathways in the UZ. Elevated levels of both ${ }^{36} \mathrm{Cl}$ and ${ }^{3} \mathrm{H}$ identified in soils elsewhere in the semi-arid southwestern United States were attributed to global fallout from aboveground testing of thermonuclear devices in the 1950s and early 1960s (Phillips et al. 1988; Scanlon 1992; Tyler et al. 1996, p. 1489; Norris et al. 1987, p. 377). The bomb-pulse ${ }^{36} \mathrm{Cl}$ "bulge" observed during these studies was restricted to the upper 1 to $2 \mathrm{~m}$ of the soil profiles. Similar profiles of ${ }^{36} \mathrm{Cl} / \mathrm{Cl}$ ratios are present in thick alluvium at Yucca Mountain (CRWMS M\&O 2000, Section 6.6.3.2). Where alluvial cover is thin or absent, bomb-pulse ${ }^{36} \mathrm{Cl}$ has entered fractures in the bedrock and rapidly penetrated to depths as great as $24 \mathrm{~m}$ in surfacebased borehole USW UZ-N11, $56 \mathrm{~m}$ in USW UZ-N53, and $77 \mathrm{~m}$ in USW UZ-N55 (FabrykaMartin et al. 1993, Table 2).

Identification of bomb-pulse ${ }^{36} \mathrm{Cl}$ in cuttings from these boreholes was complicated by the presence of ${ }^{36} \mathrm{Cl} / \mathrm{Cl}$ ratios in cuttings from borehole USW UZ-N55 that were "considerably higher than can be explained by global fallout" (Fabryka-Martin et al. 1993, p. 66) (i.e., ${ }^{36} \mathrm{Cl} / \mathrm{Cl}$ values up to $27,040 \times 10^{-15}$, Fabryka-Martin et al. 1993, Table 2). This observation led the authors to conclude that "the possibility that elevated levels in any of these holes may also be attributable to contamination cannot as yet be ruled out" and that "until the source of these elevated ${ }^{36} \mathrm{Cl}$ signals can be identified, the ${ }^{36} \mathrm{Cl} / \mathrm{Cl}$ results in the other $\mathrm{N}$-holes ${ }^{2}$ are also suspect" (Fabryka-Martin et al. 1993, p. 66). Subsequent interpretation of the data, however, indicated that the high ${ }^{36} \mathrm{Cl} / \mathrm{Cl}$ ratios measured in the cuttings were possible (Fabryka-Martin, Turin et al. 1996, Table 4-3, and Sections 4.3.3 and 5.3.1).

Further tests of core samples from borehole USW UZ-N55, in the same zones where cuttings gave very high ${ }^{36} \mathrm{Cl} / \mathrm{Cl}$ values, yielded much lower ${ }^{36} \mathrm{Cl} / \mathrm{Cl}$ values $\left(1,152 \times 10^{-15}\right.$ to $7,937 \times 10^{-15}$, Fabryka-Martin and Liu 1995, Table 3-3), leading the authors to conclude that the "difference supports-but does not prove-the hypothesis that the cuttings may have been contaminated during the drilling or collection process" (Fabryka-Martin and Liu 1995, Section 3.1.3). Soils and equipment contaminated with very high levels of ${ }^{36} \mathrm{Cl}$ from the Rover Nuclear Rocket Program in Test Cell C of the Nevada Test Site were discovered in subsequent work (FabrykaMartin, Turin et al. 1996, Table 4-3, Sections 4.3.3 and 5.3.1). Ratios of ${ }^{36} \mathrm{Cl} / \mathrm{Cl}$ as high as $227,102 \times 10^{-15}$ were obtained from soil pits within $60 \mathrm{~m}$ of the rocket tests (Fabryka-Martin, Turin et al. 1996, Table 4-3), and drilling equipment that was used in these areas was later used to drill borehole USW UZ-N55 (Fabryka-Martin, Turin et al. 1996, Section 5.3.1). However, the authors later concluded "... it is likely that this issue will never be resolved but may be a moot point because the same conclusion is reached with either set of data. Regardless of the origin of

2 "N-holes" are holes drilled for neutron logging. 
the ${ }^{36} \mathrm{Cl}$ in the cuttings, elevated ratios for the drillcore samples clearly indicate bomb-pulse ${ }^{36} \mathrm{Cl}$ at this location" (Fabryka-Martin, Turin et al. 1996, Section 5.3.1).

The subset of elevated ${ }^{36} \mathrm{Cl} / \mathrm{Cl}$ values in the northern ESF was interpreted to indicate that at least some meteoric water has percolated rapidly through the fractured TCW and the PTn into the TSw to depths of $300 \mathrm{~m}$ below the surface in the last 50 years (Fabryka-Martin, Wolfsberg et al. 1996, Section 9; Fabryka-Martin et al. 1997, Section 9; CRWMS M\&O 1998, Section 10; Wolfsberg et al. 2000, p. 349; Campbell et al. 2003, p. 43). Alternative explanations for the elevated ${ }^{36} \mathrm{Cl} / \mathrm{Cl}$ ratios were discussed, including deep, subsurface production in rocks and cosmogenic production in surface rocks and calcrete (Fabryka-Martin et al. 1997, Section 3.4). Although calcrete samples were shown to have substantial cosmogenically produced ${ }^{36} \mathrm{Cl}\left({ }^{36} \mathrm{Cl} / \mathrm{Cl}\right.$ values of $5,067 \times 10^{-15}$ and $9,772 \times 10^{-15}$ for two of three soil calcites analyzed, Fabryka-Martin et al. 1997, Table 3-3), ${ }^{36} \mathrm{Cl}$ from this source was estimated to be at least an order of magnitude less than that from the atmosphere (Fabryka-Martin et al. 1997, p. 3-10).

To simulate the differences in ${ }^{36} \mathrm{Cl}$ signatures observed in the ESF, a UZ flow and transport model was developed that incorporated a large number of geological and hydrological elements (Fabryka-Martin et al. 1997, Section 9.2; Wolfsberg et al. 2000, Section 4; Flint et al. 2001, Section 4.5; Campbell et al. 2003, Section 2). The model requires faults cutting through the PTn for rapid transport of bomb-pulse ${ }^{36} \mathrm{Cl}$ to depth within the TSw. Unless a structural discontinuity existed, percolation into the PTn would transition to matrix-dominated flow, where travel times would greatly exceed the approximately 50-year existence of bomb-pulse tracer isotopes (Wolfsberg et al. 2000, Section 4; Campbell et al. 2003, p. 46). A formal statistical approach based on log-linear models produced "a very strong association" between ESF samples with elevated ${ }^{36} \mathrm{Cl}$ and faults that cut the PTn (Campbell et al. 2003, p. 59). This analysis evaluated the relation between sites where elevated ${ }^{36} \mathrm{Cl}$ was identified and the locations of known PTncutting structures. Within the TSw, the relation between elevated ${ }^{36} \mathrm{Cl}$ occurrences and faults and shears is not evident (Figure 2-2). Because structural features were targeted for ${ }^{36} \mathrm{Cl}$ studies, approximately one-third of the LANL samples listed in Appendix A were collected from sites associated with faults and shears (DTN: LAJF831222AQ98.004 [Q]).

Differences in the amount of infiltration between the northern ESF and southern ESF also were considered important in explaining the presence or absence of elevated ${ }^{36} \mathrm{Cl}$ (CRWMS M\&O 1998 , p. 10-1; Campbell et al. 2003, p. 59). As precipitation is not likely to vary greatly across the area overlying the ESF, other factors, such as the slope and orientation of the land surface and soil thickness, were considered important in controlling differences in infiltration. FabrykaMartin et al. (1997, Figure 6-4) and CRWMS M\&O (1998, Figure 4-2c) show differences in simulated soil thicknesses between the northern ESF and southern ESF, with more occurrences of thicker soils over the southern ESF. However, simulated infiltration rates based on the numerical model of Flint et al. (1996) are similar in both areas (Fabryka-Martin et al. 1997, Figure 6-3; CRWMS M\&O 1998, Figure 4-2b; Campbell et al. 2003, Figure 1c). To explain this difference between the infiltration and ${ }^{36} \mathrm{Cl}$ models, Fabryka-Martin et al. (CRWMS M\&O 1998, p. 10-1) cited elevated chloride concentrations in pore waters from the ESF south ramp to suggest that the numerical infiltration model should be modified to allow for lower infiltration rates above the southern ESF. 
Bomb-pulse ${ }^{36} \mathrm{Cl} / \mathrm{Cl}$ ratios were reported in shallow surface deposits (less than $0.5 \mathrm{~m}$ depth) between surface-based boreholes USW UZ-N53 and USW UZ-N55, approximately $800 \mathrm{~m}$ east of ESF station 51+00; at the UE-25 NRG \#5 drill pad, near ESF station 17+00; and in soil pits near the ESF north portal (Fabryka-Martin et al. 1997, Table 4-6). In addition, elevated ${ }^{36} \mathrm{Cl} / \mathrm{Cl}$ ratios were common in shallow surface deposits above the southern ESF between ESF stations $67+00$ and 78+00 (CRWMS M\&O 1998, Table 3-5). These data confirm that bomb-pulse ${ }^{36} \mathrm{Cl}$ has not been completely removed from soil profiles and that infiltration throughout the site is likely to carry bomb-pulse ${ }^{36} \mathrm{Cl}$ into the bedrock (CRWMS M\&O 1998, p. 3-5).

Just as there are differences in the distribution of elevated ${ }^{36} \mathrm{Cl}$ in the ESF, there is a distinct spatial trend in the non-bomb-pulse ${ }^{36} \mathrm{Cl}$ data (Campbell et al. 2003, p. 57). Most samples from the northern ESF and main drift (up to about ESF station $60+00$, Figure 2-1) have ${ }^{36} \mathrm{Cl} / \mathrm{Cl}$ ratios between $500 \times 10^{-15}$ to $1,250 \times 10^{-15}$. These intermediate ${ }^{36} \mathrm{Cl} / \mathrm{Cl}$ values may be the result of a more dilute bomb-pulse signal or mixtures of the modern meteoric chloride with late Pleistocene meteoric water having higher baseline ${ }^{36} \mathrm{Cl} / \mathrm{Cl}$ values (Plummer et al. 1997, Figure 2). Campbell et al. (2003, Section 7) used statistical tests to conclude that intermediate ${ }^{36} \mathrm{Cl} / \mathrm{Cl}$ ratios are not associated with the same structural features as the elevated ${ }^{36} \mathrm{Cl} / \mathrm{Cl}$ ratios. Therefore, they deduced that the thicker PTn in the northern ESF provides greater average residence time for percolating water, resulting in a larger component of Pleistocene meteoric ${ }^{36} \mathrm{Cl}$ (Campbell et al. 2003, p. 59).

\subsubsection{Fracture Mineral Studies}

Secondary calcite and silica deposits in the ESF have been interpreted as having formed from fracture flow through the welded tuffs (Paces et al. 1996; Paces et al. 1997; Paces et al. 1998; Whelan et al. 1998; Paces et al. 2001; Whelan et al. 2002; Marshall and Futa 2003; Marshall et al. 2003). Geochemical, isotopic, and geochronological data indicate evolution of fracture flow from a meteoric source that was modified by water-rock interactions in the overlying PTn prior to percolation through a small number of fractures in the welded tuffs. Seepage of water films into cavities permitted evaporation with the resulting slow growth of secondary minerals (millimeters per million years) (Paces et al. 2004; Paces et al. 2001, p. 59; Neymark and Paces 2000, p. 158; Neymark et al. 2000, Section 5.3; Neymark et al. 2002, Section 6.7). The slow growth rates preclude identification of minerals deposited since the generation of bomb-pulse isotopes, and carbon-14 $\left({ }^{14} \mathrm{C}\right)$ and ${ }^{230} \mathrm{Th} / \mathrm{U}$ ages and ${ }^{234} \mathrm{U} /{ }^{238} \mathrm{U}$ ratios of fracture minerals from zones with elevated ${ }^{36} \mathrm{Cl} / \mathrm{Cl}$ ratios in the northern ESF are indistinguishable from those of secondary minerals outside these zones (Paces et al. 2001, p. 20, Figures 11, 14, and 16).

\subsection{PREVIOUS STUDIES OF OTHER BOMB-PULSE ISOTOPES}

Following the identification of elevated ${ }^{36} \mathrm{Cl} / \mathrm{Cl}$ ratios in the ESF, studies using other isotopes related to thermonuclear weapons testing were initiated to substantiate the bomb-pulse interpretation. Both ${ }^{14} \mathrm{C}$ and ${ }^{3} \mathrm{H}$ were produced during atmospheric testing of nuclear devices and have been analyzed in a variety of gas and water samples at Yucca Mountain (Yang et al. 1996, p. $25 ; 1998$, p. 16). The sporadic distribution of elevated concentrations of ${ }^{14} \mathrm{C}$ and ${ }^{3} \mathrm{H}$ in pore water samples from surface-based boreholes was interpreted as evidence of rapid transport of young waters to deeper parts of the UZ (Yang et al. 1996, p. 31; 1998, p. 16). More recent evaluations of the earlier pore water data have identified sampling and analytical problems with 
the ${ }^{14} \mathrm{C}$ and ${ }^{3} \mathrm{H}$ data sets. In a paper describing pore water travel times based on UZ gas data, Yang (2002, Section 4.1.2) concluded that ${ }^{14} \mathrm{C}$ concentrations reported in earlier studies "were not representative of the pore water residence time because of contamination by atmospheric ${ }^{14} \mathrm{CO}_{2}$ during drilling, resulting in apparently younger residence times." Yang (2002, Section 4.1.2) proposed using the depth-dependent variation of radiocarbon in the gas phase, which indicates that the average age of water at the repository level is several thousand years.

A re-evaluation of the analytical precision for analyses of ${ }^{3} \mathrm{H}$ in pore water produced in the USGS Yucca Mountain Project Branch (YMPB) laboratory in Denver (DTN: GS030508312272.004 [UQ]) resulted in a 22 to 31 tritium unit (TU) detection limit for reliability of significance above background levels. A similar "cutoff" for bomb-pulse values of $25 \mathrm{TU}$ was obtained by statistical analysis of previous ${ }^{3} \mathrm{H}$ results (CRWMS M\&O 2000, p. 60 and Figure 30). This larger value reduces the number of analyses that may be interpreted to indicate the presence of modern water.

Bomb-pulse technetium-99 $\left({ }^{99} \mathrm{Tc}\right)$ was detected in soil and rock samples from the shallow UZ, including samples of Bow Ridge fault gouge exposed in the ESF and cuttings from borehole USW UZ-N55. High ${ }^{36} \mathrm{Cl} / \mathrm{Cl}$ ratios also were detected in cuttings from USW UZ-N55; however, the elevated ${ }^{36} \mathrm{Cl} / \mathrm{Cl}$ ratios in USW UZ-N55 cuttings were suspected to have resulted from ${ }^{36} \mathrm{Cl}$ contamination from equipment used elsewhere on the Nevada Test Site (Fabryka-Martin and Liu 1995, Section 3.1.3; Fabryka-Martin, Turin et al. 1996, Sections 4.3.3 and 5.3.1; Fabryka-Martin et al. 1997, Section 6.2.2).

In addition, measurable levels of cesium-137 $\left({ }^{137} \mathrm{Cs}\right)$ were detected in three soil samples (0-5 centimeters [cm]) from the Midway Valley soil pits, located east of Yucca Mountain, but ${ }^{137} \mathrm{Cs}$ was not detected in a soil sample $(0-40 \mathrm{~cm})$ from the USW NRG-5 drill pad, located north of the ESF north ramp. Plutonium was detected in two soil samples (one from Midway Valley and the other from the USW NRG-5 drill pad), but plutonium was not detected in the fault gouge sample and was not analyzed for in the cuttings. These results were interpreted to indicate the immobility of cesium and plutonium in surface sediments at Yucca Mountain, limiting their use as ground-water tracers (Fabryka-Martin et al. 1997, p. 6-13, and Fabryka-Martin, Wolfsberg et al. 1996, Table 6-1).

\subsection{PEER REVIEW OF CHLORINE-36 STUDIES}

In January 1998, DOE convened a formal peer review of the ${ }^{36} \mathrm{Cl}$ and related investigations at Yucca Mountain. The Peer Review Team was tasked with reviewing the existing ${ }^{36} \mathrm{Cl}$ reports in the context of the UZ flow and transport models; evaluating the sampling approach and locations; evaluating the adequacy of the analytical approach, including the precision and accuracy of the data; and evaluating the adequacy of interpretations of ${ }^{36} \mathrm{Cl}$ and other isotope data in the context of conceptual UZ flow models. The Peer Review Team identified five major issues (YMP 1998, Section 3.2):

- Whether the bomb-pulse ${ }^{36} \mathrm{Cl} / \mathrm{Cl}$ values are real [presumably the Peer Review Team was concerned about the large ${ }^{36} \mathrm{Cl} / \mathrm{Cl}$ values], 
- Whether ${ }^{36} \mathrm{Cl} / \mathrm{Cl}$ distributions can be explained by variations in source strength with time or by mixing of waters with different ${ }^{36} \mathrm{Cl} / \mathrm{Cl}$ ratios,

- Whether ${ }^{36} \mathrm{Cl}$ anomalies are an artifact of sampling and analysis,

- Whether there is adequate integration of ${ }^{36} \mathrm{Cl}$ and other environmental tracer programs to achieve a consistent conceptual model of the UZ flow system, and

- Whether results of ${ }^{36} \mathrm{Cl}$ and other environmental tracers are effectively integrated with conceptual and numerical flow models.

The Peer Review Team concluded that bomb-pulse sources were currently the only plausible explanation for the elevated ${ }^{36} \mathrm{Cl} / \mathrm{Cl}$ values observed in the ESF (YMP 1998, Section 4.1). Contributions from other sources, primarily spallation of ${ }^{40} \mathrm{Ca}$ in surficial calcrete, were considered and dismissed. The Team also evaluated the possibility that ${ }^{36} \mathrm{Cl}$ anomalies might be artifacts of sampling and analytical practices (YMP 1998, Section 3.5) and included discussions on sample collection, extraction of chloride, and corrections to chloride and ${ }^{36} \mathrm{Cl}$ measurements. The Team accepted the conclusion that bomb-pulse ${ }^{36} \mathrm{Cl}$ entered the ground-water system through infiltration (YMP 1998, Section 3.3.2). Field and/or laboratory contamination as a source for the elevated ${ }^{36} \mathrm{Cl} / \mathrm{Cl}$ values was considered in a general sense and the Team did not see obvious evidence or "red flags" to indicate that contamination was an issue. However, the Team did acknowledge that contamination was not a primary focus of their review and it was not examined in detail (Coleman 2005).

The Peer Review Team recognized the limitations of using a single isotopic tracer to identify paths of rapid flow in the $\mathrm{UZ}$ and recommended coordination of ${ }^{36} \mathrm{Cl} / \mathrm{Cl}$ studies with studies of other isotopes and environmental tracers, including ${ }^{3} \mathrm{H}$, deuterium $\left({ }^{2} \mathrm{H}\right)$, oxygen-18 $\left({ }^{18} \mathrm{O}\right),{ }^{14} \mathrm{C}$, strontium-87/strontium-86 $\left({ }^{87} \mathrm{Sr} /{ }^{86} \mathrm{Sr}\right.$ ), and ${ }^{99} \mathrm{Tc}$ (YMP 1998, Section 3.6). The Team emphasized the importance of evaluating ${ }^{3} \mathrm{H}$ data relative to ${ }^{36} \mathrm{Cl} / \mathrm{Cl}$ ratios, but also recognized the difficulties in interpreting the ${ }^{3} \mathrm{H}$ results (YMP 1998, Section 3.6.2). In particular, the Team discussed the potential for obtaining false positive values (elevated ${ }^{3} \mathrm{H}$ values not related to fast-path fracture flow) through contamination with air from tunnel or drilling activities. Finally, the Peer Review Team recommended continuation of the ${ }^{36} \mathrm{Cl}$ studies, with suggestions on sampling strategies and integration with other isotopic and environmental tracer methods (YMP 1998, Section 4.2).

In response to the recommendations of the Peer Review Team, the USGS conducted ${ }^{3} \mathrm{H}$ analyses of pore water, $\mathrm{Sr}$ isotope analyses of pore water and pore-water salts, and uranium isotopic $\left({ }^{234} \mathrm{U} /{ }^{238} \mathrm{U}\right)$ analyses of bulk rock samples within and outside of fracture zones. Results of ${ }^{3} \mathrm{H}$ study are given elsewhere in this report (Section 5). The strontium and uranium isotopic analyses yielded equivocal results with regard to the identification of potential fast flow pathways, and the analytical data are not included in this report. All of the bulk rock samples exhibited a small depletion of approximately 5 percent in ${ }^{234} U$ relative to the secular equilibrium value of unity for ${ }^{234} U{ }^{238} U$, with no significant differences between samples collected in areas of elevated ${ }^{36} \mathrm{Cl} / \mathrm{Cl}$ and those collected elsewhere in the ESF (Gascoyne et al. 2002, p. 788). Similarly, strontium-isotope ratios of pore water and pore-water salts from different locations were in the same range regardless of associated differences in ${ }^{36} \mathrm{Cl} / \mathrm{Cl}$ values (Marshall and Futa 2003, p. 375). 
INTENTIONALLY LEFT BLANK 


\section{DESIGN AND IMPLEMENTATION OF THE VALIDATION STUDY}

Because of the potential impact of ${ }^{36} \mathrm{Cl}$ data on conceptual models of $\mathrm{UZ}$ flow and transport, DOE asked the USGS to design and implement an independent validation study. With support from the Yucca Mountain Project Management and Test Coordination Office, scientists from the USGS, LLNL, and AECL drafted a proposal that was submitted to DOE in January 1999. Collection of new data was part of the validation study, and members of the validation study team were granted wide latitude in the design of the field work and laboratory experiments. The Center for Accelerator Mass Spectrometry (CAMS) at LLNL was charged with processing and analyzing the new samples for ${ }^{36} \mathrm{Cl} / \mathrm{Cl}$ ratios.

Following recommendations of the ${ }^{36} \mathrm{Cl}$ Peer Review Team, the use of other isotopic tracers was viewed as an essential part of the validation study. Finding elevated concentrations of ${ }^{3} \mathrm{H}$ would support the interpretation of fast-paths based on elevated ${ }^{36} \mathrm{Cl} / \mathrm{Cl}$ ratios. However, substantial improvements in analytical sensitivity were required in the ${ }^{3} \mathrm{H}$ measurements for this method to be useful. Laboratory capabilities for water extraction by vacuum distillation were well established (Yang et al. 1998, p. 25). Samples of extracted pore water were sent to the University of Miami Rosenstiel School of Marine and Atmospheric Science Tritium Laboratory for ${ }^{3} \mathrm{H}$ analysis following enrichment by electrolysis. Tritium sampling and analysis are described in Section 5 of this report.

LANL's participation in the validation study included measuring ${ }^{36} \mathrm{Cl} / \mathrm{Cl}$ ratios in a few of the validation study samples. Leachates of core samples from seven validation study boreholes were analyzed prior to 2000 under the same conditions as the previous LANL ${ }^{36} \mathrm{Cl}$ studies. However, by the Fall of 2000 , substantial changes had occurred in the LANL ${ }^{36} \mathrm{Cl}$ program (Table $3-1$ ). Damage caused by the Cerro Grande fire in the Spring of 2000 necessitated lengthy shutdowns and relocation of laboratory facilities. In February 2001 , the ${ }^{36} \mathrm{Cl}$ laboratory was moved from its previous location in Technical Area 48 (Radiochemistry Site) to a laboratory in Technical Area 3 (Geochemistry and Geomaterials Research Laboratories, SM494, Room 107). The new laboratory was located in a general geosciences facility designated as a non-radiological facility. Sample processing in the new laboratory began in March 2001, and all subsequent analyses of validation study samples were conducted there. In this report, $\mathrm{LANL}{ }^{36} \mathrm{Cl} / \mathrm{Cl}$ data collected prior to 2000 are generally considered to be from the previous ${ }^{36} \mathrm{Cl} / \mathrm{Cl}$ studies, and data collected during and after 2000 are considered to be part of the ${ }^{36} \mathrm{Cl}$ validation study.

Initially, LANL's participation in the validation study was not fully integrated with other parts of the study. However, from 2000 on, LANL scientists coordinated more closely with the other validation study participants, to include analyses of the same leachates and crushed materials. This coordination was ultimately critical for producing a better understanding of the conflicting results obtained by the different investigators.

\subsection{DESIGN OF SAMPLING PROTOCOL}

Difficulties in replicating elevated ${ }^{36} \mathrm{Cl} / \mathrm{Cl}$ ratios in ESF samples led to the hypothesis that the elevated ${ }^{36} \mathrm{Cl}$ is inhomogeneously distributed in fractured rock (CRWMS M\&O 1998, p. 3-3). Therefore, for the validation study, attempts to replicate the previous analyses were based on the likelihood of finding elevated values along reaches of the ESF where numerous occurrences 
were identified by the previous analyses. Thus, the fundamental assumption of the validation study was that a sufficiently detailed re-sampling of the same areas should yield a similar proportion of elevated ${ }^{36} \mathrm{Cl} / \mathrm{Cl}$ values.

Elevated ${ }^{36} \mathrm{Cl} / \mathrm{Cl}$ ratios were reported in multiple samples from two intervals in the northern ESF. These are associated with the Drill Hole Wash fault (Figure 3-1), between ESF stations 18+96 and 19+42, and the Sundance fault (Figure 3-2), between ESF stations 34+28 and 35+93. Both intervals include northwest-trending strike-slip faults exposed in tunnel walls and in surface exposures on the east slope of Yucca Mountain. Of the seven analyses from five samples collected previously from the $100-\mathrm{m}$ interval including the Drill Hole Wash fault, five of the analyses yielded ${ }^{36} \mathrm{Cl} / \mathrm{Cl}$ values greater than the bomb-pulse threshold of $1,250 \times 10^{-15}$, with a sixth analysis very near the bomb-pulse threshold $\left(1,144 \times 10^{-15}\right)(1,880$ to $1,980 \mathrm{~m}$; Figure 3-1). From the nine samples collected at and north of the Sundance fault $(3,428$ to 3,593 m; Figure 32), 11 of 16 analyses had ${ }^{36} \mathrm{Cl} / \mathrm{Cl}$ values greater than $1,250 \times 10^{-15}$. In addition, eight of 15 analyses of samples associated with Niche \#1, which was constructed to access the Sundance fault, had ${ }^{36} \mathrm{Cl} / \mathrm{Cl}$ values greater than $1,250 \times 10^{-15}$. Five analyses from four samples of the walls of Niche \#1 had values between $540 \times 10^{-15}$ and $659 \times 10^{-15}$; whereas, eight of 10 samples obtained from boreholes drilled along the axis of the niche prior to excavation, or from the end of Niche \#1 toward the Sundance fault, yielded ${ }^{36} \mathrm{Cl} / \mathrm{Cl}$ values greater than $1,250 \times 10^{-15}$. A ninth sample had a ${ }^{36} \mathrm{Cl} / \mathrm{Cl}$ value of $1,235 \times 10^{-15}$ (CRWMS M\&O 1998, p. 3-4, Table 3-4). Because of these elevated values, the Drill Hole Wash fault zone and Sundance fault zone were targeted for validation study sample collection.

Most of the previous samples had been collected from tunnel walls shortly after excavation, between 1995 and 1997. Re-sampling of tunnel walls for the validation study was not desirable because chloride may have been lost when tunnel walls were washed and (or) if the tunnel walls were contaminated with ${ }^{36} \mathrm{Cl}$-enriched dust brought into the ESF by the ventilation system. Instead, core was sampled from 4- and 10-m-long dry-drilled boreholes spaced along the right rib (side) of the ESF at approximately 5-m intervals. Fifty new boreholes were sited across the two zones ( 10 boreholes from the Drill Hole Wash fault zone and 40 from the Sundance fault zone; Table 3-2 and Figures 3-1 and 3-2). One advantage of using a borehole sampling approach is the probability that the deeper core intervals extend beyond the zone of penetration of construction water and ventilation-induced dry-out. Therefore, the deeper intervals could be used for water extraction and ${ }^{3} \mathrm{H}$ analyses as an independent indicator of a bomb-pulse component.

Selection of sampling sites for the validation study differed from that of the previous studies, which had been based on two sampling approaches (Fabryka-Martin et al. 1996, p. 1-3). The first, referred to as "feature-based" sampling, targeted specific features such as faults, fractures, and cooling joints. These samples were collected to maximize the surface area of the targeted feature. Of the 234 feature-based samples, 35 (15 percent) had bomb-pulse ${ }^{36} \mathrm{Cl}$ values. The second sampling approach, referred to as "systematic sampling," consisted of sampling sites at $200-\mathrm{m}$ intervals between stations $5+00$ and $59+00$. The spacing was later reduced to $100-\mathrm{m}$ intervals from stations $59+00$ to $69+00$ and stations $69+50$ to $76+50$ (Fabryka-Martin et al. 1997, p. 55). According to Fabryka-Martin et al. (1997, p. 55), "The systematic sampling was designed to acquire isotopic data unbiased by any other selection criteria. These samples 
represent the rock matrix and whatever fracture fabric typifies the collection site." Of the 54 systematic samples, two (4 percent) had bomb-pulse ${ }^{36} \mathrm{Cl}$ values.

The validation study boreholes also were spaced systematically, but the spacing was on 5-m centers over the areas of interest rather than $100 \mathrm{~m}$, and at least $4 \mathrm{~m}$ of rock were penetrated. Sampling for previous work typically penetrated only a few tens of centimeters into the tunnel walls. Prior to drilling for the validation study, the original tunnel-wall sample sites were examined and the conclusion was reached that, given the number of boreholes that were to be drilled, the fracture density, and the amount of rock sampled by the boreholes, the validation study would have a high probability of accessing potential zones of fast flow. To evaluate the validation study sampling plan, fracture density data for the ESF were examined (Figure 3-3). These data were obtained by documenting individual fractures and cooling joints with traces on the tunnel wall greater than $1 \mathrm{~m}$. The two validation study target zones are characterized by distinctly different fracture densities. Fracture density data can be converted to fracture spacing along the detailed line surveys by measuring distances between successive fractures intersecting the survey line. Distributions of fracture spacing are given in Figure 3-4A for the tunnel around the Drill Hole Wash fault (ESF stations $16+00$ to $21+00$ ) and Figure 3-5A for the Sundance fault (ESF station $34+00$ to $36+00$ ). For both zones, fracture spacing is strongly skewed, with the largest frequencies having the shortest spacings. The median values for fracture spacings are $0.78 \mathrm{~m}$ for the Drill Hole Wash fault zone and $0.15 \mathrm{~m}$ for the Sundance fault zone. Because of the skewed distributions, arithmetic means are inappropriate. However, values for the $\log _{10}$ of the fracture spacings are more normally distributed and give geometric means closer to the medians (Figures 3-4B and 3-5B). These data, along with the variable fracture orientations, indicate that the 4-m-long validation study boreholes should have intersected multiple (between about 5 and 27) fractures with trace lengths greater than $1 \mathrm{~m}$. In addition to these fractures, short-trace-length fractures with trace lengths less than $1 \mathrm{~m}$ are locally important geologic and hydrologic features (Sweetkind et al. 1998, p. S231). Because short-trace-length fractures were excluded from detailed line surveys, true fracture densities throughout the ESF are underestimated by the evaluation shown in Figures 3-4 and 3-5, with the greatest disparities observed in lithophysal units (Sweetkind et al. 1998, p. S231). Thus, abundant fractures were expected in the validation study boreholes in the Sundance fault zone.

The validation study sampling approach was further supported by earlier results obtained from leachates of core samples collected from the Sundance fault zone. Elevated ${ }^{36} \mathrm{Cl} / \mathrm{Cl}$ values between $1,235 \times 10^{-15}$ and $2,038 \times 10^{-15}$ were obtained for eight of 10 samples from different intervals from three boreholes associated with Niche \#1 (boreholes ESF-MD-NICHE3566\#1, \#2, \#LT in Appendix A). Therefore, although the previously analyzed sites would not be re-sampled for the validation study, it was expected that a statistically significant percentage of the validation study analyses would contain bomb-pulse ${ }^{36} \mathrm{Cl}$.

\subsection{DESCRIPTION AND ALLOCATION OF VALIDATION STUDY CORE}

Fifty validation study boreholes were drilled between mid-March 1999 and early-October 1999. Drilling activities were conducted by the Yucca Mountain Project Management and Test Coordination Office and core documentation, preservation, and handling were performed by the Sample Management Facility. (SMF) in accordance with NWI-DS-001Q, Field Logging, Handling, \& Documenting Borehole Samples. Core intervals deeper than $2 \mathrm{~m}$ in each borehole 
were preserved for pore water extraction by packaging in Lexan® sleeves sealed inside ProtecCore ${ }^{\mathrm{TM}}$ after video logging each core run.

The video logs were examined and the core was classified on the basis of core recovery and fracturing. This classification was intended to identify zones with the greatest amounts of fracturing, which were then selected for further analysis. Assignment of mechanical classes of core was intended as a qualitative measure of the degree of fracturing and included descriptors such as "intact," "broken," "rubbly," and "shattered," in order of increasing fracture intensity (Paces 2003). Results of video logging are included in Appendix B. Most core is classified as broken to rubbly, indicating core fragments are generally less than about $7 \mathrm{~cm}$ (broken) to $2 \mathrm{~cm}$ (rubble or rubbly). These observations are consistent with the fracture densities determined from the detailed line surveys and measurements of short-trace-length fractures (Section 3.1).

The video logs formed the basis for distribution of core intervals to LLNL, USGS, and AECL. Core intervals were selected from the deeper half of the borehole to avoid both dry-out and contamination with construction water. To provide sufficient chloride for ${ }^{36} \mathrm{Cl}$ analyses, and water for ${ }^{3} \mathrm{H}$ analyses, core intervals of approximately $60 \mathrm{~cm}$ were selected. LLNL received the core with the greatest fracture densities, providing the greatest probability of including a flow path containing bomb-pulse ${ }^{36} \mathrm{Cl}$. Although samples for ${ }^{3} \mathrm{H}$ analyses may have contained fewer fractures, core intervals from the deepest parts of the boreholes were selected to minimize the effects of dry-out. Core intervals from intermediate depths $(1.2$ to $2.0 \mathrm{~m})$ in boreholes in the Sundance fault zone were selected for ${ }^{36} \mathrm{Cl} / \mathrm{Cl}$ analysis at LANL. All core intervals were distributed from the SMF shortly after the boreholes were completed.

In addition to the 50 new validation study boreholes (Figures 3-1 and 3-2; Table 3-2), samples of existing core were obtained from the same three Niche \#1 boreholes that had been analyzed previously (ESF-MD-NICHE3566\#1, ESF-MD-NICHE3566\#2, and ESF-MD-NICHE3566LT\#1). These intervals were originally requested from the SMF for ${ }^{36} \mathrm{Cl} / \mathrm{Cl}$ analyses shortly after the boreholes were completed in 1997 . Core selected for validation study analyses had remained unopened in the original SMF packaging. The 41 intervals available for the validation study were distributed between the USGS and LANL. The approximate locations of these three boreholes relative to the ESF main drift and Sundance fault are shown in Figure 3-6 (Source: USGS 1996). Because individual intervals were generally too small to supply sufficient chloride for reliable ${ }^{36} \mathrm{Cl}$ measurements, multiple intervals were combined into six samples leached at the USGS and five samples leached at LANL. Two of the LANL samples were further subdivided into coarser (6.3 to 12.5 millimeters [mm]) and finer (less than $6.3 \mathrm{~mm}$ ) fractions, resulting in a total of seven leachate analyses. These combined samples were selected to provide at least some overlap of core intervals from each borehole to facilitate a more-or-less direct comparison between USGS and LANL validation study analyses (Table 3-3 and Figure 3-7), as well as comparison between validation study results and results reported previously by LANL (CRWMS M\&O 1998, Table 3-4).

To determine whether the method of crushing affected the release of chloride during leaching, samples from borehole ESF-SAD-GTB\#1 (southern Ghost Dance fault, Alcove \#7) were crushed by hand with a hammer and steel plate, and by jaw crusher. Three samples were screened to the same particle size and leached for the same length of time. Core from ESF-SAD-GTB\#1 was selected for the crushing experiments because it was similar to the validation study core (i.e., 
both the validation study boreholes and ESF-SAD-GTB\#1 were drilled in the crystal-poor, middle nonlithophysal unit of the Topopah Spring Tuff), and because a large amount was available to the USGS in Denver. Experimental methods and results of the crushing experiments are described in Section 4.4.1.2. 
INTENTIONALLY LEFT BLANK 


\section{CHLORINE-36 MEASUREMENTS}

The validation study proceeded in three phases, beginning in late-1999 and continuing through late-2002. In Phase I, ${ }^{36} \mathrm{Cl}$ experiments were conducted at LLNL, including crushing, leaching, silver chloride $(\mathrm{AgCl})$ target preparation, and isotope analysis. Concurrent with the work at LLNL, several samples of the validation study core were analyzed at LANL in accordance with the standard analytical procedures used previously by LANL. Results from the two sets of experiments differed significantly. The active-leach protocol used by LLNL during this phase of the investigation resulted in anomalously large chloride concentrations and low ${ }^{36} \mathrm{Cl} / \mathrm{Cl}$ ratios compared to the LANL results for the validation study core and previous LANL results for tunnel-wall samples. This prompted a halt in ${ }^{36} \mathrm{Cl}$ data-collection activities and initiation of Phase II of the study to evaluate leaching protocols that would maximize the probability of identifying a meteoric chloride component. Leaching experiments were conducted on systematic and feature-based samples collected previously by LANL from the ECRB Cross Drift. Results of these experiments indicated that the release of rock chloride was minimized by passive-leach methods and that most of the meteoric chloride components were liberated after short leaching times. A final 1-hour passive-leach protocol was then adopted by all the study participants for Phase III of the validation study. In Phase III, responsibility for crushing and leaching validation study samples shifted to the USGS and LANL, although LLNL-CAMS and PRIME Lab continued to analyze the new samples. Details of the procedures used and results obtained are given in the following sections.

\subsection{PHASE I: MEASUREMENTS MADE AT LLNL}

\subsubsection{Methods}

An active-leach approach was used by LLNL during Phase I of the validation study to provide a repeatable process for extracting chloride from Yucca Mountain tuffs. The procedure involved mechanical crushing and sieving of samples to a 1- to 2-cm size fraction. Between 1.4 and $3.0 \mathrm{~kg}$ of rock were combined with 1.3 to 1.7 times that weight of de-ionized water. The mixture was placed in a stainless-steel tumbler and allowed to rotate slowly for 7 hours.

The resulting slurry was decanted from the tumbler into a stack of $150-$ to $38-\mu \mathrm{m}$ stainless steel sieves. This solution was filtered using vacuum flasks fitted with a series of filters of decreasing pore size $(25,8,0.8,0.45$, and $0.22 \mu \mathrm{m})$. Chloride was precipitated from this final, clear solution following the chemical procedures described in Appendix C. The resulting $\mathrm{AgCl}$ target was analyzed for ${ }^{36} \mathrm{Cl} / \mathrm{Cl}$ ratios by accelerator mass spectrometry (AMS) at the LLNL-CAMS facility. No procedural blanks were reported for this phase of the validation study.

\subsubsection{Results}

The active-leach method was used for 25 validation study core samples from the Sundance fault zone between ESF stations $33+89$ and $36+75$. Chloride concentrations and ${ }^{36} \mathrm{Cl} / \mathrm{Cl}$ ratios are given in Table 4-1 and plotted against borehole locations in the ESF in Figure 4-1. Chloride concentrations varied between 1.25 and $3.54 \mathrm{mg} / \mathrm{kg}$, with a median value of $2.13 \mathrm{mg} / \mathrm{kg}$ rock and a mean of $2.07 \pm 1.24 \mathrm{mg} / \mathrm{kg}$ rock (Figure $4-2 \mathrm{~A}$ ). ${ }^{36} \mathrm{Cl} / \mathrm{Cl}$ ratios range between $48 \times 10^{-15}$ and 
$248 \times 10^{-15}$, although all values but one are less than $156 \times 10^{-15}$. The median value for all 25 samples is $88 \times 10^{-15}$, and the mean is $97 \pm 86 \times 10^{-15}$ (Figure 4-2B).

Isotope ratios are commonly plotted against the reciprocal of the concentration values so that binary mixing relations are linear (Faure 1986, p. 142). On such a plot the data form a diffuse cluster with a positive slope $\left(R^{2}\right.$ value of 0.2 if sample ESF-SD-CIV\#32, with a ratio of $248 \times 10^{-15}$, is excluded), showing that leachates with higher chloride concentrations tend to have lower ${ }^{36} \mathrm{Cl} / \mathrm{Cl}$ ratios (Figure 4-3A). Results of the active-leach experiments performed at LLNL differ from the results of passive-leach experiments conducted previously at LANL (Figure 4-3B). Chloride concentrations in the 25 active leachates reported in Table 4-1 are within the range of values obtained earlier by LANL, although the median of $2.1 \mathrm{mg} / \mathrm{kg}$ for active leachates is higher than the median of $1.0 \mathrm{mg} / \mathrm{kg}$ rock calculated for the 293 passive leachates reported by LANL (Appendix A). (Note: Ten of the samples listed in Appendix A were not analyzed for chloride concentrations). The ${ }^{36} \mathrm{Cl} / \mathrm{Cl}$ values of the two data sets plot in distinct fields, with very little overlap. The median ${ }^{36} \mathrm{Cl} / \mathrm{Cl}$ value for the active leachates is $85 \times 10^{-15}$, whereas the median value for the passive leachates is $569 \times 10^{-15}$. The median value for the passive leachates, excluding the 47 samples with ${ }^{36} \mathrm{Cl} / \mathrm{Cl}$ at or over the $1,250 \times 10^{-15}$ bomb-pulse threshold, is only slightly lower $\left(531 \times 10^{-15}\right)$.

Roback et al. (2002, p. 235) demonstrated that active leaching methods released a greater proportion of rock chloride relative to meteoric chloride, thus yielding smaller ${ }^{36} \mathrm{Cl} / \mathrm{Cl}$ ratios than obtained by passive leaching methods. Similarly, measurements of chloride concentrations and ${ }^{36} \mathrm{Cl} / \mathrm{Cl}$ ratios in leachates of powdered rock samples after most of the meteoric chloride components had been removed resulted in large chloride concentrations $(7.6$ to $17.6 \mathrm{mg} / \mathrm{L})$ and small ${ }^{36} \mathrm{Cl} / \mathrm{Cl}$ values $\left(43 \times 10^{-15}\right.$ to $57 \times 10^{-15}$ ) (Fabryka-Martin, Wolfsberg et al. 1996, Table 5-4). These ${ }^{36} \mathrm{Cl} / \mathrm{Cl}$ values were interpreted to reflect ${ }^{36} \mathrm{Cl}$ produced in situ through neutron capture by stable chlorine-35 $\left({ }^{35} \mathrm{Cl}\right.$ ) (Fabryka-Martin, Turin et al. 1996, Section 4.4.3). The relation of measured rock chloride values along a projection of the regression line for the active-leach data (Figure 4-3A) provides a strong indication that the active-leach method is too aggressive and extracts too much rock chloride, which masks the meteoric chloride component.

\subsection{PHASE I: MEASUREMENTS MADE AT LANL}

\subsubsection{Methods}

Methods used by LANL for the ${ }^{36} \mathrm{Cl}$ validation study involved crushing, leaching, and chemical processing procedures similar to those used in previous LANL ${ }^{36} \mathrm{Cl}$ studies (Fabryka-Martin, Turin, et al. 1996, Section 3; Fabryka-Martin et al. 1997, Section 4; CRWMS M\&O 1998, Section 2.3). No procedural blanks were reported for this phase of the validation study.

\subsubsection{Results}

Core samples from the Sundance fault zone were selected by LANL for analysis as oversight to the active-leach experiments performed at LLNL. Chloride concentrations in these leachates are uniform, ranging from 0.23 to $0.35 \mathrm{mg} / \mathrm{kg}$ rock (Table 4-2). Measured ${ }^{36} \mathrm{Cl} / \mathrm{Cl}$ ratios range from $508 \times 10^{-15}$ to $942 \times 10^{-15}$, with no values exceeding the $1,250 \times 10^{-15}$ bomb-pulse threshold. 
These values are similar to other northern ESF samples analyzed at LANL prior to 2000 (Appendix A).

\subsection{PHASE II: LEACHING EXPERIMENTS}

Phase I results from active leaching at LLNL and passive leaching at LANL were presented at the May 1, 2000, meeting of the Nuclear Waste Technical Review Board (NWTRB) in Pahrump, Nevada. The large discrepancies in ${ }^{36} \mathrm{Cl} / \mathrm{Cl}$ values between the two data sets were debated and led to a letter from the NWTRB to the Director of the DOE OCRWM (Cohon 2000), urging that high priority be given to resolution of the disagreements. In response, the validation study participants agreed that additional work was necessary to identify a standardized leaching procedure for extracting labile meteoric chloride and minimizing releases of rock chloride.

To accommodate this work, a large sample of tuff with homogenous chloride was required for a reference sample so that comparable splits could be distributed to LANL and LLNL for leaching experiments. The ${ }^{36} \mathrm{Cl} / \mathrm{Cl}$ composition of this reference sample was not critical and could be a mixture of chloride from meteoric, bomb-pulse, or construction-water sources, as long as the mixture was uniformly distributed throughout the material. The reference sample would then be used to test the effects of leaching methods, leaching times, and particle sizes. Due to changes in personnel at LLNL during this period, no leaching experiments were conducted at LLNL.

In addition to the leaching experiments conducted at LANL, leaching experiments were also conducted at AECL to determine the distribution of chloride in validation study core samples associated with the Sundance fault zone. The goal of this work was to understand the sources and locations of chloride (and, by extension, ${ }^{36} \mathrm{Cl}$ ) in the tuff and to determine whether the difficulties in reproducing ${ }^{36} \mathrm{Cl} / \mathrm{Cl}$ ratios could be explained in terms of the sample treatment processes used. These analyses produced chloride concentrations but did not determine ${ }^{36} \mathrm{Cl} / \mathrm{Cl}$ ratios in the leachates.

\subsubsection{Preparation of the Reference Sample}

The USGS worked with the Yucca Mountain Project Management and Test Coordination Office to identify and collect a large rock sample that could be used as the reference sample (referred to as "EVAL001" by LANL). The sample (SPC00557088) consisted of two 55-gallon drums of coarse muck collected from the discharge end of the Alpine miner during construction of Niche \#5 in the ECRB Cross Drift (Figure 1-1). Niche \#5 is located within the lower part of the TSw.

The muck was shipped to Phillips Enterprises, LLC, of Golden, Colorado, where it was removed from the shipping containers, spread out on clean plastic tarps, and allowed to air-dry over a 3-day period prior to processing. The muck was then stage-crushed using a jaw crusher and screened to recover the maximum quantity from the $6.3-$ to $12.5-\mathrm{mm}$ size fraction. Approximately $136.1 \mathrm{~kg}$ of crushed and sized rock was produced in this manner, after which it was homogenized by hand mixing. The sized material was then split into ten $13.61-\mathrm{kg}$ subsamples, and each was given a final blow-down with compressed dry nitrogen to remove dust adhering to rock surfaces. Blow-down was conducted on a vibrating screen to promote 
maximum dust removal. Each sub-sample was placed in a polyethylene bag, sealed, and stored in a plastic-lined 55-gallon drum.

\subsubsection{Leaching Experiments Conducted at LANL}

During Phase II of the validation study, LANL performed a series of experiments using EVAL001 and several samples from the ECRB Cross Drift to determine the effects of leaching time, leaching method, and particle size on the release of chloride and the resulting differences in ${ }^{36} \mathrm{Cl} / \mathrm{Cl}$ ratios. The goals of these experiments were to identify the processing method that would be most effective in identifying a bomb-pulse ${ }^{36} \mathrm{Cl} / \mathrm{Cl}$ component if one is present, and to provide information to evaluate previous ${ }^{36} \mathrm{Cl} / \mathrm{Cl}$ data from the Yucca Mountain UZ.

Substantial changes occurred in the LANL ${ }^{36} \mathrm{Cl}$ program between Phases I and II of the validation study. In the Fall of 2000, a new principal investigator assumed the lead role for the Yucca Mountain ${ }^{36} \mathrm{Cl}$ studies. Personnel responsible for sample processing also changed by October 2001, after a 2-month overlap. In February 2001, the LANL ${ }^{36} \mathrm{Cl}$ laboratory was moved from its previous location in Technical Area 48 (Radiochemistry Site) to a laboratory in Technical Area 3 (Geochemistry Analytical Facility, SM494, Room 107). The new laboratory is located in a general geosciences laboratory facility designated as a non-radioactive facility. The laboratory was cleaned prior to relocating the ${ }^{36} \mathrm{Cl}$ laboratory equipment. Sample processing in the new laboratory began in March 2001 and all subsequent analyses for Phases II and III of the validation study were conducted in this laboratory. Although many of the methods used in Phases II and III were the same as those used previously by LANL, some changes were made to accommodate changing objectives of the project. Methods related to establishing a standard leaching protocol during Phase II are described below. Methods related to sample processing during Phases II and III are described in Section 4.5.1.

\subsubsection{Methods}

LANL patterned the leaching experiments after work that was done at LANL between July and December 2000. EVAL001 was split into aliquots using a geotechnical sample splitter. Some of these aliquots were crushed further to investigate the effects of particle size on leaching. Crushed aliquots were sized using an Endecotts ${ }^{\circledR}$ EFL2 mk3 Test Sieve Shaker to obtain subsamples of uniform particle-size range. Portions of some samples were pulverized to a fine powder in a pre-cleaned Bico ${ }^{\circledR}$ shatter box to determine the chloride and bromide content of the rock.

Two aliquots of EVAL001 ( -7 and -11$)$ were passively leached by leaving the rock and leachate undisturbed during leaching. To determine whether vigorous agitation during leaching liberates additional chloride from the rock, 3 splits from EVAL001 $(-8,-9,-11)$ were actively leached by placing the rock fragments into a 2-L polyethylene bottle with a sub-equal weight of de-ionized water. The bottle was shaken in a horizontal position using a Glas-Col Apparatus Company® Shaker-in-the-Round Model S500 shaker. The shaker rotated the bottle laterally $32^{\circ}$ in 0.45 seconds, before returning it to its original position. The shaker was allowed to oscillate in this manner continuously for up to 7 days. Both active- and passive-leach splits were leached for $0.5,2.0,7.0$ and 76 to 165 hours. One active-leach sample was leached for $0.05-0.12$ hours. The mass of rock leached (after combining the actively leached samples) ranged from 2.961 to 
$5.044 \mathrm{~kg}$. These rock masses yielded a minimum of $0.44 \mathrm{mg}$ of chloride (not including chloride in the tracer or procedural blank) for analysis. Chloride isotopic analyses were performed at PRIME Lab.

LANL also performed sequential leaching experiments on six samples that were collected from the ECRB Cross Drift for the pre-2000 LANL ${ }^{36} \mathrm{Cl}$ studies (Table 4-3, samples with the prefix "EXD"). For these experiments, only the passive-leach method was used, with leaching times of $0.5,2,7$ and 48 hours. After each leaching period, the water was removed and replaced with new de-ionized water. One sample (EXD-069) was separated into three size fractions prior to leaching. The 6.3 - to $12.5-\mathrm{mm}$ size fraction was used for all other samples. Rock mass typically varied between 3 and $6 \mathrm{~kg}$. In all cases, this amount of material yielded a minimum of $0.3 \mathrm{mg}$ of rock chloride (not including chloride in the tracer or blank), and in most cases considerably more rock chloride (mean of $1.2 \mathrm{mg}$, maximum of $6.8 \mathrm{mg}$ chloride). Analyses of ${ }^{36} \mathrm{Cl} / \mathrm{Cl}$ ratios were performed at LLNL-CAMS.

\subsubsection{Results}

Chloride concentrations for aliquots of the two passive-leach samples (EVAL001-7 and EVAL001-11) range from $0.11 \mathrm{mg} / \mathrm{kg}$ rock to $0.25 \mathrm{mg} / \mathrm{kg}$ rock, with a mean of $0.16 \mathrm{mg} / \mathrm{kg}$ rock for all aliquots (Table 4-3). Chloride concentrations for the active-leach splits (EVAL001-8, -9, -10) are larger, ranging from $0.15 \mathrm{mg} / \mathrm{kg}$ rock to $0.31 \mathrm{mg} / \mathrm{kg}$ rock, with a mean of $0.21 \mathrm{mg} / \mathrm{kg}$ rock. ${ }^{36} \mathrm{Cl} / \mathrm{Cl}$ ratios for the two passive-leach splits range from $492 \times 10^{-15}$ to $889 \times 10^{-15}$, with analytically indistinguishable means of $619 \times 10^{-15}$ for EVAL001-7 leachates and $585 \times 10^{-15}$ for EVAL001-11 leachates. In contrast, the ${ }^{36} \mathrm{Cl} / \mathrm{Cl}$ ratios for the active-leach splits are smaller than the passive-leach splits, with a range of $234 \times 10^{-15}$ to $501 \times 10^{-15}$ and a mean of $397 \times 10^{-15}$.

Relations between chloride concentration, ${ }^{36} \mathrm{Cl} / \mathrm{Cl}$ ratios, and time are plotted in Figures 4-4 and 4-5. These plots show the evolution of compositions with increasing leach duration and the differences in results obtained from passive and active leaching. Most passive-leach samples have smaller chloride concentrations, and all have larger ${ }^{36} \mathrm{Cl} / \mathrm{Cl}$ ratios for equivalent leaching times when compared to the active-leach samples. In all samples, the chloride concentration increases rapidly through the first 7 hours. Chloride concentrations remain constant or decrease in the longer leaches for passive-leach samples, whereas the active-leach sample shows continued increases in the release of chloride with increases in leaching time. Passive-leach samples have larger ${ }^{36} \mathrm{Cl} / \mathrm{Cl}$ ratios for equivalent leaching times compared to the active-leach sample. The largest ${ }^{36} \mathrm{Cl} / \mathrm{Cl}$ ratios were obtained in the shortest leaching time for both passiveleach samples. Passive leachates from EVAL001-7 show a consistent decrease in the ${ }^{36} \mathrm{Cl} / \mathrm{Cl}$ ratios over time, from a value of $889 \times 10^{-15}$ for the 0.5 -hour leach to a value of $493 \times 10^{-15}$ for the longest leach (Table 4-3). Data from both passive-leach EVAL001 samples converge to identical ${ }^{36} \mathrm{Cl} / \mathrm{Cl}$ ratios of approximately $575 \times 10^{-15}$ for cumulative values. Active leachates from EVAL001-8, -9 , and -10 have ${ }^{36} \mathrm{Cl} / \mathrm{Cl}$ ratios between $423 \times 10^{-15}$ and $501 \times 10^{-15}$ for the first 7 hours and a substantially smaller value of $234 \times 10^{-15}$ for the longest leaching time.

Chloride concentrations in sequential leachates of the 6.3- to 12.5-mm size fraction of ECRB Cross Drift samples varied considerably, with values ranging from $0.07 \mathrm{mg} / \mathrm{kg}$ rock to $0.66 \mathrm{mg} / \mathrm{kg}$ rock (Table 4-3). Chloride concentrations in leachates remained relatively constant for successive leaches of increasing durations in four of six samples (Figure 4-6A). As a result, 
chloride extraction rates are much greater for the initial leaches and decrease dramatically as leaching times exceed 7 hours. This is reflected in the flattening of cumulative chloride concentration curves with increased leaching time (Figure 4-6B).

Like chloride concentrations, ${ }^{36} \mathrm{Cl} / \mathrm{Cl}$ ratios show wide variations among samples, but much smaller variations for different leach durations of the same sample (Figure 4-7). ${ }^{36} \mathrm{Cl} / \mathrm{Cl}$ ratios range from $234 \times 10^{-15}$ to $924 \times 10^{-15}$ for the $6.3-$ to $12.5-\mathrm{mm}$ size fraction (Table 4-3). Most samples have relatively constant ${ }^{36} \mathrm{Cl} / \mathrm{Cl}$ ratios regardless of leach duration. Leachates of sample EXD-072 show a statistically significant change in ${ }^{36} \mathrm{Cl} / \mathrm{Cl}$ ratios as leaching progressed, with values decreasing from $924 \times 10^{-15}$ for the 0.5 -hour leach to more-or-less constant values between $676 \times 10^{-15}$ and $753 \times 10^{-15}$ in subsequent leaches. The opposite trend of small ${ }^{36} \mathrm{Cl} / \mathrm{Cl}$ ratios progressively increasing to larger values in subsequent samples was observed for EXD-049; however, these samples have large and overlapping analytical uncertainties. The other four samples show remarkably consistent ${ }^{36} \mathrm{Cl} / \mathrm{Cl}$ ratios throughout the entire 48-hour leach duration.

In an additional leaching experiment, one sample was used to evaluate the effects of different particle sizes on chloride concentrations and ${ }^{36} \mathrm{Cl} / \mathrm{Cl}$ ratios (sample EXD-069 in Table 4-3). A consistent pattern of leachable chloride concentrations was not observed for the size fractions used (Figure 4-8A). For the shortest leach duration ( 0.5 hour), the finest fraction (less than $2 \mathrm{~mm})$ had the smallest chloride concentration $(0.40 \mathrm{mg} / \mathrm{kg}$ rock), and the intermediate size fractions ( 2 to $6.3 \mathrm{~mm}$ ) had the largest chloride concentration $(0.99 \mathrm{mg} / \mathrm{kg}$ rock). However, the relatively constant chloride extracted from the coarsest fraction (6.3 to $12.5 \mathrm{~mm})$ over time resulted in the largest cumulative chloride concentration after 48 hours (Figure 4-8B).

Values of ${ }^{36} \mathrm{Cl} / \mathrm{Cl}$ in successive leaches of each size fraction also are nearly constant with leach duration (Figure 4-9). The finest size fraction has both the smallest chloride concentration and the largest ${ }^{36} \mathrm{Cl} / \mathrm{Cl}$ ratio in all sequential leachates, ranging from $317 \times 10^{-15}$ to $432 \times 10^{-15}$ (all values are within $2 \sigma$ error or very nearly so). The intermediate and coarse size fractions have smaller ${ }^{36} \mathrm{Cl} / \mathrm{Cl}$ ratios $\left(261 \times 10^{-15}\right.$ to $\left.297 \times 10^{-15}\right)$, which are distinguishable (within $2 \sigma$ error) from values for the fine fraction. Cumulative ${ }^{36} \mathrm{Cl} / \mathrm{Cl}$ ratios obtained over time for these samples are constant, indicating that ${ }^{36} \mathrm{Cl}$ and total chloride are extracted in the same proportions throughout the experiments.

\subsubsection{Discussion of Results}

Leaching experiments performed at LANL were designed to test the effects of leaching methods, leaching times, and particle size on the measured ${ }^{36} \mathrm{Cl} / \mathrm{Cl}$ ratios. Most ${ }^{36} \mathrm{Cl} / \mathrm{Cl}$ values for samples leached by the passive-leach method are consistent with derivation from the conceptualized sources listed in Table 4-4, involving salts precipitated from meteoric water less than $10 \mathrm{ka}$ or a mixture of salts less than $10 \mathrm{ka}$ and greater than $10 \mathrm{ka}$. Three samples show a decrease in ${ }^{36} \mathrm{Cl} / \mathrm{Cl}$ ratios over time, with the largest ratios corresponding to the shortest leaching time. This trend is interpreted to indicate that these samples may contain a small component of bomb-pulse or surface contaminant ${ }^{36} \mathrm{Cl}$, which is mixed with pre-bomb-pulse meteoric salts. Dilution of this elevated ${ }^{36} \mathrm{Cl}$ signal increases throughout the leaching process. Only one sample shows a substantial decrease in the ${ }^{36} \mathrm{Cl} / \mathrm{Cl}$ ratio in the final leaching step, indicating increasing input of rock chloride. Different aliquots of the reference sample, EVAL001' (which was homogenized), 
displayed a large range of ${ }^{36} \mathrm{Cl} / \mathrm{Cl}$ values in the first 0.5 hour (Table 4-3). This suggests that splits of EVAL001 have different ${ }^{36} \mathrm{Cl} / \mathrm{Cl}$ values in the most labile chloride component.

The constancy of the ${ }^{36} \mathrm{Cl} / \mathrm{Cl}$ ratios in individual samples with increasing leaching time, and the fact that they remained uniform despite the variability of measured ratios among the samples, indicate that there is only a single source of chloride in the rock or that a uniform mixture of different sources of chloride was leached. On a plot of ${ }^{36} \mathrm{Cl} / \mathrm{Cl}$ ratio versus reciprocal of chloride concentration (Figure 4-10), most samples do not show a correlation between ${ }^{36} \mathrm{Cl} / \mathrm{Cl}$ ratios and chloride concentrations, with the exception of EVAL001-8, $-9,-10$, obtained by active leaching. The small ${ }^{36} \mathrm{Cl} / \mathrm{Cl}$ ratios and the observed correlation between ${ }^{36} \mathrm{Cl} / \mathrm{Cl}$ ratios and chloride concentrations is a result of the active-leach process. Active leaching liberates more rock chloride, which dilutes the meteoric chloride and results in smaller ${ }^{36} \mathrm{Cl} / \mathrm{Cl}$ ratios.

Leachates of all aliquots of sample EXD-069 have a wide range of chloride concentrations and small ${ }^{36} \mathrm{Cl} / \mathrm{Cl}$ ratios (Figure 4-10A), and they show uniform ${ }^{36} \mathrm{Cl} / \mathrm{Cl}$ ratios in each fraction for different leach durations. The small ${ }^{36} \mathrm{Cl} / \mathrm{Cl}$ ratios in the coarser fractions of this sample are similar to many of the USGS-LLNL leachates (Section 4.4). These results imply that some samples, and perhaps rock masses in the subsurface, may be characterized by uniformly small ${ }^{36} \mathrm{Cl} / \mathrm{Cl}$ ratios. However, leachates of the finer fraction (less than $2 \mathrm{~mm}$ ) have substantially larger ${ }^{36} \mathrm{Cl} / \mathrm{Cl}$ ratios than the coarser fractions. A similar negative correlation of ${ }^{36} \mathrm{Cl} / \mathrm{Cl}$ ratios with particle size is observed in samples of Niche \#1 core analyzed at LANL. These relations contradict the conceptual model of chloride distribution described by Lu et al. (2003), as discussed in Section 4.6.2.

\subsubsection{Leaching Experiments Conducted at AECL}

As indicated in Section 4.3.2, the location and distribution of primary chloride in tuffs at Yucca Mountain is not well understood. Noble et al. (1967, p. 222) have shown that, on average, 80 percent of the chloride originally present in silicic volcanic glass is lost during formation of densely welded tuffs. Chloride liberated during devitrification may have been deposited locally during cooling of the tuffs, forming soluble minerals that would be dissolved readily by percolating water. Twenty rock samples of the crystal-poor part of the devitrified TSw from the ECRB Cross Drift have a mean value and $1 \sigma$ for chloride of $170 \pm 40 \mu \mathrm{g} / \mathrm{g}$ (Peterman and Cloke, 2002 , p. 695). The chloride concentrations in the volcanic glass contained in the tuff before devitrification were probably much larger. In an attempt to characterize the primary rock chloride, Fabryka-Martin, Wolfsberg et al. (1996, Table 5-4) leached finely ground tuff for chloride concentrations and ${ }^{36} \mathrm{Cl} / \mathrm{Cl}$ ratios. The resultant concentrations are more than a factor of 10 less than the mean rock value of $170 \mu \mathrm{g} / \mathrm{g}$, indicating that a substantial amount of the rock chloride is tightly bound and unavailable to leaching. More recently, work done at AECL has further investigated the chloride content of samples of the TSw from the validation study boreholes. This work is described below.

\subsubsection{Methods}

The crush-leach method was used to determine the chloride distribution in validation study samples and the effects of leaching time and grain size on the leachable chloride content. By 
varying the particle size and leaching time, it was thought possible to gain an understanding of the location of chloride in the rock and, hence, what might happen to infiltrated ${ }^{36} \mathrm{Cl}$ on leaching.

Samples used for the leaching experiments (Table 4-5) were from three of the dry-drilled validation study boreholes in the vicinity of the Sundance fault (fault trace at about ESF station 35+93). The proximity of the boreholes to the fault varied: ESF-SD-ClV\#2 was the farthest (about $82 \mathrm{~m}$ south of the fault trace at ESF station 36+75), ESF-SD-ClV\#14 was at an intermediate distance (about $48 \mathrm{~m}$ north of the fault trace at ESF station $35+45$ ), and ESF-SD-CIV\#9 was within $10 \mathrm{~m}$ of the fault trace on the ESF tunnel wall. The intensity of fracturing also varied within the boreholes. Core was largely intact in the $30-\mathrm{cm}$ interval from ESF-SD-ClV\#2, broken with two to three fractures in the 55-cm interval from ESF-SD-CIV\#14, and largely rubble in the 49-cm interval from ESF-SD-CIV\#9. The latter sample was selected to determine the leaching characteristics of very coarse fractions of rubblized rock.

Test parameters for the leaching experiments are listed in Table 4-5. The leaching experiments were designed to test differences in the amount of chloride extracted from different particle sizes for rock crushed by both laboratory and natural processes over different leaching times. Samples were crushed in the laboratory using a rock breaker, jaw crusher, and shatter box, if needed. No attempt was made to trim the core sample or wash its surface to remove external contaminants. In addition, experiments designed to evaluate the effects of both leaching time and particle size on naturally broken rock were performed using fragments that were hand-picked and sieved from the rubblized interval of borehole ESF-SD-ClV\#9 near the Sundance fault. Six fractions of this sample, including coarse fractions up to $60 \mathrm{~mm}$, were obtained by hand-picking and sieving without laboratory crushing.

All size fractions were leached with de-ionized water for durations ranging from 10 minutes to 72 hours, depending on the experiment. De-ionized water used for leaching had blank chloride concentrations below the detection limit of $0.15 \mathrm{mg} / \mathrm{L}$, whereas most rock leachates had concentrations at this level or higher. Also, chloride concentrations varied systematically down to the lowest values, implying that the true detection limit is probably lower than $0.15 \mathrm{mg} / \mathrm{L}$. Leaching bottles containing measured amounts of sample and water wère gently shaken occasionally and just prior to sampling to ensure the homogeneity of the leachate. Small volumes of leachate were drawn off by syringe, filtered through a $0.45-\mu \mathrm{m}$ filter, and analyzed by ion chromatography. The leaching method used by AECL was similar to that used by LANL for chloride extraction in previous ${ }^{36} \mathrm{Cl}$ studies; however, it was different from the leaching experiments conducted at LANL during the this study, where the leachate was completely removed and replaced with new de-ionized water after each leach period was complete. All chloride concentrations in leachates are expressed as milligrams per kilogram rock after correction for the water-rock ratio used in the leaching process and removal of small amounts of leachate for analysis during the leaching experiments.

\subsubsection{Results}

Three time-series experiments, lasting a total of 70 to 72 hours (Table 4-6), were conducted on two of the core samples. Leachates of the coarser fraction $(4$ to $10 \mathrm{~mm})$ of core from ESF-SD-CIV\#2 and ESF-SD-CIV\#14 attained maximum chloride concentrations of about 1 and $0.68 \mathrm{mg} / \mathrm{kg}$ rock (tests CT and 2CT in Table 4-6), and leaching of chloride was essentially 
complete (constant chloride concentrations) after 24 hours. A similar time-series experiment performed on a finer fraction (less than $0.125 \mathrm{~mm}$ ) of core from ESF-SD-ClV\#2 (FT series in Table 4-6) yielded substantially larger chloride concentrations of approximately $5 \mathrm{mg} / \mathrm{kg}$ rock (Figure 4-11). The decrease in chloride concentration in the fine fraction with time (filled diamonds in Figure 4-11) may be accounted for by analytical error (approximately \pm 5 percent).

In addition, the larger chloride concentrations in the fine-fraction leachates were obtained in much less time than those for the coarse-fraction leachates. Maximum chloride concentrations were observed in the first leachate sampled after only 10 minutes. Differences in chloride concentrations of the two coarse-fraction leachates also are apparent. Leachates from the first 10 hours show that chloride concentrations in both the CT and 2CT time-series experiments increase progressively (Figure 4-12). However, leachates from broken core at an intermediate distance from the Sundance fault (ESF-SD-ClV\#14, 2CT series) are systematically lower in chloride concentration than the intact core at a greater distance from the Sundance fault (ESF-SD-CIV\#2, CT). Concentrations of chloride in the 2CT time-series leachates are typically 50 to 70 percent of those in the CT leachates extrapolated to an equivalent time.

The particle size of the material being leached has a large but variable effect on the concentration of chloride in the leachates (Figure 4-13). In these experiments, sized fractions of core from intervals in ESF-SD-ClV\#2 (GS series) and ESF-SD-ClV\#14 (2A2 series), ranging from less than 0.063 to $12 \mathrm{~mm}$, were each leached for 24 hours. Except for the coarsest GS series fraction, resulting chloride concentrations increased progressively with decreasing particle size. Chloride concentrations continued to increase as particle size became smaller in both experiments with no indications of leveling out, implying that additional chloride would have been leached if the rock was ground to particle sizes less than $0.063 \mathrm{~mm}$.

In addition to the differences in size fractions from each core sample, differences in chloride concentrations were observed for leachates of the same size fractions between the two core samples. For the three coarser size fractions with particles between 0.25 and $4 \mathrm{~mm}$, chloride concentrations are 2.1 to 1.2 times larger in leachates of the intact core from ESF-SD-CIV\#2 than leachates of the broken core from ESF-SD-CIV\#14 (Figure 4-13). The opposite trend is present in finer size fractions, where chloride concentrations become up to 3.8 times larger in leachates of ESF-SD-CIV\#14 core relative to leachates of ESF-SD-ClV\#2. The differences in chloride concentrations in leachates of these two core intervals change progressively as particle size changes. Causes for the differences in leaching behavior of these two samples are not known.

A third set of leaching experiments was conducted on naturally rubblized core from borehole ESF-SD-ClV\#9, adjacent to the Sundance fault. Both leach duration and fragment size varied in this series of experiments (BT series in Table 4-6). Small increases in the soluble chloride concentrations corresponding to increasing leach durations are observed for the coarse fractions (Figure 4-14). However, reversals in these trends occur in the finer size fractions. A steady decrease in leachable chloride from the finest to coarsest particle sizes, and a lack of a "step" in the data, indicates that there is no preferential accumulation of chloride on rock surfaces in the fractures, as this would likely be more available to leaching solutions than chloride in the matrix. As a result, these results suggest that leaching of matrix pore fluid salts is the dominant source of chloride in both the finer and coarser size fractions. 


\subsubsection{Discussion of Results}

Time-series leaching experiments conducted at AECL on the coarser fractions of rock (4 to $10 \mathrm{~mm}$ ) indicated that extraction of leachable chloride was essentially complete after 24 hours. Crushing the rock to finer fractions shortens this leaching time to as little as 10 minutes. These results indicate that minor differences in leaching times or particle sizes would cause only minor differences in the amounts of chloride leached from rock samples. However, chloride concentrations observed in different leachates of relatively coarse tuff samples are not greatly affected by sample preparation and processing, and probably cannot explain the large differences in ${ }^{36} \mathrm{Cl} / \mathrm{Cl}$ ratios obtained by LLNL and LANL during Phase I of the validation study.

Experiments designed to determine the effects of particle size (between 6.3 to $12.5 \mathrm{~mm}$ and less than $0.063 \mathrm{~mm}$ ) on the leaching of chloride showed that more chloride was leached from the finer size fractions. Results also suggest that more leachable chloride would have been obtained if the rock had been ground to sizes less than $0.063 \mathrm{~mm}$. In general, particle size appears to have greater influence on chloride concentrations than does leaching time. This effect is likely a function of the increased surface area as particle size decreases. Values for the surface area per mass unit have been calculated assuming that particles in each size fraction have a spherical shape, a mean size between upper and lower sieve openings, and a mean bulk density of $2.25 \mathrm{~g} / \mathrm{cm}^{3}$ (Flint 2003, value for the middle nonlithophysal unit of the Topopah Spring Tuff, Table 3). Results for both the BT (natural rubble) and 2A2 (mechanically crushed) leaching series show a relatively smooth trend of increasing chloride concentrations with increasing particle surface area per mass unit (Figure 4-15). Results for the GS series leachates (core ESFSD-CIV\#2, sample names GS1-GS7, in Table 4-6) show similar increases, but with a lower slope.

The contributions from meteoric and rock chloride sources cannot be determined directly from these data; however, estimates from end-member compositions can be calculated. The concentration of chloride in pore fluids in a kilogram of rock can be calculated from the mean concentration in pore fluids (34.5 mg/L; Peterman and Marshall 2002, p. 308) corrected for the mean porosity $(0.110)$, saturation $(0.848)$, and bulk density $\left(2.25 \mathrm{~g} / \mathrm{cm}^{3}\right)$ of the crystal-poor, middle nonlithophysal unit of the Topopah Spring Tuff (mean values from Flint 2003, Table 3). This calculation reveals that a chloride concentration of $1.4 \mathrm{mg} / \mathrm{kg}$ rock is potentially available to leaching solutions. Therefore, a meteoric chloride source may provide all the chloride in leachates of rock crushed to sizes greater than about $0.5 \mathrm{~mm}$. However, rock chloride is required to provide a substantial amount of the chloride leached from rock fractions finer than $0.5 \mathrm{~mm}$. A maximum chloride concentration of about $16 \mathrm{mg} / \mathrm{kg}$ rock for the finest fraction of the $2 \mathrm{~A} 2$ series represents only about 10 percent of the total chloride present in the rock mass (mean value of $170 \mathrm{mg} / \mathrm{kg}$ rock; Peterman and Cloke 2002, Table 6). Therefore, a substantial fraction of the chloride remains tightly bound in solid phases in the rock and is unavailable for leaching from even the most finely ground samples.

A possible trend of decreasing chloride concentrations toward the Sundance fault also was noted during these leaching experiments. Concentrations of chloride in 21- to 24-hour leachates of the 4- to $10-\mathrm{mm}$ size fraction were largest for the intact core at approximately $82 \mathrm{~m}$ from the fault trace $(1.00 \mathrm{mg} / \mathrm{kg}$ for ESF-SD-ClV\#2-CT9 in Table 4-6), intermediate for the broken core at approximately $48 \mathrm{~m}$ from the fault trace $(0.53 \mathrm{mg} / \mathrm{kg}$ for ESF-SD-ClV\#14-2CT-6 in Table 4-6), 
and smallest for the naturally rubblized core from within $2 \mathrm{~m}$ of the fault trace $(0.34 \mathrm{mg} / \mathrm{kg}$ for ESF-SD-CIV\#9-2BT-4 in Table 4-6). Although these differences may be caused by random variations in the chloride content of pore fluids in the tuff, it is possible they may be caused by differential flow of fracture water and pore water across this zone. Increased percolation fluxes focused in the Sundance fault zone could cause lower chloride concentrations in the rubblized rocks due to previous natural leaching processes.

\subsubsection{Conclusions from the Phase II Leaching Experiments}

Results from the leaching experiments performed at LANL and AECL indicate that variations in particle size and leaching times can affect chloride concentrations and ${ }^{36} \mathrm{Cl} / \mathrm{Cl}$ compositions of leachates, but probably not in substantial ways. Experiments conducted at LANL using the reference sample, EVAL001, demonstrated that most passive-leach aliquots have smaller chloride concentrations and all have larger ${ }^{36} \mathrm{Cl} / \mathrm{Cl}$ ratios compared to active-leach aliquots taken at equivalent leaching times. These results confirm that active leaching is likely to extract more rock chloride compared to passive leaching, and they explain the differences between initial LLNL active-leach results and those obtained previously by LANL (Figure 4-3). Results also support the intuitive view that passive leaching and shorter leaching times favor extraction of more labile, meteoric chloride components that may contain bomb-pulse ${ }^{36} \mathrm{Cl}$. Based on these results, the active-leach method was abandoned.

Leaching experiments performed at LANL with multiple samples from the ECRB Cross Drift demonstrate the presence of a wide range of chloride concentrations and ${ }^{36} \mathrm{Cl} / \mathrm{Cl}$ ratios at different sites. However, results of the sequential leaching experiments show only minor variability in a single set of leachates. These results indicate that ${ }^{36} \mathrm{Cl} / \mathrm{Cl}$ ratios for individual samples have a tendency to remain relatively constant (typically within the range of analytical error) regardless of leach durations between 0.5 and 48 hours. Only one sample shows a statistically significant change in ${ }^{36} \mathrm{Cl} / \mathrm{Cl}$ ratios between the first leaching time (taken at $0.5 \mathrm{hr}$ ) and those for subsequent leaching times (Figure 4-5, EVAL001-7). These experiments imply either that there is only a single source of leachable chloride in the rock or that a uniform mixture of different sources of chloride was maintained in spite of variable leaching times.

Although leaching experiments conducted at AECL did not include analyses of ${ }^{36} \mathrm{Cl} / \mathrm{Cl}$ ratios, they provide information on the nature of extractable chloride in tuff samples. Rates of extraction of soluble chloride from coarser fractions of rock were greatest in the first several hours of leaching and extraction was largely complete after 24 hours. Crushing the rock to finer fractions shortened this leaching time to as little as 10 minutes. The effects of particle size were larger than the effects of leach duration. However, these experiments demonstrated that for coarser particle sizes (greater than $0.5 \mathrm{~mm}$ ), much of the chloride in leachates most likely has a meteoric source, and that large amounts of rock chloride are not likely unless the sample is more finely ground. Similar results were obtained from ${ }^{36} \mathrm{Cl}$ leaching experiments conducted at LANL.

\subsection{PHASE III: MEASUREMENTS MADE AT USGS-LLNL}

Results from the Phase II leaching experiments (Section 4.3) led to substantial modifications in the method used to leach additional validation study samples. The active-leach method used by 
LLNL in Phase I was abandoned in favor of the passive-leach method developed in Phase II to minimize contributions of rock chloride to the leachate. Also, because the leaching experiments indicated that much of the readily leachable chloride was extracted in the first several hours of passive leaching, the study participants agreed that passive leaching for short time periods was the most reliable means of obtaining labile, meteoric chloride. The study participants also agreed that adopting an approach that minimized variables in analytical procedures was an important aspect of Phase III. By minimizing the variables, each step could be evaluated separately. The first step in this process involved crushing at either the SMF or USGS, followed by leaching at the USGS, and distribution of leachates to LANL and LLNL for $\mathrm{AgCl}$ precipitation and target preparation. Targets made in each laboratory were analyzed at a single AMS facility (LLNL-CAMS). This strategy was applied to samples sent for analysis as Batch \#1. A similar strategy was applied to Batch \#2 samples, except that targets prepared at LANL were analyzed at PRIME Lab and targets prepared at LLNL were analyzed at LLNL-CAMS. Targets for Batches \#3, \#4, and \#5 were prepared and analyzed at LLNL. Table 4-7 gives the unique identification numbers assigned to leachates of samples that were crushed at the SMF or USGS, and leached at the USGS.

\subsubsection{Methods}

Processing of validation study core resumed in the Summer of 2001 on new core intervals requested from the SMF (identified as " ${ }^{36} \mathrm{Cl}$ (USGS)" in Appendix B). The heavily fractured intervals from the deepest $2 \mathrm{~m}$ of the core had been sent previously to LLNL for ${ }^{36} \mathrm{Cl}$ analysis, leaving core intervals that ranged from rubblized to intact intervals. General descriptions of the intervals prepared during core logging indicate that 11 of the 39 core intervals were relatively intact, with only about one to three fractures per foot (Table 4-8). The other 28 core intervals had fracture densities similar to the intervals selected for the original allocations.

\subsubsection{Sample Processing}

Samples of validation study core were crushed and sieved at the SMF using a jaw crusher, which was previously used only for crushing samples of TSw, and new 6.3- to 19-mm stainless-steel sieves. Crushed samples were shipped to the USGS YMPB laboratory in Denver, where they were re-sieved and the fines were removed using compressed nitrogen before leaching. For each leachate, between 0.989 and $2.399 \mathrm{~kg}$ (median of $1.788 \mathrm{~kg}$ ) of crushed rock was placed in a stainless-steel wire basket and immersed in a stainless-steel stockpot containing an approximately equal weight of de-ionized water. The basket was initially raised and lowered five times to wet all rock surfaces and then allowed to soak for 1 hour. This process approximated the passive-leach methods used in previous LANL studies, except for a substantial reduction in the 24- to 72-hour leaching times used previously. After the 1-hour leach, the basket was raised and lowered five times to rinse the rock surfaces, then removed from the pot. The leachate was filtered through a pre-rinsed $0.45-\mu \mathrm{m}$ barrel filter into two $1-\mathrm{L}$ polyethylene bottles, which were sent to LLNL (Batches \#1 to \#5) and LANL (Batches \#3 to \#5) for AgCl precipitation and target preparation. An additional $30-\mathrm{mL}$ aliquot of the leachate was filtered through a $0.2-\mu \mathrm{m}$ filter for anion analysis $\left(\mathrm{Cl}^{-1}, \mathrm{NO}_{3}{ }^{-1}, \mathrm{SO}_{4}^{-2}, \mathrm{~F}^{-1}, \mathrm{Br}^{-1}\right)$ at the USGS. 


\subsubsection{Crushing Experiments}

The USGS modified the sample processing procedures slightly near the end of the validation study in response to concerns about differences in crushing methods and their possible impact on the ${ }^{36} \mathrm{Cl}$ results. Validation study core were being crushed using a jaw crusher, whereas samples analyzed previously at LANL were generally crushed by hand using a hammer and steel plate. To evaluate the differences between mechanical crushing and hand crushing on the release of rock chloride, the USGS conducted a crushing experiment on approximately $8 \mathrm{~kg}$ of core from six intervals in borehole ESF-SAD-GTB\#1 (southern Ghost Dance fault zone, Alcove.\#7) that were combined, homogenized, and split into two aliquots. One aliquot was crushed using a hammer and steel plate and the other was passed through a mechanical jaw crusher to replicate the process used on the validation study core. In both cases, coarse fragments were crushed to pass a $19-\mathrm{mm}(3 / 4-\mathrm{inch})$ sieve. In addition to leachates from the 6.3 - to $19-\mathrm{mm}(1 / 4-$ inch to $3 / 4$-inch) size fraction for both aliquots, a third sample was used to test the effects of increasing the size range to 2 to $19 \mathrm{~mm}$ ( 10 mesh to $3 / 4$ inch).

The different crushing methods did not result in significant differences in ${ }^{36} \mathrm{Cl} / \mathrm{Cl}$ ratios (Table 4-9). For the two leachates of the $6.3-$ to $19-\mathrm{mm}(1 / 4$-inch to $3 / 4$-inch) size fraction from ESF-SAD-GTB\#1, the mechanically crushed sample yielded a slightly larger chloride concentration $(0.517 \mathrm{mg} / \mathrm{kg}$ rock $)$ and a smaller ${ }^{36} \mathrm{Cl} / \mathrm{Cl}$ ratio $\left(344 \pm 104 \times 10^{-15}\right)$ compared to the hand-crushed sample $\left(0.474 \mathrm{mg} / \mathrm{kg}\right.$ rock and $457 \pm 107 \times 10^{-15}$, respectively). However, the differences are within analytical error (Figure 4-16). The leachate from the finer fraction of hand-crushed material ( 2 to $19 \mathrm{~mm}$, [10 mesh to $3 / 4$ inch]) had a larger chloride concentration $\left(0.697 \mathrm{mg} / \mathrm{kg}\right.$ rock) than those obtained from the coarser fractions; however, the ${ }^{36} \mathrm{Cl} / \mathrm{Cl}$ ratio of $510 \pm 108 \times 10^{-15}$ was within analytical error of the other leachates.

Although core samples from outside the areas investigated for the ${ }^{36} \mathrm{Cl}$ validation study were used for these experiments, the ${ }^{36} \mathrm{Cl} / \mathrm{Cl}$ values are within the range observed for core from the Sundance fault zone (red diamonds [ESF-SD-ClV drill core] on Figure 4-17). Chloride concentrations in leachates of the ESF-SAD-GTB\#1 core from the southern part of the ESF are larger than the leachates of validation study samples located to the north. This trend is consistent with results reported previously by LANL. The median chloride concentration for 155 samples from the northern half of the ESF (stations $0+00$ to $39+00$ ) is $0.7 \mathrm{mg} / \mathrm{kg}$ rock, whereas the value for 138 samples from the southern half of the ESF (stations $39+39$ to $78+50$ ) is $1.7 \mathrm{mg} / \mathrm{kg}$ rock (Appendix A). In addition, ${ }^{36} \mathrm{Cl} / \mathrm{Cl}$ ratios for ESF-SAD-GTB\#1 core from Alcove \#7 (mean and $1 \sigma$ of $437 \pm 85 \times 10^{-15}$ ) are similar to the LANL values obtained for six samples of Alcove \#7 rocks listed in Appendix A (mean and $1 \sigma$ of $551 \pm 55 \times 10^{-15}$ ).

Results of the crushing experiments on ESF-SAD-GTB\#1 core indicate that differences in crushing and particle size are unlikely the cause of major differences in chloride concentrations and ${ }^{36} \mathrm{Cl} / \mathrm{Cl}$ ratios obtained using the validation study protocols and earlier LANL protocols. Therefore, the large differences in ${ }^{36} \mathrm{Cl} / \mathrm{Cl}$ ratios between LANL leachates with bomb-pulse values and USGS-LLNL validation study leachates $\left({ }^{36} \mathrm{Cl} / \mathrm{Cl}\right.$ ratios less than $\left.619 \times 10^{-15}\right)$ must be attributed to other causes. To evaluate the large differences in ${ }^{36} \mathrm{Cl} / \mathrm{Cl}$ ratios between LANL leachates with bomb-pulse values and USGS-LLNL leachates without bomb-pulse values, the study participants conducted additional comparative studies using intervals of the same Niche \#1 core samples that had been analyzed previously at LANL (Section 4.4.2.3). 


\subsubsection{Procedural Blanks}

Measured chloride consists of a mixture of natural chloride present in the rock sample plus chloride that is added to the rock sample and leachate during sample collection, crushing, leaching, and $\mathrm{AgCl}$ target preparation. To determine the mass of ${ }^{35} \mathrm{Cl},{ }^{36} \mathrm{Cl}$, and chlorine-37 $\left({ }^{37} \mathrm{Cl}\right)$ in a sample, the mass of chloride added during the analytical processing (process blank) must be subtracted from the measured results. At different times during the ${ }^{36} \mathrm{Cl}$ validation study, the mass of chloride and its isotopic composition were measured in de-ionized water that was processed using the leaching and target preparation procedures and run as unknown samples. In addition, the chloride isotopic composition of a blank was determined for water from the deionization system without further processing. Results of blank analyses for samples leached at the USGS and $\mathrm{AgCl}$ precipitated at LLNL are given in Table 4-10.

Concentrations of total chloride in the blank samples prepared at the USGS and analyzed at LLNL (USGS-LLNL) varied between 0.004 and $0.017 \mathrm{mg} / \mathrm{kg}$ water, with a mean of $0.0104 \pm 0.0047(1 \sigma)$. Precise measurements of ${ }^{36} \mathrm{Cl} / \mathrm{Cl}$ ratios could not be made on the small chloride concentrations of the blank samples. Individual ${ }^{36} \mathrm{Cl} / \mathrm{Cl}$ ratios ranged from $47 \pm 211(1 \sigma) \times 10^{-15}$ to $1,839 \pm 555(1 \sigma) \times 10^{-15}$. Chloride concentrations and ${ }^{36} \mathrm{Cl} / \mathrm{Cl}$ ratios in the process blanks and the water blank were similar. The mean ${ }^{36} \mathrm{Cl} / \mathrm{Cl}$ ratio of five blank measurements was $555 \pm 337$ ( 1 standard error [SE]) $\times 10^{-15}$. These data are more meaningful if they are converted to concentrations of ${ }^{36} \mathrm{Cl}$ added during sample processing. The five USGS-LLNL blanks represent between $0.47 \times 10^{-15}$ and $7.6 \times 10^{-15} \mathrm{mg}{ }^{36} \mathrm{Cl}$ added per kilogram of water used, with a mean of $3.5 \pm 3.0 \times 10^{-15}(1 \sigma) \mathrm{mg}^{36} \mathrm{Cl} / \mathrm{kg}$ water. Thus, although the ${ }^{36} \mathrm{Cl} / \mathrm{Cl}$ ratios in the blanks ranged widely, the amounts of ${ }^{36} \mathrm{Cl}$ that would be added during processing of the samples is very small.

In addition to chloride added during leaching and target preparation, both crushing and handling operations could add chloride to a sample. This contribution was not measured in previous studies because of the lack of a chloride-free material with physical properties similar to the densely welded tuffs. Methods of investigating this source of contamination were initiated at the USGS. Electronics-grade silicon was chosen because of its extremely high purity (typical metal contamination levels are less than $1 \times 10^{-11} \mathrm{~g} / \mathrm{g}$ silicon). A $3.8-\mathrm{kg}$ cylindrical (approximately $15-\mathrm{cm}$ diameter by $15-\mathrm{cm}$ height), monocrystalline silicon ingot was obtained from the DOE's National Renewable Energy Laboratory in Golden, Colorado. The ingot and all crushing equipment were cleaned with de-ionized water to remove surface contamination, then the ingot was broken into fragments using a rock hammer. Approximately half of the material was crushed using a hammer and steel plate, and the other half was crushed using a steel mortar and pestle. Both sets of material were sieved to obtain a 2- to $19-\mathrm{mm}$ size fraction and leached using the same passive-leach process used by USGS for the Niche \#1 samples (Section 4.4.2.3).

The samples were analyzed by ion chromatography using low-level detection methods $(0.01 \mathrm{mg} / \mathrm{L}$ detection limit) at the USGS National Water Quality Laboratory (NWQL) and by isotope dilution at LLNL (Table 4-11). Chloride concentrations in the two crushing blanks were only slightly larger $(0.019$ and $0.014 \mathrm{mg} / \mathrm{L})$ than the value obtained for the system leaching blank processed at the same time (less than $0.010 \mathrm{mg} / \mathrm{L}$ ). The ${ }^{36} \mathrm{Cl} / \mathrm{Cl}$ ratios in the two crushing blanks were $957 \pm 174 \times 10^{-15}$ and $1,033 \pm 249 \times 10^{-15}$. These values are within analytical uncertainty of the mean value obtained from the USGS-LLNL leaching blanks analyzed earlier in the validation 
study and consistent with meteoric values expected for Colorado (Phillips 2000, Figure 10.3). Although small amounts of chloride may be added during crushing and sieving, the added chloride does not have small ${ }^{36} \mathrm{Cl} / \mathrm{Cl}$ values that would explain the differences between small ${ }^{36} \mathrm{Cl} / \mathrm{Cl}$ ratios obtained for the USGS-LLNL validation study samples and the large ${ }^{36} \mathrm{Cl} / \mathrm{Cl}$ ratios measured previously at LANL. These results indicate that crushing at the USGS did not add substantial amounts of chloride and that added chloride has a ${ }^{36} \mathrm{Cl} / \mathrm{Cl}$ composition similar to meteoric chloride.

A similar evaluation of crushing blanks was not performed at LANL. However, two samples of Niche \#1 core that had been crushed and sieved at LANL were sent to the USGS for leaching. The samples, Niche 1-RCR-1A (approximately $1.3 \mathrm{~kg}$ ) and Niche LT-RCR-1A (approximately $0.7 \mathrm{~kg}$ ), were remnants of the $6.3-$ to $12.5-\mathrm{mm}$ size fraction that had been analyzed at LANL (Table 4-12) and had ${ }^{36} \mathrm{Cl} / \mathrm{Cl}$ ratios of $1,163 \pm 94 \times 10^{-15}$ and $1,016 \pm 87 \times 10^{-15}$, respectively. The two samples were combined into a single $2.0-\mathrm{kg}$ sample (NICHE3566\#1+NICHE3566\#LT1) at the USGS to ensure sufficient chloride for analysis, and the sample was leached without additional handling. The resulting USGS-LLNL chloride concentration ' of $0.188 \mathrm{mg} / \mathrm{kg}$ water and ${ }^{36} \mathrm{Cl} / \mathrm{Cl}$ ratio of $1,185 \pm 121 \times 10^{-15}$ (Table 4-11), are similar to values obtained by LANL, but distinctly higher than values obtained for other USGS-LLNL leachates.

\subsubsection{Results}

\subsubsection{Anions in Leachates of Validation Study Core}

The USGS used ion chromatography to measure concentrations of the soluble anions $\mathrm{Cl}^{-1}, \mathrm{Br}^{-1}$, $\mathrm{NO}_{3}{ }^{-1}$, and $\mathrm{SO}_{4}{ }^{-2}$ in leachates of validation study core, Niche \#1 core, and Alcove \#7 core, as well as leachates of the EVAL001 reference sample (Table 4-13). These data do not reflect true concentrations of pore water and are generally much more dilute than values obtained directly from water extracted from the core (Peterman and Marshall 2002, p. 308), due in part to the relatively large volumes of water used for leaching. However, all leachates of validation study core were obtained from similar amounts of the same size fractions leached for the same time periods. Therefore, measured differences in concentration should reflect natural variability rather than artifacts of laboratory processing.

Concentrations of chloride in leachates of samples from the Sundance fault zone (including Niche \#1) vary from 0.050 to $0.31 \mathrm{mg} / \mathrm{kg}$ rock, with a median value of $0.120 \mathrm{mg} / \mathrm{kg}$ rock and a mean value of $0.145 \pm 0.074(1 \sigma, 51$ analyses) $\mathrm{mg} / \mathrm{kg}$ rock (Table 4-14). Values for leachates from the Drill Hole Wash fault zone are slightly higher, with a median chloride concentration of $0.205 \mathrm{mg} / \mathrm{kg}$ rock and a mean of $0.223 \pm 0.053(1 \sigma, 10$ analyses) $\mathrm{mg} / \mathrm{kg}$ rock. Differences in mean values between the two groups of data are significant at the 95 percent confidence level (Figure 4-18). In contrast to leachate chloride concentrations, pore water chloride concentrations obtained by ultra-centrifugation of high-silica rhyolite units of the Topopah Spring Tuff are generally much larger (mean and $1 \sigma$ of $34.5 \pm 16.7 \mathrm{mg} / \mathrm{L}$; Peterman and Marshall 2002, p. 308). A mean chloride concentration of $1.4 \mathrm{mg} / \mathrm{kg}$ rock is calculated for the middle nonlithophysal unit of the Topopah Spring Tuff using the mean pore water chloride concentration and the mean pore water content of 0.093 (Flint 2003, Table 3). Chloride concentrations in leachates indicate that less than 10 percent of the total pore water chloride available in the rock is extracted during the 1-hour leaching process. 
Chloride concentrations in leachates show variations with distance across the Sundance fault zone (Figure 4-19A). Values tend to be smallest in leachates of ESF-SD-CIV core between ESF stations 35+40 and 36+00 adjacent to and north of the trace of the Sundance fault. The mean chloride concentration in leachates from this zone is $0.066 \pm 0.018 \mathrm{mg} / \mathrm{kg}$ rock $(1 \sigma, 10$ analyses $)$. Leachates of ESF-SD-ClV samples from either side of this zone have a combined mean chloride concentration of $0.151 \pm 0.066 \mathrm{mg} / \mathrm{kg}$ rock $(1 \sigma, 35$ analyses $)$, which is significantly different at the 95 percent confidence level. Similar variations across the Drill Hole Wash fault zone are not apparent (Figure 4-19B).

Relations between chloride concentration and proximity to the Sundance fault observed from ESF-SD-CIV core are complicated by results for leachates of Niche\#1 core. Although the Niche \#1 boreholes were not drilled normal to the walls of the ESF main drift, the resulting core lies within the interval between ESF stations $35+40$ and 36+00. Leachates of core from all three Niche \#1 boreholes have substantially higher chloride concentrations than the ESF-SD-CIV core, with a mean of $0.231 \pm 0.044 \mathrm{mg} / \mathrm{kg}$ rock $(1 \sigma, 6$ analyses) (Figure $4-19 \mathrm{~A})$.

Concentrations of other anions in leachates of validation study core are poorly to moderately correlated with chloride. Concentrations of $\mathrm{NO}_{3}{ }^{-1}$ in leachates of core from the Sundance fault zone (including Niche \#1) range from less than 0.04 to $0.44 \mathrm{mg} / \mathrm{kg}$ rock (Table 4-14) and are poorly correlated with chloride concentrations (Figure 4-20A). Large concentrations of $\mathrm{NO}_{3}{ }^{-1}$ are not present in leachates with small chloride concentrations; however, $\mathrm{NO}_{3}{ }^{-1}$ concentrations commonly remain small as chloride concentrations increase. In contrast, $\mathrm{SO}_{4}{ }^{-2}$ concentrations ranging from less than 0.03 to $0.51 \mathrm{mg} / \mathrm{kg}$ rock show a positive correlation with chloride concentrations (Table 4-14 and Figure 4-20B). Concentrations of $\mathrm{Br}^{-1}$ are below detection limits $(0.02 \mathrm{mg} / \mathrm{kg}$ water) for all leachates of dry-drilled validation study core. Because the construction water that was used during excavation of the ESF and ECRB was tagged with $\mathrm{LiBr}$, this result indicates the absence of substantial amounts of construction water in all samples, some of which are from depths as shallow as 0.40 to $0.60 \mathrm{~m}$ from the tunnel wall. Concentrations of $\mathrm{Br}^{-1}$ are above detection limits in analyses of two leachates of the reference sample EVAL001 $(0.18$ and $0.14 \mathrm{mg} / \mathrm{kg}$ rock, Table $4-14)$, which was collected with mining equipment that used construction water for dust suppression. Because there is no detectable $\mathrm{Br}^{-1}$ in any of the leachates of validation study core, corrections for construction water are not necessary.

\subsubsection{Chlorine-36 in Leachates of Validation Study Core}

USGS-LLNL used AMS to analyze 34 1-hour passive leachates of core samples from 29 validation study boreholes (ESF-SD-CIV) located across the Sundance fault zone (Table 4-15). Chloride concentrations range from 0.037 to $0.372 \mathrm{mg} / \mathrm{kg}$ rock, with an arithmetic mean of $0.130 \mathrm{mg} / \mathrm{kg}$ rock and a median value of $0.120 \mathrm{mg} / \mathrm{kg}$ rock. Chloride concentrations determined by isotope dilution at LLNL typically agree within error with chloride concentrations determined by ion chromatography at the USGS (Figure 4-21). All but three analyses fall in a narrower range between 0.037 and $0.197 \mathrm{mg} / \mathrm{kg}$ rock (Figure 4-22A). The three elevated values are from core locations scattered across the Sundance fault zone (Figure 4-23A). The isotope dilution data confirm the pattern of chloride distribution that was determined on the larger ion chromatography data set (compare Figure 4-23A with Figure 4-19A). 
Leachates of validation study core have ${ }^{36} \mathrm{Cl} / \mathrm{Cl}$ ratios ranging between $137 \times 10^{-15}$ and $615 \times 10^{-15}$ (ESF-SD-ClV core, excluding Niche \#1, Table 4-15). Values for the median and mean ${ }^{36} \mathrm{Cl} / \mathrm{Cl}$ are $316 \times 10^{-15}$ and $326 \times 10^{-15}$, respectively. The frequency distribution of these 34 values of ${ }^{36} \mathrm{Cl} / \mathrm{Cl}$ does not show any indication of being skewed toward high ratios (Figure 4-22B). Use of the Anderson-Darling normality test (Stephens 1974) results in a probability value of 0.141 , which indicates that the sample population cannot be distinguished from a normal distribution at the 95 percent confidence level. Unlike chloride concentrations that appear to be correlated with respect to location of the Sundance fault trace (Figure 4-19A and Figure $4-23 \mathrm{~A}),{ }^{36} \mathrm{Cl} / \mathrm{Cl}$ ratios vary randomly between ESF stations $34+95$ and $36+75$. However, ${ }^{36} \mathrm{Cl} / \mathrm{Cl}$ ratios show a general trend of decreasing values from about $540 \times 10^{-15}$ to $580 \times 10^{-15}$ at around ESF station $34+00$, to about $140 \times 10^{-15}$ to $190 \times 10^{-15}$ around ESF station $34+70$ (Figure 4-23B). To evaluate this trend, ${ }^{36} \mathrm{Cl} / \mathrm{Cl}$ ratios were plotted against borehole completion dates with analyses discriminated by batch number (Figure 4-24). Although most of the boreholes constituting this trend were completed in sequence during the first round of drilling between March and April, 1999, borehole ESF-SD-CIV\#26 at ESF station $34+73$, containing the lowest ${ }^{36} \mathrm{Cl} / \mathrm{Cl}$ values, was completed at the end of the second round of drilling in June 1999. Most other samples from the second round of drilling have substantially higher ${ }^{36} \mathrm{Cl} / \mathrm{Cl}$ ratios. Progressive contamination (or decontamination) from drilling equipment is not suspected because the Yucca Mountain Project Management and Test Coordination Office advised that new drill bits and rods were used for drilling, and because ${ }^{36} \mathrm{Cl} / \mathrm{Cl}$ ratios in core samples from the second and third rounds of drilling (September 1999) span most of the range observed in core obtained from the first round. Also, ${ }^{36} \mathrm{Cl} / \mathrm{Cl}$ ratios in different batches of leachates analyzed in different AMS runs overlap. Therefore, natural chloride compositional variations are the likely cause for the trend of monotonically decreasing ${ }^{36} \mathrm{Cl} / \mathrm{Cl}$ ratios observed between ESF stations $33+98$ and $34+73$.

The ${ }^{36} \mathrm{Cl} / \mathrm{Cl}$ ratios in leachates of validation study core do not correlate with chloride concentrations (Figure 4-17). If the relatively small ${ }^{36} \mathrm{Cl} / \mathrm{Cl}$ ratios measured in validation study core were the result of mixing chloride from meteoric and rock sources, data would plot on a mixing line between a meteoric end-member with large ${ }^{36} \mathrm{Cl} / \mathrm{Cl}$-high reciprocal chloride concentration values (small chloride concentrations) and a rock end-member with small ${ }^{36} \mathrm{Cl} / \mathrm{Cl}$-low reciprocal chloride concentration values (large chloride concentrations). Instead, ${ }^{36} \mathrm{Cl} / \mathrm{Cl}$ ratios remain uniform across the range of reciprocal chloride concentration values, indicating that small ${ }^{36} \mathrm{Cl} / \mathrm{Cl}$ ratios are as likely in the samples with the smallest concentrations as they are in the samples with the largest concentrations.

\subsubsection{Re-Analysis of Niche \#1 Core for Chlorine-36}

As part of the in situ testing for the UZ flow and transport model, 10-m-long boreholes were drilled before and after construction of Niche \#1 at ESF station 35+66 (Figure 3-6). Nine of the 10 core samples from three boreholes (ESF-MD-NICHE3566\#1, ESF-MD-NICHE3566\#2, and ESF-MD-NICHE3566LT\#1) analyzed at LANL had ${ }^{36} \mathrm{Cl} / \mathrm{Cl}$ values between $1,235 \times 10^{-15}$ and $2,038 \times 10^{-15}$ (CRWMS M\&O 1998, Table 3-4). Core intervals remaining at LANL (sealed in the original SMF packaging) were inventoried and split between LANL and USGS to span the intervals analyzed previously at LANL and to ensure that comparable samples were analyzed by the separate laboratories. 
Multiple, overlapping intervals were combined into single samples so that sufficient rock was available for leaching (Figure 3-7). After the outer surfaces of the sealed ProtecCore ${ }^{\mathrm{TM}}$ packages were rinsed with de-ionized water, intervals within individual composite samples were crushed, homogenized, sieved ( 2 to $19 \mathrm{~mm}$ at the USGS and either 6.3 to $12.5 \mathrm{~mm}$ or 2 to $12.5 \mathrm{~mm}$ at LANL), and leached at the USGS and LANL. Composited sample sizes ranged from 1.2 to $1.8 \mathrm{~kg}$. All samples were leached for 1 hour. The $\mathrm{AgCl}$ precipitates were prepared at LLNL and analyzed at LLNL-CAMS.

Chloride concentrations in leachates of the coarse material prepared at the USGS range from 0.17 to $0.27 \mathrm{mg} / \mathrm{kg}$ rock (Table 4-9). The ${ }^{36} \mathrm{Cl} / \mathrm{Cl}$ ratios from the six Niche \#1 leachates range from $226 \times 10^{-15}$ to $717 \times 10^{-15}$ and have median and mean values of $387 \times 10^{-15}$ and $401 \times 10^{-15}$ (Table 4-15). These ${ }^{36} \mathrm{Cl} / \mathrm{Cl}$ ratios are in the same range as those obtained from leachates of ESF-SD-ClV core (Figure 4-25). The means of the two sample groups (34 samples of ESF-SD-ClV core and six samples of Niche \#1 core) are indistinguishable at the 95 percent confidence level. Therefore, all leachate data for samples from the Sundance fault zone prepared at the USGS were pooled to give median and mean values for ${ }^{36} \mathrm{Cl} / \mathrm{Cl}$ of $316 \times 10^{-15}$ and $337 \times 10^{-15}$ (Table 4-15).

\subsection{PHASE III: MEASUREMENTS MADE AT LANL}

\subsubsection{Methods}

\subsubsection{Sample Processing}

Most rock samples were composed of a wide range of particle sizes, from pieces as large as $20 \mathrm{~cm}$ to dust. Therefore, samples required crushing and sieving to obtain the desired size fractions. Prior to use, all crushing and sieving equipment was thoroughly cleaned. Hammers and steel plates were cleaned by scrubbing with a wire brush, blowing with compressed air, and rinsing with de-ionized water. These steps were repeated so that no visible evidence of the prior samples remained. Sieves were cleaned by manually removing any pieces lodged in openings, scrubbing with a soft brush, blowing off with compressed air, and rinsing in de-ionized water. The table on which crushing and sieving was performed also was wiped clean with de-ionized water. Crushing and sieving were performed inside a new cardboard file box, with one side cut and folded down for access, into which a clean plastic garbage bag was placed. The crushed sample was then poured into a stack of sieves and gently shaken. Fragments of the desired size fraction were placed into a clean zip-lock bag, and the process was repeated until enough material of each size fraction was obtained. If necessary, large pieces were crushed with a hammer and steel plate in the file box. In some instances, as noted below, the dust was blown from the final fraction with dry compressed nitrogen prior to leaching.

Leaching was performed in stainless steel buckets with tight-fitting lids. These were washed thoroughly in soapy water, rinsed three times with de-ionized water, and placed upside-down on towels to dry prior to use. Samples were poured into pre-weighed buckets and re-weighed to determine sample mass by difference. A sub-equal mass of de-ionized water was added to the sample. Typically, water and sample mass differed by less than 10 percent. The de-ionized water and sample were left covered and undisturbed for the desired length of time. For this 
study, the leaching time was intentionally varied for a number of samples to determine the effects of leaching time on chloride concentrations and ${ }^{36} \mathrm{Cl} / \mathrm{Cl}$ ratios.

\subsubsection{Procedural Blanks}

Twelve procedural blanks were collected by LANL during the course of the investigation. Procedural blanks consisted of de-ionized water that was processed in the same manner as, and along side, the samples. As a result, these procedural blanks capture all the same processing steps as the rock samples, with the exception of crushing. Procedural blanks processed at LANL (Table 4-16) have low total chloride concentrations, with a mean of $0.008 \pm 0.006(1 \sigma) \mathrm{mg} / \mathrm{kg}$ water, similar to the mean value of $0.010 \pm 0.005(1 \sigma) \mathrm{mg} / \mathrm{kg}$ water for the USGS procedural blanks (Table 4-10). One blank consisting of LANL water was processed simultaneously with three blanks that consisted of USGS water that was representative of the water used to leach validation core samples. Results for the USGS water are comparable with those of the LANL water blanks. The ${ }^{36} \mathrm{Cl} / \mathrm{Cl}$ ratios have a mean of $1,994 \pm 400 \times 10^{-15}$ ( 1 standard error [SE]) (median value of $1,441 \times 10^{-15}, \mathrm{n}=12$ ). Although these values are larger than the values for the USGS blanks, the overall total mass of ${ }^{36} \mathrm{Cl}$ in the LANL blanks is small, with a range from $2.99 \times 10^{-15}$ to $25.54 \times 10^{-15} \mathrm{mg} / \mathrm{kg}$ water used (Table 4-16). These values represent a maximum of 15 percent of the total ${ }^{36} \mathrm{Cl}$ in the samples for the smallest samples analyzed, but in most cases the blank accounts for between 0.2 and 5 percent of the total mass of ${ }^{36} \mathrm{Cl}$ in the samples. The consistently small values for procedural blanks relative to the samples indicate that they do not significantly affect the results. All reported ratios are corrected for the mean of the blank values analyzed with a sample set. The corrections are generally within the uncertainty of the measurement and do not affect the interpretation.

Crushing blanks were not measured at LANL for this study; however, crushing blanks are not expected to contribute significantly to the samples because the crushing equipment was thoroughly cleaned by scrubbing with a wire brush, blowing with compressed air, and rinsing with de-ionized water prior to use. This procedure ensured that any contamination from prior samples or dust particles that accumulated during storage of the equipment was removed. Crushing typically exposed the samples to the atmosphere for up to a few hours, limiting the likelihood of ${ }^{36} \mathrm{Cl}$ contamination from this source. In contrast, sample leachates and accompanying blanks are left open to the atmosphere (to allow evaporation of the sample) for up to a week. In all instances the leaching blanks still showed very small levels of ${ }^{36} \mathrm{Cl}$. Contamination from the steel itself is not expected because the steel is not likely to contain significant ${ }^{36} \mathrm{Cl}$, distilled water-leachable components of the steel will be insignificant, and the amount of steel contamination in a sample is also very small. Thus, it is expected that the crushing process did not contribute an anomalously large amount of contamination to any of the samples. Additional arguments to support the lack of laboratory contamination in samples processed at LANL are presented in Section 6.3.4.2.

\subsubsection{Results}

\subsubsection{Chlorine-36 in Leachates of Validation Study Core}

During Phase III, samples of validation study core were crushed at the SMF and leached at the USGS. Two sub-equal volumes of leachate were split and sent to LLNL and LANL for AgCl 
target preparation and analysis. Results for the LANL splits analyzed at PRIME Lab (ESF samples from the Sundance fault zone) are shown in Table 4-12. Chloride concentrations range from $0.07 \mathrm{mg} / \mathrm{kg}$ rock to $0.32 \mathrm{mg} / \mathrm{kg}$ rock. ${ }^{36} \mathrm{Cl} / \mathrm{Cl}$ ratios range from $163 \pm 30 \times 10^{-15}$ to $640 \pm 162 \times 10^{-15}$.

\subsubsection{Chlorine-36 in ECRB Cross Drift Tunnel-Wall Samples}

Previously unreported ${ }^{36} \mathrm{Cl}$ data for 58 samples from the ECRB Cross Drift are included in this report (Table 4-17). These samples. were processed prior to the relocation of the LANL laboratory and changes in LANL personnel in 2000. These data are reported for comparison with other ECRB samples processed as part of the validation study. Leachates for most of these samples were made using the $2-$ to $20-\mathrm{mm}$ size fraction. However, three samples (EXD-064, EXD-071, and EXD-085) were collected as highly fragmented samples and processed without sieving or additional crushing. All samples were leached for 19 hours and all were greater than $4.4 \mathrm{~kg}$. Chloride concentrations range from $0.20 \mathrm{mg} / \mathrm{kg}$ rock to $3.59 \mathrm{mg} / \mathrm{kg}$ rock. ${ }^{36} \mathrm{Cl} / \mathrm{Cl}$ ratios range from $161 \pm 22 \times 10^{-15}$ to $4,890 \pm 349 \times 10^{-15}$. Eight of the 58 samples (14 percent) contain ${ }^{36} \mathrm{Cl} / \mathrm{Cl}$ values greater than $1,250 \times 10^{-15}$.

\subsubsection{Re-Analysis of Niche \#1 Core for Chlorine-36}

Multiple, nearly adjacent intervals of Niche \#1 core were combined into single samples so that sufficient rock was available for leaching (Figure 3-7). After the outer surfaces of the sealed ProtecCore ${ }^{\mathrm{TM}}$ packages were rinsed with de-ionized water, intervals within individual composite samples were crushed, homogenized, sieved (either 6.3 to $12.5 \mathrm{~mm}$ or 2 to $12.5 \mathrm{~mm}$ ), and leached at LANL. Composited sample sizes ranged from 1.2 to $1.8 \mathrm{~kg}$. All samples were leached for 1 hour. All crushing, leaching, and $\mathrm{AgCl}$ precipitation for LANL leachates was performed at LANL. Silver chloride precipitates were analyzed at LLNL-CAMS. In addition, fines (less than $6.3 \mathrm{~mm}$ ) from two of the samples crushed at LANL (Niche 1-RCR-1B and Niche LT-RCR-1B, Table 4-12) were leached at LANL and analyzed at LLNL. Chloride concentrations for leachates of the coarser material are 0.13 and $0.28 \mathrm{mg} / \mathrm{kg}$ rock (Niche LT-RCR-1A and Niche 1-RCR-3, Table 4-12). Leachates of the two finer fractions (Niche 1-RCR-1B and Niche LT-RCR-1B) have substantially larger chloride concentrations (0.69 and $0.67 \mathrm{mg} / \mathrm{kg}$ rock).

The ${ }^{36} \mathrm{Cl} / \mathrm{Cl}$ ratios obtained by LANL for composite samples of Niche \#1 core are larger than the USGS-LLNL results for overlapping composite samples of the same core (Table 4-9 and Figure 4-26). The new LANL analyses are similar to previous LANL analyses of Niche \#1 core (CRWMS M\&O 1998, Table 3-4) in that some of the ${ }^{36} \mathrm{Cl} / \mathrm{Cl}$ values exceed the $1,250 \times 10^{-15}$ bomb-pulse threshold (four of seven analyses). New LANL ${ }^{36} \mathrm{Cl} / \mathrm{Cl}$ values range from $1,016 \times 10^{-15}$ to $8,558 \times 10^{-15}$. The new analyses show a positive correlation between ${ }^{36} \mathrm{Cl} / \mathrm{Cl}$ ratios and chloride concentration (largest ${ }^{36} \mathrm{Cl} / \mathrm{Cl}$ ratios in leachates with the largest chloride concentrations). The observation of the largest ${ }^{36} \mathrm{Cl} / \mathrm{Cl}$ ratios in leachates of Niche \#1 core, which consist entirely of fine fractions (less than $6.3 \mathrm{~mm}$ ), is the opposite of the relation observed in leachates of tunnel-wall samples reported previously (Figure 4-27). Larger chloride concentrations in leachates of finer material previously have been attributed to addition of progressively more rock chloride liberated from particle surfaces as the total surface area per unit 
mass of sample increases (Fabryka Martin, Wolfsberg et al. 1996, p. 24; and this report, Section 4.3).

\subsection{DISCUSSION OF THE CHLORINE-36 MEASUREMENTS}

Analytical protocols evolved during the course of the validation study in response to preliminary results and discussions among the participants. The final passive-leach procedure was designed to maximize contributions from meteoric chloride and minimize contributions from rock chloride unrelated to $\mathrm{UZ}$ percolation. ${ }^{36} \mathrm{Cl} / \mathrm{Cl}$ ratios in the validation study samples from both USGS-LLNL and USGS-LANL generally agree within analytical error despite the analytical challenges of dealing with the low chloride concentrations in the 1-hour leachates. However, large differences in ${ }^{36} \mathrm{Cl} / \mathrm{Cl}$ ratios exist between results for Niche \#1 samples processed at the USGS and LANL, and between results obtained from USGS-LLNL leachates and those obtained previously by LANL from samples in the Sundance fault zone.

\subsubsection{Active Leaching}

The analytical procedure used by LLNL during Phase I of the validation study, which involved leaching crushed rock in a slowly rotating tumbler for 7 hours (active-leach process), resulted in leachates with relatively large chloride concentrations and small ${ }^{36} \mathrm{Cl} / \mathrm{Cl}$ ratios. Results obtained from active leaching are distinct from those obtained from passive leaching (previous LANL studies and work conducted at LANL and USGS-LLNL during Phase III) for both longer and shorter leaching times (Figures 4-3B and 4-28). The data obtained from active leaching are interpreted to be the result of adding large amounts of rock chloride during the extraction process. Consequently, the ${ }^{36} \mathrm{Cl} / \mathrm{Cl}$ ratios in the leachates cannot be used to detect the bombpulse meteoric component along the Sundance fault zone.

\subsubsection{Chloride Sources and Leaching Experiments}

Rock samples from the Yucca Mountain UZ contain chloride and ${ }^{36} \mathrm{Cl}$ from multiple sources, including ${ }^{36} \mathrm{Cl}$ potentially added to sample sites during tunnel construction and operation, and to samples during processing (Table 4-4). Lu et al. (2003, p. 3-5) discuss these sources and categorize them into "(1) leach-accessible salts or fluids (present in the inter-granular connected pores and fractures) and (2) leach-limited salts or fluids present in fluid inclusions, disconnected pores, and grain boundaries (called isolated and boundary salts)". Figure 4-29 presents a conceptual model of the effects of leaching on ${ }^{36} \mathrm{Cl} / \mathrm{Cl}$ ratios in rocks. Bomb-pulse and contaminant ${ }^{36} \mathrm{Cl}$ in a sample should be readily leachable from the rock, and chloride from these sources will be mixed during leaching. It is likely that longer leaching times will dilute a bombpulse signal. Eventually, any bomb-pulse meteoric salts, if present, will be thoroughly dissolved and the ${ }^{36} \mathrm{Cl} / \mathrm{Cl}$ ratio will reflect a mixture of salts precipitated from younger (i.e., less than $10 \mathrm{ka}$ ) and older (i.e., greater than $10 \mathrm{ka}$ ) meteoric water. Prolonged or aggressive leaching could potentially liberate older meteoric salts or rock chloride, resulting in a decrease in the ${ }^{36} \mathrm{Cl} / \mathrm{Cl}$ ratio. It is clear from this conceptual model that shorter (and less vigorous) leaching should favor extraction of the most recently deposited meteoric salts, including a bomb-pulse component, if present. However, sufficient chloride must be leached from the rock for a reliable analysis. 


\subsubsection{Procedural Blanks and Detection Limits for the Total Chloride and Chlorine-36 Analyses}

Because several results are based on leachates with low chloride concentrations, the contribution of blanks and the limits of detection of chloride and ${ }^{36} \mathrm{Cl}$ become very important in determining the validity of these data. The U.S. Environmental Protection Agency (USEPA) has a procedure for determining the "method detection limit" (MDL), which ". . . is defined as the minimum concentration of a substance that can be measured and reported with 99 percent confidence that the analyte concentration is greater than zero and is determined from analysis of a sample in a given matrix containing the analyte." (40 CFR 136, 2004, Appendix B, p. 317). The procedure is based on the analysis of detection limits presented by Glaser et al. (1981). The calculation involves determining the standard deviation of seven samples with analyte concentrations that are one to five times the assumed detection limit, and using the Student's $t$ multiplier (R. Università di Roma 1925, pp. 105-108) for the 99 percent confidence level to calculate the MDL.

Analyses of leaching blanks processed at the USGS (Table 4-10) and LANL (Table 4-16) can be used to evaluate the MDL for both laboratories because blank levels define minimum measurable concentrations in real samples. Mean concentrations of total chloride in the USGS and LANL blank samples are $0.0104 \pm 0.0047 \mathrm{mg} / \mathrm{kg}$ water $(1 \sigma)$ and $0.0087 \pm 0.0067 \mathrm{mg} / \mathrm{kg}$ water $(1 \sigma)$, respectively. For both laboratories, multiplying obtained standard deviations by the Student's $t$ factors for the 99 percent confidence level gives values of $0.020 \mathrm{mg}$ chloride $/ \mathrm{kg}$ water for the MDL of total chloride.

Five isotopic analyses of USGS blanks and nine analyses of LANL blanks yielded mean values of $3.5 \pm 3.0 \times 10^{-15}(1 \sigma)$ and $12.9 \pm 8.7 \times 10^{-15}(1 \sigma) \mathrm{mg}{ }^{36} \mathrm{Cl} / \mathrm{kg}$ water, respectively (Tables 4-10 and 4-16). Multiplying obtained standard deviations by the Student's $t$ factors for the 99 percent confidence level gives values of $11 \times 10^{-15} \mathrm{mg}{ }^{36} \mathrm{Cl} / \mathrm{kg}$ water for the MDL at USGS and $24 \times 10^{-15} \mathrm{mg}{ }^{36} \mathrm{Cl} / \mathrm{kg}$ water for the MDL at LANL. Although these MDLs are lower than most of the measured total chloride and ${ }^{36} \mathrm{Cl}$ concentrations in the validation study samples, some of the 1-hour passive-leach analyses with low ${ }^{36} \mathrm{Cl}$ concentrations obtained during Phase III of the validation study are very close to these detection limits and should be interpreted with caution. However, three USGS system blanks processed at LANL, where AgCl targets were precipitated, yielded results that are similar to USGS blanks spiked and precipitated at LLNL. These analyses yielded a mean value of $4.7 \pm 1.1 \times 10^{-15}(1 \sigma) \mathrm{mg}{ }^{36} \mathrm{Cl} / \mathrm{kg}$ water, which is in good agreement with the mean value of $3.5 \pm 3.0 \times 10^{-15}(1 \sigma) \mathrm{mg}{ }^{36} \mathrm{Cl} / \mathrm{kg}$ water for blanks processed by USGS. The close agreement of mean values for blanks analyzed at two independent laboratories indicates that chloride isotopic results are generally reproducible even at the smallest chloride concentrations.

LANL analyses with elevated ${ }^{36} \mathrm{Cl} / \mathrm{Cl}$ ratios measured during Phase II and Phase III of the validation study contain ${ }^{36} \mathrm{Cl}$ concentrations that are significantly higher than the MDL. Similar assessment of the MDL for earlier LANL results cannot be made because ${ }^{36} \mathrm{Cl}$ concentrations in blanks were not reported. 


\subsubsection{Analysis of Duplicate Samples}

Validation study samples were analyzed in several stages as work progressed. The USGS prepared the first batch of samples using the modified 1-hour passive-leach process. Sixteen leachates were each split into aliquots and sent to LANL and LLNL for independent spiking, $\mathrm{AgCl}$ precipitation, and target preparation. All $\mathrm{AgCl}$ targets were then analyzed at LLNL. All samples had small chloride concentrations, ranging from $0.069 \mathrm{mg} / \mathrm{kg}$ rock to $0.372 \mathrm{mg} / \mathrm{kg}$ rock (Table 4-18). The duplicates of the 14 analyses that were run successfully had similar chloride concentrations and ${ }^{36} \mathrm{Cl} / \mathrm{Cl}$ ratios, with no indications of inter-laboratory biases (Figure 4-30).

The duplicate analyses were used to evaluate the analytical reproducibility of ${ }^{36} \mathrm{Cl} / \mathrm{Cl}$ measurements. In addition to in-run statistics, analytical uncertainties include estimates of external precision obtained by duplicate analyses of the same material. Therefore, the external error to be added to the total analytical uncertainties is estimated from the 14 duplicate analyses given in Table 4-18. The standard deviation was determined from the duplicate pairs following the equation given by Youden (1951, p. 16):

$$
\text { Standard deviation }=\sqrt{\frac{\sum\left(R_{L L N L}-R_{L A N L}\right)^{2}}{2 n}} .
$$

where $R_{L L N L}$ and $R_{L A N L}$ are the ${ }^{36} \mathrm{Cl} / \mathrm{Cl}$ ratios obtained from the LLNL and LANL preparations, respectively, and $n$ is the number of duplicate pairs (as well as the number of degrees of freedom). The resulting value of $48 \times 10^{-15}$ is an appropriate estimate for the absolute $1 \sigma$ external error of a typical ${ }^{36} \mathrm{Cl} / \mathrm{Cl}$ measurement. This external error was propagated with the error from other sources to obtain the final estimate of $2 \sigma$ analytical uncertainty for each measurement of the USGS-LLNL ${ }^{36} \mathrm{Cl} / \mathrm{Cl}$ data (Table 4-9).

A similar comparison of results was made on splits of six Batch \#2 leachates prepared at the USGS and analyzed at LLNL-CAMS and LANL-PRIME Lab (Tables 4-9 and 4-12). Chloride concentrations and ${ }^{36} \mathrm{Cl} / \mathrm{Cl}$ ratios determined for the duplicate splits are in general agreement, although they exhibit larger deviations than the Batch 1 results obtained from a single AMS facility. Chloride concentrations in Batch 2 samples ranged from 0.071 to $0.265 \mathrm{mg} / \mathrm{kg}$ rock for the LANL-PRIME Lab analyses (mean $0.140 \pm 0.078 \mathrm{mg} / \mathrm{kg}$ rock, $1 \sigma$ ) and 0.087 to $0.333 \mathrm{mg} / \mathrm{kg}$ rock for LLNL-CAMS analyses (mean $0.171 \pm 0.089 \mathrm{mg} / \mathrm{kg}$ rock). Measured ${ }^{36} \mathrm{Cl} / \mathrm{Cl}$ ratios range from $180 \times 10^{-15}$ to $640 \times 10^{-15}$ for LANL-PRIME Lab analyses (mean $361 \pm 177 \times 10^{-15}, 1 \sigma$ ) and

from $294 \times 10^{-15}$ to $615 \times 10^{-15}$ for LLNL analyses (mean $442 \pm 132 \times 10^{-15}, 1 \sigma$ ). Standard deviation ( $1 \sigma$ external error) for ${ }^{36} \mathrm{Cl} / \mathrm{Cl}$ ratios in this set of six duplicate pairs obtained by two different laboratories is $125 \times 10^{-15}$, or about 2.5 times larger than the comparison of duplicate pairs made for analyses conducted at LLNL-CAMS. This estimate for external error was not incorporated into individual analyses because of the smaller number of analyses used for the comparison and because direct comparisons of USGS-LLNL and LANL-PRIME Lab validation study data were made only on leachates from Niche \#1, which were all analyzed at the LLNL-CAMS facility. 
Chloride concentrations and ${ }^{36} \mathrm{Cl} / \mathrm{Cl}$ analyses of passive 1-hour leachates prepared at the USGS and sent to LLNL and LANL for $\mathrm{AgCl}$ precipitation and analysis commonly agree within analytical uncertainty (squares and circles in Figure 4-28). The two groups of analyses show no systematic differences in ${ }^{36} \mathrm{Cl} / \mathrm{Cl}$ ratios ranging from $163 \times 10^{-15}$ to $721 \times 10^{-15}$ (Figure 4-31). The difference between the mean ${ }^{36} \mathrm{Cl} / \mathrm{Cl}$ ratio for the 20 leachates sent to LANL for processing $\left(307 \times 10^{-15}\right)$ and the mean ratio for 40 leachates sent to LLNL $\left(360 \times 10^{-15}\right)$ is not statistically significant at the 95 percent confidence level. The LANL results also include ${ }^{36} \mathrm{Cl} / \mathrm{Cl}$ measurements made at both LLNL-CAMS and PRIME Lab. The agreement between ${ }^{36} \mathrm{Cl} / \mathrm{Cl}$ values obtained by both laboratories on separate aliquots of the same leachates indicates that the process of $\mathrm{AgCl}$ target preparation and AMS isotope analysis does not cause significant differences in ${ }^{36} \mathrm{Cl}$ results.

\subsubsection{LANL Data from the ECRB Cross Drift}

A considerable body of ${ }^{36} \mathrm{Cl}$ data has been collected for previous studies of the ECRB Cross Drift (Table 4-17). Leaching time for the previously analyzed samples was typically 48 hours, and particle size was between 2 and $20 \mathrm{~mm}$. Results from these previous studies are compared in Figure 4-32 with results from the validation study. Both data sets agree for samples between stations $0+77$ and $20+00$ and most values range between $500 \times 10^{-15}$ and $1,000 \times 10^{-15}$. This range includes samples that were processed using different leaching times. Each data set contains at least one sample with a ${ }^{36} \mathrm{Cl} / \mathrm{Cl}$ ratio greater than $1,250 \times 10^{-15}$ (beyond ECRB Cross Drift station 21+00), which is interpreted to represent a bomb-pulse signal. In all cases, for both data sets, samples with bomb-pulse ${ }^{36} \mathrm{Cl} / \mathrm{Cl}$ ratios were collected from faults. These data are interpreted by LANL to support previous hypotheses (Fabryka-Martin et al. 1997, Section 9.3; Campbell et al. 2003, Section 9) that faults are conduits for rapid flow (less than 50 years to depths of about $300 \mathrm{~m}$ ) of meteoric water from the surface to the depths of the ECRB Cross Drift.

Sample EXD-059 (Table 4-12) yielded a ${ }^{36} \mathrm{Cl} / \mathrm{Cl}$ value of $1,309 \pm 114 \times 10^{-15}$. This value is slightly larger than the lower cutoff value $\left(1,250 \times 10^{-15}\right)$ used to detect bomb-pulse ${ }^{36} \mathrm{Cl}$ (Fabryka-Martin et al. 1997, Section 4.2.4) and is therefore used to indicate the presence of bomb-pulse ${ }^{36} \mathrm{Cl}$ in this sample. Values between $412 \times 10^{-15}$ and $671 \times 10^{-15}$ are interpreted to indicate that the chloride was derived predominantly from meteoric salts deposited in the past $10 \mathrm{ka}$ (but not in the past approximately 60 years). One sample (EXD-066), has an anomalously small ${ }^{36} \mathrm{Cl} / \mathrm{Cl}$ value of $161 \pm 22 \times 10^{-15}$ and an anomalously large chloride concentration of $3.59 \mathrm{mg} / \mathrm{kg}$; larger than any other leachates analyzed at LANL for this study by more than a factor of two. It is likely that this small ${ }^{36} \mathrm{Cl} / \mathrm{Cl}$ ratio is due to dilution of a meteoric signal by rock chloride.

\subsubsection{Comparison of Validation Study Data with Previous Chlorine-36 Data}

\subsubsection{Sundance Fault Zone}

Thirty-four analyses of samples of Niche \#1 core and samples from the Sundance fault zone between ESF stations $34+28$ and $37+00$ were reported as part of the previous studies (Appendix A). Chloride concentrations in these 48-hour leachates are larger, on average (mean of $0.55 \mathrm{mg} / \mathrm{kg}$ rock), than those obtained for the 1-hour leachates obtained during the validation 
study (mean of $0.141 \mathrm{mg} / \mathrm{kg}$ rock) (Figure 4-33A). This result is consistent with the general relations between leach duration and chloride concentration. The larger chloride concentrations from earlier LANL results show a wide range of ${ }^{36} \mathrm{Cl} / \mathrm{Cl}$ values from $388 \times 10^{-15}$ to $4,105 \times 10^{-15}$ (Figure 4-33B). LANL results obtained during Phase I for seven validation study core samples (Table 4-2) are within this range, but show no bomb-pulse values. These ${ }^{36} \mathrm{Cl} / \mathrm{Cl}$ ratios have only a limited overlap at their lower end, with the much smaller values obtained from the ${ }^{36} \mathrm{Cl}$ validation study samples analyzed by USGS-LLNL during Phase III (Figure 4-33B). The differences in ${ }^{36} \mathrm{Cl} / \mathrm{Cl}$ ratios between these two data sets are inconsistent with an interpretation that smaller ratios are caused by greater contributions from rock chloride. It would have been expected that the longer leaching times used for LANL samples would have diluted a bombpulse signal with chloride from older meteoric salts and/or rock chloride with small ${ }^{36} \mathrm{Cl} / \mathrm{Cl}$ ratios. This type of mixing relation is shown by the LANL Sundance fault zone data set (Figure 4-34) as a negative correlation between ${ }^{36} \mathrm{Cl} / \mathrm{Cl}$ ratios and chloride concentrations (that is, larger ${ }^{36} \mathrm{Cl} / \mathrm{Cl}$ ratios are present in leachates with the smallest chloride concentrations, resulting in a positive correlation with reciprocal chloride values). In contrast, USGS-LLNL leachates have a wide range of chloride concentrations, but show no correlation between ${ }^{36} \mathrm{Cl} / \mathrm{Cl}$ ratios and reciprocal chloride concentrations, resulting in the horizontal trend in Figure 4-34. Low chloride concentrations in 1-hour leachates should be particularly susceptible to contributions of rock chloride or other sources of potential low ${ }^{36} \mathrm{Cl}$ contamination. However, the ${ }^{36} \mathrm{Cl} / \mathrm{Cl}$ ratios in these leachates remain more-or-less uniformly small despite the order-of-magnitude variation in chloride concentrations.

\subsubsection{Southern Exploratory Studies Facility}

Additional evidence that contamination from a low $-{ }^{36} \mathrm{Cl} / \mathrm{Cl}$ source is not the cause for the smaller ${ }^{36} \mathrm{Cl} / \mathrm{Cl}$ ratios observed in .USGS-LLNL leachates is their similarity with data obtained for samples from the southern ESF. LANL's analysis of 125 leachates from ESF stations $45+78$ to $78+50$ (Appendix A) show large chloride concentrations, ranging from 0.3 to $11.5 \mathrm{mg} / \mathrm{kg}$ rock (Figure 4-35A). The variability of chloride concentrations increases with distance (Figure 2-1A) along the southern ESF, including the south ramp. These data define a triangular field, with the maximum chloride concentrations increasing toward the south portal (Figure $2-1 \mathrm{~A}) .{ }^{36} \mathrm{Cl} / \mathrm{Cl}$ ratios in these samples range from $140 \times 10^{-15}$ to $1,117 \times 10^{-15}$ (Figure 4-35B). These data have a median ${ }^{36} \mathrm{Cl} / \mathrm{Cl}$ value of $467 \times 10^{-15}$ and a mean value of $480 \times 10^{-15}$. Chloride concentrations in USGS-LLNL leachates are systematically lower than, and only partly overlap, the smallest values for LANL leachates from southern ESF samples (Figure 4-35A). Chlorine isotope data from USGS-LLNL leachates overlap most of the range observed for southern ESF samples (Figure 4-35B). However, the distribution of USGS-LLNL ${ }^{36} \mathrm{Cl} / \mathrm{Cl}$ values is shifted toward the lower side of the LANL southern ESF data set. The 40 analyses constituting the USGS-LLNL data set have a mean ${ }^{36} \mathrm{Cl} / \mathrm{Cl}$ value of $337 \times 10^{-15}$, which is statistically different from the LANL mean value of $480 \times 10^{-15}$ at greater than 99 percent confidence level.

\subsubsection{Comparison of USGS-LLNL Niche \#1 Data and LANL-LLNL Niche \#1 Data}

${ }^{36} \mathrm{Cl} / \mathrm{Cl}$ ratios are significantly different for samples of Niche \#1 core separately prepared and leached at the USGS and LANL. Although samples were not homogenized prior to splitting between the two facilities, alternating intervals were selected to minimize sampling differences (Figure 3-7). Six samples of the 2- to 19-mm size fraction crushed and leached at the USGS and 
analyzed at LLNL have a mean ${ }^{36} \mathrm{Cl} / \mathrm{Cl}$ value of $412 \times 10^{-15}$ (open circles in Figure 4-28). LANL crushed and leached two size fractions of Niche \#1 core which were analyzed at LLNL. Five samples of coarser material $(6.3$ to $12.5 \mathrm{~mm})$ have a mean value of $1,616 \times 10^{-15}$ (Niche 1-RCR-1A, Niche 1-RCR-2, Niche 1-RCR-3, Niche 2-RCR-1, Niche LT-RCR-1A in Table 4-12, and red triangles in Figure 4-28). Leachates of the finer fractions (less than $6.3 \mathrm{~mm}$ ) have significantly larger chloride concentrations and ${ }^{36} \mathrm{Cl} / \mathrm{Cl}$ ratios than leachates of the coarser fractions of the same material, including the largest ${ }^{36} \mathrm{Cl} / \mathrm{Cl}$ ratio $\left(8,558 \times 10^{-15}\right)$ yet reported for ESF samples (Niche 1-RCR-1B and Niche LT-RCR-1B in Table 4-12). The large ${ }^{36} \mathrm{Cl} / \mathrm{Cl}$ ratios in the new LANL analyses are consistent with previous LANL results (CRWMS M\&O 1998, Table 3-4), but the relation between the largest ${ }^{36} \mathrm{Cl} / \mathrm{Cl}$ ratios and the largest chloride concentrations differs from previous LANL results for tunnel-wall samples. Finally, one sample crushed and homogenized at LANL and sent to the USGS for leaching yielded comparable ${ }^{36} \mathrm{Cl} / \mathrm{Cl}$ ratios between the two laboratories $\left(1,016 \times 10^{-15}\right.$ and $1,163 \times 10^{-15}$ for the two LANL analyses and $1,181 \times 10^{-15}$ for the single USGS-LLNL composite sample). This elevated ${ }^{36} \mathrm{Cl} / \mathrm{Cl}$ ratio represents the largest value obtained in the USGS-LLNL data set and indicates that the USGS leaching process captured elevated ${ }^{36} \mathrm{Cl} / \mathrm{Cl}$ ratios present in the sample.

Comparisons of the new Niche \#1 results are important because they are independent of other factors that complicate direct comparisons of validation study results with previous results. The Niche \#1 data are exclusively from core samples, eliminating the possibility that bomb-pulse measurements are unique to features observed on tunnel walls. Also, the new Niche \#1 samples processed by USGS-LLNL and LANL-LLNL are more-or-less evenly distributed among the same three boreholes to achieve the goal of having equivalent material analyzed by both laboratories. Processing and analysis of the new Niche \#1 samples was also nearly identical at both laboratories. 


\section{TRITIUM MEASUREMENTS}

Tritium $\left({ }^{3} \mathrm{H}\right)$ has a half-life of 12.33 years and is produced mainly through the bombardment of nitrogen atoms with neutrons in the upper atmosphere (Solomon and Cook 2000, p. 397). This cosmogenic ${ }^{3} \mathrm{H}$ combines with oxygen to form water that enters the hydrologic system as precipitation. Levels of cosmogenic ${ }^{3} \mathrm{H}$ vary with latitude due to the shielding effects of the geomagnetic field from 3 to $6 \mathrm{TU}$ for Europe and North America to approximately $15 \mathrm{TU}$ for coastal Antarctic snow (Solomon and Cook 2000, p. 398). Beginning in 1952, concentrations of ${ }^{3} \mathrm{H}$ in the atmosphere began to increase due to nuclear weapons testing and reached peak values in 1962 and 1963 (Plummer et al., 1993, p. 258). Atmospheric ${ }^{3} \mathrm{H}$ concentrations have declined steadily since above-ground nuclear weapons testing ended in 1963, although small amounts of anthropogenic ${ }^{3} \mathrm{H}$ continue to be produced at nuclear power plants and processing facilities. Present-day ${ }^{3} \mathrm{H}$ values of precipitation at Yucca Mountain are not well constrained. Water from a perched spring near Yucca Mountain contains $6.3 \pm 0.4 \mathrm{TU}$, and this value is assumed to be close to that of present-day precipitation (Striegl et al. 1998, Table 3, p. 12-13).

\subsection{POTENTIAL SOURCES OF TRITIUM IN CORE SAMPLES FROM THE YUCCA MOUNTAIN UNSATURATED ZONE}

Pore water in the UZ at Yucca Mountain could be composed of mixtures of pre-bomb-pulse water and modern water. Modern water is defined by Clark and Fritz (1997, p. 172) as water that was recharged since the inception of nuclear testing (i.e., since 1952). Modern water may include bomb-pulse water and recent recharge. Water that entered the UZ immediately before 1952 (containing about $6 \mathrm{TU}$, similar to present-day precipitation), and remained isolated from the atmosphere would, at present, contain approximately $0.4 \mathrm{TU}$. In contrast, water with thousands of TU recharged to the UZ between 1962 and 1963 would presently contain hundreds of TU. A threshold value must be established to distinguish between modern water and prebomb-pulse water whose ${ }^{3} \mathrm{H}$ values may have been modified by sampling, extraction, and/or analytical errors. This threshold should not result in false positive values, which were a concern of the ${ }^{36} \mathrm{Cl}$ Peer Review Team in suggesting ${ }^{3} \mathrm{H}$ as a corroborating bomb-pulse isotope (YMP, 1998, Section 3.6.2). Threshold values used in interpreting the ${ }^{3} \mathrm{H}$ data are described in Section 5.4 .

Low-level concentrations of ${ }^{3} \mathrm{H}$ in small-volume pore water samples are not easy to interpret. As Lehmann et al. (1993, p. 2034) state in their discussion of atmospheric and subsurface sources of radionuclides in ground water, "One of the most vexing problems related to ${ }^{3} \mathrm{H}$ is the apparent evidence of small amounts of young water at great depths in water which should have been isolated from the atmosphere for thousands of years." They note four possible explanations for the presence of ${ }^{3} \mathrm{H}$ in otherwise old water: (1) sample contamination by younger water during collection; (2) movement of young water to depth along fast pathways; (3) subsurface production; and (4) contamination during analysis, such as from exposure to tritiated exit signs or illuminated watches. In addition, circulation of water-saturated air through the UZ at Yucca Mountain is a possible mechanism for introducing young water to large depths in the mountain. Such vapor-phase transport of ${ }^{3} \mathrm{H}$ in alluvium at relatively shallow depths has been well documented at a low-level waste disposal site near Yucca Mountain (Striegl et al. 1998, p. 1). 
The possibility of contamination during sample collection is difficult to evaluate but must be considered, at least at a low level. During and following excavation, tunnel walls in the ESF and ECRB Cross Drift were repeatedly washed with construction water that was obtained from well UE-25 J-13. Water from this well has a ${ }^{3} \mathrm{H}$ concentration of less than $0.3 \mathrm{TU}$ (DTN: GS040108312232.001 [Q]). This construction water was tagged with lithium bromide $(\mathrm{LiBr})$ at concentrations typically between 18 and $22 \mathrm{mg} / \mathrm{L}$, but not exceeding $30 \mathrm{mg} / \mathrm{L}$. Evaporation of the construction water on the surface of the tunnel walls and from within the rock next to the walls would leave $\mathrm{LiBr}$ as a salt. The absence of measurable $\mathrm{Br}^{-1}$ in leachates of validation study core samples (Table 4-13), some from depths as shallow as 0.4 to $0.6 \mathrm{~m}$, indicates that construction water is not an important contaminant of pore water samples, and therefore not of concern in determining their ${ }^{3} \mathrm{H}$ content.

Core from which water for ${ }^{3} \mathrm{H}$ measurements was extracted was obtained by a "dry drilling" technique in which compressed air was used to remove cuttings and to cool the drill bit. No measurements of the moisture content of the "dried air" are available, nor is it known what volume of air was used per meter of drill advance. Contamination of pore water extracted from core with atmospheric ${ }^{14} \mathrm{CO}_{2}$ has been documented by Yang (2002, Section 4.1.2). Some level of ${ }^{3} \mathrm{H}$ contamination is therefore possible, but this level is not known. The maximum effect of drilling contamination or natural deep atmospheric circulation of saturated air would be the complete replacement of the native pore water with modern water that has a ${ }^{3} \mathrm{H}$ concentration of approximately 6.3 TU. This is not the case for most of the samples.

In situ production of ${ }^{3} \mathrm{H}$ within the rock mass occurs primarily through a neutron-induced reaction with ${ }^{6} \mathrm{Li}$ (Andrews and Kay, 1982, p. 361). Calculations using average crustal rock compositions indicate that ${ }^{3} \mathrm{H}$ generated from subsurface production should contribute less than 0.2 TU to ground water (Lehmann et al., 1993, p. 2034).

\subsection{METHODS}

Water for ${ }^{3} \mathrm{H}$ analyses was extracted from the 50 validation study core samples and core samples from other boreholes in the ESF and ECRB Cross Drift (Figure 1-1; Appendix B). Samples from the north ramp included 11 samples of $\mathrm{TCw}$ and rocks younger than the $\mathrm{TCw}$ from boreholes in Alcove \#2 that intersect the Bow Ridge fault, three samples of PTn from the north ramp moisture study boreholes, and 10 samples of TSw from the validation study boreholes in the Drill Hole Wash fault zone. From the ESF main drift, 42 samples from the 40 validation study boreholes associated with the Sundance fault, 10 samples from the northern Ghost Dance fault zone (Alcove \#6), and five samples from the southern Ghost Dance fault zone (Alcove \#7) were used for water extraction. Twenty-three samples of TCw, PTn, and TSw were collected from the south ramp moisture study boreholes between stations $59+65$ and $75+10$. In addition, 22 pore water samples from 19 boreholes between stations $6+00$ and $25+00$ in the TSw in the ECRB were analyzed.

All boreholes were dry drilled, using compressed air. Core was video-logged and wrapped in plastic film, inserted into Lexan ${ }^{\circledR}$ tubing with caps taped onto each end, and sealed in ProtecCore ${ }^{\mathrm{TM}}$ packages. Where possible, core for ${ }^{3} \mathrm{H}$ analysis was selected from the deepest parts of the borehole to minimize the effects of dry-out and construction water contamination. 
Core was shipped and stored under refrigerated conditions until samples were ready for processing.

Pore water was extracted from the core samples by vacuum distillation (Yang et al. 1998, pp. 25-27). Water volumes ranged from 39 to $169 \mathrm{~mL}$ per sample. Samples from Alcove \#2 were processed and analyzed at the USGS YMPB laboratory in Denver using a low-energy betacounting technique with a detection limit of about $25 \mathrm{TU}$. Other samples were sent to the University of Miami, Rosenstiel School of Marine and Atmospheric Science Tritium Laboratory for low-level analysis. Details of the analytical procedure are given by Ostlund (1987, pp. 8-10). Pore water samples with low-level ${ }^{3} \mathrm{H}$ concentrations were processed using an electrolytic enrichment step in which ${ }^{3} \mathrm{H}$ concentrations are increased about 60 -fold through volume reduction. Tritium activities were measured by internal gas proportional counting of hydrogen $\left(\mathrm{H}_{2}\right)$ gas made from the water samples. Accuracy of the low-level measurement with enrichment for a 1-liter sample is $0.10 \mathrm{TU}\left(0.3 \mathrm{pCi} \mathrm{L}^{-1}\right.$ of $\left.\mathrm{H}_{2} \mathrm{O}\right)$, or 3.5 percent, whichever is greater (http://www.rsmas.miami.edu/groups/tritium/). For smaller samples, accuracy is estimated to be $1.0 \mathrm{TU}$, or 10 percent for $50 \mathrm{~mL}$ samples, and $0.4 \mathrm{TU}$, or 10 percent for $100 \mathrm{~mL}$ samples (Happell 2005). The $2 \sigma$ uncertainties given for the ${ }^{3} \mathrm{H}$ values include only counting uncertainties assigned by the laboratory and do not include a $1 \sigma$ external error of $0.36 \mathrm{TU}$ determined from replicate analyses of standards.

Multiple aliquots of five water standards with known ${ }^{3} \mathrm{H}$ concentrations ranging between 0 and $2.15 \mathrm{TU}$ were analyzed (Table 5-1). In general, the mean ${ }^{3} \mathrm{H}$ concentrations obtained for each standard are in good agreement with the accepted values. Standard deviations obtained for these replicate measurements are similar to or slightly larger than the reported analytical errors, based on counting statistics alone.

\subsection{RESULTS}

\subsubsection{Tritium in Validation Study Core Samples}

Pore water extracted from validation study core across the Drill Hole Wash fault zone and Sundance fault zone had ${ }^{3} \mathrm{H}$ concentrations ranging from less than 0.1 to $2.6 \pm 1.0$ TU (Figure 5-1 and Table 5-2). Most analyses have large uncertainties due to the small sample volumes. Collectively, ${ }^{3} \mathrm{H}$ concentrations define a skewed distribution (Figure 5-2), with a median value of $0.40 \mathrm{TU}$ and a geometric mean of $0.41 \mathrm{TU}$. One sample from the Sundance fault zone (ESF-SDClV\#18, 12.3 to $13.3 \mathrm{ft}$ [3.75 to $4.05 \mathrm{~m}$ ]) had a ${ }^{3} \mathrm{H}$ concentration of $2.6 \pm 1.0 \mathrm{TU}$, but a sample from an adjacent interval in the same borehole (10.9 to $11.8 \mathrm{ft}[3.32$ to $3.6 \mathrm{~m}])$ had a smaller ${ }^{3} \mathrm{H}$ value of $1.4 \pm 1.6 \mathrm{TU}$.

\subsubsection{Tritium in Other Core Samples from the Exploratory Studies Facility}

Pore water extracted from core sampled elsewhere in the ESF shows a wider range of ${ }^{3} \mathrm{H}$ concentrations than pore water extracted from the validation study core (Table 5-3). Eight of 11 core samples from Alcove \#2 (30 m below the surface), which intersects the highly fractured Bow Ridge fault zone, have ${ }^{3} \mathrm{H}$ concentrations ranging from $28.8 \pm 8.4 \mathrm{TU}$ to $155 \pm 11 \mathrm{TU}$. These ${ }^{3} \mathrm{H}$ concentrations, which are larger than the detection limit of about $25 \mathrm{TU}$ for this data set, are compelling evidence for the presence of bomb-pulse ${ }^{3} \mathrm{H}$ in the shallow subsurface. Elevated ${ }^{3} \mathrm{H}$ 
concentrations in these samples correlate with elevated ${ }^{36} \mathrm{Cl} / \mathrm{Cl}$ ratios observed in samples associated with the Bow Ridge fault zone, exposed nearby in the ESF tunnel walls (Appendix A).

Pore water in 7 core samples of PTn from the north ramp moisture study boreholes between stations $7+70$ and $10+69$ has ${ }^{3} \mathrm{H}$ concentrations ranging from less than $0.1 \mathrm{TU}$ to about $0.8 \mathrm{TU}$ (Table 5-3). Eighteen analyses of pore water from core samples from the northern Ghost Dance fault zone (Alcove \#6) have ${ }^{3} \mathrm{H}$ concentrations between $0.3 \pm 0.8 \mathrm{TU}$ and $2.2 \pm 1.2 \mathrm{TU}$ (Figure 5-3). Samples with elevated ${ }^{3} \mathrm{H}$ concentrations are common in the southern part of the ESF. Pore water from five core samples of TSw from borehole ESF-SAD-GTB\#1 drilled in Alcove \#7 has ${ }^{3} \mathrm{H}$ concentrations between $1.1 \pm 0.6 \mathrm{TU}$ and 3.7 $\pm 1.4 \mathrm{TU}$ (Table 5-3). Samples from the south ramp of the ESF between stations 59+65 and 75+10 typically have elevated ${ }^{3} \mathrm{H}$. Concentrations of ${ }^{3} \mathrm{H}$ in 28 samples, primarily from several exposures of faulted PTn, have a distribution that is skewed toward large values (Figure 5-4). Elevated ${ }^{3} \mathrm{H}$ concentrations also are present in the welded tuffs above and below the PTn (Figure 5-5, Source: Modified from USBR 1997). Four of the south ramp samples have ${ }^{3} \mathrm{H}$ concentrations $(8.2 \pm 1.0,12.5 \pm 1.2$, $14.3 \pm 2.0$, and $28.6 \pm 3.6 \mathrm{TU}$ ) that are above the $6 \mathrm{TU}$ value for present-day precipitation (Striegl et al. 1998, Table 3, p. 12-13).

\subsubsection{Tritium in Core Samples from the ECRB Cross Drift}

Tritium concentrations in pore water samples from welded TSw in the ECRB Cross Drift (Table 5-4 and Figure 5-6) are larger than in those from the ESF (Table 5-3 and Figure 5-3). The frequency distribution of ${ }^{3} \mathrm{H}$ values is skewed toward values as large as $10.3 \pm 1.8 \mathrm{TU}$, well above the modern atmospheric value of $6.3 \mathrm{TU}$ (Figure 5-7). No samples were obtained from the immediate vicinity of the Sundance fault, located approximately at ECRB Cross Drift station 11+35; however, samples closest to the fault (stations $10+00,12+00$, and 13+00) had low ${ }^{3} \mathrm{H}$ values. The samples closest to the Solitario Canyon fault, collected at ECRB Cross Drift station $25+00$, also had small ${ }^{3} \mathrm{H}$ concentrations. Samples with elevated ${ }^{3} \mathrm{H}$ concentrations are scattered throughout the ECRB Cross Drift and are not known to be associated with major faults.

The USGS made several attempts to replicate elevated ${ }^{3} \mathrm{H}$ concentrations observed in initial pore water extractions from boreholes ECRB-SYS-CS1500 and ECRB-SYS-CS2150. The work yielded mixed results (Table 5-4). The sample containing the largest ${ }^{3} \mathrm{H}$ concentration determined in the first set of analyses from 5.5 to $6.7 \mathrm{ft}(1.67$ to $2.04 \mathrm{~m})$ in borehole ECRB-SYS-CS2150 had a ${ }^{3} \mathrm{H}$ value of $9.8 \pm 1.0$ TU. A ${ }^{3} \mathrm{H}$ measurement from core between 3.4 and $4.1 \mathrm{ft}(1.04$ and $1.25 \mathrm{~m})$ in the same borehole yielded a value of less than $0.1 \mathrm{TU}$. The second largest ${ }^{3} \mathrm{H}$ concentration measured in the first set of analyses was from 14.4 to $17.4 \mathrm{ft}$ $(4.39$ to $5.30 \mathrm{~m})$ in borehole ECRB-SYS-CS1500, with a ${ }^{3} \mathrm{H}$ concentration of $2.5 \pm 0.8 \mathrm{TU}$. Subsequent analyses of pore water from different intervals of core $(4.3$ to $7.1 \mathrm{ft}$ [1.31 to $2.16 \mathrm{~m}$ ] and 9.5 to $12.1 \mathrm{ft}$ [2.90 to $3.69 \mathrm{~m}]$ ) from the same borehole yielded ${ }^{3} \mathrm{H}$ concentrations of $10.3 \pm 1.8 \mathrm{TU}$ and $1.5 \pm 0.8 \mathrm{TU}$, respectively. The difficulty in replicating these large values is not understood. 


\subsection{THRESHOLD VALUES FOR DETECTING MODERN WATER}

As noted in Section 5.1, a major challenge in using ${ }^{3} \mathrm{H}$ to detect modern water in the UZ is the establishment of a realistic threshold value that will minimize false positive values. This problem is not unique to the use of ${ }^{3} \mathrm{H}$ in hydrology and applies to a number of geochemical problems where the analyte of interest occurs at low concentrations, close to the method detection limits. In the following paragraphs, two alternative approaches are given for establishing the threshold value for ${ }^{3} \mathrm{H}$.

\subsubsection{USGS Establishment of a Threshold for Identifying Modern Water}

To establish a realistic threshold value for interpreting measured ${ }^{3} \mathrm{H}$ values as indicators of modern water, the USGS first evaluated the limitations of the analytical method. The USEPA has a procedure for determining the "method detection limit" (MDL). A brief description of the procedure and its application to the ${ }^{36} \mathrm{Cl}$ data was discussed in Section 4.6.3. Assuming the detection limit for low-level analysis of ${ }^{3} \mathrm{H}$ in small (about $100 \mathrm{~mL}$ ) water samples is about $0.4 \mathrm{TU}$ (Happell 2005), replicate analyses of standards with ${ }^{3} \mathrm{H}$ concentrations of $1.31,1.75$, and $1.81 \mathrm{TU}$ (Table 5-1) are suitable for evaluating the variability of the results at low levels. For this calculation, the USGS pooled the replicate analyses of the standards (Table 5-1) and calculated a standard deviation following Youden (1951, p. 16). The pooled standard deviation for these three sets of analyses $(n=16$, degrees of freedom=13) is 0.36 and the calculated MDL is 1.0 TU. The F-test (Youden 1951, p. 29-32) shows that the standard deviations for the data from three sets of standards are equal at the 95 percent confidence level. This pooling also is valid because the standard deviation is not a function of the concentration in the range of 0 to $2.0 \mathrm{TU}$, as is evident from the counting errors reported for real samples with ${ }^{3} \mathrm{H}$ values that are within this range of concentrations (i.e., the errors are not systematically larger for larger values). Values below 1.0 TU should be considered statistically indistinguishable from zero at the 99 percent confidence level and should not be interpreted as real ${ }^{3} \mathrm{H}$ concentrations.

The USEPA states that, "It is essential that all sample-processing steps of the analytical method be included in the determination of the method detection limit" (40 CFR 136, 2004, Appendix B, p. 317). Because the effects of drilling and water extraction methods were not evaluated for the validation study, this value of $\mathrm{MDL}=1.0 \mathrm{TU}$ may be an overly optimistic estimate. The USGS (Childress et al. 1999, p. 6) proposed a long-term method detection level (LT-MDL) that would incorporate additional measurement variability derived from multiple instruments, operators, calibrations, and sample preparation events. A larger number of duplicates, at least 24 per year, is required for calculation of the LT-MDL. Neither the MDL nor the LT-MDL addresses the issue of reporting levels, as pointed out by Childress et al. $(1999$, p. 7$)$, and both limits lead to a 50 percent probability of false negative values. Childress et al. (1999, p. 7$)$ further discuss various reporting levels that have been used, which are 5 to 10 times the MDL, and they cite USEPA's use of minimum level of quantitation (ML), which is 3.18 times the MDL for $n=7$ replicates.

Childress et al. (1999, p. 8) devised the laboratory reporting level (LRL) to limit the rate of false negative values to 1 percent or less. The LRL is defined as twice the LT-MDL. Using the USGS-calculated MDL of $1.0 \mathrm{TU}$ as an approximate representation of the USGS LT-MDL, the LRL for the ${ }^{3} \mathrm{H}$ data set is $2.0 \mathrm{TU}$. Analyses with concentrations between 1.0 and $2.0 \mathrm{TU}$ should 
be reported as estimates because detection in this region should have a $\leq 1$ percent probability of being a false positive value. The USGS considers the LRL of $2.0 \mathrm{TU}$ to be a reliable threshold value for the ${ }^{3} \mathrm{H}$ measurements. The statistical approach discussed below further supports the use of this $2.0 \mathrm{TU}$ threshold value.

The statistical approach that was used to estimate a threshold for bomb-pulse ${ }^{36} \mathrm{Cl} / \mathrm{Cl}$ values (Fabryka-Martin et al. 1997, Section 4.2.4) also was used to establish an independent threshold for ${ }^{3} \mathrm{H}$ in pore water extracted from ESF and ECRB Cross Drift core samples. The USGS applied this approach to the ${ }^{3} \mathrm{H}$ analyses contained in Tables 5-2, 5-3, and 5-4. These analyses were ranked by increasing ${ }^{3} \mathrm{H}$ concentration, and cumulative averages and standard deviations were calculated at each added value. The 11 samples with ${ }^{3} \mathrm{H}$ concentrations reported as less than $0.1 \mathrm{TU}$ were arbitrarily assigned concentrations between 0 and 0.1 in 0.01 increments to avoid standard deviations of zero. This artificial approach could cause the excursion of data above the curve on a plot (Figure 5-8) showing the number of standard deviations for each value from the cumulative mean. However, similar results are obtained if these 11 samples are excluded from the statistical analysis. The plot shows a relatively smooth curve for the first 108 samples in the data set (Figure 5-8). Tritium concentrations for these samples are less than 2.0 TU. After this ranking, the deviation of individual data points increases markedly, such that the probability of these values being that far from the cumulative mean of the ranked data set is less than 0.5 percent. The limit of 0.5 percent probability, known as Chauvenet's criterion, establishes a boundary for values that are likely to lie outside a sample population that is normally distributed (Taylor 1982, Chapter 6.2). The threshold value of $2.0 \mathrm{TU}$ established using Chauvenet's criterion agrees with the threshold value using the USEPA MDL and USGS LRL methods. The $2.0 \mathrm{TU}$ threshold minimizes the potential for obtaining false positive or false negative values.

\subsubsection{LANL Establishment of a Threshold.for Identifying Modern Water}

As pointed out in Section 5.1, interpretation of low-level ${ }^{3} \mathrm{H}$ concentrations in small-volume pore water samples is not straightforward. Complications may arise due to the fact that most environmental samples will be exposed to the atmosphere at some time(s) during their collection. Thus, it is difficult or impossible to completely rule out some contamination of samples. The statistical analyses of MDL and Chauvenet's criterion presented in Section 5.4.1 are used to determine a "threshold value" that will minimize false positives. These analyses result in a threshold value of $2 \mathrm{TU}$, a value that is considerably larger (by a factor of about 10) than would be expected if only in situ-produced ${ }^{3} \mathrm{H}$ were present, and a factor of 2 to 5 greater than the quoted analytical detection limit. The applicability of the statistical methods applied above in determining this threshold value is discussed below.

Although methods to determine MDLs may vary, it is agreed that determination of an MDL requires rigorous analyses of many standards of appropriate concentrations. Tritium data reported herein were analyzed by the University of Miami, Rosenstiel School of Marine and Atmospheric Science Tritium Laboratory for low-level analysis, one of two laboratories used for ${ }^{3} \mathrm{H}$ analyses by the NWQL. They report a detection limit of $0.1 \mathrm{TU}$ and a reportable accuracy and precision of $0.1 \mathrm{TU}$ or \pm 3.5 percent, whichever is larger, for $1-\mathrm{L}$ samples. Most of the samples analyzed for this study are smaller, however, and therefore larger detection limits of $0.4 \mathrm{TU}$ for $100-\mathrm{mL}$ samples and $1.0 \mathrm{TU}$ for $50-\mathrm{mL}$ samples are reported. 
The method to determine MDL, as applied in Section 5.4.1, has several requirements and assumptions, many of which are not satisfied in the analysis above, as follows.

- The data are assumed to have a normal distribution about a mean value. Although the available data do not show a normal distribution, their numbers are likely insufficient to prove or disprove such a distribution.

- The USEPA method calls for a minimum of seven analyses of the same standard; the method used by the NWQL requires at least 24 analyses per year. The maximum number of replicates of a single standard is seven (Table 5-1). Values from three standards were pooled to derive the MDL above (Section 5.4.1); however, the total number of analyses pooled is still below the minimum requirements of the NWQL.

- Choice of the appropriate standard concentrations to use for determination of MDLs is based on the assumption that at small concentrations, the standard deviation of the sample set will become constant at a small value because small differences in small instrument signals cannot be measured accurately. This is an important assumption for determination of the MDL. Standard deviations of the three sample sets used in Section 5.4.1 are not constant, nor do they show a trend with sample size. As a result, they are overly large and result in an over-estimation of the MDL.

- Finally, the USEPA method recommends an iterative process by analyzing standards with increasingly smaller concentrations to ensure robustness of the method. This was not conducted in this study.

The analysis to determine MDL, as described in Section 5.4.1, violates most of the basic requirements and assumptions of the method. The MDL of $1.0 \mathrm{TU}$ determined by this analysis is not statistically robust and should be considered a qualitative assessment.

Analysis using the NWQL, which is based on the USEPA method, is then used to arrive at a reliable threshold value for the ${ }^{3} \mathrm{H}$ measurement of $2.0 \mathrm{TU}$. It should be reiterated, however, that values between the MDL and the LRL have a $\leq 1$ percent probability of being a false positive value. Thus, values between 1.0 and 2.0 should be reported as detections.

Chauvenet's criterion is a simple test that can be used to identify data that may be considered as outliers of a normally distributed data set (Taylor 1982, Chapter 6.2). The use of Chauvenet's criterion, however, is controversial and "some scientists believe that data should never be rejected without external evidence that the measurement in question is incorrect" (Taylor, 1982, p. 169). The use of Chauvenet's criterion to evaluate potential outliers in the ${ }^{3} \mathrm{H}$ data set and the implications of the interpretations based on this approach may not be appropriate. Potential problems with this approach fall into two categories: (1) whether or not use of this statistical approach is appropriate for such a data set, and (2) the interpretation of the results of the statistical analysis presented above is not unique.

Chauvenet's criterion for rejection is typically used on data sets for which the range in values is expected to be normally distributed around a single mean value. In this case, the method is applied to a set of unknowns, for which the individual data points are not likely to have a 
common mean value. Application of the method implicitly assumes that variability in the data due to hydrogeologic heterogeneity is small compared to other sources of spread in the data. This is an invalid assumption for these geologic samples. Infiltration at Yucca Mountain is predicted to be heterogeneous due to the fractured nature of the rocks. Tritium concentrations will reflect these heterogeneities, unless a sampling scheme is carefully designed and the number of samples is sufficient to reflect a true average value. The ${ }^{3} \mathrm{H}$ data clearly reflect these heterogeneities. Many of these samples targeted features such as fault zones (e.g., the Bow Ridge fault zone, Ghost Dance fault zone), stratigraphic and/or hydrogeologic units (e.g., the Topopah Spring Tuff, PTn), or regions (e.g., the ESF south ramp). As expected, the data show a range in values from very small (equivalent to zero) to the largest values reported in this study (155 TU).

Of the ${ }^{3} \mathrm{H}$ data collected for this study, the data sets most likely to average natural hydrogeologic heterogeneities are the samples from the ECRB (Table 5-4) and the validation study core (Table 5-2). These data were obtained from cores that were drilled on regularly spaced intervals. Although the validation study boreholes were located near fault zones, it is reasonable to suggest that the random spacing of the boreholes could average geologic heterogeneities, and that this data set approximates a random sampling. On the basis of fracture density data it was expected that the validation study boreholes would intersect multiple fractures in the tuff (Section 3.1). Application of Chauvenet's criterion to this subset of the data (Figure 5-9) presents a potentially different picture than that presented for the entire data set (Figure 5-8). Figure 5-9 shows two distinct jumps in the data that rise beyond Chauvenet's criterion for outliers. The first of these jumps lies between 1.1 and $1.4 \mathrm{TU}$, values that differ from the 1.8 to $2.2 \mathrm{TU}$ cutoff obtained when the entire data set is used (Figure 5-8). This analysis illustrates the point that a different result may be obtained when a different subset of the data is selected for statistical analysis.

An alternate interpretation that unifies the ${ }^{3} \mathrm{H}$ data with analytical and geologic information follows. As shown in Figures 5-2 and 5-7, the ${ }^{3} \mathrm{H}$ data do not form a normal distribution. In all cases the data form distributions with maxima skewed to small values and long tails of larger values. However, data should form a normal distribution about a true composite detection limit. Reduced chi-squared tests performed on all of the data, and data from only the ECRB Cross Drift and validation study core samples, show best fits to normal distributions for data below approximately 1.2 TU. The mean for all data below $1.2 \mathrm{TU}$ is $0.5 \mathrm{TU}$, with a standard deviation of 0.3. The mean and standard deviation for the ECRB Cross Drift and validation study core data are 0.4 and 0.3 , respectively. These values also can be deduced by examination of histograms, which show maxima at these median values. These values are interpreted to indicate a "composite ${ }^{3} \mathrm{H}$ background" that represents the sum of all small sources of ${ }^{3} \mathrm{H}$ that may have entered the sample, either through natural processes or through sampling, processing, and analysis. This background value thus includes natural in situ ${ }^{3} \mathrm{H}$, possibly ${ }^{3} \mathrm{H}$ derived from construction water and the natural circulation of modern water vapor, and all other sources of ${ }^{3} \mathrm{H}$ contamination. These values lead to MDLs of 1.3 to $1.4 \mathrm{TU}$ at the 99 percent confidence level. These values are larger than those assigned by the analytical facility, consistent with the fact that these samples have undergone more extensive processing than have the standards. This value is also in reasonable agreement with the MDL of $1.0 \mathrm{TU}$ discussed above, but suggests a high probability that values above $1.4 \mathrm{TU}$ are true quantifiable detections. As pointed out above, analyses with concentrations between 1.0 and $2.0 \mathrm{TU}$ will have a greater than 99 percent probability of being a true positive value. 


\subsection{INTERPRETATION OF THE TRITIUM MEASUREMENTS}

Any discussion of the significance of the ${ }^{3} \mathrm{H}$ results is dependent on the estimation of the threshold value for the unambiguous detection of modern water described in Sections 5.4.1 and 5.4.2. The following paragraphs reflect differences in the two interpretations of the data.

\subsubsection{USGS Interpretation of the Tritium Measurements}

Tritium values in Alcove \#2 that are above the 25 TU detection limit (Section 2.2) indicate the presence of modern water associated with the Bow Ridge fault zone. Other locations of modern water include numerous sample sites along the south ramp of the ESF, the southern Ghost Dance fault zone (Alcove \#7), and several locations along the ECRB Cross Drift. Slightly elevated ${ }^{3} \mathrm{H}$ values are noted near the Sundance fault in the main ESF drift and near the northern Ghost Dance fault zone (Alcove \#6). These values are marginally above the $2.0 \mathrm{TU}$ threshold.

Eight pore water samples from the ECRB Cross Drift have ${ }^{3} \mathrm{H}$ values in excess of $2.0 \mathrm{TU}$. None of these locations is associated with known, through-going faults. This contrasts with observations in the ESF, where modern water occurrences are associated with faults or highly faulted zones, such as the south ramp. The lack of association of elevated ${ }^{3} \mathrm{H}$ values with faults led to a concern by the USGS about the possibility of analytical problems that may have caused the larger ${ }^{3} \mathrm{H}$ values. The attempt to replicate analyses by extracting water from adjacent intervals of core produced ambiguous results. At the present time, the USGS views the ${ }^{3} \mathrm{H}$ values in this area as suggestive but not conclusive proof of the presence of modern water.

\subsubsection{LANL Interpretation of the Tritium Measurements}

Interpretation of ${ }^{3} \mathrm{H}$ data obtained from low-level, small-volume samples is not straightforward. The problem is likely compounded for small-volume pore water samples as are presented here because of the more involved collection and processing schemes (e.g., drilling and water extraction) compared to saturated zone ground-water collection. Ideally, a composite background that incorporates the potential for higher than predicted analytical errors, as well as sample contamination, would have been rigorously determined. This campaign would involve analysis of a statistically sufficient number of standards of the appropriate composition that were subjected to all the same processing steps of the samples in all the same places. Such a campaign, which would be very difficult, time consuming, and costly, was not conducted. The robustness of the data was monitored through analysis of standards. Standards submitted along with the samples were of comparable volume and ${ }^{3} \mathrm{H}$ concentration as a large number of samples. Data from 18 standards agree well with the accepted value, indicating that these small-volume, low-concentration standards can be analyzed accurately. Two samples of dead water (zero $\left.{ }^{3} \mathrm{H}\right)$ also were analyzed accurately. These data demonstrate that the standards were handled without introducing contamination. They also demonstrate the robustness of the analytical techniques for small volume samples with small ${ }^{3} \mathrm{H}$ concentrations.

Although the data from the standards do not indicate analytical or contamination problems, they did not undergo the same sampling and extraction procedures as the samples. The actual samples will likely reflect increased analytical errors and are more susceptible to contamination when compared to the standards. The large number and skewed distribution of analyses below 
the detection limit of 0.4 for samples less than $100-\mathrm{mL}$ and 1.0 for samples less than $50 \mathrm{~mL}$ indicate that many of the samples were processed without substantial contamination.

On the basis of arguments presented above, the following guidelines for interpretation of the ${ }^{3} \mathrm{H}$ data are suggested. These guidelines are designed to not over-interpret potential false positives, while at the same time to not eliminate possibly important and accurate ${ }^{3} \mathrm{H}$ detections. Samples with ${ }^{3} \mathrm{H}$ concentrations greater than $1.4 \mathrm{TU}$ should be considered as having a greater than 99 percent probability of being a detection above a composite background value of approximately $0.5 \mathrm{TU}$, and thus indicate the presence of a component of modern water. The presence of modern water in samples with ${ }^{3} \mathrm{H}$ values between 0.5 and $1.4 \mathrm{TU}$ is equivocal, but should be considered a possibility, especially for samples greater than $1.1 \mathrm{TU}$, which have a 97.7 percent probability of being a true detection. The presence of bomb-pulse water is indicated by ${ }^{3} \mathrm{H}$ concentrations above $6 \mathrm{TU}$, the value assumed for modern precipitation (see Sections 5.0 and 5.1).

Given these guidelines, it is likely that modern water (Clark and Fritz 1997, p. 172) is present in the validation study core and ECRB tunnel samples in a number of locations. One sample with a

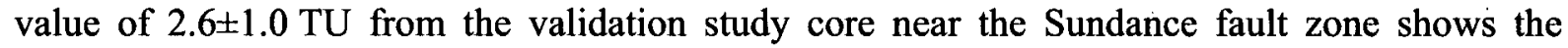
presence of modern water. The presence of modern water is suggested in four additional samples: two from the Sundance fault zone and two from the Drill Hole Wash fault zone. Most samples from Alcove \#2, near the Bow Ridge fault, show the presence of bomb-pulse water. Five samples from the northern Ghost Dance fault zone (Alcove \#6), with values between $1.4 \pm 0.08$ and $2.2 \pm 1.2 \mathrm{TU}$, show the presence of modern water. Two samples from borehole ESF-SAD-GTB\#1, drilled in Alcove \#7, with values of $1.8 \pm 1.4$ and $2.3 \pm 0.6 \mathrm{TU}$, indicate the presence of modern water. The presence of modern water is widespread in the south ramp, with 17 of 28 samples containing ${ }^{3} \mathrm{H}$ concentrations greater than $1.5 \mathrm{TU}$; five of these are greater than 6 TU. Modern water is also widely distributed in the ECRB. Eleven of 22 samples have ${ }^{3} \mathrm{H}$ concentrations greater than $1.5 \mathrm{TU}$; five of these have concentrations greater than $6 \mathrm{TU}$, indicating the presence of bomb-pulse water. 


\section{SUMMARY OF RESULTS, CONCLUSIONS, REMAINING ISSUES, AND RECOMMENDATIONS}

\subsection{SUMMARY OF RESULTS}

The ${ }^{36} \mathrm{Cl}$ validation study was conducted in three phases and involved the collection of new samples by drilling into the ESF tunnel walls so that ${ }^{36} \mathrm{Cl} / \mathrm{Cl}$ and ${ }^{3} \mathrm{H}$ measurements could be made in areas where previous studies identified elevated ${ }^{36} \mathrm{Cl} / \mathrm{Cl}$ ratios. The results of the validation study are summarized as follows:

- Results from Phase I work conducted at LLNL indicated that active leaching abraded the rock samples and extracted too much rock chloride relative to meteoric chloride ${ }^{36} \mathrm{Cl} / \mathrm{Cl}$ ratios range from $47 \times 10^{-15}$ to $248 \times 10^{-15}$; all but one value are less than $156 \times 10^{-15}$ ).

- Results from Phase I work conducted at LANL on validation core samples from the Sundance fault zone yielded ${ }^{36} \mathrm{Cl} / \mathrm{Cl}$ values consistent with analyses from previous LANL studies.

- Following a detailed series of leaching experiments in Phase II of the validation study, a 1-hour passive leaching protocol was established for processing samples in Phase III of the study. The passive leaching process extracted less rock chloride relative to meteoric chloride.

- USGS-LLNL ${ }^{36} \mathrm{Cl} / \mathrm{Cl}$ values for Phase III leachates of 34 samples of core from validation study boreholes across an area that includes the Sundance fault zone range from $137 \times 10^{-15}$ to $615 \times 10^{-15}$. These contrast with values greater than $1250 \times 10^{-15}$ reported previously for feature-based tunnel-wall samples in the same area (Figure 6-1).

- ${ }^{36} \mathrm{Cl} / \mathrm{Cl}$ ratios for Phase III leachates of validation study core prepared at the USGS and processed separately at LLNL and LANL agree within analytical error (Figure 6-1).

- LLNL analyses of six Niche \#1 core samples prepared at the USGS are statistically indistinguishable from validation study borehole data $\left({ }^{36} \mathrm{Cl} / \mathrm{Cl}\right.$ ratios range from $226 \times 10^{-15}$ to $717 \times 10^{-15}$ ).

- LLNL analyses of seven Niche \#1 core samples prepared at LANL yielded bomb-pulse values that are comparable to previous LANL data $\left({ }^{36} \mathrm{Cl} / \mathrm{Cl}\right.$ ratios range from $1,016 \times 10^{-15}$ to $8,558 \times 10^{-15}$ ).

- One LANL validation study analysis and several previous LANL analyses of samples from the ECRB Cross Drift also have ${ }^{36} \mathrm{Cl} / \mathrm{Cl}$ ratios above the $1,250 \times 10^{-15}$ bomb-pulse threshold.

- Tritium concentrations in pore water extracted from validation study core across the Drill Hole Wash fault zone and the Sundance fault zone range from less than 0.1 to $2.6 \pm 1.0 \mathrm{TU}$. 
- Tritium concéntrations in pore water extracted from samples from areas of known faulting in the north ramp, south ramp, and Alcove \#7 indicate the presence of modern water (i.e., water that entered the Yucca Mountain UZ after 1952).

- Tritium concentrations in pore water extracted from core samples from the ECRB Cross Drift range from less than 0.1 to $10.3 \pm 1.8 \mathrm{TU}$.

- The USGS and LANL established different ${ }^{3} \mathrm{H}$ thresholds for identifying modern water. The USGS value is $2.0 \mathrm{TU}$ and the LANL value is $1.4 \mathrm{TU}$.

\subsection{CONCLUSIONS}

The main conclusions of the validation study are as follows:

- USGS-LLNL did not find ${ }^{36} \mathrm{Cl} / \mathrm{Cl}$ ratios greater than $1,250 \times 10^{-15}$ in samples from the Sundance fault zone comparable to values reported previously by LANL.

- New analyses by LANL-LLNL on Niche \#1 core and ECRB Cross Drift tunnel-wall samples were consistent with results from previous LANL studies showing the presence of bomb-pulse ${ }^{36} \mathrm{Cl}$ in the ESF and ECRB Cross Drift. Analyses of these core samples by USGS-LLNL did not produce comparable results.

- With one exception, ${ }^{3} \mathrm{H}$ values in pore water from validation study core samples from the ESF do not exceed the USGS or LANL threshold values beyond the $2 \sigma$ error limits. Tritium values in pore water from two validation study core samples from the Drillhole Wash fault zone exceed the LANL threshold value of $1.4 \mathrm{TU}$.

- Regardless of whether the USGS or LANL threshold value is used, ${ }^{3} \mathrm{H}$ analyses of samples from areas of known faulting in the ESF north ramp, south ramp, and Alcove \#7 indicate the presence of modern water. Several locations in the ECRB Cross Drift that are not associated with major faults may also contain modern water; however, several attempts to replicate elevated ${ }^{3} \mathrm{H}$ values yielded ambiguous results. The difficulty in replicating these large values is not understood.

\subsection{REMAINING ISSUES}

\subsubsection{Absence of Elevated Chlorine-36/Chloride Ratios in USGS-LLNL Measurements}

Small concentrations of chloride in USGS-LLNL leachates resulted in relatively large uncertainties in ${ }^{36} \mathrm{Cl} / \mathrm{Cl}$ ratios. Use of the passive-leach protocol with short (1 hour) leaching times resulted in small chloride concentrations. Despite the large uncertainties of ${ }^{36} \mathrm{Cl} / \mathrm{Cl}$ ratios in AMS measurements of leachates with small concentrations of chloride, the replicate analyses of leachates from rocks (as well as blanks) are consistent and are considered to be reliable. However, bomb-pulse ${ }^{36} \mathrm{Cl} / \mathrm{Cl}$ ratios were not found using this technique.

Thirty-four leachates from the validation study boreholes, plus leachates of core from existing Niche \#1 boreholes, yielded a mean ${ }^{36} \mathrm{Cl} / \mathrm{Cl}$ ratio of $337 \pm 141(1 \sigma) \times 10^{-15}$ and a maximum ${ }^{36} \mathrm{Cl}$ 
ratio of $717 \pm 139 \times 10^{-15}$. This mean value contrasts with 19 of 34 LANL analyses (24 tunnel-wall samples and 10 Niche \#1 core samples), which have ${ }^{36} \mathrm{Cl} / \mathrm{Cl}$ ratios in excess of 1,250 $\times 10^{-15}$ and one value of $4,108 \times 10^{-15}$. The limited range of ${ }^{36} \mathrm{Cl} / \mathrm{Cl}$ ratios in the USGS-LLNL data over a wide range of chloride concentrations indicates that these data are not the result of mixing between distinct components with high and low ${ }^{36} \mathrm{Cl} / \mathrm{Cl}$ ratios.

In addition to a lack of bomb-pulse ${ }^{36} \mathrm{Cl}$ values, the ${ }^{36} \mathrm{Cl} / \mathrm{Cl}$ ratios determined by USGS-LLNL for samples from the Sundance fault zone are, on average, smaller than the Holocene value of about $500 \times 10^{-15}$. The USGS-LLNL results differ from the background LANL ${ }^{36} \mathrm{Cl} / \mathrm{Cl}$ values, which are higher than the Holocene value for northern ESF samples, but are closer to, although still statistically different from, the LANL values for southern ESF samples.

Whether the differences between ${ }^{36} \mathrm{Cl} / \mathrm{Cl}$ ratios determined for the validation study and those determined for the previous studies can be ascribed to differences in sampling protocol is currently a matter of professional opinion. The justification for using a borehole strategy across a broad ${ }^{36} \mathrm{Cl}$ anomaly was discussed in Section 3.1. As noted previously in this report, featurebased samples obtained from the tunnel walls allow selection of sub-samples with a greater fracture surface area per mass unit of rock than do the core samples. However, the different results obtained by USGS-LLNL and LANL-LLNL for representative core samples from the Niche \#1 boreholes demonstrate that other factors, such as laboratory contamination, also should be considered.

\subsubsection{Results for Niche \#1 Core}

Leaching experiments showed that leachates of more finely crushed material contain larger chloride concentrations than those from the more coarsely crushed material and that particle size is more important than leach duration. The increase in surface area as particle size decreases allows a greater amount of rock chloride to be extracted, resulting in a negative correlation between chloride concentration and ${ }^{36} \mathrm{Cl} / \mathrm{Cl}$ ratio. This negative correlation is observed in data for leachates from the active-leach process. In contrast, validation study leachates of Niche \#1 core crushed and processed at LANL show the opposite trend. For the five samples of the coarsest material, ${ }^{36} \mathrm{Cl} / \mathrm{Cl}$ ratios are smallest in the two samples with the smallest chloride concentration. Leachates of the fines from both of these samples also were analyzed and yielded not only larger chloride concentrations, as expected, but also much larger ${ }^{36} \mathrm{Cl} / \mathrm{Cl}$ ratios, including the largest value reported for an ESF sample $\left(8,558 \times 10^{-15}\right)$. These results are opposite of the conclusions of Lu et al. (2003), who stated that larger ${ }^{36} \mathrm{Cl} / \mathrm{Cl}$ ratios should be observed in leachates with smaller chloride concentrations from larger particle sizes. These contradictions show that the present understanding of chloride sources and mixing during leaching is inadequate.

\subsubsection{Spatial Distribution of Elevated Chlorine-36 Values and Tritium Values}

The USGS and LANL differ in their interpretations of the spatial distribution of elevated ${ }^{36} \mathrm{Cl} / \mathrm{Cl}$ ratios and ${ }^{3} \mathrm{H}$ results, as described below. 


\subsubsection{USGS Interpretation of the Spatial Distribution of Elevated Values}

The ${ }^{36} \mathrm{Cl}$ Peer Review Team recommended that future studies include analyses of other bombpulse indicators, in particular ${ }^{3} \mathrm{H}$. All of the 52 analyses of validation study core from the Drill Hole Wash fault zone and Sundance fault zone yielded ${ }^{3} \mathrm{H}$ concentrations that were either less than the $2.0 \mathrm{TU}$ background cutoff value or were indistinguishable within $2 \sigma$ analytical error. In contrast, the presence of modern water is indicated by elevated ${ }^{3} \mathrm{H}$ concentrations in the south ramp. Some water samples have ${ }^{3} \mathrm{H}$ concentrations that were substantially larger than modern atmospheric levels, indicating a bomb-pulse origin. The distribution of ${ }^{3} \mathrm{H}$ in south ramp samples contrasts with the distribution of previously reported ${ }^{36} \mathrm{Cl} / \mathrm{Cl}$ analyses from the same area. A large number of tunnel-wall samples from the ESF south ramp did not contain ${ }^{36} \mathrm{Cl} / \mathrm{Cl}$ ratios with bomb-pulse values. In addition, samples from the northern ESF show bomb-pulse ${ }^{36} \mathrm{Cl} / \mathrm{Cl}$ ratios, but ${ }^{3} \mathrm{H}$ values below the threshold value of $2.0 \mathrm{TU}$.

Similar differences between the location of elevated ${ }^{3} \mathrm{H}$ and ${ }^{36} \mathrm{Cl} / \mathrm{Cl}$ values occur in the ECRB Cross Drift. Samples with elevated ${ }^{36} \mathrm{Cl} / \mathrm{Cl}$ ratios were obtained only from areas associated with the Solitario Canyon fault and an unnamed fault near ECRB Cross Drift station 22+37. Samples with elevated ${ }^{3} \mathrm{H}$ concentrations, including two values indicative of a bomb-pulse origin, were scattered throughout the ECRB Cross Drift. In one case, samples within $4 \mathrm{~m}$ of each other contained a ${ }^{3} \mathrm{H}$ concentration of $9.8 \mathrm{TU}$ (sampled at ECRB Cross Drift station 21+49; Table 5-4), and ${ }^{36} \mathrm{Cl} / \mathrm{Cl}$ ratio of $4,890 \times 10^{-15}$ (sampled at ECRB Cross Drift station 21+54.5; Table 4-17). However, attempts to reproduce the ${ }^{3} \mathrm{H}$ measurement from core in the same borehole resulted in a value of $0.1 \mathrm{TU}$. Analyses of adjacent tunnel-wall samples at stations $21+54$ and $21+55$ (Table 4-17) had ${ }^{36} \mathrm{Cl} / \mathrm{Cl}$ values of $915 \times 10^{-15}$ and $553 \times 10^{-15}$, respectively. Additional samples at stations $22+50$ and $25+00$ (Table 5-4) had ${ }^{3} \mathrm{H}$ concentrations below $1 \mathrm{TU}$. ${ }^{3} \mathrm{H}$ measurements were not made for samples beyond station $25+00$ in the area where multiple bomb-pulse ${ }^{36} \mathrm{Cl} / \mathrm{Cl}$ values were observed, because core was not available.

\subsubsection{LANL Interpretation of the Spatial Distribution of Elevated Values}

Interpretation of ${ }^{3} \mathrm{H}$ data collected for this study relies heavily on interpretations of a threshold value, below which an analysis is not considered indicative of modern water (Section 5). If, as discussed in Section 5.4.2, a value of $1.4 \mathrm{TU}$ is taken as a lower limit for quantifiable ${ }^{3} \mathrm{H}$ values and some smaller values are accepted as possible indicators of modern water, then the comparison of the spatial differences between ${ }^{3} \mathrm{H}$ and ${ }^{36} \mathrm{Cl}$ changes substantially. Given these lower limits for ${ }^{3} \mathrm{H}$ detections, modern water was detected in at least one (value of $2.6 \pm 1.0$ ) and up to four (three ${ }^{3} \mathrm{H}$ values between 1.4 and 1.6) of 52 samples of the validation study core. These core samples were collected at 5-m spaced intervals, a collection scheme similar to that used to collect systematic samples for previous LANL ${ }^{36} \mathrm{Cl}$ studies. In these samples the occurrence of bomb-pulse ${ }^{36} \mathrm{Cl}$ is two of the 54 samples. The occurrences of modern water based on ${ }^{3} \mathrm{H}$ and ${ }^{36} \mathrm{Cl}$ for systematically collected samples are therefore in reasonable agreement.

Contrasting distributions of ${ }^{3} \mathrm{H}$ and ${ }^{36} \mathrm{Cl}$ ratios in the south ramp are readily attributable to the elevated chloride concentrations in pore water in this region. Elevated pore water chloride concentrations mask potential bomb-pulse signals through dilution ( $\mathrm{Lu}$ et al. 2003), but do not affect ${ }^{3} \mathrm{H}$ concentrations. 
Apparent differences between ${ }^{3} \mathrm{H}$ and ${ }^{36} \mathrm{Cl}$ distribution in the ECRB Cross Drift are difficult to evaluate because none of the samples were precisely collocated. Nonetheless, most ${ }^{3} \mathrm{H}$ and ${ }^{36} \mathrm{Cl}$ data from samples collocated within a few meters agree (i.e., ${ }^{3} \mathrm{H}$ is below detection and ${ }^{36} \mathrm{Cl}$ is less than $1200 \times 10^{-15}$, both values indicating pre-bomb-pulse water). As stated above, the sample pair most closely collocated (4 m apart) shows the second largest ${ }^{3} \mathrm{H}$ value $(9.8 \mathrm{TU})$ and the largest ${ }^{36} \mathrm{Cl}$ value $\left(4890 \times 10^{-15}\right)$ measured in the ECRB Cross Drift. Of other samples with either a (but not both) ${ }^{3} \mathrm{H}$ or ${ }^{36} \mathrm{Cl}$ bomb-pulse signature, none is collocated closer than $12 \mathrm{~m}$. Unfortunately, these two studies were conducted independently and thus did not emphasize collocation of samples. As a result, comparison of the spatial distribution of modern water deduced from ${ }^{3} \mathrm{H}$ and ${ }^{36} \mathrm{Cl}$ data is inconclusive. The data, however, are not contradictory, but rather suggest a rough correlation.

\subsubsection{Potential Contamination from Field and Laboratory Environments}

The USGS and LANL differ in their interpretations of the potential for contamination from field and laboratory environments, as described below.

\subsubsection{USGS Interpretation of the Potential for Contamination from Field and Laboratory Environments}

Contamination of USGS-LLNL leachates by sources with low ${ }^{36} \mathrm{Cl} / \mathrm{Cl}$ ratios or contamination of LANL leachates by sources with high ${ }^{36} \mathrm{Cl} / \mathrm{Cl}$ ratios could explain the differences in ${ }^{36} \mathrm{Cl} / \mathrm{Cl}$ ratios determined by USGS-LLNL and LANL. Analysis of laboratory blanks testing the amount and composition of chloride added during crushing, leaching, and target preparation by USGS-LLNL has not identified a source with consistently low ${ }^{36} \mathrm{Cl} / \mathrm{Cl}$ ratios (Sections 4.4.1 and 4.6). Samples analyzed by USGS-LLNL included rock crushed and sieved at the SMF, the USGS, and Phillips Enterprises LLC of Golden, Colorado, by machine and by hand. Resulting ${ }^{36} \mathrm{Cl} / \mathrm{Cl}$ ratios are similar regardless of where the sample was crushed. Therefore, contributions from a contaminant introduced during crushing would have to be similar at all three facilities and the same for both hand and machine crushing. Furthermore, the absence of a correlation between chloride concentrations and ${ }^{36} \mathrm{Cl} / \mathrm{Cl}$ ratios in the USGS-LLNL data seems to be inconsistent with mixing of multiple components with distinct compositions.

Possible contamination of samples with large ${ }^{36} \mathrm{Cl} / \mathrm{Cl}$ ratios in field and laboratory environments has been evaluated. The very high ${ }^{36} \mathrm{Cl} / \mathrm{Cl}$ ratios measured in cuttings from a surface-based borehole (USW UZ-N55), with eight of 14 leachates having. ${ }^{36} \mathrm{Cl} / \mathrm{Cl}$ ratios between $10,480 \times 10^{-15}$ and $27,040 \times 10^{-15}$ (Fabryka-Martin et al. 1993, Table 2), were likely caused by drilling or sample collection using ${ }^{36} \mathrm{Cl}$-contaminated equipment (Fabryka-Martin and Liu 1995, Section 3.1.3; Fabryka-Martin, Turin et al. 1996, Sections 4.3.3 and 5.3.1). The presence of laboratory equipment contaminated with ${ }^{36} \mathrm{Cl}$ also was mentioned in later LANL reports that presented results from ESF samples: "Although this nuclide has been found to be present at unacceptably high levels in some laboratory equipment and rooms, these items and work environments are simply avoided for routine processing" (Fabryka-Martin, Wolfsberg et al. 1996, p. 15). "A particular piece of equipment is not used to prepare samples if an excessively high ${ }^{36} \mathrm{Cl}$ level is measured in a blank prepared using it; for example, such was the case for a shatterbox that was being used to characterize the in situ halide and $\mathrm{SO}_{4}$ concentrations of Paintbrush Tuffs" (Fabryka-Martin et al. 1997, p. 4-2). Details of the nature and extent of the 
${ }^{36} \mathrm{Cl}$ contamination have not been presented. In each case, the authors indicate that ${ }^{36} \mathrm{Cl} / \mathrm{Cl}$ levels were monitored and that contamination was not "at a level to cause concern" (Fabryka-Martin, Wolfsberg et al. 1996, p. 15).

Studies of ${ }^{36} \mathrm{Cl}$ performed at other sites hosting nuclear activities have reported high blank ${ }^{36} \mathrm{Cl}$ from laboratory processing. Background values for ${ }^{36} \mathrm{Cl} / \mathrm{Cl}$ as high as $1,000,000 \times 10^{-15}$ were observed at the AECL's Chalk River Laboratories and were attributed to reactor and wastemanagement operations (Andrews et al. 1994, Section 3.2). Although special care taken during handling and processing of samples allowed background ${ }^{36} \mathrm{Cl} / \mathrm{Cl}$ limits of $10^{-15}$ to be achieved for most types of samples, rock samples remained an exception, having about 10 times higher background levels (Andrews et al. 1994, Section 3.2). Determinations of ${ }^{36} \mathrm{Cl} / \mathrm{Cl}$ ratios at the Australian Nuclear Science and Technology Organization's Lucas Heights reactor facility also have identified ${ }^{36} \mathrm{Cl} / \mathrm{Cl}$ contamination up to $10,000 \times 10^{-15}$ that was traced to neutron irradiation of ${ }^{35} \mathrm{Cl}$ in the air circulated around the High Flux Australian Reactor (Bird et al. 1990, Section 2.2). High values of ${ }^{36} \mathrm{Cl} / \mathrm{Cl}$ (up to $24,000 \times 10^{-15}$ ) also were observed in blanks stored in a desiccator for a 6-month period as well as in chloride extracted from the silica gel desiccant (Bird et al. 1990, Section 2.4). The authors attributed this contamination to vapor phase exchange of chloride. They further cite that "samples with ${ }^{36} \mathrm{Cl} / \mathrm{Cl}$ ratios on the order of $10^{-11}$ [tens of thousands $\times 10^{-15}$ ] have also been observed in radiochemistry laboratories" (citation credited to "J. Fabryka-Martin, private communication, 1989" in Bird et al. 1990, Section 2.4 and reference [10]).

Potential sources of field contamination of tunnel walls have not been fully evaluated. Contaminated soils in Jackass Flats, within a few kilometers of the north portal, contain ${ }^{36} \mathrm{Cl} / \mathrm{Cl}$ ratios two orders of magnitude larger than bomb-pulse values in the ESF (Section 2.1.1). The ESF ventilation system continually intakes unfiltered outside air, which is distributed throughout the tunnel. The amount and source of exogenous dust brought into the tunnel is currently under investigation. Another source of chlorine contamination was recently discovered. The conveyor belt covers (CRWMS M\&O 1995) contain approximately 10 percent chlorinated paraffin wax, which is 71.5 percent chlorine by weight (Skeggs 2005).

\subsubsection{LANL Interpretation of the Potential for Contamination from Field and Laboratory Environments}

Procedural blanks taken throughout the course of this study indicate that blank levels are small and do not affect the ${ }^{36} \mathrm{Cl} / \mathrm{Cl}$ ratios substantially, even for smallest sample sizes. In addition to the analytical data collected during this and previous studies by LANL, there are a number of additional reasons why it is unlikely that blanks are a cause of large ${ }^{36} \mathrm{Cl} / \mathrm{Cl}$ ratios in LANL samples including:

- Niche \#1 core with the largest ${ }^{36} \mathrm{Cl} / \mathrm{Cl}$ ratios also has the largest chloride concentrations. Therefore it would take an extremely high ${ }^{36} \mathrm{Cl}$ blank to account for these values. Furthermore, the largest measured ${ }^{36} \mathrm{Cl} / \mathrm{Cl}$ blank for samples analyzed at LANL during this study was $4,257 \times 10^{-15}$, with most being considerably less (Table 4-16). This largest blank ratio is still considerably smaller than the maximum value of $8,558 \times 10^{-15}$ for Niche \#1 core (Table 4-12). Available data preclude blank ${ }^{36} \mathrm{Cl}$ from being the reason for this large value. 
- The data from Niche \#1 follow a consistent pattern with the largest chloride concentrations and ${ }^{36} \mathrm{Cl} / \mathrm{Cl}$ ratios in the finest samples, smallest values in the coarsest fractions, and intermediate values for intermediate size fractions. This pattern is consistent for five separate samples, three of which did not undergo the same sieving sequence. It is difficult to imagine a mechanism by which the large blanks required by the sample size might manifest themselves in such a consistent fashion.

- Samples from the ESF and ECRB Cross Drift with bomb-pulse values are typically large; generally rock samples between 3 and $5 \mathrm{~kg}$ were processed. Leachate chloride concentrations are typically between 0.4 and $1.0 \mathrm{mg} / \mathrm{kg}$ (DTN: LA0305RR831222.001 [UQ], LAJF831222AQ98.004 [Q]). A 4-kg sample with $0.5 \mathrm{mg} / \mathrm{kg}$ chloride concentration and a ${ }^{36} \mathrm{Cl} / \mathrm{Cl}$ ratio of 2000 will contain approximately $4 \times 10^{-12} \mathrm{mg}{ }^{36} \mathrm{Cl}$. The mean ${ }^{36} \mathrm{Cl}$ mass for 12 blanks reported by LANL is $1.6 \times 10^{-14} \mathrm{mg}$ (Table 4-16). Thus, a typical sample with a bomb-pulse signal contains 250 times more ${ }^{36} \mathrm{Cl}$ than the mean blank. In order for a bomb-pulse measurement in a sample to be due solely to blank contamination, that blank value would have to be enormously high relative to measured values. Blanks in this study vary by a maximum of a factor of seven.

- Most samples with bomb-pulse values were found near structures; systematic samples rarely show bomb-pulse values, as discussed above (DTN: LA0305RR831222.001 [UQ], LAJF831222AQ98.004 [Q]). It is highly unlikely that anomalously elevated blanks would correlate with structures.

- The data for samples processed from the ECRB Cross Drift as part of this study (Table 4-17) compare well to those from previous studies (Appendix A), with both data sets containing bomb-pulse signals; values between $500 \times 10^{-15}$ and $1,250 \times 10^{-15}$ and values less than $500 \times 10^{-15}$. These data sets were generated by different personnel, working in different laboratories with different laboratory equipment, and processing samples by slightly different methods. Analyses were also performed by a different analytical facility. Thus, the two studies meet qualifications of an independent validation study.

- Bomb-pulse ${ }^{36} \mathrm{Cl}$ values for samples collected at Yucca Mountain, including some from the deep subsurface, have been obtained by facilities other than LANL in investigations that have spanned 20 years. Table 3-1 outlines the sample processing history of Yucca Mountain Project ${ }^{36} \mathrm{Cl}$ samples. The table corroborates arguments above that ${ }^{36} \mathrm{Cl}$ contamination from laboratory processing is not responsible for bomb-pulse values observed in Yucca Mountain samples.

\subsection{RECOMMENDATIONS}

The differences between ${ }^{36} \mathrm{Cl} / \mathrm{Cl}$ measurements obtained from previous ${ }^{36} \mathrm{Cl}$ studies and the ${ }^{36} \mathrm{Cl}$ validation study cannot be explained by presently available data. However, these data do point to areas where continued investigations may resolve many of the remaining issues outlined in Section 6.3. The following recommendations for further investigations include additional evaluations of existing work, additional analyses of blank materials and existing samples, and an independent validation study that incorporates the lessons learned, to date. 


\subsubsection{Evaluation of Field Contamination}

The USGS and Bechtel SAIC Company (BSC) are collecting dust samples from various environments at Yucca Mountain, including dust in the ESF and ECRB Cross Drift. The ${ }^{36} \mathrm{Cl} / \mathrm{Cl}$ ratios in this dust should be determined. Further, the isotopic composition of chlorine in neoprene and other potential chlorine-bearing materials used in construction should be measured. If further samples for ${ }^{3} \mathrm{H}$ measurements are collected by dry-drilling methods, sampling blanks should be designed, implemented, and monitored. For example, the ${ }^{3} \mathrm{H}$ content of moisture in the compressed air should be determined and its effect on sampling evaluated.

\subsubsection{Evaluation of Laboratory Blanks}

All stages of sample processing should be fully controlled by adequate blank measurements. Long-term environmental exposure blanks could capture sporadic ${ }^{36} \mathrm{Cl}$ contamination, if present. Also, crushing blanks remain a potential source of uncertainty in identifying possible contamination problems. Although it is difficult to evaluate crushing blanks, approaches such as those outlined in Section 4.4.1.2 would help document important aspects of sample processing that may have been unconstrained in the past. Better data on crushing blanks need to be collected using protocols that replicate previous handling and processing steps. Additional ${ }^{3} \mathrm{H}$ measurements should be made to evaluate potential contamination during all stages of pore water extraction. One approach would involve imbibing ${ }^{3} \mathrm{H}$-free water under controlled conditions into the rock sample from which water was previously extracted. Re-extraction of water for ${ }^{3} \mathrm{H}$ analyses would yield a laboratory process blank.

\subsubsection{Additional ${ }^{36} \mathrm{Cl} / \mathrm{Cl}$ Analyses of Validation Study Core and ECRB Cross Drift Core}

Validation study core used for pore water distillation and ${ }^{3} \mathrm{H}$ analysis is archived at the USGS. Although the core was dried out during vacuum distillation, the process did not remove chloride. Therefore, this core is suitable for chloride extraction. Validation study core from the Sundance fault zone, Drill Hole Wash fault zone, and ECRB Cross Drift remaining after vacuum distillation should be split and leached using previous methods, with the exception of increasing sample sizes or leaching times to increase the total amount of chloride available for ${ }^{36} \mathrm{Cl} / \mathrm{Cl}$ analysis. This test should include handling and crushing processes to detect possible differences in ${ }^{36} \mathrm{Cl} / \mathrm{Cl}$ values from USGS-LLNL and LANL. In addition, splits of these samples should be sent to an independent laboratory with no history of ${ }^{36} \mathrm{Cl}$ contamination. Also, ${ }^{36} \mathrm{Cl} / \mathrm{Cl}$ ratios should be re-analyzed in the ECRB Cross Drift samples where elevated ${ }^{3} \mathrm{H}$ values were observed.

\subsubsection{Independent Validation Study Using New Samples}

Using existing samples, the experiments outlined above may provide sufficient insight to resolve the issue of whether or not bomb-pulse ${ }^{36} \mathrm{Cl}$ is present at depth in the Yucca Mountain UZ.

However, in the event these experiments do not provide conclusive evidence, it is recommended that a third party, without previous ties to either the USGS or LANL, should be assigned the task of designing an independent validation study that includes new sample collection. This effort would have the distinct advantage of evaluating the scientific strengths and weaknesses of the work completed to date. 


\section{REFERENCES CITED}

\subsection{DOCUMENTS CITED}

Andrews, H.R.; Koslowsky, V.T.; Cornett, R.J.J.; Davies, W.G.; Greiner, B.F.; Imahori, Y.; McKay, J.W.; Milton, G.M.; and Milton, J.C.D. 1994. "AMS Measurements of ${ }^{36} \mathrm{Cl}$ at Chalk River." Nuclear Instruments and Methods in Physics Research Section B: Beam Interactions with Materials and Atoms, 92, ([1-4]), 74-78. New York, New York: North-Holland. TIC: 254091.

Andrews, J.N.; and Kay. R.L.F. 1982. "Natural Production of Tritium in Permeable Rocks." Nature, 298, (22). New York, New York: Macmillan Journals Ltd. TIC: 257453.

Bird, J.R.; Shahgholi, N.; Jenkinson, A.; Smith, A.; Fifield, L.K.; Ophel, T.; and Allan, G. 1990. "Problems of Contamination in ${ }^{36} \mathrm{Cl}$ Studies." Nuclear Instruments and Methods in Physics Research B: Beam Interactions with Materials and Atoms, 52, ([3-4]), 348-350. Amsterdam, The Netherlands: North-Holland. TIC: 254141.

BSC (Bechtel SAIC Company) 2002. Technical Work Plan for: Performance Assessment Unsaturated Zone. TWP-NBS-HS-000003, Rev. 02. Las Vegas, Nevada: Bechtel SAIC Company. ACC: MOL.20030102.0108.

Campbell, K.; Wolfsberg, A.; Fabryka-Martin, J.; and Sweetkind, D. 2003. "Chlorine-36 Data at Yucca Mountain: Statistical Tests of Conceptual Models for Unsaturated-Zone Flow." Journal of Contaminant Hydrology, 62-63, 43-61. New York, New York: Elsevier. TIC: 254205.

Childress, C.J. Oblinger; Foreman, W.T.; Connor, B.F.; and Maloney, T.J. 1999. New Reporting Procedures Based on Long-Term Method Detection Levels and Some Considerations for Interpretations of Water-Quality Data Provided by the U.S. Geological Survey National Water Quality Laboratory. Open-File Report 99-193. Reston, VA: U.S. Geological Survey. ACC: MOL.20050712.0406.

Clark, I.D. and Fritz, P. 1997. Environmental Isotopes in Hydrogeology. Boca Raton, Florida: Lewis Publishers. TIC: 233503.

Cohon, J.L. 2000. "Appreciation for Presentations Made at the May Meeting of the Nuclear Waste Technical Review Board." Letter from J.L. Cohon (NWTRB) to I. Itkin (DOE/OCRWM), June 16, 2000. ACC: HQO.20010227.0090.

Coleman, D. 2005. "Cl-36 Comments." E-mail from D. Coleman to R. Jones and C. Madore, June 15, 2005. ACC: MOL.20050629.0278.

CRWMS M\&O (Civilian Radioactive Waste Management System Management and Operating Contractor) 1995. "Transmittal of Shop Drawings, Equipment Data, Material Samples, or Manufacturer's Certificates of Compliance (SDT) for Subsurface Conveyor Belt." Transmittal No. 2A-14-CLD490. Las Vegas, Nevada: CRWMS M\&O. ACC: DRC.19961218.0002. 
CRWMS M\&O 1998. Evaluation of Flow and Transport Models of Yucca Mountain, Based on Chlorine-36 and Chloride Studies for FY98. BA0000000-01717-5700-00007, Rev. 00. Las Vegas, Nevada: CRWMS M\&O. ACC: MOL.19981208.0119.

CRWMS M\&O 2000. Analysis of Geochemical Data for the Unsaturated Zone. ANL-NBS-HS000017, Rev. 00. Las Vegas, Nevada: CRWMS M\&O. ACC: MOL.20000725.0453.

DOE (U.S. Department of Energy) 2001. Yucca Mountain Science and Engineering Report. DOE/RW-0539. Washington, D.C.: U.S. Department of Energy, Office of Civilian Radioactive Waste Management. ACC: MOL.20010524.0272.

DOE 2004. Quality Assurance Requirements and Description. DOE/RW-0333P, Rev. 16. Washington, D.C.: U.S. Department of Energy, Office of Civilian Radioactive Waste Management. ACC: MOL.20020819.0387.

DOE 2002. Yucca Mountain Science and Engineering Report. DOE/RW-0539, Rev. 1. Washington, D.C.: U.S. Department of Energy, Office of Civilian Radioactive Waste Management. ACC: MOL.20020404.0042.

Fabryka-Martin, J.T. and Liu, B. 1995. Distribution of Chlorine-36 in UZ-14, UZ-16, Perched Water, and the ESF North Ramp, Yucca Mountain, Nevada. LA-CST-TIP-95-011, R2. Draft. Los Alamos, New Mexico: Los Alamos National Laboratory. ACC: MOL.19960415.0209.

Fabryka-Martin, J.; Wightman, S.; Murphy, W.; Wickham, M.; Caffee, M.; Nimz, G.; Southon, J.; and Sharma, P. 1993. "Distribution of Chlorine-36 in the Unsaturated Zone at Yucca Mountain: An Indicator of Fast Transport Paths." Proceedings of the Topical Meeting on Site Characterization and Model Validation, FOCUS '93, September 26-29, 1993, Las Vegas, Nevada. Pages 58-68. La Grange Park, Illinois: American Nuclear Society. TIC: 102245.

Fabryka-Martin, J.; Wolfsberg, A.V.; Dixon, P.R.; Levy, S.; Musgrave, J.; and Turin, H.J. 1996. Summary Report of Chlorine-36 Studies: Sampling, Analysis and Simulation of Chlorine-36 in the Exploratory Studies Facility. Milestone 3783M. Los Alamos, New Mexico: Los Alamos National Laboratory. ACC: MOL.19970103.0047.

Fabryka-Martin, J.T.; Flint, A.L.; Sweetkind, D.S.; Wolfsberg, A.V.; Levy, S.S.; Roemer, G.J.C.; Roach, J.L.; Wolfsberg, L.E.; and Duff, M.C. 1997. Evaluation of Flow and Transport Models of Yucca Mountain, Based on Chlorine-36 Studies for FY97. LA-CST-TIP-97-010. Los Alamos, New Mexico: Los Alamos National Laboratory. ACC: MOL.19980204.0916.

Fabryka-Martin, J.T.; Turin, H.J.; Wolfsberg, A.V.; Brenner, D.; Dixon, P.R.; and Musgrave, J.A. 1996. Summary Report of Chlorine-36 Studies. LA-CST-TIP-96-003. Draft. Los Alamos, New Mexico: Los Alamos National Laboratory. ACC: MOL.19970103.0037.

Faure, G. 1986. Principles of Isotope Geology. 2nd Edition. New York, New York: John Wiley \& Sons. TIC: 237212. 
Flint, A.L.; Flint, L.E.; Bodvarsson, G.S.; Kwicklis, E.M.; and Fabryka-Martin, J. 2001. "Evolution of the Conceptual Model of Unsaturated Zone Hydrology at Yucca Mountain, Nevada." Journal of Hydrology, 247, ([1-2]), 1-30. New York, New York: Elsevier. TIC: 250932.

Flint, A.L.; Hevesi, J.A.; and Flint, L.E. 1996. Conceptual and Numerical Model of Infiltration for the Yucca Mountain Area, Nevada. Milestone 3GUI623M. Denver, Colorado: U.S. Geological Survey. ACC: MOL.19970409.0087.

Flint, L.E. 2003. "Physical and Hydraulic Properties of Volcanic Rocks from Yucca Mountain, Nevada." Water Resources Research, 39, (5), 1-1 through 1-13. Washington, D.C.: American Geophysical Union. TIC: 254297.

Gascoyne, M.; Miller, N.H.; and Neymark, L.A. 2002. "Uranium-Series Disequilibrium in Tuffs from Yucca Mountain, Nevada, as Evidence of Pore-fluid Flow Over the last Million Years." Applied Geochemistry, 17, ([6]), 781-792. [New York, New York]: Elsevier. TIC: 251901.

Glaser, J.A.; Foerst, D.L.; McKee, G.D.; Quave, S.A.; Budde, W.L. 1981. "Trace Analyses for Wastewaters." Environmental Science \& Technology, 15, (12), 1428-1435. Washington, D.C.: American Chemical Society. TIC: 257279.

Happell, J. 2005. "Detection Limit for Low Volume Samples." E-mail from J. Happell to Z. Peterman, May 12, 2005. ACC: MOL.20050629.0277.

Kwicklis, E.M.; Flint, A.L.; and Healy, R.W. 1994. "Simulation of Flow in the Unsaturated Zone Beneath Pagany Wash, Yucca Mountain." High Level Radioactive Waste Management, Proceedings of the Fifth Annual International Conference, Las Vegas, Nevada, May 22-26, 1994, 4, 2341-2351. La Grange Park, Illinois: American Nuclear Society. TIC: 210984.

Lehmann, B.E.; Davis, S.N.; and Fabryka-Martin, J.T. 1993. "Atmospheric and Subsurface Sources of Stable and Radioactive Nuclides Used for Groundwater Dating." Water Resources Research 29, (7), 2027-2040. Washington, D.C.: American Geophysical Union. TIC: 252317.

Lu, G.; Sonnenthal, E.L.; and Bodvarsson, G.S. 2003. "Implications of Halide Leaching on ${ }^{36} \mathrm{Cl}$ Studies at Yucca Mountain, Nevada." Water Resources Research, 39, (12), 3-1 through 3-15. Washington, D.C.: American Geophysical Union. TIC: 255498.

Marshall, B.D. and Futa, K. 2003. "Strontium in Pore Water from the Topopah Spring Tuff, Yucca Mountain, Nevada." Proceedings of the 10th International High-Level Radioactive Waste Management Conference (IHLRWM), March 30-April 2, 2003, Las Vegas, Nevada. Pages 373-376. La Grange Park, Illinois: American Nuclear Society. TIC: 254559.

Marshall, B.D.; Neymark, L.A.; and Peterman, Z.E. 2003. "Estimation of Past Seepage Volumes from Calcite Distribution in the Topopah Spring Tuff, Yucca Mountain, Nevada." Bodvarsson, G.S.; Ho, C.K.; and Robinson, B.A.; guest eds. Journal of Contaminant Hydrology, 62-63, 237-247. New York, New York: Elsevier. TIC: 254205. 
Montazer, P. and Wilson, W.E. 1984. Conceptual Hydrologic Model of Flow in the Unsaturated Zone, Yucca Mountain, Nevada. Water-Resources Investigations Report 84-4345. Lakewood, Colorado: U.S. Geological Survey. ACC: NNA.19890327.0051 (corrected by MOL.20030927.0200).

Moyer, T.C.; Geslin, J.K.; and Flint, L.E. 1996. Stratigraphic Relations and Hydrologic Properties of the Paintbrush Tuff Nonwelded (PTn) Hydrologic Unit, Yucca Mountain, Nevada. Open-File Report 95-397. Denver, Colorado: U.S. Geological Survey. ACC: MOL.19970204.0216.

Neymark, L.A. and Paces, J.B. 2000. "Consequences of Slow Growth for ${ }^{230} \mathrm{Th} / \mathrm{U}$ Dating of Quaternary Opals, Yucca Mountain, NV, USA." Chemical Geology, 164, ([1-2]), 143-160. Amsterdam, The Netherlands: Elsevier. TIC: 246316.

Neymark, L.A.; Amelin, Y.; Paces, J.B.; and Peterman, Z.E. 2002. "U-Pb Ages of Secondary Silica at Yucca Mountain, Nevada: Implications for the Paleohydrology of the Unsaturated Zone." Gascoyne, M. and Peterman, Z.E., eds. Applied Geochemistry, 17, (6), 709-734. New York, New York: Elsevier. TIC: 254046.

Neymark, L.A.; Amelin, Y.V.; and Paces, J.B. $2000 .{ }^{206} \mathrm{~Pb}-{ }^{230} \mathrm{Th}-{ }^{234} \mathrm{U}-{ }^{238} \mathrm{U}$ and ${ }^{207} \mathrm{~Pb}-{ }^{235} \mathrm{U}$ Geochronology of Quaternary Opal, Yucca Mountain, Nevada." Geochimica et Cosmochimica Acta, 64, (17), 2913-2928. New York, New York: Pergamon. TIC: 253360.

Noble, D.C.; Smith, V.C.; and Peck, L.C. 1967. "Loss of Halogens from Crystallized and Glassy Silicic Volcanic Rocks." Geochimica et Cosmochimica Acta, 31, (2), 215-223. New York, New York: Pergamon. TIC: 224707.

Norris, A.E.; Wolfsberg, K.; Gifford, S.K.; Bentley, H.W.; and Elmore, D. 1987. "Infiltration at Yucca Mountain, Nevada, Traced by ${ }^{36} \mathrm{Cl}$." Nuclear Instruments and Methods in Physics Research [Section B: Beam Interactions with Materials and Atoms], 29, ([1-2]), 376-379. [Amsterdam, The Netherlands]: North-Holland. TIC: 224708.

Ortiz, T.S.; Williams, R.L.; Nimick, F.B.; Whittet, B.C.; and South, D.L. 1985. A ThreeDimensional Model of Reference Thermal/Mechanical and Hydrological Stratigraphy at Yucca Mountain, Southern Nevada. SAND84-1076. Albuquerque, New Mexico: Sandia National Laboratories. ACC: MOL.19980602.0331.

Ostlund, H.G. 1987. "Tritium." Volume 7 of GEOSECS Atlantic, Pacific, and Indian Ocean Expeditions. Shorebased Data and Graphics. Pages 7-10. Washington, D.C.: National Science Foundation. TIC: 254317.

Paces, J.B. 2003. "Copies of Notes taken from SMF Videotapes." Letter from J.B. Paces (USGS) to The Record, June 19, 2003, with attachment. ACC: MOL.20030624.0226. 
Paces, J.B.; Marshall, B.D.; Whelan, J.F.; and Neymark, L.A. 1997. "Submission of Milestone: SPC23FM4, Due March 14, 1997." Memorandum from J.B. Paces, B.D. Marshall, J.F. Whelan, and L.A. Neymark (USGS) to R.W. Craig (USGS), March 14, 1997, with attachment, "Progress Report on Unsaturated Zone Stable and Radiogenic Isotope Studies." ACC: MOL.19980224.0119.

Paces, J.B.; Neymark, L.A.; Marshall, B.D.; Whelan, J.F.; and Peterman, Z.E. 1996. Letter Report: Ages and Origins of Subsurface Secondary Minerals in the Exploratory Studies Facility $(E S F)$. Milestone 3GQH450M, Results of Sampling and Age Determination. Las Vegas, Nevada: U.S. Geological Survey. ACC: MOL.19970324.0052.

Paces, J.B.; Neymark, L.A.; Marshall, B.D.; Whelan, J.F.; and Peterman, Z.E. 1998. "Inferences for Yucca Mountain Unsaturated-Zone Hydrology from Secondary Minerals." High-Level Radioactive Waste Management, Proceedings of the Eighth International Conference, Las Vegas, Nevada, May 11-14, 1998. Pages 36-39. La Grange Park, Illinois: American Nuclear Society. TIC: 238482.

Paces, J.B.; Neymark, L.A.; Marshall, B.D.; Whelan, J.F.; and Peterman, Z.E. 2001. Ages and Origins of Calcite and Opal in the Exploratory Studies Facility Tunnel, Yucca Mountain, Nevada. Water-Resources Investigations Report 01-4049. Denver, Colorado: U.S. Geological Survey. TIC: 251284 and ACC: MOL20020115.0207.

Paces, J.B.; Neymark, L.A.; Wooden, J.L.; and Persing, H.M. 2004. "Improved Spatial Resolution for U-Series Dating of Opal at Yucca Mountain, Nevada, USA, Using IonMicroprobe and Microdigestion Methods." Geochimica et Cosmochimica Acta, 68, (7), 1591-1601. [New York, New York]: Elsevier. TIC: 257287.

Peterman, Z.E. and Cloke, P.L. 2002. "Geochemistry of Rock Units at the Potential Repository Level, Yucca Mountain, Nevada (includes erratum)." Applied Geochemistry, 17, ([6,7]), 683-698, 955-958. [New York, New York]: Pergamon. TIC: 254046.

Peterman, Z.E. and Marshall, B.D. 2002. "Geochemistry of Pore Water from Densely Welded Topopah Spring Tuff at Yucca Mountain, Nevada." GSA Abstracts with Programs, 34, (6), 308. Boulder, Colorado: Geological Society of America. TIC: 254868.

Phillips, F.M. 2000. "Chlorine-36." Chapter 10 of Environmental Tracers in Subsurface Hydrology. Cook, P.G. and Herczeg, A.L., eds. Pages 299-348. Boston, Massachusetts: Kluwer Academic Publishers. TIC: 247021.

Phillips, F.M.; Mattick, J.L.; Duval, T.A.; Elmore, D.; and Kubik, P.W. 1988. "Chlorine 36 and Tritium from Nuclear Weapons Fallout as Tracers for Long-Term Liquid and Vapor Movement in Desert Soils." Water Resources Research, 24, (11), 1877-1891. Washington, D.C.: American Geophysical Union. TIC: 224125.

Plummer, L.N.; Michel, R.L.; Thurman, E.M.; and Glynn, P.D. 1993. "Environmental Tracers for Age Dating Young Ground Water." Chapter 11 of Regional Ground-Water Quality. Alley, W.M., ed. New York, New York: Van Nostrand Reinhold. TIC: 257278. 
Plummer, M.A.; Phillips, F.M.; Fabryka-Martin, J.; Turin, H.J.; Wigand, P.E.; and Sharma, P. 1997. "Chlorine-36 in Fossil Rat Urine: An Archive of Cosmogenic Nuclide Deposition During the Past 40,000 Years." Science, 277, 538-541. Washington, D.C.: American Association for the Advancement of Science. TIC: 237425.

R. Università di Roma 1925. "New Tables for Testing the Significance of Observations." Metron International Journal of Statistics, V, (3), 105-108. Rome, Italy: Università di Roma Instituto di Statistica, e Politica Economica. TIC: 257776.

Roback, R.C.; Jones, C.L.; Fabryka-Martin, J.; Roseborrough, K.; Wolfsberg, L.E.; and Dixon, P. 2002. "Effects of Sample Processing on Chlorine-36 Chlorine Ratios in Unsaturated Crystalline Rocks at Yucca Mountain Implications for Unsaturated Zone Flow." GSA Abstracts with Programs, 34, (6), Page 235. Boulder, Colorado: Geological Society of America. TIC: 254868.

Sawyer, D.A.; Fleck, R.J., Lanphere, M.A., Warren, R.G., and Broxton, D.E. 1994. "Episodic Caldera Volcanism in the Miocene Southwestern Nevada Volcanic Field: Revised Stratigraphic Framework, 40Ar/39Ar Geochronology, and Implications for Magmatism and Extension." Geol. Soc. Amer. Bull., 106 (10), Pages 1304-1318. Boulder, Colorado: Geological Society of America. TIC: 222523.

Scanlon, B.R. 1992. "Evaluation of Liquid and Vapor Water Flow in Desert Soils Based on Chlorine 36 and Tritium Tracers and Nonisothermal Flow Simulations." Water Resources Research, 28, (1), 285-297. Washington, D.C.: American Geophysical Union. TIC: 224127.

Scott, R.B. and Bonk, J. 1984. Preliminary Geologic Map of Yucca Mountain, Nye County, Nevada, with Geologic Sections. Open-File Report 84-494, Denver, Colorado: U.S. Geological Survey. ACC: HQS19880517.1443.

Skeggs, M. 2005. "MSHA Formulations: Facsimile Memorandum from Fenner Dunlop," November 10, 2005. ACC: MOL.20060213.0039.

Solomon, D.K. and Cook, P.G. 2000. ${ }^{63} \mathrm{H}$ and ${ }^{3}$ He." Environmental Tracers in Subsurface Hydrology. Cook, P.G. and Herczeg, A.L., eds. Pages 397-424. Boston, Massachusetts: Kluwer Academic Publishers. TIC: 254319.

Stephens, M.A. 1974. "EDF Statistics for Goodness of Fit and Some Comparisons." Journal of the American Statistical Association, 69, (347), 730-737. Alexandria, Virginia: American Statistical Association. TIC: 254457.

Stone, J.O.; Allan, G.L.; Fifield, L.K.; and Cresswell, R.G. 1996. "Cosmogenic Chlorine-36 from Calcium Spallation." Geochimica et Cosmochimica Acta, 60, (4), 679-692. New York, New York: Pergamon. TIC: 254135.

Striegl, R.G.; Healy, R.W.; Michel, R.L.; and Prudic, D.E. 1998. Tritium in Unsaturated Zone Gases and Air at the Amargosa Desert Research Site, and in Spring and River Water, near Beatty, Nevada, May 1997. Open-File Report 97-778. Carson City, Nevada: U.S. Geological Survey. TIC: 254318. 
Sweetkind, D.S.; Rautman, C.A.; and Singleton, W.L. 1998. "Evaluation of Short-Trace-Length Fractures at Yucca Mountain, Nevada." Eos, Transactions (Supplement), 79, S231. Washington, D.C.: American Geophysical Union. TIC: 240662.

Taylor, J.R. 1982. An Introduction to Error Analysis, The Study of Uncertainties in Physical Measurements. Mill Valley, California: University Science Books. TIC: 210357.

Tyler, S.W.; Chapman, J.B.; Conrad, S.H.; Hammermeister, D.P.; Blout, D.O.; Miller, J.J.; Sully, M.J.; and Ginanni, J.M. 1996. "Soil-Water Flux in the Southern Great Basin, United States: Temporal and Spatial Variations Over the Last 120,000 Years." Hornberger, G.M., ed. Water Resources Research, 32, (6), 1481-1499. Washington, D.C.: American Geophysical Union. TIC: 252291.

USBR (U.S. Bureau of Reclamation) 1996. ESF-Main Drift, Comparative Geology Cross Section Along Main Drift Sta. $28+94.76$ to Sta. 59+36.89. OA-46-291. Mercury, Nevada: U.S. Bureau of Reclamation. ACC: MOL.19970625.0097.

USBR 1997. ESF-South Ramp, Comparative Geology Cross Section Along the South Ramp Sta. 59+36.89 to Sta. 78+77. OA-46-301. Mercury, Nevada: U.S. Bureau of Reclamation. ACC: MOL.19980127.0397.

USGS (U.S. Geological Survey) 1996. ESF-Main Drift, Station 35+00.00 to Station 36+00.00 As Built Geology and Geotechnical Data. OA-46-234. Denver, Colorado: U.S. Geological Survey. ACC: MOL.19970106.0303.

USGS 2002. Test Plan for: Chlorine-36 Validation. SITP-02-UZ-005, Rev. 00. Denver, Colorado: U.S. Geological Survey. ACC: MOL.20020213.0179.

Whelan, J.F.; Moscati, R.J.; Allerton, S.B.M.; and Marshall, B.D. 1998. Applications of Isotope Geochemistry to the Reconstruction of Yucca Mountain, Nevada, Paleohydrology-Status of Investigations: June 1996. Open-File Report 98-83. Denver, Colorado: U.S. Geological Survey. ACC: MOL.19981012.0740.

Whelan, J.F.; Paces, J.B.; and Peterman, Z.E. 2002. "Physical and Stable-Isotope Evidence for Formation of Secondary Calcite and Silica in the Unsaturated Zone, Yucca Mountain, Nevada." Gascoyne, M. and Peterman, Z.E., eds., Applied Geochemistry, 17, (6), 735-750. New York, New York: Elsevier. TIC: 254046.

Wolfsberg, A.; Campbell, K.; and Fabryka-Martin, J. 2000. "Use of Chlorine-36 Data to Evaluate Fracture Flow and Transport Models at Yucca Mountain, Nevada." Dynamics of Fluids in Fractured Rock, Papers Selected from a Symposium held at Ernest Orlando Lawrence Berkeley National Laboratory on February 10-12, 1999. Faybishenko, B.; Witherspoon, A.; and Benson, S.M.; eds. Geophysical Monograph 122. Pages 349-362. Washington, D.C.: American Geophysical Union. TIC: 255306.

Yang, I.C. 2002. "Percolation Flux and Transport Velocity in the Unsaturated Zone, Yucca Mountain, Nevada." Gascoyne, M. and Peterman, Z.E., eds. Applied Geochemistry, 17, (6), 807817. New York, New York: Elsevier. TIC: 254046. 
Yang, I.C.; Rattray, G.W.; and Yu, P. 1996. Interpretation of Chemical and Isotopic Data from Boreholes in the Unsaturated Zone at Yucca Mountain, Nevada. Water-Resources Investigations Report 96-4058. Denver, Colorado: U.S. Geological Survey. ACC: MOL.19980528.0216.

Yang, I.C.; Yu, P.; Rattray, G.W.; Ferarese, J.S.; and Ryan, J.N. 1998. Hydrochemical Investigations in Characterizing the Unsaturated Zone at Yucca Mountain, Nevada. Water-Resources Investigations Report 98 4132. Denver, Colorado: U.S. Geological Survey. ACC: MOL.19981012.0790.

YMP (Yucca Mountain Site Characterization Project) 1998. Peer Review Report on Chlorine-36 Studies at Yucca Mountain. Las Vegas, Nevada: Yucca Mountain Site Characterization Office. ACC: MOL.19981124.0240.

Youden, W.J. 1951. Statistical Methods for Chemists. New York, New York: John Wiley \& Sons. TIC: 248814.

\subsection{CODES, STANDARDS, REGULATIONS, AND PROCEDURES}

NWI-DS-001Q, Rev. 1, ICN 1. Field Logging, Handling, and Documenting Borehole Samples. Las Vegas, Nevada: CRWMS M\&O. ACC: MOL.19960314.0007.

PA-PRO-0313, Rev. 1. Technical Reports. Las Vegas, Nevada: Bechtel SAIC Company. ACC: DOC.20060807.004.

TIP-CL-95, Rev. 0. Preparation of Samples for Chlorine-36 Analysis. Livermore, California: Lawrence Livermore National Laboratory. ACC: MOL.20000412.0094.

TIP-CL-110, Rev. 0. Use of Ion Chromatography to Determine Anion Concentrations. Livermore, California: Lawrence Livermore National Laboratory. ACC: MOL.20000301.0188.

Code of Federal Regulations, Title 40, Protection of Environment, Part 136-Guidelines Establishing Test Procedures for the Analysis of Pollutants, Appendix B-Definition and Procedure for the Determination of the Method Detection Limit-Revision 1.11. Readily available. ACC: MOL.20050411.0381.

\subsection{SOURCE DATA, LISTED BY DATA TRACKING NUMBER}

GS000608314224.004. Provisional Results: Geotechnical Data for Station 35+00 to Station 40+00, Main Drift of the ESF. (Q)

GS030508312272.003. Distribution of Chloride Ion in Yucca Mountain Tuff; Summary of Leaching Data for ESF Cores (April 2002-May 2003). (UQ)

GS030508312272.004. Statistical Parameters of the Tritium Analysis in the Denver Laboratory. (UQ)

GS030608312272.005. Anion Data from leach samples collected for the Chlorine-36 Validation Study. (Q) 
GS030608312272.006. Anion Data from Leach Samples Collected in September 2002 for the Chlorine-36 Validation Study. (UQ)

GS040108312232.001. Shut-in Pressure Monitoring Data from the Radial Boreholes in Alcove 1 of the ESF, 4/95; and Tritium Data from Borehole ESF-AL\#2-HPF\#1 in Alcove 2. (Q)

GS060308312272.001. Tritium Abundance Data from Pore Water in Core Samples from Yucca Mountain ESF Boreholes for the Period of April 30, 1998 through March 21, 2001. (Q)

GS060308312272.002. Tritium Abundance Data from Pore Water in Core Samples from Yucca Mountain ESF ECRB. (Q)

GS060383122410.001. Tritium Data from Pore Water from ESF Borehole Cores, 1998 Analyses by University of Miami. (UQ)

GS960708314224.008. Provisional Results: Geotechnical Data for Station $30+00$ to Station 35+00, Main Drift of the ESF. (Q)

GS960708314224.010. Provisional Results: Geotechnical Data for Station $40+00$ to Station 45+00, Main Drift of the ESF. (Q)

GS960908314224.014. Provisional Results-ESF Main Drift, Station 50+00 to Station 55+00. (Q)

GS961108312261.006. Gas Chemistry, ESF Alcoves \#2 and \#3, 11/95-4/96; Water Chemistry, Alcove \#2 (Tritium), Alcove \#3, and ESF Tunnel; and Pneumatic Pressure Response from Boreholes in Exploratory Studies Facility Alcoves \#2 and \#3, 10/95-5/96. (Q)

GS970208314224.003. Geotechnical Data for Station $60+00$ to Station $65+00$, South Ramp of the ESF. (Q)

GS970808314224.008. Provisional Results: Geotechnical Data for Station $65+00$ to Station 70+00, South Ramp of the ESF. (Q)

GS970808314224.010. Provisional Results: Geotechnical Data for Station 70+00 to Station $75+00$, South Ramp of the ESF. (Q)

GS970808314224.012. Provisional Results: Geotechnical Data for Station $75+00$ to Station 78+77, South Ramp of the ESF. (Q)

GS971108314224.020. Revision 1 of Detailed Line Survey Data, Station 0+60 to Station 4+00, North Ramp Starter Tunnel, Exploratory Studies Facility. (Q)

GS971108314224.021. Revision 1 of Detailed Line Survey Data, Station 4+00 to Station 8+00, North Ramp, Exploratory Studies Facility. (Q)

GS971108314224.022. Revision 1 of Detailed Line Survey Data, Station 8+00 to Station 10+00, North Ramp, Exploratory Studies Facility. (Q) 
GS971108314224.023. Revision 1 of Detailed Line Survey Data, Station 10+00 to Station 18+00, North Ramp, Exploratory Studies Facility. (Q)

GS971108314224.024. Revision 1 of Detailed Line Survey Data, Station $18+00$ to Station 26+00, North Ramp, Exploratory Studies Facility. (Q)

GS971108314224.025. Revision 1 of Detailed Line Survey Data, Station $26+00$ to Station 30+00, North Ramp and Main Drift, Exploratory Studies Facility. (Q)

GS971108314224.026. Revision 1 of Detailed Line Survey Data, Station $45+00$ to Station 50+00, Main Drift, Exploratory Studies Facility. (Q)

GS971108314224.028. Revision 1 of Detailed Line Survey Data, Station 55+00 to Station 60+00, Main Drift and South Ramp, Exploratory Studies Facility. (Q)

LA0305RR831222.001. Chlorine-36 and $\mathrm{Cl}$ in Salts Leached from Rock Samples for the Chlorine-36 Validation Study. (UQ)

LA0307RR831222.001. Chloride, Bromide, Sulfate, and Chlorine-36 Analyses of Salts Leached from Cross Drift Rock Samples in FY99 and FY00. (UQ)

LA0307RR831222.002. Chloride, Bromide, Sulfate, and Chlorine-36 Analyses of Salts Leached from ESF Chlorine-36 Validation Drillcore Samples in FY99. (UQ)

LA0509JF831222.001. Chlorine-36 Analyses of Salts Leached from ESF Niche \#3566 (Niche \#1) Drillcore. (Q)

LAJF831222AQ98.004. Chloride, Bromide, Sulfate, and Chlorine-36 Analyses of Salts Leached from ESF Rock Samples. (Q)

LL030408023121.027. Cl Abundance and Cl Ratios of Leachates from ESF Core Samples. (Q)

LL031200223121.036. Cl Abundance and Cl Ratio of Leachates from ESF Core Samples. (Q) 
APPENDIX A

CHLORIDE CONCENTRATIONS AND CHLORINE-36/CHLORIDE RATIOS IN SALTS LEACHED FROM EXPLORATORY STUDIES FACILITY ROCK SAMPLES AT LOS ALAMOS NATIONAL LABORATORY AS OF SEPTEMBER 8, 1998 
INTENTIONALLY LEFT BLANK 
Chloride Concentrations and Chlorine-36/Chloride Ratios in Salts Leached From Exploratory Studies Facility Rock Samples at Los Alamos National Laboratory as of September 8, 1998

\begin{tabular}{|c|c|c|c|c|c|c|c|c|}
\hline $\begin{array}{l}\text { Sample } \\
\text { Number }\end{array}$ & SMF Barcode Number & $\begin{array}{c}\text { Location } \\
\text { (approximate) }\end{array}$ & Sample Type & Material & $\begin{array}{c}\text { Year } \\
\text { Reported }\end{array}$ & $\begin{array}{c}\text { Leachate } \\
\text { Cl Conc. } \\
\text { (mg/kg) }\end{array}$ & \begin{tabular}{c|} 
AgCl \\
Target \\
Identifier
\end{tabular} & $\begin{array}{c}\text { Corrected } \\
{ }^{36} \mathrm{Cl} / \mathrm{Cl}\left(\times 10^{15}\right)\end{array}$ \\
\hline E001-1 & SPC00507923 & $01+98$ & Bow Ridge fault zone & Wall rock & 1996 & 2 & YM401 & $518 \pm 20$ \\
\hline E008-2 & SPC00509016 & $01+99.8$ & Bow Ridge fault zone & Breccia & 1996 & 2.6 & YM336 & $2138 \pm 137$ \\
\hline E009-2 & SPC00509017 & $01+99.8$ & Bow Ridge fault zone & Breccia & 1996 & 2 & YM337 & $2444 \pm 169$ \\
\hline E010-2 & SPC00509018 & $01+99.8$ & Bow Ridge fault zone & Rubble & 1996 & 2.3 & YM338 & $720 \pm 49$ \\
\hline E011-2 & SPC00509019 & $01+99.8$ & Bow Ridge fault zone & Rubble & 1996 & 2 & YM339 & $2378 \pm 153$ \\
\hline E012-2 & SPC00509020 & $01+99.8$ & Bow Ridge fault zone & Breccia & 1996 & 2.1 & YM340 & $2398 \pm 154$ \\
\hline E243-1 & SPC00509751 & $01+99.8$ & Bow Ridge fault zone & Breccia & 1996 & 4.4 & YM596 & $381 \pm 16$ \\
\hline E163-3 & SPC00512551 & $04+94$ & Systematic & Representative bulk material & 1996 & 2.4 & YM529 & $485 \pm 12$ \\
\hline E073-1 & SPC00504280 & $05+04$ & Fracture & Breccia & 1996 & 5.6 & YM438 & $468 \pm 19$ \\
\hline E074-1 & SPC00503866 & $05+05.5$ & Fracture & Breccia & 1996 & 11.8 & YM424 & $493 \pm 17$ \\
\hline E164-3 & SPC00512550. & $07+00$ & Systematic & Representative bulk material & 1996 & 0.6 & YM530 & $571 \pm 35$ \\
\hline E165-3 & SPC00512549 & $07+70$ & Subunit contact & Representative bulk material & 1996 & 2.9 & YM531 & $496 \pm 14$ \\
\hline E166-3 & SPC00512548 & $07+70$ & Subunit contact & Representative bulk material & 1996 & 12.2 & YM527 & $484 \pm 15$ \\
\hline E167-3 & SPC00512547 & $07+70$ & Subunit contact & Representative bulk material & 1996 & 24.1 & YM528 & $427 \pm 13$ \\
\hline E168-3 & SPC00512546 & $08+59$ & Subunit contact & Representative bulk material & 1996 & 0.8 & YM552 & $802 \pm 29$ \\
\hline E169-3 & SPC00512545 & $08+59$ & Subunit contact & Representative bulk material & 1996 & 0.6 & YM553 & $1096 \pm 40$ \\
\hline E170-3 & SPC00512544 & $08+59$ & Subunit contact & Representative bulk material & 1996 & 1.1 & YM554 & $635 \pm 23$ \\
\hline E191-2 & SPC00515104 & $08+75$ & Subunit contact & Representative bulk material & 1996 & 0.9 & YM572 & $904 \pm 28$ \\
\hline E192-2 & SPC00515105 & $08+75$ & Subunit contact & Representative bulk material & 1996 & 0.8 & YM573 & $698 \pm 20$ \\
\hline E193-2 & SPC00515106 & $08+75$ & Subunit contact & Representative bulk material & 1996 & 1.5 & YM574 & $748 \pm 21$ \\
\hline E171-1 & SPC00512554 & $08+90$ & Subunit contact & Representative bulk material & 1996 & 0.7 & YM555 & $1335 \pm 56$ \\
\hline E172-3 & SPC00512553 & $08+90$ & Subunit contact & Representative bulk material & 1996 & 0.6 & YM557 & $637 \pm 26$ \\
\hline E174-3 & SPC00512543 & $09+00$ & Systematic & Representative bulk material & 1996 & 0.6 & YM559 & $660 \pm 29$ \\
\hline E194-2 & SPC00512586 & $10+56$ & Subunit contact & Representative bulk material & 1996 & 0.7 & YM575 & $1354 \pm 45$ \\
\hline E195-2 & SPC00512587 & $10+56$ & Subunit contact & Representative bulk material & 1996 & 0.8 & YM576 & $1292 \pm 37$ \\
\hline
\end{tabular}


Chloride Concentrations and Chlorine-36/Chloride Ratios in Salts Leached From Exploratory Studies Facility Rock Samples at Los Alamos National Laboratory as of September 8, 1998

\begin{tabular}{|c|c|c|c|c|c|c|c|c|}
\hline $\begin{array}{l}\text { Sample } \\
\text { Number }\end{array}$ & SMF Barcode Number & $\begin{array}{c}\text { Location } \\
\text { (approximate) }\end{array}$ & Sample Type & Material & $\begin{array}{c}\text { Year } \\
\text { Reported }\end{array}$ & \begin{tabular}{|c|}
$\begin{array}{c}\text { Leachate } \\
\text { Cl Conc. } \\
\text { (mg/kg) }\end{array}$ \\
\end{tabular} & \begin{tabular}{c|} 
AgCl \\
Target \\
Identifier
\end{tabular} & $\begin{array}{l}\text { Corrected } \\
{ }^{36} \mathrm{Cl} / \mathrm{Cl}\left(\times 10^{15}\right)\end{array}$ \\
\hline E195-2 & SPC00512587 & $10+56$ & Subunit contact & Representative bulk material & 1996 & 0.8 & YM576 & $1292 \pm 37$ \\
\hline E197-2 & SPC00512585 & $10+62.5$ & Subunit contact & Representative bulk material & 1996 & 0.1 & YM578 & $1452 \pm 72$ \\
\hline E086-1 & SPC00510583 & $11+43$ & Bedrock & Representative bulk material & 1996 & 0.7 & YM439 & $640 \pm 29$ \\
\hline E213-1 & SPC00510792 & $12+36.5$ & Fracture & Breccia & 1996 & 0.3 & YM598 & $719 \pm 68$ \\
\hline E028-1 & SPC00503934 & $12+44$ & Cooling joints & Representative bulk material & 1996 & 0.2 & YM452 & $2629 \pm 105$ \\
\hline E214-1 & SPC00510790 & $12+44$ & Cooling joints & Representative bulk material & 1996 & 0.5 & YM599 & $751 \pm 27$ \\
\hline E215-1 & SPC00510791 & $12+49$ & Cooling joints & Representative bulk material & 1996 & 0.4 & YM600 & $668 \pm 54$ \\
\hline E029-1 & SPC00503932 & $13+00$ & Systematic & Representative bulk material & 1996 & 0.6 & YM426 & $640 \pm 28$ \\
\hline E030-2 & SPC00503931 & $13+67$ & Cooling joints & Breccia $<0.5 \mathrm{~cm}$ & 1996 & 0.5 & YM563 & $1634 \pm 85$ \\
\hline E031-3 & SPC00503930 & $14+00$ & Shear zone & Breccia $<1 \mathrm{~cm}$ & 1996 & 0.7 & YM564 & $2399 \pm 191$ \\
\hline E032-2 & SPC00503929 & $14+14$ & Shear zone & Representative bulk material & 1996 & 0.8 & YM454 & $680 \pm 45$ \\
\hline E033-1 & SPC00503928 & $14+41$ & Fault & Gouge & 1996 & 0.3 & YM427 & $876 \pm 42$ \\
\hline E034-1 & SPC00503926 & $15+00$ & Systematic & Representative bulk material & 1996 & 0.3 & YM428 & $954 \pm 51$ \\
\hline E035-1 & SPC00503925 & $15+05$ & Fracture & Breccia & 1996 & 1 & YM429 & $628 \pm 61$ \\
\hline E036-1 & SPC00509242 & $16+12$ & Cooling joint & Representative bulk material & 1996 & 1.4 & YM455 & $382 \pm 57$ \\
\hline E037-2 & SPC00509241 & $16+19$ & Fracture & Representative bulk material & 1996 & 0.5 & YM430 & $982 \pm 42$ \\
\hline E038-1 & SPC00503924 & $17+00$ & Systematic & Representative bulk material & 1996 & 0.4 & YM450 & $714 \pm 38$ \\
\hline E040-1 & SPC00503922 & $18+96$ & Broken rock & Representative bulk material & 1996 & 0.6 & YM456 & $1642 \pm 59$ \\
\hline E041-1 & SPC00503921 & $19+00$ & Systematic & Representative bulk material & 1996 & 0.6 & YM431 & $746 \pm 27$ \\
\hline E042-2 & SPC00503920 & $19+31$ & Fault zone & Breccia > $\sim 0.5 \mathrm{~cm}$ & 1996 & 0.6 & YM457 & $3023 \pm 94$ \\
\hline E042-3 & SPC00503920 & $19+31$ & Fault zone & Breccia $<\sim 0.5 \mathrm{~cm}$ & 1996 & 0.6 & YM458 & $1838 \pm 65$ \\
\hline E043-2 & SPC00503919 & $19+37$ & Fault zone & Representative bulk material & 1996 & 0.8 & YM459 & $1144 \pm 36$ \\
\hline E044-2 & SPC00503918 & $19+42$ & Breccia zone & Representative bulk material & 1996 & 0.6 & YM460 & $2290 \pm 74$ \\
\hline E045-1 & SPC00503917 & $21+00$ & Systematic & Representative bulk material & 1996 & 0.6 & YM432 & $799 \pm 29$ \\
\hline E046-1 & SPC00503916 & $22+71$ & Fracture zone & Representative bulk material & 1996 & 1 & YM461 & $864 \pm 44$ \\
\hline
\end{tabular}


Chloride Concentrations and Chlorine-36/Chloride Ratios in Salts Leached From Exploratory Studies Facility Rock Samples at Los Alamos National Laboratory as of September 8, 1998

\begin{tabular}{|c|c|c|c|c|c|c|c|c|}
\hline $\begin{array}{l}\text { Sample } \\
\text { Number }\end{array}$ & SMF Barcode Number & $\begin{array}{c}\text { Location } \\
\text { (approximate) }\end{array}$ & Sample Type & Material & $\begin{array}{c}\text { Year } \\
\text { Reported }\end{array}$ & $\begin{array}{l}\text { Leachate } \\
\text { Cl Conc. } \\
\text { (mg/kg) }\end{array}$ & $\begin{array}{c}\text { AgCl } \\
\text { Target } \\
\text { Identifier }\end{array}$ & $\begin{array}{c}\text { Corrected } \\
{ }^{36} \mathrm{Cl} / \mathrm{Cl}\left(\times 10^{15}\right)\end{array}$ \\
\hline E047-1 & SPC00509247 & $23+00$ & Systematic & Representative bulk material & 1996 & 0.8 & YM451 & $663 \pm 37$ \\
\hline E050-2 & SPC00509240 & $24+40$ & Fault zone & Breccia & 1996 & 0.4 & YM462 & $2579 \pm 94$ \\
\hline E020-1 & SPC00509220 & $24+68$ & Fracture & Representative bulk material & 1996 & 0.6 & YM448 & $814 \pm 56$ \\
\hline E051-1 & SPC00509259 & $25+00$ & Systematic & Representative bulk material & 1996 & 0.5 & YM433 & $1003 \pm 49$ \\
\hline E217-1 & SPC00510716 & $26+19$ & Cooling joints & Representative bulk material & 1996 & 0.7 & YM602 & $522 \pm 21$ \\
\hline E218-1 & SPC00510714 & $26+36$ & Fracture & Representative bulk material & 1996 & 1.2 & YM603 & $603 \pm 20$ \\
\hline E219-1 & SPC00510713 & $26+46$ & Fracture & Representative bulk material & 1996 & 0.4 & YM604 & $578 \pm 38$ \\
\hline E052-1 & SPC00509244 & $26+79$ & Shear zone & Representative bulk material & 1996 & 0.4 & YM463 & $2036 \pm 68$ \\
\hline E220-1 & SPC00510719 & $26+79$ & Fracture & Representative bulk material & 1996 & 1.3 & YM605 & $564 \pm 25$ \\
\hline E054-1 & SPC00509257 & $27+00$ & Systematic & Representative bulk material & 1996 & 0.4 & YM434 & $973 \pm 29$ \\
\hline E056-1 & SPC00509243 & $27+18$ & Fault & Representative bulk material & 1996 & 0.4 & YM565 & $1709 \pm 53$ \\
\hline E057-2 & SPC00509238 & $27+50$ & Fracture & Breccia & 1996 & 1.3 & YM435 & $779 \pm 23$ \\
\hline E058-2 & SPC00509237 & $27+66$ & Fault & Breccia & 1996 & $1.4^{\circ}$ & YM436 & $458 \pm 19$ \\
\hline E059-2 & SPC00509236 & $28+40$ & Fault & Breccia & 1996 & 2.1 & YM437 & $512 \pm 21$ \\
\hline E141-1 & SPC00503947 & $29+00$ & Systematic & Representative bulk material & 1996 & 0.4 & YM464 & $922 \pm 36$ \\
\hline E142-1 & SPC00503983 & $29+21$ & Fracture & Representative bulk material & 1996 & 0.5 & YM493 & $583 \pm 28$ \\
\hline E143-1 & SPC00503948 & $29+65$ & Fault & Representative bulk material & 1996 & 0.4 & YM494 & $1077 \pm 162$ \\
\hline E144-1 & SPC00503949 & $29+73$ & Cooling joint & Representative bulk material & 1996 & 0.2 & YM495 & $815 \pm 34$ \\
\hline E147-1 & SPC00503976 & $30+27$ & Cooling joints & Representative bulk material & 1996 & 1.7 & YM496 & $490 \pm 15$ \\
\hline E149-1 & SPC00503973 & $31+64$ & Cooling joint & Representative bulk material & 1996 & 0.7 & YM465 & $631 \pm 29$ \\
\hline E150-1 & SPC00503939 & $33+00$ & Systematic & Representative bulk material & 1996 & 0.2 & YM473 & $1341 \pm 56$ \\
\hline E152-1 & SPC00503993 & $34+28$ & Fractures & Representative bulk material & 1996 & 0.3 & YM478 & $4105 \pm 310$ \\
\hline E153-3 & SPC00503938 & $34+32$ & Cooling joints & Representative bulk material & 1996 & 0.2 & YM479 & $3261 \pm 160$ \\
\hline E154-1 & SPC00503937 & $34+71$ & Cooling joints & Breccia & 1996 & 0.3 & YM474 & $803 \pm 41$ \\
\hline E154-3 & SPC00503937 & $34+71$ & Cooling joints & Wall rock & 1996 & 0.2 & YM480 & $3794 \pm 120$ \\
\hline
\end{tabular}


Chloride Concentrations and Chlorine-36/Chloride Ratios in Salts Leached From Exploratory Studies Facility Rock Samples at Los Alamos National Laboratory as of September 8, 1998

\begin{tabular}{|c|c|c|c|c|c|c|c|c|}
\hline $\begin{array}{l}\text { Sample } \\
\text { Number }\end{array}$ & SMF Barcode Number & $\begin{array}{c}\text { Location } \\
\text { (approximate) }\end{array}$ & Sample Type & Material & $\begin{array}{c}\text { Year } \\
\text { Reported }\end{array}$ & $\begin{array}{l}\text { Leachate } \\
\text { Cl Conc. } \\
\text { (mg/kg) }\end{array}$ & \begin{tabular}{|c|} 
AgCl \\
Target \\
Identifier
\end{tabular} & $\begin{array}{l}\text { Corrected } \\
{ }^{36} \mathrm{Cl} / \mathrm{Cl}\left(\times 10^{15}\right)\end{array}$ \\
\hline E156-1 & SPC00503969 & $35+00$ & Cooling joints & Representative bulk material & 1996 & 1 & YM467 & $626 \pm 29$ \\
\hline E155-1 & SPC00503980 & $35+00$ & Systematic & Representative bulk material & 1996 & 0.5 & YM466 & $1013 \pm 60$ \\
\hline E157-3 & SPC00503994 & $35+03$ & Cooling joints & Representative bulk material & 1996 & 0.5 & YM566 & $1339 \pm 76$ \\
\hline E158-1 & SPC00503995 & $35+08$ & Cooling joints & Breccia $<\sim 0.5 \mathrm{~cm}$ & 1996 & 0.7 & YM475 & $1113 \pm 58$ \\
\hline E158-3 & SPC00503995 & $35+08$ & Cooling joints & Breccia $>-0.5 \mathrm{~cm}$ & 1996 & 0.5 & YM492 & $2671 \pm 158$ \\
\hline E160-1 & SPC00503979 & $35+45$ & Cooling joints & Representative bulk material & 1996 & 0.3 & YM481 & $3529 \pm 205$ \\
\hline E161-3 & SPC00503999 & $35+58$ & Cooling joint & Breccia > $-0.5 \mathrm{~cm}$ & 1996 & 0.5 & YM501 & $2169 \pm 80$ \\
\hline E175-1 & SPC00512511 & $35+93$ & Fault & Breccia $>\sim 0.5 \mathrm{~cm}$ & 1996 & 0.3 & YM514 & $2840 \pm 231$ \\
\hline E175-3 & SPC00512511 & $35+93$ & Fault & Breccia $<\sim 0.5 \mathrm{~cm}$ & 1996 & 0.3 & YM515 & $1674 \pm 141$ \\
\hline E176-1 & SPC00512506 & $36+55$ & Fault & Breccia > $~-0.5 \mathrm{~cm}$ & 1996 & 0.9 & YM516 & $888 \pm 27$ \\
\hline E176-3 & SPC00512506 & $36+55$ & Fault & Breccia $<\sim 0.5 \mathrm{~cm}$ & 1996 & 1 & YM517 & $604 \pm 25$ \\
\hline E177-1 & SPC00512510 & $37+00$ & Systematic & Representative bulk material & 1996 & 0.9 & YM518 & $484 \pm 15$ \\
\hline E178-1 & SPC00512504 & $37+60$ & Cooling joint & Gouge & 1996 & 1.8 & YM503 & $471 \pm 26$ \\
\hline E179-1 & SPC00512509 & $37+68$ & Cooling joint & Breccia & 1996 & 1.5 & YM504 & $363 \pm 22$ \\
\hline E179-3 & SPC0051.2509 & $37+68$ & Cooling joint & Wall rock & 1996 & 1.5 & YM519 & $397 \pm 13$ \\
\hline E182-1 & SPC00512502 & $38+79$ & Fracture & Breccia & 1996 & 0.4 & YM505 & $379 \pm 38$ \\
\hline E183-1 & SPC00512517 & $38+95$ & Cooling joint & Breccia & 1996 & 0.4 & YM506 & $745 \pm 39$ \\
\hline E184-1 & SPC00512508 & $39+00$ & Systematic & Representative bulk material & 1996 & 0.2 & YM520 & $536 \pm 29$ \\
\hline E185-1 & SPC00503944 & $39+39$ & Frac/lith cavity & Representative bulk material & 1996 & 0.3 & YM521 & $897 \pm 46$ \\
\hline E186-1 & SPC00503943 & $39+47$ & Cooling joint & Breccia/gouge & 1996 & 0.5 & YM507 & $561 \pm 34$ \\
\hline E187-1 & SPC00503946 & $39+61$ & Cooling joint & Breccia/gouge & 1996 & 0.9 & YM508 & $540 \pm 33$ \\
\hline E221-1 & SPC00510710 & $41+00$ & Systematic & Representative bulk material & 1996 & 0.6 & YM606 & $773 \pm 24$ \\
\hline E198-2 & SPC00510700 & $41+65$ & Cooling joint & Representative bulk material & 1996 & 1.9 & YM584 & $291 \pm 12$ \\
\hline E199-2 & SPC00512590 & $43+00$ & Systematic & Representative bulk material & 1996 & 0.3 & YM585 & $1042 \pm 53$ \\
\hline $\mathrm{E} 200-2$ & SPC00512589 & $43+39$ & Fault & Gouge & 1996 & 0.3 & YM586 & $967 \pm 66$ \\
\hline
\end{tabular}


Chloride Concentrations and Chlorine-36/Chloride Ratios in Salts Leached From Exploratory Studies Facility Rock Samples at Los Alamos National Laboratory as of September 8, 1998

\begin{tabular}{|c|c|c|c|c|c|c|c|c|}
\hline $\begin{array}{l}\text { Sample } \\
\text { Number }\end{array}$ & SMF Barcode Number & $\begin{array}{c}\text { Location } \\
\text { (approximate) }\end{array}$ & Sample Type & Material & $\begin{array}{c}\text { Year } \\
\text { Reported }\end{array}$ & $\begin{array}{c}\text { Leachate } \\
\text { Cl Conc. } \\
\text { (mg/kg) }\end{array}$ & $\begin{array}{c}\text { AgCI } \\
\text { Target } \\
\text { Identifier }\end{array}$ & $\begin{array}{l}\text { Corrected } \\
{ }^{36} \mathrm{Cl} / \mathrm{Cl}\left(\times 10^{15}\right)\end{array}$ \\
\hline E201-2 & SPC00512591 & $43+63$ & Cooling joint & Representative bulk material & 1996 & 0.4 & YM587 & $1974 \pm 65$ \\
\hline E202-2 & SPC00512592 & $44+20$ & Cooling joint & Representative bulk material & 1996 & 0.6 & YM588 & $3463 \pm 97$ \\
\hline E203-2 & SPC00512593 & $44+21$ & Cooling joint & Representative bulk material & 1996 & 0.4 & YM589 & $849 \pm 34$ \\
\hline E204-2 & SPC00512594 & $44+22$ & Cooling joint & Representative bulk material & 1996 & 0.6 & YM590 & $772 \pm 31$ \\
\hline E205-2 & SPC00512595 & $45+00$ & Systematic & Representative bulk material & 1996 & 0.3 & YM591 & $1514 \pm 69$ \\
\hline E207-2 & SPC00512597 & $45+79$ & Cooling joint & Representative bulk material & 1996 & 1.6 & YM592 & $593 \pm 15$ \\
\hline E211-2 & SPC00515107 & Alc 4/0+51.58 & Subunit contact & Representative bulk material & 1996 & 2.1 & YM594 & $810 \pm 24$ \\
\hline$E 210-2$ & SPC00515109 & Alc 4/0+51.58 & Subunit contact & Representative bulk material & 1996 & 4.3 & YM593 & $712 \pm 31$ \\
\hline E212-2 & SPC00515108 & Alc 4/0+51.58 & Subunit contact & Representative bulk material & 1996 & 2.9 & YM597 & $815 \pm 18$ \\
\hline E007-2 & SPC00507924 & $02+03$ & Fault zone & Wall rock & 1997 & 3.4 & YM402 & $519 \pm 13$ \\
\hline E188-2 & SPC00515100 & $08+26.5$ & Subunit contact & Representative bulk material & 1997 & 2.2 & YM569 & $766 \pm 24$ \\
\hline E189-2 & SPC00515101 & $08+26.5$ & Subunit contact & Representative bulk material & 1997 & 4.3 & YM570 & $625 \pm 17$ \\
\hline E190-2 & SPC00515102 & $08+26.5$ & Subunit contact & Representative bulk material & 1997 & 6.3 & YM571 & $647 \pm 14$ \\
\hline E244-1 & SPC00515135 & $08+38$ & Fault & Representative bulk material & 1997 & 1.5 & YM654 & $488 \pm 17$ \\
\hline E245-1 & SPC00515136 & $08+44$ & Fracture & Representative bulk material & 1997 & 1.7 & YM655 & $530 \pm 21$ \\
\hline E246-1 & SPC00515137 & $08+66$ & Fault & Representative bulk material & 1997 & 0.8 & YM656 & $475 \pm 17$ \\
\hline E247-1 & SPC00515138 & $09+32$ & Fault & Representative bulk material & 1997 & 0.6 & YM657 & $509 \pm 22$ \\
\hline E126-1 & SPC00509155 & $10+34$ & Fault zone & Representative bulk material & 1997 & 0.8 & YM671 & $633 \pm 41$ \\
\hline E128-1 & SPC00509147 & $10+40$ & Fault zone & Representative bulk material & 1997 & 1.5 & YM672 & $662 \pm 27$ \\
\hline E130-1 & SPC00509150 & $10+41$ & Fault zone & Representative bulk material & 1997 & 0.7 & YM673 & $773 \pm 40$ \\
\hline E196-2 & SPC00512588 & $10+56$ & Subunit contact & Representative bulk material & 1997 & 0.4 & YM577 & $1202 \pm 27$ \\
\hline E134-1 & SPC00510506 & $10+66$ & Fault & Representative bulk material & 1997 & 1.8 & YM728 & $747 \pm 41$ \\
\hline$E 136-1$ & SPC00510505 & $10+66.8$ & Fault & Representative bulk material & 1997 & 2.5 & YM729 & $801 \pm 33$ \\
\hline E139-1 & SPC00510510 & $10+74.2$ & Fault & Representative bulk material & 1997 & 1.4 & YM730 & $738 \pm 52$ \\
\hline E248-1 & SPC00515139 & $10+75$ & Fault & Representative bulk material & 1997 & 0.5 & YM658 & $570 \pm 37$ \\
\hline
\end{tabular}


Chloride Concentrations and Chlorine-36/Chloride Ratios in Salts Leached From Exploratory Studies Facility Rock Samples at Los Alamos National Laboratory as of September 8, 1998

\begin{tabular}{|c|c|c|c|c|c|c|c|c|}
\hline $\begin{array}{l}\text { Sample } \\
\text { Number }\end{array}$ & SMF Barcode Number & $\begin{array}{c}\text { Location } \\
\text { (approximate) }\end{array}$ & Sample Type & Material & $\begin{array}{c}\text { Year } \\
\text { Reported }\end{array}$ & $\begin{array}{l}\text { Leachate } \\
\text { Cl Conc. } \\
\text { (mg/kg) }\end{array}$ & $\begin{array}{c}\text { AgCl } \\
\text { Target } \\
\text { Identifier }\end{array}$ & $\begin{array}{l}\text { Corrected } \\
{ }^{36} \mathrm{Cl} / \mathrm{Cl}\left(\times 10^{15}\right)\end{array}$ \\
\hline E249-1 & SPC00515142 & $11+00$ & Systematic & Representative bulk material & 1997 & 0.5 & YM659 & $657 \pm 37$ \\
\hline E027-3 & SPC00503935 & $11+00$ & Systematic & Representative bulk material & 1997 & 0.3 & YM749 & $1076 \pm 52$ \\
\hline E249-3 & SPC00515142 & $11+00$ & Systematic & Representative bulk material & 1997 & 0.4 & YM733 & $672 \pm 46$ \\
\hline E249-4 & SPC00515142 & $11+00$ & Systematic & Representative bulk material & 1997 & 1 & YM734 & $912 \pm 58$ \\
\hline E250-1 & SPC00515140 & $11+43$ & Fault & Representative bulk material & 1997 & 0.4 & YM660 & $532 \pm 35$ \\
\hline E251-1 & SPC00515141 & $11+77$ & Fault & Representative bulk material & 1997 & 0.5 & YM661 & $633 \pm 38$ \\
\hline E030-1 & SPC00503931 & $13+67$ & Cooling joints & Breccia $<0.5 \mathrm{~cm}$ & 1997 & 1 & YM449 & $698 \pm 35$ \\
\hline E031-1 & SPC00503930 & $14+00$ & Shear zone & Breccia > $1 \mathrm{~cm}$ & 1997 & 0.6 & YM453 & $1039 \pm 35$ \\
\hline E216-1 & SPC00510788 & $20+71$ & Fracture & Representative bulk material & 1997 & 1.2 & YM601 & $840 \pm 38$ \\
\hline E046-4 & SPC00503916 & $22+71$ & Fracture zone & Representative bulk material & 1997 & 1.1 & PRIME & $458 \pm 24$ \\
\hline E146-4 & SPC00503987 & $30+18$ & Lith cavity & Representative bulk material & 1997 & 1.8 & PRIME & $496 \pm 24$ \\
\hline E151-4 & SPC00503990 & $33+16$ & Lith cavity & Representative bulk material & 1997 & 2.3 & PRIME & $529 \pm 24$ \\
\hline E160-4 & SPC00503979 & $35+45$ & Cooling joints & Representative bulk material & 1997 & 1.8 & PRIME & $388 \pm 17$ \\
\hline E161-1 & SPC00503999 & $35+58$ & Cooling joint & Breccia $<-0.5 \mathrm{~cm}$ & 1997 & 0.8 & YM476 & $1951 \pm 103$ \\
\hline E222-1 & SPC00510724 & $42+55$ & Shear sets & Gouge & 1997 & 3.8 & YM608 & $605 \pm 18$ \\
\hline E222-2 & SPC00510724 & $42+55$ & Shear sets & Wall rock & 1997 & 2.9 & YM609 & $531 \pm 16$ \\
\hline E206-1 & SPC00512596 & $45+78$ & Fracture zone & Representative bulk material & 1997 & 1.6 & YM731 & $525 \pm 29$ \\
\hline E208-1 & SPC00515103 & $46+18$ & Fault & Breccia & 1997 & 1.9 & YM732 & $497 \pm 26$ \\
\hline E223-1 & SPC00510728 & $47+00$ & Systematic & Representative bulk material & 1997 & 0.3 & YM610 & $734 \pm 37$ \\
\hline E225-1 & SPC00510731 & $48+56$ & Cooling joints & Breccia & 1997 & 1.2 & YM612 & $350 \pm 14$ \\
\hline E224-1 & SPC00510734 & $49+00$ & Systematic & Representative bulk material & 1997 & 1.7 & YM611 & $499 \pm 16$ \\
\hline E226-1 & SPC00510737 & $49+56$ & Cooling joint & Breccia & 1997 & 1.1 & YM613 & $450 \pm 20$ \\
\hline E226-2 & SPC00510737 & $49+56$ & Cooling joint & Wall rock & 1997 & 1 & YM614 & $456 \pm 20$ \\
\hline E227-1 & SPC00510705 & $49+89$ & Cooling joints & Breccia & 1997 & 0.5 & YM615 & $497 \pm 33$ \\
\hline E230-1 & SPC00510739 & $51+00$ & Systematic & Representative bulk material & 1997 & 0.5 & YM625 & $555 \pm 23$ \\
\hline
\end{tabular}


Chloride Concentrations and Chlorine-36/Chloride Ratios in Salts Leached From Exploratory Studies Facility Rock Samples at Los Alamos National Laboratory as of September 8, 1998

\begin{tabular}{|c|c|c|c|c|c|c|c|c|}
\hline $\begin{array}{l}\text { Sample } \\
\text { Number }\end{array}$ & SMF Barcode Number & $\begin{array}{c}\text { Location } \\
\text { (approximate) }\end{array}$ & Sample Type & Material & $\begin{array}{c}\text { Year } \\
\text { Reported }\end{array}$ & $\begin{array}{l}\text { Leachate } \\
\text { Cl Conc. } \\
\text { (mg/kg) }\end{array}$ & \begin{tabular}{c|} 
AgCl \\
Target \\
Identifier
\end{tabular} & $\begin{array}{c}\text { Corrected } \\
{ }^{36} \mathrm{Cl} / \mathrm{Cl}\left(\times 10^{15}\right)\end{array}$ \\
\hline E231-1 & SPC00510740 & $51+07$ & Cooling joints & Wall rock & 1997 & 0.5 & YM626 & $709 \pm 30$ \\
\hline E231-2 & SPC00510740 & $51+07$ & Cooling joints & Breccia & 1997 & 0.5 & YM627 & $530 \pm 26$ \\
\hline E232-1 & SPC00510741 & $51+33$ & Cooling joints & Representative bulk material & 1997 & 0.4 & YM628 & $942 \pm 42$ \\
\hline E233-1 & SPC00510742 & $51+73$ & Fracture & Representative bulk material & 1997 & 0.3 & YM629 & $647 \pm 30$ \\
\hline E234-1 & SPC00510743 & $52+43$ & Cooling joint & Representative bulk material & 1997 & 0.5 & YM630 & $291 \pm 26$ \\
\hline E235-1 & SPC00510744 & $52+46$ & Cooling joint & Representative bulk material & 1997 & 0.3 & YM631 & $596 \pm 43$ \\
\hline E236-1 & SPC00510745 & $53+00$ & Systematic & Representative bulk material & 1997 & 0.7 & YM632 & $417 \pm 17$ \\
\hline E237-1 & SPC00510746 & $53+61$ & Cooling joint & Representative bulk material & 1997 & 0.4 & YM633 & $539 \pm 27$ \\
\hline E238-1 & SPC00510747 & $54+20$ & Cooling joint & Breccia & 1997 & 1.3 & YM634 & $727 \pm 37$ \\
\hline E239-1 & SPC00510748 & $55+00$ & Systematic & Representative bulk material & 1997 & 0.3 & YM635 & $464 \pm 23$ \\
\hline E240-1 & SPC00510756 & $56+63$ & Cooling joint & Breccia & 1997 & 0.3 & YM636 & $673 \pm 42$ \\
\hline E241-1 & SPC00510754 & $56+85$ & Cooling joint & Breccia & 1997 & 0.7 & YM637 & $777 \pm 38$ \\
\hline E242-1 & SPC00510750 & $56+93$ & Cooling joint & Breccia $>2 \mathrm{~mm}$ & 1997 & 0.7 & YM638 & $664 \pm 30$ \\
\hline E242-2 & SPC00510750 & $56+93$ & Cooling joint & Breccia $<2 \mathrm{~mm}$ & 1997 & 0.9 & YM639 & $1117 \pm 49$ \\
\hline E252-1 & SPC00515143 & $57+00$ & Systematic & Representative bulk material & 1997 & 0.7 & YM641 & $388 \pm 23$ \\
\hline E253-1 & SPC00515144 & $57+27$ & Fault & Representative bulk material & 1997 & 1.7 & YM642 & $483 \pm 14$ \\
\hline E254-1 & SPC00515145 & $58+66$ & Fault & Breccia & 1997 & 0.5 & YM643 & $588 \pm 58$ \\
\hline E255-1 & SPC00515146 & $58+77$ & Subunit contact & Representative bulk material & 1997 & 3 & YM644 & $140 \pm 9$ \\
\hline E256-1 & SPC00515147 & $59+00$ & Systematic & Breccia & 1997 & 0.5 & YM645 & $347 \pm 41$ \\
\hline E256-3 & SPC00515147 & $59+00$ & Systematic & Wall rock. & 1997 & 1.7 & YM675 & $359 \pm 23$ \\
\hline E290-1 & SPC00521128 & $59+98$ & Systematic & Representative bulk material & 1997 & 0.6 & YM711 & $205 \pm 14$ \\
\hline E257-1 & SPC00515148 & $61+00$ & Systematic & Representative bulk material & 1997 & 1.2 & YM646 & $428 \pm 26$ \\
\hline E258-1 & SPC00515149 & $61+92$ & Fracture & Representative bulk material & 1997 & 0.8 & YM647 & $276 \pm 21$ \\
\hline E259-1 & SPC00515150 & $62+00$ & Systematic & Representative bulk material & 1997 & 0.5 & YM648 & $409 \pm 28$ \\
\hline E260-1 & SPC00515151 & $62+05$ & Fault & Representative bulk material & 1997 & 1.2 & YM649 & $261 \pm 13$ \\
\hline
\end{tabular}


Chloride Concentrations and Chlorine-36/Chloride Ratios in Salts Leached From Exploratory Studies Facility Rock Samples at Los Alamos National Laboratory as of September 8, 1998

\begin{tabular}{|c|c|c|c|c|c|c|c|c|}
\hline $\begin{array}{l}\text { Sample } \\
\text { Number }\end{array}$ & SMF Barcode Number & $\begin{array}{c}\text { Location } \\
\text { (approximate) }\end{array}$ & Sample Type & Material & $\begin{array}{c}\text { Year } \\
\text { Reported }\end{array}$ & $\begin{array}{l}\text { Leachate } \\
\text { Cl Conc. } \\
\text { (mg/kg) }\end{array}$ & \begin{tabular}{|c|} 
AgCl \\
Target \\
Identifier
\end{tabular} & $\begin{array}{c}\text { Corrected } \\
{ }^{36} \mathrm{Cl} / \mathrm{Cl}\left(\times 10^{15}\right)\end{array}$ \\
\hline E261-1 & SPC00515152 & $62+18$ & Fault & Representative bulk material & 1997 & 0.5 & YM650 & $749 \pm 39$ \\
\hline E262-1 & SPC00515153 & $62+71$ & Shear & Representative bulk material & 1997 & 0.4 & YM651 & $420 \pm 28$ \\
\hline E263-1 & SPC00515154 & $63+00$ & Systematic & Representative bulk material & 1997 & 3 & YM662 & $465 \pm 14$ \\
\hline E264-1 & SPC00515155 & $63+06$ & Fracture & Representative bulk material & 1997 & 1.7 & YM663 & $458 \pm 13$ \\
\hline E265-1 & SPC00515156 & $63+21$ & Fracture & Representative bulk material & 1997 & 3.3 & YM664 & $452 \pm 12$ \\
\hline E266-1 & SPC00515157 & $63+26$ & Fracture & Representative bulk material & 1997 & 3.7 & YM676 & $486 \pm 16$ \\
\hline E267-1 & SPC00515158 & $63+30$ & Fault & Representative bulk material & 1997 & 3.7 & YM677 & $427 \pm 14$ \\
\hline E269-1 & SPC00515188 & $63+73$ & Fracture & Representative bulk material & 1997 & 2.5 & YM698 & $551 \pm 14$ \\
\hline E270-1 & SPC00515187 & $63+81$ & Fracture & Representative bulk material & 1997 & 3.6 & YM678 & $439 \pm 14$ \\
\hline E271-1 & SPC00515186 & $64+00$ & Systematic & Representative bulk material & 1997 & 3.2 & YM699 & $467 \pm 20$ \\
\hline E271-1D & SPC00515186 & $64+00$ & Systematic & Representative bulk material & 1997 & 3.2 & YM707 & $438 \pm 18$ \\
\hline E272-1 & SPC00515185 & $64+34$ & Broken rock & Representative bulk material & 1997 & 1.4 & YM679 & $467 \pm 22$ \\
\hline E273-1 & SPC00515184 & $64+50$ & Broken rock & Representative bulk material & 1997 & 1.4 & YM700 & $610 \pm 22$ \\
\hline E274-1 & SPC00515182 & $64+93$ & Fracture & Representative bulk material & 1997 & 2.9 & YM701 & $491 \pm 25$ \\
\hline E275-1 & SPC00515181 & $65+00$ & Systematic & Representative bulk material & 1997 & 1.8 & YM684 & $443 \pm 19$ \\
\hline E268-1 & SPC005151.80 & $65+20$ & Fracture zone & Breccia & 1997 & 1.7 & YM683 & $468 \pm 18$ \\
\hline E276-1 & SPC00515179 & $65+56$ & Fracture zone & Breccia & 1997 & 1.6 & YM702 & $480 \pm 14$ \\
\hline E277-1 & SPC00515178 & $65+80$ & Fracture zone & Representative bulk material & 1997 & 2 & YM685 & $424 \pm 33$ \\
\hline E278-1 & SPC00515177 & $66+00$ & Systematic & Representative bulk material & 1997 & 1.5 & YM703 & $520 \pm 61$ \\
\hline E279-1 & SPC00515176 & $66+15$ & Fault & Breccia & 1997 & 1.3 & YM686 & $402 \pm 40$ \\
\hline E280-1 & SPC00515175 & $66+40$ & Fault & Representative bulk material & 1997 & 0.3 & YM687 & $238 \pm 30$ \\
\hline E281-1 & SPC00515174 & $67+00$ & Systematic & Representative bulk material & 1997 & 2.3 & YM688 & $453 \pm 18$ \\
\hline E283-1 & SPC00515172 & $67+27$ & Fault & Representative bulk material & 1997 & 3 & YM689 & $470 \pm 21$ \\
\hline E284-3 & SPC00515173 & $67+35$ & Subunit contact & Representative bulk material & 1997 & 1.3 & YM710 & $509 \pm 20$ \\
\hline E284-1 & SPC00515173 & $67+35$ & Subunit contact & Representative bulk material & 1997 & 1.8 & YM709 & $502 \pm 19$ \\
\hline
\end{tabular}


Chloride Concentrations and Chlorine-36/Chloride Ratios in Salts Leached From Exploratory Studies Facility Rock Samples at Los Alamos National Laboratory as of September 8, 1998

\begin{tabular}{|c|c|c|c|c|c|c|c|c|}
\hline $\begin{array}{l}\text { Sample } \\
\text { Number }\end{array}$ & SMF Barcode Number & $\begin{array}{c}\text { Location } \\
\text { (approximate) }\end{array}$ & Sample Type & Material & $\begin{array}{c}\text { Year } \\
\text { Reported }\end{array}$ & $\begin{array}{l}\text { Leachate } \\
\mathrm{Cl} \text { Conc. } \\
\text { (mg/kg) }\end{array}$ & $\begin{array}{c}\text { AgCl } \\
\text { Target } \\
\text { Identifier }\end{array}$ & $\begin{array}{c}\text { Corrected } \\
{ }^{36} \mathrm{Cl} / \mathrm{Cl}\left(\times 10^{15}\right)\end{array}$ \\
\hline E289-1 & SPC00515170 & $67+61$ & Fault & Gouge & 1997 & 4.4 & YM692 & $589 \pm 23$ \\
\hline E285-1 & SPC00515171 & $67+73$ & Damp zone & Representative bulk material & 1997 & 4.8 & YM680 & $468 \pm 23$ \\
\hline E286-4 & SPC00515133 & $67+87$ & Fault zone & Wall rock & 1997 & 0.7 & YM704 & $645 \pm 29$ \\
\hline E286-1 & SPC00515133 & $67+87$ & Fault zone & Clay fracture filling & 1997 & 2.2 & YM690 & $475 \pm 22$ \\
\hline E287-1 & SPC00515134 & $67+87$ & Fault & Breccia & 1997 & 2.3 & YM691 & $517 \pm 19$ \\
\hline E288-1 & SPC00515132 & $67+90$ & Fault footwall & Wall rock & 1997 & 1.1 & YM681 & $557 \pm 31$ \\
\hline E298-1 & SPC00521127 & $68+00$ & Systematic & Representative bulk material & 1997 & 1.4 & YM718 & $606 \pm 17$ \\
\hline E292-1 & SPC00521123 & $69+00$ & Systematic & Representative bulk material & 1997 & 3.1 & YM712 & $414 \pm 17$ \\
\hline E293-1 & SPC00521122 & $69+14.5$ & Fault zone & Breccia & 1997 & 2.7 & YM713 & $454 \pm 35$ \\
\hline E294-1 & SPC00521121 & $69+32.5$ & Fracture & Breccia & 1997 & 1.7 & YM714 & $474 \pm 22$ \\
\hline E295-1 & SPC00521120 & $69+41.7$ & Fracture zone & Representative bulk material & 1997 & 1.9 & YM715 & $476 \pm 21$ \\
\hline E299-1 & SPC00522221 & $69+47$ & Systematic & Representative bulk material & 1997 & 2.1 & YM750 & $441 \pm 20$ \\
\hline E300-1 & SPC00522220 & $69+68$ & Fault (?) & Representative bulk material & 1997 & 2.5 & YM763 & $354 \pm 13$ \\
\hline E300-3 & SPC00522220 & $69+68$ & Fault (?) & Representative bulk material & 1997 & 2.3 & YM764 & $376 \pm 16$ \\
\hline$E 302-1$ & SPC00522218 & $70+19$ & Fault & Representative bulk material & 1997 & 5.8 & YM736 & $327 \pm 13$ \\
\hline E303-1 & SPC00522217 & $70+36$ & Fault & Representative bulk material & 1997 & 2.3 & YM751 & $439 \pm 17$ \\
\hline E304-1 & SPC00522216 & $70+50$ & Systematic & Representative bulk material & 1997 & 5.1 & YM765 & $491 \pm 21$ \\
\hline E305-1 & SPC00522215 & $70+55.5$ & Fault & Breccia & 1997 & 4.4 & YM752 & $386 \pm 12$ \\
\hline E306-1 & SPC00522214 & $70+66$ & Fault & Breccia & 1997 & 3.3 & YM766 & $499 \pm 19$ \\
\hline E307-1 & SPC00522212 & $71+34$ & Fault & Representative bulk material & 1997 & 0.7 & YM737 & $557 \pm 29$ \\
\hline E308-1 & SPC00522213 & $71+39$ & Fault & Representative bulk material & 1997 & 1.1 & YM753 & $492 \pm 13$ \\
\hline E309-1 & SPC00522211 & $71+41$ & Fault & Representative bulk material & 1997 & 1 & YM767 & $445 \pm 21$ \\
\hline E310-1 & SPC00522210 & $71+50$ & Systematic & Representative bulk material & 1997 & 1 & YM768 & $441 \pm 21$ \\
\hline E311-1 & SPC00522209 & $72+50$ & Systematic & Representative bulk material & 1997 & 2.4 & YM754 & $459 \pm 11$ \\
\hline E312-1 & SPC00522208 & $72+69$ & Fault & Representative bulk material & 1997 & 1.6 & YM769 & $463 \pm 18$ \\
\hline
\end{tabular}


Chloride Concentrations and Chlorine-36/Chloride Ratios in Salts Leached From Exploratory Studies Facility Rock Samples at Los Alamos National Laboratory as of September 8, 1998

\begin{tabular}{|c|c|c|c|c|c|c|c|c|}
\hline $\begin{array}{l}\text { Sample } \\
\text { Number }\end{array}$ & SMF Barcode Number & $\begin{array}{c}\text { Location } \\
\text { (approximate) }\end{array}$ & Sample Type & Material & $\begin{array}{c}\text { Year } \\
\text { Reported }\end{array}$ & $\begin{array}{l}\text { Leachate } \\
\text { Cl Conc. } \\
\text { (mg/kg) }\end{array}$ & $\begin{array}{c}\text { AgCl } \\
\text { Target } \\
\text { Identifier }\end{array}$ & $\begin{array}{c}\text { Corrected } \\
{ }^{36} \mathrm{Cl} / \mathrm{Cl}\left(\times 10^{15}\right)\end{array}$ \\
\hline E313-1 & SPC00522207 & $73+48$ & Systematic & Representative bulk material & 1997 & 3.8 & YM770 & $367 \pm 14$ \\
\hline E315-1A & SPC00522205 & $74+49$ & Systematic & Representative bulk.material & 1997 & 11.5 & YM738 & $435^{\circ} \pm 16$ \\
\hline E316-1 & SPC00522202 & $74+89$ & Fault & Representative bulk material & 1997 & 5.9 & YM755 & $459 \pm 11$ \\
\hline E317-1 & SPC00522201 & $75+09$ & Subunit contact & Representative bulk material & 1997 & 1.8 & YM740 & $402 \pm 30$ \\
\hline E318-1 & SPC00522203 & $75+09.5$ & Subunit contact & Representative bulk material & 1997 & 2.3 & YM756 & $395 \pm 16$ \\
\hline E319-1 & SPC00522204 & $75+10$ & Subunit contact & Representative bulk material & 1997 & 3.6 & YM741 & $414 \pm 16$ \\
\hline E321-1 & SPC00521287 & $.75+34$ & Fault & Breccia & 1997 & 6.1 & YM757 & $476 \pm 12$ \\
\hline E323-1 & SPC00521289 & $75+53.5$ & Cooling joints & Breccia & 1997 & 3.4 & YM742 & $465 \pm 17$ \\
\hline E323-3 & SPC00521289 & $75+53.5$ & Cooling joints & Wall rock & 1997 & 4.4 & YM743 & $413 \pm 19$ \\
\hline E324-1 & SPC00521290 & $75+78$ & Fault & Breccia & 1997 & 5.4 & YM744 & $418 \pm 31$ \\
\hline E324-3 & SPC00521290 & $75+78$ & Fault & Wall rock & 1997 & 4.5 & YM745 & $322 \pm 13$ \\
\hline E325-1 & SPC00521291 & $76+30$ & Fault & Breccia & 1997 & 2.3 & YM771 & $380 \pm 20$ \\
\hline E326-1 & SPC00521292 & $76+31$ & Fault & Wall rock & 1997 & 4.7 & YM758 & $423 \pm 9$ \\
\hline E326-3 & SPC00521292 & $76+31$ & Fault & Wall rock & 1997 & 3.4 & YM759 & $419 \pm 11$ \\
\hline E327-1 & SPC00521295 & $76+50$ & Systematic & Representative bulk material & 1997 & 2.4 & YM772 & $281 \pm 12$ \\
\hline E328-1 & SPC00521294 & $76+76$ & Fault & Breccia $<\sim 1 \mathrm{~cm}$ & 1997 & 1.3 & YM746 & $334 \pm 15$ \\
\hline E328-3 & SPC00521294 & $76+76$ & Fault & Breccia > $\sim 1 \mathrm{~cm}$ & 1997 & 0.6 & YM747 & $445 \pm 20$ \\
\hline E329-1 & SPC00521293 & $77+10$ & Fault & Representative bulk material & 1997 & 0.5 & YM773 & $394 \pm 19$ \\
\hline E228-1 & SPC00510795 & Alc $2 / 0+25$ & Drill \& blast & Representative bulk material & 1997 & 0.7 & YM674 & $362 \pm 41$ \\
\hline E229-1 & SPC00510702 & Alc $3 / 014$ & Intact bedrock & Representative bulk material & 1997 & 3.4 & YM616 & $558 \pm 19$ \\
\hline E296-1 & SPC00521129 & Alc $6 / 0+95$ & Fault & Representative bulk material & 1997 & 1.6 & YM716 & $533 \pm 52$ \\
\hline E297-1 & SPC00521130 & Alc $6 / 0+98$ & Fault & Representative bulk material & 1997 & 1.8 & YM717 & $499 \pm 27$ \\
\hline E044-4 & SPC00503918 & $19+42$ & Breccia zone & Representative bulk material & 1998 & 0.7 & YM775 & $4270 \pm 159$ \\
\hline E160-7 & SPC00503979 & $35+45$ & Cooling joints & Representative bulk material & 1998 & 0.2 & YM776 & $1704 \pm 76$ \\
\hline$E 301-1$ & SPC00522219 & $69+95.8$ & Fault & Representative bulk material & 1998 & 0.6 & YM777 & $224 \pm 11$ \\
\hline
\end{tabular}


Chloride Concentrations and Chlorine-36/Chloride Ratios in Salts Leached From Exploratory Studies Facility Rock Samples at Los Alamos National Laboratory as of September 8, 1998

\begin{tabular}{|c|c|c|c|c|c|c|c|c|}
\hline $\begin{array}{l}\text { Sample } \\
\text { Number }\end{array}$ & SMF Barcode Number & $\begin{array}{c}\text { Location } \\
\text { (approximate) }\end{array}$ & Sample Type & Material & $\begin{array}{c}\text { Year } \\
\text { Reported }\end{array}$ & $\begin{array}{l}\text { Leachate } \\
\text { Cl Conc. } \\
\text { (mg/kg) }\end{array}$ & $\begin{array}{c}\text { AgCl } \\
\text { Target } \\
\text { Identifier }\end{array}$ & $\begin{array}{c}\text { Corrected } \\
{ }^{36} \mathrm{Cl} / \mathrm{Cl}\left(\times 10^{15}\right)\end{array}$ \\
\hline E306-3 & SPC00522214 & $70+66$ & Fault & Representative bulk material & 1998 & 3.3 & YM778 & $496 \pm 20$ \\
\hline E314-1 & SPC00522206 & $74+43$ & Fracture & Representative bulk material & 1998 & 2.1 & YM779 & $341 \pm 18$ \\
\hline E352-1 & SPC00524963 & $74+55.5$ & Fault & Representative bulk material & 1998 & 3.2 & YM883 & $484 \pm 21$ \\
\hline E320-1 & SPC00522200 & $75+20$ & Subunit contact & Representative bulk material & 1998 & 3.7 & YM780 & $457 \pm 20$ \\
\hline E322-1 & SPC00521288 & $75+47.5$ & Systematic & Representative bulk material & 1998 & 2.9 & YM781 & $318 \pm 81$ \\
\hline E353-1 & SPC00524964 & $76+01$ & Fracture & Representative bulk material & 1998 & 2.9 & YM884 & $515 \pm 19$ \\
\hline E354-1 & SPC00524965 & $76+08$ & Fracture & Breccia & 1998 & 1.9 & YM885 & $616 \pm 21$ \\
\hline E355-1 & SPC00524971 & $76+11.5$ & Fault & Wall rock & 1998 & 3.7 & YM886 & $473 \pm 18$ \\
\hline E356-1 & SPC00524966 & $76+11.5$ & Fault & Gouge & 1998 & 1.8 & YM887 & $570 \pm 24$ \\
\hline E335-1 & SPC00524901 & $77+19$ & Fracture & Representative bulk material & 1998 & 1.9 & YM812 & $186 \pm 9$ \\
\hline E357-1 & SPC00524967 & $77+29.5$ & Broken rock & Representative bulk material & 1998 & 0.6 & YM888 & $621 \pm 26$ \\
\hline E358-1 & SPC00524968 & $77+31$ & Broken rock & Representative bulk material & 1998 & 0.7 & YM889 & $341 \pm 14$ \\
\hline E359-1 & SPC00524969 & $77+49.5$ & Systematic & Representative bulk material & 1998 & 4.2 & YM890 & $511 \pm 23$ \\
\hline E360-1 & SPC00524970 & $78+50$ & Systematic & Representative bulk material & 1998 & 1.6 & YM891 & $973 \pm 27$ \\
\hline E337-1 & SPC00525144 & Alc $6 / 0+30$ & Systematic & Representative bulk material & 1998 & 0.9 & YM858 & $666 \pm 24$ \\
\hline E338-1 & SPC00525145 & Alc $6 / 0+60$ & Systematic & Representative bulk material & 1998 & 0.8 & YM859 & $689 \pm 22$ \\
\hline E339-1 & SPC00525130 & Alc $6 / 0+82$ & Breccia zone & Representative bulk material & 1998 & 0.6 & YM860 & $703 \pm 36$ \\
\hline E340-1 & SPC00525131 & Alc $6 / 0+93$ & Breccia zone & Representative bulk material & 1998 & 0.6 & YM861 & $1511 \pm 48$ \\
\hline E341-1 & SPC00525132 & Alc $6 / 0+97$ & Fault & Gouge & 1998 & 0.5 & YM840 & $513 \pm 23$ \\
\hline E342-1 & SPC00525135 & Alc $6 / 1+00$ & Breccia zone & Representative bulk material & 1998 & 0.7 & YM841 & $927 \pm 35$ \\
\hline E343-1 & SPC00525136 & Alc $6 / 1+05$ & Fracture zone & Representative bulk material & 1998 & 0.9 & YM842 & $1080 \pm 33$ \\
\hline E344-1 & SPC00525137 & Alc $6 / 1+10$ & Fault & Representative bulk material & 1998 & 1.5 & YM843 & $884 \pm 32$ \\
\hline E345-1 & SPC00525138 & Alc $6 / 1+17$ & Fault & Representative bulk material & 1998 & 0.9 & YM844 & $1081 \pm 37$ \\
\hline E346-1 & SPC00525139 & Alc $6 / 1+24$ & Fracture & Representative bulk material & 1998 & 1.1 & YM845 & $1130 \pm 38$ \\
\hline E347-1 & SPC00525140 & Alc $6 / 1+40$ & Fault & Representative bulk material & 1998 & 1.3 & YM862 & $455 \pm 23$ \\
\hline
\end{tabular}


Chloride Concentrations and Chlorine-36/Chloride Ratios in Salts Leached From Exploratory Studies Facility Rock Samples at Los Alamos National Laboratory as of September 8, 1998

\begin{tabular}{|c|c|c|c|c|c|c|c|c|}
\hline $\begin{array}{l}\text { Sample } \\
\text { Number }\end{array}$ & SMF Barcode Number & $\begin{array}{c}\text { Location } \\
\text { (approximate) }\end{array}$ & Sample Type & Material & $\begin{array}{c}\text { Year } \\
\text { Reported }\end{array}$ & $\begin{array}{l}\text { Leachate } \\
\text { Cl Conc. } \\
\text { (mg/kg) }\end{array}$ & \begin{tabular}{|c} 
AgCl \\
Target \\
Identifier
\end{tabular} & $\begin{array}{c}\text { Corrected } \\
{ }^{36} \mathrm{Cl} / \mathrm{Cl}\left(\times 10^{15}\right)\end{array}$ \\
\hline E348-1 & SPC00530000 & Alc $6 / 1+52$ & Fault & Breccia & 1998 & 0.5 & YM846 & $1250 \pm 65$ \\
\hline E349-1 & SPC00525141 & Alc $6 / 1+52$ & Fault & Wall rock & 1998 & 0.5 & YM847 & $3357 \pm 132$ \\
\hline E333-1 & SPC00524960 & Alc $6 / 1+53$ & Fault & Representative bulk material & 1998 & 0.5 & YM809 & $521 \pm 32$ \\
\hline E333-2 & SPC00524960 & Alc $6 / 1+53$ & Fault & Representative bulk material & 1998 & 0.5 & YM810 & $497 \pm 23$ \\
\hline E350-1 & SPC00525142 & Alc $6 / 1+60$ & Cooling joints & Representative bulk material & 1998 & 0.5 & YM848 & $1699 \pm 70$ \\
\hline E351-1 & SPC00525143 & Alc $6 / 1+68$ & Broken rock & Representative bulk material & 1998 & 0.3 & YM849 & $1792 \pm 77$ \\
\hline E351-2 & SPC00525143 & Alc $6 / 1+68$ & Broken rock & Representative bulk material & 1998 & 0.3 & YM850 & $499 \pm 20$ \\
\hline E334-1 & SPC00524959 & Alc $7 / 1+30$ & Fracture & Representative bulk material & 1998 & 1.2 & YM811 & $474 \pm 16$ \\
\hline E361-1 & SPC00524975 & Alc $7 / 1+54.5$ & Fault & Representative bulk material & 1998 & 0.9 & YM911 & $539 \pm 24$ \\
\hline E362-1 & SPC00524973 & Alc $7 / 1+67$ & Fault & Representative bulk material & 1998 & 2.1 & YM912 & $541 \pm 25$ \\
\hline E363-1 & SPC00524974 & Alc $7 / 1+67.5$ & Fault & Representative bulk material & 1998 & 1.9 & YM913 & $643 \pm 29$ \\
\hline E364-1 & SPC00524976 & Alc $7 / 1+84$ & Fault & Representative bulk material & 1998 & 1.1 & YM914 & $569 \pm 27$ \\
\hline E365-1 & SPC00524972 & Alc $7 / 2+00$ & Fault & Representative bulk material & 1998 & 1.9 & YM915 & $538 \pm 26$ \\
\hline $\begin{array}{l}\text { DCN086- } \\
2\end{array}$ & SPC01003078 & \begin{tabular}{|c|} 
ESF-MD- \\
NICHE3566\#1 (22.2- \\
$23.0)$ \\
\end{tabular} & NA & 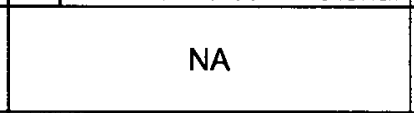 & 1998 & NA & YM903 & $1372 \pm 69$ \\
\hline $\begin{array}{l}\text { DCN007- } \\
2 / 008-1\end{array}$ & $\begin{array}{l}\text { SPC01003096 } \\
\text { SPC01003097 } \\
\text { SPC01003098 } \\
\end{array}$ & $\begin{array}{c}\text { ESF-MD- } \\
\text { NICHE3566\#1 (32.1- } \\
\text { 33.1) } \\
\end{array}$ & NA & NA & 1998 & NA & YM894 & $2008 \pm 90$ \\
\hline $\begin{array}{l}\text { DCN024- } \\
1 / 025-2\end{array}$ & $\begin{array}{l}\text { SPC01003131 } \\
\text { SPC01003132 } \\
\text { SPC01003133 } \\
\end{array}$ & $\begin{array}{c}\text { ESF-MD- } \\
\text { NICHE3566\#2 (15.7- } \\
\text { 17.1) } \\
\end{array}$ & NA & NA & 1998 & NA & YM896 & $2038 \pm 99$ \\
\hline $\begin{array}{l}\text { DCN015- } \\
2\end{array}$ & SPC01003111 & $\begin{array}{c}\text { ESF-MD- } \\
\text { NICHE3566\#2 (6.7- } \\
7.5) \\
\end{array}$ & NA & NA & 1998 & NA & YM895 & $1235 \pm 62$ \\
\hline $\begin{array}{l}\text { DCN038- } \\
1 / 039-2\end{array}$ & $\begin{array}{l}\text { SPC01004399 } \\
\text { SPC01004400 } \\
\text { SPC01004401 } \\
\text { SPC01004402 }\end{array}$ & $\begin{array}{c}\text { ESF-MD- } \\
\text { NICHE3566LT\#1 (1.7- } \\
5.0)\end{array}$ & NA & NA & 1998 & NA & YM897 & $997 \pm 49$ \\
\hline $\begin{array}{l}\text { DCN048- } \\
1 / 049-2\end{array}$ & $\begin{array}{l}\text { SPC01004420 } \\
\text { SPC01004421 }\end{array}$ & $\begin{array}{c}\text { ESF-MD- } \\
\text { NICHE3566LT\#1 }\end{array}$ & NA & NA & 1998 & NA & YM898 & $1476 \pm 75$ \\
\hline
\end{tabular}


Chloride Concentrations and Chlorine-36/Chloride Ratios in Salts Leached From Exploratory Studies Facility Rock Samples at Los Alamos National Laboratory as of September 8, 1998

\begin{tabular}{|c|c|c|c|c|c|c|c|c|}
\hline $\begin{array}{l}\text { Sample } \\
\text { Number }\end{array}$ & SMF Barcode Number & $\begin{array}{c}\text { Location } \\
\text { (approximate) }\end{array}$ & Sample Type & Material & $\begin{array}{c}\text { Year } \\
\text { Reported }\end{array}$ & \begin{tabular}{|c|} 
Leachate \\
Cl Conc. \\
(mg/kg) \\
\end{tabular} & \begin{tabular}{|c|} 
AgCl \\
Target \\
Identifier \\
\end{tabular} & $\begin{array}{c}\text { Corrected } \\
{ }^{36} \mathrm{Cl} / \mathrm{Cl}\left(\times 10^{15}\right)\end{array}$ \\
\hline & SPC01004422 & $(14.3-16.3)$ & & & & & & \\
\hline $\begin{array}{l}\text { DCN050- } \\
1 / 051-2\end{array}$ & $\begin{array}{l}\text { SPC01004424 } \\
\text { SPC01004425 } \\
\text { SPC01004426 } \\
\text { SPC01004427 }\end{array}$ & $\begin{array}{c}\text { ESF-MD- } \\
\text { NICHE3566LT\#1 } \\
(16.6-19.3)\end{array}$ & NA & NA & 1998 & NA & YM899 & $1252 \pm 68$ \\
\hline $\begin{array}{l}\text { DCN059- } \\
2 / 060-1\end{array}$ & $\begin{array}{l}\text { SPC01004445 } \\
\text { SPC01004446 } \\
\text { SPC01004447 }\end{array}$ & $\begin{array}{c}\text { ESF-MD- } \\
\text { NICHE3566LT\#1 } \\
(29.0-30.7) \\
\end{array}$ & NA & NA & 1998 & NA & YM900 & $1627 \pm 73$ \\
\hline $\begin{array}{l}\text { DCNO62- } \\
1\end{array}$ & SPC01004453 & $\begin{array}{c}\text { ESF-MD- } \\
\text { NICHE3566LT\#1 } \\
(32.1-33.1) \\
\end{array}$ & NA & NA & 1998 & NA & YM901 & $1705 \pm 87$ \\
\hline $\begin{array}{l}\mathrm{DCN} 064- \\
2\end{array}$ & SPC01004457 & $\begin{array}{c}\text { ESF-MD- } \\
\text { NICHE3566LT\#1 } \\
(34.4-35.5) \\
\end{array}$ & NA & NA & 1998 & NA & YM902 & $1335 \pm 67$ \\
\hline E331-1 & SPC00524998 & Niche $1 / 0+13.5$ & Breccia zone & Representative bulk material & 1998 & 0.2 & YM806 & $540 \pm 31$ \\
\hline E332-1 & SPC00524999 & Niche $1 / 0+13.5$ & Breccia zone & Representative bulk material & 1998 & 0.4 & YM807 & $588 \pm 37$ \\
\hline E332-2 & SPC00524999 & Niche $1 / 0+13.5$ & Breccia zone & Representative bulk material & 1998 & 0.3 & YM808 & $618 \pm 45$ \\
\hline E330-1 & SPC00524900 & Niche $1 / 0+10$ & Breccia zone & Representative bulk material & 1998 & 0.9 & YM805 & $553 \pm 29$ \\
\hline E336-1 & SPC00008073 & Niche $1 / 7+05$ & Fracture & Representative bulk material & 1998 & 0.3 & YM817 & $659 \pm 177$ \\
\hline
\end{tabular}

NOTES:

DTNs: LAJF831222AQ98.004 (Q), LA0509JF831222.001(Q)

SMF = Sample Management Facility, Alc = Alcove, NA = Not Available.

Locations (i.e., ESF stations, borehole intervals) are approximate.

The Sundance fault zone is located between ESF stations $33+89$ and $36+89$ (approx.).

Samples E331-1, E332-1, E332-2, E330-1, and E336-1 are tunnel wall samples.

Samples from ESF-MD-NICHE3566\#1, \#2, and LT1 are borehole samples.

Errors are $1 \sigma$. One-sigma analytical errors given for construction-water corrected ${ }^{36} \mathrm{Cl} / \mathrm{Cl}$ ratios are based on in-run counting statistics.

Leachate chloride concentrations are given as salts leached per kilogram of rock. 
Chloride Concentrations and Chlorine-36/Chloride Ratios in Salts Leached From

Exploratory Studies Facility Rock Samples at Los Alamos National Laboratory as of September 8, 1998

Measured ${ }^{36} \mathrm{Cl} / \mathrm{Cl}$ ratios have been corrected for the addition of a ${ }^{35} \mathrm{Cl}$ tracer and for the addition of $\mathrm{Cl}$ from construction water using the approach described in Fabryka-Martin et al. (1997, p. B-1, Section 4.2.2).

The concentration of salts extracted from each sample is only a qualitative indicator of the pore-water composition: no attempt was made to maximize the yield of the leaching process, which is probably highly variable.

The data were originally reported in Fabryka-Martin, Wolfsberg, et al. (1996), Fabryka-Martin et al. (1997), CRWMS M\&O (1998). Small differences were noted for some values contained in the cited reports and the final data reported in DTNs LAJF831222AQ98.004 and LA0509JF831222.001. These reflect final adjustments and corrections to analytical data and do not affect any of the conclusions based on these data. 


\section{REFERENCES CITED}

\section{A.1 DOCUMENTS CITED}

CRWMS M\&O (Civilian Radioactive Waste Management System Management and Operating Contractor) 1998. Evaluation of Flow and Transport Models of Yucca Mountain, Based on Chlorine-36 and Chloride Studies for FY98. BA0000000-017175700-00007, Rev. 00. Las Vegas, Nevada: CRWMS M\&O. ACC: MOL.19981208.0119.

Fabryka-Martin, J.; Wolfsberg, A.V.; Dixon, P.R.; Levy, S.; Musgrave, J.; and Turin, H.J. 1996. Summary Report of Chlorine-36 Studies: Sampling, Analysis and Simulation of Chlorine-36 in the Exploratory Studies Facility. Milestone 3783M. Los Alamos, New Mexico: Los Alamos National Laboratory. ACC: MOL.19970103.0047.

Fabryka-Martin, J.T.; Flint, A.L.; Sweetkind, D.S.; Wolfsberg, A.V.; Levy, S.S.; Roemer, G.J.C.; Roach, J.L.; Wolfsberg, L.E.; and Duff, M.C. 1997. Evaluation of Flow and Transport Models of Yucca Mountain, Based on Chlorine-36 Studies for FY97. LA-CSTTIP-97-010. Los Alamos, New Mexico: Los Alamos National Laboratory. ACC: MOL.19980204.0196.

\section{A.2 SOURCE DATA, LISTED BY DATA TRACKING NUMBER}

LA0509JF831222.001. Chlorine-36 Analyses of Salts Leached from ESF Niche \#3566 (Niche \#1) Drillcore. (Q)

LAJF831222AQ98.004. Chloride, Bromide, Sulfate, and Chlorine-36 Analyses of Salts Leached from ESF Rock Samples. (Q) 
INTENTIONALLY LEFT BLANK 
APPENDIX B

VIDEO-LOG OBSERVATIONS FROM VALIDATION STUDY BOREHOLES 
INTENTIONALLY LEFT BLANK 


\section{Video Log Observations from Validation Study Boreholes}

\begin{tabular}{|c|c|c|c|c|}
\hline $\begin{array}{l}\text { SMF Name: } \\
\text { Borehole name: } \\
\text { Completion date: } \\
\text { Total depth (ft) }\end{array}$ & $\begin{array}{l}879 \\
\text { ESF-DHW-Cl } \\
9 / 30 / 1999 \\
13.4\end{array}$ & & ESF Station $19+65$ & \\
\hline Run \#. & Interval (ft) & $\begin{array}{l}\text { Recovery } \\
\text { (ft) }\end{array}$ & Fractures/Comments & $\begin{array}{c}\text { Unrecovered } \\
\text { Core Interval } \\
\text { (ft) }\end{array}$ \\
\hline 1 & $0.0-2.9$ & 1.7 & rubble & $1.7-1.9$ \\
\hline 2 & $2.9-5.2$ & 2.1 & rubble & $5.0-5.2$ \\
\hline 3 & $5.2-7.9$ & 1.8 & rubble & $7.0-7.9$ \\
\hline 4 & $7.9-10.9$ & 2.3 & rubble & $10.2-10.9$ \\
\hline 5 & $10.9-13.4$ & 2.3 & rubble & $13.2-13.4$ \\
\hline $\begin{array}{l}\text { SMF Name: } \\
\text { Borehole name: } \\
\text { Completion date: } \\
\text { Total depth (ft) } \\
\end{array}$ & $\begin{array}{l}880 \\
\text { ESF-DHW-CI } \\
9 / 29 / 1999 \\
13.5 \\
\end{array}$ & & ESF Station $19+55$ & \\
\hline Run \# & Interval (ft) & $\begin{array}{l}\text { Recovery } \\
\text { (ft) }\end{array}$ & Fractures/Comments & $\begin{array}{c}\text { Unrecovered } \\
\text { Core Interval } \\
\text { (ft) }\end{array}$ \\
\hline 1 & $0.0-3.2$ & 1.8 & rubble & $1.8-3.2$ \\
\hline 2 & $2 \quad 3.2-5.6$ & 1.6 & rubble & $4.8-5.6$ \\
\hline 3 & $5.6-8.2$ & 2.6 & $5.6-7.2=$ rubble $; 7.2-8.2=$ fairly intact $w / 3$ fractures & none \\
\hline 4 & $8.2-10.7$ & 1.7 & rubble & $9.9-10.7$ \\
\hline 5 & $10.7-13.5$ & 0.9 & rübble & $11.6-13.5$ \\
\hline $\begin{array}{l}\text { SMF Name: } \\
\text { Borehole name: } \\
\text { Completion date: } \\
\text { Total depth (ft) }\end{array}$ & $\begin{array}{l}881 \\
\text { ESF-DHW-Cl } \\
9 / 29 / 1999 \\
13.6\end{array}$ & & ESF Station $19+50$ & \\
\hline Run \# & \# Interval (ft) & $\begin{array}{l}\text { Recovery } \\
\text { (ft) }\end{array}$ & Fractures/Comments & $\begin{array}{c}\text { Unrecovered } \\
\text { Core Interval } \\
\text { (ft) }\end{array}$ \\
\hline 1 & $10.0-3.1$ & 1.5 & rubble & $1.5-3.1$ \\
\hline 2 & $23.1-5.0$ & 1.5 & rubble & $4.6-5.0$ \\
\hline 3 & $3.0-6.9$ & 1.9 & rubble & none \\
\hline 4 & $4 \quad 6.9-8.1$ & 1.1 & rubble & $8.0-8.1$ \\
\hline 5 & $58.1-10.1$ & 1.6 & rubble & $9.7-10.1$ \\
\hline 6 & $10.1-10.6$ & 0.5 & $10.1-10.4=$ rubble $10.4-10.6=$ intact & none \\
\hline 7 & $10.6-12.0$ & 0.6 & rubble & $11.2-12.0$ \\
\hline 8 & $312.0-13.6$ & 1.3 & rubble & $13.3-13.6$ \\
\hline $\begin{array}{l}\text { SMF Name: } \\
\text { Borehole name: } \\
\text { Completion date: } \\
\text { Total depth (ft) } \\
\end{array}$ & $\begin{array}{l}906 \\
\text { ESF-DHW-CI } \\
9 / 28 / 1999 \\
13.8 \\
\end{array}$ & V\#4 & ESF Station $19+45$ & \\
\hline Run \# & $¥ \quad$ Interval (ft) & $\begin{array}{l}\text { Recovery } \\
\text { (ft) }\end{array}$ & Fractures/Comments & $\begin{array}{c}\text { Unrecovered } \\
\text { Core Interval } \\
\text { (ft) } \\
\end{array}$ \\
\hline 1 & $0.0-2.0$ & 0.9 & rubble & $0.9-2.0$ \\
\hline 2 & $2.0-3.7$ & 0.6 & rubble & $2.6-3.7$ \\
\hline 3 & $3.7-5.8$ & 0.5 & rubble & $4.2-5.8$ \\
\hline 4 & $5.8-7.6$ & 0.3 & rubble & $6.1-7.6$ \\
\hline 5 & $7.6-9.6$ & 2 & $7.6-8.5=$ rubble $8.5-8.7=$ fractured, rubbly; $9.2-9.6=$ intact & none \\
\hline 6 & $69.6-10.5$ & 0.9 & $9.6-9.9=$ rubble; $9.9-10.5=$ intact w/ 2 fractures & none \\
\hline 7 & $710.5-13.8$ & 3.2 & $\begin{array}{l}10.5-11.1=\text { fairly intact } w / 2 \text { fractures; } 11.1-11.5=\text { rubble; } 11.5-13.7= \\
\text { fairly intact } w / 6 \text { fractures }\end{array}$ & $13.7-13.8$ \\
\hline
\end{tabular}




\section{Video Log Observations from Validation Study Boreholes}

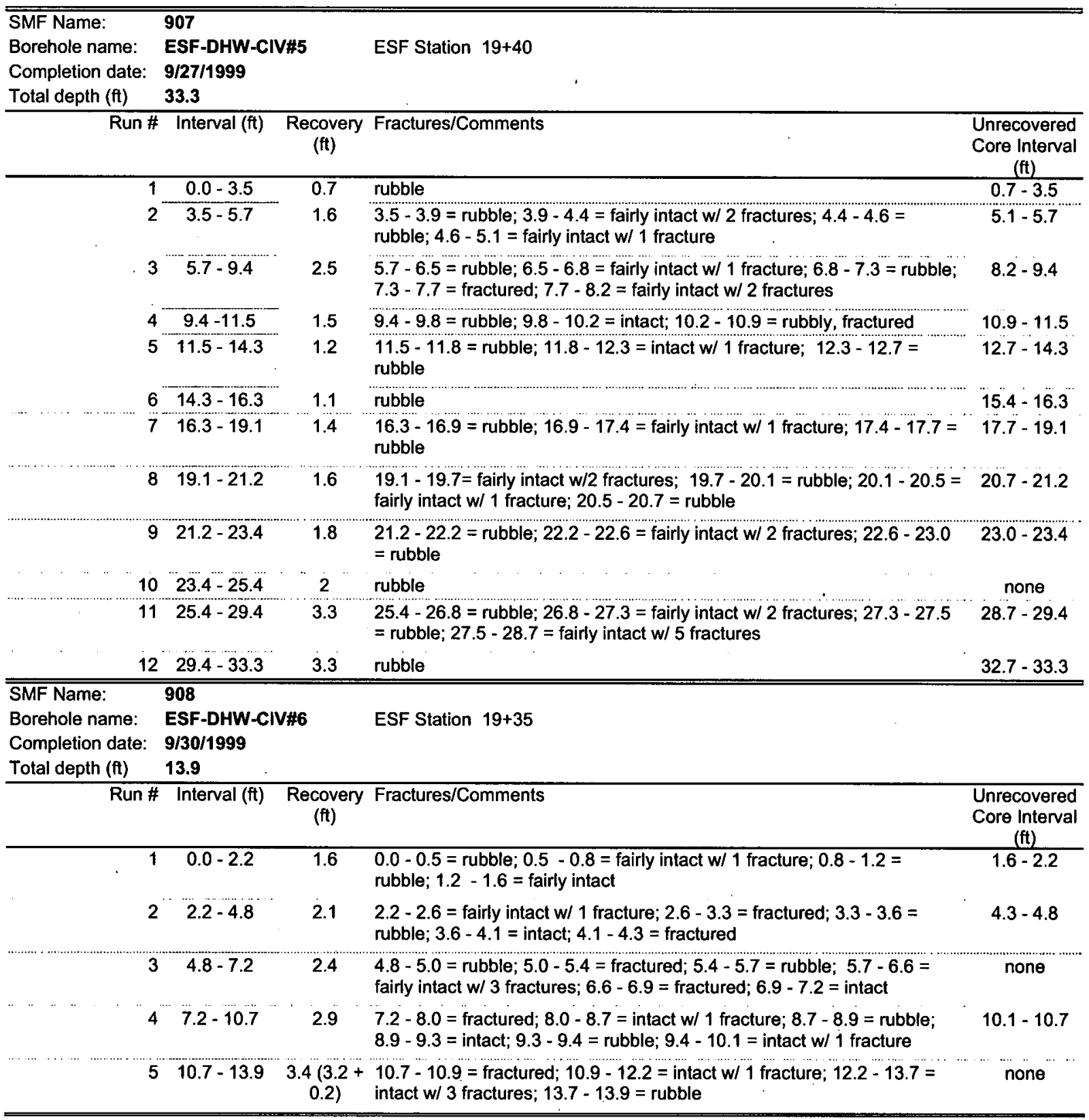




\section{Video Log Observations from Validation Study Boreholes}

\begin{tabular}{|c|c|c|c|c|}
\hline $\begin{array}{l}\text { SMF Name: } \\
\text { Borehole name: } \\
\text { Completion date: } \\
\text { Total depth (ft) }\end{array}$ & $\begin{array}{l}909 \\
\text { ESF-DHW-CIV\# } \\
10 / 5 / 1999 \\
13.6\end{array}$ & & ESF Station $19+30$ & \\
\hline \multirow[t]{6}{*}{ Run } & Interval (ft) $R$ & $\begin{array}{l}\text { Recovery } \\
\text { (ft) }\end{array}$ & Fractures/Comments & $\begin{array}{c}\text { Unrecovered } \\
\text { Core Interval } \\
\text { (ft) }\end{array}$ \\
\hline & $0.0-2.2$ & 1.2 & rubble & $1.2-2.2$ \\
\hline & $2.2-5.4$ & 2.2 & $2.2-2.5=$ rubble $; 2.5-2.7=$ intact $2.7-3.9=$ rubble $3.9-4.4=$ intact & $4.4-5.4$ \\
\hline & $5.4-7.3$ & 1.9 & rubble & none \\
\hline & $7.3 \cdot 9.6$ & 1.7 & $\begin{array}{l}7.3-7.6=\text { rubble; } 7.6-8.0=\text { intact; } 8.0-8.8=\text { rubble; } 8.8-9.0 \\
=\text { fractured }\end{array}$ & $9.0-9.6$ \\
\hline & $9.6-13.6$ & 3 & $\begin{array}{l}9.6-10.0=\text { rubble; } 10.0-12.1=\text { fairly intact } w / 6 \text { fractures; } 12.1-12.6= \\
\text { fractured, rubbly }\end{array}$ & $12.6-13.6$ \\
\hline $\begin{array}{l}\text { SMF Name: } \\
\text { Borehole name: } \\
\text { Completion date: } \\
\text { Total depth }(\mathrm{ft})\end{array}$ & $\begin{array}{l}910 \\
\text { ESF-DHW-CIV\# } \\
10 / 5 / 1999 \\
13.4\end{array}$ & & ESF Station $19+25$ & \\
\hline Run \# & Interval (ft) $\mathrm{R}$ & $\begin{array}{l}\text { Recovery } \\
\text { (ft) }\end{array}$ & Fractures/Comments & $\begin{array}{c}\text { Unrecovered } \\
\text { Core Interval } \\
\text { (ft) }\end{array}$ \\
\hline \multirow{2}{*}{ 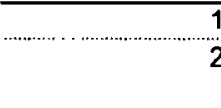 } & $0.0-1.4$ & 0.8 & fractured, broken & $0.8-1.4$ \\
\hline & $1.4 \cdot 3.8$ & 1.2 & $\begin{array}{l}1.4-1.5=\text { rubble; } 1.5-2.2=\text { fairly intact } w / 2 \text { fractures and crystal-lined } \\
\text { cavities in this section; } 2.2-2.6=\text { rubble }\end{array}$ & $2.6-3.8$ \\
\hline \multirow{3}{*}{$\begin{array}{lllll}\cdots & \ldots & \ldots & \ldots & \end{array}$} & $3.8-7.4$ & 3.2 & $\begin{array}{l}3.8-4.2=\text { fractured rubbly; } 4.2-5.0=\text { fairly intact } w / 3 \text { fractures; } 5.0- \\
6.8=\text { intact } w / 2 \text { frac. } \& 2 \text { cavities; } 6.8-7.0=\text { rubble }\end{array}$ & $7.0-7.4$ \\
\hline & $7.4-10.3$ & 1.8 & $\begin{array}{l}7.4-7.5=\text { rubble; } 7.5-7.7=\text { intact; } 7.7-8.1=\text { rubble; } 8.1-8.7=\text { intact; } \\
8.7-9.2=\text { fractured, rubbly }\end{array}$ & $9.2-10.3$ \\
\hline & $10.3-13.4$ & 2.8 & $\begin{array}{l}10.3-10.7=\text { rubble; } 10.7-11.5=\text { fairly intact w/ } 3 \text { fractures; } 11.5-11.8 \\
=\text { rubble; } 11.8-12.5=\text { fairly intact } w / 1 \text { fracture; } 12.5-12.9=\text { fractured, } \\
\text { broken; } 12.9-13.1=\text { intact }\end{array}$ & $13.1-13.4$ \\
\hline $\begin{array}{l}\text { SMF Name: } \\
\text { Borehole name: } \\
\text { Completion date: } \\
\text { Total depth (ft) }\end{array}$ & \multicolumn{2}{|c|}{$\begin{array}{l}\text { ESF-DHW-CIV\#9 } \\
10 / 6 / 1999\end{array}$} & ESF Station $19+20$ & \\
\hline Run \# & Interval (ft) & $\begin{array}{l}\text { Recovery } \\
\text { (ft) }\end{array}$ & Fractures/Comments & $\begin{array}{c}\text { Unrecovered } \\
\text { Core Interval } \\
\text { (ft) }\end{array}$ \\
\hline 1 & $0.0-2.4$ & 0.9 & rubble & $0.9-2.4$ \\
\hline 2 & $2.4-5.2$ & 2.5 & $\begin{array}{l}2.4-2.8=\text { rubble; } 2.8-3.3=\text { fairly intact } w / 1 \text { fracture; } 3.3-3.8=\text { rubble; } \\
3.8-4.5=\text { intact } w / 1 \text { fracture; } 4.5-4.9=\text { fractured, rubbly }\end{array}$ & $4.9-5.2$ \\
\hline 3 & $5.2-7.3$ & 1.7 & $\begin{array}{l}5.2-5.7=\text { fractured, broken; } 5.7-6.1=\text { fairly intact } w / 2 \text { fractures; } 6.1- \\
6.9=\text { rubble }\end{array}$ & $6.9-7.3$ \\
\hline 4 & $7.3-10.3$ & 2.2 & $\begin{array}{l}7.3-8.2=\text { rubble; } 8.2-8.7=\text { intact } w / 1 \text { fracture; } 8.7-9.5=\text { fractured, } \\
\text { rubbly }\end{array}$ & $9.5-10.3$ \\
\hline 5 & $10.3-12.3$ & 2.2 & $\begin{array}{l}10.3-10.8=\text { intact w/1 frac. \& lg. cavity; } 10.8-11.5=\text { rubble; } 11.5-12.2 \\
=\text { fairly intact } w / 4 \text { fractures; } 12.2-12.5=\text { rubble }\end{array}$ & $12.5-13.3$ \\
\hline
\end{tabular}




\section{Video Log Observations from Validation Study Boreholes}

\begin{tabular}{|c|c|c|c|c|}
\hline $\begin{array}{l}\text { SMF Name: } \\
\text { Borehole name: } \\
\text { Completion date: } \\
\text { Total depth (ft) } \\
\end{array}$ & $\begin{array}{l}912 \\
\text { ESF-DHW-CIV } \\
10 / 6 / 1999 \\
13.4\end{array}$ & /\#10 & ESF Station $19+10$ & \\
\hline & Interval (ft) & $\begin{array}{l}\text { Recovery } \\
\text { (ft) }\end{array}$ & Fractures/Comments & $\begin{array}{c}\text { Unrecovered } \\
\text { Core Interval } \\
\text { (ft) }\end{array}$ \\
\hline$\overline{1}$ & $0.0-2.4$ & 0.5 & rubble & $.05-2.4$ \\
\hline 2 & $2.4-5.3$ & 2.3 & $2.4-2.7=$ rubble; $2.7-3.7=$ fractured, broken; $3.7-4.7=$ rubble & $4.7-5.3$ \\
\hline 3 & $5.3-7.6$ & 0.6 & rubble & $5.9-7.6$ \\
\hline 4 & $7.6-10.4$ & 1.6 & $\begin{array}{l}7.6-8.2=\text { rubble; } 8.2-8.6=\text { fairly intact } w / 2 \text { fractures; } 8.6-9.0= \\
\text { rubble; } 9.0-9.2=\text { intact }\end{array}$ & $9.2-10.4$ \\
\hline 5 & $10.4-13.4$ & 2 & $\begin{array}{l}10.4-11.2=\text { fractured, rubbly; } 11.2-12.0=\text { fairly intact } w / 2 \text { fractures; } \\
12.0-12.4=\text { fractured, rubbly }\end{array}$ & $12.4: 13.4$ \\
\hline $\begin{array}{l}\text { SMF Name: } \\
\text { Borehole name: } \\
\text { Completion date: } \\
\text { Total depth (ft) }\end{array}$ & $\begin{array}{l}913 \\
\text { ESF-SD-CIV\#1 } \\
6 / 17 / 1999 \\
13.5\end{array}$ & & ESF Station $36+90$ & \\
\hline Run \# & Interval (ft) & $\begin{array}{c}\text { Recovery } \\
\text { (ft) }\end{array}$ & Description & $\begin{array}{c}\text { Unrecovered } \\
\text { Core Interval } \\
\text { (ft) }\end{array}$ \\
\hline 1 & $0.0-2.1$ & 1.4 & Broken, -1 Fracture & $1.4-2.1$ \\
\hline 2 & $2.1-4.3$ & 2.8 & Intact, 2 Fractures, Broken $(3.7-3.8)$ & $4.2-4.3$ \\
\hline 3 & $4.3-6.3$ & 2 & Broken & $6.2-6.3$ \\
\hline 4 & $6.3-8.1$ & 1.8 & Intact, 2-3 Fractures & $8.0-8.1$ \\
\hline 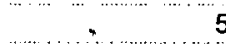 & $8.1-9.1$ & 0.9 & Broken & $9.0-9.1$ \\
\hline & $9.1-11.0$ & 1.4 & 2 Fractures, Broken & $10.5-11.0$ \\
\hline & $11.0-12.8$ & 2.1 & Broken & - \\
\hline 8 & $12.8-13.5$ & 0.9 & Broken & - \\
\hline $\begin{array}{l}\text { SMF Name: } \\
\text { Borehole name: } \\
\text { Completion date: } \\
\text { Total depth (ft) }\end{array}$ & $\begin{array}{l}914 \\
\text { ESF-SD-CIV\#2 } \\
6 / 16 / 1999 \\
13.6\end{array}$ & & ESF Station $36+75$ & \\
\hline Run & Interval (ft) & $\begin{array}{l}\text { Recovery } \\
\text { (ft) }\end{array}$ & Fractures/Comments & $\begin{array}{c}\text { Unrecovered } \\
\text { Core Interval } \\
\text { (ft) }\end{array}$ \\
\hline 1 & $0.0-2.0$ & 1.8 & Broken $(0.0-1.8)$ & $1.8-2.0$ \\
\hline 2 & $2.0-3.9$ & 1.4 & Rubbly $(2.0-2.3) ;$ Intact(2.3 - 2.7); Intact with $\sim 4$ Fractures $(2.7-3.9)$ & - \\
\hline 3 & $3.9-4.7$ & 0.9 & $\operatorname{Broken}(3.9-4.1) ; \operatorname{Intact}(4.1-4.7)$ & - \\
\hline 4 & $4.7-5.9$ & 1.2 & Rubbly $(4.7-4.8) ;$ Intact, $\sim 1$ Fracture $(4.8-5.9)$ & - \\
\hline 5 & $5.9-8.0$ & 2.1 & Intact $(5.9-6.4) ; \operatorname{Broken}(6.4-7.0) ; \operatorname{Intact}(7.0-7.6) ; \operatorname{Broken}(7.6-8.0)$ & - \\
\hline 6 & $8.0-9.9$ & 1.9 & Intact, $\sim 3$ Fractures $(8.0-9.9)$ & $\cdot-$ \\
\hline 7 & $9.9-12.0$ & 1.9 & Intact $(9.9-10.7) ;$ Intact $(10.7-11.5) ;$ Broken $(11.5-11.8)$ & $11.8-12.0$ \\
\hline 8 & $12.0-12.5$ & 0.5 & Broken & - \\
\hline 9 & $12.5-13.6$ & 0.9 & Intact, 1 Fracture $(12.5-13.4)$ & $13.4-13.6$ \\
\hline
\end{tabular}


Video Log Observations from Validation Study Boreholes

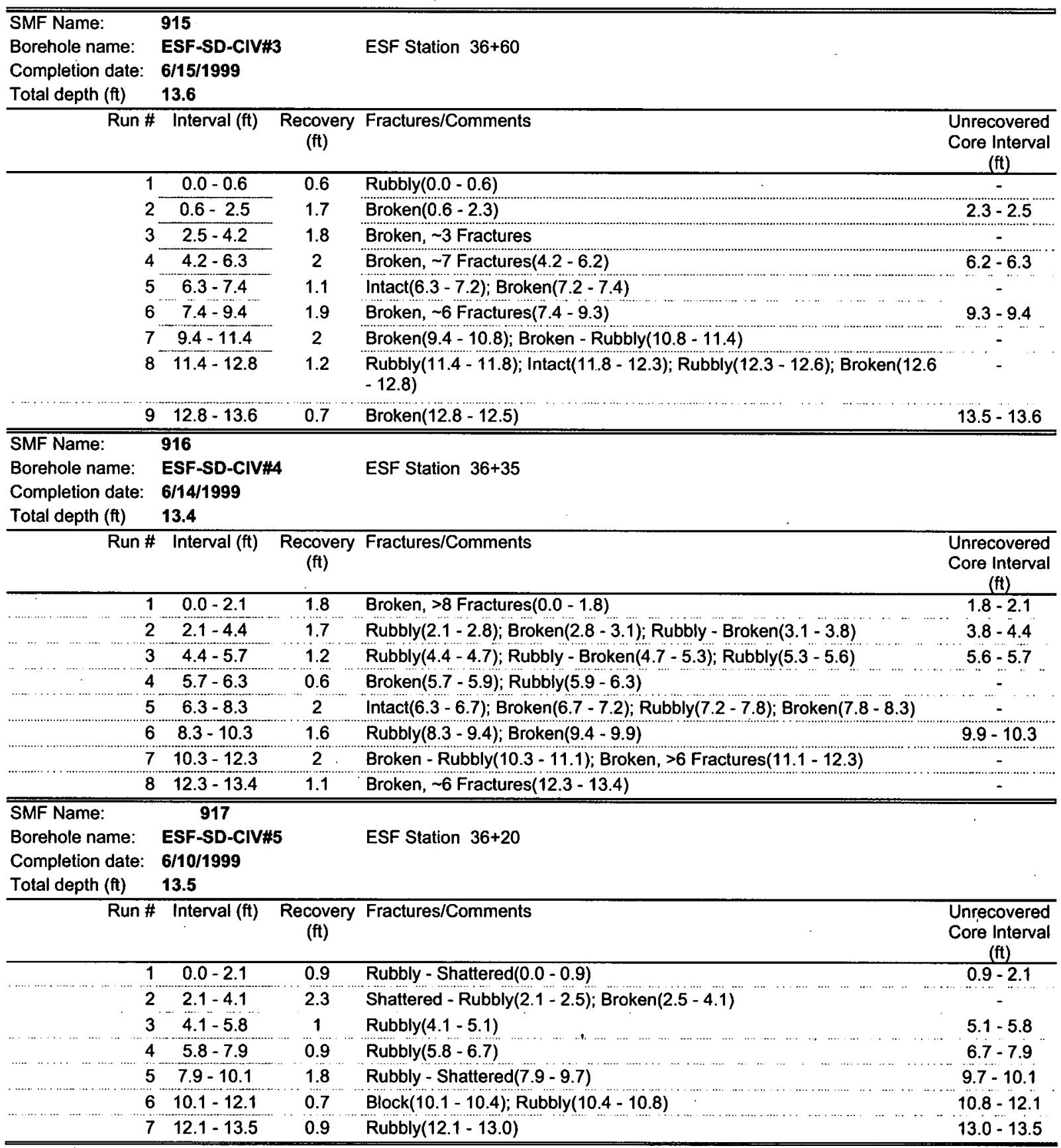




\section{Video Log Observations from Validation Study Boreholes}

\begin{tabular}{|c|c|c|c|c|}
\hline \multirow{9}{*}{$\begin{array}{l}\text { SMF Name: } \\
\text { Borehole name: } \\
\text { Completion date: } \\
\text { Total depth (ft) } \\
\text { Run } \#\end{array}$} & $\begin{array}{l}918 \\
\text { ESF-SD-CIV\#6 } \\
6 / 10 / 1999 \\
13.4\end{array}$ & & ESF Station $36+10$ & \\
\hline & Interval (ft) & $\begin{array}{l}\text { Recovery } \\
\text { (ft) }\end{array}$ & Fractures/Comments & $\begin{array}{c}\text { Unrecovered } \\
\text { Core Interval } \\
\text { (ft) }\end{array}$ \\
\hline & $0.0-2.0$ & 0.4 & Broken - Rubbly(0.0 - 0.4$)$ & $0.4-2.0$ \\
\hline & $2.0-4.0$ & 1.9 & Rubbly $(2.0-3.9)$ & $3.9-4.0$ \\
\hline & $4.0-5.7$ & 1.3 & Rubbly $(4.0-5.0)$; Intact, few hairline fractures $(5.0-5.3)$ & $5.3-5.7$ \\
\hline & $5.7-7.8$ & 1.2 & Rubbly(5.7-6.9) & $6.9-7.8$ \\
\hline & $7.8-10.8$ & 2.7 & Rubbly - Shattered (7.8 - 9.3); Broken $(9.4-10.2)$; Rubbly(10.2 - 10.5) & $10.5-10.8$ \\
\hline & $10.8-12.3$ & 1.2 & Rubbly $(10.8-11.4)$; Broken $(11.4-12.0)$ & $12.0-12.3$ \\
\hline & $12.3-12.4$ & 0 & No Core & $12.3-12.4$ \\
\hline $\begin{array}{l}\text { SMF Name: } \\
\text { Borehole name: } \\
\text { Completion date: } \\
\text { Total depth (ft) }\end{array}$ & $\begin{array}{l}919 \\
\text { ESF-SD-CIV\#7 } \\
6 / 8 / 1999 \\
13.5\end{array}$ & & ESF Station $36+05$ & \\
\hline Run \# & Interval (ft) & $\begin{array}{l}\text { Recovery } \\
\text { (ft) }\end{array}$ & Fractures/Comments & $\begin{array}{l}\text { Unrecovered } \\
\text { Core Interval } \\
\text { (ft) }\end{array}$ \\
\hline \multirow{5}{*}{ 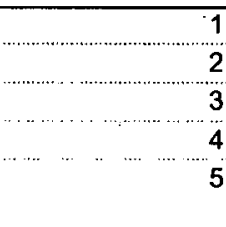 } & $0.0-2.0$ & 1.1 & Rubbly $(0.0-1.1)$ & $1.1-2.0$ \\
\hline & $2.0-3.9$ & 0.1 & Rubbly $(2.0-2.1)$ & $2.1-3.9$ \\
\hline & $3.9-6.0$ & 0.5 & 2 blocks $(3.9-4.4)$ & $4.4-6.0$ \\
\hline & $6.0-8.1$ & 2 & Broken, >12 Fractures $(6.0-8.0)$ & $8.0-8.1$ \\
\hline & $8.1-10.7$ & 1.6 & $\begin{array}{l}\text { Broken, 3 Fractures }(8.1-8.8) \text {; Rubbly }(8.8-9.4) \text {; Broken(9.4 - 9.6); } \\
\text { Rubbly(9.6 - 9.7) }\end{array}$ & $9.7-10.7$ \\
\hline \multirow{2}{*}{$\begin{array}{r}6 \\
7 \\
\end{array}$} & $10.7-11.7$ & 1 & Rubbly & $\cdots$ \\
\hline & $11.7-13.5$ & 0.6 & Rubbly $(11.7-12.3)$ & $12.3-13.5$ \\
\hline \multicolumn{2}{|c|}{$\begin{array}{ll}\text { SMF Name: } & 920 \\
\text { Borehole name: } & \text { ESF-SD-CIV\#8 } \\
\text { Completion date: } & 6 / 8 / 1999 \\
\text { Total depth (ft) } & 13.5\end{array}$} & & ESF Station $36+00$ & \\
\hline Run \# & Interval (ft) & $\begin{array}{l}\text { Recovery } \\
\text { (ft) }\end{array}$ & Fractures/Comments & $\begin{array}{l}\text { Unrecovered } \\
\text { Core Interval } \\
\text { (ft) }\end{array}$ \\
\hline \multirow{7}{*}{ 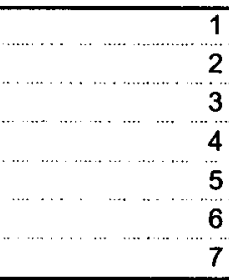 } & $0.0-2.0$ & 1.7 & Rubbly(0.0 - 1.0); Broken(1.0 - 1.7) & $1.7-2.0$ \\
\hline & $2.0-4.0$ & 1.4 & Broken - Rubbly $(2.0-2.8)$; Rubbly $(2.8-3.4)$ & $3.4-4.0$ \\
\hline & $4.0-6.0$ & 1.5 & Rubbly $(4.0-4.3)$; Block $(4.3-4.7)$; Rubbly $(4.7-5.5)$ & $5.5-6.0$ \\
\hline & $6.0-7.9$ & 0.2 & Block $(6.0-6.2)$ & $6.2-7.9$ \\
\hline & $7.9-9.9$ & 2 & Rubbly $(7.9-9.9)$ & - \\
\hline & $9.9-11.9$ & 1.9 & Rubbly $(9.9-11.8)$ & $11.8-11.9$ \\
\hline & $11.9-13.5$ & 1.1 & Rubbly(11.9-12.3); Broken(12.3 - 13.0) & $13.0-13.5$ \\
\hline
\end{tabular}




\section{Video Log Observations from Validation Study Boreholes}

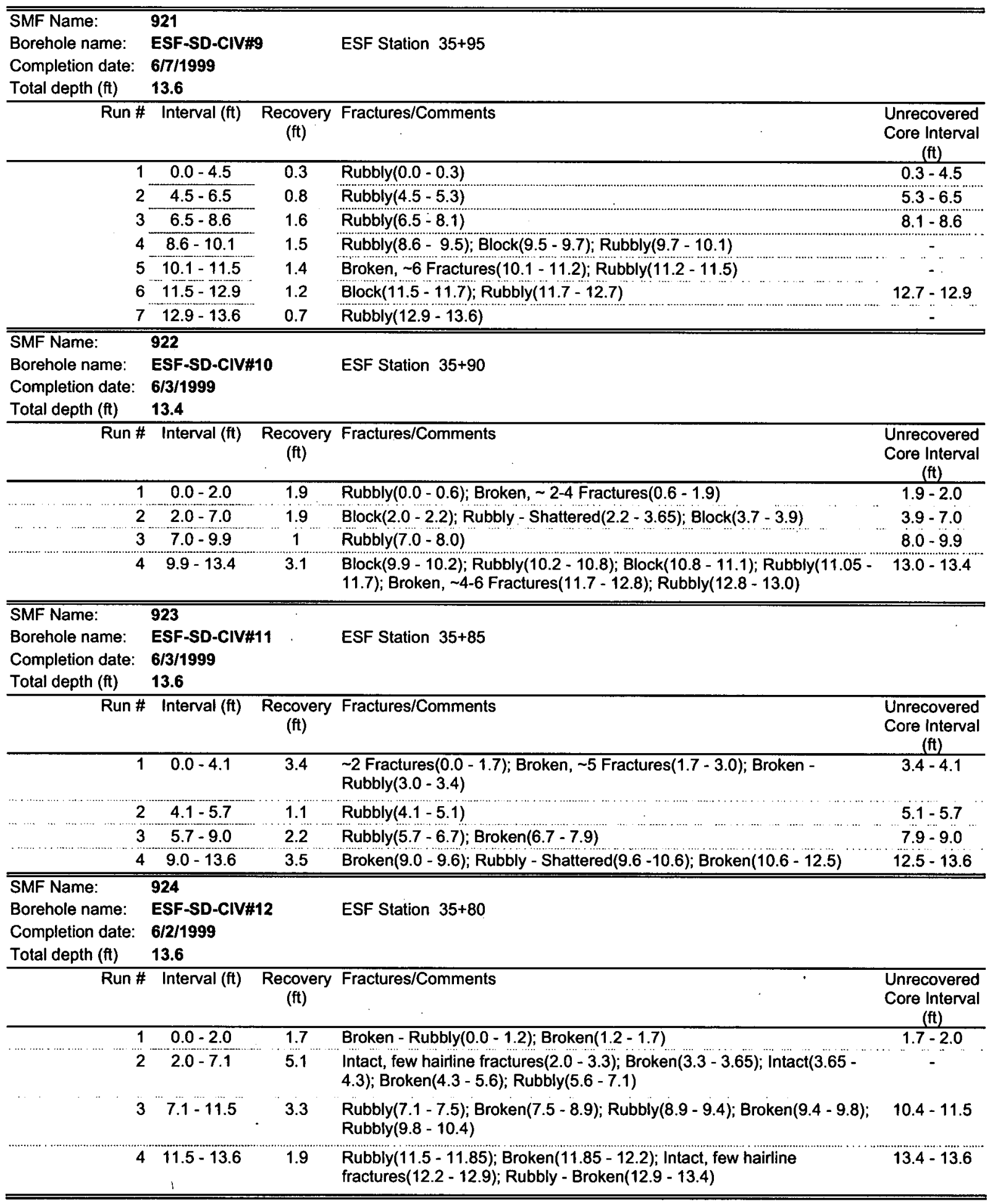




\section{Video Log Observations from Validation Study Boreholes}

\begin{tabular}{|c|c|c|c|c|}
\hline $\begin{array}{l}\text { SMF Name: } \\
\text { Borehole name: } \\
\text { Completion date: } \\
\text { Total depth (ft) } \\
\end{array}$ & \multicolumn{2}{|l|}{$\begin{array}{l}925 \\
\text { ESF-SD-CIV\#13 } \\
6 / 2 / 1999 \\
32.6\end{array}$} & \multicolumn{2}{|l|}{ ESF Station $35+75$} \\
\hline & Interval (ft) $\mathrm{F}$ & $\begin{array}{l}\text { Recovery } \\
\text { (ft) }\end{array}$ & Fractures/Comments & $\begin{array}{c}\text { Unrecovered } \\
\text { Core Interval } \\
\text { (ft) }\end{array}$ \\
\hline 1 & $0.0-1.7$ & 1.7 & Rubbly - Broken & - \\
\hline 2 & $1.7-2.9$ & 1.2 & Broken - Rubbly & - \\
\hline 3 & $2.9 \cdot 7.0$ & 3.8 & Broken, $>12$ Fractures $(2.9-6.3)$; Rubbly $(6.3-6.7)$ & $6.7-7.0$ \\
\hline 4 & $7.0-9.7$ & 1.6 & Rubbly - Broken $(7.0-8.6)$ & $8.6-9.7$ \\
\hline 5 & $9.7-13.9$ & 3 & Rubbly(9.7-11.4); Intact(11.4-11.85); Broken(11.85-12.7) & $12.7-13.9$ \\
\hline 6 & $13.9-17.0$ & 2.2 & $\begin{array}{l}\text { Broken(13.9-14.4); Rubbly(14.4-14.7); Broken(14.7-15.0); Intact(15.0 } \\
-15.8) ; \text { Broken(15.8 - 16.1) }\end{array}$ & $16.1-17.0$ \\
\hline 7 & $17.0-18.8$ & 1.3 & Intact(17.0 - 17.5); Broken(17.5 - 17.9); Rubbly(17.9 - 18.3) & $18.3-18.8$ \\
\hline 8 & $18.8-21.7$ & 2.9 & Rubbly(18.8 - 19.6); Broken(19.6 - 20.5); Broken - Rubbly(20.5 - 21.7) & - \\
\hline & $21.7-23.2$ & 1.5 & Rubbly $(21.7-23.2)$ & - \\
\hline & $23.2-24.8$ & 1.2 & Broken - Rubbly(23.2 - 24.4) & $24.4-24.8$ \\
\hline 11 & $24.8-25.3$ & 0.5 & Broken $(24.8-25.3)$ & - \\
\hline 12 & $25.3-30.0$ & 2 & Rubbly $(25.3-27.3)$ & $27.3-30.0$ \\
\hline 13 & $30.0-32.6$ & 2.3 & Rubbly $(30.0-30.6)$; Broken - Rubbly $(30.6-32.3)$ & $32.3-32.6$ \\
\hline $\begin{array}{l}\text { SMF Name: } \\
\text { Borehole name: } \\
\text { Completion date: } \\
\text { Total depth (ft) } \\
\end{array}$ & $\begin{array}{l}926 \\
\text { ESF-SD-CIV\#14 } \\
9 / 22 / 1999 \\
13.4 \\
\end{array}$ & & ESF Station $35+45$ & \\
\hline Run \# & Interval (ft) $\mathrm{F}$ & $\begin{array}{l}\text { Recovery } \\
\text { (ft) }\end{array}$ & Fractures/Comments & $\begin{array}{c}\text { Unrecovered } \\
\text { Core Interval } \\
\text { (ft) }\end{array}$ \\
\hline 1 & $0.0-2.8$ & 2.1 & Mostly Broken, Rubbly & $2.1-2.8$ \\
\hline 2 & $2.8-5.4$ & 2.6 & Mostly Rubble, Broken & - \\
\hline 3 & $5.4-6.9$ & 1.5 & Broken & - \\
\hline 4 & $6.9-8.0$ & 1 & Broken, -1 Fracture & $7.9-8.0$ \\
\hline $\begin{array}{rr}5 \\
\end{array}$ & $\frac{8.0-11.9}{11.9-13.4}$ & $\begin{array}{l}3.5 \\
1.8\end{array}$ & $\begin{array}{l}\text { Rubbly }(8.2-8.9) \text {, Broken, } 2-3 \text { Fractures } \\
\text { Broken, } 1 \text { Fracture }\end{array}$ & $\begin{array}{c}11.5-11.9 \\
-\end{array}$ \\
\hline $\begin{array}{l}\text { SMF Name: } \\
\text { Borehole name: } \\
\text { Completion date: } \\
\text { Total depth (ft) } \\
\end{array}$ & $\begin{array}{l}927 \\
\text { ESF-SD-CIV\#1S } \\
9 / 21 / 1999 \\
13.5 \\
\end{array}$ & & ESF Station $35+40$ & \\
\hline Run \# & Interval (ft) $\mathrm{F}$ & $\begin{array}{l}\text { Recovery } \\
\text { (ft) }\end{array}$ & Fractures/Comments & $\begin{array}{c}\text { Unrecovered } \\
\text { Core Interval } \\
\text { (ft) }\end{array}$ \\
\hline 1 & $0.0-1.9$ & 1.7 & Broken & $1.7-1.9$ \\
\hline 2 & $1.9-4.7$ & 2.7 & Mostly intact, Broken $(4.3-4.6)$ & $4.6-4.7$ \\
\hline 3 & $4.7-7.8$ & 2.4 & Broken, $\sim 2$ Fractures & $7.1-7.8$ \\
\hline 4 & $7.8-12.1$ & 4.1 & Broken, -3 Fractures & $11.9-12.1$ \\
\hline 5 & $12.1-13.5$ & 1.6 & Broken, $\sim 2$ Fractures & - \\
\hline
\end{tabular}


Video Log Observations from Validation Study Boreholes

\begin{tabular}{|c|c|c|c|}
\hline $\begin{array}{l}\text { SMF Name: } \\
\text { Borehole name: } \\
\text { Completion date: } \\
\text { Total depth (ft) }\end{array}$ & $\begin{array}{l}928 \\
\text { ESF-SD-CIV\#1 } \\
9 / 20 / 1999 \\
13.5 \\
\end{array}$ & & ESF Station $35+35$ \\
\hline Run \# & \# Interval (ft) & $\begin{array}{l}\text { Recovery } \\
\text { (ft) }\end{array}$ & $\begin{array}{c}\text { Unrecovered } \\
\text { Core Interval } \\
\text { (ft) }\end{array}$ \\
\hline 1 & $0.0-0.5$ & 0.2 & $0.2-0.5$ \\
\hline 2 & $0.5-1.7$ & 1.2 & 1 Fracture, Broken \\
\hline 3 & $1.7-2.5$ & 0.9 & Intact, - 1 Fracture \\
\hline 4 & $2.5-2.8$ & 0.1 & 1 Block \\
\hline 5 & $2.8-5.0$ & 2.2 & Broken \\
\hline 6 & $5.0-6.7$ & 1.7 & Broken, -1 Fracture \\
\hline 7 & $6.7-7.4$ & 0.6 & $7.3-7.4$ \\
\hline 8 & $7.4-9.3$ & 1.8 & $9.2-9.3$ \\
\hline 9 & $99.3-13.5$ & 3.9 & $13.2-13.5$ \\
\hline $\begin{array}{l}\text { SMF Name: } \\
\text { Borehole name: } \\
\text { Completion date: } \\
\text { Total depth }(\mathrm{ft}) \\
\end{array}$ & $\begin{array}{l}929 \\
\text { ESF-SD-CIV\#1 } \\
9 / 17 / 1999 \\
13.3 \\
\end{array}$ & & ESF Station $35+30$ \\
\hline Run A & \# Interval (ft) & $\begin{array}{l}\text { Recovery } \\
\text { (ft) }\end{array}$ & $\begin{array}{c}\text { Unrecovered } \\
\text { Core Interval } \\
\text { (ft) }\end{array}$ \\
\hline 1 & $0.0-0.5$ & 0.5 & Broken \\
\hline 2 & $0.5-2.6$ & 2.1 & Broken, -3 Fractures \\
\hline 3 & $2.6-4.7$ & 2.1 & Broken \\
\hline 4 & $4.7-5.5$ & 0.8 & Broken, Blocky \\
\hline ............ & $5.5-6.9$ & $1: 4$ & Broken, Some Fractures? (Video black out) \\
\hline .. . . . . & $6.9-8.5$ & 1.6 & Broken, -2 Fractures \\
\hline 7 & $8.5-9.8$ & 0.8 & Broken \\
\hline 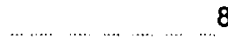 & $89.8-10.5$ & 0.9 & Broken, Blocky \\
\hline $\begin{array}{r}9 \\
\end{array}$ & $910.5-13.3$ & 2.7 & Broken, 2-3 Fractures \\
\hline $\begin{array}{l}\text { SMF Name: } \\
\text { Borehole name: } \\
\text { Completion date: } \\
\text { Total depth (ft) }\end{array}$ & $\begin{array}{l}930 \\
\text { ESF-SD-CIV\#1 } \\
9 / 16 / 1999 \\
13.5 \\
\end{array}$ & 18 & ESF Station $35+25$ \\
\hline Run \# & \# Interval (ft) & $\begin{array}{c}\text { Recovery } \\
\text { (ft) }\end{array}$ & $\begin{array}{c}\text { Unrecovered } \\
\text { Core Interval } \\
\text { (ft) }\end{array}$ \\
\hline 1 & $0.0-2.1$ & 1.9 & Broken, $\sim 2$ Fractures \\
\hline 2 & $2.1-3.6$ & 1.5 & Broken, -3 Fractures \\
\hline 3 & $3.6-5.7$ & 2.1 & $5.6-5.7$ \\
\hline & $5.7-7.5$ & 1.5 & $7.2-7.5$ \\
\hline 5 & $7.5-8.8$ & 1.2 & Broken, -2 Fractures? \\
\hline 6 & $68.8-10.2$ & 1.4 & Broken \\
\hline 7 & $7 \quad 10.2-10.9$ & 0.7 & Broken \\
\hline 8 & $810.9-12.6$ & 0.9 & $11.8-12.6$ \\
\hline 9 & $912.6-13.5$ & 1.2 & Rubbly - Broken \\
\hline
\end{tabular}


Video Log Observations from Validation Study Boreholes

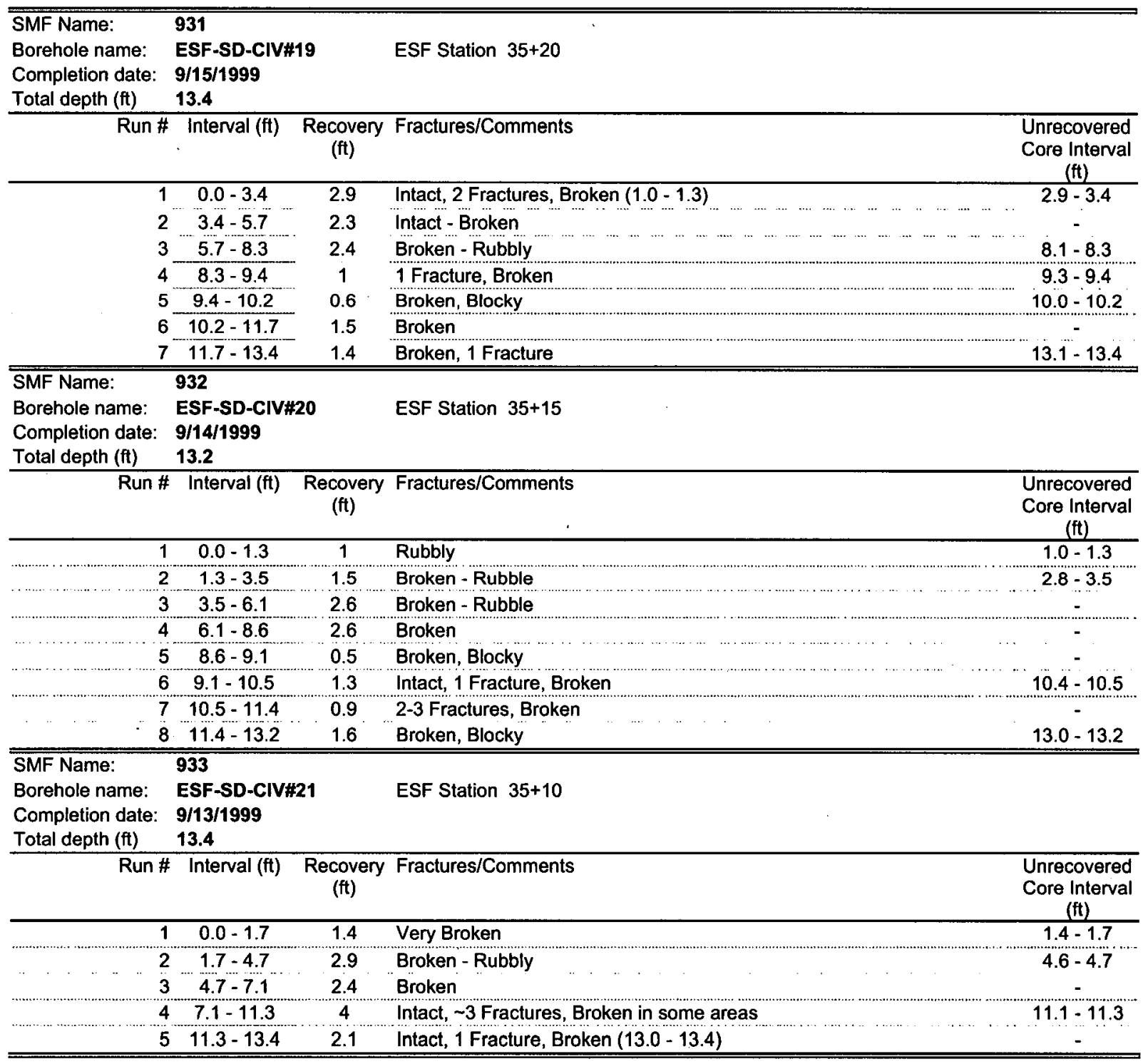




\section{Video Log Observations from Validation Study Boreholes}

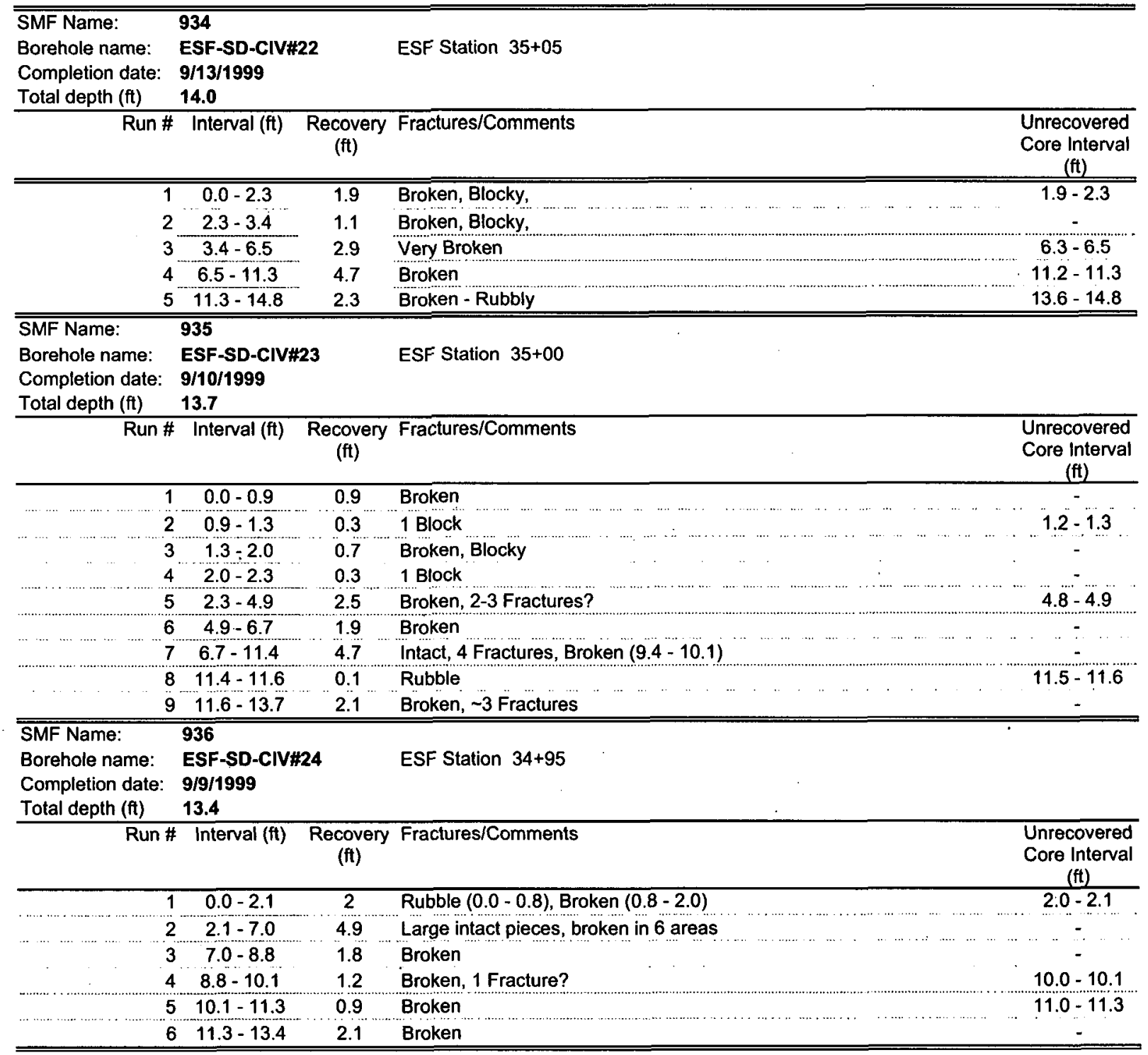




\section{Video Log Observations from Validation Study Boreholes}

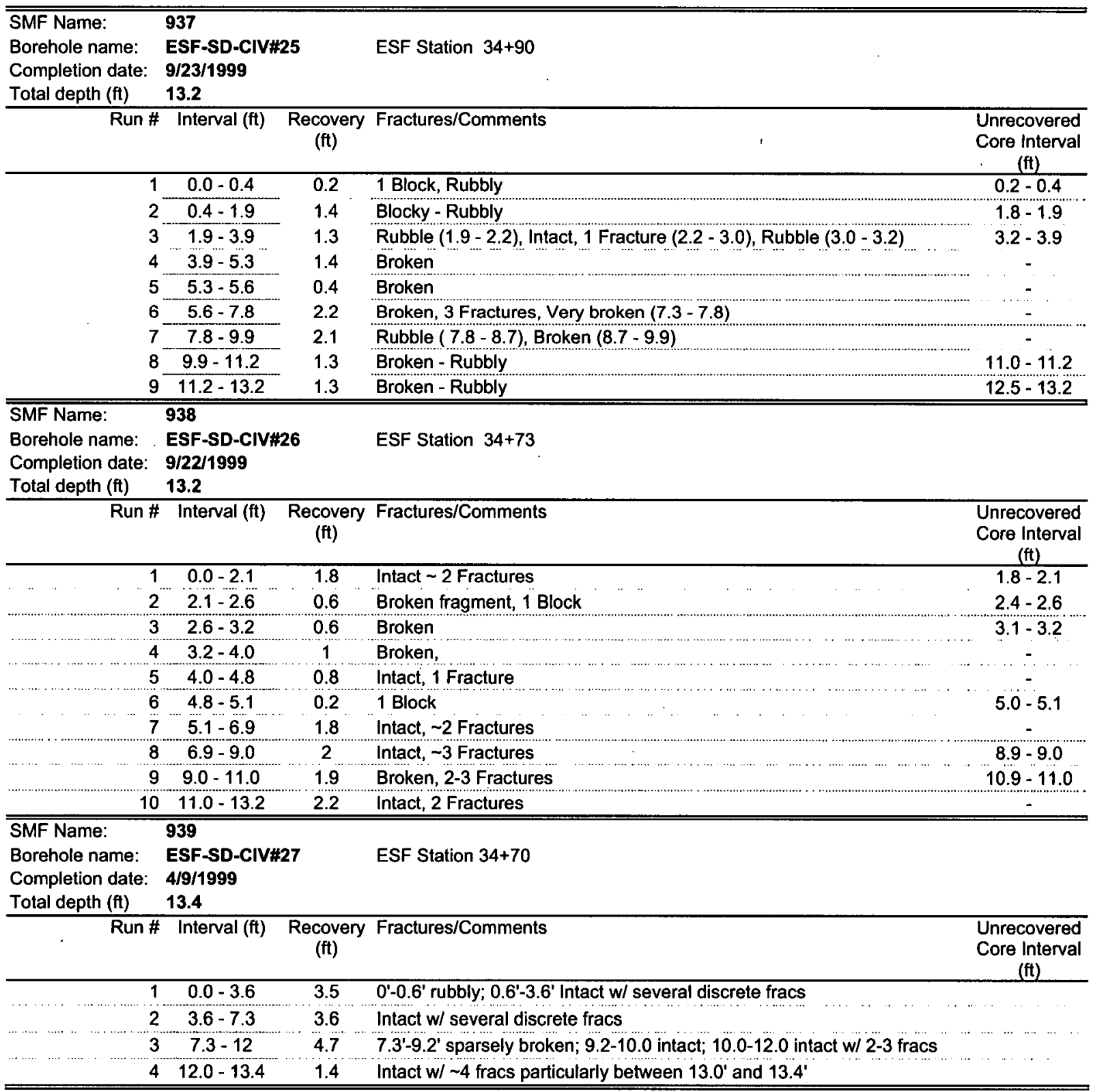




\section{Video Log Observations from Validation Study Boreholes}

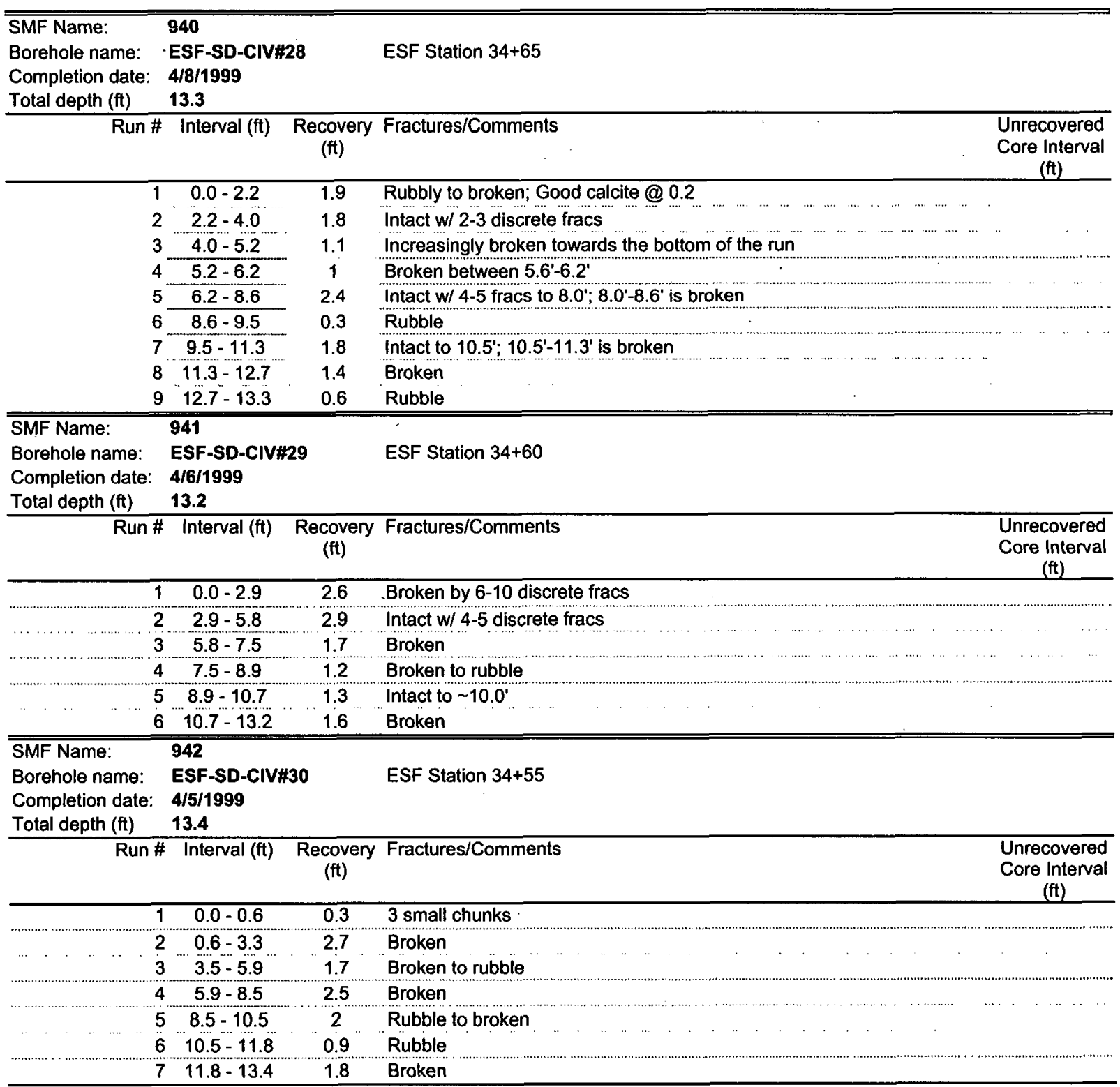




\section{Video Log Observations from Validation Study Boreholes}

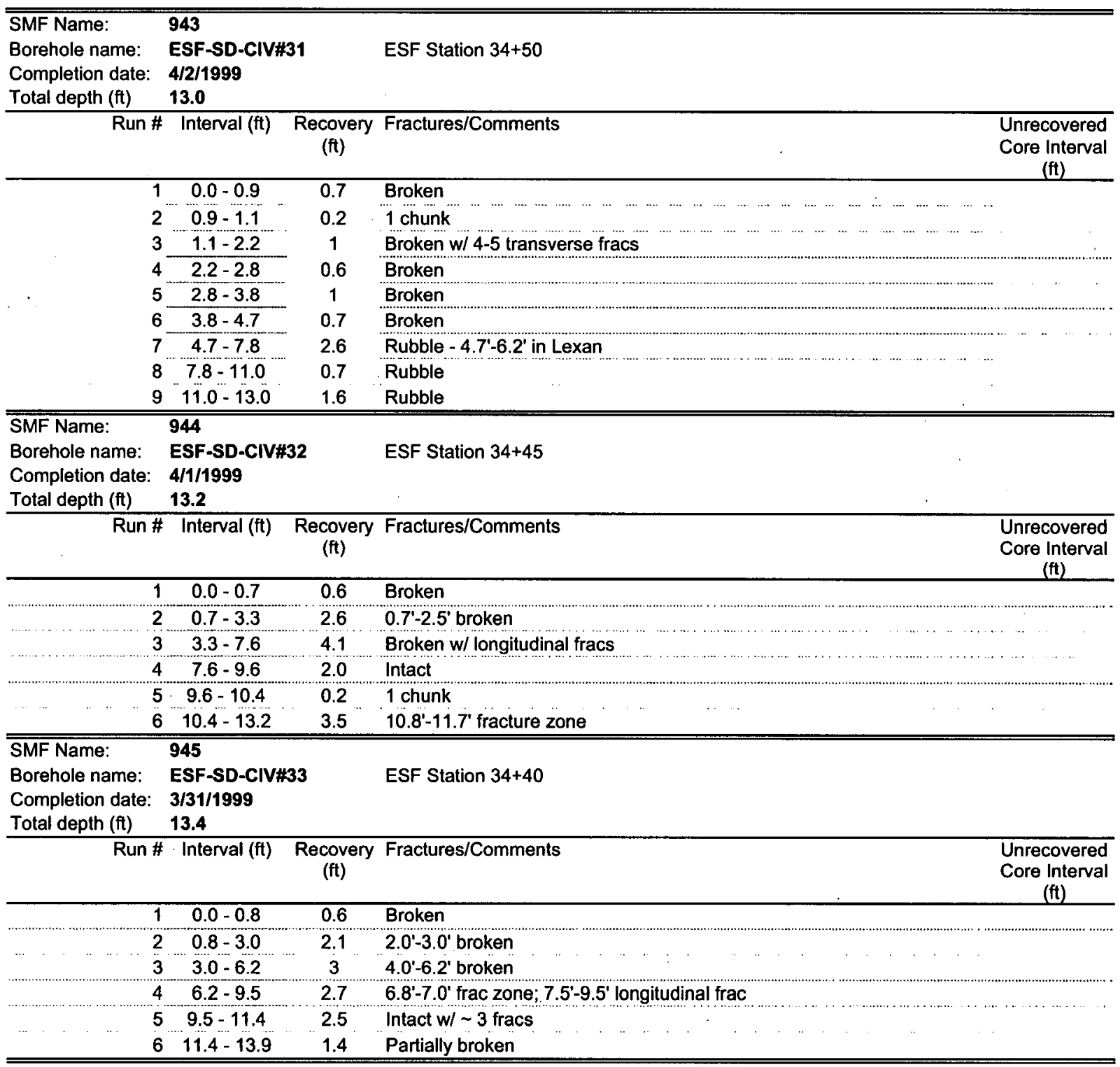




\section{Video Log Observations from Validation Study Boreholes}

\begin{tabular}{|c|c|c|c|}
\hline $\begin{array}{l}\text { SMF Name: } \\
\text { Borehole name: } \\
\text { Completion date: } \\
\text { Total depth (ft) }\end{array}$ & $\begin{array}{l}946 \\
\text { ESF-SD-CIV\#34 } \\
3 / 30 / 1999 \\
13.3\end{array}$ & & ESF Station $34+35$ \\
\hline Run \# & Interval (ft) & $\begin{array}{l}\text { Recovery } \\
\text { (ft) }\end{array}$ & $\begin{array}{l}\text { Unrecovered } \\
\text { Core Interval } \\
\quad(\mathrm{ft})\end{array}$ \\
\hline 1 & $0.0-1.4$ & 0.9 & Broken \\
\hline 2 & $1.4-1.8$ & 0.4 & Broken \\
\hline 3 & $1.8-2.1$ & 0.3 & Broken \\
\hline 4 & $2.1-2.5$ & 0.3 & Broken \\
\hline 5 & $2.5-3.0$ & 0.6 & Broken \\
\hline 6 & $3.0-3.8$ & 0.5 & Rubbly \\
\hline 7 & $3.8-4.8$ & 1. & Intact $w / \sim 3$ fracs \\
\hline 8 & $4.8-7.7$ & 2.8 & 4.8'-6.6' rubbly; $6.6^{\prime}-7.7^{\prime}$ intact $w / 22-3$ fracs \\
\hline 9 & $7.7-8.3$ & 0.5 & Rubbly \\
\hline 10 & $8.3-13.3$ & 4.7 & $8.3^{\prime}-10.0^{\prime}$ rubbly; $10.4^{\prime}-10.5^{\prime}$ frac zone; $11.2^{\prime}-13.0^{\prime}$ longitudinal frac \\
\hline $\begin{array}{l}\text { SMF Name: } \\
\text { Borehole name: } \\
\text { Completion date: } \\
\text { Total depth (ft) }\end{array}$ & $\begin{array}{l}947 \\
\text { ESF-SD-CIV\#35 } \\
3 / 26 / 1999 \\
13.3\end{array}$ & & ESF Station $34+30$ \\
\hline Run \# & Interval (ft) & $\begin{array}{l}\text { Recovery } \\
\text { (ft) }\end{array}$ & $\begin{array}{l}\text { Unrecovered } \\
\text { Core Interval } \\
\text { (ft) }\end{array}$ \\
\hline 1 & $0.0-2.2$ & 1.9 & Broken \\
\hline 2 & $2.2-4.0$ & 1.8 & Broken to rubbly \\
\hline ............. & $4.0-6.3$ & 1.9 & Intact $w / \sim 2$ fracs \\
\hline 4 & $6.3-6.4$ & 0.1 & 1 chunk \\
\hline$\cdots \quad . \quad \frac{5}{6}$ & $\begin{array}{l}6.4-8.5 \\
8.5-9.2\end{array}$ & $\begin{array}{l}2.1 \\
0.5\end{array}$ & $\begin{array}{l}6.3^{\prime}-8.5^{\prime} \text { broken } \\
\text { Several chunks }\end{array}$ \\
\hline 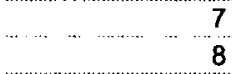 & $\frac{9.2-11.4}{11.4-11.6}$ & $\begin{array}{l}2.4 \\
0.2\end{array}$ & $\begin{array}{l}10.5^{\prime}-10.7^{\prime} \text { broken zone; } 11.2^{\prime}-11.4^{\prime} \text { broken } \\
1 \text { chunk }\end{array}$ \\
\hline $\begin{array}{rr}9 \\
\quad \\
& 10\end{array}$ & $\begin{array}{l}11.6-12.8 \\
12.8-13.3\end{array}$ & $\begin{array}{l}1.2 \\
0.5\end{array}$ & 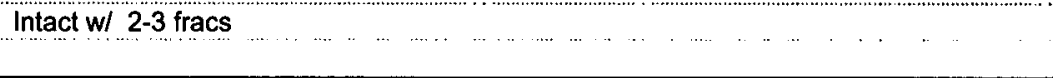 \\
\hline $\begin{array}{l}\text { SMF Name: } \\
\text { Borehole name: } \\
\text { Completion date: } \\
\text { Total depth (ft) }\end{array}$ & $\begin{array}{l}948 \\
\text { ESF-SD-CIV\#36 } \\
3 / 25 / 1999 \\
13.3 \\
\end{array}$ & & ESF Station $34+25$ \\
\hline Run \# & Interval (ft) & $\begin{array}{l}\text { Recovery } \\
\text { (ft) }\end{array}$ & $\begin{array}{l}\text { Unrecovered } \\
\text { Core Interval } \\
\text { (ft) }\end{array}$ \\
\hline $\begin{array}{llll}. & \cdots & . & .\end{array}$ & $\begin{array}{c}0.0-1.0 \\
1.0-2.7 \\
2.7-3.4\end{array}$ & $\begin{array}{l}0.7 \\
1.4 \\
0.8\end{array}$ & $\begin{array}{l}\text { Rubbly } \\
1.4^{\prime}-2.4^{\prime} \text { broken } \\
\sim 4 \text { fracs - broken }\end{array}$ \\
\hline 4 & $3.4-3.9$ & 0.5 & Rubbly \\
\hline 5 & $3.9-6.7$ & 2.8 & Broken \\
\hline 6 & $6.7-9.1$ & 2.4 & $6.7^{\prime}-7.0^{\prime}$ rubbly; $7.0^{\prime}-9.1^{\prime}$ intact $w / \sim 4$ fracs \\
\hline 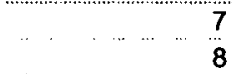 & $\begin{array}{r}9.1-9.4 \\
9.4-10.6\end{array}$ & 0.3 & $\begin{array}{l}1 \text { chunk } \\
\text { Intact } w / \sim 5 \text { fracs }\end{array}$ \\
\hline 9 & $10.6-10.8$ & 0.2 & 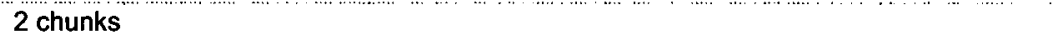 \\
\hline 10 & $10.8-13.3$ & 1.3 & 10.8'-12.1' rubbly \\
\hline
\end{tabular}




\section{Video Log Observations from Validation Study Boreholes}

\begin{tabular}{|c|c|c|c|c|}
\hline $\begin{array}{l}\text { SMF Name: } \\
\text { Borehole name: } \\
\text { Completion date: } \\
\text { Total depth (ft) }\end{array}$ & $\begin{array}{l}949 \\
\text { ESF-SD-CIV\#37 } \\
3 / 24 / 1999 \\
13.3 \\
\end{array}$ & & ESF Station $34+20$ & \\
\hline Run \# & $\exists$ Interval (ft) $\quad R$ & $\begin{array}{l}\text { Recovery } \\
\text { (ft) }\end{array}$ & Fractures/Comments & $\begin{array}{c}\text { Unrecovered } \\
\text { Core Interval } \\
\text { (ft) }\end{array}$ \\
\hline 1 & $0.0-0.3$ & 0.3 & 2 blocks & \\
\hline 2 & $0.3-0.9$ & 0.4 & 4 blocks & \\
\hline 3 & $0.9-1.3$ & 0.6 & 1 block & \\
\hline 4 & $1.3-5.9$ & 4 & $1.3^{\prime}-3.0^{\prime}$ broken; $3.0^{\prime}-5.9$ intact w/2-3 discrete fracs & \\
\hline 5 & $5.9-8.9$ & 3.8 & Intact $w /-7$ discrete fracs & \\
\hline 6 & $8.9-9.7$ & $?$ & $?$ & \\
\hline 7 & $9.7-13.3$ & 3.3 & $9.7^{\prime}-11.2^{\prime}$ intact $w / \sim 3$ fracs; $11.4^{\prime}-13.0^{\prime}$ broken & \\
\hline $\begin{array}{l}\text { SMF Name: } \\
\text { Borehole name: } \\
\text { Completion date: } \\
\text { Total depth (ft) }\end{array}$ & $\begin{array}{l}950 \\
\text { ESF-SD-CIV\#38 } \\
3 / 23 / 1999 \\
13.2 \\
\end{array}$ & & ESF Station $34+10$ & \\
\hline Run \# & $\#$ Interval (ft) $\quad F$ & $\begin{array}{l}\text { Recovery } \\
\text { (ft) }\end{array}$ & Fractures/Comments & $\begin{array}{c}\text { Unrecovered } \\
\text { Core Interval } \\
\text { (ft) }\end{array}$ \\
\hline 1 & $0.0-0.6$ & 0.4 & Broken @ top & \\
\hline 2 & $0.6-1.4$ & 0.8 & 4 fracs over 0.8 ' - "broken" & \\
\hline 3 & $1.4-6.1$ & 4.4 & $1.4^{\prime}-2.6^{\prime}$ intact; $2.6^{\prime}-5.0^{\prime}$ broken to rubbly; $5.0^{\prime}-6.1^{\prime}$ intact & \\
\hline 4 & $6.1-9.1$ & 2.9 & Intact w/ 4-5 discrete fracs & \\
\hline 5 & $59.1-11.0$ & 1.7 & $9.1^{\prime}-9.8^{\prime}$ rubbly; $10.5^{\prime}-10.8^{\prime}$ broken & \\
\hline 6 & $611.0-12.7$ & 1.7 & $11.0^{\prime}-11.5^{\prime}$ imbricate slices; $11.5^{\prime}-12.7^{\prime}$ intact & \\
\hline 7 & $712.7-13.2$ & 0.4 & $?$ & \\
\hline $\begin{array}{l}\text { SMF Name: } \\
\text { Borehole name: } \\
\text { Completion date: } \\
\text { Total depth (ft) }\end{array}$ & $\begin{array}{l}951 \\
\text { ESF-SD-CIV\#39 } \\
3 / 19 / 1999 \\
13.4 \\
\end{array}$ & & ESF Station $33+98$ & \\
\hline Run \# & $\#$ Interval (ft) $\mathrm{F}$ & $\begin{array}{l}\text { Recovery } \\
\text { (ft) }\end{array}$ & Fractures/Comments & $\begin{array}{c}\text { Unrecovered } \\
\text { Core Interval } \\
\text { (ft) }\end{array}$ \\
\hline & $0.0-2.1$ & 1.9 & Intact - 3 total (1@0.2 \& 2@2.1) & \\
\hline 2 & $2.1-5.7$ & 3.6 & Intact to $\sim 5.0^{\prime}$; broken btwn 5.0-5.3; rubbly btwn 5.4-5.7 & \\
\hline 3 & $\begin{array}{l}5.7-7.8 \\
7.8-8.0\end{array}$ & $\begin{array}{c}2.1 \\
0\end{array}$ & $\begin{array}{l}\text { Rubbly btwn }-6.6^{\prime}-6.9^{\prime} ; 2-3 \text { fracs @ } 7.3^{\prime} \\
\text { Unrecovered }\end{array}$ & \\
\hline 5 & $8.0-9.3$ & & Broken btwn 8.6'-9.3' & \\
\hline$-\frac{6}{7}$ & $\begin{array}{l}6 \\
6.3-10.1 \\
7 \quad 10.1-11.0\end{array}$ & $\begin{array}{l}0.8 \\
0.7\end{array}$ & $\begin{array}{l}\text { Rubbly } \\
\text { Broken }\end{array}$ & \\
\hline 8 & $811.0-13.4$ & 2.4 & Broken btwn 11.0'-12.1'; broken btwn 12.6'-13.4' & \\
\hline
\end{tabular}


Video Log Observations from Validation Study Boreholes

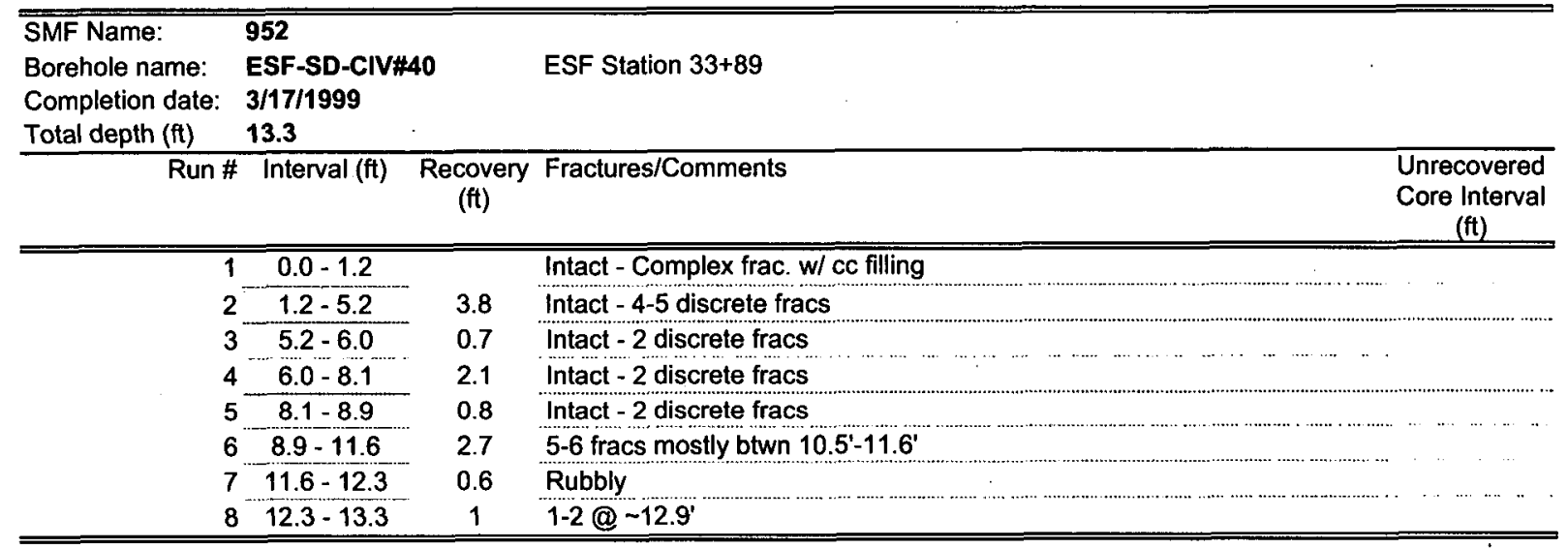

Source: Paces (2003)

NOTE: The information contained in this Appendix is not considered to be data, and it has not been collected under any formal QA procedure. 
INTENTIONALLY LEFT BLANK 


\section{APPENDIX C}

\section{ACCELERATOR MASS SPECTROMETRY METHODS}


INTENTIONALLY LEFT BLANK 


\section{C1. OVERVIEW OF YUCCA MOUNTAIN PROJECT CHLORINE-36 WORK AT LAWRENCE LIVERMORE NATIONAL LABORATORY}

Work at Lawrence Livermore National Laboratory (LLNL) supporting the chlorine-36 $\left({ }^{36} \mathrm{Cl}\right)$ validation study was carried out in two phases, each involving somewhat different techniques, approaches, and personnel. The first phase occurred primarily in 2000, with the work consisting of active leaching of rock core samples, chlorine extraction from the leachate, chlorine concentration determination of the leachate by ion chromatography, and measurement of the ${ }^{36} \mathrm{Cl} / \mathrm{Cl}$ ratio of the chlorine by accelerator mass spectrometry (AMS). The second phase occurred during the latter part of 2001 and continued through 2002 , with the work consisting of chlorine extraction from leachates that were prepared by passive leaching at the U.S. Geological Survey (USGS) in Denver, chlorine concentration determination by measurement of the ${ }^{35} \mathrm{Cl} /{ }^{37} \mathrm{Cl}$ ratio by $\mathrm{AMS}$ in isotopically spiked samples, and measurement of the ${ }^{36} \mathrm{Cl} / \mathrm{Cl}$ ratio of the chlorine by AMS. The AMS measurements were made at the Center for Accelerator Mass Spectrometry (CAMS) at LLNL.

The primary differences between the work in 2000 and that in 2001 and 2002 were that (1) the leachates were derived from active (about 7 hours) leaching at LLNL in 2000, whereas they were derived from passive leaching (about 1 hour) at the USGS in 20012002 , and (2) chlorine concentration determination was by ion chromatography in 2000 , but was by isotope dilution AMS in 2001-2002. The difference in method of chlorine concentration measurement had no effect on the outcome of the project, and precisions are regarded as similar between the ion chromatography and the AMS methods. Both methods produced agreement between aliquots measured at the USGS and with independent samples measured at Los Alamos National Laboratory (LANL). The difference in leaching techniques (active versus passive), however, did affect the outcome of the project. Active leaching produced a far higher concentration of chlorine than passive leaching, resulting in more chlorine being available for AMS analysis. This produced lower and more unstable AMS ion beam currents, which ultimately produced lower statistical analytical precision, as well as lower confidence in the replicability of the analysis. The latter concern, however, is lessened by the generally good replication of the two split aliquots prepared by LANL and LLNL, respectively, measured in the November 2001 AMS run.

In 2001-2002, silver chloride (AgCl) samples were prepared at LANL and sent to LLNL for measurement of ${ }^{36} \mathrm{Cl} / \mathrm{Cl}$ and ${ }^{35} \mathrm{Cl} /{ }^{37} \mathrm{Cl}$ by AMS. The samples were treated for AMS analysis in every respect like the $\mathrm{AgCl}$ samples prepared at LLNL. Therefore, the discussion below concerning the procedures for AMS analysis of LLNL $\mathrm{AgCl}$ samples also applies to the LANL $\mathrm{AgCl}$ samples.

Active and passive leaching procedures are discussed in the body of this report. This appendix discusses the details of the LLNL procedures for chlorine extraction, chlorine concentration measurement, and ${ }^{36} \mathrm{Cl} / \mathrm{Cl}$ and ${ }^{35} \mathrm{Cl} /{ }^{37} \mathrm{Cl}$ determination by AMS. 


\section{C2. PROCEDURES FOR CHLORINE EXTRACTION FROM LEACHATE}

\section{C2.1 Year 2000 Procedures}

Leachate solutions produced in 2000 ranged in size from about 2100 to about $3800 \mathrm{~g}$. Of this liquid, one or two small (about $50 \mathrm{~mL}$ ) aliquots were removed for ion chromatography chlorine concentration analysis. The remainder was then weighed and a pre-weighed amount of chlorine carrier $\left({ }^{36} \mathrm{Cl}\right.$-free chloride salt) was added to the solution. The main purpose of this carrier was to increase the mass of chloride in the solution to facilitate chlorine extraction. The carrier chlorine itself was measured for its ${ }^{36} \mathrm{Cl}$ content during every AMS run to ensure that no additional ${ }^{36} \mathrm{Cl}$ was being added to the sample during carrier addition. Because of the very large amount of liquid involved, extraction of chloride relied on pumping the sample through an ion-exchange column containing AG-4X anion resin, using a peristaltic pump. The column was initially conditioned using three applications of $40 \mathrm{~mL}$ of high-purity nitric acid $\left(\mathrm{HNO}_{3}\right.$ in two $1 \mathrm{~N}$ applications and one $2.5 \mathrm{~N}$ application). After all the leachate had passed through the column, chloride was eluted by applying three elution rinses of $40 \mathrm{~mL} 1 \mathrm{~N}$ high-purity ammonium hydroxide $\left(\mathrm{NH}_{4} \mathrm{OH}\right)$ solution. Chlorine was then extracted from this solution using the chlorine extraction procedure described below, which also was used in 2001-2002.

\section{C2.2 Years 2001-2002 Procedures}

There were three main differences in procedures used between 2000 and 2001-2002. First, in 2001-2002, the leachate solution was created at the USGS and smaller sample sizes than those of 2000 were available (less than 2 L). It was felt that the smaller sample sizes did not require the anion column extraction method, and so this was not used. Second, no leachate aliquot was removed at LLNL for chlorine concentration analysis, although aliquots were removed and analyzed in Denver by the USGS. Third, for 20012002, the chlorine isotope tracer with a known ${ }^{35} \mathrm{Cl}^{37} \mathrm{Cl}$ ratio (TIP-CL-95, Preparation of Samples for Chlorine-36 Analysis) was added to the sample, and this was used in the AMS analysis for isotope dilution chlorine concentration determination. Other than these differences, the chlorine extraction procedures for both project phases were similar, as described in the following paragraphs.

\section{C2.3 Chloride Extraction Procedures}

The leachate solution was placed in an appropriately sized pre-cleaned glass beaker (typically $250 \mathrm{~mL}$ in 2000, and $1 \mathrm{~L}$ in 2001-2002). In 2001-2002, the sample was weighed prior to being placed in the beaker (in 2000, the sample was weighed prior to being passed through the anion column). In 2001-2002, the tracer solution was then added to the leachate (the carrier was added prior to the columns in 2000). The sample was then acidified by addition of concentrated high-purity nitric acid $\left(\mathrm{HNO}_{3}\right)$. Silver was added to the solution in the form of a 5 percent solution of silver nitrate $\left(\mathrm{AgNO}_{3}\right)$. Under acidic conditions, $\mathrm{AgNO}_{3}$ is dissociated while $\mathrm{AgCl}$ becomes insoluble, leading to the precipitation of $\mathrm{AgCl}$. The sample was then left to sit overnight, covered with parafilm and enclosed in a Plexiglass ${ }^{\circledR}$ hood for contamination protection, during which time the 
$\mathrm{AgCl}$ flocculated to the bottom of the beaker. The leachate solution (now chlorine-free) was then carefully removed from the beaker, leaving behind the $\mathrm{AgCl}$ precipitate. The precipitate was dissolved in less than $40 \mathrm{~mL}$ of a $1: 1$ solution of ultra-clean Milli- ${ }^{\circledR}$ deionized water (resistivity greater than 17.5 megaohm-cm) and concentrated high-purity ammonium hydroxide $\left(\mathrm{NH}_{4} \mathrm{OH}\right)$, and this solution was transferred to capped centrifuge tubes for further processing. The sample was then reprecipitated using $\mathrm{HNO}_{3}$ and centrifuged before the supernate was poured off. The precipitate was washed twice with ultra-clean Milli- ${ }^{\circledR}$ water, each time vortexing to break up the precipitate in the centrifuge tube and centrifuging to re-assemble the $\mathrm{AgCl}$ in the bottom of the tube. After each of the washings, the supernate water was poured off and after the first washing replaced with about $6 \mathrm{~mL}$ of clean Milli- $\mathrm{Q}^{\circledR}$ water. After the second washing, the sample was redissolved in a less than $10 \mathrm{~mL}$ solution of $1: 1 \mathrm{NH}_{4} \mathrm{OH}$ (as above), filtered through a pre-cleaned $0.45-\mu \mathrm{m}$ cellulose nitrate membrane syringe filter attached to the tip of a 10-mL capacity medical-grade syringe. $\mathrm{HNO}_{3}$ was added to the sample until $\mathrm{AgCl}$ again precipitated. The precipitate was then washed three times in Milli- $Q^{\circledR}$ water, using the vortexing/centrifuging alternation used for the first water washings. After the final washing, the sample was dried overnight in its centrifuge tube in a small laboratory convection oven at about $70^{\circ} \mathrm{C}$. The dried $\mathrm{AgCl}$ sample was then ready for mounting into an AMS target for ${ }^{36} \mathrm{Cl}$ analysis. Although chlorine extraction procedures were somewhat different at LANL, the final product - the $\mathrm{AgCl}$ sample-was the same, and it was this sample that was sent to LLNL for AMS analysis. Therefore, from this stage onward, the sample handing and analysis was the same for samples originating at LANL and samples processed at LLNL.

Typically, between four and seven samples were prepared simultaneously. With each preparation episode, one to three chemical extraction blanks were prepared. These samples were treated exactly like the actual samples, except that ultra-clean Milli- $Q^{\circledR}$ water was used instead of a leachate solution. The same amount of reagents, $\mathrm{AgNO}_{3}$, and carrier or tracer solutions were added to the chemical extraction blanks as were added to the samples. These blanks were then analyzed by AMS during the sample runs to determine the amount of ${ }^{36} \mathrm{Cl}$ being added to the sample by the reagents, $\mathrm{AgNO}_{3}$, and carrier/tracer. The amount of ${ }^{36} \mathrm{Cl}$ added, as determined by this measurement, was subtracted from the measured values of each actual sample during data reduction. The ${ }^{36} \mathrm{Cl} / \mathrm{Cl}$ ratios reported by LLNL (DTNs: LL030408023121.027 [Q] and LL031200223121.036 [Q]) reflect this subtraction. In all cases, the amount subtracted was extremely minor, because very little ${ }^{36} \mathrm{Cl}$ was ever detected in the blanks.

\section{C3. PROCEDURES FOR DETERMINATION OF LEACHATE CHLORINE CONCENTRATIONS}

\section{C3.1 Year 2000 Procedures}

Chlorine concentrations were measured by ion chromatography at LLNL in 2000 . Details of the analytical procedure are described in TIP-CL-110, Use of Ion Chromatography to Determine Anion Concentrations, and will not be discussed here. However, a few of the most pertinent points will be mentioned. 
The instrument used was a commercially available Dionex AI-450 Ion Chromatograph, using the imbedded Dionex PeakNet software for instrument control and data reduction. The eluent used was a solution of $\mathrm{NaHCO}_{3}+\mathrm{Na}_{2} \mathrm{CO}_{3}$, in about 1:1 molar amounts. The microbore piston option was used, allowing a liquid flow of $0.3 \mathrm{~mL} / \mathrm{min}$ at a pressure of about $1,400 \mathrm{psi}$. This produced an anion column retention time for chlorine of about $3.8 \mathrm{~min}$. Total collection time for the ion chromatography spectrum was $14 \mathrm{~min}$.

Sample concentrations were derived by reference to standard solutions with nominal values of $0.3,0.5,2.0$, and $3.0 \mu \mathrm{g} / \mathrm{g}$ chlorine. The standards were prepared from a commercially available NIST-traceable (confirmation vs. SRM 3182) $1,000 \mu \mathrm{g} / \mathrm{g}$ stock standard solution. Preparation of standard solutions used for the ion chromatography calibration curve was done using a 100 -g capacity, 0.1 percent sensitivity (quantities greater than $0.4 \mathrm{~g}$ ) analytical balance. Because the analyses were done over a short time period, the same calibration curve could be used for all of the analyses. The calibration was by peak area (as opposed to height), with a linear fit curve forced through the origin. The $r^{2}$ value of the fit of the calibration standards to the curve was 0.9796 .

Due to time pressures, all samples were analyzed only once, which was allowed by the controlling technical implementation procedure (TIP-CL-110). Analytical precision can be assessed only by replication of standard solutions run as unknowns, including some standards not used to derive the calibration curve. Replication ranges from about 6 percent at the $0.5 \mu \mathrm{g} / \mathrm{g}$ chlorine level, to about 2 percent at the $3.0 \mu \mathrm{g} / \mathrm{g}$ level. Most samples analyzed by ion chromatography in this project had concentrations greater than $1 \mu \mathrm{g} / \mathrm{g}$, and an analytical error of 5 percent $(2 \sigma)$ has been assigned to all of the analyses. This appears to be sufficiently conservative.

\section{C3.2 Years 2001-2002 Procedures}

The procedure used for chlorine concentration determination in 2001-2002 was isotope dilution mass spectrometry using the LLNL AMS. The method employs the variation between the measured ${ }^{35} \mathrm{Cl} /{ }^{37} \mathrm{Cl}$ ratio in the sample and the measured ${ }^{35} \mathrm{Cl} /{ }^{37} \mathrm{Cl}$ ratio in the tracer that has been added to the sample (as discussed above). The measured deviation from the tracer ${ }^{35} \mathrm{Cl} /{ }^{37} \mathrm{Cl}$ value $(\sim 0.9$ in this project) is due to the addition of the natural chlorine in the leachate (with the terrestrial natural ${ }^{35} \mathrm{Cl} /{ }^{37} \mathrm{Cl}$ ratio of 3.127 ). The magnitude of the deviation is directly related to the concentration of chloride in the leachate sample. Therefore, the leachate sample concentration can be calculated from the magnitude of the deviation. This method is the same as the standard isotope dilution method commonly used in mass spectrometry, and the calculations used to derive the sample chlorine concentration from the measured values also are commonly recognizable.

For this project, the tracer ${ }^{35} \mathrm{Cl} /{ }^{37} \mathrm{Cl}$ ratio was measured in one or more tracer-only ("blank") samples during the course of each run. To account for instrumental mass bias, the value was normalized to the ${ }^{35} \mathrm{Cl} /{ }^{37} \mathrm{Cl}$ ratio measured in the AMS standards (LLNL111) used during the run. It was this in-run value for the tracer that was used during data reduction. 
The conditions of the AMS analysis pertinent to the isotope dilution ${ }^{35} \mathrm{Cl} /{ }^{37} \mathrm{Cl}$ measurement are discussed in the following section.

\section{C4. PROCEDURES FOR DETERMINATION OF CHLORINE-36/CHLORINE AND CHLORINE-35/CHLORINE-37 BY ACCELERATOR MASS SPECTROMETRY}

Samples were analyzed for chlorine isotopes using the FN tandem accelerator mass spectrometer housed within the CAMS at LLNL. Samples are ionized to negative chlorine ions by bombardment with a cesium ion beam in the instrument source. The negative ions are then extracted, using a positive electrical potential, into the beamline of the instrument where the ion particles are accelerated to $8.3 \mathrm{MV}$ within the tandem accelerator. In the center of the tandem, the ion beam is passed through a thin carbon foil, which breaks up molecular species that can be mass/energy interferences and also strips electrons from the chlorine anions, creating positively charged chlorine cations. The cations are then further accelerated by a negative electrical potential to the end of the tandem unit, where they pass into a long instrument beamline with extensive beam focusing, steering, and deflecting capabilities, including two $90^{\circ}$ curvature massseparation magnets that separate the three chlorine isotopes into separate streams. The stream (beam) that finally makes it to the detector is composed almost entirely of ${ }^{36} \mathrm{Cl}$ ions, although an unwanted amount of interfering ${ }^{36} \mathrm{~S}$ can still be present. The ions are detected through 5 -fold coincident detection of electrons given off during collisional interaction between the ions and gas within the detector (i.e., five coincident detections equals one ${ }^{36} \mathrm{Cl}$ count). This provides the mechanism for discriminating between ${ }^{36} \mathrm{Cl}$ ions and ${ }^{36} \mathrm{~S}$ ions within the detector, because the very small difference in mass between the two species produces different energy loss during gas interaction and therefore different coincident detection patterns. Two Faraday cups located between the two $90^{\circ}$ magnets measure the currents of ${ }^{35} \mathrm{Cl}$ and ${ }^{37} \mathrm{Cl}$. The ratio of the currents normalized to those of the standards is the ${ }^{35} \mathrm{Cl} /{ }^{37} \mathrm{Cl}$ ratio.

Procedures specific to the ${ }^{36} \mathrm{Cl}$ analyses for the two phases of this project are given in the following sections.

\section{C4.1 Year 2000 Procedures}

The AMS procedures used in 2000 are identical to those used in 2001-2002, except that the ${ }^{35} \mathrm{Cl} /{ }^{37} \mathrm{Cl}$ ratio was not measured because the isotope dilution technique for chlorine concentration determination was not used. A substantial difference between the two phases of the project exists, however, due to the larger $\mathrm{AgCl}$ sample sizes obtained in 2000. The larger $\mathrm{AgCl}$ sizes were due both to the higher chlorine concentrations of the leachates and to the larger leachate volumes available for analysis. Larger amounts of $\mathrm{AgCl}$ result in larger and more stable AMS beam currents, resulting in greater analytical precision and probably greater replicability of results. This is discussed more fully below with respect to the procedures for 2001-2002. 


\section{C4.2 Years 2001-2002 Procedures}

In order to place $\mathrm{AgCl}$ samples within the $\mathrm{AMS}$ instrument for analysis, the $\mathrm{AgCl}$ must be placed in stainless steel holders, commonly called "targets." The targets are small, hollow cylinders about 1 inch long, with an inner diameter opening of about 0.25 inches. They are closed at one end, giving them the appearance of a small bullet casing (shell). The center of the target is filled with silver bromide $(\mathrm{AgBr})$, which eliminates ${ }^{36} \mathrm{Cl}$ and ${ }^{36} \mathrm{~S}$ contamination derived from the stainless steel and acts as a thermal and electrical buffer to the $\mathrm{AgCl}$ during analysis. A small hole is drilled into the center of the $\mathrm{AgBr}$ into which the $\mathrm{AgCl}$ of the sample is packed.

It has been standard practice through the years to use at least about $3 \mathrm{mg} \mathrm{AgCl}$ for an analysis. This amount was available in 2000, but not in 2001-2002. During this second phase, samples as small as $0.3 \mathrm{mg} \mathrm{AgCl}$ were used, for which a smaller diameter hole was drilled in the target $\mathrm{AgBr}$. The purpose of drilling smaller diameter holes is to slow ionization and prolong the analysis as much as possible, so that several determinations of a single sample can be made during the course of the analytical day. This produces better between-determination statistics and more confidence in the individual determinations. However, this method has at least three important negative effects. First, the smaller diameter of the sample hole produces a smaller ion "cloud" in the source, resulting in smaller beam currents for the same extraction potential. A typical ${ }^{37} \mathrm{Cl}$ beam current (measured in the Faraday cup) for a $3 \mathrm{mg} \mathrm{AgCl}$ sample is about $20 \mu \mathrm{A}$, whereas beam currents for 2001-2002 samples (small holes) were often in the 1 to $5 \mu \mathrm{A}$ range. Because extraction potential cannot be substantially increased without causing electrical instability within the source, beam currents cannot be "artificially" increased for small samples. For the same duration of analysis, smaller beam currents produce fewer ${ }^{36} \mathrm{Cl}$ detector counts than normal beam currents, and this of course produces poorer within-determination statistical precision (most simplistically, $1 / V_{n}$ ). Second, the smaller samples were expended during the course of analysis, so that more ${ }^{36} \mathrm{Cl}$ counts (better statistics) could not be achieved simply by running the sample for a longer period. This also produced fewer individual determinations during the course of the run (the larger samples analyzed in 2000 typically had three to five determinations during the course of the run, while 2001-2002 samples often had only one or two determinations). Third, the cesium beam that causes the initial ionization "rasters" over the small region of the $\mathrm{AgCl}$ in the target and this cannot be made smaller to accommodate the smaller diameter holes. Inevitably, the cesium beam "rasters" outside of the $\mathrm{AgCl}$ area into the $\mathrm{AgBr}$ area. When this happens, chlorine ionization decreases. The fluctuation in ionization causes a fluctuation in beam current. The efficiency of the various mass and energy filters in the AMS beamline depends on the strength of the beam current, such that beam instability translates into greater within-determination variability in the various isotope beam currents. That is, the measured variability in the ${ }^{36} \mathrm{Cl} / \mathrm{Cl}$ or the ${ }^{35} \mathrm{Cl} /{ }^{37} \mathrm{Cl}$ ratios increases with increased variability in beam current. Due to the intrinsic differences in beam sizes for the chlorine isotopes, this effect is more pronounced for the ${ }^{36} \mathrm{Cl} / \mathrm{Cl}$ ratio than for the ${ }^{35} \mathrm{Cl}^{37} \mathrm{Cl}$ ratio. Therefore, the precision of the ${ }^{35} \mathrm{Cl} /{ }^{37} \mathrm{Cl}$ ratio is not substantially worsened. 
These three effects can be summarized by saying that smaller sample sizes lead to increased analytical uncertainty for ${ }^{36} \mathrm{Cl} / \mathrm{Cl}$. This problem is compounded by the fact that the LLNL AMS facility has had little experience with replication of small (less than $1 \mathrm{mg}$ $\mathrm{AgCl})$ samples or standards over time. Replication of samples or standards over the course of months or years produces an understanding of the instrumental variability that can occur, which would take the form of a time integrated assessment of true analytical precision. The facility experience gathered over more than a decade of analyzing ${ }^{36} \mathrm{Cl}$ from samples greater in size than $3 \mathrm{mg} \mathrm{AgCl}$ indicates that the true analytical uncertainty is better than \pm 5 percent for ${ }^{36} \mathrm{Cl} / \mathrm{Cl}$ ratios. This is believed to apply to the samples for the 2000 phase of this project, but for samples for 2001-2002, the true analytical uncertainty may not bethat low. However, the reasonably good sample replication achieved during the November 2001 AMS run between the samples chemically processed at LANL and their aliquot splits chemically processed at LLNL demonstrated that the uncertainty is not exceedingly large. In all cases, the analytical precisions reported (DTNs: LL030408023121.027 [Q] and LL031200223121.036 [Q]) are the within-run analytical precisions as derived through the statistical treatment of the data reduction code used for all years of this project (FUDGER3.1), and reflect what are commonly referred to as "counting statistics."

The AMS ${ }^{36} \mathrm{Cl} / \mathrm{Cl}$ standard used for the project ("LLNL111") is a NIST-traceable ${ }^{36} \mathrm{Cl}$ standard, which has been gravimetrically adjusted with ${ }^{36} \mathrm{Cl}$-free chloride salt to produce a ${ }^{36} \mathrm{Cl} / \mathrm{Cl}$ ratio of $111 \times 10^{-15}$. This was done using a $100 \mathrm{~g}$ capacity, 0.1 percent sensitivity (quantities greater than $0.4 \mathrm{~g}$ ) analytical balance. The final ${ }^{36} \mathrm{Cl} / \mathrm{Cl}$ ratio was confirmed against the original standard material as well as several in-house standards that have been in use for many years.

Data reduction for both phases of this project was accomplished in two basic steps. The first used the computer program FUDGER3.1, which was developed at LLNL. The program reads the original data file from the AMS instrument and allows the analyst to assess the quality of each individual determination through examination of variables, such as sulfur count rates, total ${ }^{36} \mathrm{Cl}$ counts registered, and individual determination deviations relative to other determinations for that sample on that day. Individual determinations, including LLNL111 determinations, can be deleted from the data set on this basis. The program then normalizes each sample determination relative to determinations for the LLNL111 standard, using a weighted average (based on the precision of the standard determination) of the four LLNL111 determinations made closest in time to that of the sample. The values for each determination are then averaged, weighting them relative to the precision of the determination. These values are then output as a tab-delimited text file. The second step in data reduction involves derivation of final ${ }^{36} \mathrm{Cl} / \mathrm{Cl}$ ratios, ${ }^{35} \mathrm{Cl} /{ }^{37} \mathrm{Cl}$ ratios, and chlorine concentrations by Excel spreadsheet calculations incorporating the required external data (e.g., leachate sample size, amount of added carrier/tracer, and blank subtraction). All steps in the calculations are included in the spreadsheets submitted to the Yucca Mountain Project Technical Data Management System (TDMS) database (DTNs: LL030408023121.027 [Q] and LL031200223121.036 [Q]). 


\section{C.5 REFERENCES CITED}

\section{C.5.1 CODES, STANDARDS, REGULATIONS AND PROCEDURES}

TIP-CL-95, Rev. 0. Preparation of Samples for Chlorine-36 Analysis. Livermore, California: Lawrence Livermore National Laboratory. ACC: MOL.20000412.0094.

TIP-CL-110, Rev. 0. Use of Ion Chromatography to Determine Anion Concentrations. Livermore, California: Lawrence Livermore National Laboratory. ACC: MOL.20000301.0188.

\section{C.5.2 SOURCE DATA, LISTED BY DATA TRACKING NUMBER}

LL030408023121.027. Cl Abundance and $\mathrm{Cl}$ Ratios of Leachates from ESF Core Samples. Submittal date: 04/17/2003. (Q)

LL031200223121.036. Cl Abundance and $\mathrm{Cl}$ Ratio of Leachates from ESF Core Samples. Submittal date: 12/03/2003. (Q) 

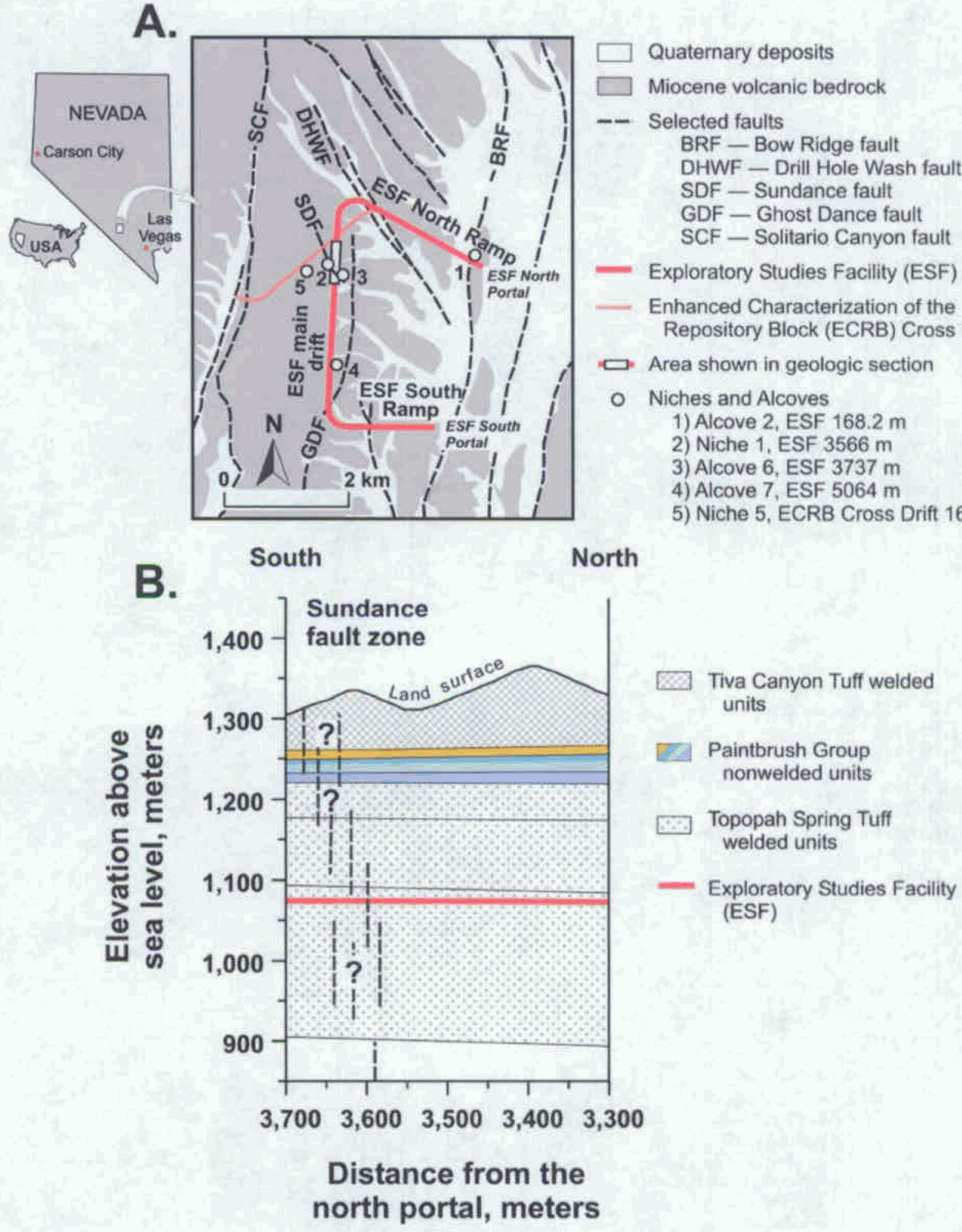

Sources: Modified from DOE (2002, Figure 1-14) and USBR (1996)

Figure 1-1. Generalized Map of Central Yucca Mountain (A) and Schematic Geologic Section along the ESF Showing the Sundance Fault Zone Validation Study Area (B) 

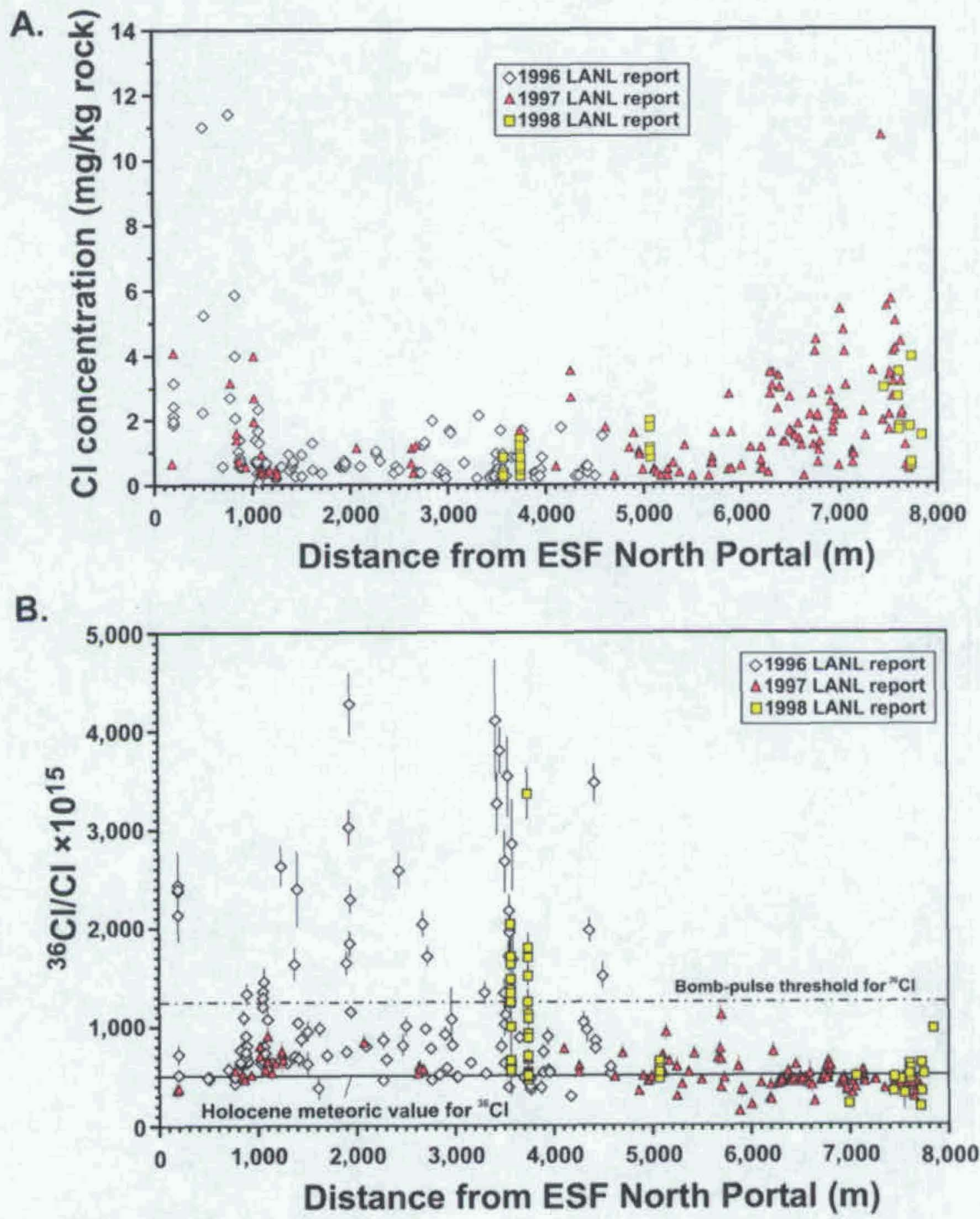

DTNs: LAJF831222AQ98.004 (Q), LA0509JF831222.001 (Q)

NOTES: $\quad E S F=$ Exploratory Studies Facility. Error bars are 20 . The 1996 report is Fabryka-Martin, Wolfsberg et al. (1996). The 1997 report is Fabryka-Martin et al. (1997). The 1998 report is CRWMS M\&O (1998).

Figure 2-1. Distribution of Chloride Concentrations (A) and ${ }^{36} \mathrm{Cl} / \mathrm{Cl}$ Ratios $(B)$ in the ESF, as Reported by LANL in 1996, 1997, and 1998 


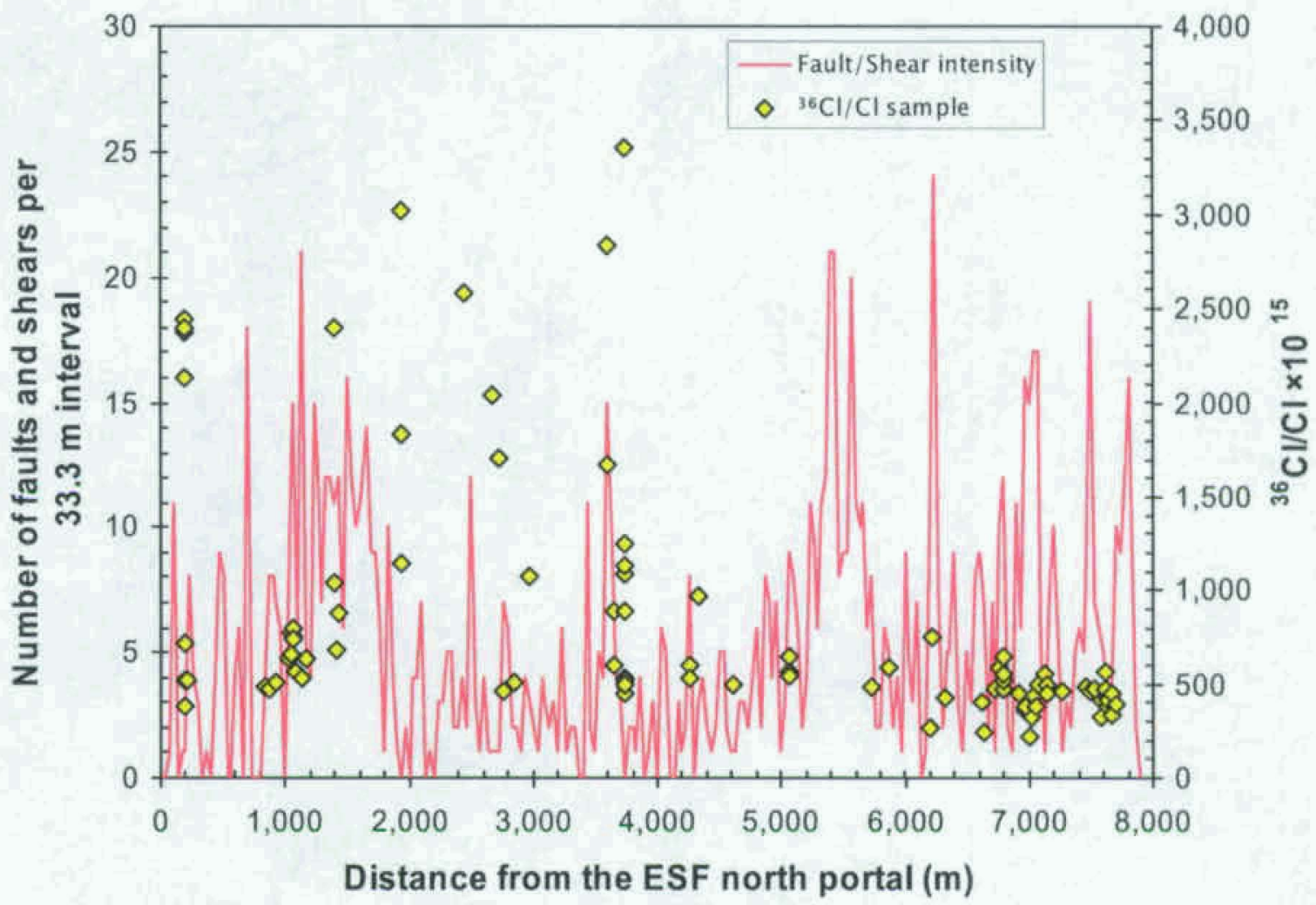

DTNs: GS960708314224.008 (Q), GS960708314224.010 (Q), GS000608314224.004 (Q), GS960908314224.014 (Q). GS970208314224.003 (Q), GS970808314224.008 (Q), GS970808314224.010 (Q), GS970808314224.012 (Q). GS971108314224.020 (Q), GS971108314224.021 (Q), GS971108314224.022 (Q), GS971108314224.023 (Q), GS971108314224.024 (Q), GS971108314224.025 (Q), GS971108314224.026 (Q), GS971108314224.028 (Q)

See also Appendix $A$, which contains information regarding sample numbers, locations, and types.

NOTE: $\quad E S F=$ Exploratory Studies Facility.

Figure 2-2. Relations between Fault/Shear Intensity as Mapped in the ESF and ${ }^{36} \mathrm{Cl} / \mathrm{CI}$ Ratios for Samples Described as Localities Associated with Faults or Shears 

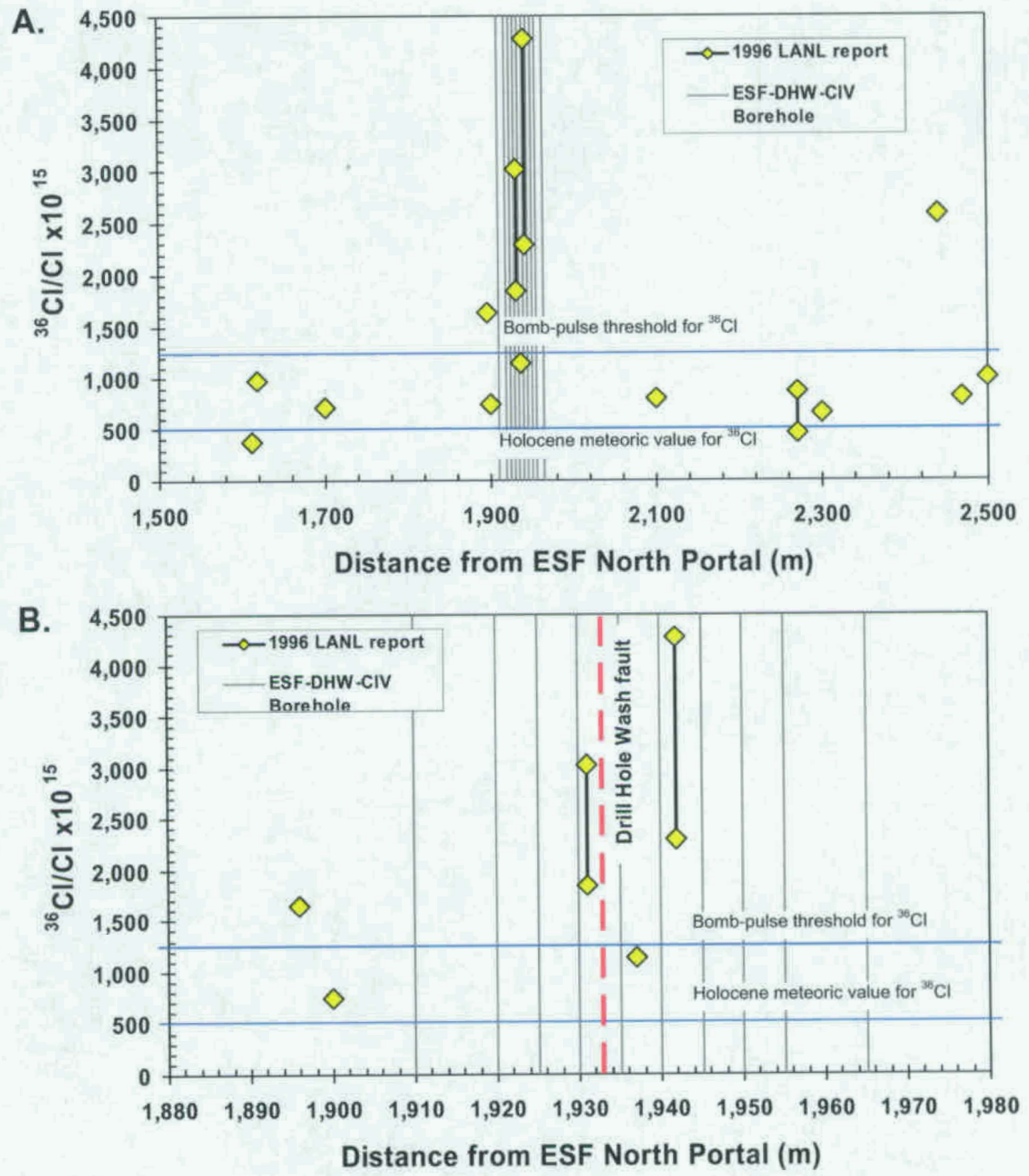

DTN: LAJF831222AQ98.004 (Q)

NOTES: ESF $=$ Exploratory Studies Facility, LANL $=$ Los Alamos National Laboratory. The 1996 LANL report is Fabryka-Martin, Wolfsberg, et al, (1996).

Figure 3-1. Distribution of ${ }^{36} \mathrm{Cl}$ along the Drill Hole Wash Fault Zone in the ESF, between 1,500 and 2,500 meters $(A)$ and between 1,880 and 1,980 meters (B), as Reported by LANL in 1996 


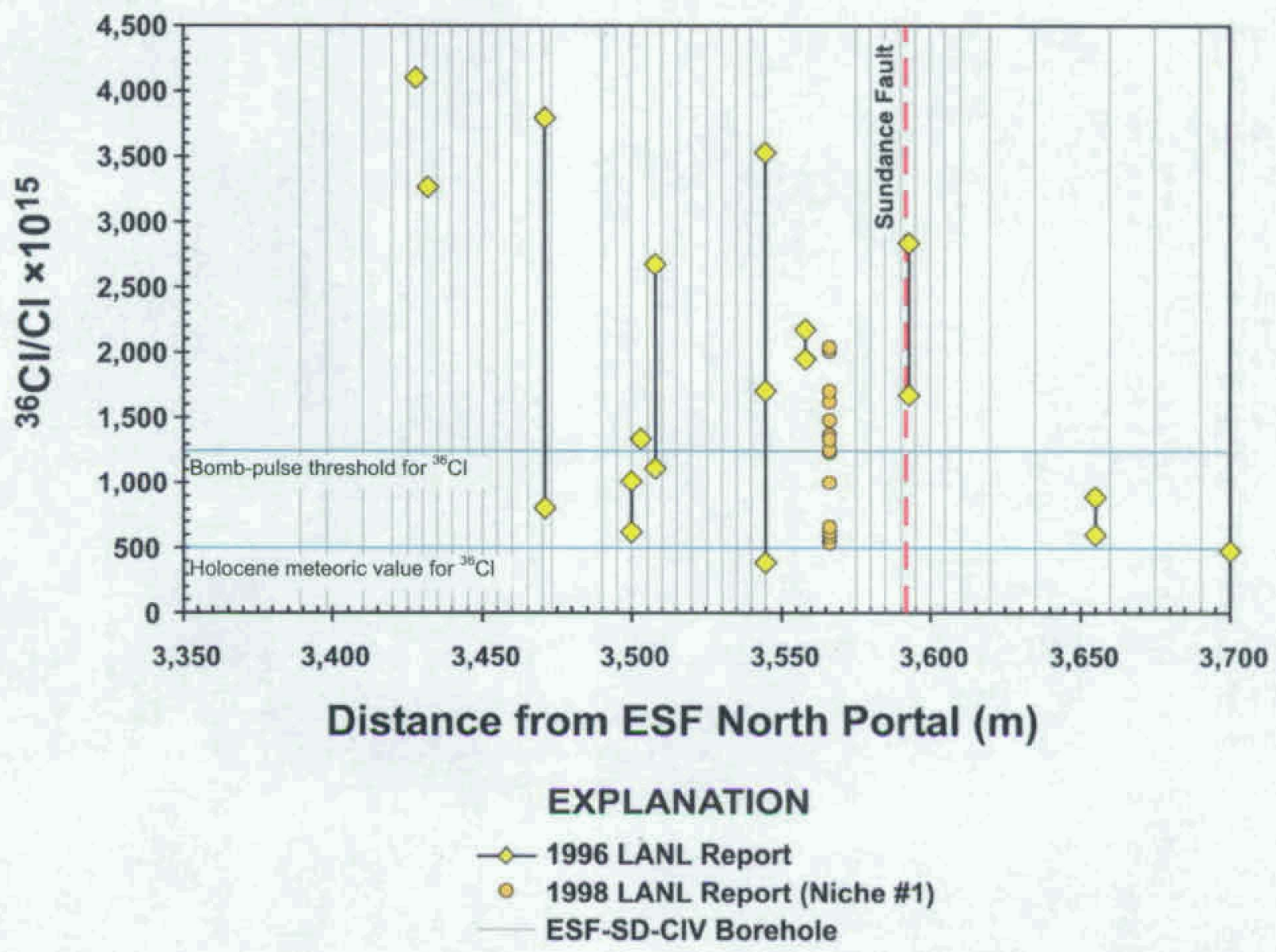

DTNs: LAJF831222AQ98.004 (Q), LA0509JF831222.001(Q)

NOTES: ESF $=$ Exploratory Studies Facility, LANL $=$ Los Alamos National Laboratory. The 1996 LANL report is Fabryka-Martin, Wolfsberg et al. (1996), and the 1998 LANL report is CRWMS M\&O (1998).

Figure 3-2. Distribution of ${ }^{36} \mathrm{Cl}$ in and adjacent to the Sundance Fault in the ESF, as Reported by LANL in 1996 and 1998 


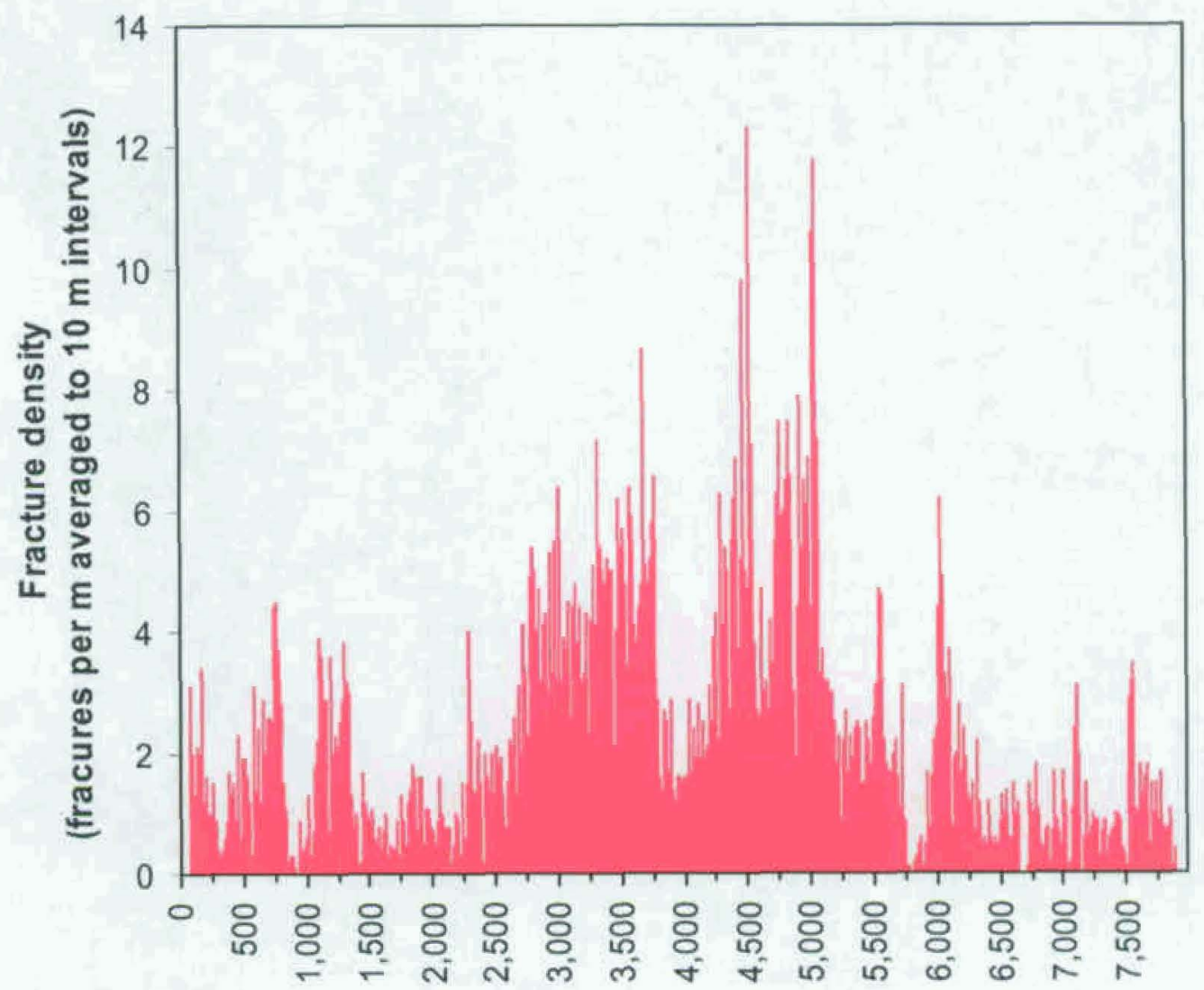

\section{Distance from ESF north portal (m)}

DTNs: GS960708314224.008 (Q), GS960708314224.010 (Q), GS000608314224.004 (Q), GS960908314224.014 (Q), GS970208314224.003 (Q), GS970808314224.008 (Q), GS970808314224.010 (Q). GS970808314224.012 (Q), GS971108314224.020 (Q), GS971108314224.021 (Q), GS971108314224.022(Q). GS971108314224.023 (Q), GS971108314224.024 (Q), GS971108314224.025 (Q), GS971108314224.026 (Q), GS971108314224.028 (Q)

NOTE: $\quad$ ESF $=$ Exploratory Studies Facility.

Figure 3-3. Distribution of Fracture Densities in the ESF 


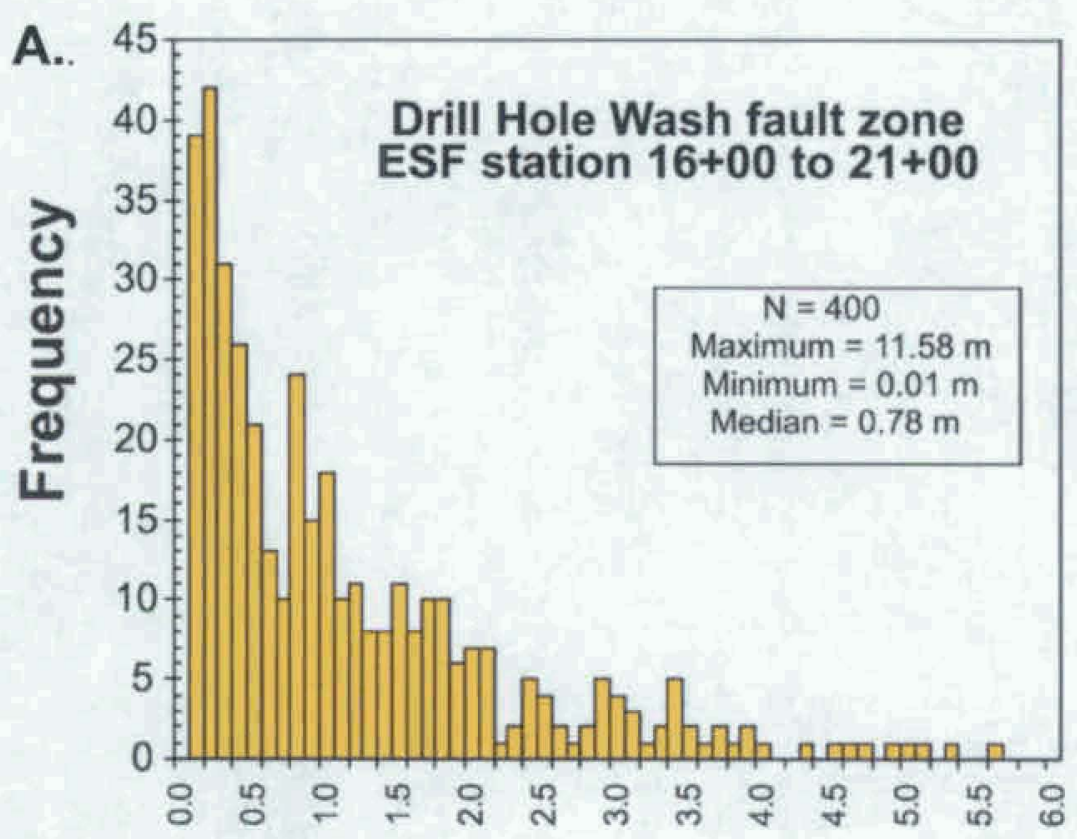

Spacing between fractures $(\mathrm{m})$

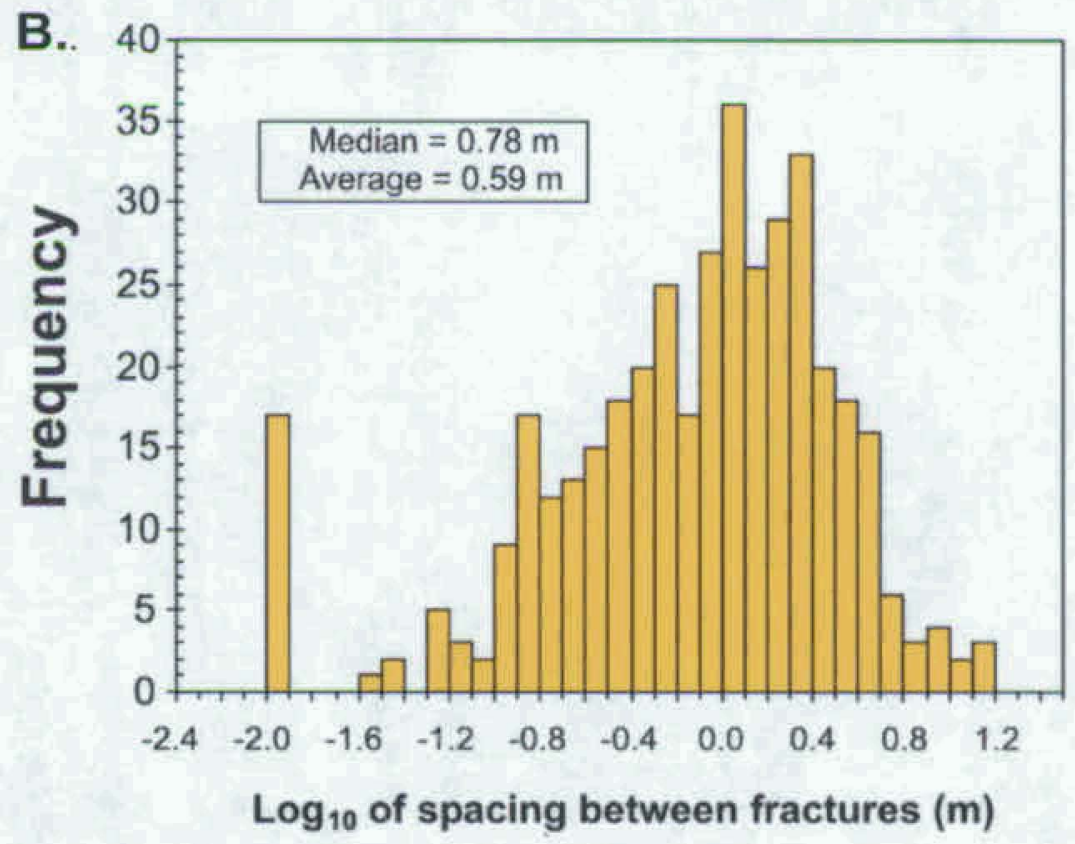

NOTE: ESF $=$ Exploratory Studies Facility.

DTNs: GS971108314224.023 (Q), GS971108314224.024 (Q)

Figure 3-4. Histograms Showing Linear Spacing (A) and Log Spacing (B) between Fractures and Cooling Joints Longer than 1 Meter, Measured from Detailed Line Surveys between ESF Stations $16+00$ and $21+00$ 

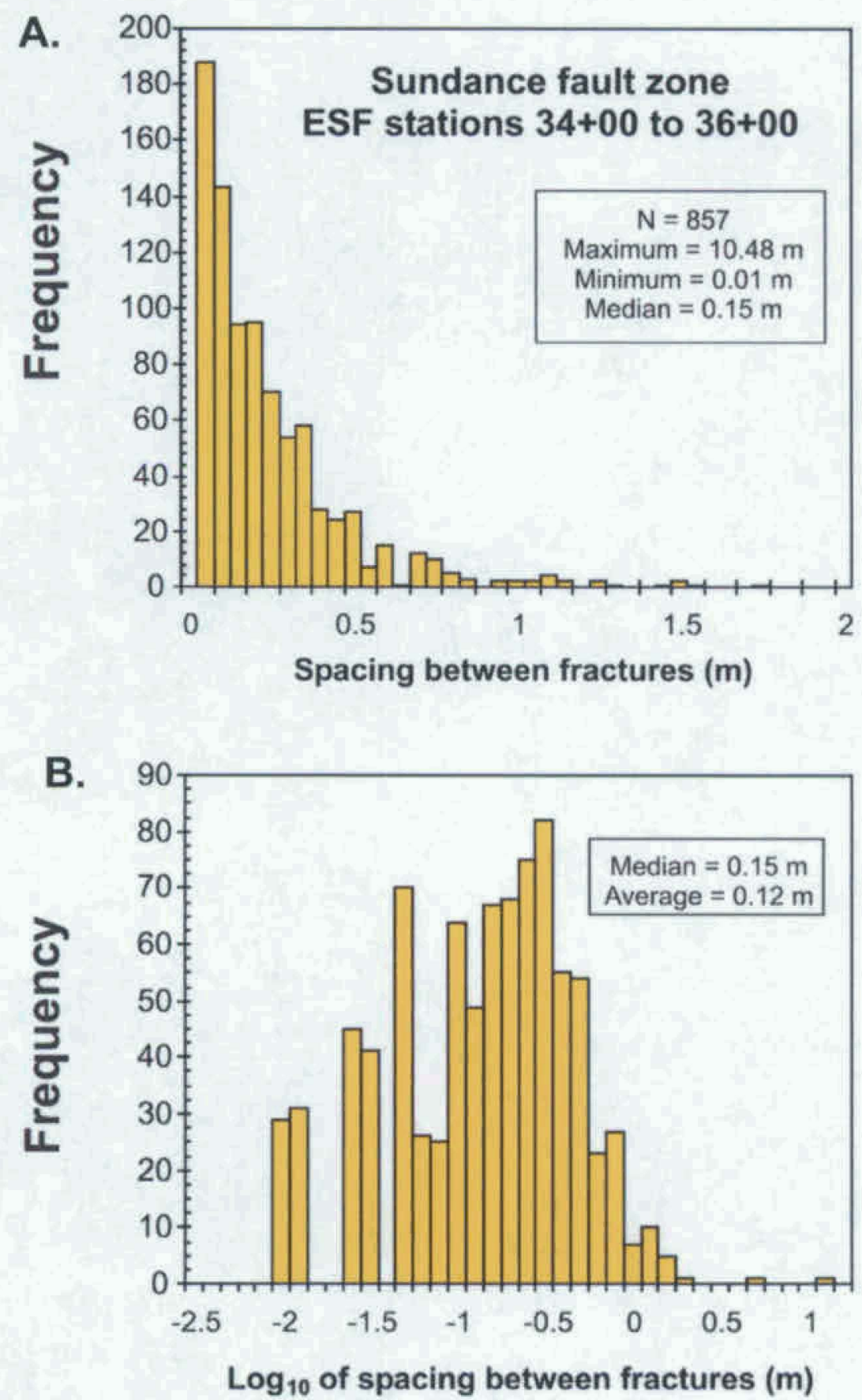

NOTE: $\quad$ ESF $=$ Exploratory Studies Facility.

DTNs: GS000608314224.004 (Q), GS960708314224.008 (Q)

Figure 3-5. Histograms Showing the Linear Spacing (A) and Log Spacing (B) between Fractures and Cooling Joints Longer than 1 Meter, Measured from Detailed Line Surveys between ESF Stations $34+00$ and $36+00$ 


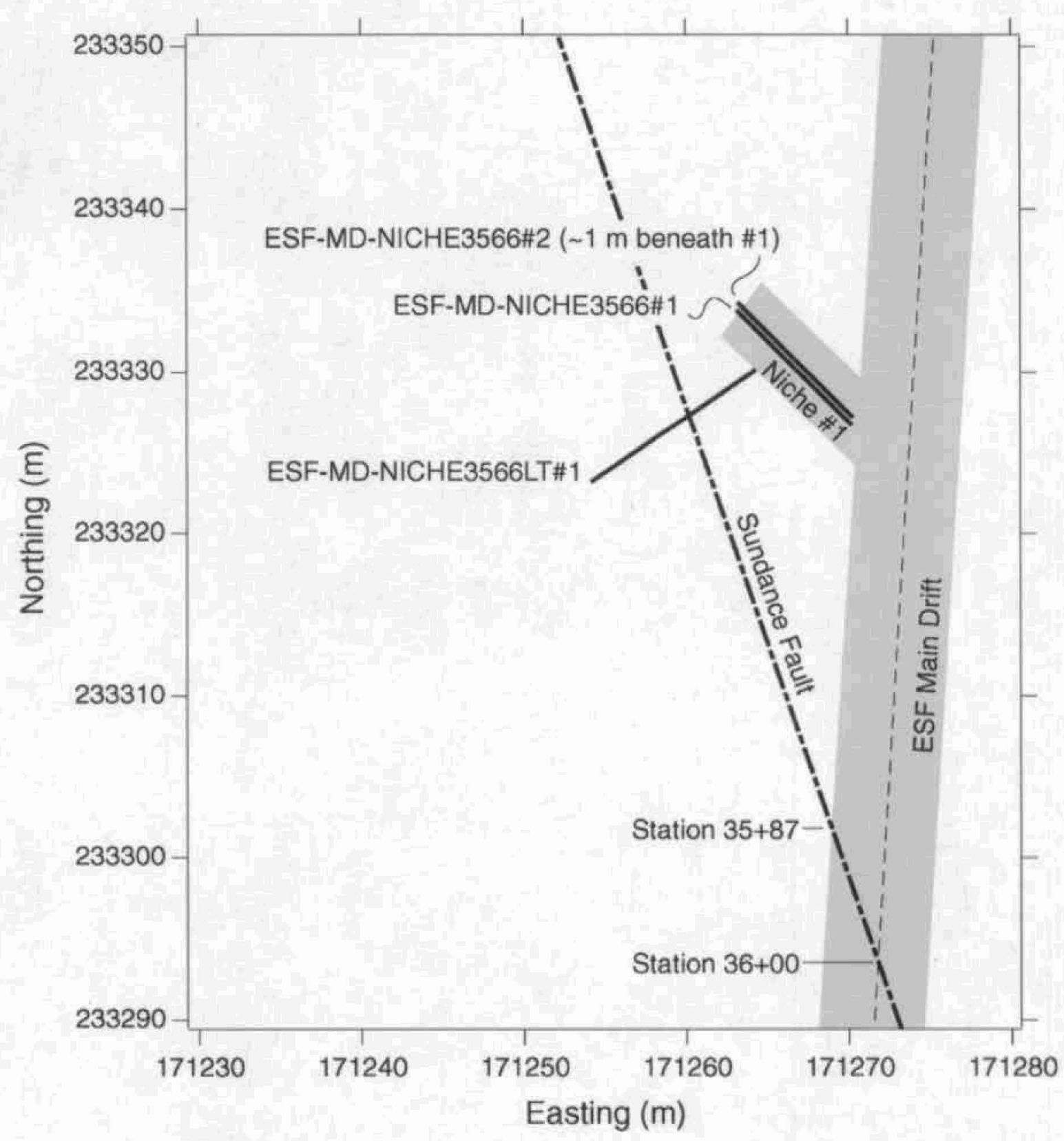

Source: USGS (1996)

NOTES: ESF = Exploratory Studies Facility. Station values represent hundreds of meters from the north portal of the ESF. Projection of the Sundance fault is estimated from tunnel-wall intersections shown on fullperiphery map (USGS 1996). Coordinates are Nevada State Plane (NAD27) in meters.

Figure 3-6. Schematic Map Showing General Relations of Niche \#1 to the ESF Main Drift and Sundance Fault, and the Orientations of Niche Boreholes Used for the Validation Study 


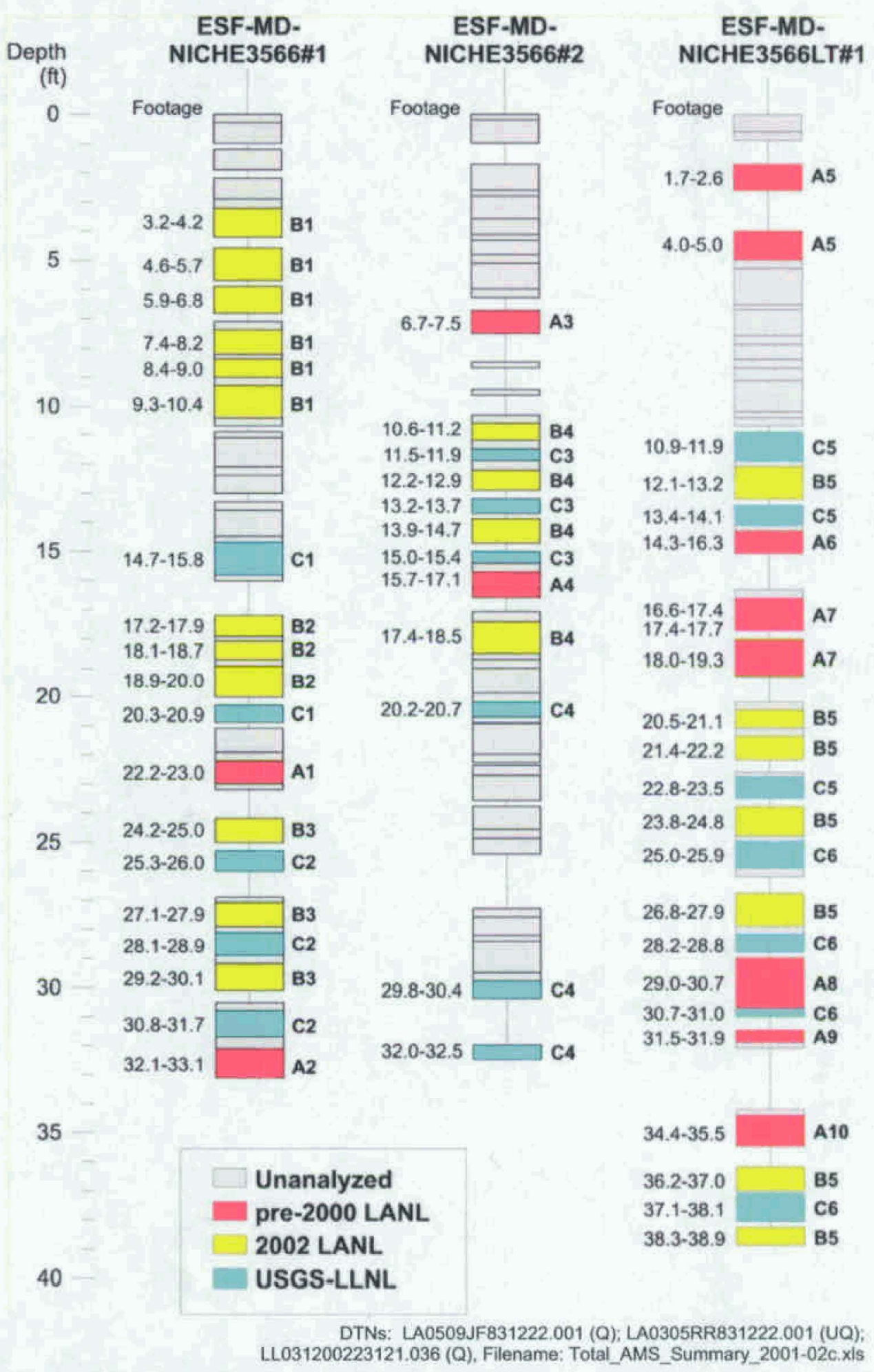

NOTES: LANL = Los Alamos National Laboratory, LLNL = Lawrence Livermore National Laboratory,

USGS $=$ U.S. Geological Survey. Bold alphanumeric symbols to the right of individual core intervals are reference codes identifying multiple core intervals composited into single samples, keyed to sample details given in Table 3-3.

Figure 3-7. Distribution of Niche \#1 Core Intervals Used for the Validation Study 


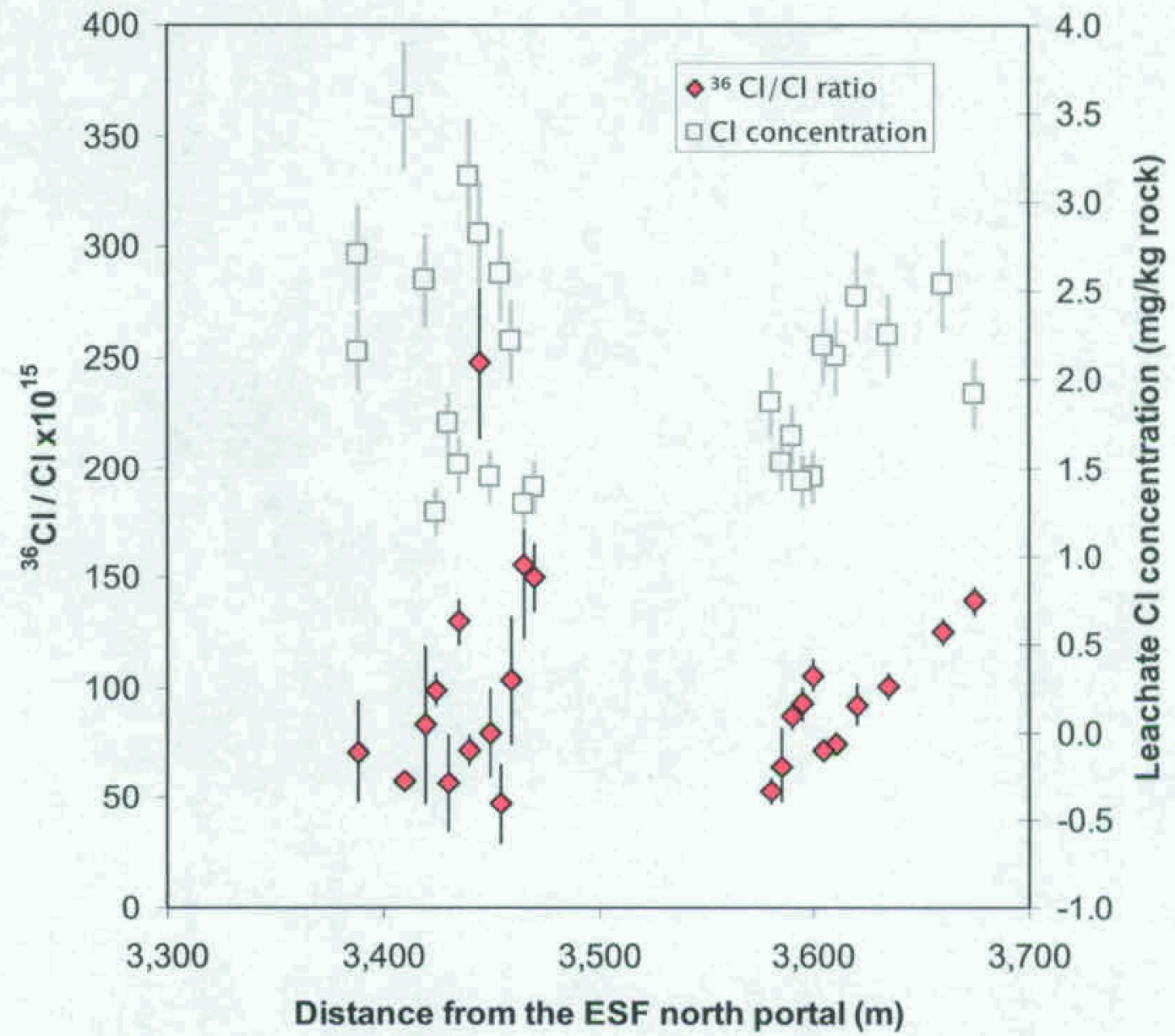

DTN: LL030408023121.027 (Q), Filename: Total_AMS_Summary_2000.xls

NOTES: ESF $=$ Exploratory Studies Facility. Error bars represent $2 \sigma$ analytical uncertainties, Borehole locations are listed in Table 3-2.

Figure 4-1. Chloride Concentrations and ${ }^{36} \mathrm{Cl} / \mathrm{CI}$ Ratios in Active Leachates of Validation Study Samples Processed and Analyzed at LLNL during Phase I 


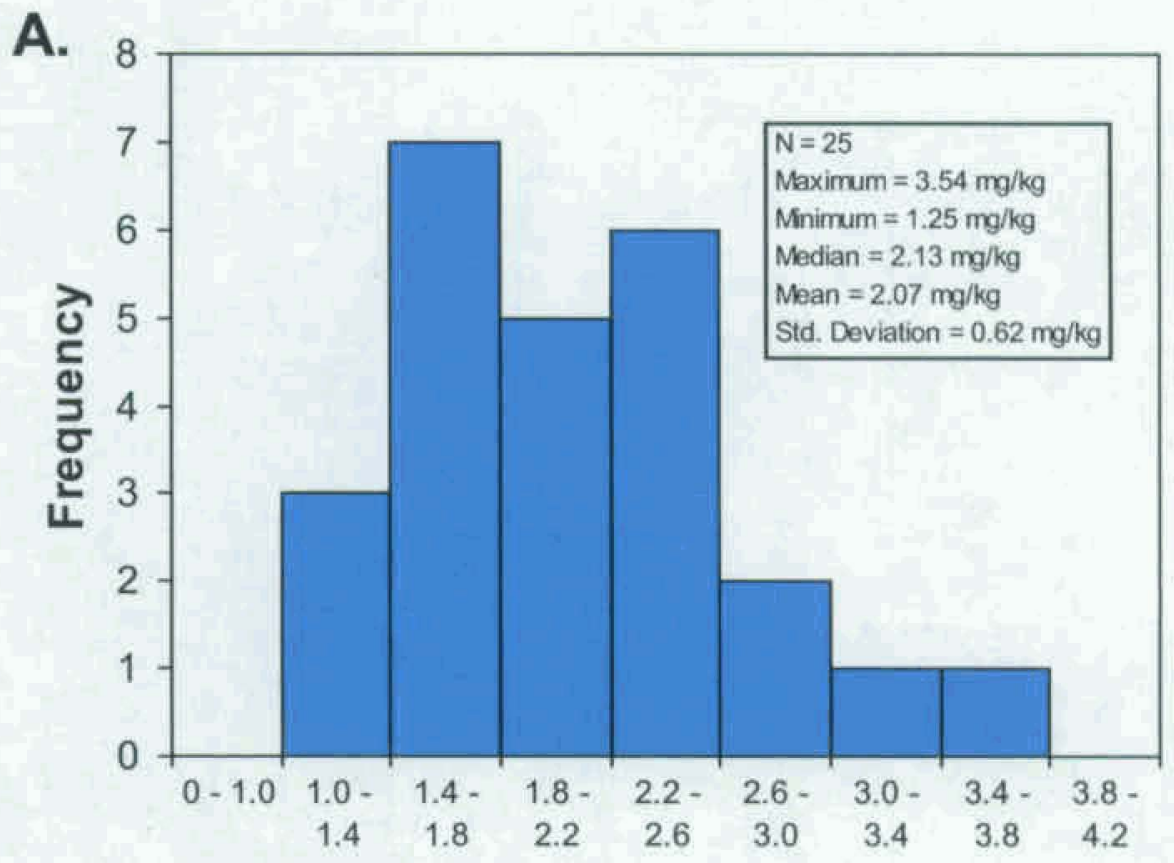

Leachate $\mathrm{Cl}$ concentration $(\mathrm{mg} / \mathrm{kg}$ rock)

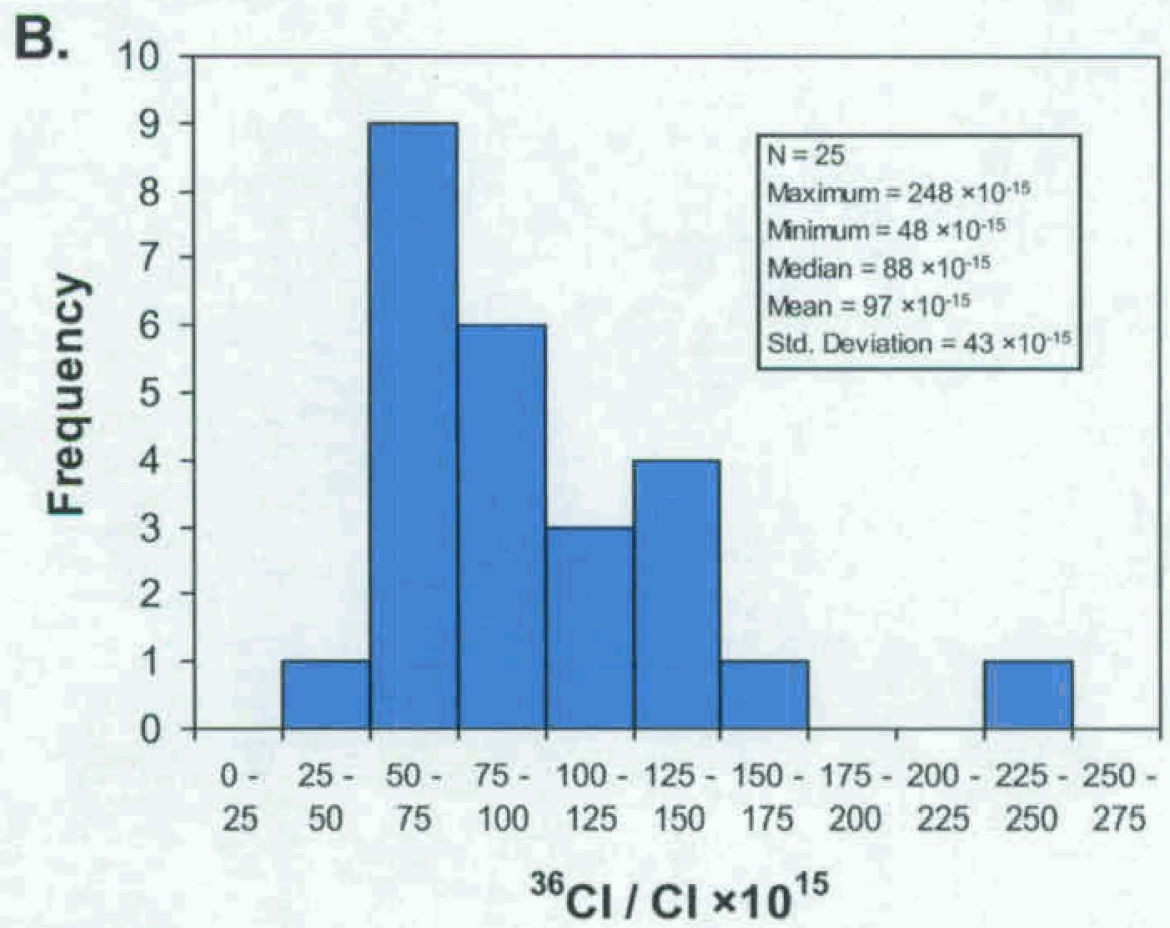

DTN: LL030408023121.027(Q)

Figure 4-2. Distribution of Chloride Concentrations $(\mathrm{A})$ and ${ }^{36} \mathrm{Cl} / \mathrm{Cl}$ Ratios $(\mathrm{B})$ in Active Leachates of Validation Study Samples Processed and Analyzed at LLNL during Phase I 

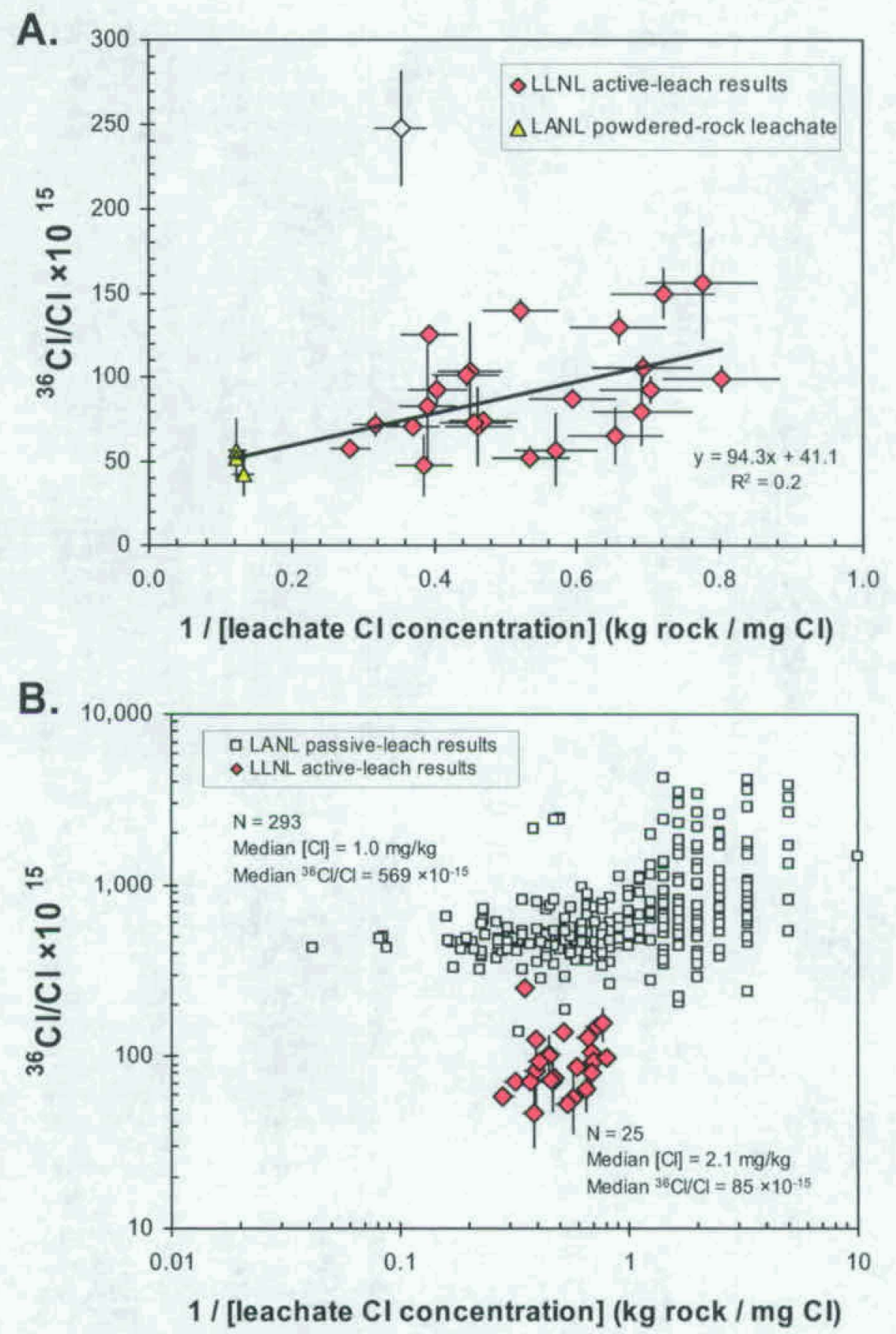

DTNs: LL030408023121.027 (Q), LAJF831222AQ98.004 (Q)

NOTES: LANL = Los Alamos National Laboratory, LLNL = Lawrence Livermore National Laboratory. The open diamond in (A) is a statistical outlier that was not included in the regression. LANL data in (A) are from Table 5-4 of Fabryka-Martin, Wolfsberg et al. (1996). LANL data in (B) are listed in Appendix A. LLNL data in (B) are listed in Table 4-1. Error bars are 20.

Figure 4-3. Relations between Reciprocal Chloride Concentrations and ${ }^{36} \mathrm{Cl} / \mathrm{Cl}$ Ratios in Active Leachates of Validation Study Samples Processed and Analyzed at LLNL during Phase I (A), and for Passive Leachates of ESF Samples Reported Previously by LANL (B) 


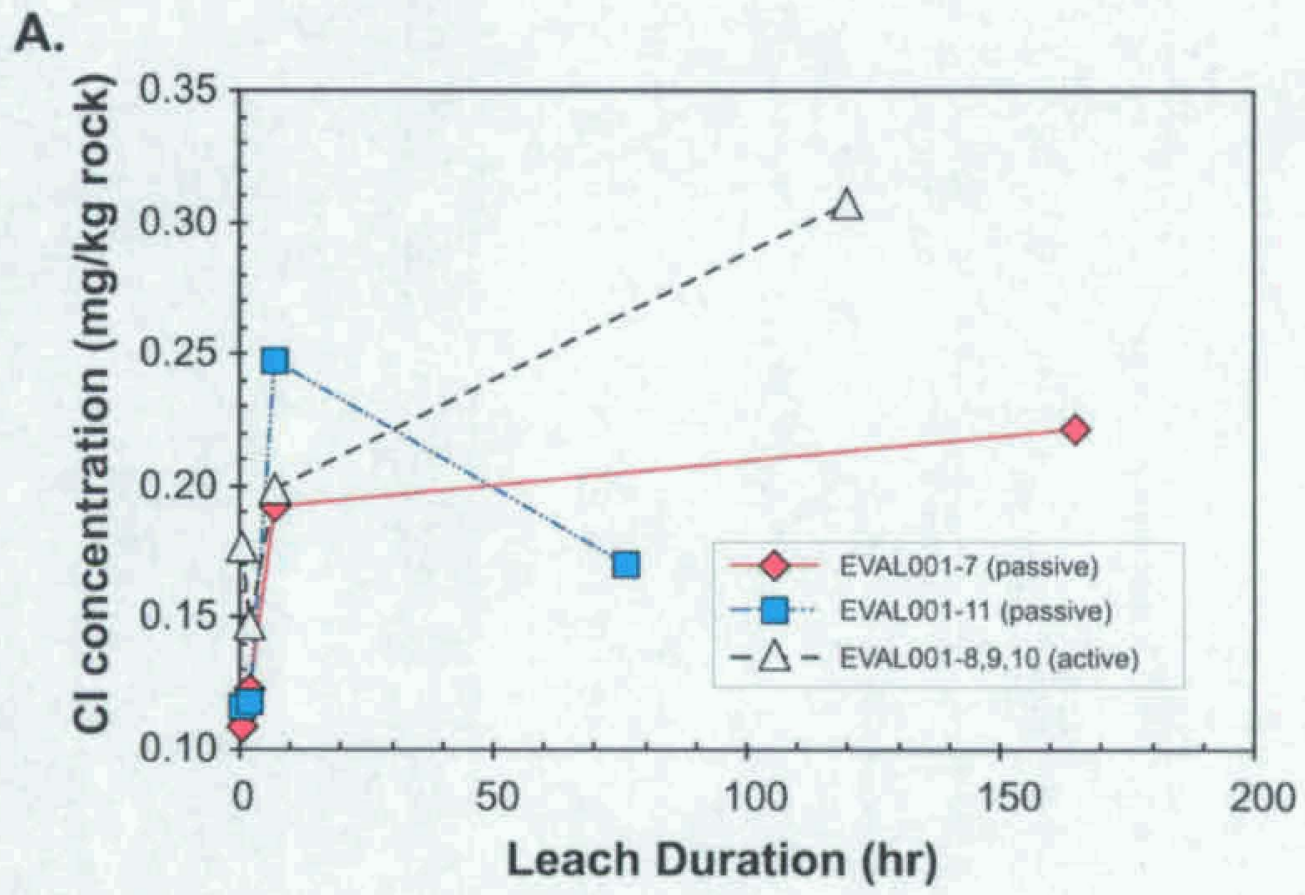

B.

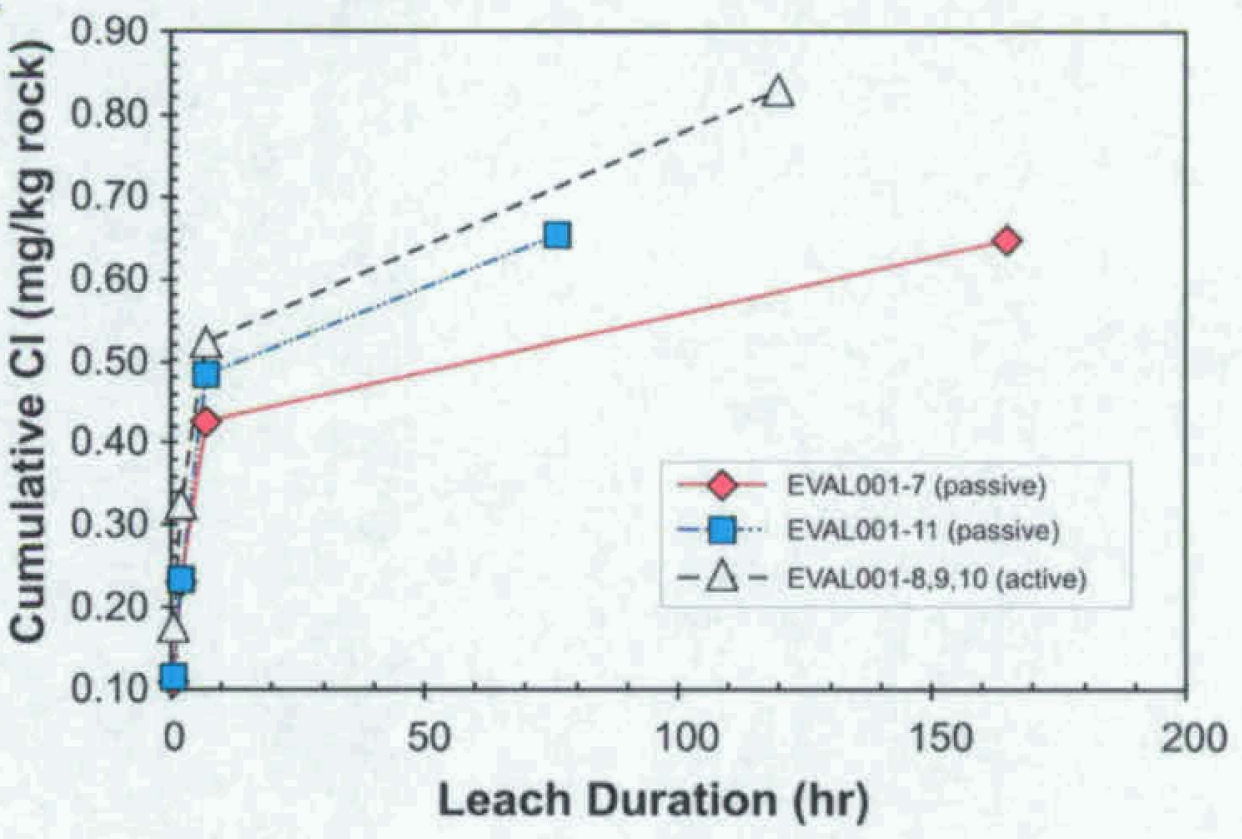

DTN: LA0305RR831222.001 (UQ)

NOTES: The figures plot measured and cumulative chloride concentrations or ${ }^{36} \mathrm{Cl} / \mathrm{Cl}$ values against leach duration. Cumulative values are derived by sequentially summing respective values from previous leach increments.

Figure 4-4. Relations between Chloride Concentrations (A) and Cumulative Chloride Concentrations (B) Plotted against Leach Duration for Sequential Leachates of Reference Sample EVAL001 Leached at LANL by Passive and Active Methods during Phase II 
A.

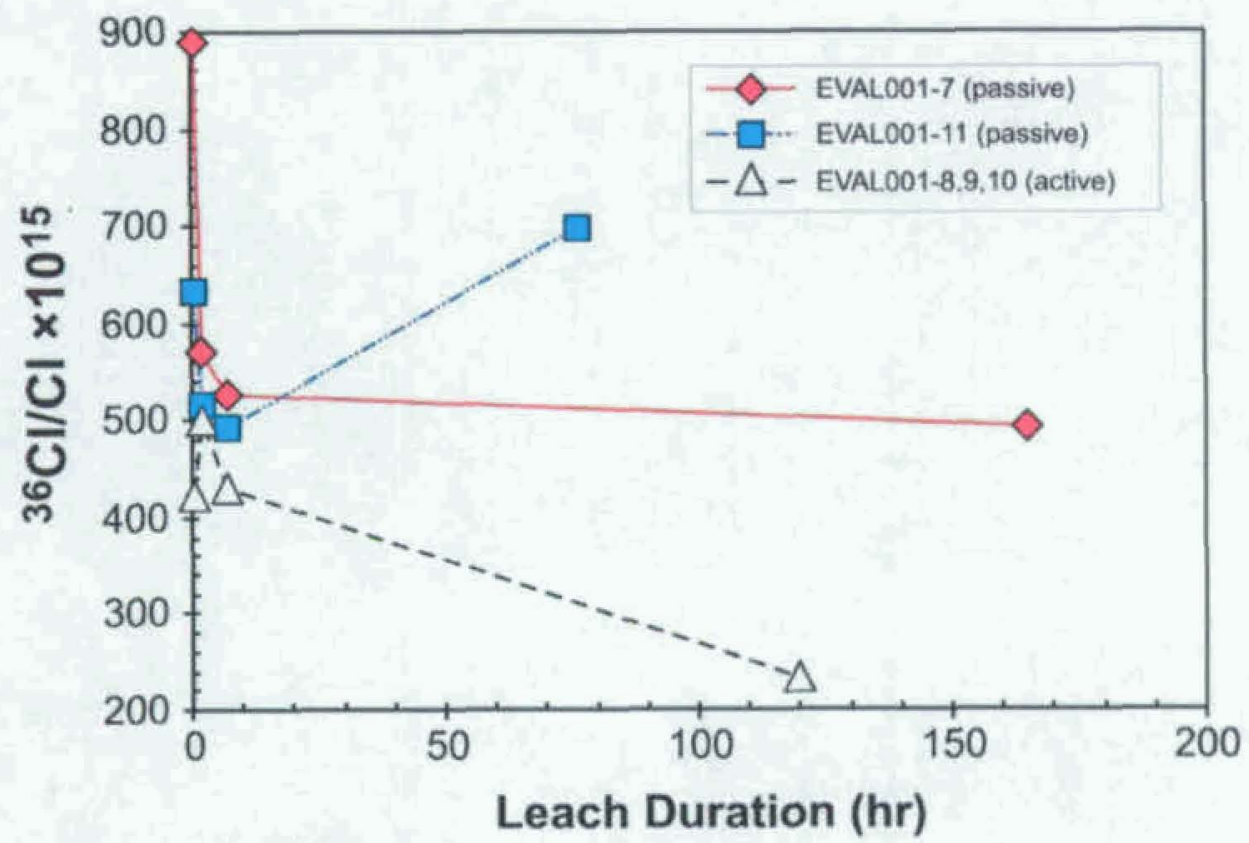

B.

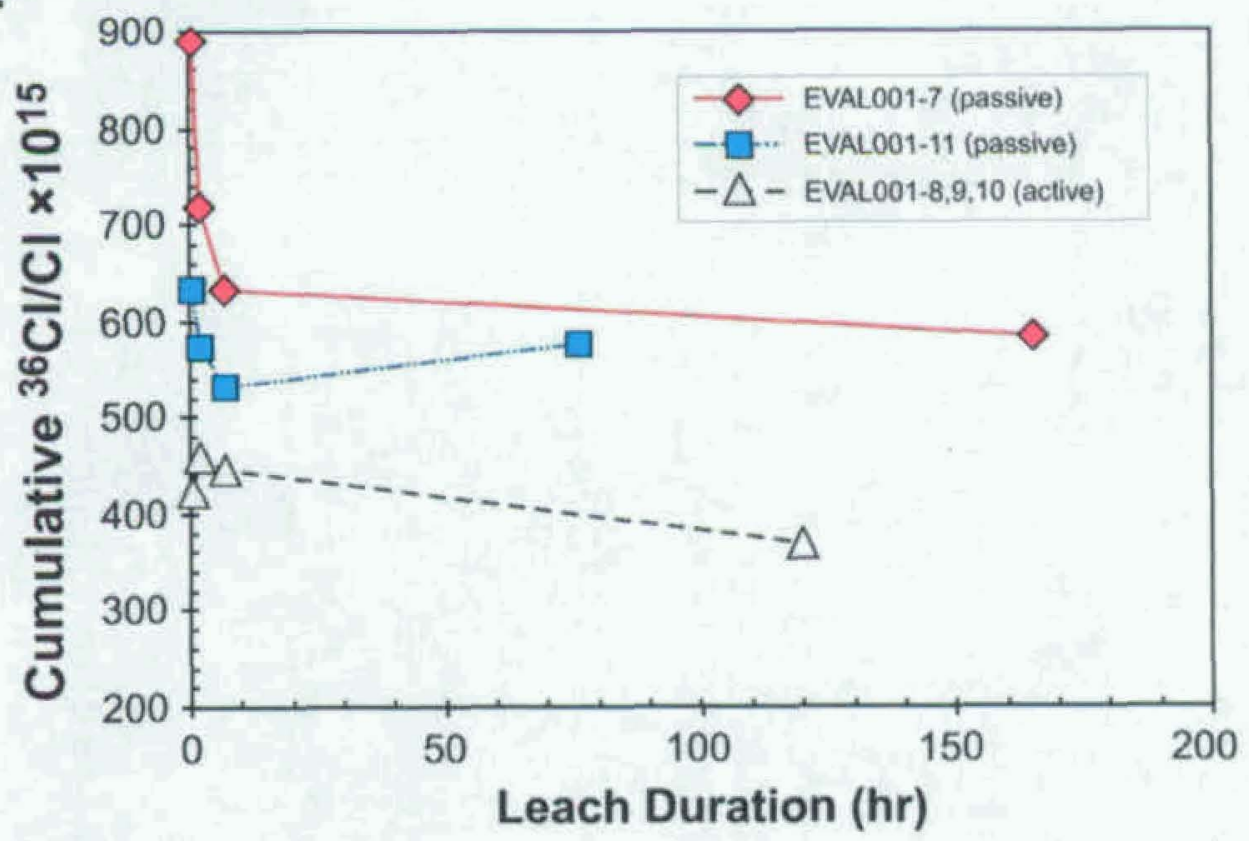

DTN: LA0305RR831222.001 (UQ)

NOTES: The figures plot measured and cumulative chloride concentrations or ${ }^{36} \mathrm{Cl} / \mathrm{Cl}$ values against leach duration. Cumulative values are derived by sequentially summing respective values from previous leach increments.

Figure 4-5. Relations between ${ }^{36} \mathrm{Cl} / \mathrm{CI}$ Ratios (A) and Cumulative ${ }^{36} \mathrm{Cl} / \mathrm{CI}$ Ratios (B) Plotted against Leach Duration for Sequential Leachates of Reference Sample EVAL001 Leached at LANL by Passive and Active Methods during Phase II 

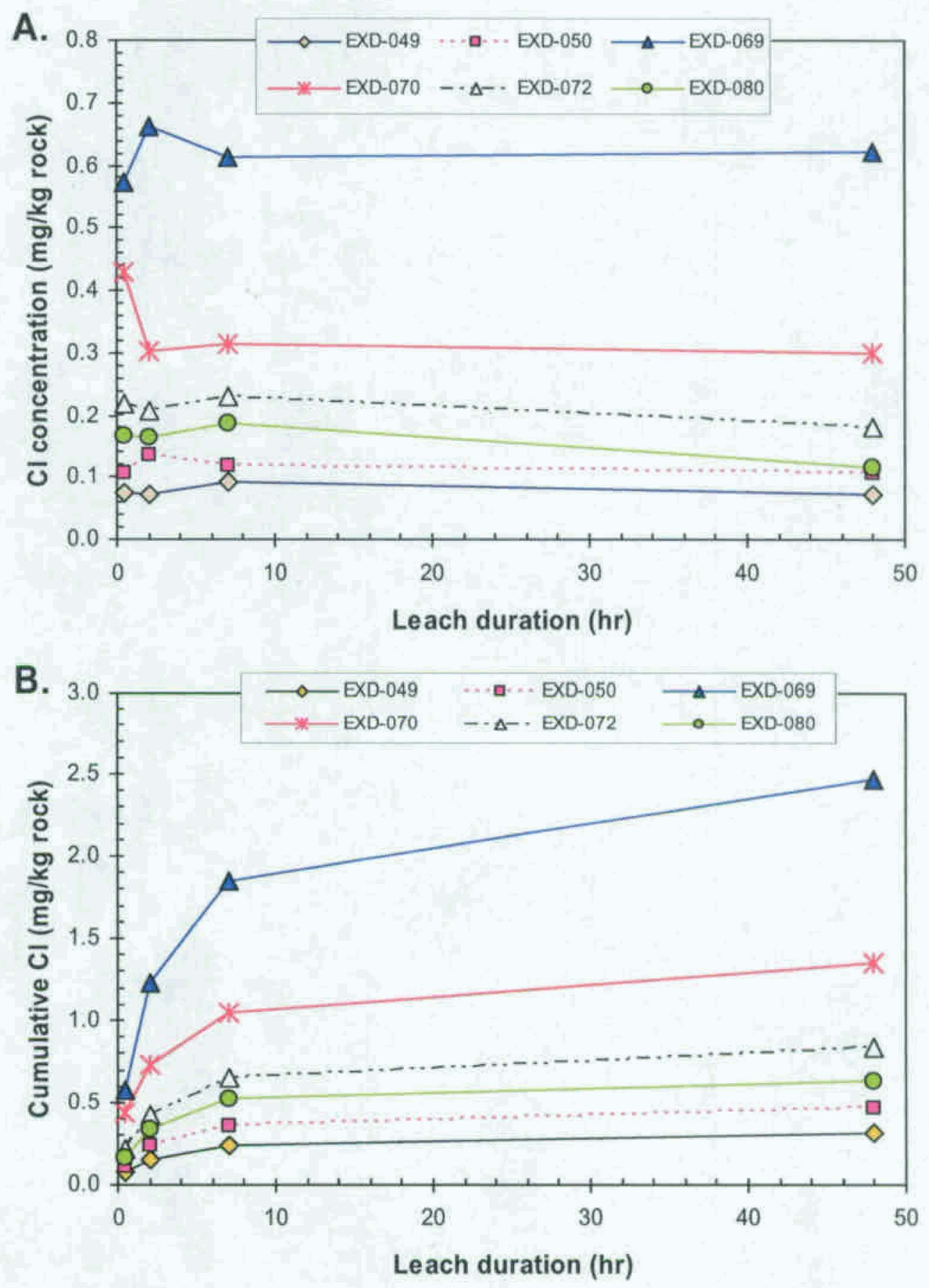

DTN: LA0305RR831222.001 (UQ)

NOTES: The figures plot measured and cumulative chloride concentrations or ${ }^{36} \mathrm{Cl} / \mathrm{Cl}$ values against leach duration. Cumulative values are derived by sequentially summing respective values from previous leach increments.

Figure 4-6. Relations between Chloride Concentrations ( $A$, showing all data) and Cumulative Chloride Concentrations ( $\mathrm{B}$, showing a subset of the data at a larger scale) Plotted against Leach Duration for Sequential Passive Leachates of the 6.3- to 12.5-mm Fraction of Six Samples from the ECRB Cross Drift Analyzed at LANL during Phase II 

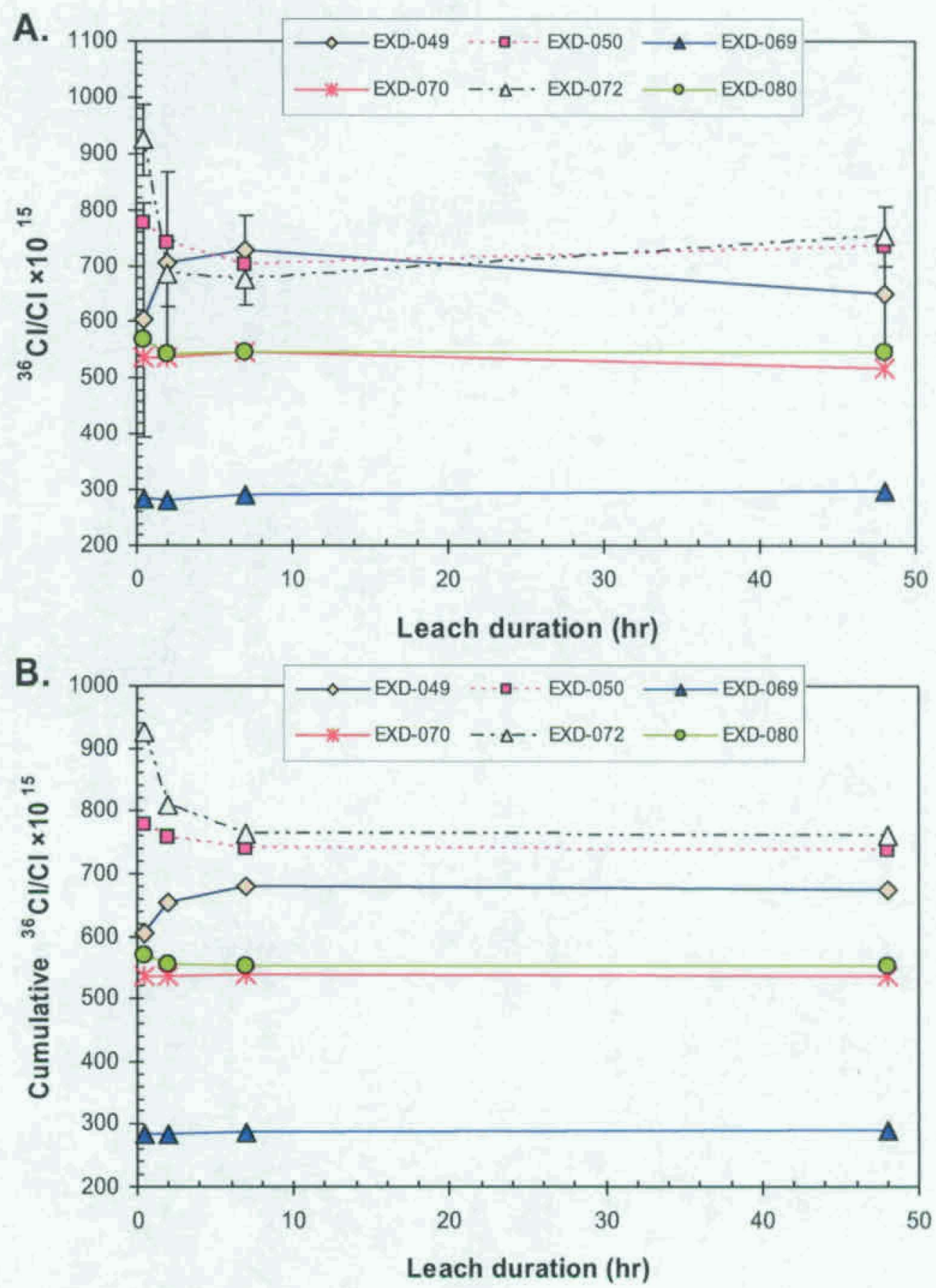

DTN: LA0305RR831222.001 (UQ)

NOTES: The figures plot measured and cumulative chloride concentrations or ${ }^{36} \mathrm{Cl} / \mathrm{Cl}$ values against leach duration. Cumulative values are derived by sequentially summing respective values from previous leach increments.

Figure 4-7. Relations between ${ }^{36} \mathrm{Cl} / \mathrm{CI}$ Ratios (A) and Cumulative ${ }^{36} \mathrm{Cl} / \mathrm{CI}$ Ratios (B) Plotted against Leach Duration for Sequential Passive Leachates of the 6.3- to 12.5-mm Fraction of Six Samples from the ECRB Cross Drift Analyzed at LANL during Phase II 

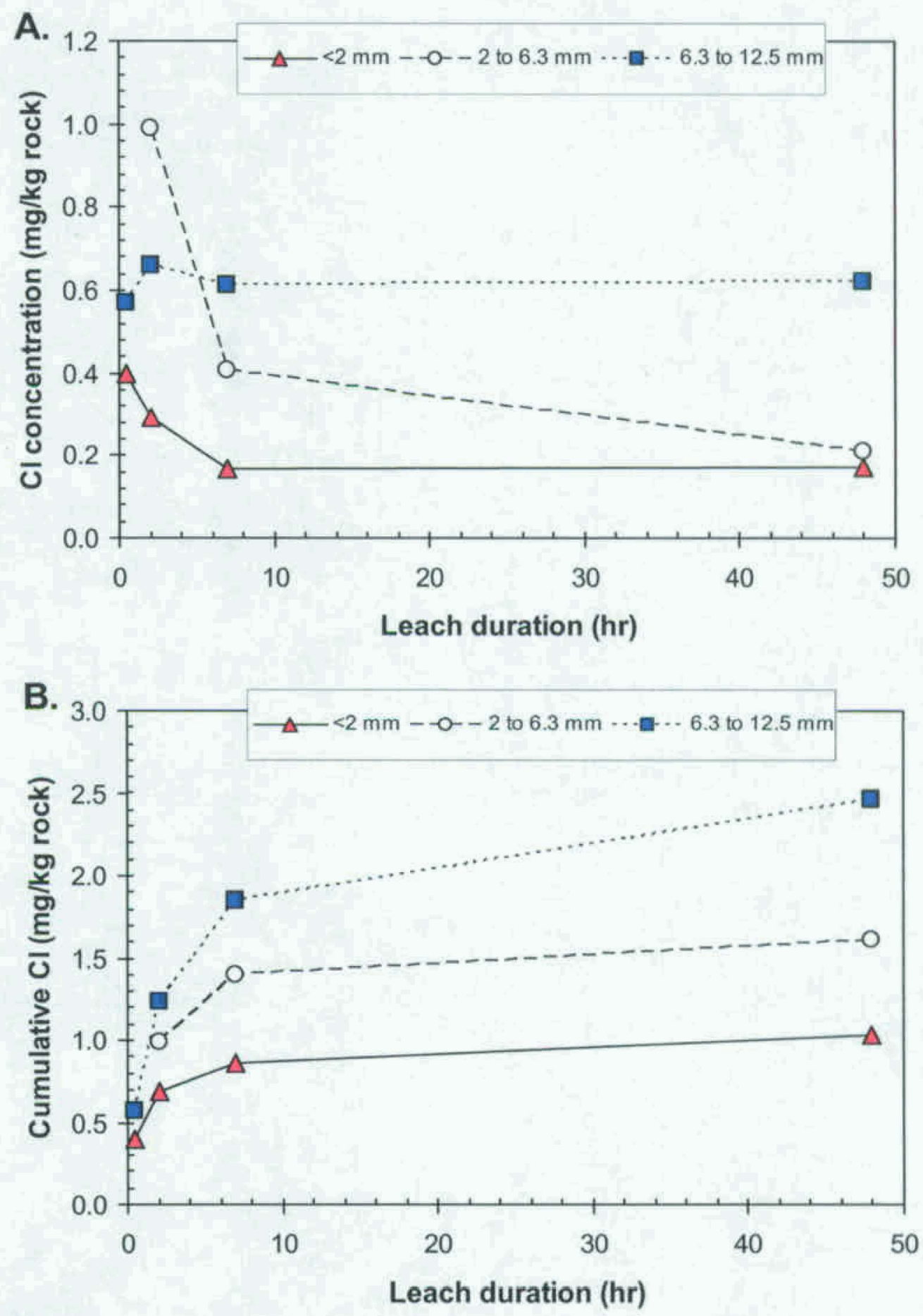

DTN: LA0305RR831222.001 (UQ)

NOTES: The figures plot measured and cumulative chloride concentrations or ${ }^{36} \mathrm{Cl} / \mathrm{Cl}$ values against leach duration. Cumulative values are derived by sequentially summing respective values from previous leach increments.

Figure 4-8. Relations between Chloride Concentrations (A) and Cumulative Chloride Concentrations (B) Plotted against Leach Duration for Passive Leachates of Different Size Fractions of ECRB Cross Drift Sample EXD-069 Analyzed at LANL during Phase II 

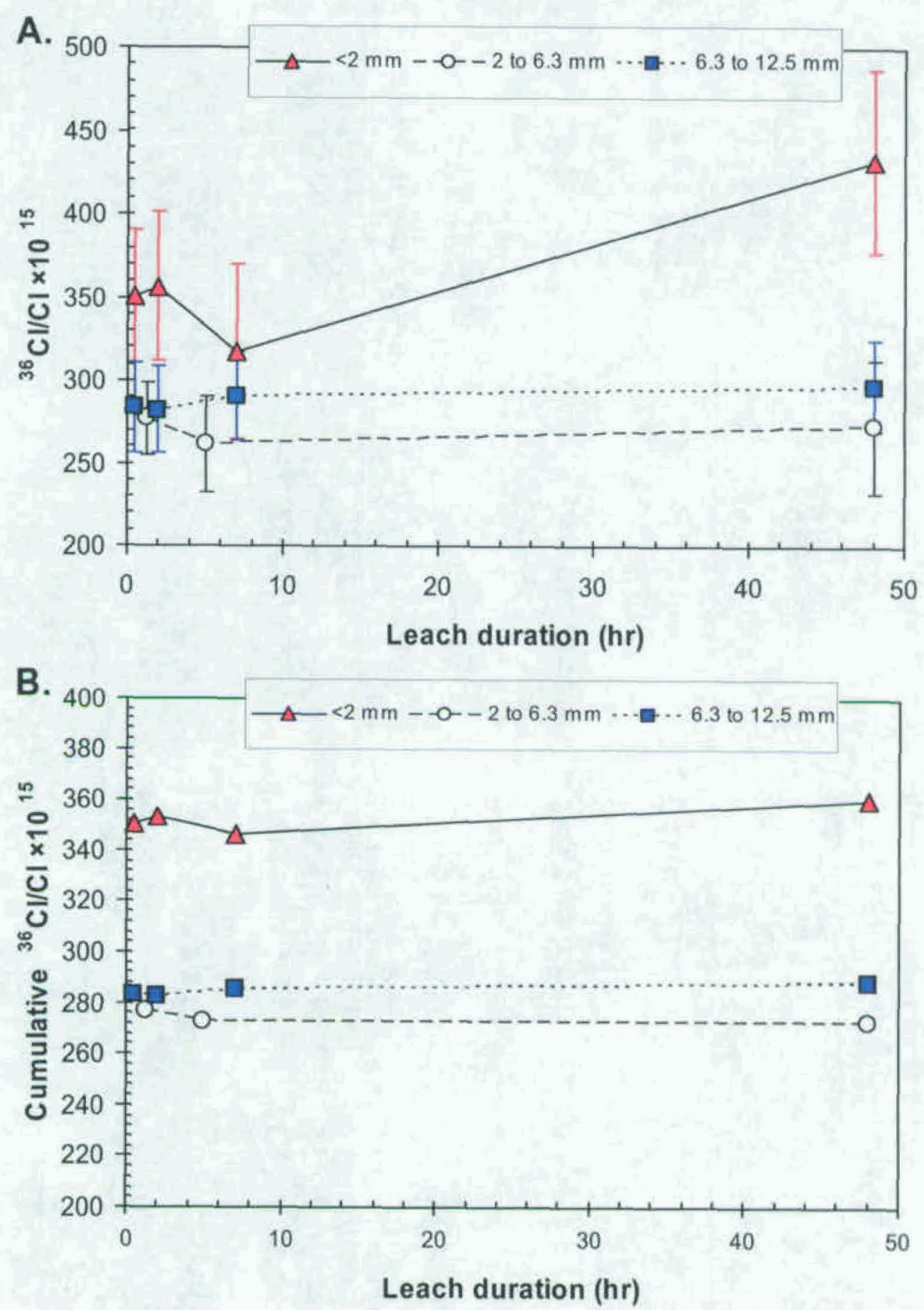

DTN: LA0305RR831222.001 (UQ)

NOTES: The figures plot measured and cumulative chloride concentrations or ${ }^{36} \mathrm{Cl} / \mathrm{Cl}$ values against leach duration. Cumulative values are derived by sequentially summing respective values from previous leach increments.

Figure 4-9. Relations between ${ }^{36} \mathrm{Cl} / \mathrm{CI}$ Ratios (A) and Cumulative ${ }^{36} \mathrm{Cl} / \mathrm{Cl}$ Ratios (B) Plotted against Leach Duration for Passive Leachates of Different Size Fractions of ECRB Cross Drift Sample EXD-069 Analyzed at LANL during Phase II 

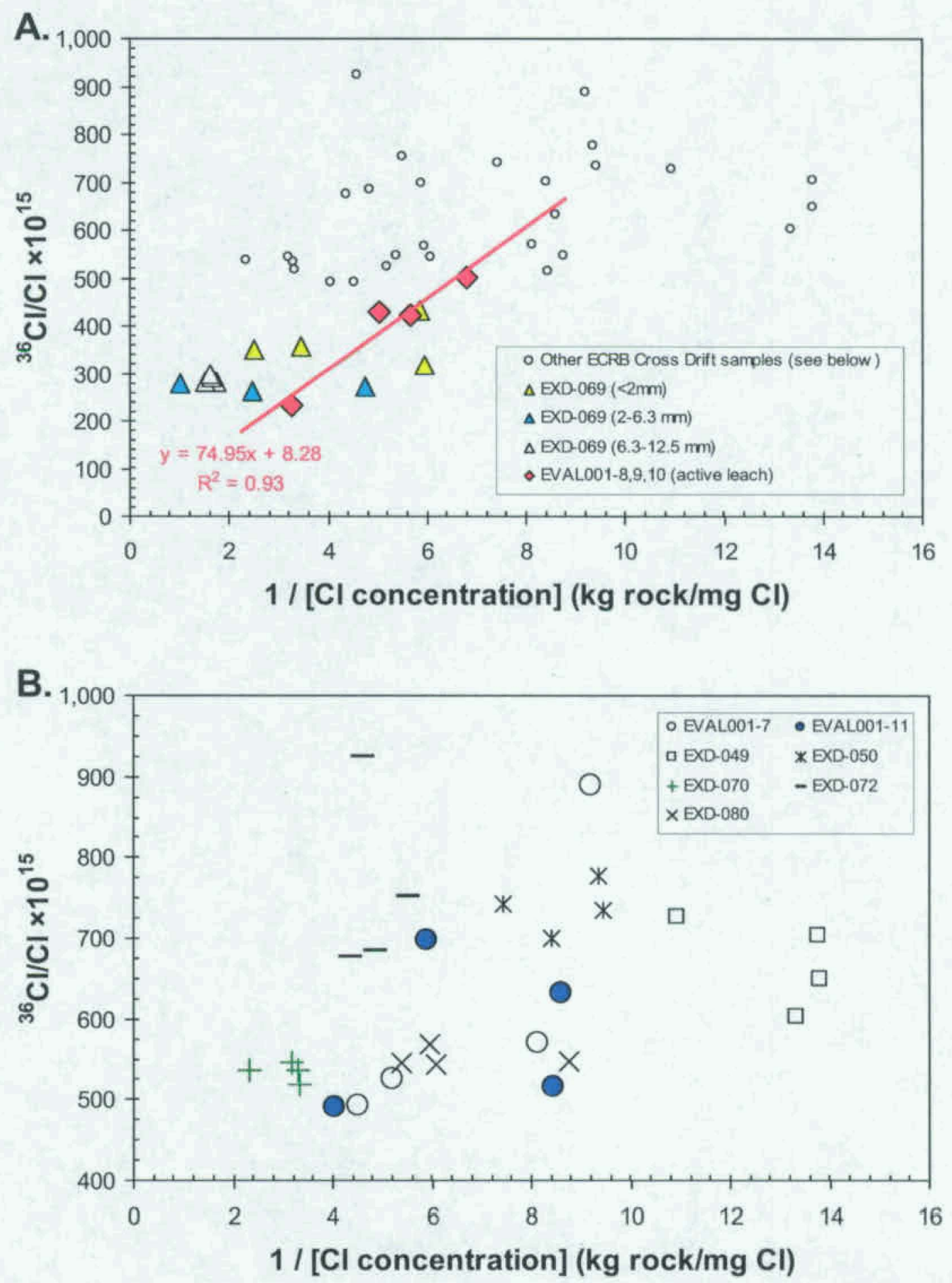

DTN: LA0305RR831222.001 (UQ)

NOTES: $\quad E C R B=$ Enhanced Characterization of the Repository Block. All samples are shown in (A), and a selected subset of samples is shown in (B).

Figure 4-10. Relations between ${ }^{36} \mathrm{Cl} / \mathrm{Cl}$ Ratios and Reciprocal Chloride Concentrations in Sequential Leachates of Reference Sample EVAL001 and ECRB Cross Drift Samples Analyzed at LANL during Phase II 


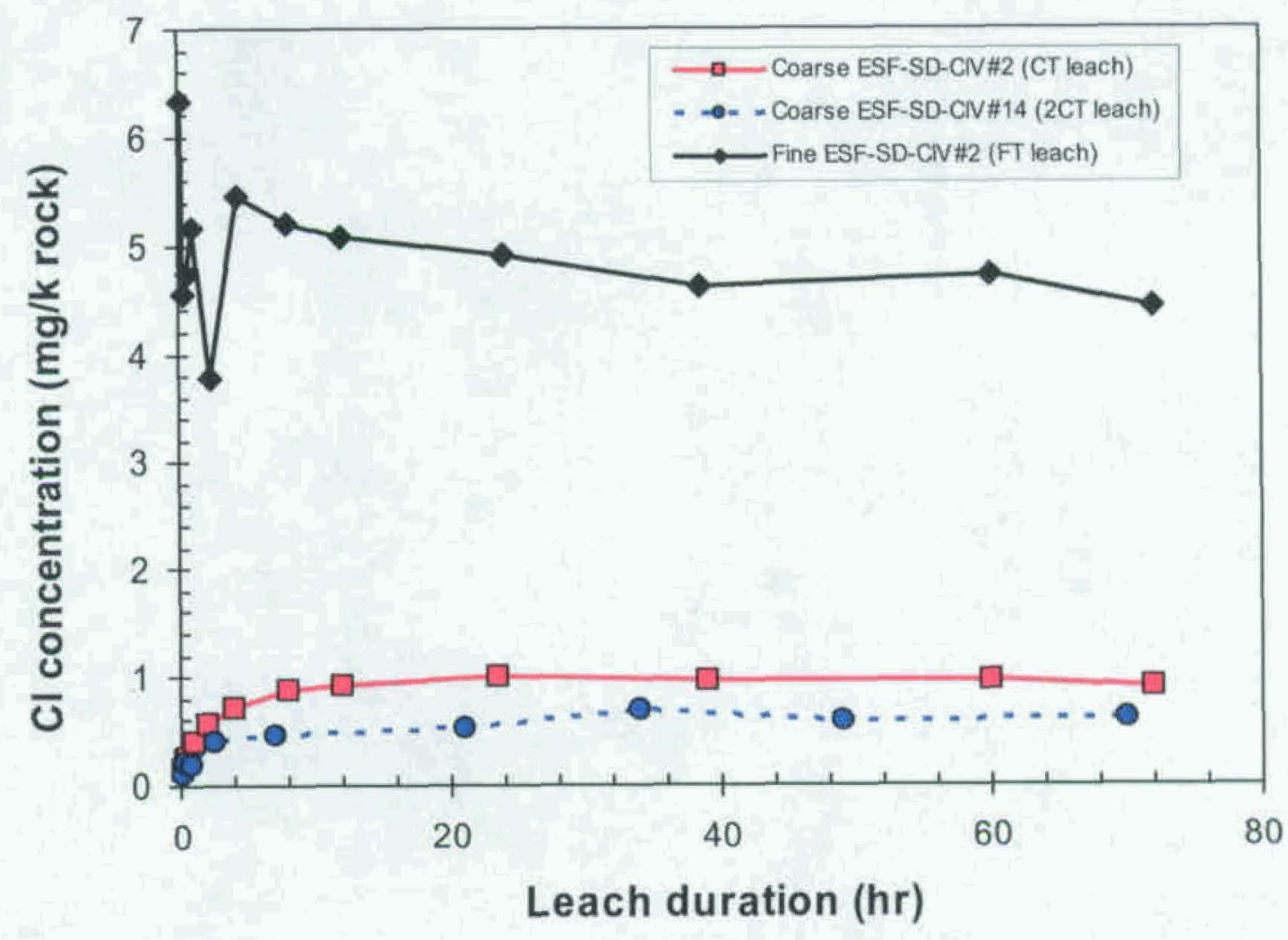

DTN: GS030508312272.003 (UQ)

Figure 4-11. Effect of Particle Size on Leach Duration and Chloride Concentration for Two Size Fractions of Tuff from Unfractured (CT and FT series, \#2) and Relatively Unfractured (2CT series, \#14) Core Samples Analyzed at AECL during Phase II 


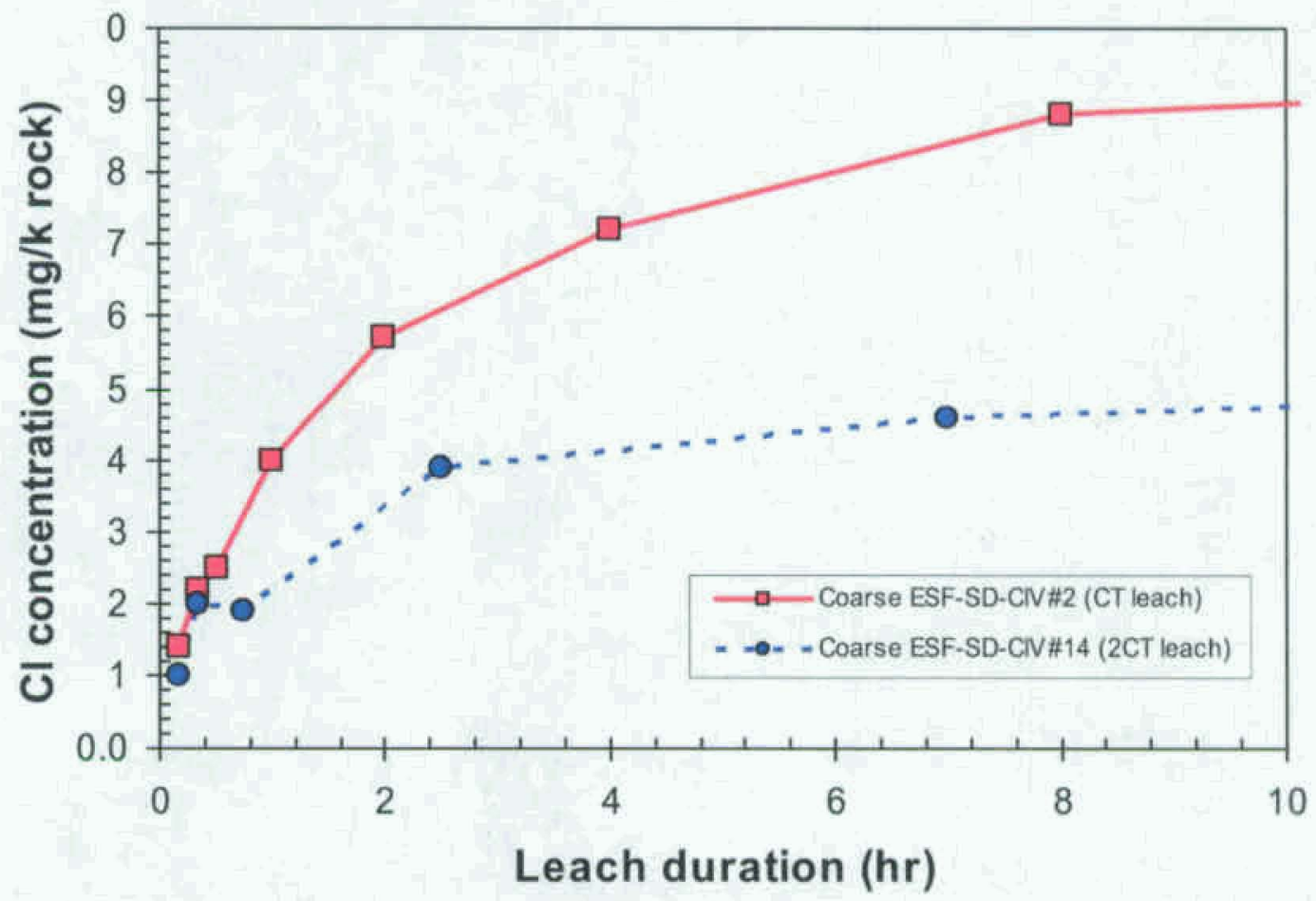

DTN: GS030508312272.003 (UQ)

Figure 4-12. Detail from Figure 4-11 Showing the Changes in Chloride Concentrations in the First Few Hours of Two Leaching Tests on the Coarse Tuff 


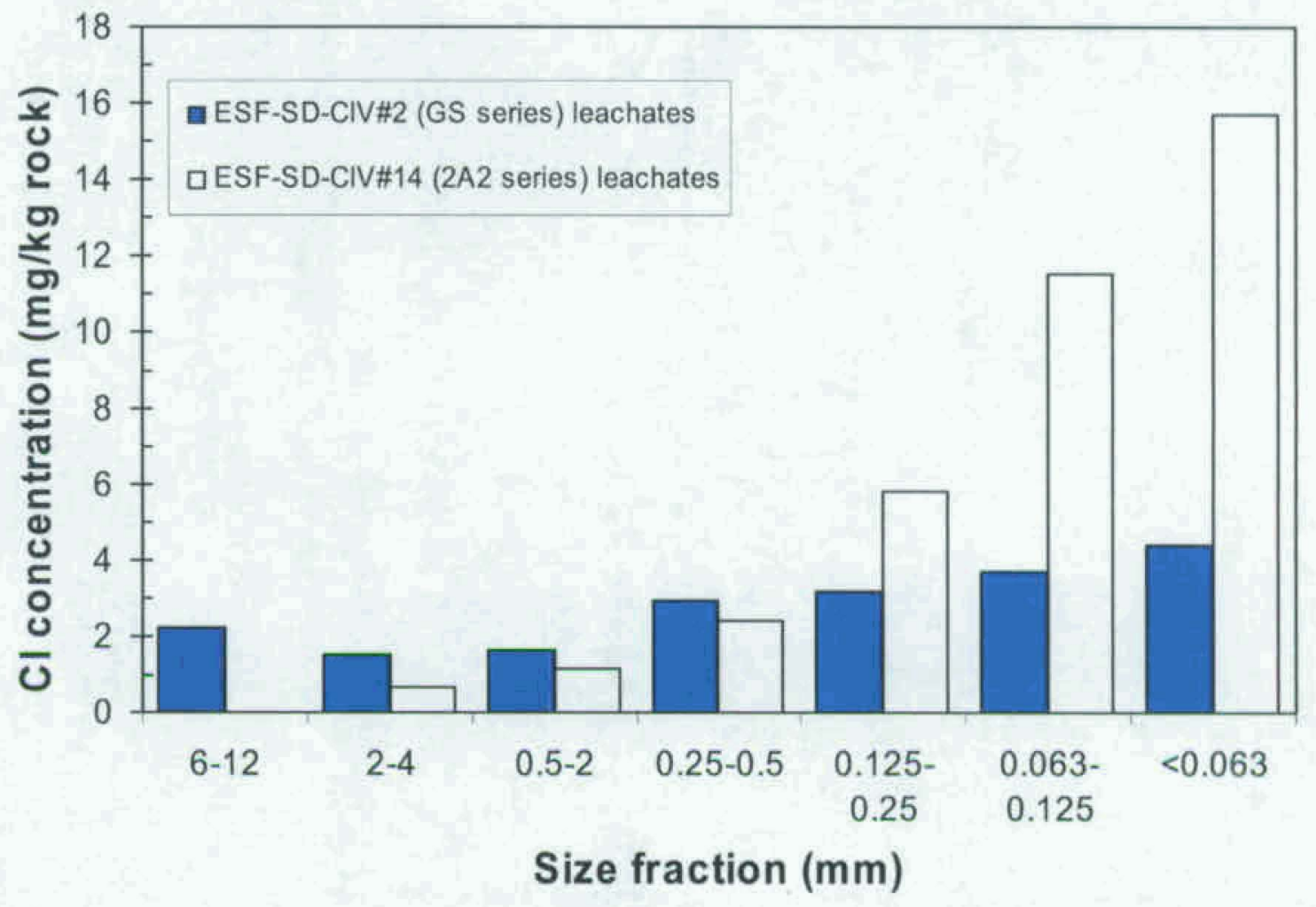

DTN: GS030508312272.003 (UQ)

Figure 4-13. Effect of Particle Size on Chloride Concentrations in Phase II Leachates of Intact Core from Borehole ESF-SD-CIV\#2 (GS series in Table 4-6) and Broken Core from Borehole ESF-SD-CIV\#14 (2A2 series in Table 4-6) 


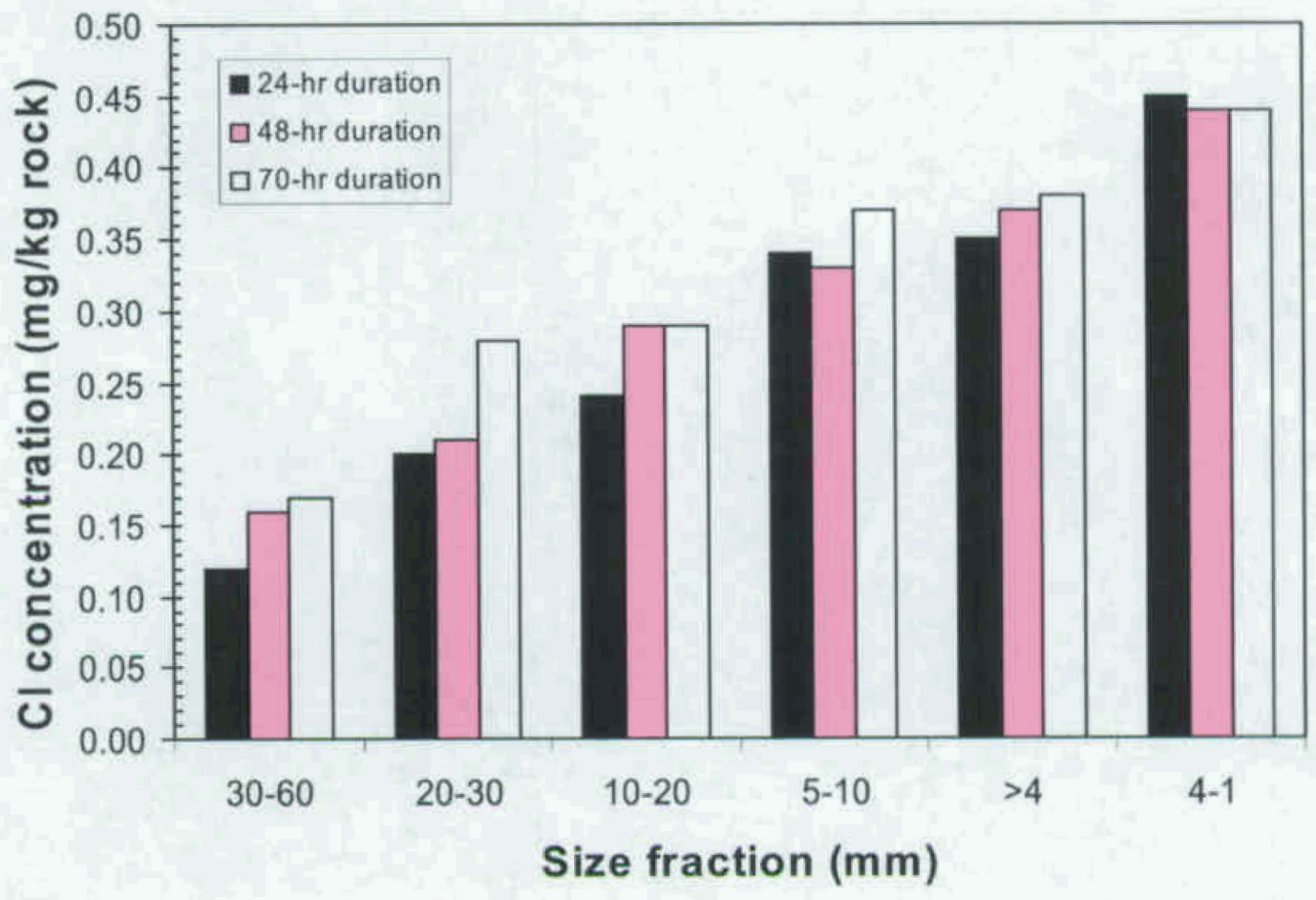

DTN: GS030508312272.003 (UQ)

Figure 4-14. Effect of Particle Size and Leach Duration on Rubblized Core Fragments from Borehole ESF-SD-CIV\#9 (BT series in Table 4-6) 


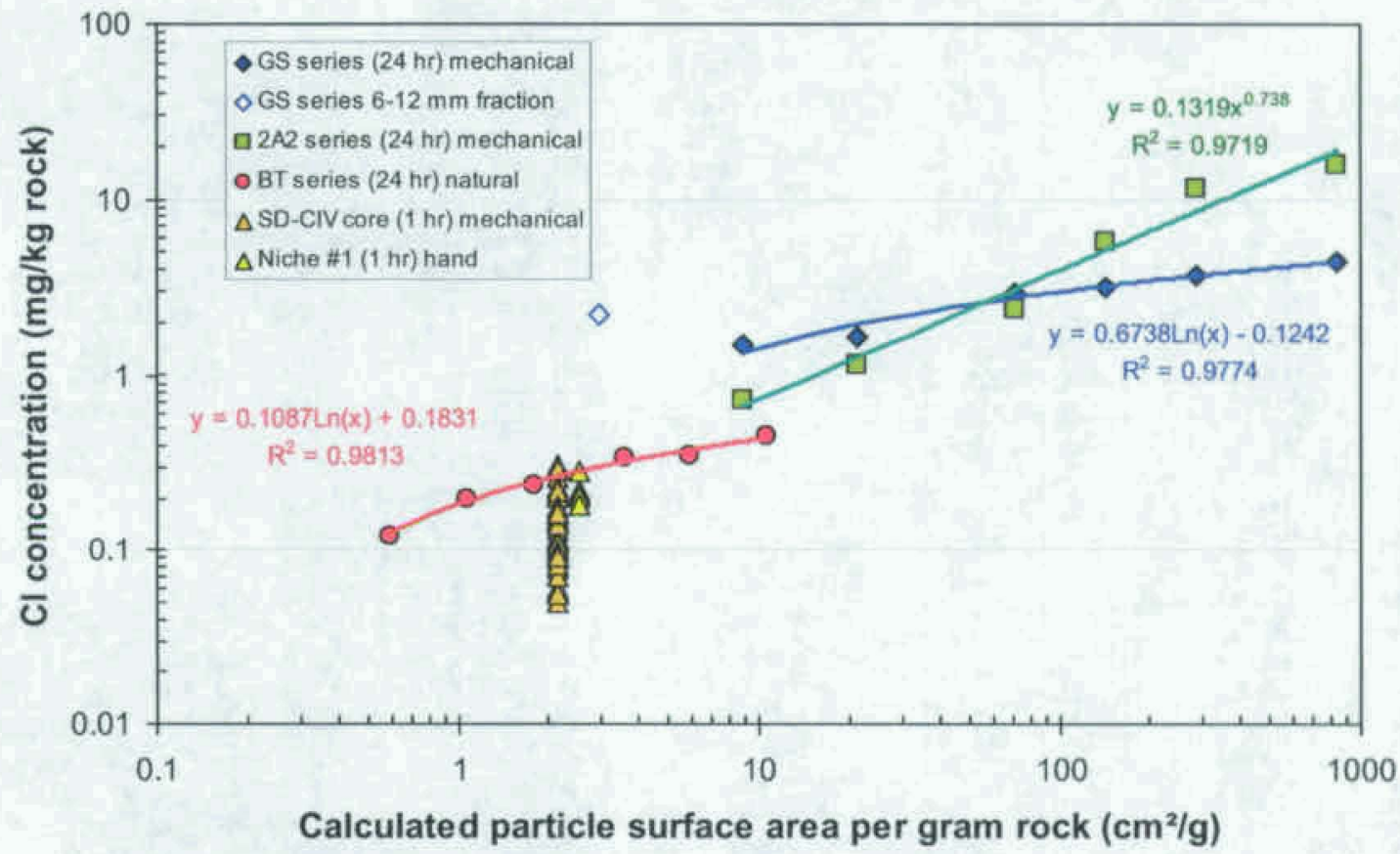

DTNs: GS030508312272.003 (UQ); LL031200223121.036 (Q), Filename: Total_AMS_Summary_2001-02c.xis

NOTES: Particle surface area per gram rock is calculated assuming spherical particles with radius equal to half the difference between upper and lower sieve opening dimensions and a density of $2.25 \mathrm{~g} / \mathrm{cm}^{3}$ to calculate particle mass, number of spheres per gram, and, finally, total surface area per gram. The open diamond is a statistical outlier that was not included in the regression.

Figure 4-15. Comparison of Chloride Concentrations in Phase II Leachates of Core Samples from ESF-SD-CIV and Niche \#1 Boreholes in the Sundance Fault Zone 


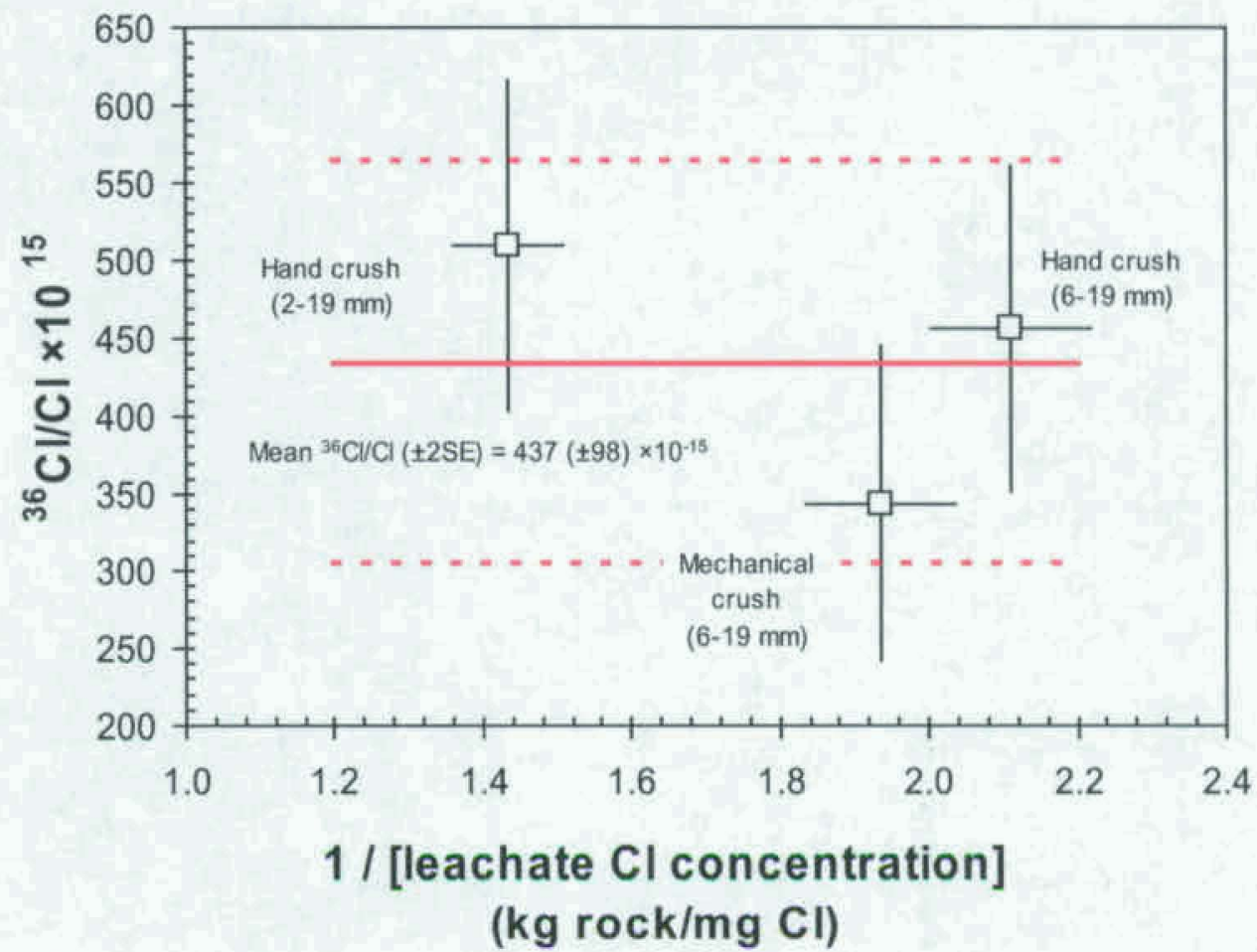

DTN: LL031200223121.036 (Q), Filename: Total_AMS_Summary_2001-02c xls NOTES: $S E=$ standard error. Error bars are 20 .

Figure 4-16. Relations between Chloride Concentrations and ${ }^{36} \mathrm{CI} / \mathrm{CI}$ Ratios in Phase III Leachates of Core Samples from Borehole ESF-SAD-GTB\#1 

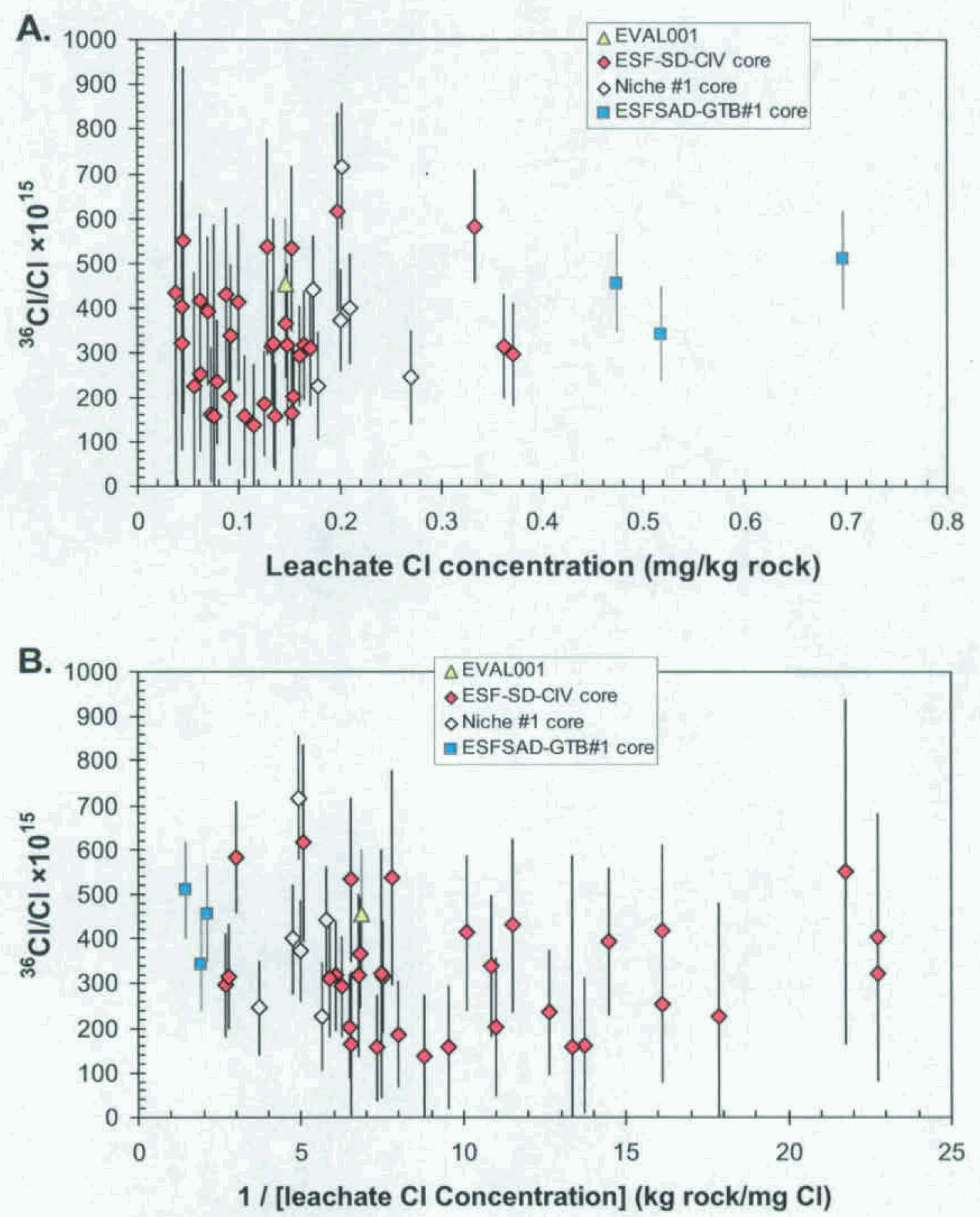

DTN: LL031200223121.036 (Q), Filename: Total_AMS_Summary_2001-02c.xis; GS030608312272.005 (Q) NOTE: $\quad$ Error bars are $2 \sigma$.

Figure 4-17. Relations between ${ }^{36} \mathrm{C} / / \mathrm{CI}$ Ratios and Chloride Concentrations (A) and Reciprocal Chloride Concentrations (B) in Phase III Leachates of Validation Study Samples Leached at the USGS and Analyzed at LLNL 

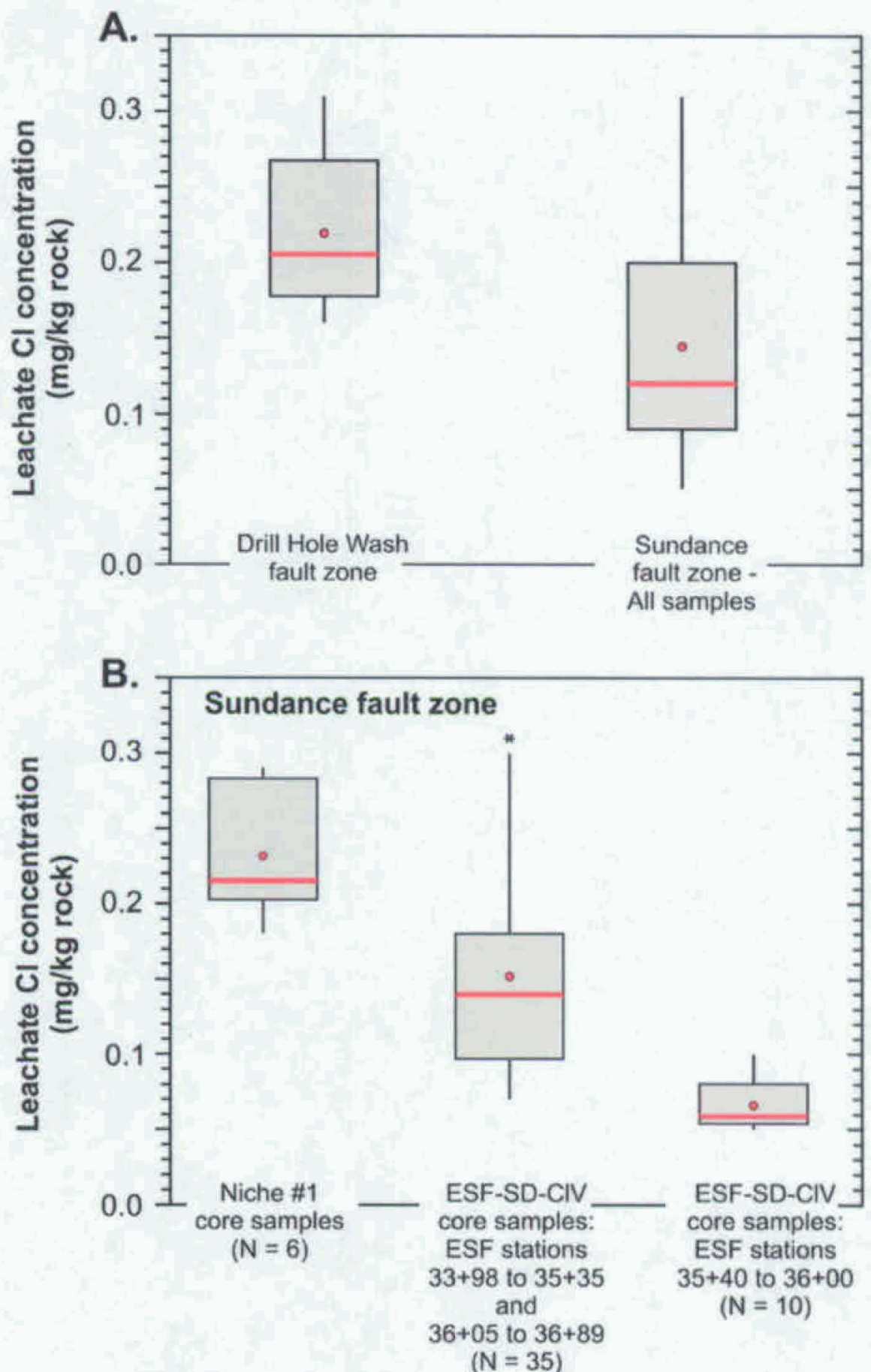

DTN: GS030608312272.005(Q)

NOTES: ESF $=$ Exploratory Studies Facility. The median value is given by the dark red line; mean value is given by the red-filled circle. The middle $50 \%$ of the data are within the gray-filled boxes and the upper-and lower-most quartiles are represented by the lines on either side of the boxes. Statistical outliers are shown as asterisks. Data from the DTN were converted from $\mathrm{mg} / \mathrm{L}$ to $\mathrm{mg} / \mathrm{kg}$ (see Table 4-13).

Figure 4-18. Box Plots of Chloride Concentration Data Comparing Phase III Leachates of Core Samples from the Drill Hole Wash and Sundance Fault Zones (A), and from Different Samples within the Sundance Fault Zone (B) 

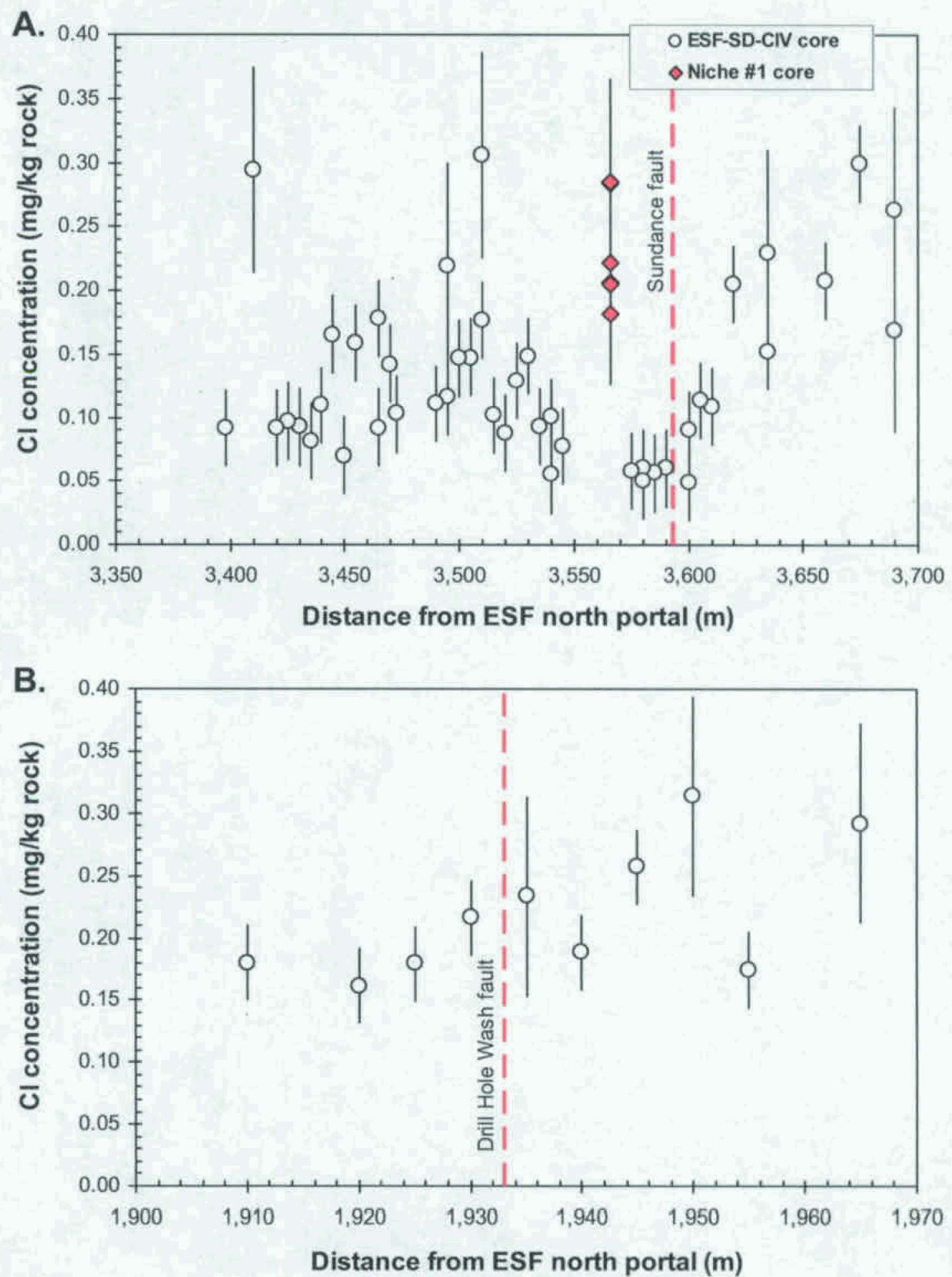

DTN: GS030608312272.005 (Q)

NOTES: ESF $=$ Exploratory Studies Facility. Error bars are 2o. Data from the DTN were converted from mg/L to $\mathrm{mg} / \mathrm{kg}$ (see Table 4-13). Borehole locations are listed in Table 3-2.

Figure 4-19. Concentrations of Chloride Determined by lon Chromatography in Phase III Leachates of Validation Study Core Samples and Niche \#1 Core Samples from the Sundance Fault Zone (A) and Drill Hole Wash Fault Zone (B) 

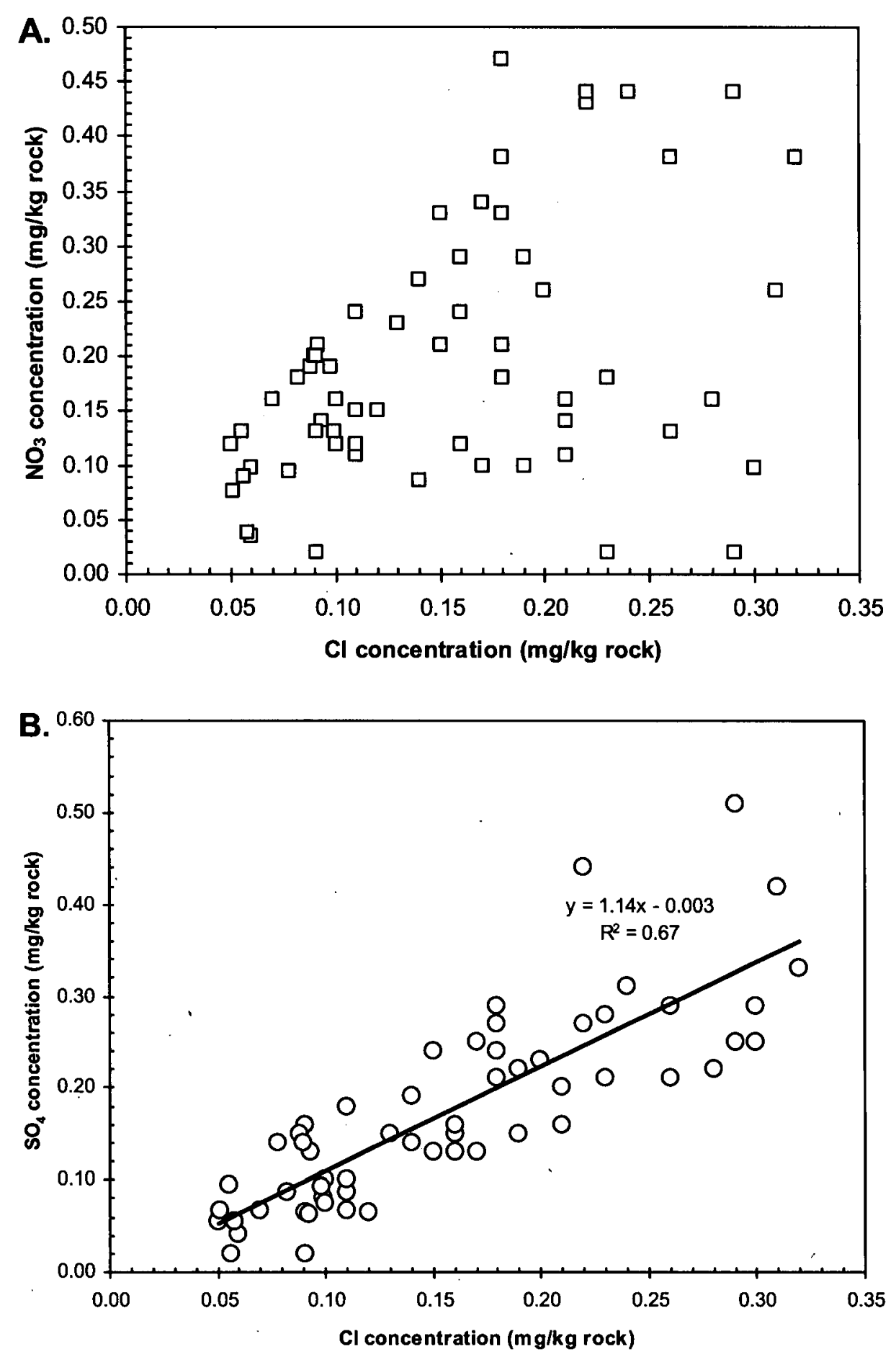

DTN: GS030608312272.005(Q)

NOTES: Linear-regression curve is shown in $(B)$ with straight-line equations and $R^{2}$ values. Data from the DTN were converted from $\mathrm{mg} / \mathrm{L}$ to $\mathrm{mg} / \mathrm{kg}$ (see Table 4-13).

Figure 4-20. Comparison Chloride Concentrations in Phase III Leachates of Validation Study Core Leached at the USGS, with $\mathrm{NO}_{3}$ Concentrations (A) and $\mathrm{SO}_{4}$ Concentrations (B) 


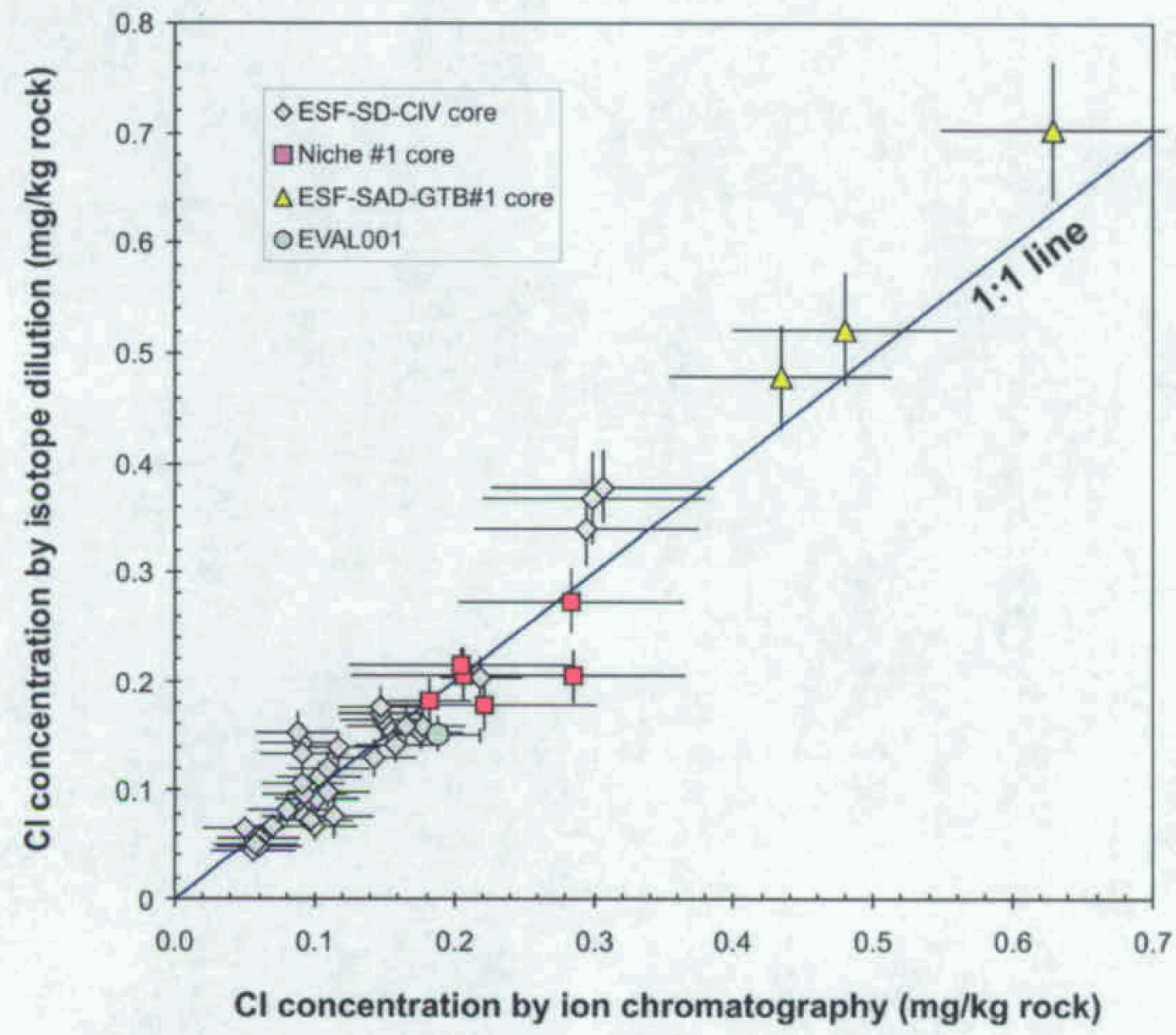

DTNs: LL031200223121.036 (Q), Filename: Total_AMS_Summary_2001-02c.xis; GS030608312272.005 (Q) NOTES: Error bars are $2 \sigma$. Data from the DTN were converted from $\mathrm{mg} / \mathrm{L}$ to $\mathrm{mg} / \mathrm{kg}$ (see Table 4-13).

Figure 4-21. Comparison of Chloride Concentrations in Phase III Leachates of Validation Study Samples Analyzed by lon Chromatography at the USGS and by Isotope Dilution at LLNL 

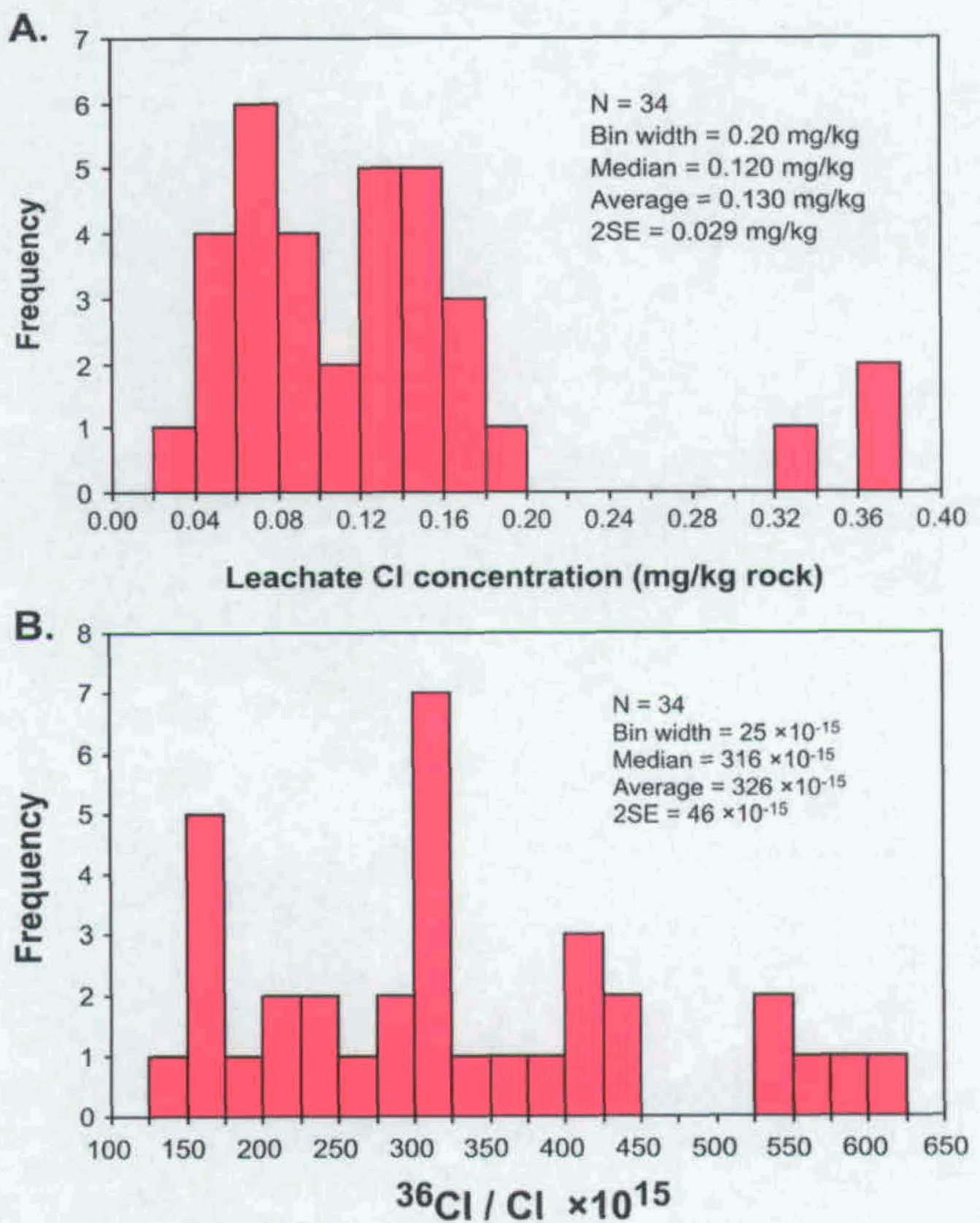

DTN: LL031200223121.036 (Q), Filename: Total_AMS_Summary_2001-02c.xls

NOTE: $\quad \mathrm{SE}=$ standard error.

Figure 4-22. Histograms Showing Chloride Concentrations $(\mathrm{A})$ and ${ }^{36} \mathrm{Cl} / \mathrm{Cl}$ Ratios $(B)$ in Phase III Leachates of Validation Study Samples Leached at the USGS and Analyzed at LLNL 


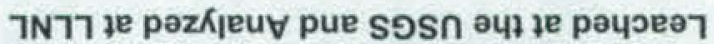

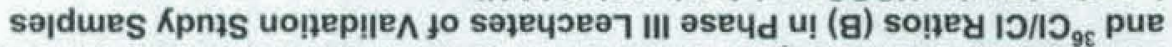

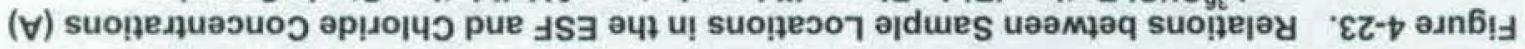

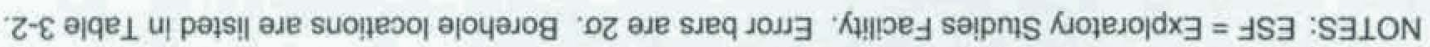

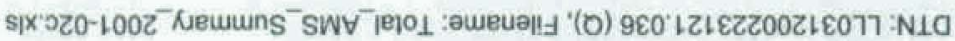

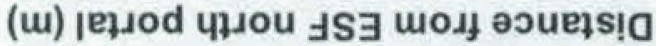

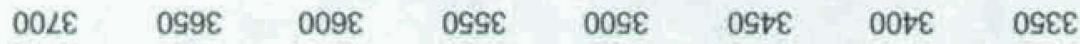

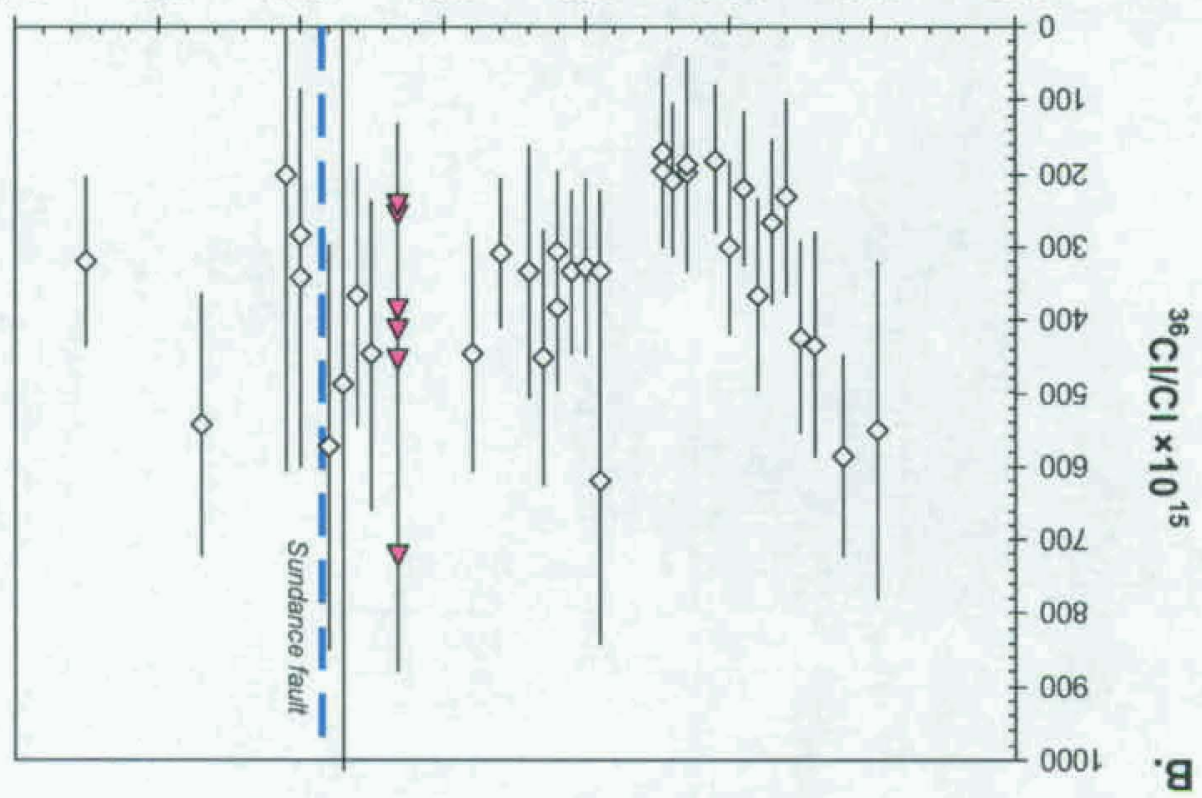

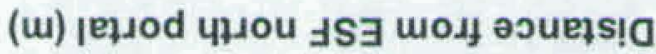

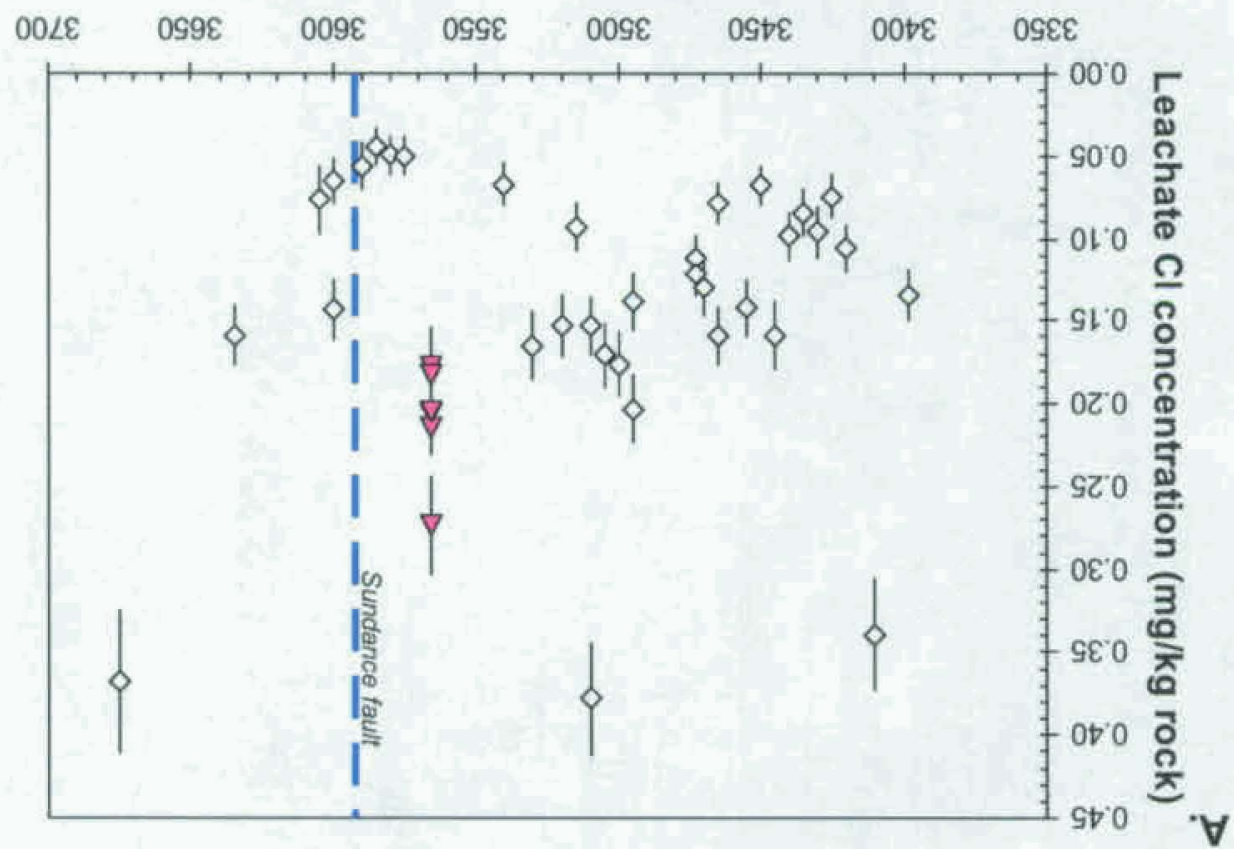




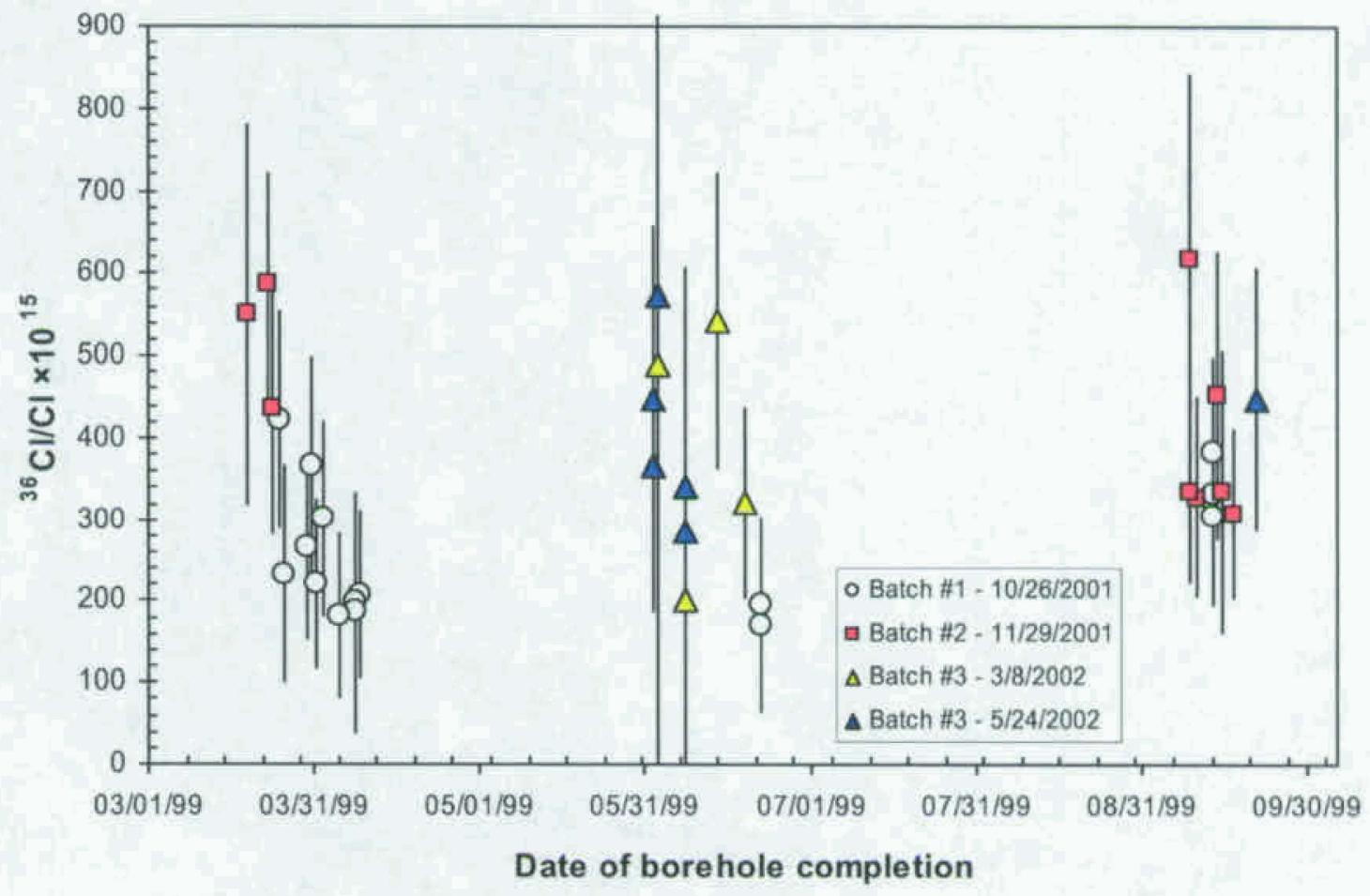

DTN: LL031200223121.036 (Q), Filename: Total_AMS_Summary_2001-02c.xIs

NOTES: Data are grouped by leaching batch number and analysis date. Borehole completion dates are listed in Table 3-2. Error bars are 20 .

Figure 4-24. Relations between Borehole Completion Dates and ${ }^{36} \mathrm{Cl} / \mathrm{Cl}$ Ratios in Phase III Leachates of Validation Study Samples Leached at the USGS and Analyzed at LLNL 


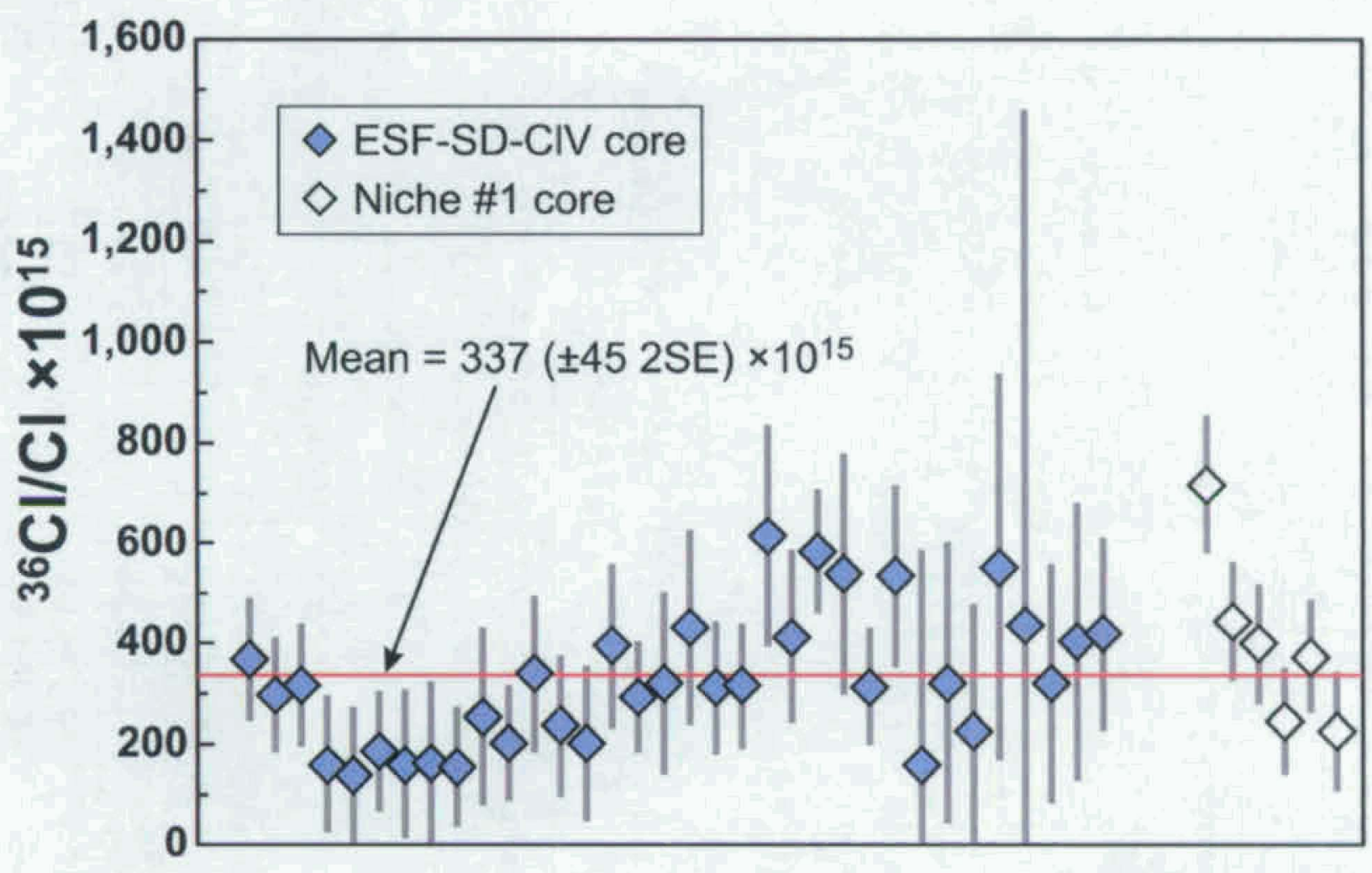

DTN: LL031200223121.036 (Q), Filename: Total_AMS_Summary_2001-02c.x/s

NOTES: SE $=$ standard error .

Error bars are $2 \sigma$.

Data are shown (from left to right) in order presented in Table 4-9.

Figure 4-25. Histogram Showing ${ }^{36} \mathrm{CI} / \mathrm{Cl}$ Ratios in Phase III Leachates of ESF-SD-CIV and Niche \#1 Core Samples Prepared at the USGS and Analyzed at LLNL 

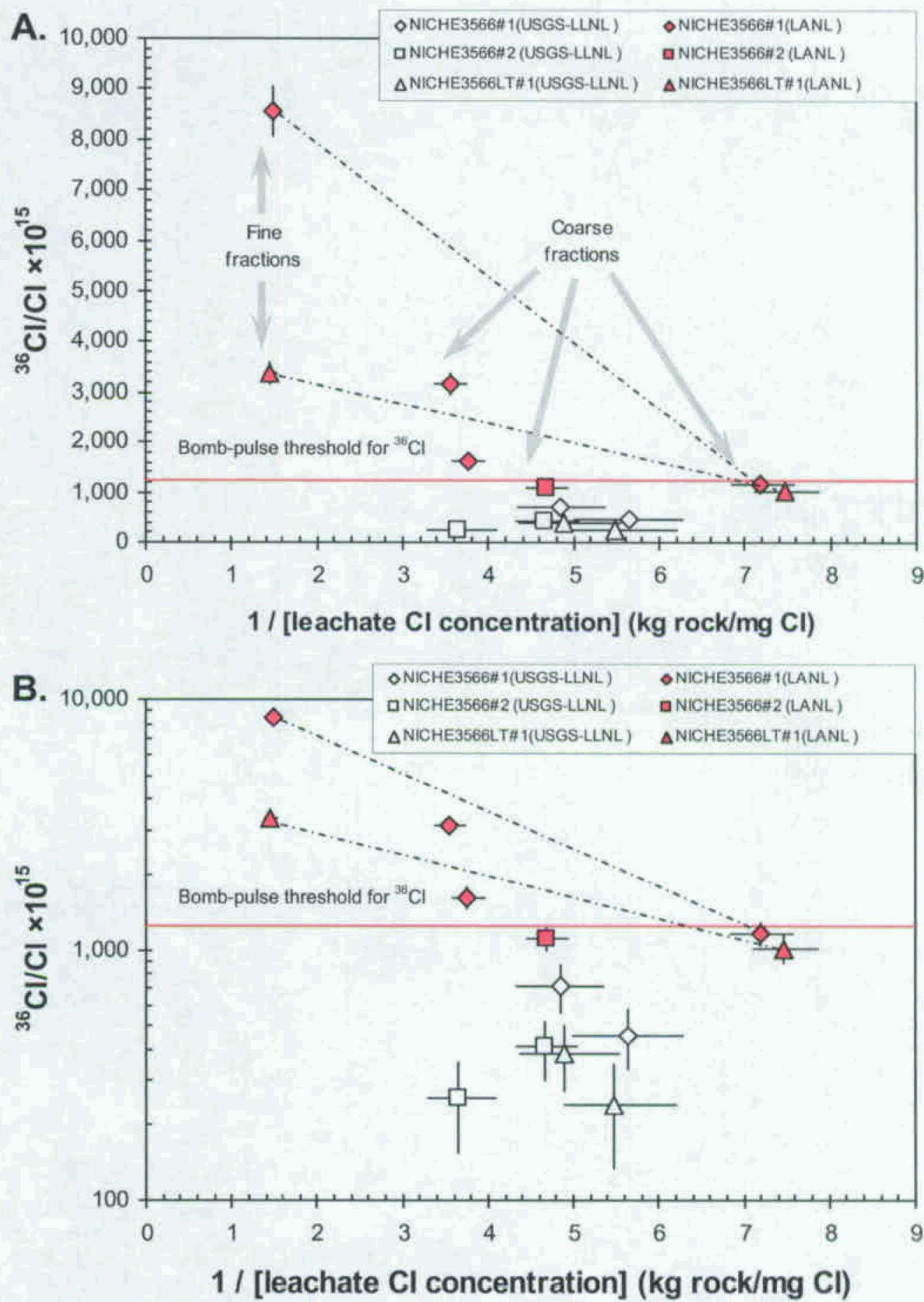

DTNs: LL.031200223121.036 (Q), Filename: Total_AMS_Summary_2001-02c.xIs; LA0305RR831222.001 (UQ)

NOTES: LANL $=$ Los Alamos National Laboratory, LLNL = Lawrence Livermore National Laboratory, USGS $=$ U.S. Geological Survey. The dash-dot lines in $(A)$ and $(B)$ are drawn between different size fractions of the same samples. Error bars are $2 \sigma$.

Figure 4-26. Relations between Reciprocal Chloride Concentrations and ${ }^{36} \mathrm{CI} / \mathrm{CI}$ Ratios in Phase III Leachates of Niche \#1 Core Samples as Linear (A) and Semi-Log (B) Plots 


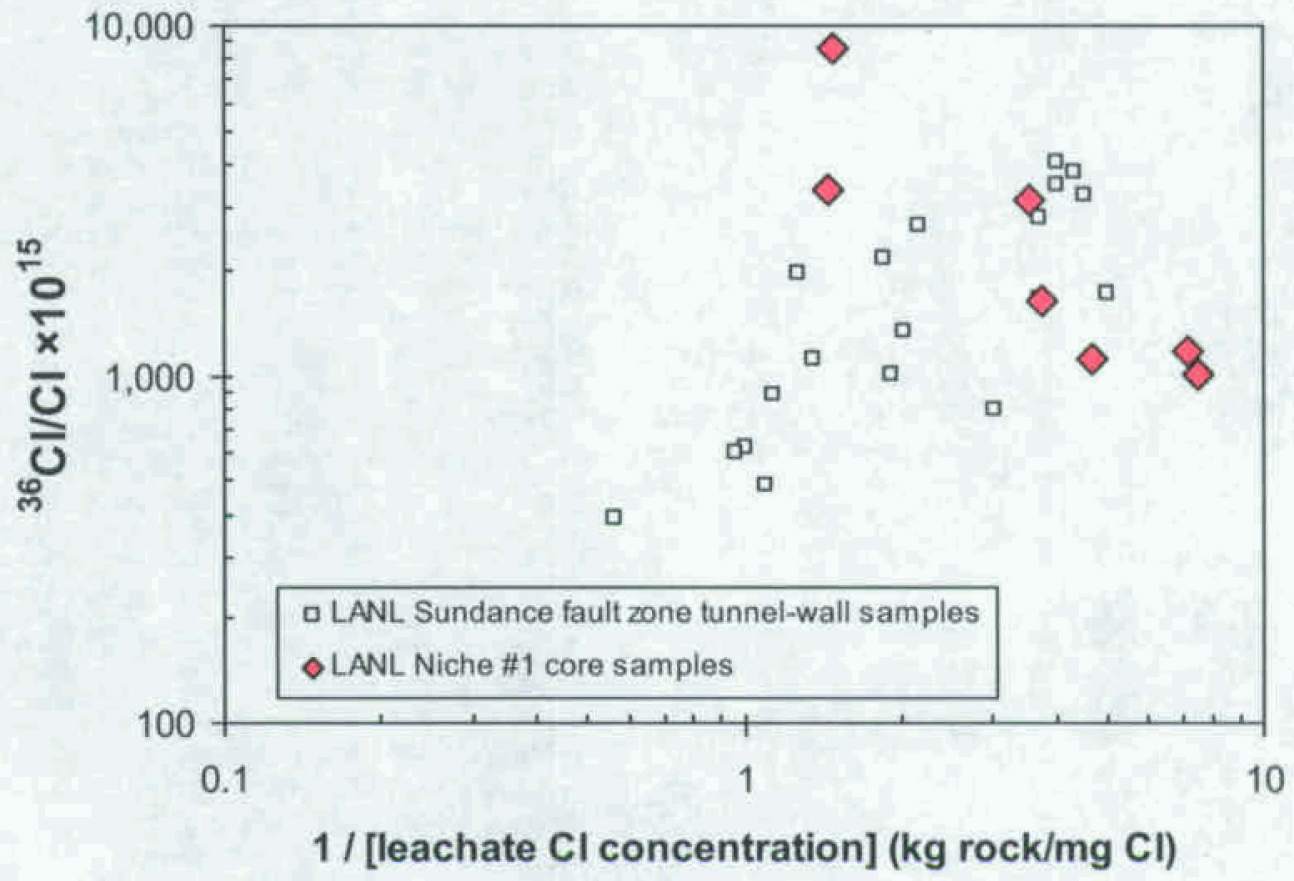

DTNs: LA0305RR831222.001 (UQ), LAJFB31222AQ98.004 (Q)

NOTE: LANL $=$ Los Alamos National Laboratory.

Figure 4-27. Comparison of Reciprocal Chloride Concentrations and ${ }^{36} \mathrm{CI} / \mathrm{CI}$ Ratios in Phase III Leachates of Samples from ESF Tunnel Walls (Sundance Fault Zone between Stations $34+28$ and $37+00$ ) and Niche \#1 Core 


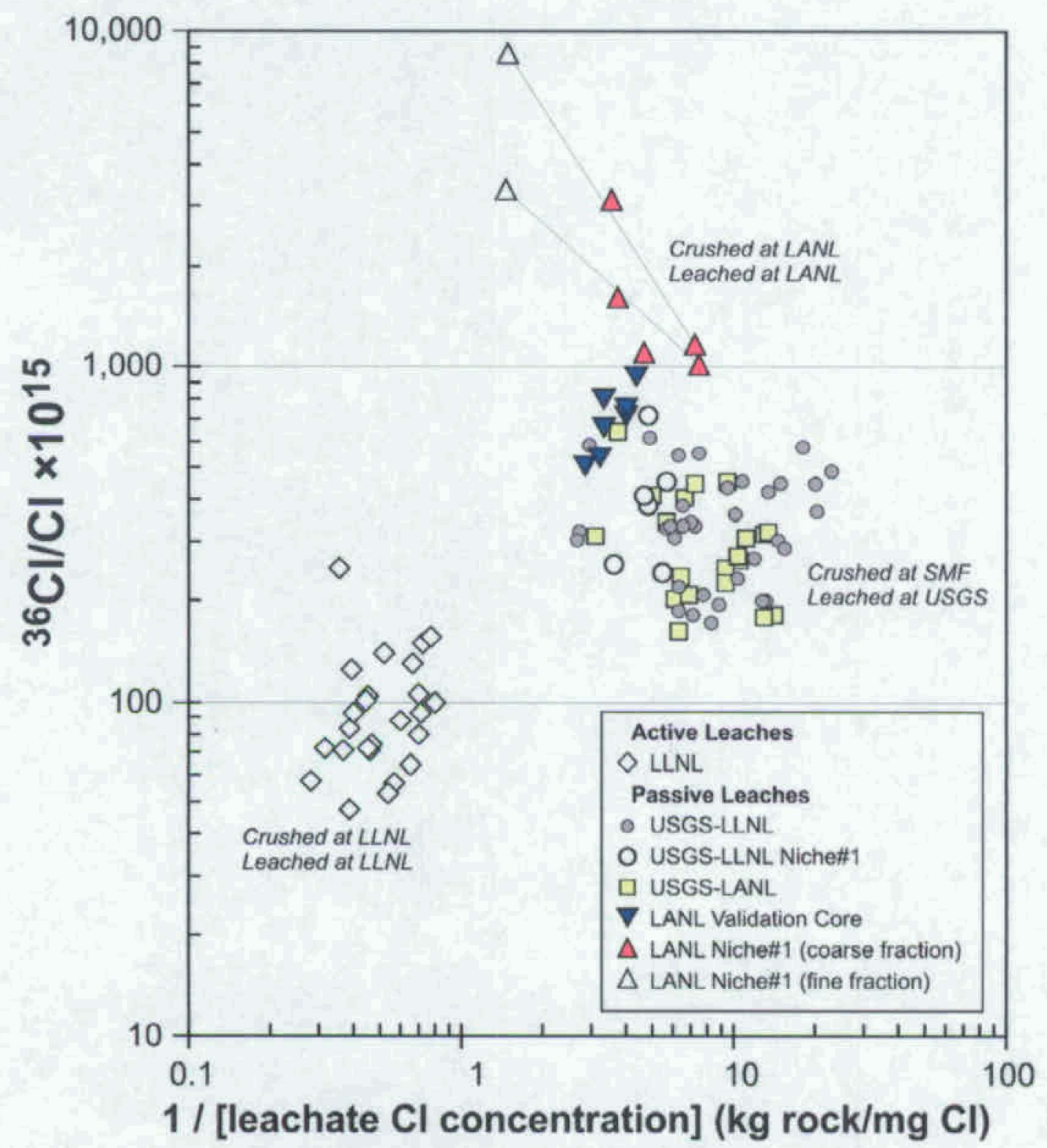

DTNs: LL031200223121.036 (Q), Filename: Total_AMS_Summary_2001-02c.xls); LL030408023121.027 (Q); LA0305RR831222.001(UQ)

NOTES: LANL = Los Alamos National Laboratory, LLNL = Lawrence Livermore National Laboratory, SMF = Sample Management Facility, USGS = U.S. Geological Survey.

Figure 4-28. Relations between Reciprocal Chloride Concentrations and ${ }^{36} \mathrm{Cl} / \mathrm{Cl}$ Ratios in Phase III Leachates of Validation Study Samples from the Sundance Fault Zone within the ESF 


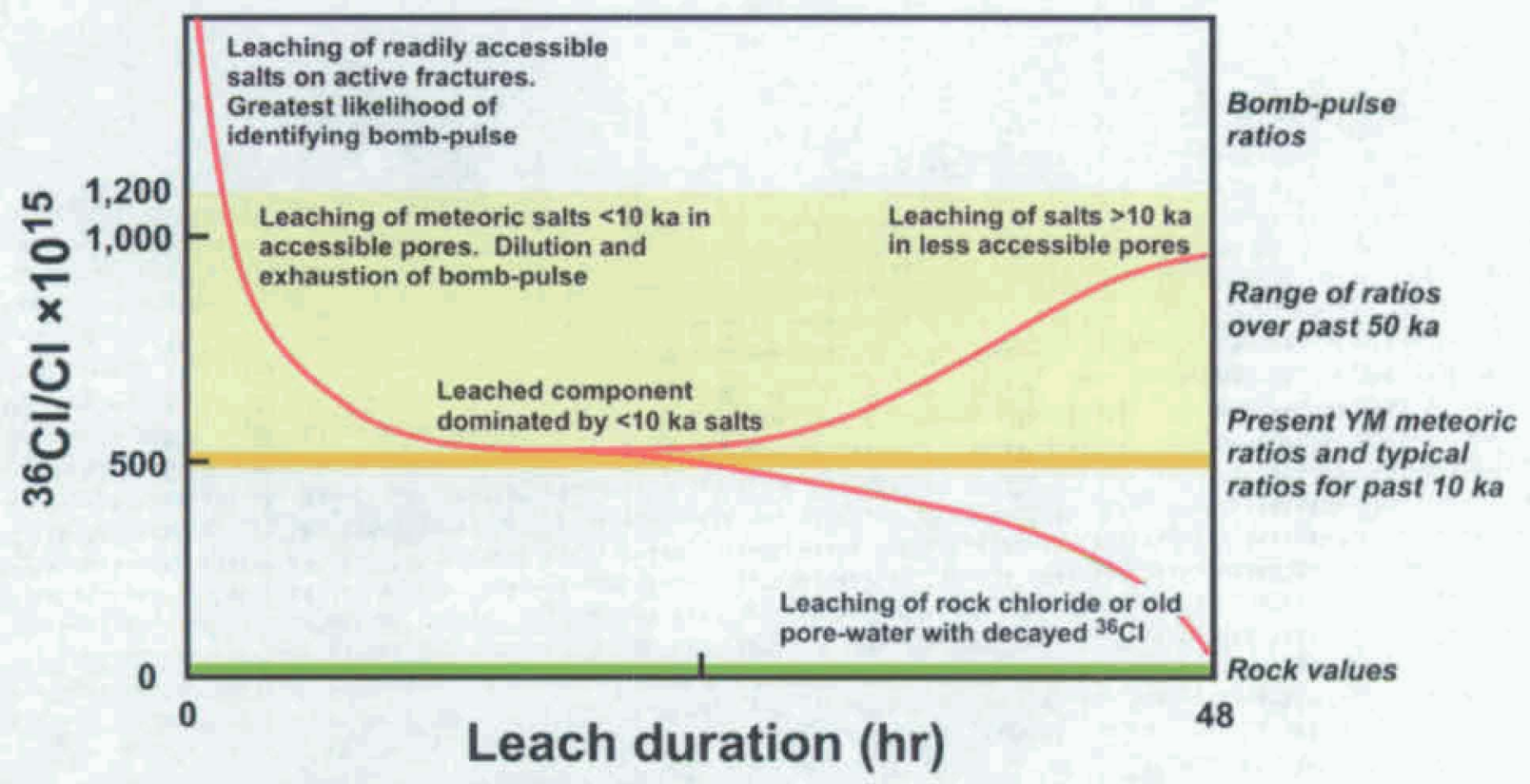

Source: Conceptualization based on chloride sources described in Fabryka-Martin et al.

(1997. Section 9); for illustration purposes only

NOTES: $\quad Y M=$ Yucca Mountain.

The red line shows a possible evolution pathway as leaching progresses; however, results of leaching experiments typically show sub-horizontal trends with only minor changes in ${ }^{36} \mathrm{Cl} / \mathrm{Cl}$ ratios (Section 4.3).

Figure 4-29. Conceptual Model of the Isotopic Evolution of ${ }^{36} \mathrm{CI} / \mathrm{Cl}$ Ratios in Passively Leached Solutions with Time 


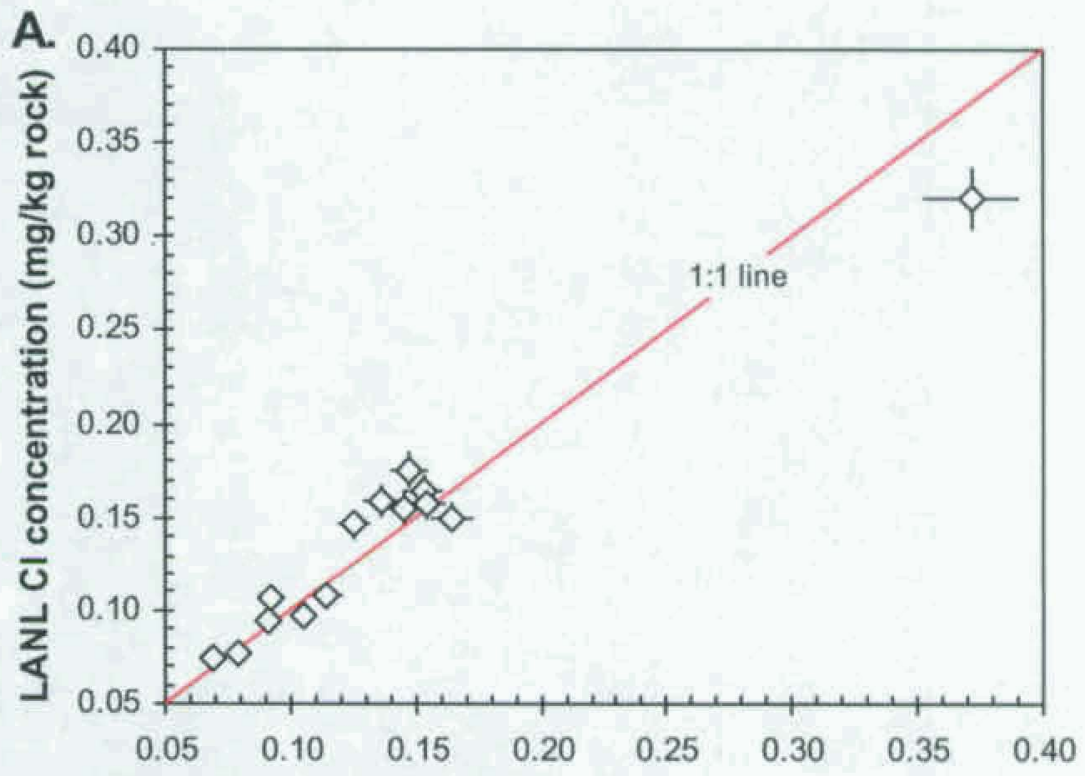

LLNL Cl concentration (mg/kg rock)

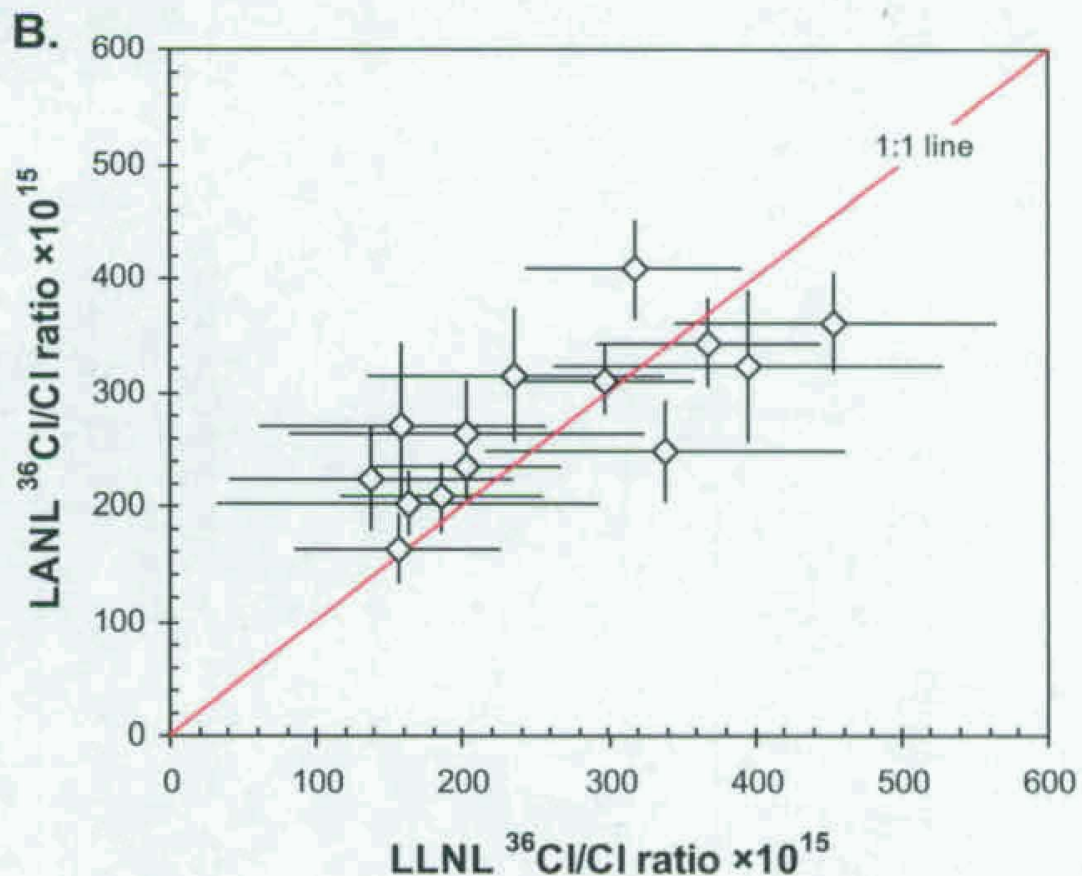

DTNs: LA0305RR831222.001 (UQ); LL031200223121.036 (Q), Filename; Total_AMS_Summary_2001-02c-xis

NOTES: $\quad$ LANL $=$ Los Alamos National Laboratory, LLNL = Lawrence Livermore National Laboratory. All targets were analyzed at the LLNL Center for Accelerator Mass Spectrometry (CAMS) facility. Sources of error (shown as $2 \sigma$ error bars) include in-run counting statistics, background and spike corrections, and corrections from blank.

Figure 4-30. Comparison of Chloride Concentrations (A) and ${ }^{36} \mathrm{Cl} / \mathrm{Cl}$ Ratios (B) in Aliquots of Validation Study Samples Passively Leached for 1 Hour at the USGS and Sent to LLNL and LANL for AgCl Target Preparation 

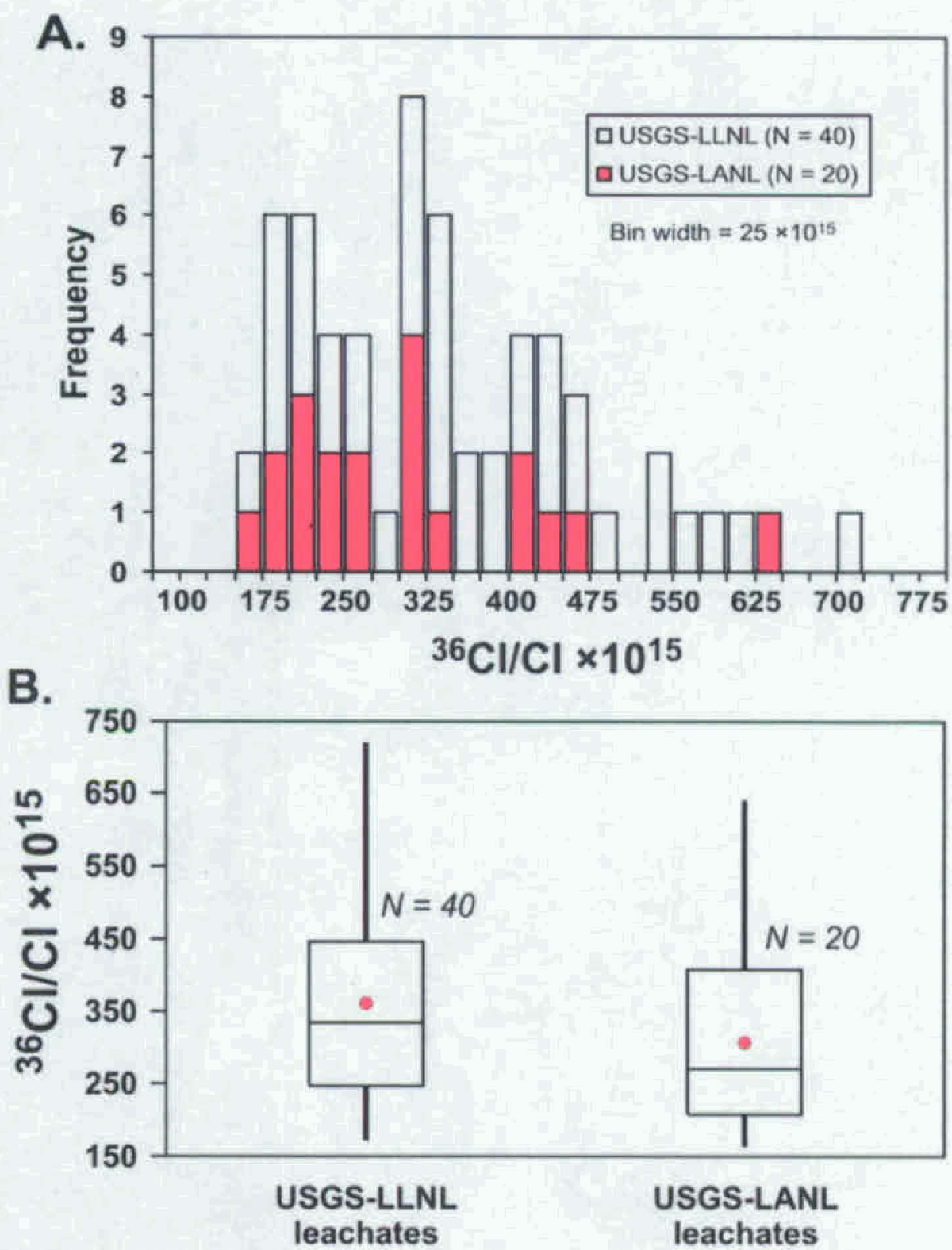

DTNs: LL031200223121.036 (Q), Filename: Total_AMS_Summary_2001-02c.xis); LA0305RR831222.001 (UQ)

NOTES: $\quad$ LANL $=$ Los Alamos National Laboratory, LLNL = Lawrence Livermore National Laboratory,

USGS = U.S. Geological Survey. Box plot shows range of data (vertical lines), middle two quartiles (boxes), median values (horizontal lines), and mean values (filled circles).

Figure 4-31. Frequency Distribution (A) and Box Plot (B) of ${ }^{36} \mathrm{CI} / \mathrm{Cl}$ Ratios in Leachates of Validation Study Core Leached at the USGS and Sent to LLNL and LANL for AgCl Precipitation and Analysis 


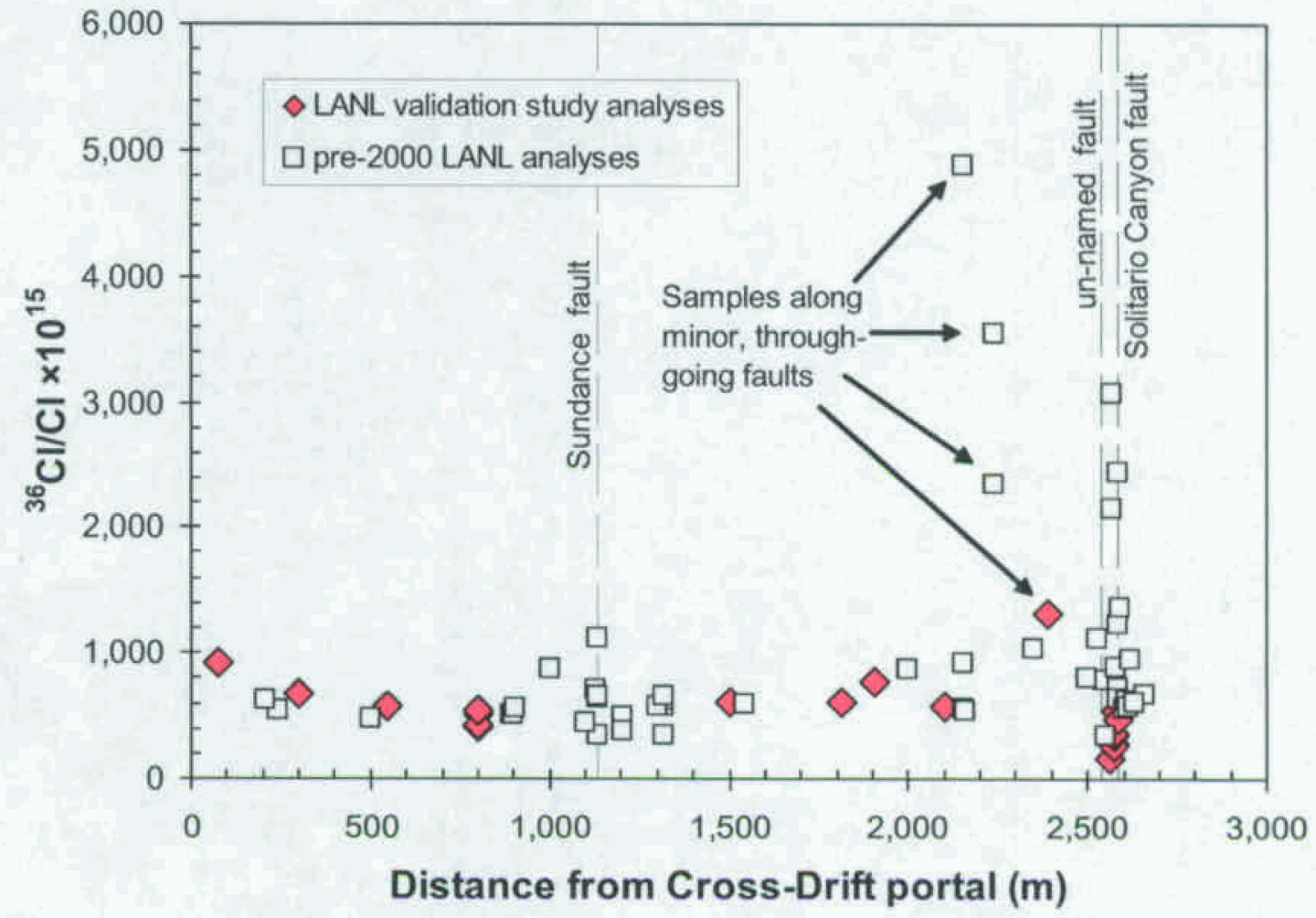

DTN: LA0305RR831222.001(UQ)

NOTES: $\quad$ LANL $=$ Los Alamos National Laboratory. Results of all sequential leachates processed between 0.5 and 2 hours are included.

Figure 4-32. Relations between ${ }^{36} \mathrm{CI} / \mathrm{CI}$ Ratios Determined at LANL and Distance in the ECRB Cross Drift 


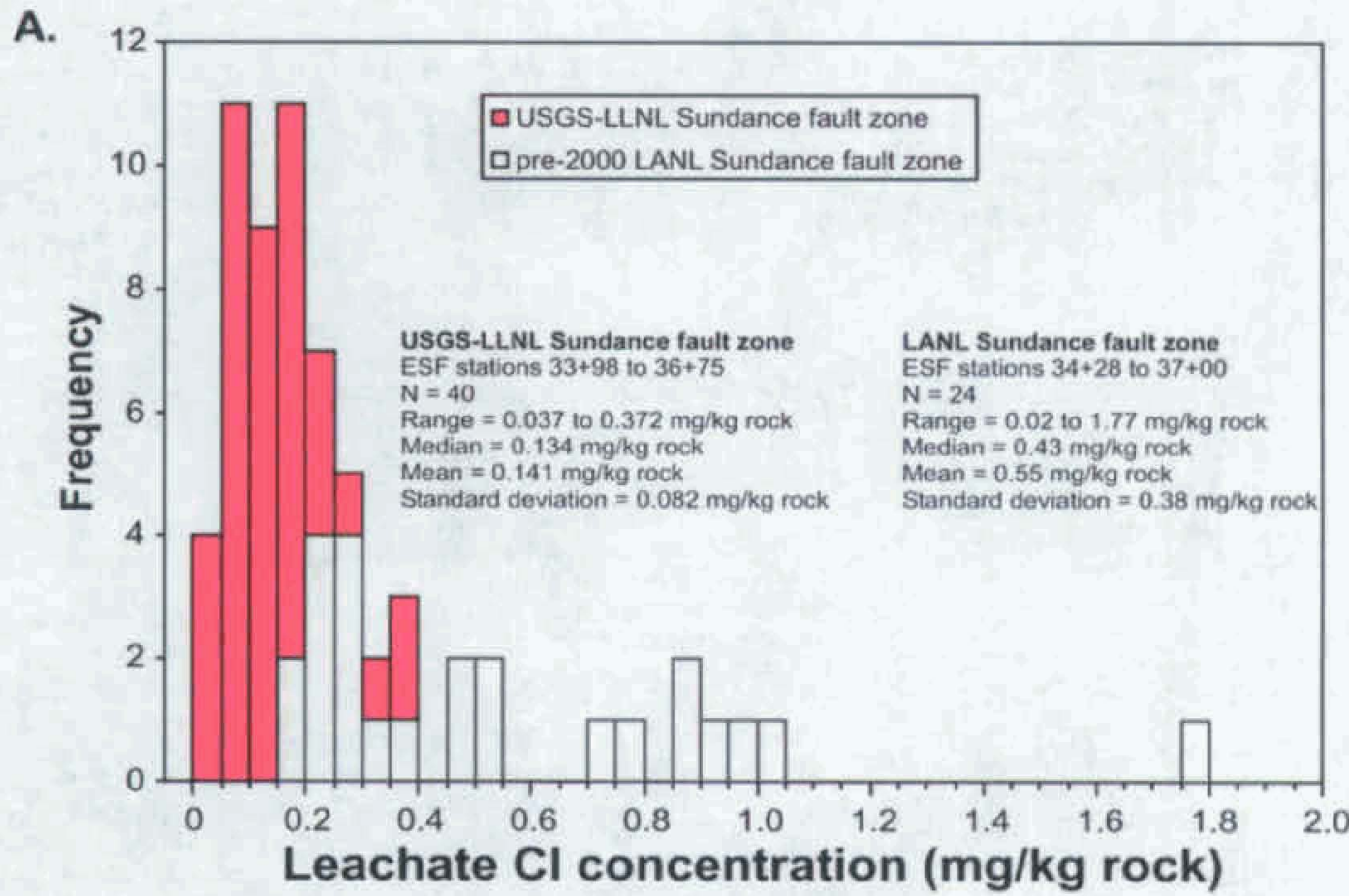

B.

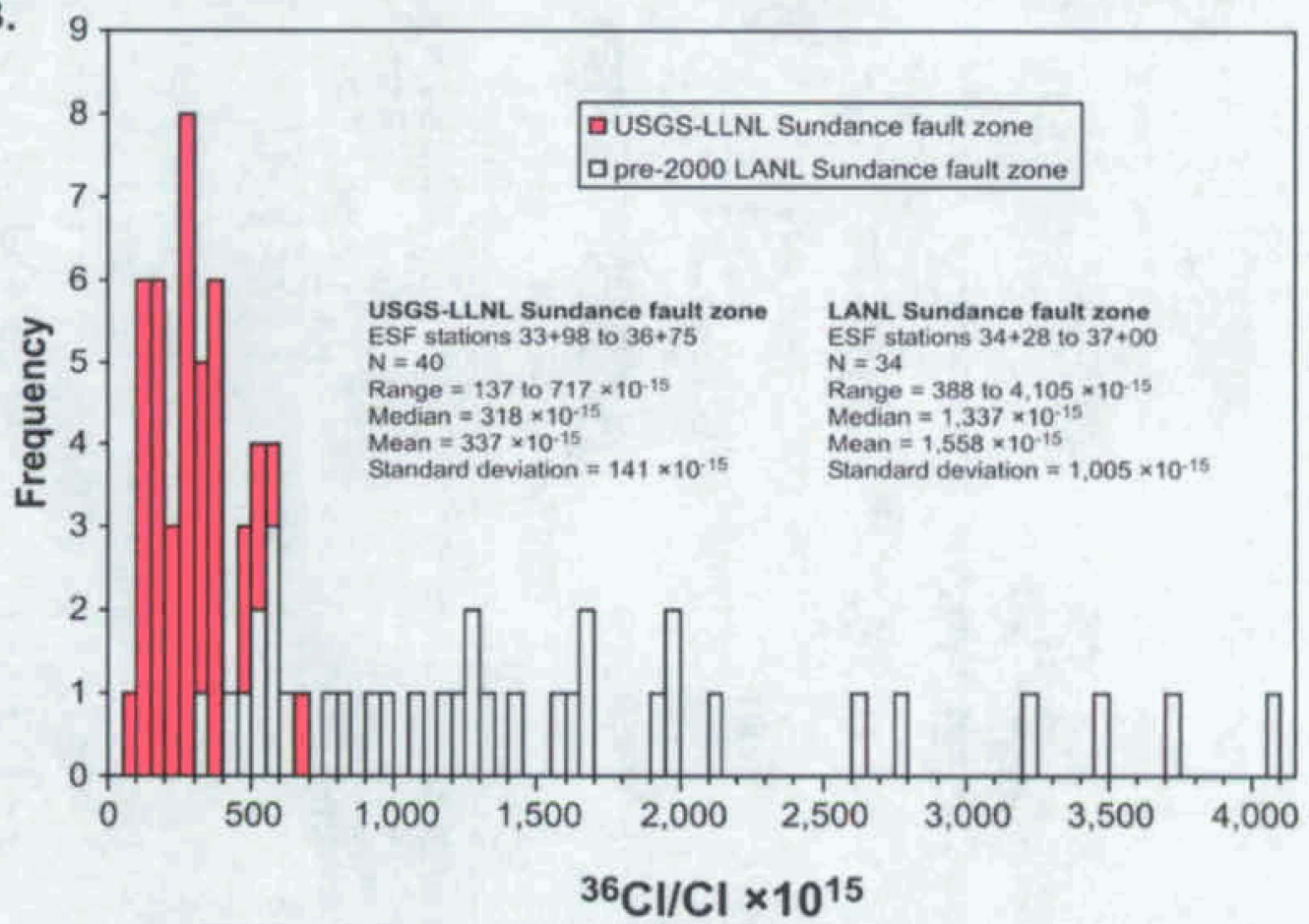

DTNs: LL031200223121.036 (Q), Filename: Total AMS Summary 2001-02c.xls; LAJF831222AQ98,004 (Q); LA0509JF831222.001 (Q)

NOTES: $\quad$ ESF $=$ Exploratory Studies Facility, $\mathrm{LANL}=\mathrm{Los}$ Alamos National Laboratory, LLNL $=$ Lawrence Livermore National Laboratory, USGS = U.S. Geological Survey.

Figure 4-33. Distribution of Chloride Concentrations $(A)$ and ${ }^{36} \mathrm{Cl} / \mathrm{Cl}$ Ratios $(B)$ in Leachates of Samples from the Sundance Fault Zone within the ESF 


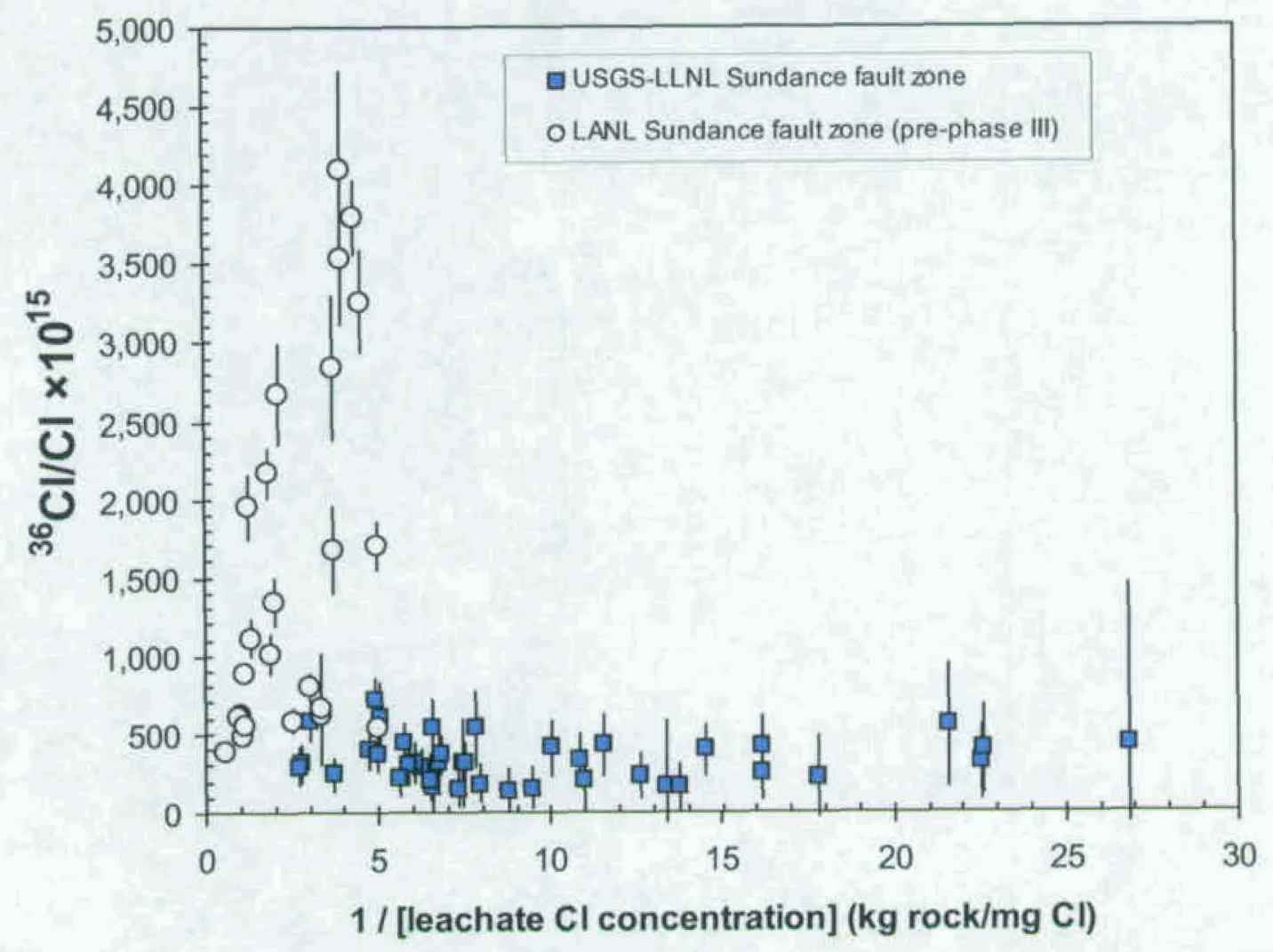

DTNs: LL031200223121.036 (Q). Filename: Total_AMS_Summary_2001-02c.xis); LAJF831222AQ98.004 (Q)

NOTES: $\quad$ LANL = Los Alamos National Laboratory, LLNL = Lawrence Livermore National Laboratory, USGS $=$ U.S. Geological Survey.

Figure 4-34. Relations between Reciprocal Chloride Concentrations and ${ }^{36} \mathrm{CI} / \mathrm{Cl}$ Ratios in Leachates of Samples from the Sundance Fault Zone 

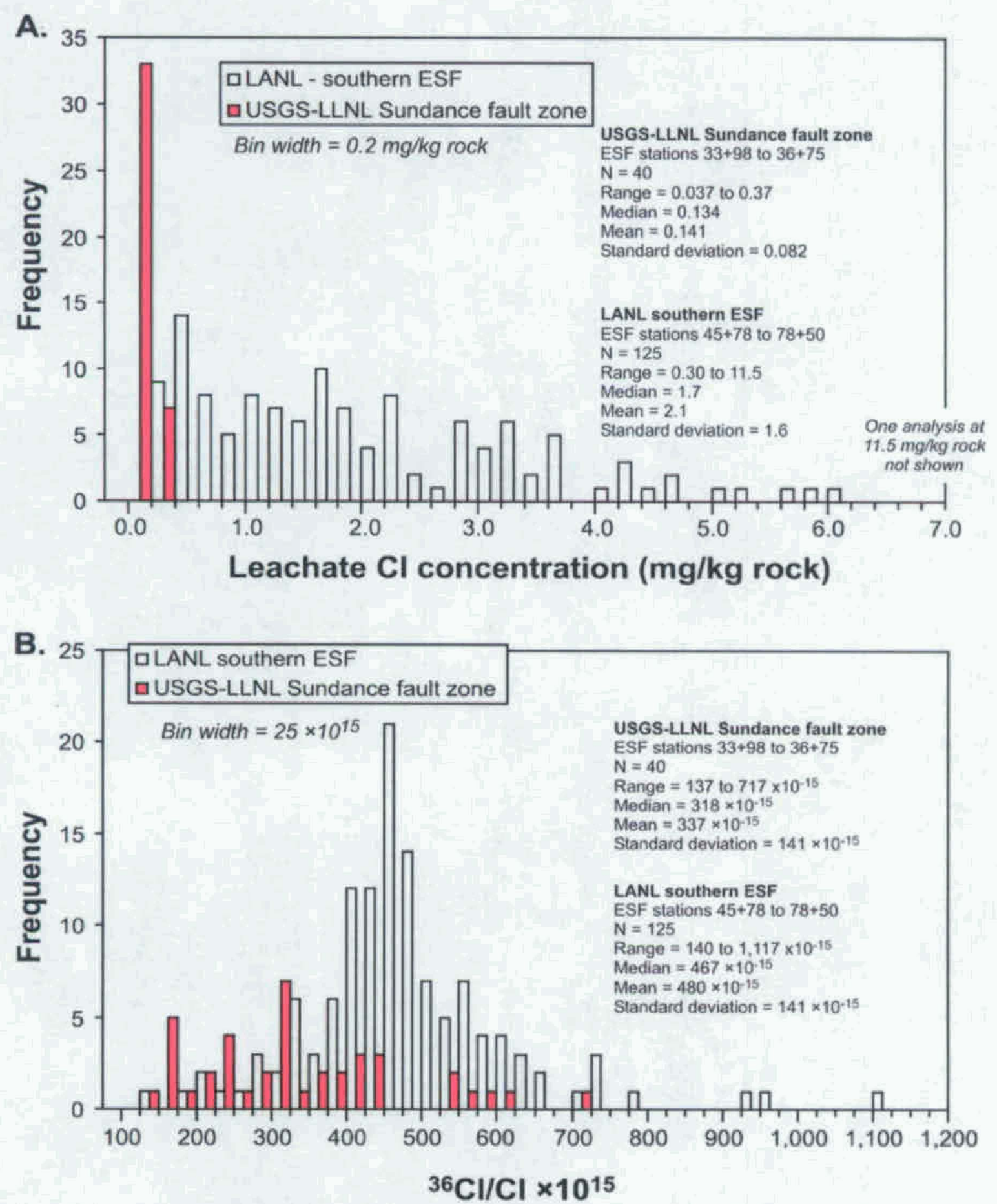

DTNs: LAJF831222AQ98.004 (Q); LL.031200223121.036 (Q), Filename: Total AMS Summary 2001-02c.xls NOTES: ESF $=$ Exploratory Studies Facility, LANL $=$ Los Alamos National Laboratory, LLNL = Lawrence Livermore National Laboratory, USGS $=$ U.S. Geological Survey.

Figure 4-35. Distribution of Chloride Concentrations $(A)$ and ${ }^{36} \mathrm{Cl} / \mathrm{Cl}$ Ratios $(B)$ in Leachates of USGS-LLNL Samples from the Sundance Fault Zone and LANL Samples from the Southern ESF 

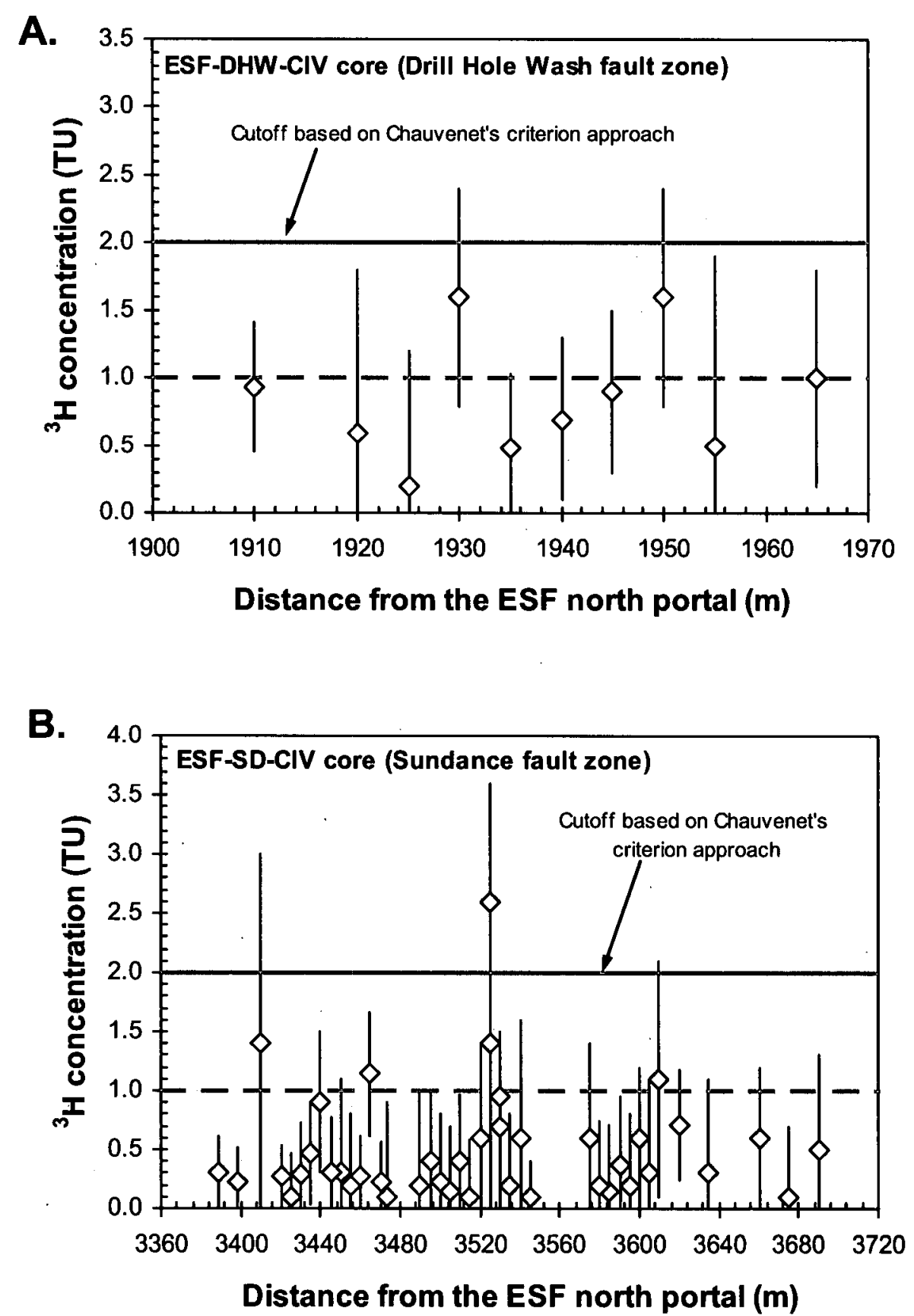

DTN: GS060308312272.001(Q)

NOTES: ESF = Exploratory Studies Facility, TU = tritium unit. Dashed horizontal line represents 1 TU background cutoff proposed initially. Solid horizontal line represents 2 TU background cutoff based on statistical criterion from $135{ }^{3} \mathrm{H}$ measurements. Error bars are $2 \sigma$. Borehole locations are listed in Table 3-2.

Figure 5-1. Distribution of Tritium Concentrations in Samples of Pore Water Extracted from Validation Study Core along the Drill Hole Wash Fault Zone (A) and Sundance Fault Zone (B) 


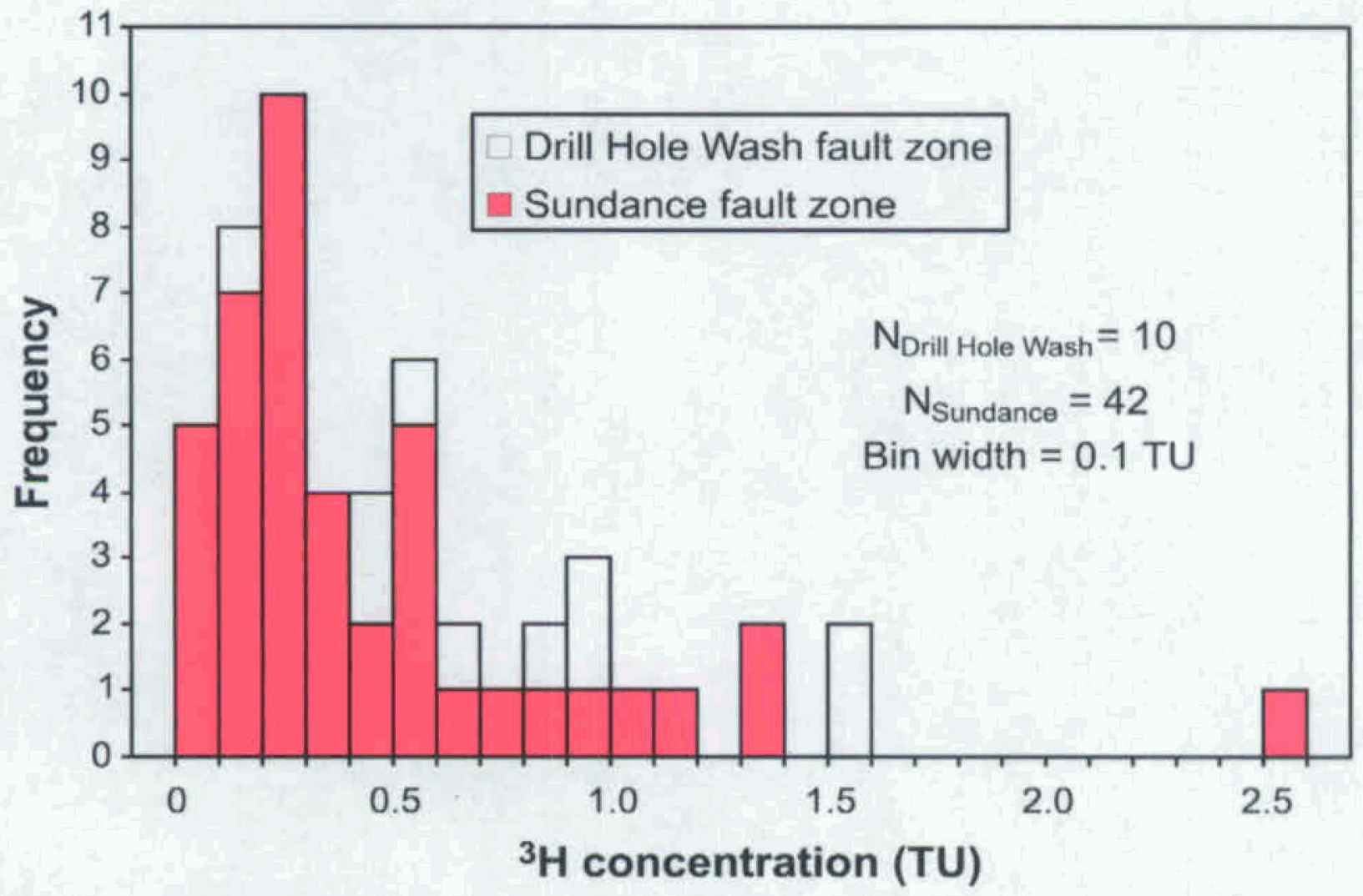

DTN: GS060308312272.001 (Q)

NOTE: $\quad$ TU $=$ tritium unit.

Figure 5-2. Frequency Distribution of Tritium Concentrations in Pore Water from Validation Study Core Samples 

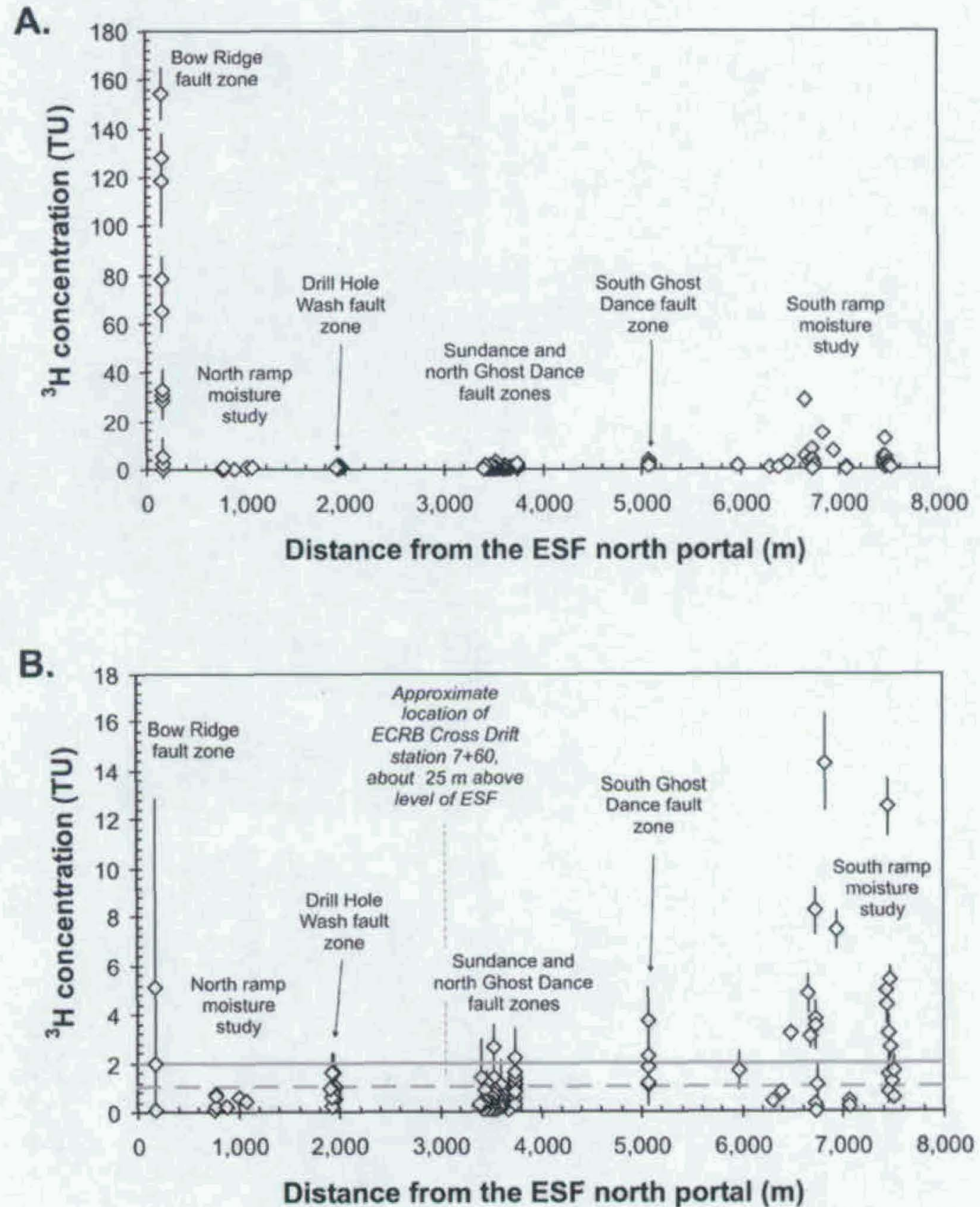

DTNs: GS040108312232.001 (Q), GS060308312272.001 (Q). GS060383122410.001(Q), GS961108312261,006 (Q)

NOTES: $E C R B=$ Enhanced Characterization of the Repository Block, ESF $=$ Exploratory Studies Facility, TU $=$ tritium unit. The same data are plotted at both full scale $(A)$ to show large ${ }^{3} \mathrm{H}$ concentrations in samples from the Bow Ridge fault zone and one sample from the South Ramp Moisture Study area, and at a reduced scale $(B)$ to show variations in low- $\mathrm{H}$ concentration samples. Error bars are $2 \sigma$. Dashed horizontal line represents 1 TU background cutoff proposed initially. Solid horizontal line represents $2 \mathrm{TU}$ background cutoff based on statistical criterion from $135^{3} \mathrm{H}$ measurements. Borehole locations are listed in Table 3-2.

Figure 5-3. Distribution of Tritium Concentrations Plotted at Full Scale (A) and at a Reduced Scale (B) in Samples of Pore Water Extracted from Drill Core throughout the ESF 


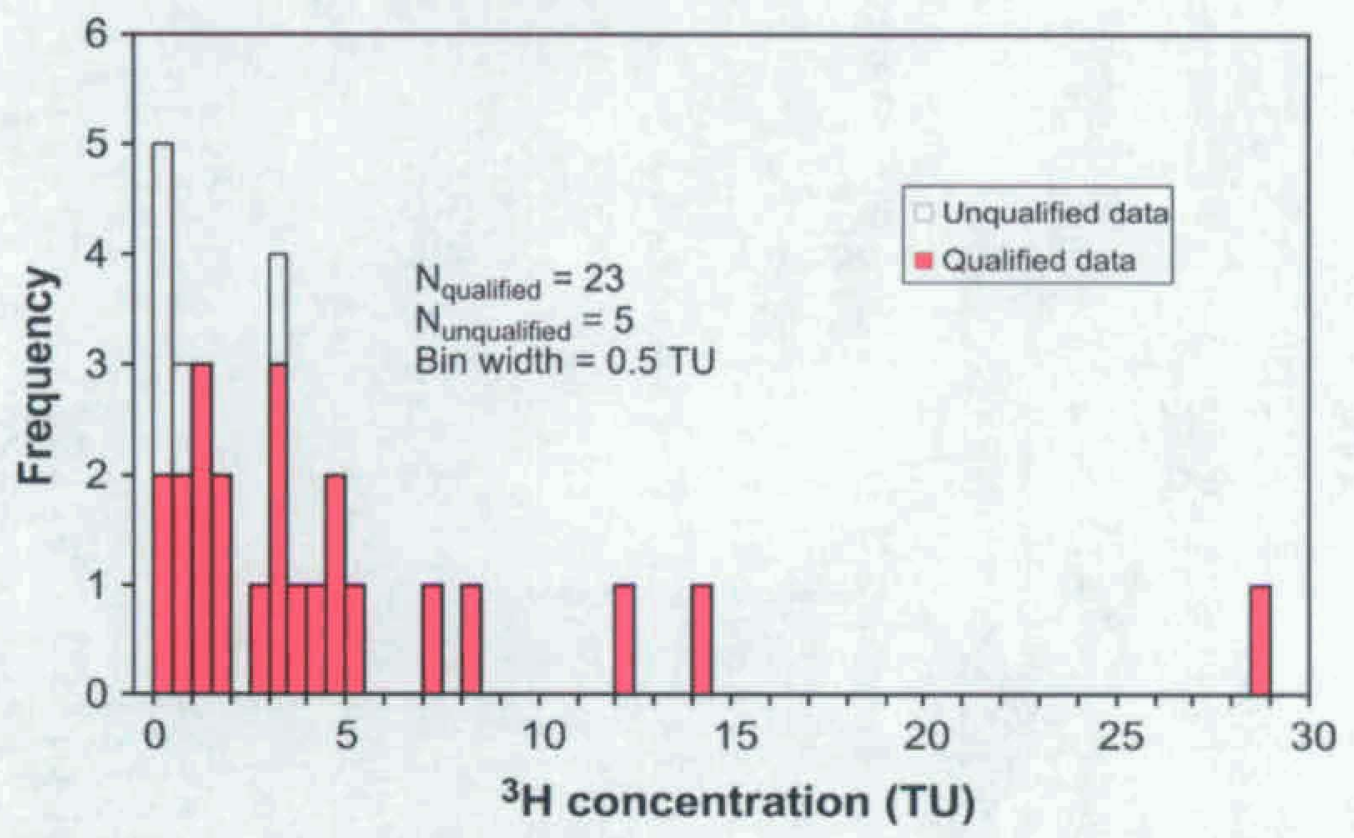

DTNs: GS060308312272.001(Q), GS060383122410.001 (UQ)

NOTE: $\quad$ TU $=$ tritium unit.

Figure 5-4. Frequency Distribution of Tritium Concentrations in Pore Water from Boreholes along the ESF South Ramp 


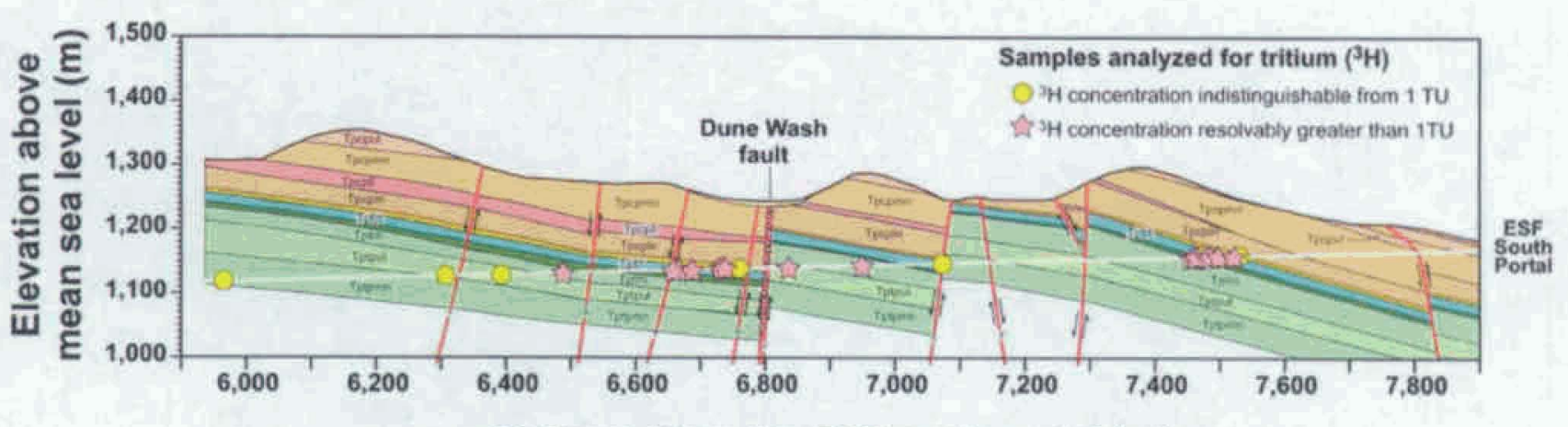

Quaternary

\section{Distance from the ESF north portal $(m)$}

\begin{tabular}{|c|c|}
\hline Onc & fal and collivial deposits \\
\hline \multicolumn{2}{|c|}{ Paintbrush Group (Miocene) } \\
\hline \multicolumn{2}{|c|}{ Tiva Canyon Tuff } \\
\hline \multicolumn{2}{|c|}{ Crystal-poor menter } \\
\hline Tpcopd & Upper ithophysal zone \\
\hline Tecpum & $\begin{array}{l}\text { Upper inephysat zone and middt } \\
\text { noplithephysal zone undivided }\end{array}$ \\
\hline Tpcorni & Middie nontithophysal zone \\
\hline Trapet & Lower Nthophysal rone \\
\hline Trepin & Lower nonithogtryal zone \\
\hline Tpcoser & Crystal-poor vitric zone \\
\hline
\end{tabular}

Bedded tuffs

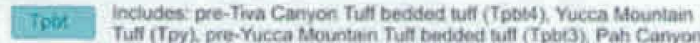

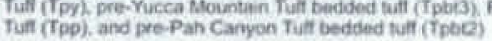

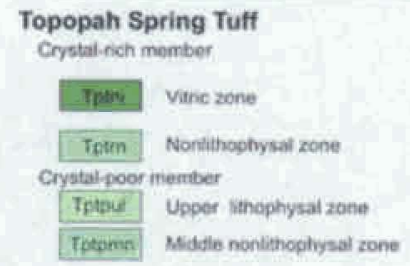

Source: Modified from USBR (1997)

DTNs: GS060308312272.001 (Q), GS060383122410.001 (UQ)

NOTES: ESF = Exploratory Studies Facility, $\mathrm{TU}=$ tritium unit. Sample locations are from Table 5-3.

Figure 5-5. Geologic Section of the ESF South Ramp Showing Locations of Samples Analyzed for Tritium 


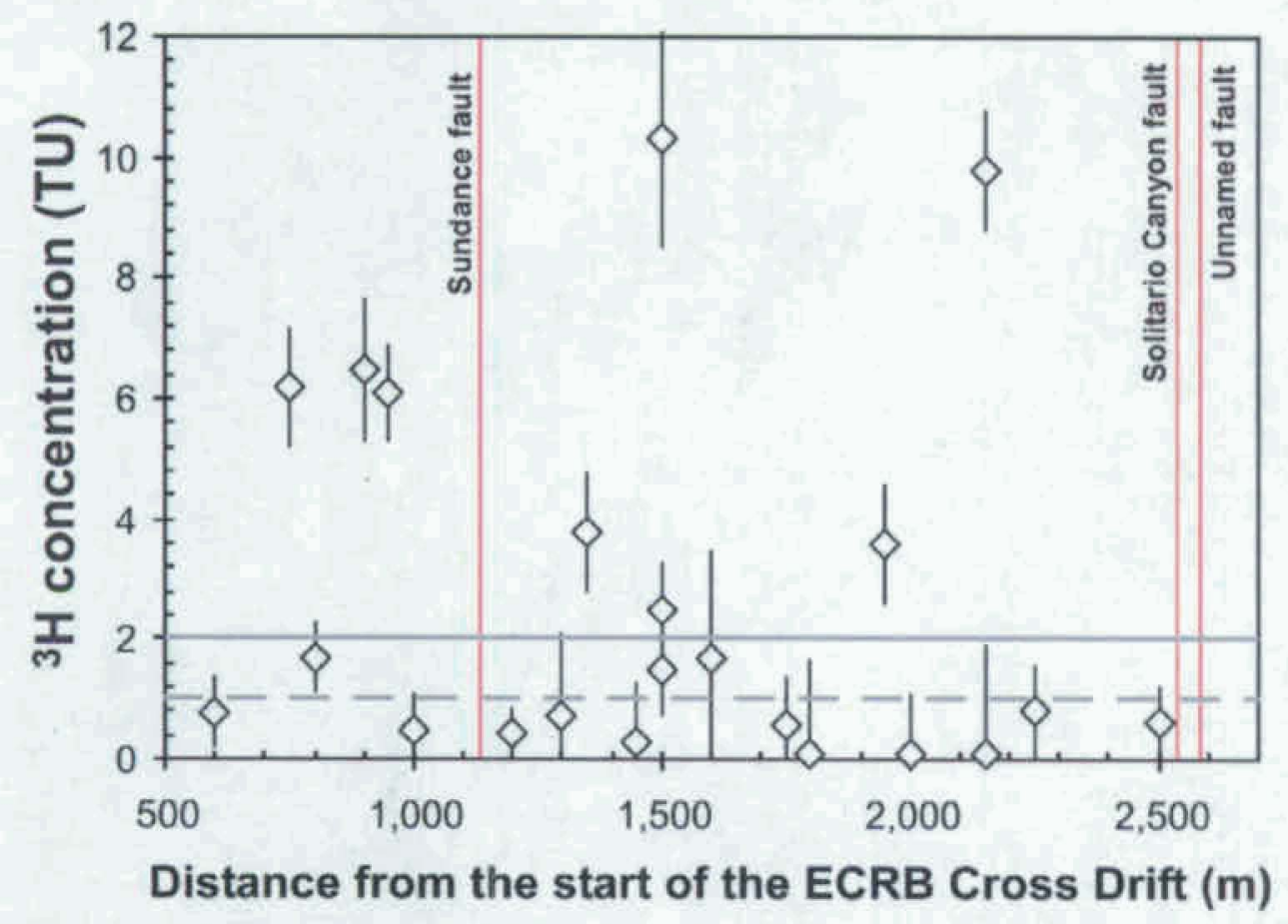

DTN: G\$060308312272.002 (Q)

NOTES: $E C R B=$ Enhanced Characterization of the Repository Block, TU $=$ tritium unit. Major faults are shown as vertical red lines. Error bars are $2 \sigma$. Dashed horizontal line represents 1 TU background cutoff proposed initially. Solid horizontal line represents 2 TU background cutoff based on statistical criterion from 135 tritium measurements.

Figure 5-6. Distribution of Tritium Concentrations in Samples of Pore Water Extracted from Drill Core along the ECRB Cross Drift 


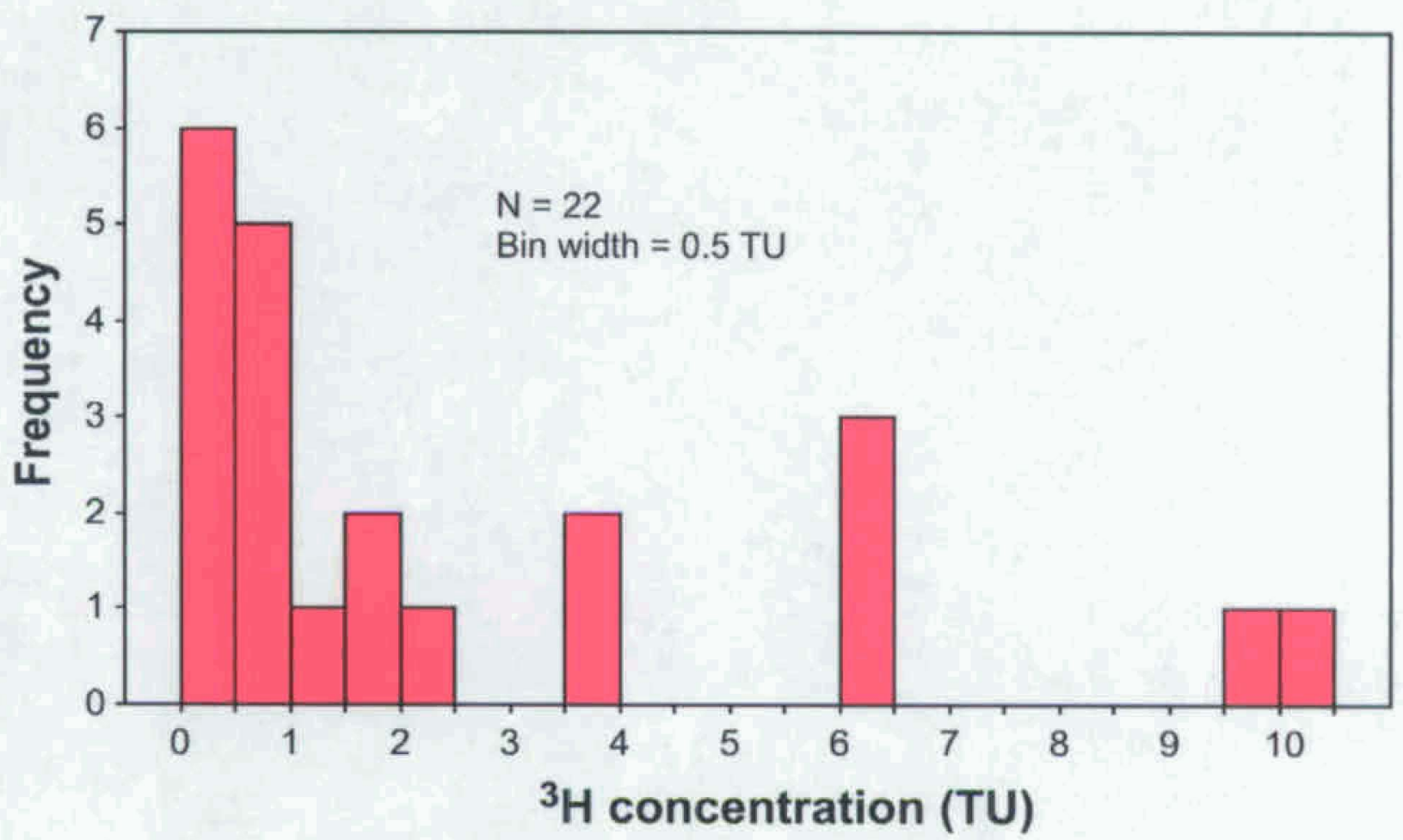

DTN: GS060308312272.002 (Q)

NOTE: $\quad$ TU $=$ tritium unit.

Figure 5-7. Frequency Distribution of Tritium Concentrations in Pore Water from ECRB Cross Drift Drill Core 


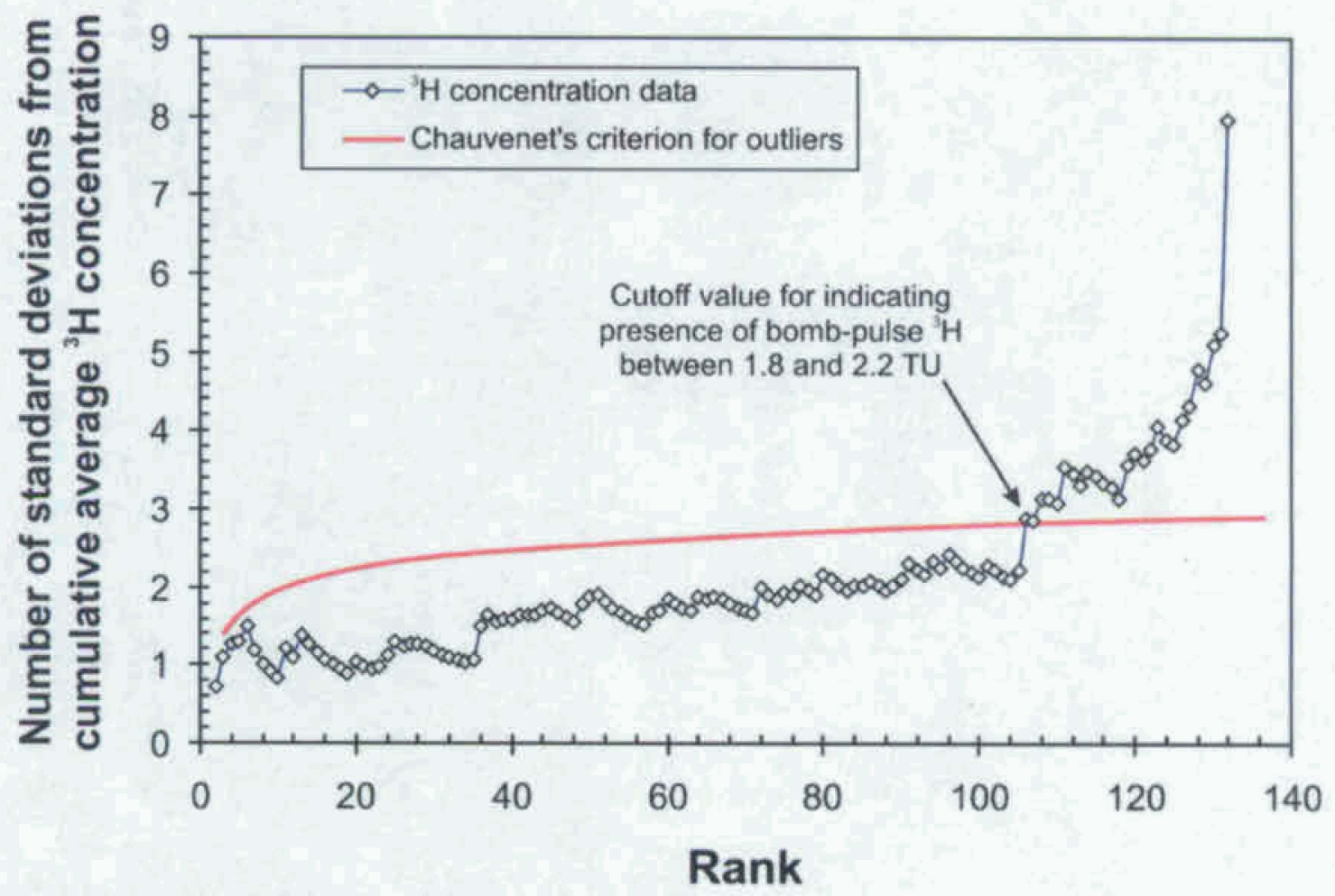

DTNs: GS060308312272.001 (Q), GS060383122410.001 (UQ), GS060308312272.002 (Q);

Chauvenet's criterion plotted as in Fabryka-Martin et al. (1997, Figure 4-6)

NOTE: $\quad T U=$ tritium unit.

Figure 5-8. Application of Chauvenet's Criterion to Establish a Cutoff Tritium Concentration for Identifying the Presence of Bomb-Pulse Tritium in Samples from the ESF and ECRB Cross Drift (USGS) 


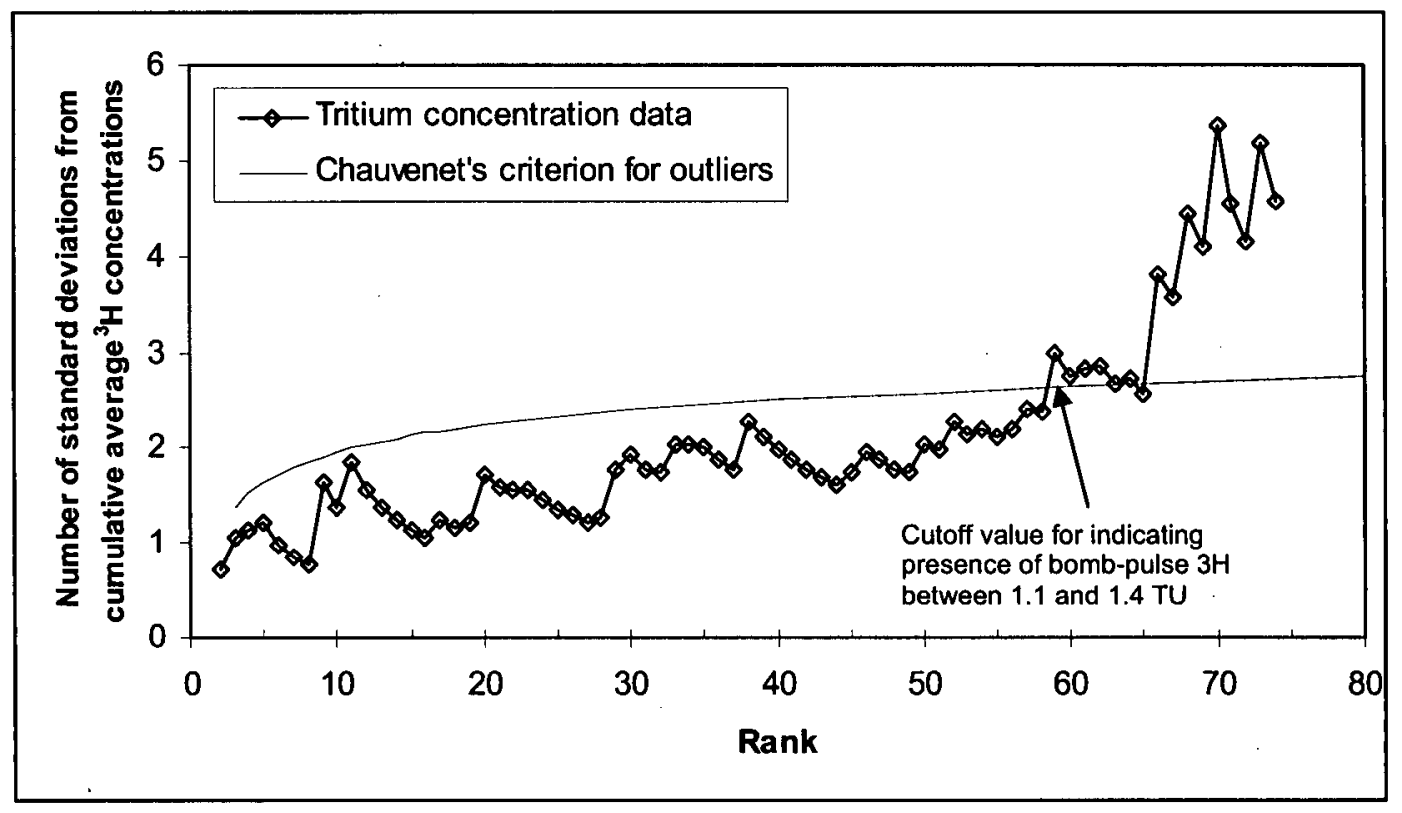

DTNs: GS060308312272.001 (Q), GS060308312272.002 (Q);

Chauvenet's criterion plotted as in Fabryka-Martin et al. (1997, Figure 4-6)

NOTE: $\quad$ TU $=$ tritium unit.

Figure 5-9. Application of Chauvenet's Criterion to Establish a Cutoff Tritium Concentration for Identifying the Presence of Bomb-Pulse Tritium in Validation Study Boreholes and ECRB Cross Drift Samples (LANL) 


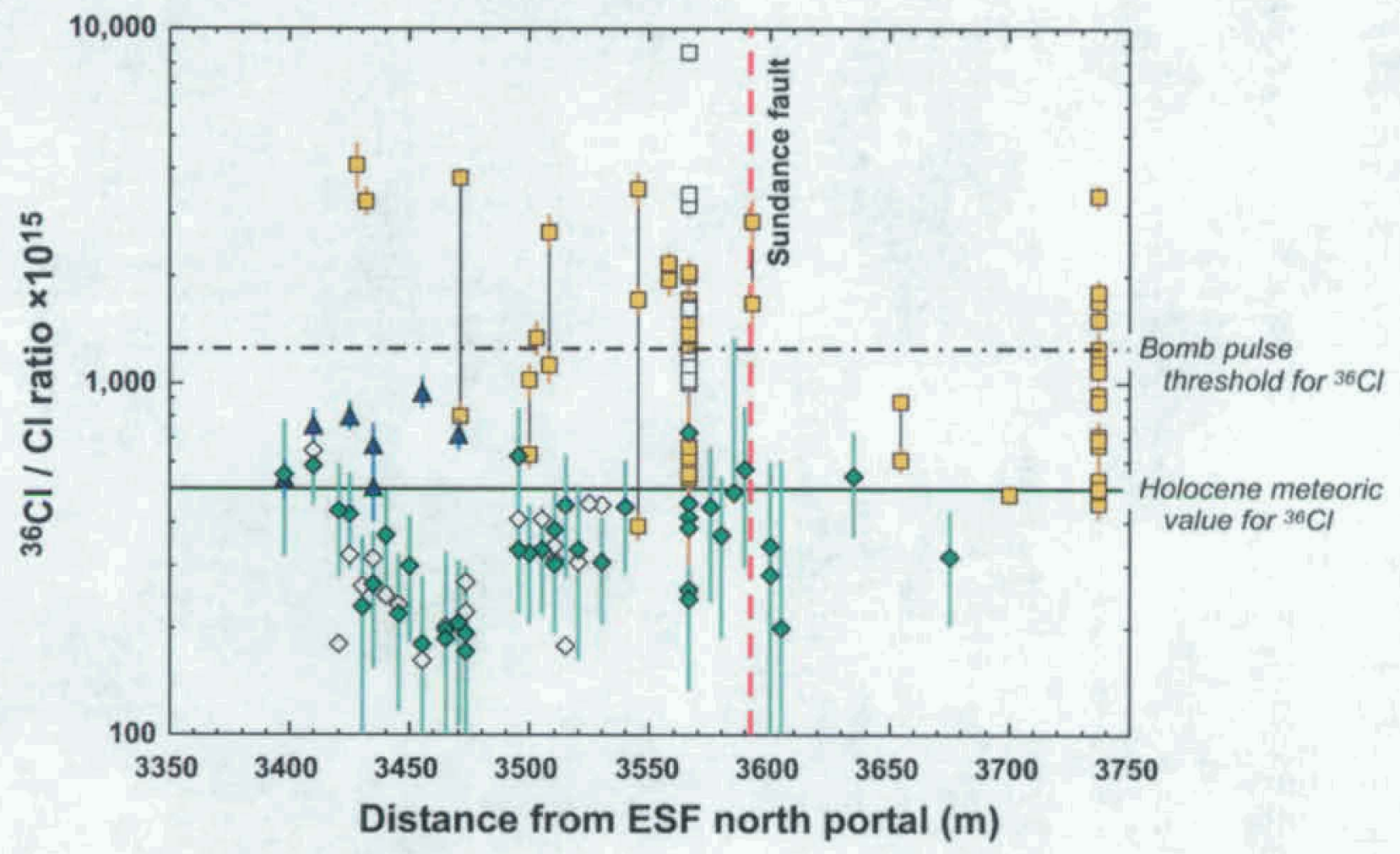

$\square$ LANL (Validation Phase III)

USGS-LLNL (Validation Phase III)

$\diamond$ USGS-LANL (Validation Phase III)

$\triangle$ LANL (Validation Phase I)

$\square$ LANL (through 1998)

DTNs: LA0305RR831222.001 (UQ); LA0307RR831222,002 (UQ); LA0509JF831222.001 (Q); LAJF831222AQ98.004 (Q);

LL031200223121.036 (Q), Filename: Total_AMS_Summary_2001-02c.xis

NOTES: ESF $=$ Exploratory Studies Facility.

Error bars are $2 \sigma$.

USGS-LLNL = Samples leached at USGS, processed (i.e., target preparation) at LLNL, and analyzed at LLNL.

USGS-LANL = Samples leached at USGS, processed (i.e., target preparation and spiking) at LANL, and analyzed at LLNL. Errors for these data are similar to USGS-LLNL data (error bars are not shown for these data because they overlap with error bars shown for the USGS-LLNL data).

Figure 6-1. Relations between ${ }^{36} \mathrm{Cl} / \mathrm{CI}$ Ratios in Validation Study Samples from the Sundance Fault Zone and ${ }^{36} \mathrm{CI} / \mathrm{Cl}$ Ratios in Samples from the Same Area Reported by LANL in 1996, 1997, and 1998. 
INTENTIONALLY LEFT BLANK 
Table 3-1. Chronology of Locations and Personnel Directly Involved in the Preparation and Analysis of LANL ${ }^{36} \mathrm{CI}$ Samples

\begin{tabular}{|c|c|c|c|c|}
\hline $\begin{array}{c}\text { Principal Investigator } \\
\text { of }{ }^{36} \mathrm{Cl} \text { Activity }\end{array}$ & $\begin{array}{l}\text { Location of } \\
\text { Sample } \\
\text { Preparation }\end{array}$ & $\begin{array}{l}\text { Laboratory } \\
\text { Supervisor of } \\
\text { Sample } \\
\text { Preparation } \\
\end{array}$ & $\begin{array}{l}\text { Analytical } \\
\text { Facility }\end{array}$ & Examples of ${ }^{36} \mathrm{Cl} / \mathrm{Cl}$ Results \\
\hline $\begin{array}{l}\text { Kurt Wolfsberg } \\
\text { (until 1984) }\end{array}$ & $\begin{array}{l}\text { Hydro Geo } \\
\text { Chem (Tucson) }\end{array}$ & Harold Bentley & \multirow[t]{2}{*}{$\begin{array}{l}\text { University of } \\
\text { Rochester }\end{array}$} & \multirow{2}{*}{$\begin{array}{l}\text { Background ratios and bomb- } \\
\text { pulse in soil profiles; bomb- } \\
\text { pulse in UZ-1 cuttings; bomb- } \\
\text { pulse in G Tunnel samples; } \\
\text { in situ ratios in Yucca Mountain } \\
\text { tuff. }\end{array}$} \\
\hline $\begin{array}{l}\text { Ted Norris } \\
(1984-1990)\end{array}$ & $\begin{array}{l}\text { Hydro Geo } \\
\text { Chem (Tucson) }\end{array}$ & $\begin{array}{l}\text { Seth Gifford } \\
(1984-1988) \\
\text { Songlin Cheng } \\
(1988-1990) \\
\text { Sun Maida } \\
(1990-1992)\end{array}$ & & \\
\hline \multirow[t]{4}{*}{$\begin{array}{l}\text { June Fabryka-Martin } \\
(1990-2000)\end{array}$} & $\begin{array}{l}\text { Hydro Geo } \\
\text { Chem (Tucson, } \\
\text { until 1994) }\end{array}$ & $\begin{array}{l}\text { Susan Maida } \\
(1990-1992) \\
\text { Scott Wightman } \\
(1992-1995)\end{array}$ & $\begin{array}{l}\text { University of } \\
\text { Rochester (until } \\
\text { 1992); LLNL } \\
\text { (1992-1994); } \\
\text { PRIME Lab } \\
\text { (1993-2000) }\end{array}$ & $\begin{array}{l}\text { Inter-laboratory comparisons of } \\
\text { blanks, standards, samples; } \\
\text { background ratio and bomb- } \\
\text { pulse in soil profiles; bomb- } \\
\text { pulse in neutron hole cuttings. }\end{array}$ \\
\hline & $\begin{array}{l}\text { LANL (May } 1994 \\
\text { until May 2000, } \\
\text { TA-48, Bidg. 45) }\end{array}$ & $\begin{array}{l}\text { Scott Wightman } \\
\text { Beiling Liu } \\
\text { Paul Dixon } \\
\text { Jeff Roach } \\
\text { Robert Roback }\end{array}$ & PRIME Lab & $\begin{array}{l}\text { Bomb-pulse in the ESF; bomb- } \\
\text { pulse in runoff; and bomb- } \\
\text { pulse in the ECRB Cross Drift. }\end{array}$ \\
\hline & PRIME Lab & Stephen Vogt & PRIME Lab & Rock ${ }^{36} \mathrm{Cl} / \mathrm{Cl}$ (no bomb-pulse). \\
\hline & $\begin{array}{l}\text { New Mexico } \\
\text { Tech (Socorro) }\end{array}$ & Mitch Plummer & PRIME Lab & $\begin{array}{l}\text { Pack rat samples; background } \\
\text { and bomb-pulse ratios. }\end{array}$ \\
\hline $\begin{array}{l}\text { Robert Roback } \\
\text { (2000- present) }\end{array}$ & $\begin{array}{l}\text { LANL (TA-03, } \\
\text { Bldg. 215) }\end{array}$ & $\begin{array}{l}\text { Robert Roback } \\
\text { Catherine Jones }\end{array}$ & $\begin{array}{l}\text { PRIME Lab } \\
\text { LLNL }\end{array}$ & $\begin{array}{l}\text { ECRB Cross Drift, one sample } \\
\text { with bomb-pulse; ESF } \\
\text { Niche \#1; validation study } \\
\text { core; sequential leaching } \\
\text { experiments. }\end{array}$ \\
\hline
\end{tabular}

Compiled by R.C. Roback May 26, 2005 
Table 3-2. Validation Study Boreholes

\begin{tabular}{|c|c|c|c|c|}
\hline Fault Zone & Borehole Identifier & ESF Station & $\begin{array}{c}\text { Date } \\
\text { Completed }\end{array}$ & $\begin{array}{c}\text { Total Depth } \\
\text { (m) }\end{array}$ \\
\hline \multirow[t]{12}{*}{ Sundance } & ESF-SD-CIV\#1 & $36+89$ & $06 / 17 / 99$ & 4 \\
\hline & ESF-SD-CIV\#2 & $36+74$ & $06 / 16 / 99$ & 4 \\
\hline & ESF-SD-CIV\#3 & $36+59$ & $06 / 15 / 99$ & 4 \\
\hline & ESF-SD-CIV\#4 & $36+35$ & $06 / 14 / 99$ & 4 \\
\hline & ESF-SD-CIV\#5 & $36+20$ & $06 / 10 / 99$ & 4 \\
\hline & ESF-SD-CIV\#6 & $36+10$ & $06 / 10 / 99$ & 4 \\
\hline & ESF-SD-CIV\#7 & $36+05$ & $06 / 08 / 99$ & 4 \\
\hline & ESF-SD-CIV\#8 & $36+00$ & $06 / 08 / 99$ & 4 \\
\hline & ESF-SD-CIV\#9 & $35+95$ & $06 / 07 / 99$ & 4 \\
\hline & ESF-SD-CIV\#10 & $35+90$ & $06 / 03 / 99$ & 4 \\
\hline & ESF-SD-CIV\#11 & $35+85$ & $06 / 03 / 99$ & 4 \\
\hline & ESF-SD-CIV\#12 & $35+80$ & $06 / 02 / 99$ & 4 \\
\hline \multirow[t]{28}{*}{. } & ESF-SD-CIV\#13 & $35+75$ & $06 / 02 / 99$ & 10 \\
\hline & ESF-SD-CIV\#14 & $35+45$ & $09 / 22 / 99$ & 4 \\
\hline & ESF-SD-CIV\#15 & $35+40$ & $09 / 21 / 99$ & 4 \\
\hline & ESF-SD-CIV\#16 & $35+35$ & $09 / 20 / 99$ & 4 \\
\hline & ESF-SD-CIV\#17 & $35+31$ & $09 / 17 / 99$ & 4 \\
\hline & ESF-SD-CIV\#18 & $35+25$ & $09 / 16 / 99$ & 4 \\
\hline & ESF-SD-CIV\#19 & $35+20$ & $09 / 15 / 99$ & 4 \\
\hline & ESF-SD-CIV\#20 & $35+15$ & $09 / 14 / 99$ & 4 \\
\hline & ESF-SD-CIV\#21 & $35+10$ & $09 / 13 / 99$ & 4 \\
\hline & ESF-SD-CIV\#22 & $35+05$ & $09 / 13 / 99$ & 4 \\
\hline & ESF-SD-CIV\#23 & $35+00$ & $09 / 10 / 99$ & 4 \\
\hline & ESF-SD-CIV\#24 & $34+95$ & $09 / 09 / 99$ & 4 \\
\hline & ESF-SD-CIV\#25 & $34+90$ & $09 / 23 / 99$ & 4 \\
\hline & ESF-SD-CIV\#26 & $34+73$ & $09 / 22 / 99$ & 4 \\
\hline & ESF-SD-CIV\#27 & $34+70$ & $04 / 09 / 99$ & 4 \\
\hline & ESF-SD-CIV\#28 & $34+65$ & $04 / 08 / 99$ & 4 \\
\hline & ESF-SD-CIV\#29 & $34+60$ & $04 / 06 / 99$ & 4 \\
\hline & ESF-SD-CIV\#30 & $34+55$ & $04 / 05 / 99$ & 4 \\
\hline & ESF-SD-CIV\#31 & $34+50$ & $04 / 02 / 99$ & 4 \\
\hline & ESF-SD-CIV\#32 & $34+45$ & $04 / 01 / 99$ & 4 \\
\hline & ESF-SD-CIV\#33 & $34+40$ & $03 / 31 / 99$ & 4 \\
\hline & ESF-SD-CIV\#34 & $34+35$ & $03 / 30 / 99$ & 4 \\
\hline & ESF-SD-CIV\#35 & $34+30$ & $03 / 26 / 99$ & 4 \\
\hline & ESF-SD-CIV\#36 & $34+25$ & $03 / 25 / 99$ & 4 \\
\hline & ESF-SD-CIV\#37 & $34+20$ & $03 / 24 / 99$ & 4 \\
\hline & ESF-SD-CIV\#38 & $34+10$ & $03 / 23 / 99$ & 4 \\
\hline & ESF-SD-CIV\#39 & $33+99$ & $03 / 19 / 99$ & 4 \\
\hline & ESF-SD-CIV\#40 & $33+89$ & $03 / 17 / 99$ & 4 \\
\hline
\end{tabular}


Table 3-2. Validation Study Boreholes (continued)

\begin{tabular}{|c|l|c|c|c|}
\hline \multicolumn{1}{c|}{ Fault Zone } & \multicolumn{1}{|c|}{ Borehole Identifier } & ESF Station & $\begin{array}{c}\text { Date } \\
\text { Completed }\end{array}$ & $\begin{array}{c}\text { Total Depth } \\
(\mathbf{m})\end{array}$ \\
\hline \multirow{3}{*}{ Drill Hole Wash } & ESF-DHW-CIV\#1 & $19+65$ & $09 / 30 / 99$ & 4 \\
\cline { 2 - 5 } & ESF-DHW-CIV\#2 & $19+55$ & $09 / 29 / 99$ & 4 \\
\cline { 2 - 5 } & ESF-DHW-CIV\#3 & $19+50$ & $09 / 29 / 99$ & 4 \\
\cline { 2 - 5 } & ESF-DHW-CIV\#4 & $19+45$ & $09 / 28 / 99$ & 4 \\
\cline { 2 - 5 } & ESF-DHW-CIV\#5 & $19+40$ & $09 / 27 / 99$ & 10 \\
\cline { 2 - 5 } & ESF-DHW-CIV\#6 & $19+35$ & $09 / 30 / 99$ & 4 \\
\cline { 2 - 5 } & ESF-DHW-CIV\#7 & $19+30$ & $10 / 05 / 99$ & 4 \\
\cline { 2 - 5 } & ESF-DHW-CIV\#8 & $19+25$ & $10 / 05 / 99$ & 4 \\
\cline { 2 - 5 } & ESF-DHW-CIV\#9 & $19+20$ & $10 / 06 / 99$ & 4 \\
\cline { 2 - 5 } & ESF-DHW-CIV\#10 & $19+10$ & $10 / 06 / 99$ & 4 \\
\hline
\end{tabular}

Source: Paces (2003); surveyed borehole locations (i.e., ESF station numbers) from DTN: LL031200223121.036 (Q)

Note: ESF = Exploratory Studies Facility . 
Table 3-3. Core Samples from Niche \#1 Boreholes

\begin{tabular}{|c|c|c|c|c|c|c|}
\hline \begin{tabular}{|c|} 
Figure 3-7 \\
Reference \\
Number \\
\end{tabular} & $\begin{array}{c}\text { Sample } \\
\text { Identifier }\end{array}$ & $\begin{array}{l}\text { Borehole } \\
\text { Identifier }\end{array}$ & $\begin{array}{c}\text { Interval } \\
\text { (ft) }\end{array}$ & $\begin{array}{c}\text { SMF Barcode } \\
\text { Identifier }\end{array}$ & Laboratory & Data Source \\
\hline A1 & DCN086-2 & $\begin{array}{l}\text { ESF-MD- } \\
\text { NICHE3566\#1 }\end{array}$ & $22.2-23.0$ & SPC01003078 & LANL & LA0509JF831222.001 (Q) \\
\hline A2 & $\begin{array}{l}\text { DCNO07-2/008- } \\
1\end{array}$ & $\begin{array}{l}\text { ESF-MD- } \\
\text { NICHE3566\#1 }\end{array}$ & $32.1-33.1$ & $\begin{array}{l}\text { SPC01003096 } \\
\text { SPC01003097 } \\
\text { SPC01003098 }\end{array}$ & LANL & LA0509JF831222.001 (Q) \\
\hline A3 & DCN015-2 & $\begin{array}{l}\text { ESF-MD- } \\
\text { NICHE3566\#2 }\end{array}$ & $6.7-7.5$ & SPC01003111 & LANL & LA0509JF831222.001 (Q) \\
\hline A4 & $\begin{array}{l}\text { DCNO24-1/025- } \\
2\end{array}$ & $\begin{array}{l}\text { ESF-MD- } \\
\text { NICHE3566\#2 }\end{array}$ & $15.7-17.1$ & $\begin{array}{l}\text { SPC01003131 } \\
\text { SPC01003132 } \\
\text { SPC01003133 }\end{array}$ & LANL & LA0509JF831222.001 (Q) \\
\hline A5 & $\begin{array}{l}\text { DCN038-1/039- } \\
2\end{array}$ & $\begin{array}{l}\text { ESF-MD- } \\
\text { NICHE3566LT\#1 }\end{array}$ & $1.7-5.0$ & $\begin{array}{l}\text { SPC01004399 } \\
\text { SPC01004400 } \\
\text { SPC01004401 } \\
\text { SPC01004402 }\end{array}$ & LANL & LA0509JF831222.001 (Q) \\
\hline A6 & $\begin{array}{l}\text { DCNO48-1/049- } \\
2\end{array}$ & $\begin{array}{l}\text { ESF-MD- } \\
\text { NICHE3566LT\#1 }\end{array}$ & $14.3-16.3$ & $\begin{array}{l}\text { SPC01004420 } \\
\text { SPC01004421 } \\
\text { SPC01004422 }\end{array}$ & LANL & LA0509JF831222.001 (Q) \\
\hline A7 & $\begin{array}{l}\text { DCN050-1/051- } \\
2\end{array}$ & $\begin{array}{l}\text { ESF-MD- } \\
\text { NICHE3566LT\#1 }\end{array}$ & $16.6-19.3$ & $\begin{array}{l}\text { SPC01004424 } \\
\text { SPC01004425 } \\
\text { SPC01004426 } \\
\text { SPC01004427 }\end{array}$ & LANL & LA0509JF831222.001 (Q) \\
\hline A8 & $\begin{array}{l}\text { DCNO59-2/060- } \\
1\end{array}$ & $\begin{array}{l}\text { ESF-MD- } \\
\text { NICHE3566LT\#1 }\end{array}$ & $29.0-30.7$ & $\begin{array}{l}\text { SPC01004445 } \\
\text { SPC01004446 } \\
\text { SPC01004447 }\end{array}$ & LANL & LA0509JF831222.001 (Q) \\
\hline A9 & DCN062-1 & $\begin{array}{l}\text { ESF-MD- } \\
\text { NICHE3566LT\#1 }\end{array}$ & $32.1-33.1$ & SPC01004453 & LANL & LA0509JF831222.001 (Q) \\
\hline A10 & DCN064-2 & \begin{tabular}{|l|} 
ESF-MD- \\
NICHE3566LT\#1
\end{tabular} & $34.4-35.5$ & SPC01004457 & LANL & LA0509JF831222.001 (Q) \\
\hline B1 & $\begin{array}{l}\text { Niche 1-RCR- } \\
1 \mathrm{~A} \\
\text { Niche } \\
1 \text { 1-RCR- } \\
1 \mathrm{~B}\end{array}$ & $\begin{array}{l}\text { ESF-MD- } \\
\text { NICHE3566\#1 }\end{array}$ & \begin{tabular}{r|}
$3.2-4.2$ \\
$4.6-5.7$ \\
$5.9-6.8$ \\
$7.4-8.2$ \\
$8.4-9.0$ \\
$9.3-10.4$
\end{tabular} & $\begin{array}{l}\text { SPC } 01003045 \\
\text { SPC01003048 } \\
\text { SPC01003050 } \\
\text { SPC01003053 } \\
\text { SPC01003055 } \\
\text { SPC } 01003057\end{array}$ & LANL & $\begin{array}{c}\text { LA0305RR831222.001 } \\
\text { (UQ) }\end{array}$ \\
\hline B2 & Niche 1-RCR-2 & $\begin{array}{l}\text { ESF-MD- } \\
\text { NICHE3566\#1 }\end{array}$ & $\begin{array}{l}17.2-17.9 \\
18.1-18.7 \\
18.9-20.0\end{array}$ & $\begin{array}{l}\text { SPC01003068 } \\
\text { SPC01003070 } \\
\text { SPC01003072 }\end{array}$ & LANL & $\begin{array}{l}\text { LA0305RR831222.001 } \\
\text { (UQ) }\end{array}$ \\
\hline B3 & Niche 1- RCR-3 & $\begin{array}{l}\text { ESF-MD- } \\
\text { NICHE3566\#1 }\end{array}$ & $\begin{array}{l}24.2-25.0 \\
27.1-27.9 \\
29.2-30.1 \\
\end{array}$ & $\begin{array}{l}\text { SPC01003082 } \\
\text { SPC01003087 } \\
\text { SPC01003091 } \\
\end{array}$ & LANL & $\begin{array}{c}\text { LA0305RR831222.001 } \\
\text { (UQ) }\end{array}$ \\
\hline B4 & Niche 2-RCR-1 & $\begin{array}{l}\text { ESF-MD- } \\
\text { NICHE3566\#2 }\end{array}$ & $\begin{array}{l}10.6-11.2 \\
12.2-12.9 \\
13.9-14.7 \\
17.4-18.5\end{array}$ & $\begin{array}{l}\text { SPC01003119 } \\
\text { SPC01003123 } \\
\text { SPC01003127 } \\
\text { SPC01003135 }\end{array}$ & LANL & $\begin{array}{l}\text { LA0305RR831222.001 } \\
\text { (UQ) }\end{array}$ \\
\hline B5 & $\begin{array}{l}\text { Niche LT-RCR- } \\
1 \mathrm{~A} \\
\text { Niche LT-RCR- } \\
1 \mathrm{~B}\end{array}$ & $\begin{array}{l}\text { ESF-MD- } \\
\text { NICHE3566LT\#1 }\end{array}$ & $\begin{array}{l}12.1-13.2 \\
20.5-21.1 \\
21.4-22.2 \\
23.8-24.8 \\
26.8-27.9 \\
36.2-37.0 \\
38.3-38.9\end{array}$ & $\begin{array}{l}\text { SPC01004416 } \\
\text { SPC01004431 } \\
\text { SPC01004433 } \\
\text { SPC01004437 } \\
\text { SPC01004441 } \\
\text { SPC01004460 } \\
\text { SPC01004464 }\end{array}$ & LANL & $\begin{array}{c}\text { LA0305RR831222.001 } \\
\text { (UQ) }\end{array}$ \\
\hline
\end{tabular}


Table 3-3. Core Samples from Niche \#1 Boreholes (continued)

\begin{tabular}{|c|c|c|c|c|c|c|}
\hline \begin{tabular}{|c|} 
Figure 3-7 \\
Reference \\
Number
\end{tabular} & $\begin{array}{l}\text { Sample } \\
\text { Identifier }\end{array}$ & $\begin{array}{l}\text { Borehole } \\
\text { Identifier }\end{array}$ & $\begin{array}{c}\text { Interval } \\
\text { (ft) }\end{array}$ & $\begin{array}{l}\text { SMF Barcode } \\
\text { Identifier }\end{array}$ & Laboratory & Data Source \\
\hline C1 & $\begin{array}{l}\text { ESF-MD- } \\
\text { NICHE3566\#1 } \\
\left(14.7^{\prime}-20.9^{\prime}\right)\end{array}$ & $\begin{array}{l}\text { ESF-MD- } \\
\text { NICHE3566\#1 }\end{array}$ & $\begin{array}{l}14.7-15.8 \\
20.3-20.9\end{array}$ & $\begin{array}{l}\text { SPC01003066 } \\
\text { SPC01003074 }\end{array}$ & $\begin{array}{l}\text { USGS- } \\
\text { LLNL }\end{array}$ & $\begin{array}{c}\text { LLO31200223121.036 } \\
\text { (Q) }\end{array}$ \\
\hline $\mathrm{C} 2$ & $\begin{array}{l}\text { ESF-MD- } \\
\text { NICHE3566\#1 } \\
\left(25.3^{\prime}-31.7^{\prime}\right) \\
\end{array}$ & $\begin{array}{l}\text { ESF-MD- } \\
\text { NICHE3566\#1 }\end{array}$ & $\begin{array}{l}25.3-26.0 \\
28.1-28.9 \\
30.8-31.7\end{array}$ & $\begin{array}{l}\text { SPC01003084 } \\
\text { SPC01003089 } \\
\text { SPC01003094 }\end{array}$ & $\begin{array}{l}\text { USGS- } \\
\text { LLNL }\end{array}$ & $\begin{array}{c}\text { LLO31200223121.036 } \\
\text { (Q) }\end{array}$ \\
\hline C3 & \begin{tabular}{|l} 
ESF-MD- \\
NICHE3566\#2 \\
$\left(11.5^{\prime}-15.4^{\prime}\right)$
\end{tabular} & $\begin{array}{l}\text { ESF-MD- } \\
\text { NICHE3566\#2 }\end{array}$ & $\begin{array}{l}11.5-11.9 \\
13.2-13.7 \\
15.0-15.4\end{array}$ & $\begin{array}{l}\text { SPC01003121 } \\
\text { SPC01003125 } \\
\text { SPC01003129 }\end{array}$ & $\begin{array}{l}\text { USGS- } \\
\text { LLNL }\end{array}$ & $\begin{array}{l}\text { LL031200223121.036 } \\
\text { (Q) }\end{array}$ \\
\hline $\mathrm{C} 4^{\prime}$ & \begin{tabular}{|l} 
ESF-MD- \\
NICHE3566\#2 \\
$\left(20.2^{\prime}-32.5^{\prime}\right)$
\end{tabular} & $\begin{array}{l}\text { ESF-MD- } \\
\text { NICHE3566\#2 }\end{array}$ & $\begin{array}{l}20.2-20.7 \\
29.8-30.4 \\
32.0-32.5\end{array}$ & $\begin{array}{l}\text { SPC01003140 } \\
\text { SPC01003155 } \\
\text { SPC01003156 }\end{array}$ & $\begin{array}{l}\text { USGS- } \\
\text { LLNL }\end{array}$ & $\begin{array}{c}\text { LL031200223121.036 } \\
\text { (Q) }\end{array}$ \\
\hline C5 & \begin{tabular}{|l|} 
ESF-MD- \\
NICHE3566\#LT1 \\
$\left(10.9^{\prime}-23.5^{\prime}\right)$
\end{tabular} & $\begin{array}{l}\text { ESF-MD- } \\
\text { NICHE3566LT\#1 }\end{array}$ & $\begin{array}{l}10.9-11.9 \\
13.4-14.1 \\
22.8-23.5\end{array}$ & $\begin{array}{l}\text { SPC01004414 } \\
\text { SPC01004418 } \\
\text { SPC01004435 }\end{array}$ & $\begin{array}{l}\text { USGS- } \\
\text { LLNL }\end{array}$ & $\begin{array}{c}\text { LLO31200223121.036 } \\
\text { (Q) }\end{array}$ \\
\hline $\mathrm{C} 6$ & $\begin{array}{l}\text { ESF-MD- } \\
\text { NICHE3566\#LT1 } \\
\left(25.0^{\prime}-38.1^{\prime}\right)\end{array}$ & $\begin{array}{l}\text { ESF-MD- } \\
\text { NICHE3566LT\#1 }\end{array}$ & $\begin{array}{l}25.0-25.9 \\
28.2-28.8 \\
31.5-31.9 \\
37.1-38.1\end{array}$ & $\begin{array}{l}\text { SPC01004439 } \\
\text { SPC01004443 } \\
\text { SPC01004451 } \\
\text { SPC01004462 }\end{array}$ & $\begin{array}{l}\text { USGS- } \\
\text { LLNL }\end{array}$ & $\begin{array}{c}\text { LLO31200223121.036 } \\
\text { (Q) }\end{array}$ \\
\hline
\end{tabular}

NOTES: $\quad$ LANL = Los Alamos National Laboratory, LLNL = Lawrence Livermore National Laboratory, SMF = Sample Management Facility, USGS = U.S. Geological Survey.

SMF barcode identifiers and intervals are contained in the data record package for the associated DTN. 


\begin{tabular}{|c|c|c|c|c|c|c|c|c|c|c|}
\hline Sample Name & $\begin{array}{l}\text { SMF Barcode } \\
\text { Identifier }\end{array}$ & $\begin{array}{l}\text { Interval } \\
\text { Leached } \\
\text { (ft) }\end{array}$ & $\begin{array}{l}\text { CAMS } \\
\text { Number }\end{array}$ & $\begin{array}{l}\text { Date AMS } \\
\text { Analyzed }\end{array}$ & $\underset{\text { Rock Mass }}{\text { Ros }}$ & $\begin{array}{l}\text { Leachate } \\
\text { Mass (g) }\end{array}$ & $\begin{array}{c}\text { Leachate } \\
{[\mathrm{cl}]} \\
(\mathbf{m g} / \mathbf{k g})\end{array}$ & $\begin{array}{c}\mathrm{Cl} \text { Concen- } \\
\text { tration } \\
\text { (mg/kg } \\
\text { rock) }\end{array}$ & $\begin{array}{l}{ }^{36} \mathrm{Cl} / / \mathrm{Cl} \\
\times 10^{15}\end{array}$ & $2 \sigma \times 10^{15}$ \\
\hline ESF-SD-CIV\#27 & SPC02016025 & $7.3-9.2$ & CL7684 & $1 / 14 / 00$ & $3,000.0$ & $4,002.4$ & 1.04 & 1.39 & 151 & 15 \\
\hline ESF-SD-CIV\#32 & SPC02016116 & $3.3-5.6$ & CL7685 & $1 / 14 / 00$ & $3,000.6$ & $4,001.2$ & 2.12 & 2.83 & 248 & 34 \\
\hline ESF-SD-CIV\#33 & SPC02016117 & $3.0-5.4$ & CL7686 & $1 / 14 / 00$ & $3,000.1$ & $3,999.7$ & 2.36 & 3.15 & 72 & 7 \\
\hline ESF-SD-CIV\#34 & SPC02015957 & $8.3-10.5$ & CL7687 & $1 / 14 / 00$ & $3,000.0$ & $4,001.1$ & 1.14 & 1.52 & 130 & 10 \\
\hline ESF-SD-CIV\#28 & $\begin{array}{l}\text { SPC02016022 } \\
\text { SPC02016023 }\end{array}$ & $\begin{array}{l}11.3-12.7 \\
12.7-13.3\end{array}$ & CL7775 & $2 / 17 / 00$ & $2,000.1$ & $3,001.6$ & 1.83 & 2.75 & 112 & 33 \\
\hline ESF-SD-CIV\#29 & $\begin{array}{l}\text { SPC02015993 } \\
\text { SPC02015994 }\end{array}$ & $\begin{array}{l}6.5-7.5 \\
7.5-8.7\end{array}$ & CL7776 & $2 / 17 / 00$ & $2,506.1$ & $3,500.1$ & 1.59 & 2.22 & 103 & 28 \\
\hline ESF-SD-CIV\#37 & SPC02016031 & $11.2-13.0$ & CL7777 & $2 / 17 / 00$ & $2,000.7$ & $2,996.3$ & 1.71 & 2.56 & 83 & 36 \\
\hline ESF-SD-CIV\#30 & SPC02015999 & $8.5-10.5$ & CL7779 & $2 / 17 / 00$ & $2,504.1$ & $3,511.6$ & 1.85 & 2.59 & 48 & 18 \\
\hline ESF-SD-CIV\#35 & SPC02016119 & $2.2-4.0$ & CL7780 & $2 / 17 / 00$ & $2,302.6$ & $3,306.2$ & 1.22 & 1.75 & 57 & 21 \\
\hline ESF-SD-CIV\#40 & $\begin{array}{l}\text { SPC02015925 } \\
\text { SPC02015926 }\end{array}$ & $\begin{array}{l}10.5-11.6 \\
11.6-12.2\end{array}$ & CL7781 & $2 / 17 / 00$ & $2,064.1$ & $3,039.3$ & 1.47 & 2.16 & 71 & 23 \\
\hline ESF-SD-CIV\#2 & SPC02016280 & $6.6-8.0$ & CL7918 & $4 / 21 / 00$ & $2,000.7$ & $3,002.8$ & 1.28 & 1.92 & 139 & 6 \\
\hline ESF-SD-CIV\#3 & $\begin{array}{l}\text { SPC02016290 } \\
\text { SPC02016291 } \\
\text { SPC02016292 }\end{array}$ & $\begin{array}{l}11.4-12.3 \\
12.3-12.8 \\
12.8-13.5\end{array}$ & CL7919 & $4 / 21 / 00$ & $1,999.6$ & $3,002.1$ & 1.69 & 2.54 & 125 & 6 \\
\hline ESF-SD-CIV\#4 & $\begin{array}{l}\text { SPC02016293 } \\
\text { SPC02016294 }\end{array}$ & $\begin{array}{l}6.3-7.3 \\
7.3-8.3\end{array}$ & CL7920 & $4 / 21 / 00$ & $1,999.4$ & $3,001.1$ & 1.50 & 2.25 & 101 & 6 \\
\hline ESF-SD-CIV\#5 & $\begin{array}{l}\text { SPC03017201 } \\
\text { SPC03017202 }\end{array}$ & $\begin{array}{l}4.1-5.1 \\
5.8-6.7\end{array}$ & CL7921 & $4 / 21 / 00$ & $1,500.8$ & $2,506.8$ & 1.48 & 2.47 & 93 & 9 \\
\hline ESF-SD-CIV\#6 & $\begin{array}{l}\text { SPC03017203 } \\
\text { SPC02016303 }\end{array}$ & $\begin{array}{l}5.7-6.9 \\
7.8-9.3\end{array}$ & CL7922 & $4 / 21 / 00$ & $1,999.4$ & $2,999.9$ & 1.42 & 2.13 & 75 & 5 \\
\hline
\end{tabular}


Table 4-1. Chloride Concentrations and ${ }^{36} \mathrm{Cl} / \mathrm{Cl}$ Ratios in Active Leachates Prepared and Analyzed at LLNL during Phase I (continued)

\begin{tabular}{|c|c|c|c|c|c|c|c|c|c|c|}
\hline Sample Name & $\begin{array}{l}\text { SMF Barcode } \\
\text { Identifier }\end{array}$ & $\begin{array}{c}\text { Interval } \\
\text { Leached } \\
\text { (ft) }\end{array}$ & $\begin{array}{l}\text { CAMS } \\
\text { Number }\end{array}$ & $\begin{array}{l}\text { Date AMS } \\
\text { Analyzed }\end{array}$ & $\begin{array}{c}\text { Rock Mass } \\
\text { (g) }\end{array}$ & $\begin{array}{l}\text { Leachate } \\
\text { Mass (g) }\end{array}$ & $\begin{array}{c}\text { Leachate } \\
{[\mathrm{Cl}]} \\
\text { (mg/kg) }\end{array}$ & $\begin{array}{c}\text { Cl Concen- } \\
\text { tration } \\
\text { (mg/kg } \\
\text { rock) }\end{array}$ & $\begin{array}{l}{ }^{36} \mathrm{Cl} / \mathrm{Cl} \\
\times 10^{15}\end{array}$ & $20 \times 10^{15}$ \\
\hline ESF-SD-CIV\#7 & SPC02016267 & $6.5-8.0$ & CL7923 & $4 / 21 / 00$ & $1,999.0$ & $3,004.7$ & 1.46 & 2.19 & 73 & 4 \\
\hline ESF-SD-CIV\#8 & SPC02016273 & $9.9-11.8$ & CL7924 & $4 / 21 / 00$ & $1,999.5$ & $3,008.6$ & 0.96 & 1.44 & 106 & 7 \\
\hline ESF-SD-CIV\#9 & SPC02016276 & $8.6-10.1$ & CL7925 & $4 / 21 / 00$ & $1,800.4$ & $2,812.2$ & 0.91 & 1.42 & 93 & 7 \\
\hline ESF-SD-CIV\#10 & SPC02016255 & $9.9-11.2$ & CL7926 & $4 / 21 / 00$ & $1,579.0$ & $2,506.3$ & 1.06 & 1.68 & 88 & 6 \\
\hline ESF-SD-CIV\#11 & SPC02016259 & $9.0-10.2$ & CL7927 & $4 / 21 / 00$ & $1,670.1$ & $2,631.1$ & 0.97 & 1.53 & 65 & 16 \\
\hline ESF-SD-CIV\#12 & $\begin{array}{l}\text { SPC02016264 } \\
\text { SPC02016265 }\end{array}$ & $\begin{array}{c}8.4-9.4 \\
9.4-10.4\end{array}$ & CL7928 & $4 / 21 / 00$ & $2,046.6$ & $3,015.4$ & 1.27 & 1.87 & 53 & 6 \\
\hline ESF-SD-CIV\#31 & SPC02016115 & $4.7-6.2$ & CL7929 & $4 / 21 / 00$ & $1,859.8$ & $2,862.2$ & 0.94 & 1.45 & 80 & 20 \\
\hline ESF-SD-CIV\#36 & $\begin{array}{l}\text { SPC02015947 } \\
\text { SPC02015948 }\end{array}$ & $\begin{array}{l}10.6-10.8 \\
10.8-12.1\end{array}$ & CL7930 & $4 / 21 / 00$ & $1,780.2$ & $2,772.1$ & 0.80 & 1.25 & 99 & 7 \\
\hline ESF-SD-CIV\#38 & SPC02015940 & $9.1-10.8$ & CL7931 & $4 / 21 / 00$ & $2,005.0$ & $3,074.1$ & 2.31 & 3.54 & 58 & 4 \\
\hline ESF-SD-CIV\#39 & SPC02015930 & $9.3-10.1$ & CL7932 & $4 / 21 / 00$ & $1,358.2$ & $2,328.3$ & 1.58 & 2.71 & 71 & 5 \\
\hline
\end{tabular}

DTN: LL030408023121.027 (Q), GS030608312272.005 (Q)

NOTES: AMS = accelerator mass spectrometer, CAMS = Center for Accelerator Mass Spectrometry, SMF = Sample Management Facility.

Chloride concentrations have a uniform $2 \sigma$ uncertainty of $5 \%$ of the stated value. 
Table 4-2. Chloride, Bromide, and Sulfate Concentrations, and ${ }^{36} \mathrm{Cl} / \mathrm{Cl}$ Ratios in Leachates of Validation Study Core Samples Analyzed at LANL during Phase I

\begin{tabular}{|c|c|c|c|c|c|c|c|c|c|c|}
\hline \multirow{2}{*}{$\begin{array}{l}\text { Sample } \\
\text { Identifier }\end{array}$} & \multirow{2}{*}{$\begin{array}{l}\text { SMF Barcode } \\
\text { Identifier }\end{array}$} & \multirow{2}{*}{$\begin{array}{l}\text { Borehole } \\
\text { Identifier }\end{array}$} & \multirow{2}{*}{$\begin{array}{c}\text { Interval } \\
\text { Used } \\
\text { (ft) }\end{array}$} & \multirow{2}{*}{$\begin{array}{c}\text { ESF } \\
\text { Station }\end{array}$} & \multirow{2}{*}{$\begin{array}{c}\text { AgCl } \\
\text { Target } \\
\text { Identifier }\end{array}$} & \multicolumn{3}{|c|}{$\begin{array}{c}\text { Concentration } \\
\text { (mg/kg rock) }\end{array}$} & \multirow{2}{*}{$\begin{array}{c}\mathrm{SO}_{4} \\
\mathrm{Cl}\end{array}$} & \multirow{2}{*}{$\begin{array}{c}\text { Measured } \\
{ }^{36} \mathrm{Cl} / \mathrm{Cl} \\
\times 10^{15} \\
(2 \sigma)\end{array}$} \\
\hline & & & & & & $\mathrm{Cl}^{-1}$ & $B r^{-1}$ & $\mathrm{SO}_{4}^{-1}$ & & \\
\hline VAL01-1 & SPC02016192 & ESF-SD-CIV\#27 & $4.2-5.9$ & $34+70$ & YM1000 & 0.25 & ND & 0.32 & 1.28 & $717 \pm 74$ \\
\hline VAL02-1 & SPC02016193 & ESF-SD-CIV\#30 & $3.8-5.0$ & $34+55$ & YM1001 & 0.23 & ND & 0.26 & 1.13 & $942 \pm 104$ \\
\hline VAL03-1 & SPC02016194 & ESF-SD-CIV\#34 & $5.7-6.6$ & $34+35$ & YM1002 & 0.30 & ND & 0.38 & 1.27 & $665 \pm 100$ \\
\hline VAL03-2 & SPC02016194 & ESF-SD-CIV\#34 & $4.8-5.7$ & $34+35$ & YM1003 & 0.35 & ND & 0.34 & 0.97 & $508 \pm 108$ \\
\hline VAL04-1 & SPC02016195 & ESF-SD-CIV\#36 & $3.9-5.4$ & $34+25$ & YM1004 & 0.30 & ND & 0.33 & 1.10 & $806 \pm 78$ \\
\hline VAL05-1 & SPC02016196 & ESF-SD-CIV\#38 & $4.5-5.8$ & $34+10$ & YM1005 & 0.25 & ND & 0.44 & 1.76 & $758 \pm 88$ \\
\hline VALO6-1 & SPC02016197 & ESF-SD-CIV\#39 & $3.5-5.7$ & $33+99$ & YM1006 & 0.31 & ND & 0.52 & 1.68 & $538 \pm 70$ \\
\hline
\end{tabular}

DTN: LA0307RRB31222.002 (UQ)

NOTES: $\quad$ ESF = Exploratory Studies Facility, ND = not detected, SMF = Sample Management Facility.

Concentration of salts extracted from each sample is only a qualitative indicator of the sample's salt content. Because the focus of this activity is on determining anion ratios, no attempt has been made to maximize the yield of the leaching process, which is probably highly variable.

Measured ${ }^{36} \mathrm{Cl} / \mathrm{Cl}$ ratios have been corrected for the addition of ${ }^{35} \mathrm{Cl}$ tracer.

Interval used for chloride, bromide, sulfate, and ${ }^{36} \mathrm{Cl} / \mathrm{Cl}$ analysis is smaller than the interval traceable to the SMF barcode number; a.portion of each core sample was removed in the laboratory and set aside for other analyses. 
Table 4-3. Chloride Concentrations and ${ }^{36} \mathrm{Cl} / \mathrm{Cl}$ Ratios for Sequential Leachates of Reference Sample EVAL001 and Six Samples from the ECRB Cross Drift Analyzed at LANL during Phase II

\begin{tabular}{|c|c|c|c|c|c|c|c|c|c|c|c|}
\hline $\begin{array}{l}\text { Sample or } \\
\text { Aliquot } \\
\text { Identifier }\end{array}$ & $\begin{array}{c}\text { SMF Barcode } \\
\text { Identifier }\end{array}$ & $\begin{array}{c}\text { Leach } \\
\text { Duration } \\
(\mathrm{hr})\end{array}$ & $\begin{array}{c}\text { Leach } \\
\text { Type }\end{array}$ & $\begin{array}{c}\text { Size } \\
\text { Fraction } \\
(\mathbf{m m})\end{array}$ & $\begin{array}{c}\text { LANL } \\
\text { Identifier }\end{array}$ & $\begin{array}{c}\text { AMS } \\
\text { Facility }\end{array}$ & $\begin{array}{c}\text { AMS } \\
\text { Identifier }\end{array}$ & $\begin{array}{c}\text { Date } \\
\text { Analyzed }\end{array}$ & $\begin{array}{c}\mathrm{Cl} \\
\text { Concen- } \\
\text { tration } \\
\text { (mg/kg } \\
\text { rock) }\end{array}$ & $\begin{array}{c}\text { Sample } \\
+ \text { Blank } \\
{ }^{36} \mathrm{Cl} / \mathrm{Cl} \\
\times 10^{15}\end{array}$ & $\begin{array}{c}\text { Sample } \\
{ }^{36} \mathrm{Cl} / \mathrm{Cl} \\
\times 10^{15} \\
(2 \sigma)\end{array}$ \\
\hline $\begin{array}{l}\text { EVAL001-7 } \\
(30)\end{array}$ & SPC00536900 & 0.5 & Passive & $6.3-12.5$ & YM2000 & PRIME & $\begin{array}{c}\text { T01- } \\
0604,5 \mathrm{~A}\end{array}$ & $5 / 21 / 2001$ & 0.11 & 838 & $889 \pm 141$ \\
\hline $\begin{array}{l}\text { EVALO01-7 } \\
(120)\end{array}$ & SPC00536900 & 2 & Passive & $|6.3-12.5|$ & YM2001 & PRIME & $\begin{array}{c}\text { T01- } \\
0605,5 \mathrm{~A}\end{array}$ & $5 / 21 / 2001$ & 0.12 & 557 & $570 \pm 110$ \\
\hline $\begin{array}{l}\text { EVAL001-7 } \\
(420)\end{array}$ & SPC 00536900 & 7 & Passive & $6.3-12.5$ & YM2002 & PRIME & $\begin{array}{c}\text { T01- } \\
0606,5 \mathrm{~A}\end{array}$ & $5 / 21 / 2001$ & 0.19 & 519 & $526 \pm 57$ \\
\hline \begin{tabular}{|l|} 
EVALO01-7 \\
$(9900)$
\end{tabular} & SPC00536900 & 165 & Passive & $6.3-12.5$ & YM2003 & PRIME & $\begin{array}{c}\text { T01- } \\
0607,5 \mathrm{~A} \\
\end{array}$ & $5 / 21 / 2001$ & 0.22 & 488 & $493 \pm 126$ \\
\hline $\begin{array}{l}\text { EVAL001-11 } \\
(30)\end{array}$ & SPC00536900 & 0.5 & Passive & $6.3-12.5$ & YM2008 & PRIME & $\begin{array}{c}\text { T01- } \\
0612,5 \mathrm{~A}\end{array}$ & $5 / 21 / 2001$ & 0.12 & 609 & $633 \pm 78$ \\
\hline $\begin{array}{l}\text { EVAL001-11 } \\
\text { (120) }\end{array}$ & SPC00536900 & 2 & Passive & $6.3-12.5$ & YM2009 & PRIME & $\begin{array}{c}\text { T01- } \\
0613,5 \mathrm{~A}\end{array}$ & $5 / 21 / 2001$ & 0.12 & 505 & $516 \pm 69$ \\
\hline $\begin{array}{l}\text { EVAL001-11 } \\
(420) \\
\end{array}$ & SPC00536900 & 7 & Passive & $6.3-12.5$ & YM2010 & PRIME & $\begin{array}{c}\text { T01- } \\
0614,5 \mathrm{~A} \\
\end{array}$ & $5 / 21 / 2001$ & 0.25 & 488 & $492 \pm 40$ \\
\hline $\begin{array}{l}\text { EVAL001-11 } \\
(4560)\end{array}$ & SPC00536900 & 76 & Passive & $6.3-12.5$ & YM2011 & PRIME & $\begin{array}{c}\text { T01- } \\
0615,5 \mathrm{~A}\end{array}$ & $5 / 21 / 2001$ & 0.17 & 680 & $698 \pm 137$ \\
\hline $\begin{array}{l}\text { EVAL001- } \\
8,9,10 \\
(30)\end{array}$ & SPC00536900. & 0.5 & Active & $6.3-12.5$ & YM2004 & PRIME & $\begin{array}{c}\text { T01- } \\
0608,5 \mathrm{~A}\end{array}$ & $5 / 21 / 2001$ & 0.18 & 420 & $423 \pm 79$ \\
\hline $\begin{array}{l}\text { EVAL001- } \\
8,9,10 \\
(120)\end{array}$ & SPC00536900 & 2 & Active & $6.3-12.5$ & YM2005 & PRIME & $\begin{array}{c}\text { T01- } \\
0609,5 \mathrm{~A}\end{array}$ & $5 / 21 / 2001$ & 0.15 & 490 & $501 \pm 126$ \\
\hline $\begin{array}{l}\text { EVAL001- } \\
8,9,10 \\
(420)\end{array}$ & SPC00536900| & 7 & Active & $6.3-12.5$ & YM2006 & PRIME & $\begin{array}{c}\text { T01- } \\
0610,5 \mathrm{~A}\end{array}$ & $5 / 21 / 2001$ & 0.20 & 427 & $430 \pm 88$ \\
\hline $\begin{array}{l}\text { EVAL001- } \\
8,9,10 \\
(3-7)\end{array}$ & SPC00536900 & $\begin{array}{l}0.05- \\
0.12\end{array}$ & Active & $6.3-12.5$ & YM2007 & PRIME & $\begin{array}{c}\text { T01- } \\
0611,5 \mathrm{~A}\end{array}$ & $5 / 21 / 2001$ & 0.31 & 243 & $234 \pm 40$ \\
\hline $\begin{array}{l}\text { EXD-049 (0.5 } \\
\text { hr) }\end{array}$ & SPC00521148 & 0.5 & Passive & $6.3-12.5$ & YM2051 & CAMS & CL9724 & $11 / 29 / 2001$ & 0.08 & 638 & $603 \pm 208$ \\
\hline EXD-049 (2 hr) & SPC00521148 & 2 & Passive & $6.3-12.5$ & YM2052 & CAMS & CL9725 & $11 / 29 / 2001$ & 0.07 & 735 & $704 \pm 164$ \\
\hline EXD-049 (7 hr) & SPC00521148 & 7 & Passive & $6.3-12.5$ & YM2053 & CAMS & CL9726 & $11 / 29 / 2001$ & 0.09 & 752 & $726 \pm 64$ \\
\hline \begin{tabular}{|l|} 
EXD-049 \\
$(48 \mathrm{hr})$ \\
\end{tabular} & SPC00521148 & 48 & Passive & $6.3-12.5$ & YM2054 & CAMS & CL9727 & $11 / 29 / 2001$ & 0.07 & 683 & $650 \pm 93$ \\
\hline \begin{tabular}{|l|} 
EXD-050 \\
$0.25-0.5^{\prime \prime}$ \\
$(0.5 \mathrm{hr})$
\end{tabular} & SPC00521147 & 0.5 & Passive & $6.3-12.5$ & YM2095 & CAMS & CL10134 & $5 / 23 / 2002$ & 0.11 & 842 & $777 \pm 102$ \\
\hline \begin{tabular}{|l|} 
EXD-050 \\
$0.25-0.5^{\prime \prime}(2 \mathrm{hr})$ \\
\end{tabular} & SPC00521147 & 2 & Passive & $6.3-12.5$ & YM2096 & CAMS & CL10135 & $5 / 23 / 2002$ & 0.13 & 793 & $742 \pm 95$ \\
\hline \begin{tabular}{|l|} 
EXD-050 \\
$0.25-0.5^{\prime \prime}(7 \mathrm{hr})$ \\
\end{tabular} & SPC00521147 & 7 & Passive & $6.3-12.5$ & YM2097 & CAMS & CL10136 & $5 / 23 / 2002$ & 0.12 & 758 & $701 \pm 87$ \\
\hline \begin{tabular}{|l|} 
EXD-050 \\
$0.25-0.5^{\prime \prime}$ \\
$(48 \mathrm{hr})$
\end{tabular} & SPC00521147 & 48 & Passive & $6.3-12.5$ & YM2098 & CAMS & CL10137 & $5 / 23 / 2002$ & 0.11 & 796 & $735 \pm 92$ \\
\hline $\begin{array}{l}\text { EXD-069 } \\
<2 \mathrm{~mm} \\
(0.5 \mathrm{hr})\end{array}$ & SPC00541213 & 0.5 & Passive & $<2.0$ & YM2084A & CAMS & CL10123 & $5 / 23 / 2002$ & 0.40 & 382 & $351 \pm 40$ \\
\hline $\begin{array}{l}\text { EXD-069 } \\
<2 \mathrm{~mm}(2 \mathrm{hr})\end{array}$ & SPC00541213 & 2 & Passive & $<2.0$ & YM2085 & CAMS & CL10124 & $5 / 23 / 2002$ & 0.29 & 394 & $356 \pm 44$ \\
\hline
\end{tabular}


Table 4-3. Chloride Concentrations and ${ }^{36} \mathrm{CI} / \mathrm{CI}$ Ratios in Sequential Leachates of Reference Sample EVAL001 and Six Samples from the ECRB Cross Drift Analyzed at LANL during Phase II (continued)

\begin{tabular}{|c|c|c|c|c|c|c|c|c|c|c|c|}
\hline $\begin{array}{l}\text { Sample or } \\
\text { Aliquot } \\
\text { Identifier }\end{array}$ & $\begin{array}{c}\text { SMF Barcode } \\
\text { Identifier }\end{array}$ & $\begin{array}{c}\text { Leach } \\
\text { Duration } \\
\text { (hr) }\end{array}$ & $\begin{array}{c}\text { Leach } \\
\text { Type }\end{array}$ & $\begin{array}{c}\text { Size } \\
\text { Fraction } \\
\text { (mm) }\end{array}$ & LANL & $\begin{array}{c}\text { AMS } \\
\text { Facility }\end{array}$ & $\begin{array}{c}\text { AMS } \\
\text { Identifier }\end{array}$ & $\begin{array}{c}\text { Date } \\
\text { Analyzed }\end{array}$ & \begin{tabular}{|c|}
$\mathbf{C l}$ \\
Concen- \\
tration \\
(mg/kg \\
rock)
\end{tabular} & $\begin{array}{c}\text { Sample } \\
+ \text { Blank } \\
{ }^{36} \mathrm{Cl} / \mathrm{Cl} \\
\times 10^{15}\end{array}$ & $\begin{array}{c}\text { Sample } \\
{ }^{36} \mathrm{Cl} / \mathrm{Cl} \mid \\
\times 10^{15} \\
(2 \sigma)\end{array}$ \\
\hline $\begin{array}{l}\text { EXD-069 } \\
<2 \mathrm{~mm}(7 \mathrm{hr})\end{array}$ & SPC00541213 & 7 & Passive & $<2.0$ & YM2086 & CAMS & CL10125 & $5 / 23 / 2002$ & 0.17 & 370 & $317 \pm 52$ \\
\hline $\begin{array}{l}\text { EXD-069 } \\
<2 \mathrm{~mm}(48 \mathrm{hr})\end{array}$ & SPC00541213 & 48 & Passive & $<2.0$ & YM2087 & CAMS & CL10126 & $5 / 23 / 2002$ & 0.17 & 477 & $432 \pm 55$ \\
\hline $\begin{array}{l}\text { EXD-069 } \\
2 \mathrm{~mm}-0.25 " \\
(0.5 / 2 \mathrm{hr}) \\
\end{array}$ & SPC00541213 & $0.5 / 2$ & Passive & $2.0-6.3$ & YM2088 & CAMS & CL10127 & $5 / 23 / 2002$ & 0.99 & 282 & $277 \pm 22$ \\
\hline $\begin{array}{l}\text { EXD-069 } \\
2 \mathrm{~mm}-0.25 " \\
(7 \mathrm{hr})\end{array}$ & SPC00541213 & 7 & Passive & $2.0-6.3$ & YM2089 & CAMS & CL10128 & $5 / 23 / 2002$ & 0.41 & 283 & $261 \pm 29$ \\
\hline \begin{tabular}{|l|} 
EXD-069 \\
$2 \mathrm{~mm}-0.25 "$ \\
$(48 \mathrm{hr})$ \\
\end{tabular} & SPC00541213 & 48 & Passive & $2.0-6.3$ & YM2090 & CAMS & CL10129 & $5 / 23 / 2002$ & 0.21 & 313 & $273 \pm 40$ \\
\hline \begin{tabular}{|l} 
EXD-069 \\
$0.25-0.5^{\prime \prime}$ \\
$(0.5 \mathrm{hr})$
\end{tabular} & SPC00541213 & 0.5 & Passive & $6.3-12.5$ & YM2091 & CAMS & CL10130 & $5 / 23 / 2002$ & 0.57 & 300 & $284 \pm 28$ \\
\hline \begin{tabular}{|l|} 
EXD-069 \\
$0.25-0.5 "$ \\
$(2 \mathrm{hr})$ \\
\end{tabular} & SPC00541213 & 2 & Passive & $6.3-12.5$ & YM2092 & CAMS & CL10131 & $5 / 23 / 2002$ & 0.66 & 297 & $282 \pm 27$ \\
\hline \begin{tabular}{|l|} 
EXD-069 \\
$0.25-0.5^{\prime \prime}$ \\
$(7 \mathrm{hr})$ \\
\end{tabular} & SPC00541213 & 7 & Passive & $6.3-12.5$ & YM2093 & CAMS & CL10132 & $5 / 23 / 2002$ & 0.61 & 305 & $290 \pm 27$ \\
\hline $\begin{array}{c}\text { EXD-069 } \\
0.25-0.5^{\prime \prime} \\
(48 \mathrm{hr}) \\
\end{array}$ & SPC 00541213 & 48 & Passive & $6.3-12.5$ & YM2094 & CAMS & CL10133 & $5 / 23 / 2002$ & 0.62 & 312 & $297 \pm 27$ \\
\hline $\begin{array}{l}\text { EXD-070 } \\
(0.5 \mathrm{hr})\end{array}$ & SPC00541215 & 0.5 & Passive & $6.3-12.5$ & YM2055 & CAMS & CL9728 & $11 / 29 / 2001$ & 0.43 & 543 & $536 \pm 39$ \\
\hline EXD-070 (2 hr) & SPC00541215 & 2 & Passive & $6.3-12.5$ & YM2056 & CAMS & CL9729 & $11 / 29 / 2001$ & 0.30 & 544 & $536 \pm 40$ \\
\hline EXD-070 $(7 \mathrm{hr})$ & $S P C 00541215$ & 7 & Passive & $6.3-12.5$ & YM2057 & CAMS & CL9730 & $11 / 29 / 2001$ & 0.31 & 553 & $545 \pm 40$ \\
\hline \begin{tabular}{|l|} 
EXD-070 \\
$(48 \mathrm{hr})$ \\
\end{tabular} & SPC00541215 & 48 & Passive & $6.3-12.5$ & YM2058 & CAMS & CL9731 & $11 / 29 / 2001$ & 0.30 & 526 & $518 \pm 40$ \\
\hline $\begin{array}{l}\text { EXD-072 } \\
(0.5 \mathrm{hr})\end{array}$ & SPC00521171 & 0.5 & Passive & $6.3-12.5$ & YM2059 & CAMS & CL.9732 & $11 / 29 / 2001$ & 0.22 & 936 & $924 \pm 64$ \\
\hline EXD-072 (2 hr) & SPC00521171 & 2 & Passive & $6.3-12.5$ & YM2060 & CAMS & CL9733 & $11 / 29 / 2001$ & 0.21 & 697 & $685 \pm 58$ \\
\hline EXD-072 $(7 \mathrm{hr})$ & SPC00521171 & 7 & Passive & $6.3-12.5$ & YM2061 & CAMS & CL9734 & $11 / 29 / 2001$ & 0.23 & 687 & $676 \pm 47$ \\
\hline $\begin{array}{l}\text { EXD-072 } \\
(48 \mathrm{hr})\end{array}$ & SPC00521171 & 48 & Passive & $6.3-12.5$ & YM2062 & CAMS & CL9735 & $11 / 29 / 2001$ & 0.18 & 766 & $753 \pm 53$ \\
\hline $\begin{array}{l}\text { EXD-080 (0.5 } \\
\mathrm{hr})\end{array}$ & SPC00533393 & 0.5 & Passive & $6.3-12.5$ & YM2063 & CAMS & CL9736 & $11 / 29 / 2001$ & 0.17 & 586 & $568 \pm 45$ \\
\hline EXD-080 $(2 \mathrm{hr})$ & SPC00533393 & 2 & Passive & $6.3-12.5$ & YM2064 & CAMS & CL9737 & $11 / 29 / 2001$ & 0.16 & 559 & $543 \pm 44$ \\
\hline
\end{tabular}


Table 4-3. Chloride Concentrations and ${ }^{36} \mathrm{Cl} / \mathrm{Cl}$ Ratios in Sequential Leachates of Reference Sample EVAL001 and Six Samples from the ECRB Cross Drift Analyzed at LANL during Phase II (continued)

\begin{tabular}{|c|c|c|c|c|c|c|c|c|c|c|c|}
\hline $\begin{array}{l}\text { Sample or } \\
\text { Aliquot } \\
\text { Identifier }\end{array}$ & $\begin{array}{c}\text { SMF Barcode } \\
\text { Identifier }\end{array}$ & $\begin{array}{c}\text { Leach } \\
\text { Duration } \\
\text { (hr) }\end{array}$ & $\begin{array}{l}\text { Leach } \\
\text { Type }\end{array}$ & $\begin{array}{c}\text { Size } \\
\text { Fraction } \\
\text { (mm) }\end{array}$ & $\begin{array}{l}\text { LANL } \\
\text { Identifier }\end{array}$ & $\begin{array}{c}\text { AMS } \\
\text { Facility }\end{array}$ & $\begin{array}{c}\text { AMS } \\
\text { Identifier }\end{array}$ & $\begin{array}{c}\text { Date } \\
\text { Analyzed }\end{array}$ & $\begin{array}{c}\mathrm{Cl} \\
\text { Concen- } \\
\text { tration } \\
\text { (mg/kg } \\
\text { rock) }\end{array}$ & $\begin{array}{c}\text { Sample } \\
+ \text { Blank } \\
{ }^{38} \mathrm{Cl} / \mathrm{Cl} \mid \\
\times 10^{15}\end{array}$ & $\begin{array}{c}\text { Sample } \\
{ }^{36} \mathrm{Cl} / \mathrm{Cl} \\
\times 10^{15} \\
(2 \sigma)\end{array}$ \\
\hline EXD-080 (7 hr) & SPC00533393 & 7 & Passive & $6.3-12.5$ & YM2065 & CAMS & CL9738 & $11 / 29 / 2001$ & 0.19 & 560 & $546 \pm 41$ \\
\hline $\begin{array}{l}\text { EXD-080 } \\
(48 \mathrm{hr})\end{array}$ & SPC00533393 & 48 & Passive & $6.3-12.5$ & YM2066 & CAMS & CL9739 & $11 / 29 / 2001$ & 0.11 & 569 & $546 \pm 60$ \\
\hline
\end{tabular}

DTN: GS030608312272.005 (Q), LA0305RR831222.001 (UQ)

NOTES: $\quad$ AMS $=$ accelerator mass spectrometer, CAMS $=$ Center for Accelerator Mass Spectrometry, LANL $=$ Los Alamos National Laboratory, PRIME = Purdue Rare Isotope Measurement Laboratory, SMF = Sample Management Facility.

Chloride concentrations have a uniform $2 \sigma$ uncertainty of $5 \%$ of the stated value.

For LANL Identifier YM2088, the 0.5-hour and 2-hour leachates were combined.

The sample or aliquot identifier is from the LANL DTN, which reports the size fraction in inches. The text refers to the size fraction column of the table, which reports the size fraction in millimeters. The numbers in parentheses for the EVAL001 samples are the leach duration, in minutes. 
Table 4-4. Possible Sources for ${ }^{36} \mathrm{Cl} / \mathrm{Cl}$ Ratios in Tuff Samples from Yucca Mountain

\begin{tabular}{|c|c|c|c|}
\hline${ }^{36} \mathrm{Cl} / \mathrm{Cl}$ Source & ${ }^{36} \mathrm{Cl} / \mathrm{Cl}$ Ratio & $\begin{array}{l}\text { Likely Location } \\
\text { in Rock }\end{array}$ & References \\
\hline Bomb-pulse & More than $1,200 \times 10^{-15}$ & $\begin{array}{l}\text { Active, throughgoing } \\
\text { fractures and } \\
\text { connected pores }\end{array}$ & $\begin{array}{l}\text { Fabryka-Martin et al. (1997, } \\
\text { Section 4.2.4) }\end{array}$ \\
\hline $\begin{array}{l}\text { Meteoric water } \\
\text { younger than } 10 \mathrm{ka}\end{array}$ & About $500 \times 10^{-15}$ & $\begin{array}{l}\text { Active fractures, } \\
\text { connected pores, but } \\
\text { potentially less so than } \\
\text { above }\end{array}$ & $\begin{array}{l}\text { Fabryka-Martin et al. (1993, } \\
\text { Section IV.A) }\end{array}$ \\
\hline $\begin{array}{l}\text { Meteoric water older } \\
\text { than } 10 \mathrm{ka}\end{array}$ & $\begin{array}{l}\text { About } 700 \text { to } \\
\text { about } 1,100 \times 10^{-15}\end{array}$ & $\begin{array}{l}\text { Less active fractures } \\
\text { and pores than above }\end{array}$ & $\begin{array}{l}\text { Fabryka-Martin et al. (1997, } \\
\text { Section 3.1.2) }\end{array}$ \\
\hline Rock chloride & Less than about $50 \times 10^{-15}$ & $\begin{array}{l}\text { As mineral component } \\
\text { and fluid inclusions }\end{array}$ & $\begin{array}{l}\text { Fabryka-Martin, Wolfsberg et } \\
\text { al. (1996, Table 5-4) and } \\
\text { Fabryka-Martin et al. (1997, } \\
\text { Section 3.4.1) }\end{array}$ \\
\hline "Old" meteoric salts & $\begin{array}{l}0 \text { to } 1,100 \times 10^{-15} \\
\text { (depending on age and the } \\
301,000-y e a r \\
\text { half-life of }{ }^{36} \mathrm{Cl} \text { ) }\end{array}$ & $\begin{array}{l}\text { Least accessible pores, } \\
\text { clogged pores, } \\
\text { insoluble salts }\end{array}$ & $\begin{array}{l}\text { Fabryka-Martin et al. (1997, } \\
\text { Section 9) }\end{array}$ \\
\hline $\begin{array}{l}\text { Contamination } \\
\text { introduced during } \\
\text { sampling and } \\
\text { processing }\end{array}$ & $\begin{array}{l}\text { Wide range, depending on } \\
\text { the source of contamination }\end{array}$ & $\begin{array}{l}\text { Surfaces of rock } \\
\text { fragments }\end{array}$ & $\begin{array}{l}\text { Fabryka-Martin et al. (1997, } \\
\text { Section 3.3) }\end{array}$ \\
\hline
\end{tabular}


Table 4-5. Dry-Drilled Core Samples Used in Chloride Leaching Experiments Conducted at AECL during Phase II

\begin{tabular}{|c|c|c|c|c|c|c|c|}
\hline Core Number & $\begin{array}{l}\text { SMF Barcode } \\
\text { Identifier }\end{array}$ & $\begin{array}{c}\text { Location } \\
\text { (ESF } \\
\text { station) }\end{array}$ & $\begin{array}{c}\text { Core } \\
\text { Interval } \\
\text { (ft) }\end{array}$ & $\begin{array}{l}\text { Experiment } \\
\text { Designation }\end{array}$ & $\begin{array}{l}\text { Parameter } \\
\text { Tested }\end{array}$ & $\begin{array}{c}\text { Size } \\
\text { Fraction } \\
(\mathrm{mm})\end{array}$ & $\begin{array}{l}\text { Leach } \\
\text { Duration }\end{array}$ \\
\hline \multirow[t]{3}{*}{ ESF-SD-CIV \#2 } & \multirow[t]{3}{*}{ SPC02016282 } & \multirow[t]{3}{*}{$36+74$} & \multirow[t]{3}{*}{$9.9-10.9$} & CT & Duration & $4-10$ & $\begin{array}{l}10 \mathrm{~min}- \\
72 \mathrm{hr}\end{array}$ \\
\hline & & & & FT & Duration & $<0.125$ & $\begin{array}{l}10 \mathrm{~min}- \\
72 \mathrm{hr}\end{array}$ \\
\hline & & & & GS & Particle size & $<0.063-12$ & $24 \mathrm{hr}$ \\
\hline \multirow[t]{2}{*}{$\begin{array}{c}\text { ESF-SD-CIV } \\
\# 14\end{array}$} & \multirow[t]{2}{*}{ SPC03017135 } & \multirow[t]{2}{*}{$35+45$} & \multirow[t]{2}{*}{$9.7-11.5$} & $2 \mathrm{~A} 2$ & Particle size & $<0.063-4$ & $24 \mathrm{hr}$ \\
\hline & & & & $2 \mathrm{CT}$ & Duration & $4-10$ & $\begin{array}{l}10 \mathrm{~min}- \\
70 \mathrm{hr}\end{array}$ \\
\hline \multirow[t]{3}{*}{ ESF-SD-CIV \#9 } & \multirow[t]{3}{*}{ SPC02016275 } & \multirow[t]{3}{*}{$35+95$} & \multirow[t]{3}{*}{$6.5-8.1$} & 2BT & Particle size & $1-60$ & $24 \mathrm{hr}$ \\
\hline & & & & 3BT & Particle size & $1-60$ & $48 \mathrm{hr}$ \\
\hline & & & & 4BT & Particle size & $1-60$ & $72 \mathrm{hr}$ \\
\hline
\end{tabular}

DTN: GS030508312272.003 (UQ)

NOTES: ESF $=$ Exploratory Studies Facility, SMF = Sample Management Facility.

Only a portion of the SPC02016282 interval was used in the chloride leaching experiments. 
Table 4-6. Summary Data for Core Samples Analyzed at AECL during Phase II

\begin{tabular}{|c|c|c|c|c|}
\hline Core Used & Sample Name & $\begin{array}{c}\text { Leach Duration } \\
\text { (hr) }\end{array}$ & $\begin{array}{c}\text { Particle Size } \\
(\mathrm{mm})\end{array}$ & $\begin{array}{c}\mathrm{Cl}^{-1} \text { Concentration } \\
\text { (mg/kg) }\end{array}$ \\
\hline \multirow[t]{12}{*}{ ESF-SD-CIV\#2 } & CT 1 & 0.17 & $4-10$ & 0.14 \\
\hline & СT2 & 0.33 & $4-10$ & 0.22 \\
\hline & СT3 & 0.5 & $4-10$ & 0.25 \\
\hline & CT4 & 1.0 & $4-10$ & 0.4 \\
\hline & CT5 & 2.0 & $4-10$ & 0.57 \\
\hline & CT6 & 4.0 & $4-10$ & 0.72 \\
\hline & CT7 & 8.0 & $4-10$ & 0.88 \\
\hline & СТ8 & 12.0 & $4-10$ & 0.91 \\
\hline & СТ9 & 23.5 & $4-10$ & 1.0 \\
\hline & CT10 & 39.0 & $4-10$ & 0.96 \\
\hline & CT11 & 60.0 & $4-10$ & 0.97 \\
\hline & CT12 & 72.0 & $4-10$ & 0.9 \\
\hline \multirow[t]{9}{*}{ ESF-SD-CIV\#14 } & $2 \mathrm{CT}-1$ & 0.17 & $4-10$ & 0.1 \\
\hline & 2CT-2 & 0.34 & $4-10$ & 0.2 \\
\hline & $2 \mathrm{CT}-3$ & 0.75 & $4-10$ & 0.19 \\
\hline & $2 \mathrm{CT}-4$ & 2.5 & $4-10$ & 0.39 \\
\hline & 2 CT -5 & 7.0 & 4-10 & 0.46 \\
\hline & $2 \mathrm{CT}-6$ & 21.0 & $4-10$ & 0.53 \\
\hline & $2 \mathrm{CT}-7$ & 34.0 & $4-10$ & 0.68 \\
\hline & $2 C T-8$ & 49.0 & $4-10$ & 0.58 \\
\hline & 2 CT-9 & 70.0 & $4-10$ & 0.6 \\
\hline \multirow[t]{12}{*}{ ESF-SD-CIV\#2 } & FT1 & 0.17 & $<0.125$ & 6.34 \\
\hline & $\mathrm{FT} 2$ & 0.33 & $<0.125$ & 4.56 \\
\hline & FT3 & 0.67 & $<0.125$ & 4.74 \\
\hline & FT4 & 1.0 & $<0.125$ & 5.17 \\
\hline & FT5 & 2.25 & $<0.125$ & 3.79 \\
\hline & FT6 & 4.33 & $<0.125$ & 5.46 \\
\hline & FT7 & 8.0 & $<0.125$ & 5.2 \\
\hline & FT8 & 12.0 & $<0.125$ & 5.08 \\
\hline & FT9 & 24.0 & $<0.125$ & 4.92 \\
\hline & FT10 & 38.5 & $<0.125$ & 4.61 \\
\hline & FT11 & 60.0 & $<0.125$ & 4.72 \\
\hline & FT12 & 72.0 & $<0.125$ & 4.42 \\
\hline
\end{tabular}


Table 4-6. Summary Data for Core Samples Analyzed at AECL during Phase II (continued)

\begin{tabular}{|c|c|c|c|c|}
\hline Core Used & Sample Name & $\begin{array}{c}\text { Leach Duration } \\
\text { (hr) }\end{array}$ & $\begin{array}{c}\text { Particle Size } \\
(\mathbf{m m})\end{array}$ & $\begin{array}{c}\mathrm{Cl}^{-1} \text { Concentration } \\
(\mathrm{mg} / \mathrm{kg})\end{array}$ \\
\hline \multirow[t]{7}{*}{ ESF-SD-CIV\#2 } & GS1 & 24.0 & $6-12$ & 2.2 \\
\hline & GS2 & 24.0 & $2-4$ & 1.5 \\
\hline & GS3 & 24.0 & $0.5-2$ & 1.64 \\
\hline & GS4 & 24.0 & $0.25-0.5$ & 2.93 \\
\hline & GS5 & 24.0 & $0.125-0.25$ & 3.18 \\
\hline & GS6 & 24.0 & $0.063-0.125$ & 3.7 \\
\hline & GS7 & 24.0 & $<0.063$ & 4.39 \\
\hline \multirow[t]{6}{*}{ ESF-SD-CIV\#14 } & $2 \mathrm{~A} 2-1$ & 24.0 & $2-4$ & 0.72 \\
\hline & 2 A2-2 & 24.0 & $0.5-2$ & 1.15 \\
\hline & 2A2-3 & 24.0 & $0.25-0.5$ & 2.4 \\
\hline & $2 \mathrm{~A} 2-4$ & 24.0 & $0.125-0.25$ & 5.83 \\
\hline & $2 A 2-5$ & 24.0 & $0.063-0.125$ & 11.56 \\
\hline & $2 A 2-6$ & 24.0 & $<0.063$ & 15.73 \\
\hline \multirow[t]{6}{*}{ ESF-SD-CIV\#9 } & $2 \mathrm{BT}-1$ & 24.0 & $30-60$ & 0.12 \\
\hline & $2 \mathrm{BT}-2$ & 24.0 & $20-30$ & 0.2 \\
\hline & $2 \mathrm{BT}-3$ & 24.0 & $10-20$ & 0.24 \\
\hline & $2 \mathrm{BT}-4$ & 24.0 & $5-10$ & 0.34 \\
\hline & $2 \mathrm{BT}-5$ & 24.0 & $>4$ & 0.35 \\
\hline & $2 \mathrm{BT}-6$ & 24.0 & $1-4$ & 0.45 \\
\hline \multirow[t]{6}{*}{ ESF-SD-CIV\#9 } & 3BT-1 & 48.0 & $30-60$ & 0.16 \\
\hline & 3BT-2 & 48.0 & $20-30$ & 0.21 \\
\hline & 3BT-3 & 48.0 & $10-20$ & 0.29 \\
\hline & 3BT-4 & 48.0 & $5-10$ & 0.33 \\
\hline & $3 \mathrm{BT}-5$ & 48.0 & $>4$ & 0.37 \\
\hline & 3BT-6 & 48.0 & $1-4$ & 0.44 \\
\hline \multirow[t]{6}{*}{ ESF-SD-CIV\#9 } & 4BT-1 & 72.0 & $30-60$ & 0.17 \\
\hline & 4BT-2 & 72.0 & $20-30$ & 0.28 \\
\hline & 4BT-3 & 72.0 & $10-20$ & 0.29 \\
\hline & $4 \mathrm{BT}-4$ & 72.0 & $5-10$ & 0.37 \\
\hline & 4BT-5 & 72.0 & $>4$ & 0.38 \\
\hline & 4BT-6 & 72.0 & $1-4$ & 0.44 \\
\hline
\end{tabular}

DTN: GS030508312272.003 (UQ)

NOTE: Sample name includes experiment designation (Table 4-5) and number. 
Table 4-7. Processing History of Validation Study Core Samples Leached at the USGS during Phase III

\begin{tabular}{|c|c|c|c|c|c|c|c|c|c|}
\hline $\begin{array}{l}\text { Batch } \\
\text { No. }\end{array}$ & Sample Identifier & $\begin{array}{l}\text { Interval } \\
\text { (ft) }\end{array}$ & $\begin{array}{l}\text { SMF Barcode } \\
\text { Identifier. }\end{array}$ & $\begin{array}{c}\text { Rock } \\
\text { Mass } \\
\text { (g) }\end{array}$ & $\begin{array}{c}\text { Water } \\
\text { Mass } \\
\text { (g) }\end{array}$ & $\begin{array}{l}\text { USGS-IC } \\
\text { Barcode } \\
\text { Identifier }\end{array}$ & $\begin{array}{c}\mathrm{Cl} \\
\text { Precip. } \\
\text { and } \\
\text { Target } \\
\text { Prep. } \\
\end{array}$ & $\begin{array}{l}{ }^{36} \mathrm{Cl} \text { Barcode } \\
\text { Identifier }\end{array}$ & $\begin{array}{l}\text { AMS } \\
\text { Facility }\end{array}$ \\
\hline \multirow[t]{2}{*}{1} & \multirow{2}{*}{ EVAL001 } & \multirow{2}{*}{ NA } & \multirow{2}{*}{ SPC00557088 } & \multirow{2}{*}{1,871} & \multirow{2}{*}{2,098} & \multirow{2}{*}{ SPC00536900 } & LLNL & SPC00536901 & \multirow{2}{*}{ LLNL } \\
\hline & & & & & & & LANL & SPC00536902 & \\
\hline \multirow[t]{2}{*}{1} & \multirow{2}{*}{ ESF-SD-CIV\#33 } & \multirow{2}{*}{$9.9-11.4$} & \multirow{2}{*}{ SPC02016014 } & \multirow{2}{*}{1,787} & \multirow{2}{*}{2,057} & \multirow{2}{*}{ SPC00536903 } & LLNL & SPC00536904 & \multirow{2}{*}{ LLNL } \\
\hline & & & & & & & LANL & SPC00536905 & \\
\hline \multirow[t]{2}{*}{1} & \multirow{2}{*}{ ESF-SD-CIV\#28 } & \multirow{2}{*}{$6.2-8.0$} & \multirow{2}{*}{ SPC02016017 } & \multirow{2}{*}{1,893} & \multirow{2}{*}{2,130} & \multirow{2}{*}{ SPC00536906 } & LLNL & SPC00536907 & \multirow{2}{*}{ LLNL } \\
\hline & & & & & & & LANL & SPC00536908 & \\
\hline \multirow[t]{2}{*}{1} & \multirow{2}{*}{ ESF-SD-CIV\#36 } & \multirow{2}{*}{$\begin{array}{l}5.4-6.7 \\
8.1-9.1 \\
9.1-9.4\end{array}$} & \multirow{2}{*}{$\begin{array}{l}\text { SPC01014834 } \\
\text { SPC02015944 } \\
\text { SPC02015945 }\end{array}$} & \multirow{2}{*}{2,002} & \multirow{2}{*}{2,038} & \multirow{2}{*}{ SPC00536909 } & LLNL & SPC00536910 & \multirow{2}{*}{ LLNL } \\
\hline & & & & & & & LANL & SPC00536911 & \\
\hline \multirow[t]{2}{*}{1} & I hlank (8/20/01) & NA & $N A$ & $N A$ & 2034 & SPC00536012 & LLNL & SPC00536913 & INI \\
\hline & 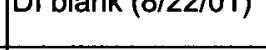 & INA & NA & NA & 2,034 & STCUUS36912 & LANL & SPC00536914 & LLNL \\
\hline 1 & IECF Cn CIMH21 & $2.8-3.8$ & SPC01014835 & 1796 & 2014 & 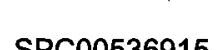 & LLNL & SPC00536916 & 11 \\
\hline & 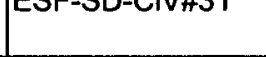 & & SPC01 & 1,100 & 2,014 & STCUOS30915 & LANL & SPC00536917 & LLIVL \\
\hline 1 & FSF-SD-CIV\#21 & $113-130$ & SPC03017095 & 1935 & 2115 & SPC00536918 & LLNL & SPC00536919 & $\| \mathrm{NI}$ \\
\hline & & $11.3-13.0$ & & 1,935 & 2,715 & SPC 00536918 & LANL & SPC00536920 & LLNL \\
\hline 1 & ESF-SD-CIV\#30 & $6.4-8.4$ & SPC02015998 & 1965 & 2092 & 36921 & LLNL & SPC00536922 & $\| \mathrm{N}$ \\
\hline & & & & 1,000 & 2,002 & & LANL & SPC00536923 & CLIVL \\
\hline 1 & IFSF-SD_CIV\#32 & $76-95$ & SPC02016007 & 2310 & 2089 & SPCOn536924 & LLNL & SPC00536925 & IN! \\
\hline & 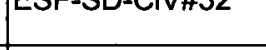 & $1.0-9.0$ & SP 60201000 & | 2,010 & 2,009 & STCU0030924 & LANL & SPC00536926 & LEIVL \\
\hline 1 & FSF_SD_CIYHO2R & 4. & SP & 2333 & 2134 & $\operatorname{SPC}$ & LLNL & SPC00536928 & N" \\
\hline & |EST-SD-CIVHZO & & $\mathrm{SP}$ & [2,000 & 2,1004 & or 20000002 & LANL & SPC00536929 & LEIVL \\
\hline 1 & & $\begin{array}{l}2.1-2.4 \\
2.4-3.0\end{array}$ & $\begin{array}{l}\text { SPC01014830 } \\
\text { SPC01014831 }\end{array}$ & 399 & 2103 & $\operatorname{sPc}$ & LLNL & SPC00536931 & \\
\hline & & $\begin{array}{l}3.0-3.5 \\
3.8-4.8\end{array}$ & $\begin{array}{l}\text { SPC01014832 } \\
\text { SPC01014833 }\end{array}$ & & & & LANL & SPC00536932 & \\
\hline
\end{tabular}


Table 4-7. Processing History of Validation Study Core Samples Leached at the USGS during Phase III (continued)

\begin{tabular}{|c|c|c|c|c|c|c|c|c|c|}
\hline $\begin{array}{l}\text { Batch } \\
\text { No. }\end{array}$ & Sample Identifier & $\begin{array}{l}\text { Interval } \\
\text { (ft) }\end{array}$ & $\begin{array}{c}\text { SMF Barcode } \\
\text { Identifier }\end{array}$ & $\begin{array}{c}\text { Rock } \\
\text { Mass } \\
\text { (g) }\end{array}$ & $\begin{array}{c}\text { Water } \\
\text { Mass } \\
\text { (g) }\end{array}$ & $\begin{array}{l}\text { USGS-IC } \\
\text { Barcode } \\
\text { Identifier }\end{array}$ & $\begin{array}{c}\mathrm{Cl} \\
\text { Precip. } \\
\text { and } \\
\text { Target } \\
\text { Prep. }\end{array}$ & $\begin{array}{l}{ }^{36} \mathrm{CI} \text { Barcode } \\
\text { Identifier }\end{array}$ & $\begin{array}{l}\text { AMS } \\
\text { Facility }\end{array}$ \\
\hline \multirow[t]{2}{*}{1} & \multirow{2}{*}{ ESF-SD-CIV\#22 } & \multirow{2}{*}{$4.5-6.3$} & \multirow{2}{*}{ SPC01014821 } & \multirow{2}{*}{1,840} & \multirow{2}{*}{2,096} & \multirow{2}{*}{ SPC00536933 } & LLNL & SPC00536934 & \multirow{2}{*}{ LLNL } \\
\hline & & & & & & & LANL & SPC00536935 & \\
\hline \multirow[t]{2}{*}{1} & \multirow{2}{*}{ ESF-SD-CIV\#21 } & \multirow{2}{*}{$2.8-4.6$} & \multirow{2}{*}{ SPC01014819 } & \multirow{2}{*}{1,736} & \multirow{2}{*}{2,049} & \multirow{2}{*}{ SPC00536936 } & LLNL & SPC00536937 & \multirow{2}{*}{ LLNL } \\
\hline & & & & & & & LANL & SPC00536938 & \\
\hline \multirow[t]{2}{*}{1} & \multirow{2}{*}{ DI blank (8/24/01) } & \multirow{2}{*}{ NA } & \multirow{2}{*}{ NA } & \multirow{2}{*}{ NA } & \multirow{2}{*}{2,061} & \multirow{2}{*}{ SPC00536939 } & LLNL & SPC00536940 & \multirow{2}{*}{ LLNL } \\
\hline & & & & & & & LANL & SPC00536941 & \\
\hline \multirow[t]{2}{*}{1} & \multirow{2}{*}{ ESF-SD-CIV\#35 } & \multirow{2}{*}{$6.4-8.5$} & \multirow{2}{*}{ SPC02015949| } & \multirow{2}{*}{2,366} & \multirow{2}{*}{2,135} & \multirow{2}{*}{ SPC00536942 } & LLNL & SPC00536943 & \multirow{2}{*}{ LLNL } \\
\hline & & & & & & & LANL & SPC00536944 & \\
\hline 1 & ESF-SD-CIV\#27 & $100-120$ & SPC02016027| & 2211 & $\ldots$ & SPC00536945 & LLNL & SPC00536946 & INI \\
\hline & & & & & & & LANL & SPC00536947 & LEIVL \\
\hline 1 & 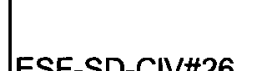 & $\begin{array}{l}3.0-4.0 \\
4.0-4.8\end{array}$ & \begin{tabular}{|l|} 
SPC01014822 \\
SPC01014823
\end{tabular} & 1689 & 2010 & SPCOn536048 & LLNL & SPC00536949 & IN" \\
\hline & $\sqrt{\pi}+0$ & $\begin{array}{l}4.8-5.0 \\
5.1-6.3\end{array}$ & \begin{tabular}{|l|} 
SPC01014824 \\
SPC01014825
\end{tabular} & 1,000 & $2,0+0$ & OT & LANL & SPC00536950 & LLIVL \\
\hline 1 & IFSF- & $\begin{array}{l}3.0-4.0 \\
4.0-4.8\end{array}$ & $\mid$\begin{tabular}{|l|} 
SPC01014822 \\
SPC01014823
\end{tabular} & 1700 & 2044 & SPC 00536951 & LLNL & SPC00536952 & LLNL \\
\hline & & $\begin{array}{l}4.8-5.0 \\
5.1-6.3\end{array}$ & $\mid \begin{array}{l}\text { SPC01014824 } \\
\text { SPC01014825 }\end{array}$ & Pros & | & (5) & LANL & SPC00536953 & \\
\hline 1 & DI system water & NA & NA & NA & NA & NA & LLNL & SPC00516600 & $\|N\|$ \\
\hline & sample $(8 / 28 / 01)$ & NA & 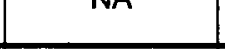 & $T \sqrt{A}$ & TNA & $\mathrm{INA}$ & LANL & SPC00516601 & LETIVL \\
\hline 2 & ESF-SD-CIV\#24 & $40-66$ & SPC01015063 & 1863 & 2054 & SPC00536954 & LLNL & SPC00536955 & LLNL \\
\hline & & & & & & & LANL & SPC00536956 & PRIME \\
\hline 2 & FSF-SD_CIV\#38 & $1.4-3.3$ & SPC01015068 & 1959 & 2076 & SPC00536957 & LLNL & SPC00536958 & LLNL \\
\hline & & & 069 & & $2, \infty$, & 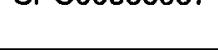 & LANL & SPC00536959 & PRIME \\
\hline 2 & ESF-SD-CIV\#37 & 3 & SPC01015066 & 1726 & 2055 & SP & LLNL & SPC00536961 & LLNL \\
\hline & & & & $1, r<0$ & 2,000 & 0000 & LANL & SPC00536962 & PRIME \\
\hline 2 & USGS system & NA & NA & NA & 2057 & SPC00536963 & LLNL & SPC00516602 & LLNL \\
\hline & blank $(9 / 5 / 01)$ & INA & m & 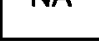 & (2,0) & or 200000000 & LANL & SPC00516603 & PRIME \\
\hline
\end{tabular}


Table 4-7. Processing History of Validation Study Core Samples Leached at the USGS during Phase III (continued)

\begin{tabular}{|c|c|c|c|c|c|c|c|c|c|}
\hline $\begin{array}{l}\text { Batch } \\
\text { No. }\end{array}$ & Sample Identifier & $\begin{array}{l}\text { Interval } \\
\text { (ft) }\end{array}$ & $\begin{array}{c}\text { SMF Barcode } \\
\text { Identifier }\end{array}$ & $\begin{array}{l}\text { Rock } \\
\text { Mass } \\
\text { (g) }\end{array}$ & $\begin{array}{c}\text { Water } \\
\text { Mass } \\
\text { (g) }\end{array}$ & $\begin{array}{l}\text { USGS-IC } \\
\text { Barcode } \\
\text { Identifier }\end{array}$ & $\begin{array}{c}\text { Cl } \\
\text { Precip. } \\
\text { and } \\
\text { Target } \\
\text { Prep. } \\
\end{array}$ & $\begin{array}{l}{ }^{36} \mathrm{CI} \text { Barcode } \\
\text { Identifier }\end{array}$ & $\begin{array}{l}\text { AMS } \\
\text { Facility }\end{array}$ \\
\hline \multirow[t]{2}{*}{2} & \multirow{2}{*}{ ESF-SD-CIV\#39 } & \multirow{2}{*}{$2.1-3.5$} & \multirow{2}{*}{ SPC01015071 } & \multirow{2}{*}{1,665} & \multirow{2}{*}{2,045} & \multirow{2}{*}{ SPC00516604 } & LLNL & SPC00516605 & LLNL \\
\hline & & & & & & & LANL & SPC00516606 & PRIME \\
\hline \multirow[t]{2}{*}{2} & \multirow{2}{*}{ ESF-SD-CIV\#24 } & \multirow{2}{*}{$2.1-4.0$} & \multirow{2}{*}{ SPC01015062 } & \multirow{2}{*}{1,907} & \multirow{2}{*}{2,050} & \multirow{2}{*}{ SPC00516607 } & LLNL & SPC00516608 & LLNL \\
\hline & & & & & & & LANL & SPC00516609 & PRIME \\
\hline \multirow[t]{2}{*}{2} & \multirow{2}{*}{ ESF-SD-CIV\#23 } & \multirow{2}{*}{$\begin{array}{l}4.8-5.9 \\
5.9-6.7 \\
\end{array}$} & \multirow{2}{*}{\begin{tabular}{|l} 
SPC01015060 \\
SPC01015061
\end{tabular}} & \multirow{2}{*}{1,788} & \multirow{2}{*}{2,048} & \multirow{2}{*}{ SPC00516610 } & LLNL & SPC00516611 & LLNL \\
\hline & & & & & & & LANL & SPC00516612 & failed \\
\hline \multirow[t]{2}{*}{2} & \multirow{2}{*}{ ESF-SD-CIV\#20 } & \multirow{2}{*}{$\begin{array}{l}3.8-5.1 \\
5.1-6.1\end{array}$} & \multirow{2}{*}{\begin{tabular}{|l|} 
SPC01015057 \\
SPC01015058
\end{tabular}} & \multirow{2}{*}{1,909} & \multirow{2}{*}{2,054} & \multirow{2}{*}{ SPC00516613 } & LLNL & SPC00516614 & LLNL \\
\hline & & & & & & & LANL & SPC00516615 & PRIME \\
\hline \multirow[t]{2}{*}{2} & \multirow{2}{*}{ ESF-SD-CIV\#19 } & \multirow{2}{*}{$\begin{array}{l}4.4-5.7 \\
5.7-6.4\end{array}$} & \multirow{2}{*}{$\begin{array}{l}\text { SPC01015054 } \\
\text { SPC01015055 }\end{array}$} & \multirow{2}{*}{2,193} & \multirow{2}{*}{2,072} & & LLNL & SPC00516617 & LLNL \\
\hline & & & & & & 8 & LANL & SPC00516618 & PRIME \\
\hline 2 & & & & 1700 & 57 & & LLNL & SPC00516620 & LLNL \\
\hline & & & 301 & 1,199 & $2,05 /$ & 0 & LANL & SPC00516621 & PRIME \\
\hline 2 & & $3.9-4.7$ & 047 & & & & LLNL & SPC00516623 & LLNL \\
\hline & ESF & & $\begin{array}{l}\text { SPC01015048 } \\
\text { SPC01015049 }\end{array}$ & 2,276 & 2,061 & 22 & LANL & SPC00516624 & PRIME \\
\hline 3 & ESF-SD-CIV\#2 & & \begin{tabular}{|l|} 
SPC01015393 \\
SPC01015394
\end{tabular} & 2,322 & 2,051 & SPC00516640 & LLNL & $\begin{array}{l}\text { SPC00516641 } \\
\text { SPC00516642 }\end{array}$ & LLNL \\
\hline 3 & ESF-SD-CIV\#4 & $\begin{array}{l}4 \\
5\end{array}$ & \begin{tabular}{|l|} 
SPC01015399 \\
SPC01015400
\end{tabular} & 1,562 & 1,890 & SPC00516691 & LLNL & $\begin{array}{l}\text { SPC00516692 } \\
\text { SPC00516693 }\end{array}$ & LLNL \\
\hline 3 & ESF-SD-CIV\#7 & $\begin{array}{l}3.9-4.4 \\
6.0-6.5\end{array}$ & $\begin{array}{l}\text { SPC01015406 } \\
\text { SPC01015407 }\end{array}$ & 989 & 1,107 & SPC00516643 & LLNL & $\begin{array}{l}\text { SPC00516644 } \\
\text { SPC00516645 }\end{array}$ & LLNL \\
\hline 3 & ESF-SD-CIV\#11 & $2.4-3.4$ & \begin{tabular}{|l|} 
SPC01015475 \\
SPC01015476
\end{tabular} & 1,513 & 1,955 & SPC00516670 & LLNL & \begin{tabular}{|l|} 
SPC00516671 \\
SPC00516672
\end{tabular} & LLNL \\
\hline 3 & ESF-SD-CIV\#8 & $2.0-3.4$ & SPC01015408 & 1,198 & 1,448 & SPC00516625 & LLNL & \begin{tabular}{|l|} 
SPC00516626 \\
SPC00516627
\end{tabular} & LLNL \\
\hline 3 & ESF-SD-CIV\#8 & $\begin{array}{l}4.0-5.5 \\
6.0-6.2\end{array}$ & \begin{tabular}{|l|} 
SPC01015409 \\
SPC01015410
\end{tabular} & 1,360 & 1,700 & SPC01015124 & LLNL & \begin{tabular}{|l} 
SPC01015125 \\
SPC01015126
\end{tabular} & LLNL \\
\hline
\end{tabular}


Table 4-7. Processing History of Validation Study Core Samples Leached at the USGS during Phase III (continued)

\begin{tabular}{|c|c|c|c|c|c|c|c|c|c|}
\hline $\begin{array}{c}\text { Batch } \\
\text { No. }\end{array}$ & Sample Identifier & $\begin{array}{l}\text { Interval } \\
\text { (ft) }\end{array}$ & $\begin{array}{c}\text { SMF Barcode } \\
\text { Identifier }\end{array}$ & $\begin{array}{c}\text { Rock } \\
\text { Mass } \\
\text { (g) }\end{array}$ & $\begin{array}{l}\text { Water } \\
\text { Mass } \\
\text { (g) }\end{array}$ & $\begin{array}{l}\text { USGS-IC } \\
\text { Barcode } \\
\text { Identifier }\end{array}$ & $\begin{array}{c}\text { Cl } \\
\text { Precip. } \\
\text { and } \\
\text { Target } \\
\text { Prep. } \\
\end{array}$ & $\begin{array}{l}{ }^{36} \mathrm{CI} \text { Barcode } \\
\text { Identifier }\end{array}$ & $\begin{array}{l}\text { AMS } \\
\text { Facility }\end{array}$ \\
\hline 3 & ESF-SD-CIV\#10 & $2.0-3.9$ & SPC01015473 & 1,046 & 1,489 & SPC00516697 & LLNL & $\begin{array}{l}\text { SPC01015119 } \\
\text { SPC01015120 }\end{array}$ & LLNL \\
\hline 3 & ESF-SD-CIV\#12 & $2.0-4.4$ & SPC01015478 & 2,202 & 2,039 & SPC00516667 & LLNL & $\begin{array}{l}\text { SPC00516668 } \\
\text { SPC00516669 }\end{array}$ & LLNL \\
\hline 3 & ESF-SD-CIV\#13 & $2.9-4.5$ & SPC01015483 & 1,858 & 2,018 & SPC00516682 & LLNL & $\begin{array}{l}\text { SPC } 00516683 \\
\text { SPC00516684 }\end{array}$ & LLNL \\
\hline 3 & ESF-SD-CIV\#15 & $\begin{array}{l}4.7-6.0 \\
6.0-6.6\end{array}$ & \begin{tabular}{|l|} 
SPC01015494 \\
SPC01015495
\end{tabular} & 2,073 & 2,052 & SPC00516652 & LLNL & \begin{tabular}{|l|} 
SPC00516653 \\
SPC00516654
\end{tabular} & LLNL \\
\hline 4 & $\begin{array}{l}\text { ESF-SAD-GTB\#1 } \\
\text { hand crush } \\
\text { (10 mesh-3/4") }\end{array}$ & $\begin{array}{l}178.5-179.1 \\
179.3-180.1 \\
180.8-181.8 \\
182.0-183.2 \\
183.9-184.8 \\
185.0-186.3 \\
\end{array}$ & $\begin{array}{l}\text { SPC } 01002899 \\
\text { SPC01002901 } \\
\text { SPC01002903 } \\
\text { SPC01002905 } \\
\text { SPC } 01002907 \\
\text { SPC01002909 } \\
\end{array}$ & 1,605 & 1,853 & SPC01015160 & LLNL & \begin{tabular}{|l|} 
SPC01015161 \\
SPC01015162
\end{tabular} & LLNL \\
\hline 4 & $\begin{array}{l}\text { ESF-SAD-GTB\#1 } \\
\text { hand crush } \\
\left(1 / 4-3 / 4^{\prime \prime}\right)\end{array}$ & $\begin{array}{l}178.5-179.1 \\
179.3-180.1 \\
180.8-181.8 \\
182.0-183.2 \\
183.9-184.8 \\
185.0-186.3 \\
\end{array}$ & \begin{tabular}{|l|} 
SPC 01002899 \\
SPC01002901 \\
SPC01002903 \\
SPC01002905 \\
SPC01002907 \\
SPC01002909 \\
\end{tabular} & 1,477 & 1,534 & SPC01015163 & LLNL & $\begin{array}{l}\text { SPC01015164 } \\
\text { SPC01015165 }\end{array}$ & LLNL \\
\hline 4 & $\begin{array}{l}\text { ESF-SAD-GTB\#1 } \\
\text { mechanical crush } \\
\left(1 / 4-3 / 4^{\prime \prime}\right)\end{array}$ & $\begin{array}{l}178.5-179.1 \\
179.3-180.1 \\
180.8-181.8 \\
182.0-183.2 \\
183.9-184.8 \\
185.0-186.3\end{array}$ & $\begin{array}{l}\text { SPC01002899 } \\
\text { SPC01002901 } \\
\text { SPC01002903 } \\
\text { SPC01002905 } \\
\text { SPC01002907 } \\
\text { SPC01002909 }\end{array}$ & 1,706 & 1,802 & SPC01015169 & LLNL & \begin{tabular}{|l|} 
SPC01015170 \\
SPC01015171
\end{tabular} & LLNL \\
\hline 5 & $\begin{array}{l}\text { ESF-MD- } \\
\text { NICHE3566\#1 }\end{array}$ & $\begin{array}{l}25.3-26.0 \\
28.1-28.9 \\
30.8-31.7\end{array}$ & \begin{tabular}{|l|} 
SPC 01003084 \\
SPC01003089 \\
SPC01003094
\end{tabular} & 1,358 & 1,456 & SPC01015172 & LLNL & $\begin{array}{l}\text { SPC01015173 } \\
\text { SPC01015174 }\end{array}$ & LLNL \\
\hline
\end{tabular}


Table 4-7. Processing History of Validation Study Core Samples Leached at the USGS during Phase III (continued)

\begin{tabular}{|c|c|c|c|c|c|c|c|c|c|}
\hline $\begin{array}{l}\text { Batch } \\
\text { No. }\end{array}$ & Sample Identifier & $\begin{array}{c}\text { Interval } \\
\text { (ft) }\end{array}$ & $\begin{array}{c}\text { SMF Barcode } \\
\text { Identifier }\end{array}$ & $\begin{array}{l}\text { Rock } \\
\text { Mass } \\
(\mathrm{g})\end{array}$ & $\begin{array}{l}\text { Water } \\
\text { Mass } \\
\text { (g) }\end{array}$ & $\begin{array}{l}\text { USGS-IC } \\
\text { Barcode } \\
\text { Identifier }\end{array}$ & $\begin{array}{c}\text { Cl } \\
\text { Precip. } \\
\text { and } \\
\text { Target } \\
\text { Prep. }\end{array}$ & ${ }^{36} \mathrm{Cl}$ Barcode & $\begin{array}{l}\text { AMS } \\
\text { Facility }\end{array}$ \\
\hline 5 & $\begin{array}{l}\text { ESF-MD- } \\
\text { NICHE3566\#2 }\end{array}$ & $\begin{array}{l}20.2-20.7 \\
29.8-30.4 \\
32.0-32.5\end{array}$ & $\begin{array}{l}\text { SPC01003140 } \\
\text { SPC01003155 } \\
\text { SPC01003156 }\end{array}$ & 1,312 & 1,446 & SPC01015175 & LLNL & $\begin{array}{l}\text { SPC01015176 } \\
\text { SPC01015177 }\end{array}$ & LLNL \\
\hline 5 & $\begin{array}{l}\text { ESF-MD- } \\
\text { NICHE3566\#2 }\end{array}$ & $\begin{array}{l}11.5-11.9 \\
13.2-13.7 \\
15.0-15.4\end{array}$ & \begin{tabular}{|l} 
SPC01003121 \\
SPC01003125 \\
SPC01003129
\end{tabular} & 1,153 & 1,419 & SPC01015181 & LLNL & $\begin{array}{l}\text { SPC01015182 } \\
\text { SPC01015183 }\end{array}$ & LLNL \\
\hline 5 & $\begin{array}{l}\text { ESF-MD- } \\
\text { NICHE3566\#1 }\end{array}$ & $\begin{array}{l}14.7-15.8 \\
20.3-20.9\end{array}$ & $\begin{array}{l}\text { SPC01003066 } \\
\text { SPC01003074 }\end{array}$ & 1,435 & 1,511 & SPC01015184 & LLNL & $\begin{array}{l}\text { SPC01015185 } \\
\text { SPC01015186 }\end{array}$ & LLNL \\
\hline 5 & $\begin{array}{l}\text { ESF-MD- } \\
\text { NICHE3566\#LT1 }\end{array}$ & $\begin{array}{l}10.9-11.9 \\
13.4-14.1 \\
22.8-23.5\end{array}$ & \begin{tabular}{|l} 
SPC01004414 \\
SPC01004418 \\
SPC01004435
\end{tabular} & 1,339 & 1,454 & SPC01015187 & LLNL & $\begin{array}{l}\text { SPC01015188 } \\
\text { SPC01015189 }\end{array}$ & LLNL \\
\hline 5 & $\begin{array}{l}\text { ESF-MD- } \\
\text { NICHE3566\#LT1 }\end{array}$ & $\begin{array}{l}25.0-25.9 \\
28.2-28.8 \\
31.5-31.9 \\
37.1-38.1\end{array}$ & $\begin{array}{l}\text { SPC01004439 } \\
\text { SPC01004443 } \\
\text { SPC01004451 } \\
\text { SPC01004462 }\end{array}$ & 1,665 & 1,737 & SPC01015190 & LLNL & $\begin{array}{l}\text { SPC01015191 } \\
\text { SPC01015192 }\end{array}$ & LLNL \\
\hline 5 & $\begin{array}{l}\text { USGS system } \\
\text { blank (6/24/02) }\end{array}$ & NA & NA & NA & 1,615 & SPC01015193 & LLNL & $\begin{array}{l}\text { SPC01015194 } \\
\text { SPC01015195 }\end{array}$ & LLNL \\
\hline
\end{tabular}

DTNs: LL031200223121.036 (Q), Filename: Total_AMS_Summary_2001-02c.xls; GS030608312272.005 (Q);LA0305RR831222.001 (UQ)

NOTES: $A M S=$ accelerator mass spectrometer, $\mathrm{DI}=$ deionized water; $I C=$ ion chromatography, LANL $=$ Los Alamos National Laboratory, LLNL = Lawrence Livermore National Laboratory, NA = not applicable, PRIME = Purdue Rare Isotope Measurement Laboratory, SMF = Sample Management Facility, USGS = U.S. Geological Survey.

Samples were crushed at either the SMF or USGS, and leached at the USGS. The leachates were distributed to LANL and LLNL for $\mathrm{AgCl}$ precipitation and target preparation.

For Batch \#1 samples, targets prepared at LANL and LLNL were analyzed at LLNL-CAMS. For Batch \#2, the targets prepared at LANL were analyzed at PRIME Lab and targets prepared at LLNL were analyzed at LLNL-CAMS. Targets for Batches \#3, \#4, and \#5 were prepared and analyzed at LLNL.

The weight of water was not recorded for ESF-SD-CIV\#27. The concentration was calculated assuming a 1:1 water to rock mass ratio (MOL.20030626.0093, p. 2).

DI blank (8/28/01) was an unfiltered 1-L sample from the DI water system.

Due to high ${ }^{36} \mathrm{~S}$, which interferes with ${ }^{36} \mathrm{Cl}$ measurements, LLNL did not report data for CIV\#18 (see file YMP_CI35-36-37C.Nov_29_01.xls in LL031200223121.036). The USGS data for CIV\#18 are located in GS0 30608312272.005 . 
Table 4-8. Validation Study Core Intervals Chosen for Passive Leaching at the USGS during Phase III

\begin{tabular}{|c|c|c|}
\hline Sample Identifier & $\begin{array}{l}\text { Interval } \\
\text { (ft) }\end{array}$ & Comments \\
\hline ESF-SD-CIV\#2 & $\begin{array}{l}4.7-5.9 \\
5.9-6.6\end{array}$ & $\begin{array}{l}\text { Rubbly (4.7-4.8); intact, about } 1 \text { fracture (4.8-5.9), intact (5.9- } \\
6.4) ; \text { broken }(6.4-7.0)\end{array}$ \\
\hline ESF-SD-CIV\#4 & $\begin{array}{l}4.4-5.6 \\
5.7-6.3\end{array}$ & $\begin{array}{l}\text { Rubbly (4.4-4.7); rubbly-broken (4.7-5.3); rubbly (5.3-5.6); } \\
\text { broken (5.7-5.9); rubbly (5.9-6.3) }\end{array}$ \\
\hline ESF-SD-CIV\#7 & $\begin{array}{l}3.9-4.4 \\
6.0-6.5 \\
\end{array}$ & 2 blocks $(3.9-4.4) ;$ broken, more than 12 fractures $(6.0-8.0)$ \\
\hline ESF-SD-CIV\#8 & $2.0-3.4$ & Broken-rubbly (2.0-2.8); rubbly (2.8-3.4) \\
\hline ESF-SD-CIV\#8 & $\begin{array}{l}4.0-5.5 \\
6.0-6.2 \\
\end{array}$ & $\begin{array}{l}\text { Rubbly (4.0-4.3); block (4.3-4.7); rubbly (4.7-5.5); block (6.0- } \\
6.2)\end{array}$ \\
\hline ESF-SD-CIV\#10 & $2.0-3.9$ & Block (2.0-2.2); rubbly-shattered (2.2-3.65); block (3.7-3.9) \\
\hline ESF-SD-CIV\#11 & $\begin{array}{l}2.4-3.4 \\
4.1-5.2\end{array}$ & $\begin{array}{l}\text { Broken, about } 5 \text { fractures }(1.7-3.0) \text {; broken-rubbly (3.0-3.4); } \\
\text { rubbly (4.1-5.1) }\end{array}$ \\
\hline ESF-SD-CIV\#12 & $2.0-4.4$ & $\begin{array}{l}\text { Intact, few hairline fractures (2.0-3.3); broken (3.3-3.65); intact } \\
(3.65-4.3) \text {; broken (4.3-4.4) }\end{array}$ \\
\hline ESF-SD-CIV\#13 & $2.9-4.5$ & Broken, more than 12 fractures (2.9-6.3) \\
\hline ESF-SD-CIV\#15 & $\begin{array}{l}4.7-6.0 \\
6.0-6.6\end{array}$ & Broken, about 2 fractures \\
\hline ESF-SD-CIV\#17 & $3.9-6.4$ & Broken, blocky \\
\hline ESF-SD-CIV\#18 & $3.6-5.6$ & Broken \\
\hline ESF-SD-CIV\#19 & $4.4-6.4$ & Intact-broken-rubbly \\
\hline ESF-SD-CIV\#20 & $3.8-6.1$ & Broken-rubble \\
\hline ESF-SD-CIV\#21 & $11.3-13.0$ & Intact, 1 fracture \\
\hline ESF-SD-CIV\#21 & $2.8-4.6$ & Broken-rubbly \\
\hline ESF-SD-CIV\#22 & $4.5-6.3$ & Very broken \\
\hline ESF-SD-CIV\#23 & $4.8-6.7$ & Broken \\
\hline ESF-SD-CIV\#24 & $2.1-4.0$ & Large intact pieces, broken in 6 areas \\
\hline ESF-SD-CIV\#24 & $4.0-6.6$ & Large intact pieces, broken in 6 areas \\
\hline ESF-SD-CIV\#26 & $3.0-6.3$ & Broken \\
\hline ESF-SD-CIV\#27 & $10.0-12.0$ & $10.0-12.0$ intact with 2 to 3 fractures \\
\hline ESF-SD-CIV\#28 & $4.0-6.2$ & $\begin{array}{l}\text { Increasingly broken toward the bottom of the run; broken from } \\
5.6-6.2\end{array}$ \\
\hline ESF-SD-CIV\#28 & $6.2-8.0$ & Intact with 4 to 5 fractures to 8.0 \\
\hline ESF-SD-CIV\#30 & $6.4-8.4$ & Broken \\
\hline ESF-SD-CIV\#31 & $2.8-4.5$ & Broken \\
\hline ESF-SD-CIV\#32 & $7.6-9.5$ & Intact \\
\hline ESF-SD-CIV\#33 & $9.9-11.4$ & Intact with about 3 fractures \\
\hline ESF-SD-CIV\#34 & $2.1-4.8$ & $\begin{array}{l}\text { Broken (2.1-3.0); rubbly (3.0-3.8); intact with about } 3 \text { fractures } \\
(3.8-4.8)\end{array}$ \\
\hline ESF-SD-CIV\#35 & $6.4-8.5$ & Broken \\
\hline ESF-SD-CIV\#36 & $5.4-9.4$ & $\begin{array}{l}\text { Broken (5.4-6.7); rubbly (6.7-7.0); intact with about } 4 \text { fractures } \\
(7.0-9.1), 9.1-9.4 \text {, one chunk }\end{array}$ \\
\hline ESF-SD-CIV\#37 & $3.6-5.3$ & 3.0-5.9 intact with 2 to 3 discrete fractures \\
\hline ESF-SD-CIV\#38 & $1.4-3.9$ & 1:4-2.6 intact; $2.6-3.9$ broken to rubbly \\
\hline ESF-SD-CIV\#39 & $2.1-3.5$ & Intact \\
\hline
\end{tabular}

DTN: LL031200223121.036 (Q), Filename: Total_AMS_Summary_2001-02c.xls

NOTE: "Comments" are from the video log observations described in Appendix B. 
Table 4-9. Chloride Concentrations and ${ }^{36} \mathrm{CI} / \mathrm{Cl}$ Ratios in Core Samples Leached and Analyzed at USGS-LLNL during Phase III

\begin{tabular}{|c|c|c|c|c|c|c|c|c|c|c|c|}
\hline \multirow[b]{2}{*}{$\begin{array}{l}\text { Batch } \\
\text { No. }\end{array}$} & \multirow[b]{2}{*}{$\begin{array}{l}\text { Sample } \\
\text { Identifier }\end{array}$} & \multirow[b]{2}{*}{$\begin{array}{l}\text { SMF Barcode } \\
\text { Identifier for } \\
\text { LLNL Sample }\end{array}$} & \multirow[b]{2}{*}{$\begin{array}{c}\text { Water } \\
\text { Mass } \\
\text { Used for } \\
\text { Analysis } \\
(\mathrm{kg})\end{array}$} & \multirow[b]{2}{*}{$\begin{array}{l}\text { Date } \\
\text { Submitted } \\
\text { to LLNL }\end{array}$} & \multicolumn{4}{|c|}{ AMS Results } & \multicolumn{3}{|c|}{ Final Results } \\
\hline & & & & & CAMS ID & $\begin{array}{c}\text { Sulfur } \\
\text { Figure } \\
\text { of } \\
\text { Merit }\end{array}$ & $\begin{array}{c}\text { Leachate } \mathrm{Cl} \\
\text { Concentration } \\
\text { (mg/kg water) }\end{array}$ & $\begin{array}{l}{ }^{36} \mathrm{Cl} / \mathrm{Cl} \\
\times 10^{15}\end{array}$ & $\begin{array}{c}\text { Leachate Cl } \\
\text { Concen- } \\
\text { tration } \\
\text { (mg/kg } \\
\text { water) } \\
\end{array}$ & $\begin{array}{c}\text { Leachate } \mathrm{Cl} \\
\text { Concen- } \\
\text { tration } \\
\text { (mg/kg } \\
\text { rock) } \\
\end{array}$ & $\begin{array}{l}{ }^{36} \mathrm{Cl} / / \mathrm{Cl} \\
\times 10^{15}\end{array}$ \\
\hline 1 & EVAL001 & SPC00536901 & 0.900 & $08 / 28 / 01$ & CL9634 & 0.99 & $0.140 \pm 0.007$ & $462 \pm 40$ & $0.130 \pm 0.008$ & $0.146 \pm 0.009$ & $454 \pm 145$ \\
\hline 1 & $\begin{array}{r}\text { ESF-SL } \\
(2.8-\end{array}$ & SPC00536919 & 0.900 & 08/28/01 & CL9640 & 0.98 & $0.145 \pm 0.007$ & $381 \pm 21$ & $0.135 \pm 0.008$ & $0.147 \pm 0.009$ & $368 \pm 122$ \\
\hline 1 & $\begin{array}{r}\text { ESF-SD } \\
(11.3\end{array}$ & SPC00536937 & 0.900 & 08/28/01 & CL9646 & 0.97 & $0.325 \pm 0.016$ & $305 \pm 25$ & $0.315 \pm 0.016$ & $0.372 \pm 0.019$ & $297 \pm 114$ \\
\hline 1 & $\begin{array}{c}\text { ESF-SD-CIV\#22 } \\
(4.5-6.3)\end{array}$ & SPC00536934 & 0.900 & 08/28/01 & CL9645 & 0.99 & $0.154 \pm 0.008$ & $333 \pm 22$ & $0.144 \pm 0.009$ & $0.165 \pm 0.010$ & $317 \pm 121$ \\
\hline 1 & $\begin{array}{l}\text { ESF-SD-CIV\#26-1 } \\
(3.0-6.3)\end{array}$ & SPC00536949 & 0.900 & 08/28/01 & CL.9650 & 0.83 & $0.097 \pm 0.005$ & $200 \pm 23$ & $0.087 \pm 0.006$ & $0.105 \pm 0.008$ & $159 \pm 137$ \\
\hline 1 & $\begin{array}{c}\text { ESF-SD-CIV\#26-2 } \\
(3.0-6.3)\end{array}$ & SPC00536952 & 0.900 & 08/28/01 & CL9651 & 0.87 & $0.105 \pm 0.005$ & $177 \pm 27$ & $0.095 \pm 0.006$ & $0.114 \pm 0.008$ & $137 \pm 136$ \\
\hline 1 & $\begin{array}{c}\text { ESF-SD-CIV\#27 } \\
(10.0-12.0) \\
\end{array}$ & SPC00536946 & 0.900 & 08/28/01 & CL9649 & 0.97 & $0.149 \pm 0.007$ & $211 \pm 21$ & $0.139 \pm 0.008$ & $0.125 \pm 0.007$ & $186 \pm 118$ \\
\hline 1 & $\begin{array}{r}\text { ESF-SD- } \\
(4.0-6 \\
\end{array}$ & SPC00536928 & 0.900 & 08/28/01 & CL9643 & 0.85 & $0.089 \pm 0.004$ & $204 \pm 32$ & $0.079 \pm 0.006$ & $0.073 \pm 0.005$ & $160 \pm 150$ \\
\hline 1 & $\begin{array}{c}\text { ESF-SD-CIV\#28 } \\
(6.2-8.0)\end{array}$ & PC00536907 & 0.900 & 08/28/01 & CL9636 & 0.92 & $0.146 \pm 0.007$ & $189 \pm 55$ & $0.136 \pm 0.008$ & $0.153 \pm 0.009$ & $163 \pm 161$ \\
\hline 1 & $\begin{array}{c}\text { ESF-SD-CIV\#30 } \\
(6.4-8.4)\end{array}$ & PC00536922 & 0.900 & $08 / 28 / 01$ & CL9641 & 0.98 & $38 \pm 0.007$ & $185 \pm 19$ & 0.008 & $0.136 \pm 0.009$ & $156 \pm 119$ \\
\hline 1 & $\begin{array}{c}\text { ESF-SD-CIV\#31 } \\
(2.8-4.5)\end{array}$ & 00536916 & 300 & 08/28/01 & 39 & 0.98 & 3 & 27 & 0.005 & 06 & 176 \\
\hline 1 & $\begin{array}{c}\text { ESF-SD-CIV\#32 } \\
(7.6-9.5)\end{array}$ & 25 & 0.900 & 08/28/01 & 2 & 0.98 & 99 & \pm 22 & \pm 0.010 & 0.154 & \pm 115 \\
\hline 1 & $\begin{array}{c}\text { ESF-SD-CIV\#33 } \\
(9.9-11.4) \\
\end{array}$ & 4 & 0.900 & 08/28/01 & 35 & 0.99 & 05 & \pm 34 & \pm 0.006 & .007 & 156 \\
\hline 1 & $\begin{array}{c}\text { ESF-SD-CIV\#34 } \\
(2.1-4.8)\end{array}$ & 1 & 0.900 & 08/28/01 & 4 & 0.97 & 005 & $267 \pm 26$ & $0.090 \pm 0.006$ & $0.079 \pm 0.006$ & $236 \pm 139$ \\
\hline 1 & $\begin{array}{c}\text { ESF-SD-CIV\#35 } \\
(6.4-8.5)\end{array}$ & SPC00536943 & 0.900 & 08/28/01 & CL9648 & 0.95 & $0.111 \pm 0.006$ & $235 \pm 43$ & $0.101 \pm 0.007$ & $0.091 \pm 0.007$ & $203 \pm 154$ \\
\hline 1 & $\begin{array}{c}\text { ESF-SD-CIV\#36 } \\
(5.4-6.7 / 8.1-9.4)\end{array}$ & SPC00536910 & 0.900 & 08/28/01 & CL9637 & 0.96 & $0.078 \pm 0.004$ & $416 \pm 31$ & $0.068 \pm 0.006$ & $0.069 \pm 0.006$ & $395 \pm 164$ \\
\hline
\end{tabular}


Table 4-9. Chloride Concentrations and ${ }^{36} \mathrm{CI} / \mathrm{CI}$ Ratios in Core Samples Leached and Analyzed at USGS-LLNL during Phase III (continued)

\begin{tabular}{|c|c|c|c|c|c|c|c|c|c|c|c|}
\hline \multirow[b]{2}{*}{$\begin{array}{l}\text { Batch } \\
\text { No. }\end{array}$} & \multirow[b]{2}{*}{$\begin{array}{l}\text { Sample } \\
\text { Identifier }\end{array}$} & \multirow[b]{2}{*}{$\begin{array}{l}\text { SMF Barcode } \\
\text { Identifier for } \\
\text { LLNL Sample }\end{array}$} & \multirow[b]{2}{*}{$\begin{array}{c}\text { Water } \\
\text { Mass } \\
\text { Used for } \\
\text { Analysis } \\
\text { (kg) }\end{array}$} & \multirow[b]{2}{*}{$\begin{array}{l}\text { Date } \\
\text { Submitted } \\
\text { to LLNL }\end{array}$} & \multicolumn{4}{|c|}{ AMS Results } & \multicolumn{3}{|c|}{ Final Results } \\
\hline & & & & & CAMS ID & $\begin{array}{c}\text { Sulfur } \\
\text { Figure } \\
\text { of } \\
\text { Merit }\end{array}$ & $\begin{array}{c}\text { Leachate } \mathrm{Cl} \\
\text { Concentration } \\
\text { (mg/kg water) }\end{array}$ & $\begin{array}{l}{ }^{36} \mathrm{Cl} / \mathrm{Cl} \\
\times 10^{15}\end{array}$ & $\begin{array}{c}\text { Leachate } \mathrm{Cl} \\
\text { Concen- } \\
\text { tration } \\
\text { (mg/kg } \\
\text { water) }\end{array}$ & \begin{tabular}{|c|} 
Leachate $\mathrm{Cl}$ \\
Concen- \\
tration \\
(mg/kg \\
rock)
\end{tabular} & $\begin{array}{l}{ }^{36} \mathrm{Cl} / \mathrm{Cl} \\
\times 10^{15}\end{array}$ \\
\hline 2 & $\begin{array}{c}\text { ESF-SD-CIV\#17 } \\
(3.9-6.4) \\
\end{array}$ & SPC00516623 & 0.900 & $09 / 10 / 01$ & CL9722 & 0.99 & $0.186 \pm 0.009$ & $308 \pm 15$ & $0.176 \pm 0.010$ & $0.160 \pm 0.009$ & $294 \pm 111$ \\
\hline 2 & $\begin{array}{c}\text { ESF-SD-CIV\#19 } \\
(4.4-6.4)\end{array}$ & SPC00516617 & 0.900 & $09 / 10 / 01$ & CL9720 & 0.88 & $0.166 \pm 0.008$ & $333 \pm 68$ & $0.156 \pm 0.009$ & $0.148 \pm 0.008$ & $319 \pm 182$ \\
\hline 2 & $\begin{array}{c}\text { ESF-SD-CIV\#20 } \\
(3.8-6.1)\end{array}$ & SPC00516614 & 0.900 & $09 / 10 / 01$ & CL9719 & 0.96 & $0.090 \pm 0.005$ & $444 \pm 61$ & $0.080 \pm 0.006$ & $0.087 \pm 0.007$ & $430 \pm 194$ \\
\hline 2 & $\begin{array}{c}\text { ESF-SD-CIV\#23 } \\
(4.8-6.7) \\
\end{array}$ & SPC00516611 & 0.900 & $09 / 10 / 01$ & CL9718 & 0.95 & $0.158 \pm 0.008$ & $327 \pm 33$ & $0.148 \pm 0.009$ & $0.170 \pm 0.010$ & $311 \pm 131$ \\
\hline 2 & $\begin{array}{c}\text { ESF-SD-CIV\#24 } \\
(2.1-4.0)\end{array}$ & SPC00516608 & 0.900 & $09 / 10 / 01$ & CL9717 & 0.98 & $0.133 \pm 0.007$ & $333 \pm 21$ & $0.123 \pm 0.008$ & $0.133 \pm 0.009$ & $315 \pm 124$ \\
\hline 2 & $\begin{array}{c}\text { ESF-SD-CIV\#24 } \\
(4.6-6.6)\end{array}$ & SPC00536955 & 0.900 & $09 / 10 / 01$ & CL9712 & 0.98 & $0.189 \pm 0.009$ & $611 \pm 90$ & $0.179 \pm 0.010$ & $0.197 \pm 0.011$ & $615 \pm 221$ \\
\hline 2 & $\begin{array}{c}\text { ESF-SD-CIV\#37 } \\
(3.6-5.3)\end{array}$ & SPC00936961 & 0.900 & $09 / 10 / 01$ & CL9714 & 0.95 & $0.093 \pm 0.005$ & $428 \pm 48$ & $0.089 \pm 0.011$ & $0.099 \pm 0.008$ & $413 \pm 173$ \\
\hline 2 & $\begin{array}{c}\text { ESF-SD-CIV\#38 } \\
(1.4-3.9)\end{array}$ & SPC00536958 & 0.900 & $09 / 10 / 01$ & CL9713 & 0.99 & $0.324 \pm 0.016$ & $582 \pm 31$ & $0.314 \pm 0.016$ & $0.333 \pm 0.017$ & $583 \pm 125$ \\
\hline 2 & $\begin{array}{c}\text { ESF-SD-CIV\#39 } \\
(2.1-3.5) \\
\end{array}$ & SPC00516605 & 0.900 & $09 / 10 / 01$ & CL9716 & 0.92 & $0.114 \pm 0.006$ & $540 \pm 93$ & $0.104 \pm 0.007$ & $0.128 \pm 0.009$ & $538 \pm 241$ \\
\hline 3 & $\begin{array}{c}\text { ESF-SD-CIV\#2 } \\
(4.7-6.6)\end{array}$ & SPC00516641 & 0.900 & $01 / 25 / 02$ & CL10023 & 1 & $0.421 \pm 0.021$ & $320 \pm 30$ & $0.411 \pm 0.021$ & $0.363 \pm 0.019$ & $314 \pm 117$ \\
\hline 3 & $\begin{array}{c}\text { ESF-SD-CIV\#4 } \\
(4.4-6.3)\end{array}$ & SPC00516692 & 0.877 & $01 / 25 / 02$ & CL10024 & 1 & $0.136 \pm 0.007$ & $535 \pm 63$ & $0.126 \pm 0.008$ & $0.153 \pm 0.010$ & $533 \pm 182$ \\
\hline 3 & $\begin{array}{c}\text { ESF-SD-CIV\#7 } \\
(3.9-4.4)\end{array}$ & SPC00516644 & 0.900 & $01 / 25 / 02$ & CL10025 & 1 & $0.077 \pm 0.004$ & $211 \pm 175$ & $0.067 \pm 0.006$ & $0.075 \pm 0.006$ & $159 \pm 428$ \\
\hline 3 & $\begin{array}{c}\text { ESF-SD-CIV\#8 } \\
(2.0-3.4)\end{array}$ & SPC00516627 & 0.411 & $01 / 25 / 02$ & CL10117 & 0.8 & $0.121 \pm 0.006$ & $341 \pm 116$ & $0.111 \pm 0.007$ & $0.134 \pm 0.009$ & $322 \pm 279$ \\
\hline 3 & $\begin{array}{c}\text { ESF-SD-CIV\#8 } \\
(4.0-6.2)\end{array}$ & SPC01015126 & 0.513 & $01 / 25 / 02$ & CL10118 & 0.98 & $0.055 \pm 0.003$ & $286 \pm 71$ & $0.045 \pm 0.005$ & $0.056 \pm 0.006$ & $226 \pm 255$ \\
\hline 3 & $\begin{array}{c}\text { ESF-SD-CIV\#10 } \\
(2.0-3.9)\end{array}$ & SPC01015120 & 0.470 & $01 / 25 / 02$ & CL10119 & 0.97 & $0.043 \pm 0.002$ & $553 \pm 112$ & $0.033 \pm 0.004$ & $0.046 \pm 0.006$ & $552 \pm 386$ \\
\hline 3 & $\begin{array}{l}\text { ESF-SD-CIV\#11 } \\
(2.4-5.2)\end{array}$ & SPC00516671 & 0.900 & $01 / 25 / 02$ & CL10026 & 1 & $0.039 \pm 0.002$ & $465 \pm 367$ & $0.029 \pm 0.004$ & $0.037 \pm 0.006$ & $\begin{array}{r}434 \\
\pm 1,026 \\
\end{array}$ \\
\hline 3 & $\begin{array}{c}\text { ESF-SD-CIV\#12 } \\
(2.0-4.4)\end{array}$ & $\begin{array}{l}\text { SPC00516668 } \\
\text { SPC00516669 }\end{array}$ & 0.936 & $01 / 25 / 02$ & CL10120 & 0.95 & $0.058 \pm 0.003$ & $362 \pm 64$ & $0.048 \pm 0.005$ & $0.044 \pm 0.005$ & $321 \pm 238$ \\
\hline
\end{tabular}


Table 4-9. Chloride Concentrations and ${ }^{36} \mathrm{Cl} / \mathrm{Cl}$ Ratios in Core Samples Leached and Analyzed at USGS-LLNL during Phase III (continued)

\begin{tabular}{|c|c|c|c|c|c|c|c|c|c|c|c|}
\hline \multirow[b]{2}{*}{$\begin{array}{l}\text { Batch } \\
\text { No. }\end{array}$} & \multirow[b]{2}{*}{$\begin{array}{l}\text { Sample } \\
\text { Identifier }\end{array}$} & \multirow[b]{2}{*}{$\begin{array}{l}\text { SMF Barcode } \\
\text { Identifier for } \\
\text { LLNL Sample. }\end{array}$} & \multirow[b]{2}{*}{$\begin{array}{c}\text { Water } \\
\text { Mass } \\
\text { Used for } \\
\text { Analysis } \\
\text { (kg) }\end{array}$} & \multirow[b]{2}{*}{$\begin{array}{l}\text { Date } \\
\text { Submitted } \\
\text { to LLNL }\end{array}$} & \multicolumn{4}{|c|}{ AMS Results } & \multicolumn{3}{|c|}{ Final Results } \\
\hline & & & & & CAMS ID & $\begin{array}{l}\text { Sulfur } \\
\text { Figure } \\
\text { of Merit }\end{array}$ & $\begin{array}{l}\text { Leachate } \mathrm{Cl} \\
\text { Concentration } \\
\text { (mg/kg water) }\end{array}$ & $\begin{array}{l}{ }^{36} \mathrm{CI} / \mathrm{Cl} ! \\
\times 10^{15}\end{array}$ & $\begin{array}{c}\text { Leachate } \mathrm{Cl} \\
\text { Concen- } \\
\text { tration } \\
\text { (mg/kg } \\
\text { water) }\end{array}$ & $\begin{array}{c}\text { Leachate } \mathrm{Cl} \\
\text { Concen- } \\
\text { tration } \\
\text { (mg/kg } \\
\text { rock) }\end{array}$ & $\begin{array}{l}{ }^{36} \mathrm{Cl} / \mathrm{Cl} \\
\times 10^{15}\end{array}$ \\
\hline 3 & $\begin{array}{c}\text { ESF-SD-CIV\#13 } \\
(2.9-4.5)\end{array}$ & $\begin{array}{l}\text { SPC00516683 } \\
\text { SPC00516684 }\end{array}$ & 0.938 & $01 / 25 / 02$ & CL10121 & 0.95 & $0.051 \pm 0.003$ & $433 \pm 75$ & $0.041 \pm 0.005$ & $0.044 \pm 0.005$ & $403 \pm 278$ \\
\hline 3 & $\begin{array}{c}\text { ESF-SD-CIV\#15 } \\
(4.7-6.6)\end{array}$ & $\begin{array}{l}\text { SPC00516653 } \\
\text { SPC00516654 }\end{array}$ & 0.977 & $01 / 25 / 02$ & CL10122 & 0.97 & $0.073 \pm 0.004$ & $437 \pm 49$ & $0.063 \pm 0.006$ & $0.062 \pm 0.006$ & $418 \pm 193$ \\
\hline 4 & $\begin{array}{c}\text { ESF-SAD-GTB\#1 } \\
\text { hand crush } \\
(1 / 4 "-3 / 4 ")\end{array}$ & SPC01015164 & 0.900 & $06 / 12 / 02$ & CL10323 & 0.99 & $0.47 \pm 0.023$ & $460 \pm 14$ & $0.456 \pm 0.023$ & $0.474 \pm 0.024$ & $457 \pm 107$ \\
\hline 4 & $\begin{array}{c}\text { ESF-SAD-GTB\#1 } \\
\text { mech. crush } \\
\left(1 / 4 "-3 / 4^{\prime \prime}\right)\end{array}$ & SPC01015170 & 0.900 & $06 / 12 / 02$ & CL10324 & 0.98 & $0.50 \pm 0.025$ & $348 \pm 13$ & $0.489 \pm 0.025$ & $0.517 \pm 0.027$ & $344 \pm 104$ \\
\hline 5 & $\begin{array}{l}\text { ESF-MD- } \\
\text { NICHE3566\#1 } \\
(14.7-20.9) \\
\end{array}$ & SPC01015185 & 0.900 & $06 / 26 / 02$ & CL10329 & 0.92 & $0.202 \pm 0.010$ & $709 \pm 36$ & $0.192 \pm 0.011$ & $0.202 \pm 0.011$ & $717 \pm 139$ \\
\hline 5 & $\begin{array}{l}\text { ESF-MD- } \\
\text { NICHE3566\#1 } \\
(25.3-31.7) \\
\end{array}$ & SPC01015173 & 0.900 & $06 / 26 / 02$ & CL10326 & 0.98 & $0.172 \pm 0.009$ & $450 \pm 20$ & $0.162 \pm 0.010$ & $0.173 \pm 0.011$ & $443 \pm 119$ \\
\hline 5 & $\begin{array}{l}\text { ESF-MD- } \\
\text { NICHE3566\#2 } \\
(11.5-15.4) \\
\end{array}$ & SPC01015182 & 0.900 & $06 / 26 / 02$ & CL10328 & 0.76 & $0.181 \pm 0.009$ & $408 \pm 26$ & $0.171 \pm 0.006$ & $0.210 \pm 0.007$ & $399 \pm 120$ \\
\hline 5 & $\begin{array}{l}\text { ESF-MD- } \\
\text { NICHE3566\#2 } \\
(20.2-32.5)\end{array}$ & SPC01015176 & 0.900 & $06 / 26 / 02$ & CL10327 & 0.91 & $0.255 \pm 0.013$ & $257 \pm 14$ & $0.245 \pm 0.014$ & $0.270 \pm 0.015$ & $245 \pm 106$ \\
\hline
\end{tabular}


Table 4-9. Chloride Concentrations and ${ }^{36} \mathrm{CI} / \mathrm{CI}$ Ratios in Core Samples Leached and Analyzed at USGS-LLNL during Phase III (continued)

\begin{tabular}{|c|c|c|c|c|c|c|c|c|c|c|c|}
\hline \multirow[b]{2}{*}{$\begin{array}{l}\text { Batch } \\
\text { No. }\end{array}$} & \multirow[b]{2}{*}{$\begin{array}{l}\text { Sample } \\
\text { Identifier }\end{array}$} & \multirow[b]{2}{*}{$\begin{array}{l}\text { SMF Barcode } \\
\text { Identifier for } \\
\text { LLNL Sample }\end{array}$} & \multirow[b]{2}{*}{$\begin{array}{c}\text { Water } \\
\text { Mass } \\
\text { Used for } \\
\text { Analysis } \\
\text { (kg) }\end{array}$} & \multirow[b]{2}{*}{$\begin{array}{l}\text { Date } \\
\text { Submitted } \\
\text { to LLNL }\end{array}$} & \multicolumn{4}{|c|}{ AMS Results } & \multicolumn{3}{|c|}{ Final Results } \\
\hline & & & & & CAMS ID & $\begin{array}{l}\text { Sulfur } \\
\text { Figure } \\
\text { of Merit }\end{array}$ & $\begin{array}{c}\text { Leachate } \mathrm{Cl} \\
\text { Concentration } \\
\text { (mg/kg water) }\end{array}$ & $\begin{array}{l}{ }^{36} \mathrm{Cl} / \mathrm{Cl} \\
\times 10^{15}\end{array}$ & $\begin{array}{c}\text { Leachate CI } \\
\text { Concen- } \\
\text { tration } \\
\text { (mg/kg } \\
\text { water) }\end{array}$ & \begin{tabular}{|c|} 
Leachate Cl \\
Concen- \\
tration \\
(mg/kg \\
rock)
\end{tabular} & $\begin{array}{l}{ }^{36} \mathrm{Cl} / \mathrm{Cl} \\
\times 10^{15}\end{array}$ \\
\hline 5 & $\begin{array}{l}\text { ESF-MD- } \\
\text { NICHE3566\#LT1 } \\
(10.9-23.5)\end{array}$ & SPC01015188 & 0.900 & $06 / 26 / 02$ & CL10330 & 0.98 & $0.195 \pm 0.010$ & $384 \pm 17$ & $0.185 \pm 0.011$ & $0.201 \pm 0.012$ & $374 \pm 113$ \\
\hline 5 & $\begin{array}{l}\text { ESF-MD- } \\
\text { NICHE3566\#LT1 } \\
(25.0-38.1)\end{array}$ & SPC01015191 & 0.900 & $06 / 26 / 02$ & CL10331 & 0.78 & $0.180 \pm 0.009$ & $244 \pm 25$ & $0.170 \pm 0.010$ & $0.178 \pm 0.010$ & $226 \pm 119$ \\
\hline
\end{tabular}

DTN: LL031200223121.036 (Q), Filename: Total_AMS_Summary_2001-02c.xls

NOTES: AMS = accelerator mass spectrometer, CAMS = Center for Accelerator Mass Spectrometry, ID = identifier, LLNL = Lawrence Livermore National Laboratory, SMF = Sample Management Facility, U.S. Geological Survey.

Sample Identifier includes core interval (in parentheses)

Measured isotope ratios were normalized to a NIST-traceable standard (LLNL111) with a ${ }^{36} \mathrm{Cl} / \mathrm{Cl}$ ratio of $1.11 \times 10^{-13}$ and a ${ }^{35} \mathrm{Cl}{ }^{37} \mathrm{Cl}$. ratio of 3.127 Measured ${ }^{36} \mathrm{Cl} /{ }^{37} \mathrm{Cl}$ ratios were not corrected for spurious counts at mass-36 attributed to ${ }^{36} \mathrm{~S}$ present in the sample because of the small amounts of sulfur present (sulfur figure of merit values greater than 0.75 correspond to sulfur corrections less than $25 \%$ ). AMS isotope ratios were corrected for background values measured in chemical processing blanks included in the same AMS runs and for the addition of spike. Analytical errors for AMS results are $2 \sigma$ for leachate chloride concentrations relative to water and $1 \sigma$ for ${ }^{36} \mathrm{Cl} / \mathrm{Cl}$. Final results given in the last three columns are also corrected for leach-process blanks. Because equal volumes of water were used for blank determinations and for sample leaching, the process blank correction was done by subtracting concentrations of total chloride and ${ }^{36} \mathrm{Cl}$ in blank (the weighted average values for the five blank analyses are given in Table 4-10) from measured AMS concentrations of total $\mathrm{Cl}$ and ${ }^{36} \mathrm{Cl}$ in the sample. For example, for EVAL001:

Final $\mathrm{Cl}$ conc. $(\mathrm{mg} / \mathrm{kg}$ water) $=$ AMS Cl conc. $0.140(\mathrm{mg} / \mathrm{kg}$ water) - Blank Cl conc. 0.010 (mg/kg water) $=0.130(\mathrm{mg} / \mathrm{kg}$ water);

Final ${ }^{36} \mathrm{Cl}$ conc. $(\mathrm{mg} / \mathrm{kg}$ water $)=\left[\mathrm{AMS} \mathrm{Cl}\right.$ conc. $0.140(\mathrm{mg} / \mathrm{kg}$ water $\left.) \times \mathrm{AMS}{ }^{36} \mathrm{Cl} / \mathrm{Cl} 462 \times 10^{-15}\right]-\left[\mathrm{Blank} \mathrm{Cl}\right.$ conc. $0.010(\mathrm{mg} / \mathrm{kg}$ water $\left.) \times 555 \times 10^{-15}\right]=$ $59.0 \times 10^{-15}(\mathrm{mg} / \mathrm{kg}$ water $)$;

Final ${ }^{36} \mathrm{Cl} / \mathrm{Cl}=$ Final ${ }^{36} \mathrm{Cl}$ conc. $59.0 \times 10^{-15}(\mathrm{mg} / \mathrm{kg}$ water $) \div$ Final $\mathrm{Cl}$ conc. $0.130(\mathrm{mg} / \mathrm{kg}$ water $)=454 \times 10^{-15}$

Errors for blank-corrected values were propagated assuming statistical independence of errors for blanks and samples using the general equation:

$$
\sigma_{f\left(x_{1}, \ldots, x_{n}\right)}^{2}=\sum_{i=1}^{n}\left(\frac{\partial f}{\partial x_{i}}\right)^{2} \sigma_{x_{i}}^{2}
$$


Table 4-9. Chloride Concentrations and ${ }^{36} \mathrm{CI} / \mathrm{Cl}$ Ratios in Core Samples Leached and Analyzed at USGS-LLNL during Phase III (continued)

The final uncertainty for isotope ratios (total $2 \sigma$ error) includes an external error derived from duplicate analyses of 14 leachates given in Table 4-10. Analytical errors for final results are $2 \sigma$ for leachate chloride concentrations relative to both water and rock.

Samples were crushed at either the SMF or USGS, and leached at the USGS. The leachates were distributed to LANL and LLNL for AgCI precipitation and target preparation. For Batch \#1 samples, targets prepared at LANL and LLNL were analyzed at LLNL-CAMS. For Batch \#2, the targets prepared at LANL were analyzed at PRIME Lab and targets prepared at LLNL were analyzed at LLNL-CAMS. Targets for Batches \#3, \#4, and \#5 were prepared and analyzed at LLNL. 
Table 4-10. Concentrations and Chloride Isotopic Compositions of Procedural Blanks Obtained for Passive Leaching at the USGS and Chloride Precipitation and Analysis at LLNL during Phase III

\begin{tabular}{|c|c|c|c|c|c|c|}
\hline \multirow[b]{2}{*}{ Sample Identifier } & \multirow{2}{*}{$\begin{array}{l}\text { SMF Barcode } \\
\text { Identifier for } \\
\text { LLNL Sample }\end{array}$} & \multirow{2}{*}{$\begin{array}{c}\text { Water } \\
\text { Mass } \\
\text { Analyzed } \\
(\mathbf{k g})\end{array}$} & \multicolumn{2}{|c|}{$\begin{array}{c}\text { AMS Results } \\
\text { (corrected for } \\
\text { background and spike) }\end{array}$} & \multirow{2}{*}{$\begin{array}{c}\text { Mass of } \\
{ }^{36} \mathrm{Cl} \\
\text { in Blank } \\
(\mathrm{mg}) \\
\times 10^{15}\end{array}$} & \multirow{2}{*}{$\begin{array}{c}\text { Conc. of }{ }^{36} \mathrm{Cl} \text { in } \\
\text { Blank (mg/kg } \\
\text { water) } \\
\times 10^{15}\end{array}$} \\
\hline & & & $\begin{array}{c}\text { Cl Conc. in } \\
\text { Blank } \\
\text { (mg/kg } \\
\text { water) } \\
\end{array}$ & $\begin{array}{l}{ }^{36} \mathrm{Cl} / \mathrm{Cl} \\
\times 10^{15}\end{array}$ & & \\
\hline DI blank & SPC00536913 & 0.900 & 0.004 & $1,839 \pm 555$ & 6.9 & $7.6 \pm 2.3$ \\
\hline DI blank & SPC00536940 & 0.900 & 0.010 & $47 \pm 211$ & 0.42 & $0.47 \pm 2.1$ \\
\hline $\begin{array}{c}\text { DI system water } \\
\text { sample }\end{array}$ & SPC00516600 & 0.900 & 0.017 & $110 \pm 118$ & 1.7 & $1.8 \pm 2.0$ \\
\hline $\begin{array}{c}\text { USGS } \\
\text { system blank }\end{array}$ & SPC00516602 & 0.900 & 0.009 & $626 \pm 126$ & 5.1 & $5.7 \pm 1.2$ \\
\hline $\begin{array}{c}\text { USGS } \\
\text { system blank }\end{array}$ & SPC01015194 & 0.900 & 0.01 & $152 \pm 148$ & 1.6 & $1.8 \pm 1.8$ \\
\hline \multicolumn{3}{|c|}{ Arithmetic mean } & 0.010 & 555 & 3.1 & 3.5 \\
\hline \multicolumn{3}{|c|}{ Standard deviation } & 0.005 & 754 & 2.7 & 3.0 \\
\hline \multicolumn{3}{|c|}{ Standard error } & 0.002 & 337 & 1.2 & 1.3 \\
\hline
\end{tabular}

DTN: LL031200223121.036 (Q), Filename: Total_AMS_Summary_2001-02c.xls

NOTES: $\quad$ AMS $=$ accelerator mass spectrometer, $\mathrm{DI}=$ deionized water, $\mathrm{LLNL}=$ Lawrence Livermore National Laboratory, SMF = Sample Management Facility, USGS = U.S. Geological Survey.

Analytical errors are $1 \sigma$ for ${ }^{36} \mathrm{Cl} / \mathrm{Cl}$ (corrected for background and spike) and concentration of ${ }^{36} \mathrm{Cl}$ in blank.

$\mathrm{Cl}$ conc. in blank $(\mathrm{mg} / \mathrm{kg}$ water $)=$ Mass $\mathrm{Cl}$ in blank $(\mathrm{mg}) \div$ Water mass analyzed $(\mathrm{kg})$.

Mass of ${ }^{36} \mathrm{Cl}$ in blank $(\mathrm{mg})=$ Water mass analyzed $(\mathrm{kg}) \times \mathrm{Cl}$ conc. in blank $(\mathrm{mg} / \mathrm{kg}$ water) $\mathrm{X}$ ${ }^{36} \mathrm{Cl} / \mathrm{Cl} \times 10^{-15}$.

Conc. of ${ }^{36} \mathrm{Cl}$ in blank $(\mathrm{mg} / \mathrm{kg}$ water $)=$ Mass of ${ }^{36} \mathrm{Cl}$ in blank $(\mathrm{mg}) \div$ Water mass analyzed $(\mathrm{kg})$. 
Table 4-11. Chloride Concentrations and ${ }^{36} \mathrm{CI} / \mathrm{CI}$ Ratios Measured during Phase III at USGS-LLNL in Silicon Crushing Blanks, System Process Blanks, and a Composite Sample of Niche \#1 Core Crushed and Sieved at LANL

\begin{tabular}{|c|c|c|c|c|c|c|c|}
\hline \multirow[b]{2}{*}{ Sample Identifier } & \multirow[b]{2}{*}{$\begin{array}{l}\text { SMF Barcode } \\
\text { Identifier }\end{array}$} & \multirow[b]{2}{*}{$\begin{array}{c}\text { Mass of } \\
\text { Rock } \\
(\mathrm{kg})\end{array}$} & \multirow[b]{2}{*}{$\begin{array}{l}\text { Mass of } \\
\text { Water } \\
\text { (kg) }\end{array}$} & \multirow{2}{*}{$\begin{array}{c}\mathrm{Cl} \text { Conc. } \\
\text { (mg/kg } \\
\text { water) by } \\
\text { lon } \\
\text { Chroma- } \\
\text { tography }\end{array}$} & \multirow{2}{*}{$\begin{array}{c}\text { Cl Conc. } \\
\text { (mg/kg } \\
\text { rock) by } \\
\text { lon } \\
\text { Chroma- } \\
\text { tography }\end{array}$} & \multicolumn{2}{|c|}{$\begin{array}{l}\text { Corrected for } \mathrm{Cl} \text { and }{ }^{36} \mathrm{Cl} \\
\text { Measured in Chemistry } \\
\text { Process Blank CL10348 }\end{array}$} \\
\hline & & & & & & $\begin{array}{c}\text { Cl Conc. } \\
\text { (mg/kg } \\
\text { water) by } \\
\text { Isotope } \\
\text { Dilution }\end{array}$ & $\begin{array}{c}{ }^{36} \mathrm{Cl} / \mathrm{Cl} \\
\times 10^{15} \\
(1 \sigma)\end{array}$ \\
\hline Silicon blank (plate) & $\begin{array}{l}\text { SPC01015196 } \\
\text { SPC01015197 }\end{array}$ & 1.571 & 1.824 & 0.019 & 0.022 & 0.028 & $957 \pm 174$ \\
\hline Silicon blank (mortar) & $\begin{array}{l}\text { SPC01015202 } \\
\text { SPC01015203 }\end{array}$ & 1.792 & 1.952 & 0.014 & 0.015 & 0.047 & $1,033 \pm 249$ \\
\hline Leaching blank (9/4/02) & $\begin{array}{l}\text { SPC01015199 } \\
\text { SPC01015200 }\end{array}$ & NA & 1.907 & $<0.010$ & NA & 0.02 & $1,077 \pm 252$ \\
\hline $\begin{array}{l}\text { Mix of NICHE3566\#1 } \\
\text { and NICHE3566\#LT1 }\end{array}$ & SPC01015205 & 2.000 & 2.005 & 0.114 & 0.114 & 0.188 & $1,185 \pm 121$ \\
\hline
\end{tabular}

DTNs: LL031200223121.036 (Q), Filename: Total_AMS_Summary_2001-02c.xls; GS030608312272.006 (UQ)

NOTES:

LANL = Los Alamos National Laboratory, LLNL = Lawrence Livermore National Laboratory, NA = not applicable, SMF = Sample Management Facility, USGS = U.S. Geological Survey.

$\mathrm{Cl}$ concentrations and ${ }^{36} \mathrm{Cl} / \mathrm{Cl}$ ratios corrected for values measured in the Lawrence Livermore National Laboratory Chemistry Process Blank CL10348 run in the same batch having $0.006 \pm 0.002 \mathrm{mg} \mathrm{Cl} / \mathrm{kg}$ water and a ${ }^{36} \mathrm{Cl} / \mathrm{Cl}$ ratio of $2,388 \pm 634(1 \sigma) \times 10^{-15}$.

$\mathrm{Cl}$ conc. $(\mathrm{mg} / \mathrm{kg}$ rock $)=[\mathrm{Cl}$ conc. $(\mathrm{mg} / \mathrm{kg}$ water $)] \times$ [Mass of water $(\mathrm{kg}) \div$ Mass of rock $(\mathrm{kg})]$. 


\begin{tabular}{|c|c|c|c|c|c|c|c|c|c|}
\hline $\begin{array}{l}\text { Sample or Aliquot } \\
\text { Identifier }\end{array}$ & $\begin{array}{l}\text { SMF Barcode } \\
\text { Identifier }\end{array}$ & Sample Location & $\begin{array}{l}\text { LANL } \\
\text { Identifier }\end{array}$ & $\begin{array}{l}\text { AMS } \\
\text { Facility }\end{array}$ & AMS Identifier & $\begin{array}{c}\text { Date } \\
\text { Analyzed }\end{array}$ & $\left|\begin{array}{c}\text { cl Concen- } \\
\text { tration (mg/kg } \\
\text { rock) }\end{array}\right|$ & $\begin{array}{c}\text { Sample + } \\
\text { Blank }{ }^{36} \mathrm{C} C / \mathrm{Cl} \\
\times 10^{15}\end{array}$ & $\begin{array}{c}\text { Sample }{ }^{36} \mathrm{Cl} / \mathrm{C} \\
\times 10^{15} \\
(2 \sigma)\end{array}$ \\
\hline EVAL001 & SPC00536902 & ESF Sundance fault zone & YM2033 & CAMS & CL9659 & $10 / 26 / 2001$ & 0.15 & 380 & $361 \pm 42$ \\
\hline \begin{tabular}{|l|} 
ESF-SD-CIV\#34 \\
$(2.1-4.8)$
\end{tabular} & SPC00536932 & ESF Sundance fault zone & YM2030 & CAMS & CL9656 & $10 / 26 / 2001$ & 0.08 & 347 & $315 \pm 59$ \\
\hline \begin{tabular}{|l|} 
ESF-SD-CIV\#22 \\
$(4.5-6.3)$
\end{tabular} & SPC00536935 & ESF Sundance fault zone & YM2032 & CAMS & CL9658 & $10 / 26 / 2001$ & 0.15 & 426 & $408 \pm 43$ \\
\hline $\begin{array}{l}\text { ESF-SD-CIV\#28 } \\
(6.2-8.0) \\
\end{array}$ & SPC00536908 & ESF Sundance fault zone & YM2034 & CAMS & CL9660 & $10 / 26 / 2001$ & 0.16 & 225 & $203 \pm 28$ \\
\hline \begin{tabular}{|l|} 
ESF-SD-CIV\#32 \\
$(7.6-9.5)$
\end{tabular} & SPC00536926 & ESF Sundance fault zone & YM2035 & CAMS & CL9661 & $10 / 26 / 2001$ & 0.16 & 254 & $235 \pm 29$ \\
\hline $\begin{array}{l}\text { ESF-SD-CIV\#21 } \\
(2.8-4.6) \\
\end{array}$ & SPC00536938 & ESF Sundance fault zone & YM2036 & CAMS & CL9662 & $10 / 26 / 2001$ & 0.18 & 362 & $344 \pm 38$ \\
\hline \begin{tabular}{|l|} 
ESF-SD-CIV\#35 \\
$(6.4-8.5)$ \\
\end{tabular} & SPC00536944 & ESF Sundance fault zone & YM2037 & CAMS & CL9663 & $10 / 26 / 2001$ & 0.09 & 292 & $264 \pm 45$ \\
\hline $\begin{array}{l}\text { ESF-SD-CIV\#21 } \\
(11.3-13.0)\end{array}$ & SPC00536920 & ESF Sundance fault zone & YM2038 & CAMS & CL9664 & $10 / 26 / 2001$ & 0.32 & 320 & $310 \pm 29$ \\
\hline $\begin{array}{l}\text { ESF-SD-CIV\#30 } \\
(6.4-8.4)\end{array}$ & SPC00536923 & ESF Sundance fault zone & YM2039 & CAMS & CL9665 & $10 / 26 / 2001$ & 0.16 & 186 & $163 \pm 30$ \\
\hline $\begin{array}{l}\text { ESF-SD-CIV\#27 } \\
(10.0-12.0)\end{array}$ & SPC00536947 & ESF Sundance fault zone & YM2040 & CAMS & CL9666 & $10 / 26 / 2001$ & 0.15 & 230 & $208 \pm 29$ \\
\hline \begin{tabular}{|l} 
ESF-SD-CIV\#33 \\
$(9.9-11.4)$
\end{tabular} & SPC00536905 & ESF Sundance fault zone & YM2041 & CAMS & CL9667 & $10 / 26 / 2001$ & 0.11 & 282 & $249 \pm 44$ \\
\hline \begin{tabular}{|l|} 
ESF-SD-CIV\#26-1 \\
$(3.0-6.3)$ \\
\end{tabular} & SPC00536950 & ESF Sundance fault zone & YM2047 & CAMS & CL9673 & $10 / 26 / 2001$ & 0.10 & 307 & $270 \pm 72$ \\
\hline $\begin{array}{l}\text { ESF-SD-CIV\#26-2 } \\
(3.0-6.3)\end{array}$ & SPC00536953 & ESF Sundance fault zone & YM2048 & CAMS & CL9674 & $10 / 26 / 2001$ & 0.11 & 260 & $225 \pm 44$ \\
\hline $\begin{array}{l}\text { ESF-SD-CIV\#36 } \\
(5.4-9.4)\end{array}$ & SPC00536911 & ESF Sundance fault zone & YM2049 & CAMS & CL9675 & $10 / 26 / 2001$ & 0.07 & 360 & $322 \pm 66$ \\
\hline $\begin{array}{l}\text { ESF-SD-CIV\#24 } \\
(4.6-6.6)\end{array}$ & SPC00536956 & ESF Sundance fault zone & YM2071 & PRIME & R02-0200,5A & $8 / 21 / 2002$ & 0.20 & 471 & $410 \pm 151$ \\
\hline $\begin{array}{l}\text { ESF-SD-CIV\#38 } \\
(1.4-3.9) \\
\end{array}$ & SPC00536959 & ESF Sundance fault zone & YM2072 & PRIME & $\mathrm{R} 02-0201,5 \mathrm{~A}$ & $8 / 21 / 2002$ & 0.26 & 666 & $640 \pm 162$ \\
\hline $\begin{array}{l}\text { ESF-SD-CIV\#37 } \\
(3.6-5.3)\end{array}$ & SPC00536962 & ESF Sundance fault zone & YM2073 & PRIME & $R 02-0202,5 \mathrm{~A}$ & $8 / 21 / 2002$ & 0.07 & 409 & $180 \pm 204$ \\
\hline $\begin{array}{l}\text { ESF-SD-CIV\#20 } \\
(3.8-6.1)\end{array}$ & SPC00516615 & ESF Sundance fault zone & YM2078 & PRIME & R02-0207,5A & $8 / 21 / 2002$ & 0.08 & 371 & $180 \pm 208$ \\
\hline
\end{tabular}


Table 4-12. Chloride Concentrations and ${ }^{36} \mathrm{CI} / \mathrm{Cl}$ Ratios in Leachates of Validation Study Samples Analyzed at LANL during Phase III (continued)

\begin{tabular}{|c|c|c|c|c|c|c|c|c|c|}
\hline $\begin{array}{c}\begin{array}{c}\text { Sample or Aliquot } \\
\text { Identifier }\end{array} \\
\end{array}$ & $\begin{array}{l}\text { SMF Barcode } \\
\text { Identifier }\end{array}$ & Sample Location & $\begin{array}{l}\text { LANL } \\
\text { Identifier }\end{array}$ & $\begin{array}{l}\text { AMS } \\
\text { Facility }\end{array}$ & AMS Identifier & $\begin{array}{c}\text { Date } \\
\text { Analyzed }\end{array}$ & \begin{tabular}{|c|}
$\begin{array}{c}\text { Cl Concen- } \\
\text { tration }(\mathrm{mg} / \mathrm{kg} \\
\text { rock) }\end{array}$ \\
\end{tabular} & $\begin{array}{c}\text { Sample + } \\
\text { Blank }{ }^{56} \mathrm{C} / / \mathrm{Cl} \\
\times 10^{15} \\
\end{array}$ & $\begin{array}{c}\text { Sample }{ }^{{ }^{36} \mathrm{Cl} / \mathrm{Cl}} \times \\
\times 10^{15} \\
(2 \sigma) \\
\end{array}$ \\
\hline $\begin{array}{l}\text { ESF-SD-CIV\#19 } \\
(4.4-6.4)\end{array}$ & SPC00516618 & ESF Sundance fault zone & YM2079 & PRIME & $\mathrm{R} 02-0208,5 \mathrm{~A}$ & $8 / 21 / 2002$ & 0.09 & 432 & $308 \pm 194$ \\
\hline $\begin{array}{l}\text { ESF-SD-CIV\#18 } \\
(3.6-5.6) \\
\end{array}$ & SPC00516621 & ESF Sundance fault zone & YM2080 & PRIME & $\mathrm{R} 02-0209,5 \mathrm{~A}$ & $8 / 21 / 2002$ & 0.10 & 557 & $456 \pm 283$ \\
\hline $\begin{array}{l}\text { ESF-SD-CIV\#17 } \\
(3.9-6.4) \\
\end{array}$ & SPC00516624 & ESF Sundance fault zone & YM2081 & PRIME & $\mathrm{R} 02-0210,5 \mathrm{~A}$ & $8 / 21 / 2002$ & 0.14 & 519 & $450 \pm 210$ \\
\hline Niche 1-RCR-1A & $\begin{array}{l}\text { SPC01003045, } \\
\text { SPC01003048, } \\
\text { SPC01003050, } \\
\text { SPC01003053, } \\
\text { SPC01003055, } \\
\text { SPC } 01003057\end{array}$ & ESF Niche \#1 & YM2103 & CAMS & CL10291 & $7 / 30 / 2002$ & 0.14 & 1,194 & $1,163 \pm 94$ \\
\hline Niche 1-RCR-1B & $\begin{array}{l}\text { SPC01003045, } \\
\text { SPC01003048, } \\
\text { SPC01003050, } \\
\text { SPC01003053, } \\
\text { SPC01003055, } \\
\text { SPC01003057 }\end{array}$ & ESF Niche \#1 & YM2104 & CAMS & CL10292 & $7 / 30 / 2002$ & 0.67 & 8,530 & $\begin{array}{c}8,558 \pm 485 \\
.\end{array}$ \\
\hline Niche 1-RCR-2 & $\begin{array}{l}\text { SPC01003068, } \\
\text { SPC01003070, } \\
\text { SPC01003072 }\end{array}$ & ESF Niche \#1 & YM2105 & CAMS & CL10293 & $7 / 30 / 2002$ & 0.27 & 1,636 & $1,624 \pm 120$ \\
\hline Niche 1- RCR-3 & $\begin{array}{l}\text { SPC01003082, } \\
\text { SPC01003087, } \\
\text { SPC01003091 }\end{array}$ & ESF Niche \#1 & YM2106 & CAMS & CL10294 & $7 / 30 / 2002$ & 0.28 & 3,164 & $3,166 \pm 199$ \\
\hline Niche 2-RCR-1 & $\begin{array}{l}\text { SPC01003119, } \\
\text { SPC01003123, } \\
\text { SPC01003127, } \\
\text { SPC01003135 }\end{array}$ & ESF Niche \#1 & YM2107 & CAMS & CL10295 & $7 / 30 / 2002$ & 0.21 & 1,130 & $1,109 \pm 78$ \\
\hline Niche LT-RCR-1A & $\begin{array}{l}\text { SPC01004416, } \\
\text { SPC01004431, } \\
\text { SPC01004433, } \\
\text { SPC01004437, } \\
\text { SPC01004441, } \\
\text { SPC01004460, } \\
\text { SPC01004464 }\end{array}$ & ESF Niche \#1 & YM2108 & CAMS & CL10296 & $7 / 30 / 2002$ & 0.13 & 1,050 & $1,016 \pm 87$ \\
\hline
\end{tabular}


Table 4-12. Chloride Concentrations and ${ }^{36} \mathrm{Cl} / \mathrm{Cl}$ Ratios in Leachates of Validation Study Samples Analyzed at LANL during Phase III (continued)

\begin{tabular}{|c|c|c|c|c|c|c|c|c|c|}
\hline $\begin{array}{l}\text { Sample or Aliquot } \\
\text { Identifier }\end{array}$ & $\begin{array}{l}\text { SMF Barcode } \\
\text { Identifier }\end{array}$ & Sample Location & $\begin{array}{l}\text { LANL } \\
\text { Identifier }\end{array}$ & $\begin{array}{l}\text { AMS } \\
\text { Facility }\end{array}$ & $\begin{array}{c}\text { AMS } \\
\text { Identifier }\end{array}$ & $\begin{array}{c}\text { Date } \\
\text { Analyzed }\end{array}$ & $\begin{array}{l}\text { Cl Concen- } \\
\text { tration } \\
\text { (mg/kg rock) }\end{array}$ & $\begin{array}{c}\text { Sample + } \\
\text { Blank }{ }^{36} \mathrm{Cl} / \mathrm{Cl} \\
\times 10^{15}\end{array}$ & $\begin{array}{l}\text { Sample } \\
{ }^{36} \mathrm{Cl} / \mathrm{Cl} \times 10^{15} \\
(2 \sigma)\end{array}$ \\
\hline Niche LT-RCR-1B & $\begin{array}{l}\text { SPC01004416, } \\
\text { SPC01004431, } \\
\text { SPC01004433, } \\
\text { SPC01004437, } \\
\text { SPC01004441, } \\
\text { SPC01004460, } \\
\text { SPC01004464 }\end{array}$ & ESF Niche \#1 & YM2109 & CAMS & CL10297 & $7 / 30 / 2002$ & 0.69 & 3,388 & $3,390 \pm 196$ \\
\hline EXD046-1 & SPC00521151 & ECRB Cross Drift & YM2012 & PRIME & T01-0852,5A & $12 / 7 / 2001$ & 0.53 & 603 & $607 \pm 51$ \\
\hline EXD052-1 & SPC00521144 & ECRB Cross Drift & YM2013 & PRIME & T01-0853,5A & $12 / 7 / 2001$ & 0.38 & 568 & $574 \pm 56$ \\
\hline EXD059-1 & SPC00521138 & ECRB Cross Drift & YM2014 & PRIME & T01-0854,5A & $12 / 7 / 2001$ & 0.30 & 1,274 & $1,309 \pm 114$ \\
\hline EXD066-1 & SPC00541211 & ECRB Cross Drift & YM2015 & PRIME & T01-0855,5A & $12 / 7 / 2001$ & 3.59 & 162 & $161 \pm 22$ \\
\hline EXD071-1 & SPC00541216 & ECRB Cross Drift & YM2016 & PRIME & T01-0856,5A & $12 / 7 / 2001$ & 0.59 & 472 & $474 \pm 46$ \\
\hline EXD076-1 & SPC00533396 & ECRB Cross Drift & YM2017 & PRIME & T01-0857,5A & $12 / 7 / 2001$ & 0.37 & 663 & $671 \pm 75$ \\
\hline EXD084-1 & SPC00521175 & ECRB Cross Drift & YM2018 & PRIME & T01-0858,5A & $12 / 7 / 2001$ & 0.74 & 511 & $513 \pm 57$ \\
\hline EXD085-1 & SPC00521174 & ECRB Cross Drift & YM2019 & PRIME & T01-0859,5A & $12 / 7 / 2001$ & 1.12 & 412 & $412 \pm 35$ \\
\hline EXD086-1 & SPC00521176 & ECRB Cross Drift & YM2020 & PRIME & T01-0860,5A & $12 / 7 / 2001$ & 0.92 & 548 & $550 \pm 179$ \\
\hline $\begin{array}{l}\text { T200-1 } \\
\text { (EXD085-1 split) }\end{array}$ & SPC00521174 & ECRB Cross Drift & YM2022 & PRIME & T01-0874,5A & $12 / 7 / 2001$ & 1.59 & 434 & $434 \pm 43$ \\
\hline
\end{tabular}

DTN: LA0305RR831222.001 (UQ)

NOTES: $\quad$ AMS $=$ accelerator mass spectrometer, CAMS = Center for Accelerator Mass Spectrometry, ECRB $=$ Enhanced Characterization of the Repository Block, ESF = Exploratory Studies Facility, LANL = Los Alamos National Laboratory, PRIME = Purdue Rare Isotope Measurement Laboratory, SMF = Sample Management Facility. Chloride concentrations have a uniform $2 \sigma$ uncertainty of $5 \%$ of the stated value. 


\begin{tabular}{|c|c|c|c|c|c|c|c|c|c|}
\hline $\begin{array}{l}\text { Batch } \\
\text { No. }\end{array}$ & Sample Identifier & $\begin{array}{l}\text { SMF Barcode } \\
\text { Identifier }\end{array}$ & $\begin{array}{c}\begin{array}{c}\text { Mass of } \\
\text { Rock } \\
(\mathbf{k g})\end{array} \\
\end{array}$ & $\begin{array}{c}\text { Mass of } \\
\text { Water } \\
(\mathrm{kg})\end{array}$ & $\begin{array}{c}\mathrm{F}^{-1} \\
(\mathrm{mg} / \mathrm{kg})\end{array}$ & $\begin{array}{c}\mathrm{Cl}^{-1} \\
(\mathrm{mg} / \mathrm{kg})\end{array}$ & $\begin{array}{c}\mathrm{Br}-1 \\
(\mathrm{mg} / \mathrm{kg})\end{array}$ & $\begin{array}{c}\mathrm{NO}_{3}^{-1} \\
(\mathrm{mg} / \mathrm{kg})\end{array}$ & $\begin{array}{c}\mathrm{SO}_{4}^{-2} \\
(\mathrm{mg} / \mathrm{kg})\end{array}$ \\
\hline 1 & EVAL001 (bulk rock material) & SPC00536900 & 1.871 & 2.098 & ND & 0.19 & 0.18 & 0.15 & 0.22 \\
\hline 1 & ESF-SD-CIV\#33 (9.9-11.4) & SPC00536903 & 1.787 & 2.057 & ND & 0.11 & $<0.02$ & 0.24 & 0.10 \\
\hline 1 & ESF-SD-CIV\#28 (6.2-8.0) & SPC00536906 & 1.893 & 2.130 & ND & 0.18 & $<0.02$ & 0.21 & 0.21 \\
\hline 1 & \begin{tabular}{|l|} 
ESF-SD-CIV\#36 \\
$(5.4-6.7 / 8.1-9.4)$ \\
\end{tabular} & SPC00536909 & 2.002 & 2.038 & ND & 0.098 & $<0.02$ & 0.19 & 0.092 \\
\hline 1 & SYSTEM BLANK $(8 / 22 / 01)$ & SPC00536912 & 2.034 & 2.034 & ND & $<0.04$ & $<0.02$ & $<0.03$ & $<0.03$ \\
\hline 1 & $\begin{array}{l}\text { SYSTEM BLANK }(8 / 22 / 01) \\
\text { rerun }\end{array}$ & SPC00536912 & 2.034 & 2.034 & ND & $<0.04$ & $<0.02$ & $<0.03$ & $<0.03$ \\
\hline 1 & ESF-SD-CIV\#31 (2.8-4.5) & SPC00536915 & 1.786 & 2.014 & ND & 0.070 & $<0.02$ & 0.16 & .0 .067 \\
\hline 1 & ESF-SD-CIV\#21 (11.3-13.0) & SPC00536918 & 1.935 & 2.115 & ND & 0.31 & $<0.02$ & 0.26 & 0.42 \\
\hline 1 & ESF-SD-CIV\#30 (6.4-8.4) & SPC00536921 & 1.965 & 2.092 & ND & 0.15 & $<0.02$ & 0.26 & 0.13 \\
\hline 1. & $\begin{array}{l}\begin{array}{c}\text { ESF-SD-CIV\#30 (6.4-8.4) } \\
\text { rerun }\end{array} \\
\end{array}$ & SPC00536921 & 1.965 & 2.092 & ND & 0.17 & $<0.02$ & 0.22 & 0.14 \\
\hline 1 & ESF-SD-CIV\#32 (7.6-9.5) & SPC00536924 & 2.310 & 2.089 & ND & 0.16 & $<0.02$ & 0.29 & 0.16 \\
\hline 1 & ESF-SD-CIV $\# 28(4.0-6.2)$ & SPC00536927 & 2.333 & 2.134 & ND & 0.091 & $<0.02$ & 0.13 & 0.065 \\
\hline 1 & ESF-SD-CIV\#34 (2.1-4.8) & SPC00536930 & 2.399 & 2.103 & ND & 0.082 & $<0.02$ & 0.18 & 0.087 \\
\hline 1 & ESF-SD-CIV\#22 (4.5-6.3) & SPC00536933 & 1.840 & 2.096 & ND & 0.15 & $<0.02$ & 0.21 & 0.24 \\
\hline 1 & ESF-SD-CIV\#21 (2.8-4.6) & SPC00536936 & 1.736 & 2.049 & ND & 0.18 & $<0.02$ & 0.18 & 0.27 \\
\hline 1 & SYSTEM BLANK (8/24/01) & SPC00536939 & 2.061 & 2.061 & ND & $<0.04$ & $<0.02$ & $<0.03$ & $<0.03$ \\
\hline 1 & ESF-SD-CIV\#35 (6.4-8.5) & SPC00536942 & 2.366 & 2.135 & ND & 0.090 & $<0.02$ & 0.20 & 0.14 \\
\hline 1 & ESF-SD-CIV\#27 (10.0-12.0) & SPC00536945 & 2.211 & $\ldots$ & ND & 0.14 & $<0.02$ & 0.086 & 0.19 \\
\hline 1 & ESF-SD-CIV\#26-1 (3.0-6.3) & SPC00536948 & 1.688 & 2.040 & ND & 0.095 & $<0.02$ & 0.13 & 0.10 \\
\hline 1 & ESF-SD-CIV\#26-2 (3.0-6.3) & SPC00536951 & 1.700 & 2.044 & ND & 0.11 & $<0.02$ & 0.11 & 0.10 \\
\hline 2 & ESF-SD-CIV\#24 (4.6-6.6) & SPC00536954 & 1.863 & 2.054 & ND & 0.22 & $<0.02$ & 0.44 & 0.44 \\
\hline 2 & ESF-SD-CIV\#38 (1.4-3.9) & SPC00536957 & 1.959 & 2.076 & ND & 0.30 & $<0.02$ & 1.6 & 0.28 \\
\hline 2 & $\begin{array}{c}\text { ESF-SD-CIV\#38 (1.4-3.9) } \\
\text { rerun }\end{array}$ & 0536957 & 1.959 & 2.076 & ND & 0.30 & $<0.02$ & 1.6 & 0.31 \\
\hline 2 & ESF-SD-CIV\#37 (3.6-5.3) & SPC00536960 & 1.726 & 2.055 & ND & 0.092 & $<0.02$ & 0.21 & 0.063 \\
\hline 2 & SYSTEM BLANK $(9 / 5 / 01)$ & SPC00536963 & 2.057 & 2.057 & ND & $<0.04$ & $<0.02$ & 0.092 & $<0.03$ \\
\hline 2 & $\begin{array}{c}\text { SYSTEM BLANK }(9 / 5 / 01) \\
\text { rerun }\end{array}$ & 0536963 & NA & 2.057 & ND & $\leq 0.04$ & $\leq 0.02$ & 0.041 & $\leq 0.03$ \\
\hline 2 & ESF-SD-CIV\#39 (2.1-3.5) & SPC00516604 & 1.665 & 2.045 & ND & 0.091 & $<0.02$ & 0.20 & $<0.04$ \\
\hline
\end{tabular}


Table 4-13. Concentrations of Anions in Leachates of Validation Study Samples Analyzed by lon Chromatography at the USGS during Phase III (continued)

\begin{tabular}{|c|c|c|c|c|c|c|c|c|c|}
\hline $\begin{array}{c}\text { Batch } \\
\text { No. }\end{array}$ & Sample Identifier & $\begin{array}{l}\text { SMF Barcode } \\
\text { Identifier }\end{array}$ & $\begin{array}{c}\text { Mass of Rock } \\
(\mathrm{kg})\end{array}$ & $\begin{array}{c}\text { Mass of } \\
\text { Water } \\
(\mathrm{kg})\end{array}$ & $\begin{array}{c}F^{-1} \\
(\mathrm{mg} / \mathrm{kg})\end{array}$ & $\begin{array}{c}\mathrm{Cl}^{-1} \\
(\mathrm{mg} / \mathrm{kg})\end{array}$ & $\begin{array}{c}B r^{-1} \\
(\mathrm{mg} / \mathrm{kg})\end{array}$ & $\begin{array}{c}\mathrm{NO}_{3}{ }^{-1} \\
(\mathrm{mg} / \mathrm{kg})\end{array}$ & $\begin{array}{c}\mathrm{SO}_{4}^{-2} \\
(\mathrm{mg} / \mathrm{kg})\end{array}$ \\
\hline 2 & ESF-SD-CIV\#24 (2.1-4.0) & SPC00516607 & 1.907 & 2.050 & ND & 0.12 & $<0.02$ & 0.15 & 0.064 \\
\hline 2 & ESF-SD-CIV\#23 (4.8-6.7) & SPC00516610 & 1.788 & 2.048 & ND & 0.15 & $<0.02$ & 0.33 & 0.13 \\
\hline 2 & ESF-SD-CIV\#20 (3.8-6.1) & SPC00516613 & 1.909 & 2.054 & ND & 0.10 & $<0.02$ & 0.16 & 0.075 \\
\hline 2 & ESF-SD-CIV\#19 (4.4-6.4) & SPC00516616 & 2.193 & 2.072 & ND & 0.088 & $<0.02$ & 0.19 & 0.15 \\
\hline 2 & ESF-SD-ClV\#18 (3.6-5.6) & SPC00516619 & 1.799 & 2.057 & ND & 0.13 & $<0.02$ & 0.23 & 0.15 \\
\hline 2 & ESF-SD-CIV\#17 (3.9-6.4) & SPC00516622 & 2.276 & 2.061 & ND & 0.14 & $<0.02$ & 0.27 & 0.14 \\
\hline 3 & ESF-SD-CIV\#8 (2.0-3.4) & SPC00516625 & 1.198 & 1.448 & ND & 0.091 & $<0.02$ & $<0.04$ & 0.16 \\
\hline 3 & ESF-SD-CIV\#6 (2.0-5.2) & SPC00516628 & 2.087 & 2.013 & ND & 0.11 & $<0.02$ & 0.15 & 0.18 \\
\hline 3 & ESF-SD-CIV\#1 (4.2-6.2) & SPC00516631 & 2.364 & 2.051 & ND & 0.18 & $<0.02$ & 0.095 & 0.13 \\
\hline 3 & ESF-SD-CIV\#1 (4.2-6.2) rerun & SPC00516631 & 2.364 & 2.051 & ND & 0.16 & $<0.02$ & 0.10 & 0.12 \\
\hline 3 & ESF-SD-CIV\#1 (1.4-3.7) & SPC00516634 & 2.312 & 2.093 & ND & 0.26 & $<0.02$ & 0.13 & 0.21 \\
\hline 3 & SYSTEM BLANK $(10 / 31 / 01)$ & SPC00516637 & 2.050 & 2.050 & ND & $<0.04$ & $<0.02$ & 0.032 & $<0.03$ \\
\hline 3 & $\begin{array}{l}\text { SYSTEM BLANK }(10 / 31 / 01) \\
\text { rerun }\end{array}$ & SPC00516637 & 2.050 & 2.050 & ND & $<0.04$ & $<0.02$ & 0.039 & $<0.03$ \\
\hline 3 & $\begin{array}{l}\text { SYSTEM BLANK }(10 / 31 / 01) \\
\text { rerun }\end{array}$ & SPC00516637 & 2.050 & 2.050 & ND & 0.049 & $<0.02$ & $<0.03$ & $<0.03$ \\
\hline 3 & ESF-SD-CIV\#2 (4.7-6.6) & SPC00516640 & 2.322 & 2.051 & ND & 0.30 & $<0.02$ & 0.097 & 0.25 \\
\hline 3 & ESF-SD-CIV\#7 (3.9-6.5) & SPC00516643 & 0.989 & 1.107 & ND & 0.11 & $<0.02$ & 0.11 & 0.067 \\
\hline 3 & ESF-SD-CIV\#4 (2.1-3.8) & SPC00516646 & 1.530 & 1.495 & ND & 0.23 & $<0.02$ & 0.18 & 0.21 \\
\hline 3 & ESF-SD-CIV\#14 (4.6-6.4) & SPC00516649 & 1.907 & 2.057 & ND & 0.078 & $<0.02$ & 0.094 & 0.14 \\
\hline 3 & ESF-SD-CIV\#15 (4.7-6.6) & SPC00516652 & 2.073 & 2.052 & ND & 0.099 & $<0.02$ & 0.13 & 0.081 \\
\hline 3 & EVAL001 (bulk rock material) & SPC00516655 & 2.315 & 2.041 & ND & 0.17 & 0.15 & 0.19 & 0.20 \\
\hline 3 & \begin{tabular}{|l|} 
EVAL001 (bulk rock material) \\
rerun
\end{tabular} & SPC00516655 & 2.315 & 2.041 & ND & 0.17 & 0.12 & 0.19 & 0.17 \\
\hline 3 & ESF-DHW-CIV\#5 (3.5-6.5) & SPC00516658 & 1.362 & 1.966 & ND & 0.19 & $<0.03$ & 0.29 & 0.22 \\
\hline 3 & SYSTEM BLANK (1/4/02) & SPC00516661 & 2.103 & 2.103 & ND & $<0.04$ & $<0.02$ & 0.036 & $<0.03$ \\
\hline 3 & \begin{tabular}{|l|} 
SYSTEM BLANK (1/4/02) \\
rerun
\end{tabular} & SPC00516661 & 2.103 & 2.103 & ND & $<0.04$ & $<0.02$ & $<0.03$ & $<0.03$ \\
\hline 3 & ESF-SD-CIV\#16 (4.3-5.0) & SPC00516664 & 1.804 & 1.948 & ND & 0.093 & $<0.02$ & 0.14 & 0.13 \\
\hline 3 & ESF-SD-CIV\#12 (2.0-4.4) & SPC00516667 & 2.202 & 2.039 & ND & 0.060 & $<0.02$ & 0.035 & 0.041 \\
\hline 3 & ESF-SD-CIV\#11 (2.4-5.2) & SPC00516670 & 1.513 & 1.955 & ND & 0.056 & $<0.03$ & 0.089 & $<0.04$ \\
\hline 3 & ESF-DHW-CIV\#8 (3.8-6.4) & SPC00516673 & 1.758 & 2.004 & ND & 0.18 & $<0.02$ & 0.35 & 0.25 \\
\hline
\end{tabular}


Table 4-13. Concentrations of Anions in Leachates of Validation Study Samples Analyzed by lon Chromatography at the USGS during Phase III (continued)

\begin{tabular}{|c|c|c|c|c|c|c|c|c|c|}
\hline $\begin{array}{l}\text { Batch } \\
\text { No. }\end{array}$ & Sample Identifier & $\begin{array}{l}\text { SMF Barcode } \\
\text { Identifier }\end{array}$ & $\begin{array}{c}\text { Mass of Rock } \\
(\mathbf{k g})\end{array}$ & $\begin{array}{c}\text { Mass of } \\
\text { Water } \\
(\mathrm{kg})\end{array}$ & $\begin{array}{c}F^{-1} \\
(\mathrm{mg} / \mathrm{kg})\end{array}$ & $\begin{array}{c}\mathrm{Cl}^{-1} \\
(\mathbf{m g} / \mathbf{k g})\end{array}$ & $\begin{array}{c}\mathrm{Br}^{-1} \\
(\mathrm{mg} / \mathrm{kg})\end{array}$ & $\begin{array}{c}\mathrm{NO}_{3}^{-1} \\
(\mathrm{mg} / \mathrm{kg})\end{array}$ & $\begin{array}{c}\mathrm{SO}_{4}^{-2} \\
(\mathrm{mg} / \mathrm{kg})\end{array}$ \\
\hline 3 & $\begin{array}{l}\text { ESF-DHW-CIV\#8 (3.8-6.4) } \\
\text { rerun }\end{array}$ & SPC00516673 & 1.758 & 2.004 & ND & 0.17 & $<0.02$ & 0.40 & 0.23 \\
\hline 3 & ESF-DHW-CIV\#9 (2.4-4.9) & SPC00516676 & 1.468 & 1.903 & ND & 0.17 & $<0.03$ & 0.34 & 0.25 \\
\hline 3 & ESF-DHW-CIV\#3 (3.1-6.9) & SPC00516679 & 1.605 & 2.037 & ND & 0.32 & $<0.03$ & 0.38 & 0.33 \\
\hline 3 & ESF-SD-CIV\#13 (2.9-4.5) & SPC00516682 & 1.858 & 2.018 & ND & 0.058 & $<0.02$ & 0.038 & 0.056 \\
\hline 3 & ESF-DHW-CIV\#1 (2.9-5.0) & SPC00516685 & 1.092 & 1.514 & ND & 0.29 & $<0.03$ & 0.44 & 0.25 \\
\hline 3 & ESF-DHW-CIV\#7 (2.2-6.5) & SPC00516688 & 1.659 & 2.028 & ND & 0.22 & $<0.02$ & 0.43 & 0.27 \\
\hline 3 & ESF-SD-CIV\#4 (4.4-6.3) & SPC00516691 & 1.562 & 1.890 & ND & 0.16 & $<0.02$ & 0.12 & 0.15 \\
\hline 3 & SYSTEM BLANK (1/7/02) & SPC00516694 & 2.045 & 2.045 & ND & $<0.04$ & $<0.02$ & $<0.03$ & $<0.03$ \\
\hline 3 & $\begin{array}{l}\text { SYSTEM BLANK }(1 / 7 / 02) \\
\text { rerun }\end{array}$ & SPC00516694 & 2.045 & 2.045 & ND & $<0.04$ & $<0.02$ & $<0.03$ & $<0.03$ \\
\hline 3 & ESF-SD-CIV\#10 (2.0-3.9) & SPC00516697 & 1.046 & 1.489 & ND & 0.060 & $<0.03$ & 0.097 & 0.63 \\
\hline 3 & ESF-DHW-CIV\#10 (2.4-4.7) & SPC01015121 & 1.156 & 1.497 & ND & 0.18 & $<0.03$ & 0.47 & 0.27 \\
\hline 3 & ESF-SD-CIV\#8 (4.0-6.2) & SPC01015124 & 1.360 & 1.700 & ND & 0.050 & $<0.03$ & 0.12 & 0.056 \\
\hline 3 & ESF-SD-CIV\#25 (5.6-6.7) & SPC01015127 & 1.264 & 1.500 & ND & 0.11 & $<0.02$ & 0.12 & 0.087 \\
\hline 3 & SYSTEM BLANK (1/8/02) & SPC01015130 & 2.042 & 2.042 & ND & $<0.04$ & $<0.02$ & $<0.03$ & $<0.03$ \\
\hline 3 & ESF-SD-CIV\#15 (2.3-4.2) & SPC01015135 & 2.109 & $2: 446$ & ND & 0.055 & $<0.02$ & 0.13 & 0.095 \\
\hline 3 & ESF-DHW-CIV\#2 (3.2-6.5) & SPC01015138 & 1.434 & 1.962 & ND & 0.18 & $<0.03$ & 0.33 & 0.29 \\
\hline 3 & ESF-SD-CIV\#12 (4.4-6.5) & SPC01015141 & 2.137 & 2.306 & ND & 0.051 & $<0.02$ & 0.076 & 0.067 \\
\hline 3 & SYSTEM BLANK (1/9/02) & SPC01015144 & 2.094 & 2.094 & ND & $<0.04$ & $<0.02$ & $<0.03$ & $<0.03$ \\
\hline 3 & ESF-SD-CIV\#5 (1.8-4.1) & SPC01015147 & 1.433 & 2.039 & ND & 0.20 & $<0.03$ & 0.26 & 0.23 \\
\hline 3 & ESF-DHW-CIV\#6 (2.2-6.6) & SPC01015150 & 2.483 & 2.552 & ND & 0.23 & $<0.02$ & 0.43 & 0.30 \\
\hline 3 & $\begin{array}{l}\text { ESF-DHW-CIV\#6 (2.2-6.6) } \\
\text { rerun }\end{array}$ & SPC01015150 & 2.483 & 2.552 & ND & 0.25 & $<0.02$ & 0.45 & 0.32 \\
\hline 3 & SYSTEM BLANK (1/10/02) & SPC01015153 & 2.108 & 2.108 & ND & $<0.04$ & $<0.02$ & 0.038 & 0.035 \\
\hline 3 & \begin{tabular}{|c|} 
SYSTEM BLANK $(1 / 10 / 02)$ \\
rerun
\end{tabular} & SPC01015153 & 2.108 & 2.108 & ND & 0.043 & $<0.02$ & 0.037 & $<0.03$ \\
\hline 3 & ESF-SD-CIV\#3 (4.2-6.2) & SPC01015156 & 2.177 & 2.389 & ND & 0.21 & $<0.02$ & 0.11 & 0.20 \\
\hline 3 & ESF-DHW-CIV\#4 (2.0-6.1) & SPC01015159 & 0.663 & 1.144 & ND & 0.26 & $<0.03$ & 0.38 & 0.29 \\
\hline 4 & $\begin{array}{c}\text { ESF-SAD-GTB\#1 hand crush } \\
(10 \text { mesh-3/4") }\end{array}$ & SPC01015160 & 1.605 & 1.853 & 0.060 & 0.62 & $<0.02$ & 0.11 & 0.50 \\
\hline 4 & $\begin{array}{l}\text { ESF-SAD-GTB\#1 hand crush } \\
\left(1 / 4^{\prime \prime}-3 / 4^{\prime \prime}\right)\end{array}$ & SPC01015163 & 1.477 & 1.534 & 0.047 & 0.44 & $<0.02$ & $<0.03$ & 0.48 \\
\hline
\end{tabular}


Table 4-13. Concentrations of Anions in Leachates of Validation Study Samples Analyzed by lon Chromatography at the USGS during Phase III (continued)

\begin{tabular}{|c|c|c|c|c|c|c|c|c|c|}
\hline $\begin{array}{l}\text { Batch } \\
\text { No. }\end{array}$ & Sample Identifier & $\begin{array}{l}\text { SMF Barcode } \\
\text { Identifier }\end{array}$ & $\begin{array}{c}\text { Mass of } \\
\text { Rock } \\
\text { (kg) }\end{array}$ & $\begin{array}{c}\text { Mass of } \\
\text { Water } \\
(\mathrm{kg}) \\
\end{array}$ & $\begin{array}{c}F^{-1} \\
(\mathbf{m g} / \mathbf{k g})\end{array}$ & $\begin{array}{c}\mathrm{Cl}^{-1} \\
(\mathrm{mg} / \mathrm{kg})\end{array}$ & $\begin{array}{c}\mathrm{Br}^{-1} \\
(\mathrm{mg} / \mathrm{kg})\end{array}$ & $\begin{array}{c}\mathrm{NO}_{3}^{-1} \\
(\mathrm{mg} / \mathrm{kg})\end{array}$ & $\begin{array}{c}\mathrm{SO}_{4}^{-2} \\
(\mathrm{mg} / \mathrm{kg})\end{array}$ \\
\hline 4 & SYSTEM BLANK $(6 / 10 / 02)$ & SPC01015166 & 1.571 & 1.571 & 0.033 & $<0.04$ & $<0.02$ & $<0.03$ & $<0.03$ \\
\hline 4 & $\begin{array}{l}\text { ESF-SAD-GTB\#1 mech. crush } \\
\left(1 / 4^{\prime \prime}-3 / 4^{\prime \prime}\right)\end{array}$ & SPC01015169 & 1.706 & 1.802 & 0.055 & 0.48 & $<0.02$ & $<0.03$ & 0.41 \\
\hline 5 & $\begin{array}{l}\text { ESF-MD-NICHE3566\#1 } \\
(25.3-31.7)\end{array}$ & SPC01015172 & 1.358 & 1.456 & 0.086 & 0.23 & $<0.02$ & $<0.03$ & 0.28 \\
\hline 5 & $\begin{array}{l}\text { ESF-MD-NICHE3566\#2 } \\
(20.2-32.5)\end{array}$ & SPC01015175 & 1.312 & 1.446 & 0.064 & 0.29 & $<0.02$ & $<0.03$ & 0.51 \\
\hline 5 & SYSTEM BLANK $(6 / 24 / 02)$ & SPC01015178 & 1.539 & 1.539 & 0.038 & $<0.04$ & $<0.02$ & $<0.03$ & $<0.03$ \\
\hline 5 & $\begin{array}{l}\text { ESF-MD-NICHE3566\#2 } \\
(11.5-15.4)\end{array}$ & SPC01015181 & 1.153 & 1.419 & 0.11 & 0.21 & $<0.02$ & 0.14 & 0.16 \\
\hline 5 & $\begin{array}{l}\text { ESF-MD-NICHE3566\#1 } \\
(14.7-20.9)\end{array}$ & SPC01015184 & 1.435 & 1.511 & 0.075 & 0.21 & $<0.02$ & 0.16 & $<0.03$ \\
\hline 5 & $\begin{array}{l}\text { ESF-MD-NICHE3566\#LT1 } \\
(10.9-23.5)\end{array}$ & SPC01015187 & 1.339 & 1.454 & 0.073 & 0.28 & $<0.02$ & 0.16 & 0.22 \\
\hline 5 & $\begin{array}{l}\text { ESF-MD-NICHE3566\#LT1 } \\
(25.0-38.1)\end{array}$ & SPC01015190 & 1.665 & 1.737 & 0.095 & 0.19 & $<0.02$ & 0.10 & 0.15 \\
\hline 5 & SYSTEM BLANK $(6 / 24 / 02)$ & SPC01015193 & 1.615 & 1.615 & 0.042 & 0.076 & $<0.02$ & $<0.03$ & $<0.03$ \\
\hline
\end{tabular}

NOTES:

DTN: GS030608312272.005(Q)

NA = not applicable, ND = not determined, SMF = Sample Management Facility, USGS = U.S. Geological Survey.

Samples were crushed at either the SMF or USGS, and leached at the USGS. The leachates were distributed to LANL and LLNL for AgCl

precipitation and target preparation. For Batch \#1 samples, targets prepared at LANL and LLNL were analyzed at LLNL-CAMS. For Batch \#2, the targets prepared at LANL were analyzed at PRIME Lab and targets prepared at LLNL were analyzed at LLNL-CAMS. Targets for Batches \#3, \#4, and \#5 were prepared and analyzed at LLNL.

Data are ordered by leaching sequence. Concentrations are given with respect to the amount of rock used after passive-leaching for 1 hour. Lessthan symbols indicate concentrations that are less than the ion-chromatography method detection limits after correction for water/rock ratio.

Analytical errors $(2 \sigma)$ for $\mathrm{Cl}$ are $\pm 0.03 \mathrm{mg} / \mathrm{kg}$ for concentrations $<0.2 \mathrm{mg} / \mathrm{kg}$ and $\pm 0.08 \mathrm{mg} / \mathrm{kg}$ for concentrations $>0.2 \mathrm{mg} / \mathrm{kg}$. Fluorine analyses were not determined prior to June 2002 because of insufficient peak separations.

Data reported in $\mathrm{mg} / \mathrm{L}$ in the DTN have been converted to $\mathrm{mg} / \mathrm{kg}$ in this table to normalize the concentration data to mass of rock:ion concentration in $\mathrm{mg} / \mathrm{kg}=$ (ion concentration in $\mathrm{mg} / \mathrm{L}$ ) * (mass of water in $\mathrm{kg}) \div$ (mass of rock in $\mathrm{kg}$ ). Assumes $1 \mathrm{~kg}$ water $=1 \mathrm{~L}$ of water.

For ESF-SD-CIV\#27, the mass of water was assumed to be $2000 \mathrm{~g}$ 
Table 4-14. Summary of Anion Concentrations in Leachates of Validation Study Samples Analyzed by lon Chromatography at the USGS during Phase III

\begin{tabular}{|c|c|c|c|c|c|c|}
\hline Sample Identifier & Sample Grouping & $\begin{array}{c}F^{-1} \\
(\mathrm{mg} / \mathbf{k g})\end{array}$ & $\begin{array}{c}\mathrm{Cl}^{-1} \\
(\mathrm{mg} / \mathrm{kg})\end{array}$ & $\begin{array}{c}\mathrm{Br}^{-1} \\
(\mathrm{mg} / \mathrm{kg})\end{array}$ & $\begin{array}{c}\mathrm{NO}_{3}^{-1} \\
(\mathrm{mg} / \mathrm{kg})\end{array}$ & $\begin{array}{c}\mathrm{SO}_{4}^{-2} \\
(\mathrm{mg} / \mathrm{kg})\end{array}$ \\
\hline \multirow{6}{*}{$\begin{array}{l}\text { Drill Hole Wash fault zone } \\
\text { (ESF-DHW-CIV series) }\end{array}$} & $\mathrm{n}=$ & 0 & 10 & 10 & 10 & 10 \\
\hline & Maximum $=$ & ND & 0.32 & $<0.02$ & 0.47 & 0.33 \\
\hline & Minimum = & ND & 0.17 & $<0.02$ & 0.29 & 0.22 \\
\hline & Median = & NA & 0.205 & $<0.02$ & 0.380 & 0.270 \\
\hline & Average $=$ & NA & 0.223 & $<0.02$ & 0.388 & 0.272 \\
\hline & Standard Deviation = & NA & 0.053 & $<0.02$ & 0.057 & 0.034 \\
\hline \multirow{6}{*}{$\begin{array}{l}\text { Sundance fault zone, } \\
\text { Incl. Niche \#1 } \\
\text { (ESF-SD-CIV series) }\end{array}$} & $\mathrm{n}=$ & 6 & 51 & 51 & 50 & 49 \\
\hline & Maximum = & 0.11 & 0.31 & $<0.02$ & 0.44 & 0.51 \\
\hline & Minimum $=$ & 0.064 & 0.050 & $<0.02$ & $<0.04$ & $<0.03$ \\
\hline & Median = & 0.081 & 0.120 & $<0.02$ & 0.145 & 0.140 \\
\hline & Average $=$ & 0.084 & 0.145 & $<0.02$ & 0.156 & 0.157 \\
\hline & Standard Deviation = & 0.017 & 0.074 & $<0.02$ & 0.082 & 0.104 \\
\hline \multirow{6}{*}{$\begin{array}{l}\text { Southern Ghost Dance fault } \\
\text { zone (Alcove \#7) } \\
\text { (ESF-SAD-GTB\#1) }\end{array}$} & $\mathrm{n}=$ & 3 & 3 & 3 & 3 & 3 \\
\hline & Maximum = & 0.06 & 0.62 & $<0.02$ & 0.11 & 0.50 \\
\hline & Minimum $=$ & 0.05 & 0.44 & $<0.02$ & $<0.04$ & 0.41 \\
\hline & Median $=$ & NA & NA & NA & NA & NA \\
\hline & Average $=$ & 0.054 & 0.513 & $<0.02$ & 0.050 & 0.463 \\
\hline & Standard Deviation $=$ & 0.007 & 0.95 & $<0.02$ & 0.052 & 0.047 \\
\hline \multirow[t]{6}{*}{ EVÁL001 } & $n=$ & 0 & 2 & 2 & 2 & 2 \\
\hline & Maximum $=$ & ND & 0.19 & 0.18 & 0.19 & 0.22 \\
\hline & Minimum = & ND & 0.17 & 0.14 & 0.15 & 0.19 \\
\hline & Median $=$ & NA & NA & NA & NA & NA \\
\hline & Average $=$ & NA & NA & NA & NA & NA \\
\hline & Standard Deviation = & NA & NA & NA & NA & NA \\
\hline
\end{tabular}

NOTES: $N A=$ not applicable, $N D=$ not determined, USGS $=$ U.S. Geological Survey.

Statistics are compiled from data given in Table 4-13 with samples grouped by area (i.e., Drill Hole Wash fault zone, Sundance fault zone, southern Ghost Dance fault zone, and EVAL001).

Re-runs of ion chromatography determinations have been averaged to yield a single value for each sample.

Concentrations reported as less than the method detection limit (MDL) have been assigned a value of $0.5 \times \mathrm{MDL}$ for statistical analysis. 
Table 4-15. Summary of Chloride Concentrations and ${ }^{36} \mathrm{CI} / \mathrm{CI}$ Ratios in Core Samples Leached and Analyzed at USGS-LLNL during Phase III

\begin{tabular}{|c|c|c|c|}
\hline Sample Identifier & Sample Grouping & $\begin{array}{c}\text { Leachate } \mathrm{Cl} \\
\text { Concentration } \\
\text { (mg/kg rock) }\end{array}$ & ${ }^{36} \mathrm{Cl} / \mathrm{Cl} \times 10^{15}$ \\
\hline \multirow{8}{*}{$\begin{array}{l}\text { ESF-SD-CIV core } \\
\text { (excludes Niche \#1) }\end{array}$} & $n=$ & 34 & 34 \\
\hline & Minimum $=$ & 0.037 & 137 \\
\hline & Maximum $=$ & 0.372 & 615 \\
\hline & $\begin{array}{r}\text { Anderson-Darling P-Value }= \\
\text { (Distribution) }=\end{array}$ & $\begin{array}{r}0.000 \\
\text { (non-normal) }\end{array}$ & $\begin{array}{c}0.108 \\
\text { (normal) }\end{array}$ \\
\hline & Median $=$ & 0.120 & 316 \\
\hline & Arithmetic Mean = & 0.130 & 326 \\
\hline & Standard Deviation $=$ & 0.083 & 134 \\
\hline & $2 \times$ Standard Error $=$ & 0.029 & 46 \\
\hline \multirow[t]{8}{*}{ Niche \#1 } & $\mathrm{n}=$ & 6 & 6 \\
\hline & Minimum = & 0.173 & 226 \\
\hline & Maximum $=$ & 0.270 & 717 \\
\hline & $\begin{array}{r}\text { Anderson-Darling P-Value }= \\
\text { (Distribution) }=\end{array}$ & $\begin{array}{r}0.133 \\
\text { (normal) } \\
\end{array}$ & $\begin{array}{c}0.287 \\
\text { (normal) }\end{array}$ \\
\hline & Median $=$ & 0.201 & 387 \\
\hline & Arithmetic Mean $=$ & 0.206 & 401 \\
\hline & Standard Deviation $=$ & 0.035 & 177 \\
\hline & $2 \times$ Standard Error $=$ & 0.028 & 145 \\
\hline \multirow{8}{*}{$\begin{array}{l}\text { All Sundance fault zone } \\
\text { (pooled data) }\end{array}$} & $\mathrm{n}=$ & 40 & 40 \\
\hline & Minimum = & 0.037 & 137 \\
\hline & Maximum $=$ & 0.372 & 717 \\
\hline & $\begin{array}{r}\text { Anderson-Darling P-Value }= \\
\text { (Distribution) }=\end{array}$ & $\begin{array}{c}0.003 \\
\text { (non-normal) } \\
\end{array}$ & $\begin{array}{c}0.125 \\
\text { (normal) }\end{array}$ \\
\hline & Median $=$ & 0.120 & 316 \\
\hline & Arithmetic Mean $=$ & 0.141 & 337 \\
\hline & Standard Deviation $=$ & 0.082 & 141 \\
\hline & $2 \times$ Standard Error $=$ & 0.026 & 45 \\
\hline
\end{tabular}

DTN: Calculated from data in LL031200223121.036 (Q), Filename: Total_AMS_Summary_2001-02c.xls

NOTE: Statistics are compiled from data given in Table 4-9, with samples grouped by area (i.e., ESF-SD-CIV, Niche \#1, and Sundance fault zone). 
Table 4-16. Mass of Total Chloride, ${ }^{36} \mathrm{Cl} / \mathrm{Cl}$ Ratios, and Mass of ${ }^{36} \mathrm{Cl}$ Present in Validation Study Blanks Processed at LANL during Phase III

\begin{tabular}{|c|c|c|c|c|c|c|c|c|c|c|c|}
\hline $\begin{array}{l}\text { Sample or } \\
\text { Aliquot } \\
\text { Identifier }\end{array}$ & $\begin{array}{c}\text { SMF } \\
\text { Barcode } \\
\text { Identifier }\end{array}$ & $\begin{array}{c}\text { LANL } \\
\text { Identifier }\end{array}$ & $\begin{array}{c}\text { AMS } \\
\text { Facility }\end{array}$ & $\begin{array}{c}\text { AMS } \\
\text { Identifier }\end{array}$ & $\begin{array}{c}\text { Date } \\
\text { Analyzed }\end{array}$ & $\begin{array}{c}\text { Water } \\
\text { Mass } \\
\text { Analyzed } \\
(\mathbf{k g})\end{array}$ & $\begin{array}{c}\text { Mass Cl } \\
\text { in Blank } \\
(\mathrm{mg})\end{array}$ & $\begin{array}{c}\text { Cl Conc. } \\
\text { in Blank } \\
\text { (mg/kg } \\
\text { water) }\end{array}$ & $\begin{array}{c}{ }^{36} \mathrm{Cl} / \mathrm{Cl} \\
\times 10^{15}\end{array}$ & $\begin{array}{c}\text { Mass } \\
{ }^{36} \mathrm{Cl} \text { in } \\
\text { Blank } \\
(\mathrm{mg}) \\
\times 10^{15} \\
\end{array}$ & $\begin{array}{c}\text { Conc. }{ }^{36} \mathrm{Cl} \\
\text { in Blank } \\
\text { (mg/kg } \\
\text { water) } \\
\times 10^{15} \\
\end{array}$ \\
\hline $\begin{array}{c}\text { Procedural } \\
\text { blank } \\
\text { (USGS } \\
\text { water) }\end{array}$ & $\mid \begin{array}{c}\text { SPC } \\
00536914\end{array}$ & YM2042 & CAMS & CL9668 & $10 / 26 / 2001$ & 0.899 & 0.0048 & 0.0053 & 1,022 & 4.91 & 5.46 \\
\hline $\begin{array}{c}\text { Procedural } \\
\text { blank } \\
\text { (USGS } \\
\text { water) }\end{array}$ & $\mid \begin{array}{c}\text { SPC } \\
00536941\end{array}$ & YM2043 & CAMS & CL9669 & $10 / 26 / 2001$ & 0.950 & 0.0048 & 0.0051 & 630 & 3.02 & 3.18 \\
\hline $\begin{array}{c}\text { Procedural } \\
\text { blank } \\
\text { (USGS } \\
\text { water) }\end{array}$ & $\mid \begin{array}{c}\text { SPC } \\
00516601\end{array}$ & YM2046 & CAMS & CL9672 & $10 / 26 / 2001$ & 0.923 & 0.0046 & 0.0050 & 1,095 & 5.04 & 5.46 \\
\hline $\begin{array}{c}\text { Procedural } \\
\text { blank } \\
\text { (PB 301) }\end{array}$ & NA & YM2021 & $\begin{array}{c}\text { PRIME } \\
\text {. }\end{array}$ & $\begin{array}{c}W 01-0861 \\
5 A\end{array}$ & $12 / 7 / 2001$ & 3.934 & 0.052 & 0.013 & 396 & 20.59 & 5.23 \\
\hline $\begin{array}{c}\text { Procedural } \\
\text { blank } \\
\text { (PB 303) }\end{array}$ & NA & YM2031 & CAMS & CL9657 & $10 / 26 / 2001$ & 0.930 & 0.0024 & 0.0027 & 1,158 & 2.78 & 2.99 \\
\hline $\begin{array}{c}\text { Procedural } \\
\text { blank } \\
\text { (PB 305) }\end{array}$ & NA & YM2068 & CAMS & CL9741 & $11 / 29 / 2001$ & 0.500 & 0.0034 & 0.0068 & 3,756 & 12.77 & 25.54 \\
\hline $\begin{array}{c}\text { Procedural } \\
\text { blank } \\
\text { (PB 306) }\end{array}$ & NA & YM2082 & PRIME & $\begin{array}{c}\text { R02- } \\
0211,5 \mathrm{~A}\end{array}$ & $8 / 21 / 2002$ & 1.000 & 0.022 & 0.022 & 920 & 20.24 & 20.24 \\
\hline $\begin{array}{c}\text { Procedural } \\
\text { blank } \\
\text { (PB 307) }\end{array}$ & NA & YM2099 & CAMS & CL10138 & $5 / 23 / 2002$ & 1.804 & 0.0254 & 0.014 & 1,724 & 43.79 & 24.27 \\
\hline $\begin{array}{c}\text { Procedural } \\
\text { blank } \\
\text { (PB 308) }\end{array}$ & NA & YM2100 & CAMS & CL10139 & $5 / 23 / 2002$ & 1.567 & 0.0097 & 0.0062 & 3,722 & 36.10 & 23.04 \\
\hline $\begin{array}{c}\text { Procedural } \\
\text { blank } \\
\text { (PB 309) }\end{array}$ & NA & YM2110 & CAMS & CL10298 & $7 / 30 / 2002$ & 0.967 & 0.0041 & 0.0042 & 3,349 & 13.73 & 14.2 \\
\hline
\end{tabular}


Table 4-16. Mass of Total Chloride, ${ }^{36} \mathrm{Cl} / \mathrm{Cl}$ Ratios, and Mass of ${ }^{36} \mathrm{CI}$ Present in Validation Study Blanks Processed at LANL during Phase III (continued)

\begin{tabular}{|c|c|c|c|c|c|c|c|c|c|c|c|}
\hline $\begin{array}{l}\text { Sample or } \\
\text { Aliquot } \\
\text { Identifier }\end{array}$ & $\begin{array}{c}\text { SMF } \\
\text { Barcode } \\
\text { Identifier }\end{array}$ & $\begin{array}{c}\text { LANL } \\
\text { Identifier }\end{array}$ & $\begin{array}{c}\text { AMS } \\
\text { Facility }\end{array}$ & $\begin{array}{c}\text { AMS } \\
\text { Identifier }\end{array}$ & $\begin{array}{c}\text { Date } \\
\text { Analyzed }\end{array}$ & $\begin{array}{c}\text { Water } \\
\text { Mass } \\
\text { Analyzed } \\
(\mathrm{kg})\end{array}$ & $\begin{array}{c}\text { Mass CI } \\
\text { in Blank } \\
\text { (mg) }\end{array}$ & $\begin{array}{c}\text { Cl Conc. } \\
\text { in Blank } \\
\text { ( } \mathrm{mg} / \mathbf{k g} \\
\text { water) }\end{array}$ & $\begin{array}{c}{ }^{36} \mathrm{Cl} / \mathrm{Cl} \\
\times 10^{15}\end{array}$ & $\begin{array}{c}\text { Mass } \\
{ }^{36} \mathrm{Cl} \text { in } \\
\text { Blank } \\
(\mathrm{mg}) \\
\times 10^{15} \\
\end{array}$ & $\begin{array}{c}\text { Conc. }{ }^{36} \mathrm{Cl} \\
\text { in Blank } \\
\text { (mg/kg } \\
\text { water) } \\
\times 10^{15} \\
\end{array}$ \\
\hline $\begin{array}{l}\text { Procedural } \\
\text { blank } \\
\text { (PB 310) }\end{array}$ & NA & YM2111 & CAMS & CL10299 & $7 / 30 / 2002$ & 0.951 & 0.0035 & 0.0037 & 4,257 & 14.90 & 15.67 \\
\hline $\begin{array}{c}\text { Procedural } \\
\text { blank } \\
\text { (PB 311) }\end{array}$ & NA & YM2112 & CAMS & CL10300 & $7 / 30 / 2002$ & 0.999 & 0.0053 & 0.0053 & 1,897 & 10.05 & 10.05 \\
\hline \multicolumn{8}{|c|}{ Arithmetic mean } & 0.008 & 1,994 & 15.7 & 12.9 \\
\hline \multicolumn{8}{|c|}{ Standard deviation } & 0.006 & 1,387 & 13.0 & 8.7 \\
\hline \multicolumn{8}{|c|}{ Standard error } & 0.002 & 400 & 3.7 & 2.5 \\
\hline
\end{tabular}

DTN: LA0305RR831222.001 (UQ)

NOTES: $\quad$ AMS $=$ accelerator mass spectrometer, CAMS $=$ Center for Accelerator Mass Spectrometry, LANL $=$ Los Alamos National Laboratory, NA = not applicable, PRIME = Purdue Rare Isotope Measurement Laboratory, SMF = Sample Management Facility, USGS = U.S. Geological Survey.

Analytical errors are $1 \sigma$ for ${ }^{36} \mathrm{Cl} / \mathrm{Cl}$ (corrected for background and spike) and concentration of ${ }^{36} \mathrm{Cl}$ in blank.

$\mathrm{Cl}$ conc. in blank $(\mathrm{mg} / \mathrm{kg}$ water $)=$ Mass $\mathrm{Cl}$ in blank $(\mathrm{mg}) \div$ Water mass analyzed $(\mathrm{kg})$.

Mass of ${ }^{36} \mathrm{Cl}$ in Blank $(\mathrm{mg})=$ Water mass analyzed $(\mathrm{kg}) \times \mathrm{Cl}$ Conc. in blank $(\mathrm{mg} / \mathrm{kg}$ water $) \times$ ${ }^{36} \mathrm{Cl} / \mathrm{Cl} \times 10^{-15}$.

Conc. ${ }^{36} \mathrm{Cl}$ in blank $(\mathrm{mg} / \mathrm{kg}$ water $)=$ Mass ${ }^{36} \mathrm{Cl}$ in blank $(\mathrm{mg}) \div$ Water mass analyzed $(\mathrm{kg})$. 
Table 4-17. Chloride, Bromide, and Sulfate Concentrations, and ${ }^{36} \mathrm{CI} / \mathrm{Cl}$ Ratios in Leachates of ECRB Cross Drift Samples Analyzed at LANL during Phase III

\begin{tabular}{|c|c|c|c|c|c|c|c|c|c|c|c|}
\hline \multirow[b]{2}{*}{$\begin{array}{l}\text { Sample } \\
\text { Identifier }\end{array}$} & \multirow[b]{2}{*}{$\begin{array}{c}\text { SMF Barcode } \\
\text { Identifier }\end{array}$} & \multirow{2}{*}{\begin{tabular}{|c|} 
Distance \\
from \\
Start of \\
ECRB \\
Cross \\
Drift (m) \\
\end{tabular}} & \multirow[b]{2}{*}{$\begin{array}{c}\text { Sample } \\
\text { Type }\end{array}$} & \multirow[b]{2}{*}{$\begin{array}{c}\text { Description } \\
\text { of Sampled } \\
\text { Feature }\end{array}$} & \multirow[b]{2}{*}{$\begin{array}{c}\mathrm{AgCl} \\
\text { Target ID }\end{array}$} & \multicolumn{3}{|c|}{$\begin{array}{c}\text { Concentration } \\
\text { (mg/kg rock) }\end{array}$} & \multirow[b]{2}{*}{$\begin{array}{c}\mathrm{Brl} \\
\mathrm{Cl}\end{array}$} & \multirow[b]{2}{*}{$\left|\begin{array}{c}\mathrm{SO}, 4 \\
\mathrm{Cl}\end{array}\right|$} & \multirow{2}{*}{$\begin{array}{c}\text { Measured } \\
{ }^{36} \mathrm{Cl} / \mathrm{Cl} \\
\times 10^{15} \\
(2 \sigma) \\
\end{array}$} \\
\hline & & & & & & $\mathrm{Cl}^{-1}$ & $\mathrm{Br}^{-1}$ & $\mathrm{SO}_{4}^{-2}$ & & & \\
\hline EXD001-1 & SPC00504392 & 2,545 & Opportunistic & $\begin{array}{l}\text { Highly fractured } \\
\text { bedrock, } \\
\text { Solitario } \\
\text { Canyon fault } \\
\text { zone } \\
\end{array}$ & YM960 & 0.65 & 0.362 & 0.90 & 0.558 & 1.4 & $789 \pm 66$ \\
\hline EXD002-1 & SPC00504390| & 2,550 & Opportunistic & $\begin{array}{l}\text { Fractured rock } \\
\text { and gouge, } \\
\text { Solitario } \\
\text { Canyon fault } \\
\text { zone }\end{array}$ & YM961 & 2.12 & 0.674 & 2.51 & 0.318 & 1.2 & $342 \pm 42$ \\
\hline EXD003-1 & SPC00524980 & $1,135.5$ & $\begin{array}{l}\text { Fault } \\
\text { transect }\end{array}$ & $\begin{array}{l}\text { Breccia from } \\
\text { Sundance fault } \\
\text { zone }\end{array}$ & YM962 & 0.72 & 0.005 & 0.59 & 0.006 & 0.8 & $347 \pm 32$ \\
\hline EXD004-1 & SPC00524981 & 1,137 & $\mid \begin{array}{l}\text { Fault } \\
\text { transect }\end{array}$ & \begin{tabular}{|l|} 
Fractured wall \\
rock adjacent to \\
Sundance fault \\
zone \\
\end{tabular} & YM963 & 0.25 & 0.089 & 0.39 & 0.358 & 1.6 & $1,124 \pm 171$ \\
\hline EXD005-1 & SPC00524977 & 1,317 & $\begin{array}{l}\text { Fault } \\
\text { transect }\end{array}$ & Breccia & YM964 & 0.40 & 0.080 & 0.97 & 0.199 & 2.4 & $582 \pm 79$ \\
\hline EXD006-1 & SPC00524978 & 1,318 & $\begin{array}{l}\text { Fault } \\
\text { transect }\end{array}$ & $\begin{array}{l}\text { Breccia in fault } \\
\text { zone }\end{array}$ & YM965 & 0.57 & 0.018 & 0.46 & 0.031 & 0.8 & $343 \pm 57$ \\
\hline EXD007-1 & SPC00533390 & 1,320 & $\begin{array}{l}\text { Fault } \\
\text { transect }\end{array}$ & $\begin{array}{l}\text { Fractured wall } \\
\text { rock near fault }\end{array}$ & YM1008 & 0.27 & 0.102 & 0.54 & 0.377 & 2.0 & $624 \pm 62$ \\
\hline EXD008-1 & SPC00533387 & 2,154 & $\begin{array}{l}\text { Fault } \\
\text { transect }\end{array}$ & $\begin{array}{l}\text { Breccia in fault } \\
\text { zone }\end{array}$ & YM968 & 0.49 & 0.015 & 0.70 & 0.031 & 1.4 & $915 \pm 97$ \\
\hline EXD009-1 & SPC00538284 & $2,154.5$ & $\begin{array}{l}\text { Fault } \\
\text { transect }\end{array}$ & $\begin{array}{l}\text { Breccia in fault } \\
\text { zone }\end{array}$ & YM969 & 0.59 & 0.011 & 0.61 & 0.018 & 1.0 & $4,890 \pm 349$ \\
\hline EXD010-1 & SPC00533388 & 2,155 & $\mid \begin{array}{l}\text { Fault } \\
\text { transect }\end{array}$ & $\begin{array}{l}\text { Fractured rock } \\
\text { in fault hanging } \\
\text { wall }\end{array}$ & YM1043 & 0.41 & 0.050 & 0.85 & 0.123 & 2.1 & $553 \pm 34$ \\
\hline EXD011-1 & SPC00533389 & 2,162 & Other fault & $\begin{array}{l}\text { Breccia from } \\
\text { minor fault }\end{array}$ & YM1032 & 0.50 & NA & 1.16 & NA & 2.3 & $550 \pm 59$ \\
\hline EXD012-1 & SPC00538283 & 2,238 & Other fault & $\begin{array}{l}\text { Breccia in fault } \\
\text { zone }\end{array}$ & YM970 & 0.51 & 0.014 & 0.45 & 0.027 & 0.9 & $2,349 \pm 210$ \\
\hline EXD012-3 & SPC00538283 & 2,238 & Other fault & \begin{tabular}{|l} 
Breccia in fault \\
zone
\end{tabular} & YM1009 & 0.92 & NA & 0.97 & NA & 1.1 & $3,549 \pm 500$ \\
\hline EXD013-1 & SPC00538282 & 2,348 & Other fault & $\begin{array}{l}\text { Fault with } 3 \mathrm{~m} \\
\text { offset }\end{array}$ & YM971,B & 0.71 & 0.063 & 0.75 & 0.088 & 1.1 & $1,043 \pm 74$ \\
\hline EXD014-1 & SPC00538281 & 2,445 & Other fault & $\begin{array}{l}\text { Fault with } 2.5 \mathrm{~m} \\
\text { offset }\end{array}$ & YM1044 & 0.34 & 0.150 & 0.56 & 0.442 & 1.6 & $550 \pm 51$ \\
\hline EXD015-1 & SPC00538279 & 2,500 & $\begin{array}{l}\begin{array}{l}\text { Systematic } \\
\text { feature }\end{array} \\
\end{array}$ & $\begin{array}{l}\text { Fault with } 0.4 \mathrm{~m} \\
\text { offset }\end{array}$ & YM1045 & 0.23 & 0.117 & 0.56 & 0.510 & 2.4 & $812 \pm 72$ \\
\hline EXD016-1 & SPC00538280 & $2,530.5$ & $\mid \begin{array}{l}\text { Fault } \\
\text { transect }\end{array}$ & $\begin{array}{l}\text { Fractured rock } \\
\text { between } 2 \\
\text { faults }\end{array}$ & YM972 & 0.65 & $\mathrm{NA}$ & 1.15 & NA & 1.8 & $1,122 \pm 89$ \\
\hline EXD017-1 & SPC00538275 & 2,570 & $\mid \begin{array}{l}\text { Fault } \\
\text { transect }\end{array}$ & $\begin{array}{l}\text { Solitario } \\
\text { Canyon fault } \\
\text { zone }\end{array}$ & YM973 & 0.52 & NA & 0.87 & NA & 1.7 & $2,158 \pm 175$ \\
\hline EXD017-3 & SPC00538275 & 2,570 & $\begin{array}{l}\text { Fault } \\
\text { transect }\end{array}$ & $\begin{array}{l}\text { Solitario } \\
\text { Canyon fault } \\
\text { zone }\end{array}$ & YM1010 & 0.80 & $\mathrm{NA}$ & 1.12 & NA & 1.4 & $3,068 \pm 258$ \\
\hline
\end{tabular}


Table 4-17. Chloride, Bromide, and Sulfate Concentrations, and ${ }^{36} \mathrm{Cl} / \mathrm{CI}$ Ratios in Leachates of ECRB Cross Drift Samples Analyzed at LANL during Phase III (continued)

\begin{tabular}{|c|c|c|c|c|c|c|c|c|c|c|c|}
\hline \multirow[b]{2}{*}{$\begin{array}{l}\text { Sample } \\
\text { Identifier }\end{array}$} & \multirow[b]{2}{*}{$\begin{array}{c}\text { SMF Barcode } \\
\text { Identifier }\end{array}$} & \multirow{2}{*}{$\begin{array}{c}\text { Distance } \\
\text { from } \\
\text { Start of } \\
\text { ECRB } \\
\text { Cross } \\
\text { Drift }(m)\end{array}$} & \multirow[b]{2}{*}{$\begin{array}{l}\text { Sample } \\
\text { Type }\end{array}$} & \multirow[b]{2}{*}{$\begin{array}{l}\text { Description } \\
\text { of Sampled } \\
\text { Feature }\end{array}$} & \multirow[b]{2}{*}{$\begin{array}{c}\mathrm{AgCl} \\
\text { Target ID }\end{array}$} & \multicolumn{3}{|c|}{$\begin{array}{c}\text { Concentration } \\
\text { (mg/kg rock) }\end{array}$} & \multirow[b]{2}{*}{$\begin{array}{l}\mathrm{Brl} \\
\mathrm{Cl}\end{array}$} & \multirow[b]{2}{*}{$\begin{array}{c}\mathrm{SO}_{4} \\
\mathrm{Cl}\end{array}$} & \multirow[b]{2}{*}{\begin{tabular}{|c|}
${ }^{\text {Measured }}{ }^{\mathrm{c}}$ \\
${ }^{36} \mathrm{Cl} / \mathrm{Cl}$ \\
$\times 10^{15}$ \\
$(2 \sigma)$
\end{tabular}} \\
\hline & & & & & & $\mathrm{Cl}^{-1}$ & $\mathrm{Br}^{-1}$ & $\mathrm{SO}_{4}^{-2}$ & & & \\
\hline EXD018-1 & SPC00538273 & 2,580 & $\begin{array}{l}\text { Fault } \\
\text { transect }\end{array}$ & \begin{tabular}{|l|} 
Solitario \\
Canyon fault \\
zone
\end{tabular} & YM974 & 0.69 & 0.020 & 0.95 & 0.029 & 1.4 & $890 \pm 109$ \\
\hline EXD019-1 & SPC00538270 & 2,585 & Other fault & \begin{tabular}{|l|} 
Brecciated \\
footwall of \\
Solitario \\
Canyon fault \\
\end{tabular} & YM975 & 0.87 & 0.029 & 0.92 & 0.034 & 1.1 & $2,447 \pm 205$ \\
\hline EXD020-1 & SPC00538280 & $2,530.5$ & Other fault & \begin{tabular}{|l|} 
Solitario \\
Canyon fault \\
plane
\end{tabular} & YM1046 & 0.79 & 0.094 & 2.06 & 0.119 & 2.6 & $720 \pm 43$ \\
\hline EXD020-3 & SPC00538271 & 2,586 & Other fault & \begin{tabular}{|l|} 
Solitario \\
Canyon fault \\
plane
\end{tabular} & YM1033 & 0.52 & 0.055 & $\mid 0.92$ & 0.105 & 1.8 & $641 \pm 67$ \\
\hline EXD021-1 & SPC00538272 & $2,586.5$ & Other fault & $\begin{array}{l}\text { Brecciated } \\
\text { hanging wall } \\
\text { of Solitario } \\
\text { Canyon fault }\end{array}$ & YM976 & 1.83 & 0.134 & 1.76 & 0.073 & 1.0 & $1,227 \pm 82$ \\
\hline EXD022-1 & $\begin{array}{c}\text { SPC00538269 } \\
.\end{array}$ & 2,590 & \begin{tabular}{|l} 
Fault \\
transect
\end{tabular} & \begin{tabular}{|l|} 
Solitario \\
Canyon fault \\
zone
\end{tabular} & YM977 & 0.83 & 0.110 & 1.16 & 0.133 & 1.4 & $1,360 \pm 113$ \\
\hline EXD023-1 & SPC00524985 & 2,600 & $\begin{array}{l}\text { Fault } \\
\text { transect }\end{array}$ & \begin{tabular}{|l|} 
Solitario \\
Canyon fault \\
zone
\end{tabular} & YM1047 & 0.69 & 0.084 & $\mid 0.69$ & 0.121 & 1.0 & $554 \pm 34$ \\
\hline EXD024-1 & SPC00538276 & 2,610 & $\mid \begin{array}{l}\text { Fault } \\
\text { transect }\end{array}$ & \begin{tabular}{|l|} 
Solitario \\
Canyon fault \\
zone
\end{tabular} & YM1048 & 0.74 & 0.205 & $\mid 0.68$ & 0.277 & 0.9 & $618 \pm 41$ \\
\hline EXD025-1 & SPC00538277 & 2,621 & Other fault & \begin{tabular}{|l|} 
Solitario \\
Canyon fault \\
zone \\
\end{tabular} & YM978 & 0.65 & 0.032 & 0.82 & 0.050 & 1.3 & $954 \pm 96$ \\
\hline EXD026-1 & SPC00538278 & 2,658 & Other fault & \begin{tabular}{|l|} 
Solitario \\
Canyon fault \\
zone
\end{tabular} & YM1034 & 0.45 & 0.090 & 0.59 & 0.200 & 1.3 & $680 \pm 63$ \\
\hline EXD028-1 & SPC00521169 & 892.5 & Other fault & \begin{tabular}{|l|} 
Junction of \\
normal and \\
reverse faults
\end{tabular} & YM1035 & 1.04 & 0.089 & 1.59 & 0.086 & 1.5 & $517 \pm 46$ \\
\hline EXD029-1 & SPC00521168 & 901 & QAVQC & No structures & YM1049 & 1.52 & 0.060 & 2.48 & 0.039 & 1.6 & $505 \pm 40$ \\
\hline EXD030-1 & SPC00521167 & 904 & $\begin{array}{l}\text { Systematic } \\
\text { feature }\end{array}$ & Fault & YM1050 & 0.82 & 0.062 & 1.51 & 0.076 & 1.8 & $566 \pm 38$ \\
\hline EXD031-1 & SPC00521166 & 1,004 & $\begin{array}{l}\text { Systematic } \\
\text { feature }\end{array}$ & \begin{tabular}{|l|} 
Set of \\
parallel \\
fractures
\end{tabular} & YM1011 & 0.67 & 0.178 & 0.70 & 0.265 & 1.0 & $873 \pm 128$ \\
\hline EXD032-1 & SPC00521165 & 1,102 & $\begin{array}{l}\text { Systematic } \\
\text { feature }\end{array}$ & $\begin{array}{l}\text { High-angle } \\
\text { fracture }\end{array}$ & YM1051 & 0.26 & 0.037 & 0.47 & 0.143 & 1.8 & $440 \pm 57$ \\
\hline EXD033-1 & SPC00521164 & $1,130.5$ & \begin{tabular}{|l} 
Fault \\
transect
\end{tabular} & $\begin{array}{l}\text { Cooling joint } \\
\text { network }\end{array}$ & YM1036 & 0.31 & NA & 0.57 & NA & 1.8 & $707 \pm 50$ \\
\hline EXD034-1 & SPC00521163 & 1,133 & $\mid \begin{array}{l}\text { Fault } \\
\text { transect }\end{array}$ & \begin{tabular}{|l|} 
Cooling joint \\
that trends \\
toward \\
Sundance \\
fault zone \\
\end{tabular} & YM1052 & 0.50 & 0.142 & 1.02 & 0.284 & 2.1 & $643 \pm 46$ \\
\hline EXD035-1 & SPC00521162 & 1,135 & Other fault & $\begin{array}{l}\text { Footwall of } \\
\text { Sundance } \\
\text { fault }\end{array}$ & YM1037 & 0.35 & 0.034 & 0.58 & 0.096 & 1.7 & $661 \pm 68$ \\
\hline
\end{tabular}


Table 4-17. Chloride, Bromide, and Sulfate Concentrations and ${ }^{36} \mathrm{Cl} / \mathrm{Cl}$ Ratios in Leachates of ECRB Cross Drift Samples Analyzed at LANL during Phase III (continued)

\begin{tabular}{|c|c|c|c|c|c|c|c|c|c|c|c|}
\hline \multirow[b]{2}{*}{$\begin{array}{l}\text { Sample } \\
\text { Identifier }\end{array}$} & \multirow[b]{2}{*}{$\begin{array}{l}\text { SMF Barcode } \\
\text { Identifier }\end{array}$} & \multirow{2}{*}{$\begin{array}{l}\text { Distance from } \\
\text { Start of ECRB } \\
\text { Cross Drift (m) }\end{array}$} & \multirow[b]{2}{*}{$\begin{array}{l}\text { Sample } \\
\text { Type }\end{array}$} & \multirow[b]{2}{*}{$\begin{array}{c}\text { Description } \\
\text { of Sampled } \\
\text { Feature }\end{array}$} & \multirow[b]{2}{*}{$\underset{\text { Target ID }}{\mathrm{AgCl}}$} & \multicolumn{3}{|c|}{$\begin{array}{c}\text { Concentration } \\
\text { (mg/kg rock) }\end{array}$} & \multirow[b]{2}{*}{$\begin{array}{l}\mathrm{Brl} \\
\mathrm{Cl}\end{array}$} & \multirow[b]{2}{*}{$\begin{array}{c}\mathrm{SO}_{4} \\
\mathrm{Cl}\end{array}$} & \multirow[b]{2}{*}{$\begin{array}{c}\begin{array}{c}\text { Measured } \\
{ }^{36} \mathrm{Cl} / \mathrm{Cl} \\
\times 10^{15} \\
(2 \sigma)\end{array} \\
\end{array}$} \\
\hline & & & & & & $\mathrm{Cl}^{-1}$ & $\mathrm{Br}^{-1}$ & $\mathrm{SO}_{4}^{-2}$ & & & \\
\hline EXD037-1 & SPC00521160 & $1,201.5$ & $\begin{array}{l}\text { Systematic } \\
\text { feature }\end{array}$ & $\begin{array}{l}\text { Broken rock } \\
\text { from hanging } \\
\text { wall of } \\
\text { Sundance } \\
\text { fault zone } \\
\end{array}$ & YM1038 & 0.70 & NA & 0.81 & NA & 1.2 & $490 \pm 43$ \\
\hline EXD037-3 & SPC00521160 & $1,201.5$ & $\begin{array}{l}\text { Systematic } \\
\text { feature }\end{array}$ & \begin{tabular}{|l|} 
Broken rock \\
from hanging \\
wall of \\
Sundance \\
fault zone \\
\end{tabular} & YM1039 & 0.53 & 0.093 & 0.83 & 0.176 & 1.6 & $497 \pm 34$ \\
\hline EXD038-1 & SPC00521159 & 1,205 & Other feature & Fracture set & YM1040 & 0.48 & NA & 0.70 & NA & 1.5 & $385 \pm 33$ \\
\hline EXD039-1 & SPC00521158 & 1,301 & $\begin{array}{l}\text { Systematic } \\
\text { feature }\end{array}$ & $\begin{array}{l}\text { Fracture set } \\
\text { with no offset }\end{array}$ & YM1041 & 0.20 & NA & 0.63 & NA & 3.2 & $569 \pm 38$ \\
\hline EXD040-1 & SPC00521157 & 1,316 & $\mid \begin{array}{l}\text { Fault } \\
\text { transect }\end{array}$ & $\begin{array}{l}\text { Cooling joint } \\
\text { and fracture } \\
\text { set: fault } \\
\text { footwall }\end{array}$ & YM1042 & 0.59 & 0.111 & 0.73 & 0.188 & 1.2 & $658 \pm 60$ \\
\hline EXD046-1 & SPC00521151 & 1,500 & -- & -- & YM2012 & 0.53 & -- & --- & -- & $\cdots$ & $607 \pm 51$ \\
\hline EXD047-1 & SPC00521150 & $1,542.5$ & $\begin{array}{l}\text { Systematic } \\
\text { feature }\end{array}$ & $\begin{array}{l}\text { Fault (shear) } \\
\text { with unknown } \\
\text { offset }\end{array}$ & YM1012 & 1.16 & 0.222 & 1.61 & 0.191 & 1.4 & $589 \pm 52$ \\
\hline EXD051-1 & SPC00521146 & 2,000 & $\begin{array}{l}\text { Systematic } \\
\text { feature }\end{array}$ & \begin{tabular}{|l|} 
Highly \\
fractured rock \\
next to \\
throughgoing \\
fracture
\end{tabular} & YM1013 & 0.63 & 0.106 & 0.94 & 0.169 & 1.5 & $878 \pm 74$ \\
\hline EXD052-1 & SPC00521144 & 2,100 & -- & -- & YM2013 & 0.38 & --- & -- & $\ldots$ & $\cdots$ & $574 \pm 56$ \\
\hline EXD059-1 & SPC00521138 & 2,387 & -- & -- & YM2014 & 0.30 & $\ldots$ & --- & $\cdots$ & -- & $1,309 \pm 114$ \\
\hline EXD063-1 & SPC00521132 & 2,612 & Other fault & Shear zone & YM1014 & 0.86 & 0.037 & 1.08 & 0.043 & 1.3 & $570 \pm 44$ \\
\hline EXD064-1 & SPC00521131 & $2,630.5$ & Other fault & $\begin{array}{l}\text { Hanging wall } \\
\text { of Solitario } \\
\text { Canyon fault } \\
\text { zone }\end{array}$ & YM1015 & 0.45 & 0.030 & 0.72 & 0.066 & 1.6 & $612 \pm 59$ \\
\hline EXD066-1 & SPC00541211 & 2,560 & -- & -- & YM2015 & 3.59 & --- & --- & $\cdots$ & -- & $161 \pm 22$ \\
\hline EXD071-1 & SPC00541216 & 2,585 & -- & $\cdots$ & YM2016 & 0.59 & --- & -- & -- & -- & $474 \pm 46$ \\
\hline EXD075-1 & SPC00533397 & 206 & $\begin{array}{l}\text { Systematic } \\
\text { feature }\end{array}$ & Fracture & YM1016 & 1.26 & 0.128 & 2.12 & 0.102 & 1.7 & $629 \pm 52$ \\
\hline EXD076-1 & SPC00533396 & 300 & -- & $\ldots$ & YM2017 & 0.37 & -- & -- & -- & -- & $671 \pm 75$ \\
\hline EXD078-1 & SPC00533395 & 499 & Other fault & \begin{tabular}{|l|} 
Possible \\
north end of \\
Ghost Dance \\
fault; gouge \\
zone
\end{tabular} & YM1017 & 3.12 & 0.020 & 3.56 & 0.006 & 1.1 & $481 \pm 42$ \\
\hline EXD084-1 & SPC00521175 & Alcove \#8 & -- & - & YM2018 & 0.74 & -- & --- & $\cdots$ & $\ldots$ & $513 \pm 57$ \\
\hline EXD085-1 & SPC00521174 & Alcove \#8 & Other fault & - & YM2019 & 1.12 & -- & --- & -- & -- & $412 \pm 35$ \\
\hline
\end{tabular}


Table 4-17. Chloride, Bromide, and Sulfate Concentrations and ${ }^{36} \mathrm{CI} / \mathrm{CI}$ Ratios in Leachates of ECRB Cross Drift Samples Analyzed at LANL during Phase III (continued)

\begin{tabular}{|c|c|c|c|c|c|c|c|c|c|c|c|}
\hline \multirow[b]{2}{*}{$\begin{array}{l}\text { Sample } \\
\text { Identifier }\end{array}$} & \multirow[b]{2}{*}{$\begin{array}{l}\text { SMF Barcode } \\
\text { Identifier }\end{array}$} & \multirow{2}{*}{$\begin{array}{l}\text { Distance from } \\
\text { Start of ECRB } \\
\text { Cross Drift (m) }\end{array}$} & \multirow[b]{2}{*}{$\begin{array}{c}\text { Sample } \\
\text { Type }\end{array}$} & \multirow[b]{2}{*}{$\begin{array}{c}\text { Description } \\
\text { of Sampled } \\
\text { Feature }\end{array}$} & \multirow[b]{2}{*}{$\begin{array}{c}\mathrm{AgCl} \\
\text { Target ID }\end{array}$} & \multicolumn{3}{|c|}{$\begin{array}{c}\text { Concentration } \\
\text { (mg/kg rock) }\end{array}$} & \multirow[b]{2}{*}{$\begin{array}{l}\mathrm{Brl} \\
\mathrm{Cl}\end{array}$} & \multirow[b]{2}{*}{$\mathrm{SO}_{4}$} & \multirow[b]{2}{*}{$\begin{array}{c}\text { Measured } \\
{ }^{36} \mathrm{Cl} / \mathrm{Cl} \\
\times 10^{15} \\
(2 \sigma)\end{array}$} \\
\hline & & & & & & $\mathrm{Cl}^{-1}$ & $\mathbf{B r}^{-1}$ & $\mathrm{SO}_{4}^{-2}$ & & & \\
\hline $\begin{array}{l}\text { T200-1 } \\
\text { (EXD085-1 } \\
\text { split) }\end{array}$ & SPC00521174 & Alcove \#8 & Other fault & --- & YM2022 & 1.59 & $\cdots$ & --- & --- & - & $434 \pm 43$ \\
\hline EXD086-1 & SPC00521176 & Alcove \#8 & $\begin{array}{l}\text { Fracture } \\
\text { feature }\end{array}$ & --- & YM2020 & 0.92 & $\cdots$ & -- & --- & -- & $550 \pm 179$ \\
\hline
\end{tabular}

DTNs: LA0305RR831222.001 (UQ), LA0307RR831222.001 (UQ)

NOTES:

ECRB = Enhanced Characterization of the Repository Block, ID = identifier, LANL = Los Alamos National Laboratory, SMF = Sample Management Facility.

Concentration of salts extracted from each sample is only a qualitative indicator of the sample's salt content.

Because the focus of this activity is on determining anion ratios, no attempt has been made to maximize the yield of the leaching process, which is probably highly variable.

Measured ${ }^{36} \mathrm{Cl} / \mathrm{Cl}$ ratios have been corrected for the addition of ${ }^{35} \mathrm{Cl}$ tracer. 


\begin{tabular}{|c|c|c|c|c|c|c|c|c|}
\hline \multirow[b]{2}{*}{ Sample Identifier } & \multicolumn{4}{|c|}{ USGS-LLNL-LLNL } & \multicolumn{4}{|c|}{ USGS-LANL-LLNL } \\
\hline & $\begin{array}{l}\text { Barcode Identifier } \\
\text { for LLNL Sample }\end{array}$ & $\begin{array}{c}\text { CAMS } \\
\text { Identifier }\end{array}$ & $\begin{array}{l}\text { Leachate Cl } \\
\text { Concentration } \\
\text { (mg/kg rock) }\end{array}$ & $\begin{array}{l}{ }^{36} \mathrm{Cl} / \mathrm{CI} \\
\times 10^{15} \\
(2 \sigma)\end{array}$ & $\begin{array}{l}\text { Barcode Identifier } \\
\text { for LANL Sample }\end{array}$ & $\begin{array}{c}\text { CAMS } \\
\text { Identifier }\end{array}$ & $\begin{array}{l}\text { Leachate } \mathrm{Cl} \\
\text { Concentration } \\
\text { (mg/kg rock) }\end{array}$ & $\begin{array}{l}{ }^{36} \mathrm{Cl} / \mathrm{CI} \\
\times 10^{15} \\
(2 \sigma)\end{array}$ \\
\hline EVAL001 & SPC00536901 & CL9634 & 0.146 & $454 \pm 109$ & SPC00536902 & CL9659 & 0.15 & $361 \pm 42$ \\
\hline $\begin{array}{l}\text { ESF-SD-CIV\#21 } \\
(2.8-4.6)\end{array}$ & SPC00536919 & CL9640 & 0.147 & $368 \pm 76$ & SPC00536938 & CL9662 & 0.18 & $344 \pm 38$ \\
\hline $\begin{array}{l}\text { ESF-SD-CIV\#21 } \\
(11.3-13.0)\end{array}$ & SPC00536937 & CL9646 & 0.372 & $297 \pm 61$ & SPC00536920 & CL9664 & 0.32 & $310 \pm 29$ \\
\hline $\begin{array}{l}\text { ESF-SD-CIV\#22 } \\
(4.5-6.3)\end{array}$ & SPC00536934 & CL9645 & 0.165 & $317 \pm 73$ & SPC00536935 & CL9658 & 0.15 & $408 \pm 43$ \\
\hline $\begin{array}{l}\text { ESF-SD-CIV\#26-1 } \\
(3.0-6.3)\end{array}$ & SPC00536949 & CL9650 & 0.105 & $159 \pm 98$ & SPC00536950 & CL9673 & 0.10 & $270 \pm 72$ \\
\hline $\begin{array}{l}\text { ESF-SD-CIV\#26-2 } \\
(3.0-6.3)\end{array}$ & SPC00536952 & CL9651 & 0.114 & $137 \pm 96$ & SPC00536953 & CL9674 & 0.11 & $225 \pm 44$ \\
\hline $\begin{array}{l}\text { ESF-SD-CIV\#27 } \\
(10.0-12.0)\end{array}$ & SPC00536946 & CL9649 & 0.125 & $186 \pm 69$ & SPC00536947 & CL9666 & 0.15 & $208 \pm 29$ \\
\hline $\begin{array}{l}\text { ESF-SD-CIV\#28 } \\
(6.2-8.0)\end{array}$ & SPC00536907 & CL9636 & 0.153 & $163 \pm 129$ & SPC00536908 & CL9660 & 0.16 & $203 \pm 28$ \\
\hline $\begin{array}{l}\text { ESF-SD-CIV\#30 } \\
(6.4-8.4)\end{array}$ & SPC00536922 & CL9641 & 0.136 & $156 \pm 70$ & SPC00536923 & CL9665 & 0.16 & $163 \pm 30$ \\
\hline $\begin{array}{l}\text { ESF-SD-CIV\#32 } \\
(7.6-9.5)\end{array}$ & SPC00536925 & CL9642 & 0.154 & $203 \pm 64$ & SPC00536926 & CL9661 & 0.16 & $235 \pm 29$ \\
\hline $\begin{array}{l}\text { ESF-SD-CIV\#33 } \\
(9.9-11.4)\end{array}$ & SPC00536904 & CL9635 & 0.092 & $339 \pm 123$ & SPC00536905 & CL9667 & 0.11 & $249 \pm 44$ \\
\hline $\begin{array}{l}\text { ESF-SD-CIV\#34 } \\
(2.1-4.8)\end{array}$ & SPC00536931 & CL9644 & 0.079 & $236 \pm 101$ & SPC00536932 & CL9656 & 0.08 & $315 \pm 59$ \\
\hline
\end{tabular}


Table 4-18. Chloride Concentrations and ${ }^{36} \mathrm{Cl} / \mathrm{Cl}$ Ratios in Duplicate Analyses Used to Calculate External Error in ${ }^{36} \mathrm{Cl} / \mathrm{Cl}$ Ratios during Phase III (continued)

\begin{tabular}{|c|c|c|c|c|c|c|c|c|}
\hline \multirow[b]{2}{*}{ Sample Identifier } & \multicolumn{4}{|c|}{ USGS-LLNL-LLNL } & \multicolumn{4}{|c|}{ USGS-LANL-LLNL } \\
\hline & $\begin{array}{l}\text { Barcode Identifier for } \\
\text { LLNL Sample }\end{array}$ & $\begin{array}{l}\text { CAMS } \\
\text { Identifier }\end{array}$ & $\begin{array}{c}\text { Leachate } \mathrm{Cl} \\
\text { Concentration } \\
\text { (mg/kg rock) }\end{array}$ & $\begin{array}{c}{ }^{36} \mathrm{Cl} / \mathrm{Cl} \\
\times 10^{15} \\
(2 \sigma) \\
\end{array}$ & $\begin{array}{c}\text { Barcode } \\
\text { Identifier for } \\
\text { LLNL Sample } \\
\end{array}$ & $\begin{array}{l}\text { CAMS } \\
\text { Identifier }\end{array}$ & $\begin{array}{c}\text { Leachate } \mathrm{Cl} \\
\text { Concentration } \\
\text { (mg/kg rock) }\end{array}$ & $\begin{array}{c}{ }^{36} \mathrm{Cl} / \mathrm{Cl} \\
\times 10^{15} \\
(2 \sigma) \\
\end{array}$ \\
\hline $\begin{array}{l}\text { ESF-SD-CIV\#36 } \\
(5.4-6.7 / 8.1-9.4)\end{array}$ & SPC00536910. & CL9637 & 0.069 & $395 \pm 133$ & SPC00536911 & CL9675 & 0.07 & $322 \pm 66$ \\
\hline
\end{tabular}

DTNs: LL031200223121.036 (Q), Filename: Total_AMS_Summary_2001-02c.xls; LA0305RR831222.001 (UQ)

NOTES: $\quad$ AMS $=$ accelerator mass spectrometer, CAMS $=$ Center for Accelerator Mass Spectrometry, LANL = Los Alamos National Laboratory LLNL = Lawrence Livermore National Laboratory, USGS = U.S. Geological Survey.

All data were generated for aliquots of leachates obtained at the USGS and analyzed at LLNL-CAMS. Silver chloride (AgCl) targets were prepared either at LLNL (first set of columns) or LANL (second set of columns).

Errors listed for the USGS-LLNL-LLNL ${ }^{36} \mathrm{Cl} / \mathrm{Cl}$ data do not include external errors (see Section 4.6.4). 
Table 5-1. Tritium Concentrations in Water Standards with Known Values

\begin{tabular}{|c|c|c|c|c|c|}
\hline Standard Name & $\begin{array}{c}\text { Date Sample Submitted } \\
\text { for Analysis }\end{array}$ & $\begin{array}{c}\text { Volume } \\
\text { Used (mL) }\end{array}$ & $\begin{array}{c}\text { Accepted }{ }^{3} \mathrm{H} \\
\text { Concentration (TU) }\end{array}$ & $\begin{array}{c}\text { Measured }{ }^{3} \mathrm{H} \\
\text { Concentration (TU) }\end{array}$ & $\begin{array}{l}\text { 1o Analytical } \\
\text { Error (TU) }\end{array}$ \\
\hline$D$ & $3 / 6 / 2000$ & 100 & 2.15 & 1.7 & 0.4 \\
\hline \multirow[t]{9}{*}{$H$} & 10/29/1999 & 110 & 1.81 & 2.09 & 0.26 \\
\hline & $10 / 29 / 1999$ & 118 & 1.81 & 2.24 & 0.24 \\
\hline & $4 / 26 / 2000$ & 114 & 1.81 & 1.4 & 0.3 \\
\hline & $4 / 26 / 2000$ & 115 & 1.81 & 1.91 & 0.24 \\
\hline & $5 / 10 / 2002$ & 112 & 1.81 & 1.45 & 0.26 \\
\hline & $8 / 2 / 2002$ & 115 & 1.81 & 1.7 & 0.3 \\
\hline & $8 / 2 / 2002$ & 115 & 1.81 & 1.8 & 0.3 \\
\hline & Average & \multicolumn{4}{|c|}{1.80} \\
\hline & Standard Deviation & \multicolumn{4}{|c|}{0.31} \\
\hline \multirow[t]{6}{*}{$E$} & $3 / 30 / 2000$ & 104 & 1.75 & 1.84 & 0.25 \\
\hline & $6 / 28 / 2000$ & 107 & 1.75 & 2.2 & 0.3 \\
\hline & $7 / 19 / 2000$ & 107 & 1.75 & 1.59 & 0.24 \\
\hline & $9 / 7 / 2000$ & 111 & 1.75 & 1.7 & 0.8 \\
\hline & Average & \multicolumn{4}{|c|}{1.83} \\
\hline & Standard Deviation & \multicolumn{4}{|c|}{0.27} \\
\hline \multirow[t]{8}{*}{ L } & $4 / 17 / 2001$ & 125 & 1.31 & 1.73 & 0.25 \\
\hline & $4 / 10 / 2002$ & 112 & 1.31 & 1.24 & 0.2 \\
\hline & $10 / 29 / 1999$ & 110 & 1.31 & 1.04 & 0.17 \\
\hline & $10 / 29 / 1999$ & 108 & 1.31 & 1.18 & 0.16 \\
\hline & $2 / 7 / 2000$ & 87 & 1.31 & 0.85 & 0.29 \\
\hline & $2 / 7 / 2000$ & 89 & 1.31 & 2.1 & 0.4 \\
\hline & Average & \multicolumn{4}{|c|}{1.36} \\
\hline & Standard Deviation & \multicolumn{4}{|c|}{0.47} \\
\hline \multirow[t]{3}{*}{ Dead Water } & $8 / 2 / 2002$ & 110 & 0 & 0.2 & 0.2 \\
\hline & $8 / 2 / 2002$ & 119 & 0 & -0.1 & 0.3 \\
\hline & Average & \multicolumn{4}{|c|}{0.05} \\
\hline
\end{tabular}

DTNs: GS060308312272.001 (Q) (MOL.20020926.0121), GS060308312272.002 (Q) (MOL.20030331.0364) 
Table 5-2. Tritium Concentrations in Pore Water Extracted from Validation Study Core Samples

\begin{tabular}{|c|c|c|c|c|c|}
\hline SMF Barcode Identifier & Borehole Name & $\begin{array}{c}\text { ESF } \\
\text { Station }\end{array}$ & Feature & $\begin{array}{l}\text { Interval Used } \\
\text { (ft) }\end{array}$ & $\begin{array}{c}{ }^{3} H \\
\text { Concentration } \\
\text { (TU) }(2 \sigma)\end{array}$ \\
\hline $\begin{array}{l}\text { SPC03017174 } \\
\text { SPC03017175 }\end{array}$ & ESF-DHW-CIV\#1 & $19+65$ & $\begin{array}{l}\text { Drill Hole } \\
\text { Wash fault }\end{array}$ & $10.9-13.2^{\mathrm{a}}$ & $1.00 \pm 0.80$ \\
\hline $\begin{array}{l}\text { SPC03017162 } \\
\text { SPC03017163 }\end{array}$ & ESF-DHW-CIV\#2 & $19+55$ & $\begin{array}{l}\text { Drill Hole } \\
\text { Wash fault }\end{array}$ & $6.5-8.2^{a}$ & $0.50 \pm 1.40$ \\
\hline SPC03017171 & ESF-DHW-CIV\#3 & $19+50$ & $\begin{array}{l}\text { Drill Hole } \\
\text { Wash fault }\end{array}$ & $12.0-13.3$ & $1.60 \pm 0.80$ \\
\hline $\begin{array}{l}\text { SPC03017159 } \\
\text { SPC03017160 }\end{array}$ & ESF-DHW-CIV\#4 & $19+45$ & $\begin{array}{l}\text { Drill Hole } \\
\text { Wash fault }\end{array}$ & $12.3-13.7^{b}$ & $0.90 \pm 0.60$ \\
\hline $\begin{array}{l}\text { SPC03017150 } \\
\text { SPC03017151 }\end{array}$ & ESF-DHW-CIV\#5 & $19+40$ & $\begin{array}{l}\text { Drill Hole } \\
\text { Wash fault }\end{array}$ & $26.7-28.7^{a}$ & $0.70 \pm 0.60$ \\
\hline SPC03017180 & ESF-DHW-CIV\#6 & $19+35$ & $\begin{array}{l}\text { Drill Hole } \\
\text { Wash fault }\end{array}$ & $12.2-13.9$ & $0.48 \pm 0.56$ \\
\hline SPC03017184 & ESF-DHW-CIV\#7 & $19+30$ & $\begin{array}{l}\text { Drill Hole } \\
\text { Wash fault }\end{array}$ & $9.6-11.0$ & $1.60 \pm 0.80$ \\
\hline SPC03017190 & ESF-DHW-CIV\#8 & $19+25$ & $\begin{array}{l}\text { Drill Hole } \\
\text { Wash fault }\end{array}$ & $11.7-13.1$ & $0.20 \pm 1.00$ \\
\hline SPC03017198 & ESF-DHW-CIV\#9 & $19+20$ & $\begin{array}{l}\text { Drill Hole } \\
\text { Wash fault }\end{array}$ & $11.5-12.5$ & $0.60 \pm 1.20$ \\
\hline SPC03017194 & ESF-DHW-CIV\#10 & $19+10$ & $\begin{array}{l}\text { Drill Hole } \\
\text { Wash fault }\end{array}$ & $11.2-12.4$ & $0.94 \pm 0.48$ \\
\hline SPC02016331 & ESF-SD-CIV\#1 & $36+89$ & $\begin{array}{l}\text { Sundance } \\
\text { fault }\end{array}$ & $11.5-12.6$ & $0.50 \pm 0.80$ \\
\hline SPC02016281 & ESF-SD-CIV\#2 & $36+74$ & $\begin{array}{l}\text { Sundance } \\
\text { fault }\end{array}$ & $8.0-9.9$ & $0.10 \pm 0.60$ \\
\hline SPC02016289 & ESF-SD-CIV\#3 & $36+59$ & $\begin{array}{l}\text { Sundance } \\
\text { Fault }\end{array}$ & $10.7-11.4$ & $0.60 \pm 0.60$ \\
\hline $\begin{array}{l}\text { SPC02016297 } \\
\text { SPC02016298 }\end{array}$ & ESF-SD-CIV\#4 & $36+35$ & $\begin{array}{l}\text { Sundance } \\
\text { fault }\end{array}$ & $11.8-13.4^{b}$ & $0.30 \pm 0.80$ \\
\hline $\begin{array}{l}\text { SPC02016299 } \\
\text { SPC02016300 }\end{array}$ & ESF-SD-CIV\#5 & $36+20$ & $\begin{array}{l}\text { Sundance } \\
\text { fault }\end{array}$ & $7.9-9.7^{\mathrm{a}}$ & $0.71 \pm 0.46$ \\
\hline SPC02016304 & ESF-SD-CIV\#6 & $36+10$ & $\begin{array}{l}\text { Sundance } \\
\text { fault }\end{array}$ & $9.3-10.5$ & $1.10 \pm 1.00$ \\
\hline SPC02016268 & ESF-SD-CIV\#7. & $36+05$ & $\begin{array}{l}\text { Sundance } \\
\text { fault }\end{array}$ & $8.1-9.7$ & $0.30 \pm 0.80$ \\
\hline $\begin{array}{l}\text { SPC02016271 } \\
\text { SPC02016272 }\end{array}$ & ESF-SD-CIV\#8 & $36+00$ & $\begin{array}{l}\text { Sundance } \\
\text { fault }\end{array}$ & $7.9-9.9^{a}$ & $0.60 \pm 0.60$ \\
\hline SPC02016277 & ESF-SD-CIV\#9 & $35+95$ & $\begin{array}{l}\text { Sundance } \\
\text { fault }\end{array}$ & $10.1-11.5$ & $0.20 \pm 0.60$ \\
\hline SPC02016257 & ESF-SD-CIV\#10 & $35+90$ & $\begin{array}{l}\text { Sundance } \\
\text { fault }\end{array}$ & $11.8-13.0$ & $0.37 \pm 0.58$ \\
\hline $\begin{array}{l}\text { SPC02016260 } \\
\text { SPC02016261 }\end{array}$ & ESF-SD-CIV\#11 & $35+85$ & $\begin{array}{l}\text { Sundance } \\
\text { fault }\end{array}$ & $11.0-12.5^{a, b}$ & $0.15 \pm 0.56$ \\
\hline SPC02016266 & ESF-SD-CIV\#12 & $35+80$ & $\begin{array}{l}\text { Sundance } \\
\text { fault }\end{array}$ & $11.8-13.4^{b}$ & $0.20 \pm 0.54$ \\
\hline $\begin{array}{l}\text { SPC02016252 } \\
\text { SPC02016253 }\end{array}$ & ESF-SD-CIV\#13 & $35+75$ & $\begin{array}{l}\text { Sundance } \\
\text { fault }\end{array}$ & $30.5-32.3^{a, b}$ & $0.60 \pm 0.80$ \\
\hline SPC03017136 & ESF-SD-CIV\#14 & $35+45$ & $\begin{array}{l}\text { Sundance } \\
\text { fault }\end{array}$ & $11.6-13.4$ & $<0.1 \pm 0.30$ \\
\hline SPC03017132 & ESF-SD-CIV\#15 & $35+40$ & $\begin{array}{l}\text { Sundance } \\
\text { fault }\end{array}$ & $12.0-13.5^{b}$ & $0.60 \pm 1.00$ \\
\hline $\begin{array}{l}\text { SPC03017124 } \\
\text { SPC03017125 }\end{array}$ & ESF-SD-CIV\#16 & $35+35$ & $\begin{array}{l}\text { Sundance } \\
\text { fault }\end{array}$ & $12.0-13.2^{a, b}$ & $0.20 \pm 0.60$ \\
\hline
\end{tabular}


Table 5-2. Tritium Concentrations in Pore Water Extracted from Validation Study Core Samples (continued)

\begin{tabular}{|c|c|c|c|c|c|}
\hline SMF Barcode Identifier & Borehole Name & $\begin{array}{c}\text { ESF } \\
\text { Station }\end{array}$ & Feature & $\begin{array}{l}\text { Interval Used } \\
\text { (ft) }\end{array}$ & $\begin{array}{c}{ }^{3} \mathrm{H} \\
\text { concentration } \\
\text { (TU) }(2 \sigma)\end{array}$ \\
\hline SPC03017107 & ESF-SD-CIV\#17 & $35+31$ & $\begin{array}{l}\text { Sundance } \\
\text { fault }\end{array}$ & $10.5-12.0$ & $0.95 \pm 0.52$ \\
\hline SPC03017108 & ESF-SD-CIV\#17 & $35+31$ & $\begin{array}{l}\text { Sundance } \\
\text { fault }\end{array}$ & $12.0-13.2$ & $0.70 \pm 0.80$ \\
\hline SPC03017113 & ESF-SD-CIV\#18 & $35+25$ & $\begin{array}{l}\text { Sundance } \\
\text { fault }\end{array}$ & $10.9-11.8$ & $1.40 \pm 1.60$ \\
\hline SPC03017114 & ESF-SD-CIV\#18 & $35+25$ & $\begin{array}{l}\text { Sundance } \\
\text { fault }\end{array}$ & $12.3-13.5$ & $2.60 \pm 1.00$ \\
\hline SPC03017119 & ESF-SD-CIV\#19 & $35+20$ & $\begin{array}{l}\text { Sundance } \\
\text { fault }\end{array}$ & $11.7-13.1$ & $0.60 \pm 0.80$ \\
\hline $\begin{array}{l}\text { SPC03017101 } \\
\text { SPC03017102 }\end{array}$ & ESF-SD-CIV\#20 & $35+15$ & $\begin{array}{l}\text { Sundance } \\
\text { fault }\end{array}$ & $10.5-13.0^{\mathrm{a}}$ & $<0.1 \pm 0.48$ \\
\hline SPC03017094 & ESF-SD-CIV\#21 & $35+10$ & $\begin{array}{l}\text { Sundance } \\
\text { fault }\end{array}$ & $9.8-11.1$ & $0.40 \pm 0.56$ \\
\hline SPC03017088 & ESF-SD-CIV\#22 & $35+05$. & $\begin{array}{l}\text { Sundance } \\
\text { fault }\end{array}$ & $10.4-11.2^{b}$ & $0.15 \pm 0.54$ \\
\hline SPC03017085 & ESF-SD-CIV\#23 & $35+00$ & $\begin{array}{l}\text { Sundance } \\
\text { fault }\end{array}$ & $12.6-13.7$ & $0.22 \pm 0.58$ \\
\hline SPC03017080 & ESF-SD-CIV\#24 & $34+95$ & $\begin{array}{l}\text { Sundance } \\
\text { fault }\end{array}$ & $12.1-13.4$ & $0.40 \pm 0.60$ \\
\hline SPC02016342 & ESF-SD-CIV\#25 & $34+90$ & $\begin{array}{l}\text { Sundance } \\
\text { fault }\end{array}$ & $8.7-9.9$ & $0.20 \pm 0.80$ \\
\hline SPC02016339 & ESF-SD-CIV\#26 & $34+73$ & $\begin{array}{l}\text { Sundance } \\
\text { fault }\end{array}$ & $12.2-13.2$ & $0.10 \pm 0.80$ \\
\hline SPC02016028 & ESF-SD-ClV\#27 & $34+70$ & $\begin{array}{l}\text { Sundance } \\
\text { fault }\end{array}$ & $12.0-13.4$ & $0.22 \pm 0.34$ \\
\hline $\begin{array}{l}\text { SPC02016018 } \\
\text { SPC02016019 } \\
\text { SPC02016021 } \\
\end{array}$ & ESF-SD-CIV\#28 & $34+65$ & $\begin{array}{l}\text { Sundance } \\
\text { fault }\end{array}$ & $8.0-11.3^{c}$ & $1.14 \pm 0.52$ \\
\hline SPC02015996 & ESF-SD-CIV\#29 & $34+60$ & $\begin{array}{l}\text { Sundance } \\
\text { fault }\end{array}$ & $10.7-12.2^{b}$ & $0.28 \pm 0.34$ \\
\hline SPC02016001 & ESF-SD-CIV\#30 & $34+55$ & $\begin{array}{l}\text { Sundance } \\
\text { fault }\end{array}$ & $12.2-13.4^{b}$ & $0.20 \pm 0.60$ \\
\hline $\begin{array}{l}\text { SPC02016004 } \\
\text { SPC02016005 }\end{array}$ & ESF-SD-CIV\#31 & $34+50$ & $\begin{array}{l}\text { Sundance } \\
\text { fault }\end{array}$ & $11.0-12.6^{a}$ & $0.30 \pm 0.80$ \\
\hline SPC02016010 & ESF-SD-CIV\#32 & $34+45$ & $\begin{array}{l}\text { Sundance } \\
\text { fault }\end{array}$ & $11.6-13.2^{\mathrm{b}}$ & $0.31 \pm 0.46$ \\
\hline SPC02016036 & ESF-SD-CIV\#33 & $34+40$ & $\begin{array}{l}\text { Sundance } \\
\text { fault }\end{array}$ & $7.7-8.9$ & $0.90 \pm 0.60$ \\
\hline SPC02016034 & ESF-SD-CIV\#34 & $34+35$ & $\begin{array}{l}\text { Sundance } \\
\text { fault }\end{array}$ & $10.5-12.0^{b}$ & $0.46 \pm 0.42$ \\
\hline SPC02015951 & ESF-SD-CIV\#35 & $34+30$ & $\begin{array}{l}\text { Sundance } \\
\text { fault }\end{array}$ & $10.0-11.4^{b}$ & $0.29 \pm 0.44$ \\
\hline SPC02015943 & ESF-SD-CIV\#36 & $34+25$ & $\begin{array}{l}\text { Sundance } \\
\text { fault }\end{array}$ & $6.7-8.1$ & $<0.1 \pm 0.36$ \\
\hline SPC02015936 & ESF-SD-CIV\#37 & $34+20$ & $\begin{array}{l}\text { Sundance } \\
\text { fault }\end{array}$ & $9.7-11.2$ & $0.28 \pm 0.26$ \\
\hline SPC02015941 & ESF-SD-CIV\#38 & $34+10$ & $\begin{array}{l}\text { Sundance } \\
\text { fault }\end{array}$ & $11.0-12.5^{b}$ & $1.40 \pm 1.60$ \\
\hline
\end{tabular}


Table 5-2. Tritium Concentrations in Pore Water Extracted from Validation Study Core Samples (continued)

\begin{tabular}{|c|l|c|l|l|r|}
\hline SMF Barcode Identifier & Borehole Name & $\begin{array}{c}\text { ESF } \\
\text { Station }\end{array}$ & Feature & $\begin{array}{c}\text { Interval Used } \\
\text { (ft) }\end{array}$ & $\begin{array}{c}{ }^{3} \mathbf{H} \\
\text { concentration } \\
\text { (TU) (2 } \sigma)\end{array}$ \\
\hline \hline SPC02015932 & ESF-SD-CIV\#39 & $33+99$ & $\begin{array}{l}\text { Sundance } \\
\text { fault }\end{array}$ & $11.2-12.7^{\mathrm{b}}$ & $0.23 \pm 0.28$ \\
\hline SPC02015927 & ESF-SD-CIV\#40 & $33+89$ & $\begin{array}{l}\text { Sundance } \\
\text { fault }\end{array}$ & $12.3-13.3$ & $0.30 \pm 0.32$ \\
\hline
\end{tabular}

DTN: GS060308312272.001(Q)

NOTES: $\quad$ ESF $=$ Exploratory Studies Facility, SMF $=$ Sample Management Facility, $\mathrm{TU}=$ tritium unit.

a Adjacent intervals combined to obtain sufficient sample volume.

b Interval used for tritium analysis is smaller than the interval traceable to the SMF barcode identifier; a portion of the core sample was removed in the laboratory and set aside for other analyses.

c Non-adjacent intervals combined to obtain sufficient sample volume. 
Table 5-3. Tritium Concentrations in Pore Water Extracted from ESF Core Samples

\begin{tabular}{|c|c|c|c|c|c|}
\hline $\begin{array}{l}\text { SMF Barcode } \\
\text { Number }\end{array}$ & Borehole Name & $\begin{array}{c}\text { ESF } \\
\text { Station }\end{array}$ & Feature & $\begin{array}{l}\text { Interval } \\
\text { Used } \\
\text { (ft) }\end{array}$ & \begin{tabular}{|c|}
${ }^{3} H$ \\
Concentration \\
(TU) $(2 \sigma)$
\end{tabular} \\
\hline SPC00046007 & ESF-AL\#2-HPF\#1 & $01+68$ & $\begin{array}{l}\text { Bow Ridge } \\
\text { fault }\end{array}$ & $16.4-16.7^{b}$ & $<0.1$ \\
\hline SPC00046009 & ESF-AL\#2-HPF\#1 & $01+68$ & $\begin{array}{l}\text { Bow Ridge } \\
\text { fault }\end{array}$ & $23.2-23.5^{\mathrm{b}}$ & $2.0 \pm 7.8$ \\
\hline SPC00046012 & ESF-AL\#2-HPF\#1 & $01+68$ & $\begin{array}{l}\text { Bow Ridge } \\
\text { fault }\end{array}$ & $27.8-28.0^{b}$ & $5.1 \pm 7.8$ \\
\hline SPC00046014 & ESF-AL\#2-HPF\#1 & $01+68$ & $\begin{array}{l}\text { Bow Ridge } \\
\text { fault }\end{array}$ & $34.3-34.6^{\mathrm{b}}$ & $28.8 \pm 8.4$ \\
\hline SPC00046017 & ESF-AL\#2-HPF\#1 & $01+68$ & $\begin{array}{l}\text { Bow Ridge } \\
\text { fault }\end{array}$ & $47.2-47.6^{b}$ & $30.9 \pm 8.4$ \\
\hline SPC00046018. & ESF-AL\#2-HPF\#1 & $01+68$ & $\begin{array}{l}\text { Bow Ridge } \\
\text { fault }\end{array}$ & $50.5-50.7$ & $118 \pm 19$ \\
\hline SPC00046019 & ESF-AL\#2-HPF\#1 & $01+68$ & $\begin{array}{l}\text { Bow Ridge } \\
\text { fault }\end{array}$ & $55.4-55.7$ & $128 \pm 10$ \\
\hline SPC00046022 & ESF-AL\#2-HPF\#1 & $01+68$ & $\begin{array}{l}\text { Bow Ridge } \\
\text { fault }\end{array}$ & $58.9-59.0^{\mathrm{b}}$ & $78.6 \pm 9.4$ \\
\hline SPC00046025 & ESF-AL\#2-HPF\#1 & $01+68$ & $\begin{array}{l}\text { Bow Ridge } \\
\text { fault }\end{array}$ & $61.2-61.3^{b}$ & $65.3 \pm 9.2$ \\
\hline SPC00046030 & ESF-AL\#2-HPF\#1 & $01+68$ & $\begin{array}{l}\text { Bow Ridge } \\
\text { fault }\end{array}$ & $68.6-68.9^{b}$ & $155 \pm 11$ \\
\hline SPC00046032 & ESF-AL\#2-HPF\#1 & $01+68$ & $\begin{array}{l}\text { Bow Ridge } \\
\text { fault }\end{array}$ & $83.6-83.8^{b}$ & $32.9 \pm 8.6$ \\
\hline SPC01004381 & $\begin{array}{l}\text { ESF-LPCA- } \\
\text { MOISTSTDY\#2 }\end{array}$ & $10+28$ & North Ramp & $6.4-7.0$ & $<0.1$ \\
\hline SPC01004190 & \begin{tabular}{|l|} 
ESF-NR:- \\
MOISTSTDY\#3 \\
\end{tabular} & $07+68$ & North Ramp & 4.4-5.0 & $0.20 \pm 0.80$ \\
\hline $\begin{array}{l}\text { SPC01004175 } \\
\text { SPC01004179 }\end{array}$ & \begin{tabular}{|l|} 
ESF-NR- \\
MOISTSTDY\#4 \\
\end{tabular} & $07+73$ & North Ramp & $4.2-6.9^{c}$ & $0.76 \pm 0.24$ \\
\hline $\begin{array}{l}\text { SPC01004175 } \\
\text { SPC01004179 }\end{array}$ & $\begin{array}{l}\text { ESF-NR- } \\
\text { MOISTSTDY\#4 }\end{array}$ & $07+73$ & North Ramp & $4.2-6.9^{c}$ & $0.66 \pm 0.20$ \\
\hline $\begin{array}{l}\text { SPC01004240 } \\
\text { SPC01004244 } \\
\end{array}$ & $\begin{array}{l}\text { ESF-NR- } \\
\text { MOISTSTDY\#10 }\end{array}$ & $08+80$ & North Ramp & $4.0-6.5^{b, c}$ & $0.22 \pm 0.30$ \\
\hline SPC01004301 & $\begin{array}{l}\text { ESF-NR- } \\
\text { MOISTSTDY\#13 }\end{array}$ & $10+07$ & North Ramp & $4.3-5.1$ & $0.55 \pm 0.30$ \\
\hline SPC01004340 & \begin{tabular}{|l|} 
ESF-NR- \\
MOISTSTDY\#16 \\
\end{tabular} & $10+70$ & North Ramp & $5.8-6.6$ & $0.44 \pm 0.30$ \\
\hline SPC01001947 & ESF/NAD/GTB\#1A & $37+37$ & $\begin{array}{l}\text { Northern } \\
\text { Ghost Dance } \\
\text { fault }\end{array}$ & $114.0-115.0$ & $0.50 \pm 0.60$ \\
\hline $\begin{array}{l}\text { SPC01001960 } \\
\text { SPC01001962 }\end{array}$ & ESF/NAD/GTB\#1A & $37+37$ & \begin{tabular}{|l|} 
Northern \\
Ghost Dance \\
fault
\end{tabular} & $\begin{array}{l}120.3^{\mathrm{b}} \\
121.6^{\mathrm{c}}\end{array}$ & $1.0 \pm 0.8$ \\
\hline $\begin{array}{l}\text { SPC01001975 } \\
\text { SPC01001976 } \\
\end{array}$ & ESF/NAD/GTB\#1A & $37+37$ & $\begin{array}{l}\text { Northern } \\
\text { Ghost Dance } \\
\text { fault }\end{array}$ & $\begin{array}{l}127.0- \\
129.0^{c}\end{array}$ & $1.6 \pm 1.2$ \\
\hline $\begin{array}{l}\text { SPC01002037 } \\
\text { SPC01002038 }\end{array}$ & ESF/NAD/GTB\#1A & $37+37$ & $\begin{array}{l}\text { Northern } \\
\text { Ghost Dance } \\
\text { fault }\end{array}$ & $\begin{array}{l}165.8- \\
166.7^{a}\end{array}$ & $0.8 \pm 1.0$ \\
\hline $\begin{array}{l}\text { SPC01003300 } \\
\text { SPC01003302 }\end{array}$ & ESF-AL6-NDR-MF\#1 & $37+37$ & $\begin{array}{l}\text { Northern } \\
\text { Ghost Dance } \\
\text { fault }\end{array}$ & $53.9-55.6^{c}$ & $1.3 \pm 1.0$ \\
\hline $\begin{array}{l}\text { SPC01003455 } \\
\text { SPC01003457 }\end{array}$ & ESF-AL6-NDR-MF\#2 & $37+37$ & $\begin{array}{l}\text { Northern } \\
\text { Ghost Dance } \\
\text { fault }\end{array}$ & $42.3-43.9^{c}$ & $1.6 \pm 1.4$ \\
\hline
\end{tabular}


Table 5-3. Tritium Concentrations in Pore Water Extracted from ESF Core Samples (continued)

\begin{tabular}{|c|c|c|c|c|c|}
\hline $\begin{array}{l}\text { SMF Barcode } \\
\text { Identifier }\end{array}$ & Borehole Name & $\begin{array}{c}\text { ESF } \\
\text { Station }\end{array}$ & Feature & $\begin{array}{l}\text { Interval } \\
\text { Used } \\
\text { (ft) }\end{array}$ & $\begin{array}{c}{ }^{3} \mathrm{H} \\
\text { Concentration } \\
\text { (TU) }(2 \sigma)\end{array}$ \\
\hline $\begin{array}{l}\text { SPC01003458 } \\
\text { SPC01003460 }\end{array}$ & $\begin{array}{l}\text { ESF-AL6-NDR- } \\
\text { MF\#02 }\end{array}$ & $37+37$ & $\begin{array}{l}\text { Northern } \\
\text { Ghost Dance } \\
\text { fault }\end{array}$ & $47.3-49.0^{\mathrm{b}, \mathrm{c}}$ & $1.2 \pm 0.4$ \\
\hline $\begin{array}{l}\text { SPC01003462 } \\
\text { SPC01003464 }\end{array}$ & $\begin{array}{l}\text { ESF-AL6-NDR- } \\
\text { MF\#02 }\end{array}$ & $37+37$ & $\begin{array}{l}\text { Northern } \\
\text { Ghost Dance } \\
\text { fault }\end{array}$ & $49.3-51.3^{c}$ & $1.1 \pm 1.0$ \\
\hline $\begin{array}{l}\text { SPC01003468 } \\
\text { SPC01003470 }\end{array}$ & $\begin{array}{l}\text { ESF-AL6-NDR- } \\
\text { MF\#02 }\end{array}$ & $37+37$ & $\begin{array}{l}\text { Northern } \\
\text { Ghost Dance } \\
\text { fault }\end{array}$ & $55.3-57.0^{\mathrm{C}}$ & $1.0 \pm 1.2$ \\
\hline $\begin{array}{l}\text { SPC01003478 } \\
\text { SPC01003480 }\end{array}$ & $\begin{array}{l}\text { ESF-AL6-NDR- } \\
\text { MF\#02 }\end{array}$ & $37+37$ & $\begin{array}{l}\text { Northern } \\
\text { Ghost Dance } \\
\text { fault }\end{array}$ & $61.1-62.9^{c}$ & $0.9 \pm 1.4$ \\
\hline $\begin{array}{l}\text { SPC01001916 } \\
\text { SPC01001918 } \\
\text { SPC01001920 } \\
\end{array}$ & $\begin{array}{l}\text { ESF-NAD- } \\
\text { GTB\#1A }\end{array}$ & $37+37$ & \begin{tabular}{|l|} 
Northern \\
Ghost Dance \\
fault
\end{tabular} & $98.4-101.0^{\mathrm{C}}$ & $1.4 \pm 0.8$ \\
\hline $\begin{array}{l}\text { SPC01001964 } \\
\text { SPC01001966 }\end{array}$ & $\begin{array}{l}\text { ESF-NAD- } \\
\text { GTB\#1A }\end{array}$ & $37+37$ & \begin{tabular}{|l|}
$\begin{array}{l}\text { Northern } \\
\text { Ghost Dance } \\
\text { fault }\end{array}$ \\
\end{tabular} & $122.1-123.8^{c}$ & $1.2 \pm 0.8$ \\
\hline $\begin{array}{l}\text { SPC01001968 } \\
\text { SPC01001970 } \\
\text { SPC01001971 } \\
\end{array}$ & $\begin{array}{l}\text { ESF-NAD- } \\
\text { GTB\#1A }\end{array}$ & $37+37$ & $\begin{array}{l}\begin{array}{l}\text { Northern } \\
\text { Ghost Dance } \\
\text { fault }\end{array} \\
\end{array}$ & $124.4-126.0^{c}$ & $1.2 \pm 0.8$ \\
\hline $\begin{array}{l}\text { SPC01001980 } \\
\text { SPC01001982 }\end{array}$ & $\begin{array}{l}\text { ESF-NAD- } \\
\text { GTB\#1A }\end{array}$ & $37+37$ & \begin{tabular}{|l|} 
Northern \\
Ghost Dance \\
fault
\end{tabular} & $130.2-131.9^{c}$ & $0.8 \pm 1.4$ \\
\hline $\begin{array}{l}\text { SPC01001991 } \\
\text { SPC01001993 } \\
\text { SPC01001995 } \\
\text { SPC01001998 } \\
\end{array}$ & $\begin{array}{l}\text { ESF-NAD- } \\
\text { GTB\#1A }\end{array}$ & $37+37$ & $\begin{array}{l}\text { Northern } \\
\text { Ghost Dance } \\
\text { fault }\end{array}$ & $137.0-142.0^{c}$ & $0.3 \pm 0.8$ \\
\hline $\begin{array}{l}\text { SPC01002042 } \\
\text { SPC01002045 }\end{array}$ & $\begin{array}{l}\text { ESF-NAD- } \\
\text { GTB\#1A }\end{array}$ & $37+37$ & $\begin{array}{l}\text { Northern } \\
\text { Ghost Dance } \\
\text { fault }\end{array}$ & $168.0-169.8^{c}$ & $0.8 \pm 1.0$ \\
\hline $\begin{array}{l}\text { SPC01003284 } \\
\text { SPC01003286 }\end{array}$ & ESF-NDR-MF\#1 & $37+37$ & $\begin{array}{l}\text { Northern } \\
\text { Ghost Dance } \\
\text { fault }\end{array}$ & $44 \cdot 2-46.0^{b, c}$ & $1.6 \pm 1.0$ \\
\hline $\begin{array}{l}\text { SPC01003292 } \\
\text { SPC01003294 } \\
\text { SPC01003296 } \\
\end{array}$ & ESF-NDR-MF\#1 & $37+37$ & $\begin{array}{l}\text { Northern } \\
\text { Ghost Dance } \\
\text { fault }\end{array}$ & $48.9-50.9^{b, c}$ & $2.2 \pm 1.2$ \\
\hline SPC01002776 & ESF/SAD/GTB\#1 & $50+64$ & $\begin{array}{l}\text { Southern } \\
\text { Ghost Dance } \\
\text { fault }\end{array}$ & $103.4-104.1$ & $3.7 \pm 1.4$ \\
\hline $\begin{array}{l}\text { SPC01002800 } \\
\text { SPC01002802 }\end{array}$ & ESF/SAD/GTB\#1 & $50+64$ & $\begin{array}{l}\text { Southern } \\
\text { Ghost Dance } \\
\text { fault }\end{array}$ & $\begin{array}{l}124.3^{-} \\
125.9^{c}\end{array}$ & $1.1 \pm 0.6$ \\
\hline $\begin{array}{l}\text { SPC01002879 } \\
\text { SPC01002897 }\end{array}$ & ESF/SAD/GTB\#1 & $50+64$ & $\begin{array}{l}\text { Southern } \\
\text { Ghost Dance } \\
\text { fault }\end{array}$ & $\begin{array}{l}175.4- \\
177.0^{c}\end{array}$ & $1.8 \pm 1.4$ \\
\hline $\begin{array}{l}\text { SPC01002956 } \\
\text { SPC01002958 }\end{array}$ & ESF/SAD/GTB\#1 & $50+64$ & $\begin{array}{l}\text { Southern } \\
\text { Ghost Dance } \\
\text { fault }\end{array}$ & $\begin{array}{l}214.5- \\
216.9^{c} \\
\end{array}$ & $2.3 \pm 0.6$ \\
\hline SPC01002754 & ESF/SAD/GTB\#1 & $50+64$ & $\begin{array}{l}\text { Southern } \\
\text { Ghost Dance } \\
\text { fault }\end{array}$ & $85.1-86.0$ & $1.2 \pm 1.0$ \\
\hline $\begin{array}{l}\text { SPC01004630 } \\
\text { SPC01004634 } \\
\end{array}$ & $\begin{array}{l}\text { ESF-SR- } \\
\text { MOISTSTDY\#3 }\end{array}$ & $59+65$ & South Ramp & $2.9-5.7^{c}$ & $1.7 \pm 0.8$ \\
\hline
\end{tabular}


Table 5-3. Tritium Concentrations in Pore Water Extracted from ESF Core Samples (continued)

\begin{tabular}{|c|c|c|c|c|c|}
\hline $\begin{array}{l}\text { SMF Barcode } \\
\text { Identifier }\end{array}$ & Borehole Name & $\begin{array}{c}\text { ESF } \\
\text { Station }\end{array}$ & Feature & $\begin{array}{l}\text { Interval } \\
\text { Used } \\
\text { (ft) }\end{array}$ & $\begin{array}{c}{ }^{3} \mathrm{H} \\
\text { Concentration } \\
\text { (TU) }(2 \sigma)\end{array}$ \\
\hline $\begin{array}{l}\text { SPC01004661 } \\
\text { SPC01004665 }\end{array}$ & \begin{tabular}{|l|} 
ESF-SR- \\
MOISTSTDY\#5 \\
\end{tabular} & $63+00$ & South Ramp & $3.6-6.5^{b, c}$ & $0.42 \pm 0.3$ \\
\hline $\begin{array}{l}\text { SPC01004672 } \\
\text { SPC01004676 }\end{array}$ & \begin{tabular}{|l} 
ESF-SR- \\
MOISTSTDY\#6
\end{tabular} & $63+89$ & South Ramp & $2.6-7.0^{c}$ & $0.81 \pm 0.28$ \\
\hline $\begin{array}{l}\text { SPC01004686 } \\
\text { SPC01004690 } \\
\end{array}$ & $\begin{array}{l}\text { ESF-SR- } \\
\text { MOISTSTDY\#7 }\end{array}$ & $64+80$ & South Ramp & $3.8-7.0^{c}$ & $3.2 \pm 0.4$ \\
\hline $\begin{array}{l}\text { SPC01004726 } \\
\text { SPC01004728 } \\
\end{array}$ & $\begin{array}{l}\text { ESF-SR- } \\
\text { MOISTSTDY\#10 }\end{array}$ & $66+48$ & South Ramp & $2.4-6.4^{c}$ & $28.6 \pm 3.6$ \\
\hline $\begin{array}{l}\text { SPC01004759 } \\
\text { SPC01004763 }\end{array}$ & $\begin{array}{l}\text { ESF-SR- } \\
\text { MOISTSTDY\#11 }\end{array}$ & $66+58$ & South Ramp & $3.2-6.9^{c}$ & $4.8 \pm 0.8$ \\
\hline SPC01004805 & \begin{tabular}{|l|} 
ESF-SR- \\
MOISTSTDY\#13
\end{tabular} & $66+80$ & South Ramp & $6.0-6.8$ & $3.1 \pm 0.5$ \\
\hline $\begin{array}{l}\text { SPC01004786 } \\
\text { SPC01004790 } \\
\end{array}$ & \begin{tabular}{|l|} 
ESF-SR- \\
MOISTSTDY\#16
\end{tabular} & $67+21$ & South Ramp & $4.6-6.8^{b, c}$ & $8.2 \pm 1.0$ \\
\hline $\begin{array}{l}\text { SPC01002407 } \\
\text { SPC01002409 } \\
\end{array}$ & $\begin{array}{l}\text { ESF-SR- } \\
\text { MOISTSTDY\#1 }\end{array}$ & $67+22$ & South Ramp & $2.1 \div 3.6^{c}$ & $0.3 \pm 0.3$ \\
\hline $\begin{array}{l}\text { SPC01002421 } \\
\text { SPC01002423 } \\
\end{array}$ & \begin{tabular}{|l} 
ESF-SR- \\
MOISTSTDY\#2
\end{tabular} & $67+20$ & South Ramp & $2.2-3.9^{c}$ & $0.03 \pm 0.2$ \\
\hline SPC01004821 & $\begin{array}{l}\text { ESF-SR- } \\
\text { MOISTSTDY\#17 }\end{array}$ & $67+30$ & South Ramp & $5.8-6.7$ & $3.8 \pm 0.6$ \\
\hline SPC01004821 & $\begin{array}{l}\text { ESF-SR- } \\
\text { MOISTSTDY\#17 } \\
\end{array}$ & $67+30$ & South Ramp & $5.8-6.7$ & $3.5 \pm 1.0$ \\
\hline $\begin{array}{l}\text { SPC01004831 } \\
\text { SPC01004835 }\end{array}$ & $\begin{array}{l}\text { ESF-SR- } \\
\text { MOISTSTDY\#18 }\end{array}$ & $67+48$ & South Ramp & $4.6-6.7^{c}$ & $1.1 \pm 0.8$ \\
\hline $\begin{array}{l}\text { SPC01004844 } \\
\text { SPC01004848 }\end{array}$ & $\begin{array}{l}\text { ESF-SR- } \\
\text { MOISTSTDY\#19 }\end{array}$ & $68+26$ & South Ramp & $4.5-6.9^{c}$ & $14.3 \pm 2.0$ \\
\hline $\begin{array}{l}\text { SPC01004858 } \\
\text { SPC01004862 } \\
\end{array}$ & \begin{tabular}{|l|} 
ESF-SR- \\
MOISTSTDY\#20
\end{tabular} & $69+37$ & South Ramp & $4.2-6.8^{c}$ & $7.4 \pm 0.8$ \\
\hline SPC01005233 & $\begin{array}{l}\text { ESF-SR- } \\
\text { MOISTSTDY\#23 }\end{array}$ & $70+59$ & South Ramp & $16.2-17.0$ & $0.45 \pm 0.30$ \\
\hline SPC01005233 & $\begin{array}{l}\text { ESF-SR- } \\
\text { MOISTSTDY\#23 }\end{array}$ & $70+59$ & South Ramp & $16.2-17.0$ & $0.25 \pm 0.32$ \\
\hline $\begin{array}{l}\text { SPC01004967 } \\
\text { SPC01004970 }\end{array}$ & \begin{tabular}{|l|} 
ESF-SR- \\
MOISTSTDY\#25
\end{tabular} & $74+35$ & South Ramp & $5.0-6.9^{c}$ & $4.4 \pm 0.8$ \\
\hline $\begin{array}{l}\text { SPC01005175 } \\
\text { SPC01005179 }\end{array}$ & \begin{tabular}{|l|} 
ESF-SR- \\
MOISTSTDY\#26 \\
\end{tabular} & $74+41$ & South Ramp & $7.4-9.6^{c}$ & $4.9 \pm 0.5$ \\
\hline SPC01004921 & $\begin{array}{l}\text { ESF-SR- } \\
\text { MOISTSTDY\#27 }\end{array}$ & $74+44$ & South Ramp & $5.9-6.8$ & $1.5 \pm 0.8$ \\
\hline $\begin{array}{l}\text { SPC01004930 } \\
\text { SPC01004936 }\end{array}$ & $\begin{array}{l}\text { ESF-SR- } \\
\text { MOISTSTDY\#28 }\end{array}$ & $74+47$ & South Ramp & $2.5-6.8^{c}$ & $3.2 \pm 0.8$ \\
\hline $\begin{array}{l}\text { SPC01004949 } \\
\text { SPC01004953 } \\
\end{array}$ & $\begin{array}{l}\text { ESF-SR- } \\
\text { MOISTSTDY\#29 }\end{array}$ & $74+54$ & South Ramp & $4.5-6.8^{c}$ & $0.77 \pm 0.46$ \\
\hline $\begin{array}{l}\text { SPC01005033 } \\
\text { SPC01005037 }\end{array}$ & $\begin{array}{l}\text { ESF-SR- } \\
\text { MOISTSTDY\#30 }\end{array}$ & $74+60$ & South Ramp & $3.8-6.7^{\mathrm{c}}$ & $12.5 \pm 1.2$ \\
\hline $\begin{array}{l}\text { SPC01004981 } \\
\text { SPC01004985 }\end{array}$ & $\begin{array}{l}\text { ESF-SR- } \\
\text { MOISTSTDY\#31 }\end{array}$ & $74+66$ & South Ramp & $4.7-7.0^{\mathrm{c}}$ & $5.4 \pm 0.6$ \\
\hline SPC01005054 & $\begin{array}{l}\text { ESF-SR- } \\
\text { MOISTSTDY\#33 }\end{array}$ & $74+77$ & South Ramp & $5.9-6.9$ & $2.7 \pm 0.6$ \\
\hline SPC01005012 & $\begin{array}{l}\text { ESF-SR- } \\
\text { MOISTSTDY\#34 }\end{array}$ & $74+82$ & South Ramp & $5.9-6.8$ & $1.2 \pm 0.5$ \\
\hline
\end{tabular}


Table 5-3. Tritium Concentrations in Pore Water Extracted from ESF Core Samples (continued)

\begin{tabular}{|c|l|c|c|c|c|}
\hline $\begin{array}{c}\text { SMF Barcode } \\
\text { Identifier }\end{array}$ & Borehole Name & $\begin{array}{c}\text { ESF } \\
\text { Station }\end{array}$ & Feature & $\begin{array}{c}\text { Interval } \\
\text { Used } \\
\text { (ft) }\end{array}$ & $\begin{array}{c}{ }^{\mathbf{3}} \mathbf{H} \\
\text { Concentration } \\
\text { (TU) (2 } \sigma \text { ) }\end{array}$ \\
\hline \hline SPC01005099 & $\begin{array}{l}\text { ESF-SR- } \\
\text { MOISTSTDY\#38 }\end{array}$ & $75+03$ & South Ramp & $5.9-6.8$ & $1.7 \pm 0.6$ \\
\hline SPC01005113 & $\begin{array}{l}\text { ESF-SR- } \\
\text { MOISTSTDY\#40 }\end{array}$ & $75+10$ & South Ramp & $5.9-6.9$ & $0.58 \pm 0.32$ \\
\hline
\end{tabular}

DTNs: GS060308312272.001 (Q), GS040108312232.001 (Q), GS961108312261.006 (Q), GS060383122410.001 (UQ)

NOTES: ESF = Exploratory Studies Facility, SMF = Sample Management Facility; $T U$ = tritium unit.

a Adjacent intervals combined to obtain sufficient sample volume.

b Interval used for tritium analysis is smaller than the interval traceable to the SMF barcode identifier; a portion of the core sample was removed in the laboratory and set aside for other analyses. Non-adjacent intervals combined to obtain sufficient sample volume. 
Table 5-4. Tritium Concentrations in Pore Water Extracted from ECRB Cross Drift Core Samples

\begin{tabular}{|c|c|c|c|c|c|}
\hline $\begin{array}{l}\text { SMF Barcode } \\
\text { Identifier }\end{array}$ & Borehole Name & $\begin{array}{c}\text { ECRB } \\
\text { Station }\end{array}$ & $\begin{array}{c}\text { Interval Used } \\
\text { (ft) }\end{array}$ & $\begin{array}{l}\text { Volume of } \\
\text { Water } \\
\text { Extracted } \\
(\mathrm{mL})\end{array}$ & $\begin{array}{l}{ }^{3} H \text { Concentration } \\
\text { (TU) }(2 \sigma)\end{array}$ \\
\hline $\begin{array}{l}\text { SPC02013439 } \\
\text { SPC02013442 }\end{array}$ & ECRB-SYS-CS0600 & $06+01$ & $3.2-6.0^{a}$ & 120 & $0.79 \pm 0.58$ \\
\hline $\begin{array}{l}\text { SPC02013547 } \\
\text { SPC02013543 } \\
\end{array}$ & ECRB-SYS-CS0750 & $07+50$ & $3.6-6.2^{a}$ & 100.4 & $6.2 \pm 1.0$ \\
\hline $\begin{array}{l}\text { SPC02013530 } \\
\text { SPC02013534 }\end{array}$ & ECRB-SYS-CS0800 & $08+00$ & $2.9-5.8^{\mathrm{a}}$ & 80.4 & $1.7 \pm 0.6$ \\
\hline $\begin{array}{l}\text { SPC02013613 } \\
\text { SPC02013617 }\end{array}$ & ECRB-SYS-CS0900 & $09+01$ & $3.5-6.4^{a}$ & 78.8 & $6.5 \pm 1.2$ \\
\hline $\begin{array}{l}\text { SPC02013628 } \\
\text { SPC02013624 } \\
\end{array}$ & ECRB-SYS-CS0950 & $09+50$ & $2.8-5.6^{a}$ & 64.4 & $6.1 \pm 0.8$ \\
\hline SPC02013695 & ECRB-SYS-CS1000 & $10+00$ & $17.4-18.2$ & 80.8 & $0.5 \pm 0.6$ \\
\hline $\begin{array}{l}\text { SPC02014326 } \\
\text { SPC02014330 } \\
\text { SPC02014334 }\end{array}$ & ECRB-SYS-CS1200 & $11+99$ & $2.9-6.9^{\mathrm{a}}$ & 109 & $0.41 \pm 0.46$ \\
\hline $\begin{array}{l}\text { SPC02014285 } \\
\text { SPC02014289 }\end{array}$ & ECRB-SYS-CS1300 & $13+01$ & $3.0-5.5^{a}$ & 50 & $0.7 \pm 1.4$ \\
\hline $\begin{array}{l}\text { SPC02014299 } \\
\text { SPC02014303 }\end{array}$ & ECRB-SYS-CS1350 & $13+51$ & $3.6-6.4^{a}$ & 82.6 & $3.80 \pm 1.00$ \\
\hline $\begin{array}{l}\text { SPC02014349 } \\
\text { SPC02014353 }\end{array}$ & ECRB-SYS-CS1450 & $14+50$ & $4.0-6.5^{a}$ & 56 & $0.3 \pm 1.0$ \\
\hline $\begin{array}{l}\text { SPC02014381 } \\
\text { SPC02014385 }\end{array}$ & ECRB-SYS-CS1500 & $14+99$ & $14.4-17.4^{a}$ & 79.4 & $2.5 \pm 0.8$ \\
\hline $\begin{array}{l}\text { SPC02014361 } \\
\text { SPC02014365 }\end{array}$ & ECRB-SYS-CS1500 & $14+99$ & $4.3-7.1^{a}$ & 98 & $10.3 \pm 1.8$ \\
\hline $\begin{array}{l}\text { SPC02014371 } \\
\text { SPC02014375 } \\
\end{array}$ & ECRB-SYS-CS1500 & $14+99$ & $9.5-12.1^{a}$ & 51 & $1.5 \pm 0.8$ \\
\hline SPC02014406 & ECRB-SYS-CS1600 & $16+00$ & $3.4-4.3$ & 54 & $1.7 \pm 1.8$ \\
\hline $\begin{array}{l}\text { SPC02014436 } \\
\text { SPC02014440 }\end{array}$ & ECRB-SYS-CS1750 & $17+50$ & $3.3-5.9^{a}$ & 78.3 & $0.6 \pm 0.8$ \\
\hline $\begin{array}{l}\text { SPC02014450 } \\
\text { SPC02014454 }\end{array}$ & ECRB-SYS-CS1800 & $18+01$ & $3.6-6.1^{\mathrm{a}}$ & 51 & $0.1 \pm 1.6$ \\
\hline $\begin{array}{l}\text { SPC02014486 } \\
\text { SPC02014490 } \\
\end{array}$ & ECRB-SYS-CS1950 & $19+50$ & $4.0-6.5^{a}$ & 104 & $3.6 \pm 1.0$ \\
\hline SPC02014623 & ECRB-SYS-CS2000 & $19+99$ & $11.0-11.9$ & 63.7 & $0.1 \pm 1.0$ \\
\hline SPC02014661 & ECRB-SYS-CS2150 & $21+49$ & $3.4-4.1$ & 62 & $<0.1$ \\
\hline SP.C02014665 & ECRB-SYS-CS2150 & $21+49$ & $5.5-6.7$ & 67.7 & $9.8 \pm 1.0$ \\
\hline SPC02014683 & ECRB-SYS-CS2250 & $22+50$ & $2.9-3.9$ & 65 & $0.8 \pm 0.8$ \\
\hline $\begin{array}{l}\text { SPC02014774 } \\
\text { SPC02014778 }\end{array}$ & ECRB-SYS-CS2500 & $25+00$ & $16.7-19.8^{a}$ & 72.4 & $0.64 \pm 0.6$ \\
\hline
\end{tabular}

DTN: GS060308312272.002 (Q)

NOTES: $E C R B=$ Enhanced Characterization of the Repository Block, SMF = Sample Management Facility,

$\mathrm{TU}=$ tritium unit.

a Adjacent intervals combined to obtain sufficient sample volume. 